\title{
Experimental Investigation of Compressive Failure of Truncated Conical Ice Specimens
}

\author{
By \\ (C) Kashfi Binte Habib \\ A thesis submitted to the \\ School of Graduate Studies \\ in partial fulfillment of the requirements for the degree of \\ Master of Engineering \\ Faculty of Engineering and Applied Science \\ Memorial University of Newfoundland
}

May, 2014

St. John's

Newfoundland 


\section{Abstract}

In total, twenty-eight (28) small-scale ice indentation tests have been carried out to study the compressive failure of polycrystalline ice during indentation and to explore the link between various parameters that influence the ice failure processes, using ice specimens having a truncated conical geometry. Taper angle, temperature, indentation rate, indenter shape and grain size are considered as controlled variables in this research program. For the experiments, three geometric configurations (with taper angles of $13^{\circ}, 21^{\circ}, 30^{\circ}$ ) have been used, conducted at temperatures of $-10^{\circ} \mathrm{C}$ and $-5^{\circ} \mathrm{C}$. Indentation rates of $0.1 \mathrm{~mm} / \mathrm{s}, 1$ $\mathrm{mm} / \mathrm{s}$ and $10 \mathrm{~mm} / \mathrm{s}$ have been considered using two indenter shapes (a flat plate and a spherical indenter). Two grain size ranges were considered for these tests. The total force and pressure were found to show dependencies on the indentation rate. The force becomes higher and failure process changes from brittle to ductile as indentation rate decreases. For example, in case of the $21^{\circ}$ taper angle ice sample, maximum ice loads were $20 \mathrm{kN}$ and $145 \mathrm{kN}$ and peak pressures were $8 \mathrm{MPa}$ and $18 \mathrm{MPa}$ for indentation speeds of $10 \mathrm{~mm} / \mathrm{s}$ and $0.1 \mathrm{~mm} / \mathrm{s}$ respectively. The total force also depends on the taper angle of ice sample. The loads increase as the ice samples become flatter. So, the $13^{\circ}$ ice sample was stronger than the $30^{\circ}$ ice sample. Different shaped indenters also observed to have distinct experimental outputs. Tests that were done using the spherical indenter show lower forces than the tests that were done using the flat indenter. Effects of temperature reveal that the warm tests show a greater tendency to ductile failure than cold 
tests having same parameters. The ice samples with smaller ice seeds need more force to fail compared to ice samples with bigger ice seeds. To observe the microstructural modification, horizontal and vertical thin-sections of the damaged ice adjacent to the indenter have been examined. Ice particles were collected from the testing area following each experiment to observe the influence of different factors on the particle size distributions. The effect of each variable on observed failure processes and associated loads are presented in the thesis. 


\section{Acknowledgement}

I would first like to express my sincere gratitude to my supervisors Dr. Rocky Taylor, Dr. Ian Jordaan and Dr. Stephen Bruneau for their support, guidance and instruction throughout the research program. I am truly grateful for their skilled advice in the areas of ice mechanics, compressive failure, experimentation, data analysis and writing the thesis. I would like to show the deepest appreciation to C-CORE and all the employees for providing an excellent place and environment to pursue my Master's degree. I would also like to thank Mr. Andrew Macneill of C-CORE for his time to design the spherical indenter, Mr. Craig Mitchell of Memorial University of Newfoundland for his assistance and guidance to execute the laboratory work and also Mr. Andrew Manuel, Mr. Sander Dragt and Mr. Brian O’Rourke for their assistance during laboratory testing.

A special thanks to Research and Development Corporation of Newfoundland and Labrador (RDC), The C-CORE-Centre for Arctic Resource Development (CARD) and Memorial University of Newfoundland for the financial supports provided to complete the research.

I would like to thank STePS ${ }^{2}$ research group and Memorial University of Newfoundland for allowing me to use the instrumentations, apparatus and the facilities of the cold room.

I would like to thank my parents Muhammad Habibur Rahman and Jahida Khatoon for all the hard work they did and providing unconditional support throughout my life. 
Everything I have accomplished today is because of them. Special thanks to my husband Muntaser Rahman for the assistance and encouragement he provides in all that I do. This would not have been possible without his inspiration and support. I also thank my family, in-laws and friends for keeping their faith on me. 


\section{Table of Contents}

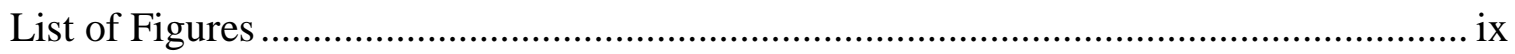

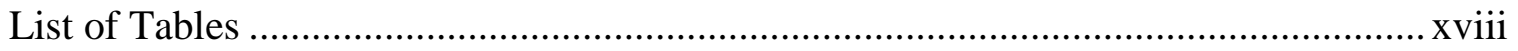

List of Abbreviations and Symbols............................................................................ xix

Chapter 1: Introduction ............................................................................................. 1

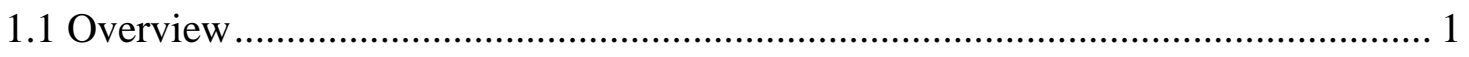

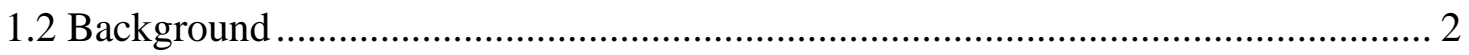

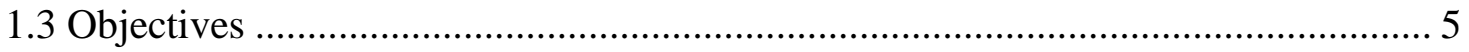

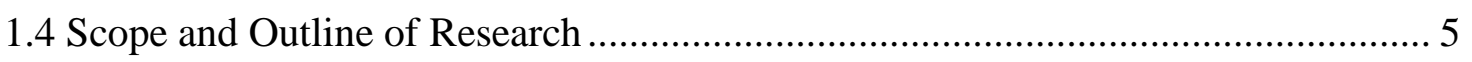

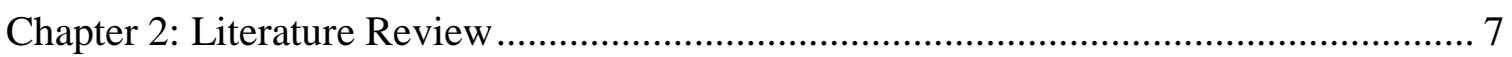

2.1 Ice Mechanics ………………………………….......................................... 7

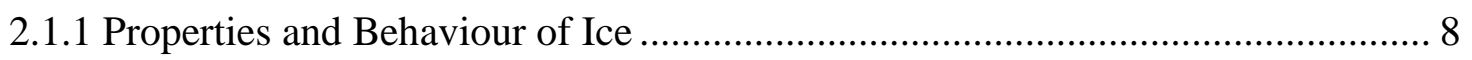

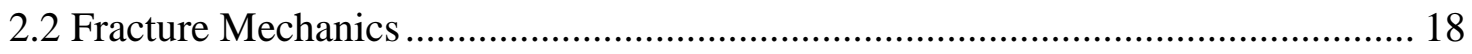

2.2.1 Linear Elastic Fracture Mechanics .................................................................. 19

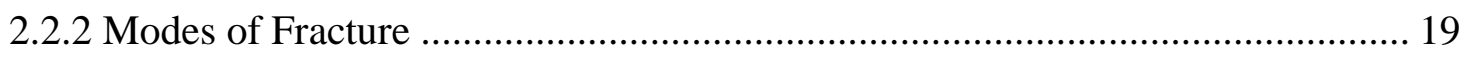

2.2.3 Energy-Release Rate ..................................................................................... 21

2.2.4 Critical Energy Release Rate...................................................................... 23

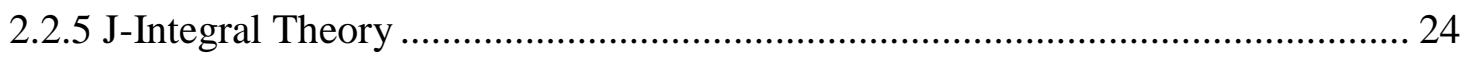

2.3 Compressive Ice Failure ……………………........................................... 25

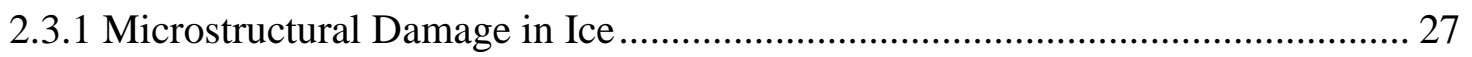

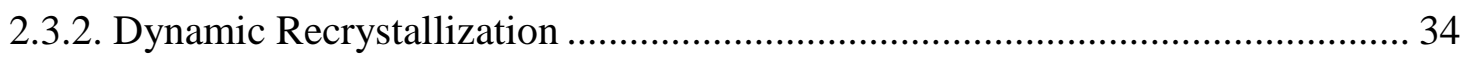

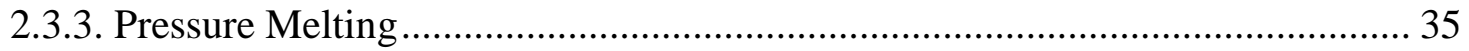




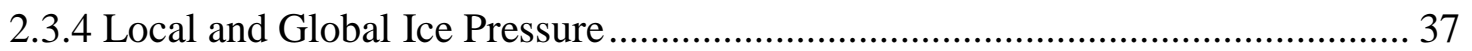

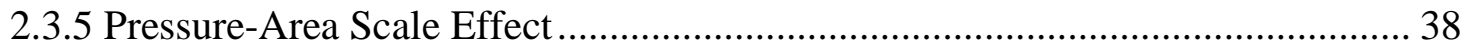

2.4 Review of Previous Indentation Test Programs...................................................... 40

Chapter 3: Experimental Methodology ……………………................................... 52

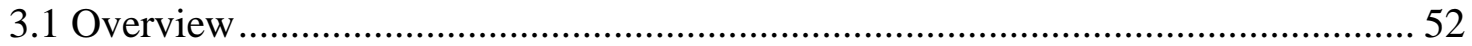

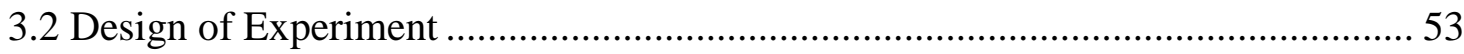

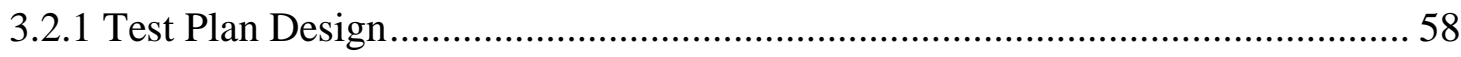

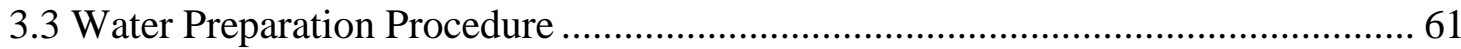

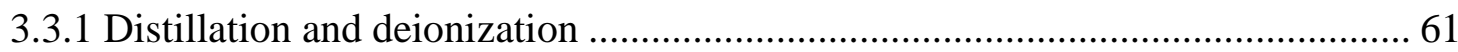

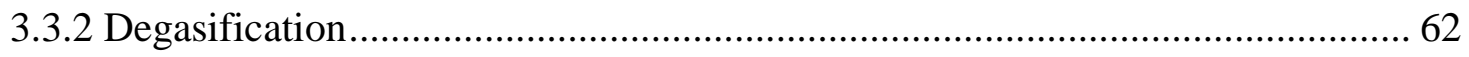

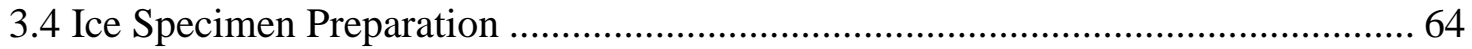

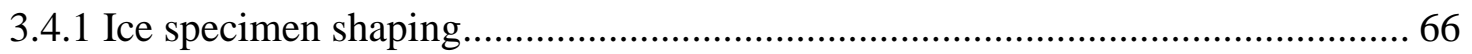

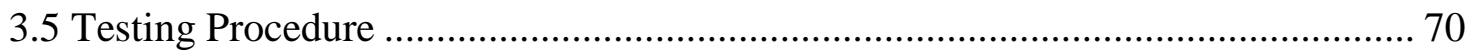

3.6 Spall Collection and Weighing ........................................................................ 72

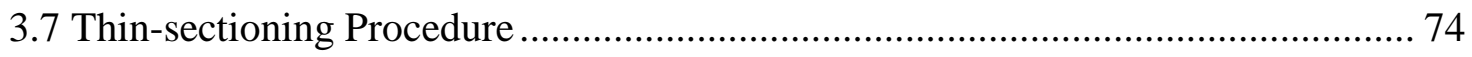

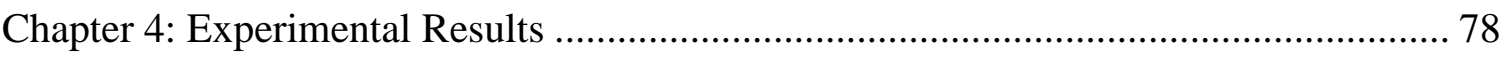

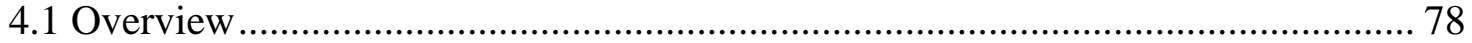

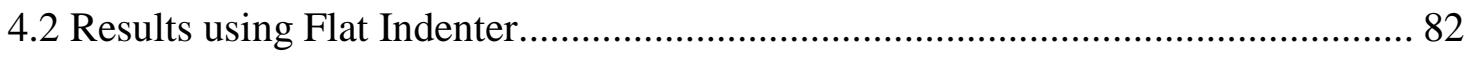

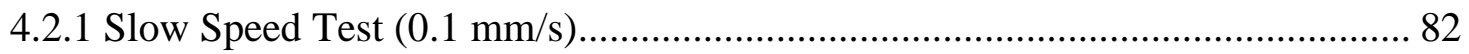

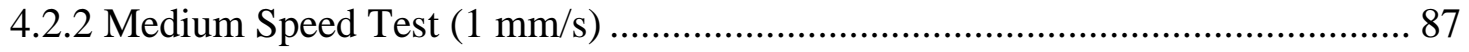

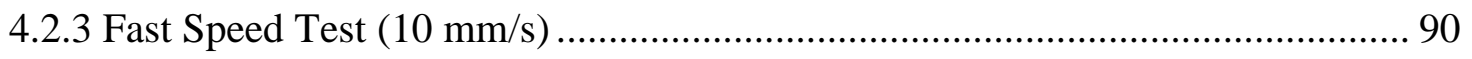

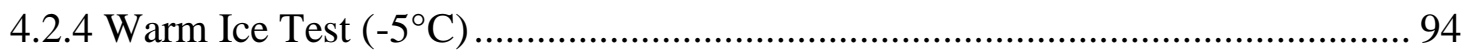

4.3 Result Using Spherical Indenter ........................................................................ 98

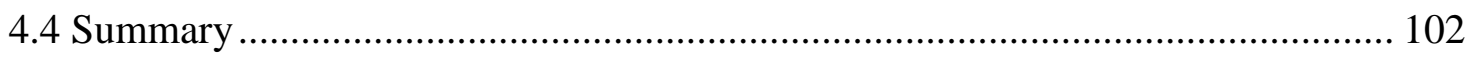

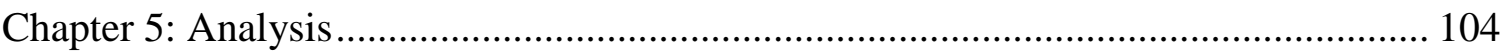

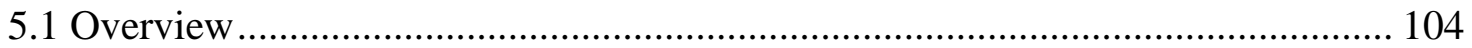

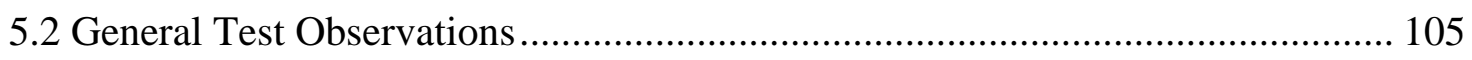

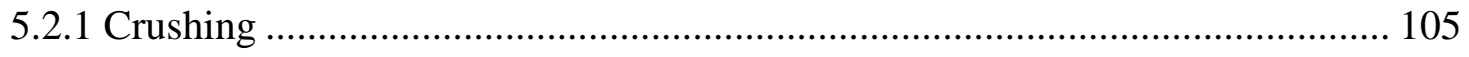




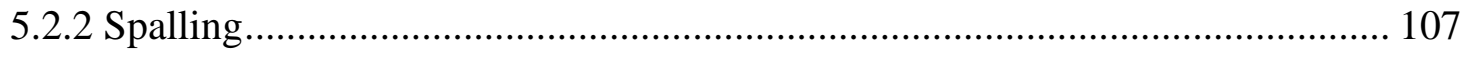

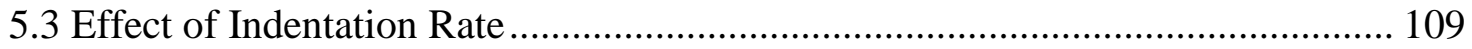

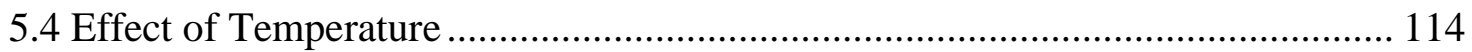

5.5 Effect of Indenter Shape ………................................................................ 118

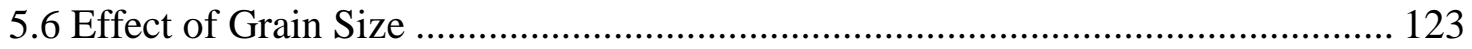

5.7 Effect of the Taper Angle of Ice Sample ....................................................... 128

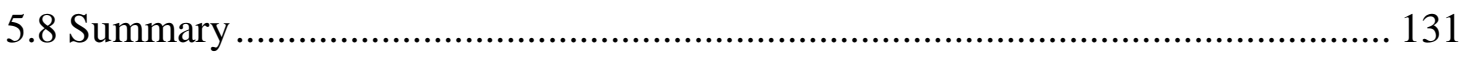

Chapter 6: Discussion and Conclusion ........................................................................ 133

6.1 Summary and Conclusion ................................................................................. 133

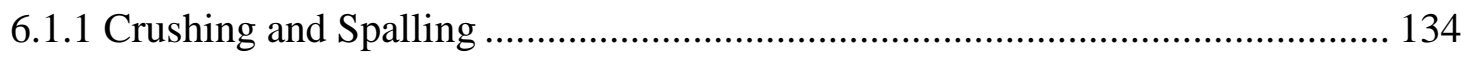

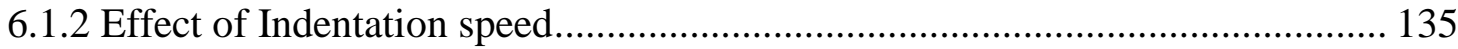

6.1.3 Effect of Temperature …………............................................................... 136

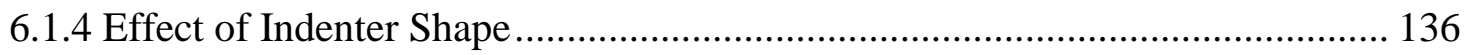

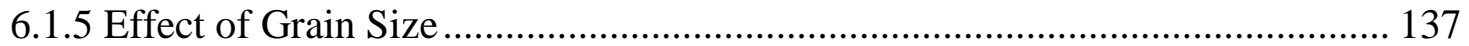

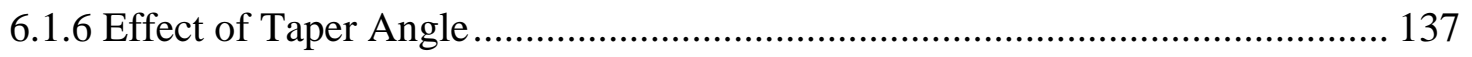

6.2 Recommendation for Future Work ………………...................................... 138

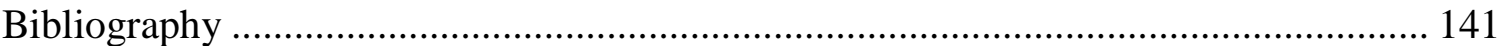

APPENDIX A - Test Results: Total Load and Pressure, After Crushing Pictures, Spall Distributions and Thin-section Photographs.............................................................. 152

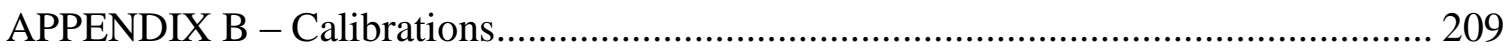




\section{List of Figures}

Figure 1. 1: Schematic representation of the link between load cycling and layer

dynamics, after Jordaan, 2001 (Jordaan et al., 2008)..................................................... 4

Figure 2.1: Typical constant strain rate creep curves for ice (Nadreau and Michel, 1984)

Figure 2.2: Typical creep curves for ice under constant stress (Nadreau and Michel, 1984)

Figure 2.3: Schematics of (a) Kelvin unit (b) Maxwell unit......................................... 12

Figure 2.4: Burgers model consisting of a Maxwell unit and Kelvin Unit in series......... 13

Figure 2.5: Modes of Fracture (a) Mode I, (b) Mode II and (c) Mode III (Anderson, 1995)

Figure 2.6: Cracked plane subjected to a biaxial stress state (Anderson, 1995) ............... 21

Figure 2.7: Illustration of J-integral around the crack tip ……….................................. 25

Figure 2.8: Schematic illustration of the main processes of spalling, extrusion and high-

pressure zone formation (Jordaan, 2001) .................................................................... 27 
Figure 2.9: Crack formation due to grain boundary sliding for (a) triple point junction of the grains and (b) 'wing' cracks between two grain boundaries (Jordaan and McKenna, 1988) 30

Figure 2.10: Test results from Hobson's Choice Ice Island experiments (Frederking et al., 1990, modified from Taylor, 2010) 31

Figure 2.11: Ductile failure observed for slow test $(\mathrm{v}=0.03 \mathrm{~cm} / \mathrm{s})$ during Rae Point experiments with $1 \mathrm{~m}^{2}$ spherical indenter (Masterson et al., 1999). 32

Figure 2.12: Brittle failure observed for fast test $(\mathrm{v}=1.0 \mathrm{~cm} / \mathrm{s})$ during Rae Point experiments with $1 \mathrm{~m}^{2}$ spherical indenter (Masterson et al., 1999).................................. 33

Figure 2.13: Plot of pressure melting data for ice (Taylor, 2010) …………………........ 36

Figure 2.14: Illustrations of (a) global interaction area and (b) local design area (Jordaan et al., 2005a) 37

Figure 2.15: (a) Load trace data; arrows indicate large load drop. (b) Ice surface showing a dark grey, cross-shaped zone at the center in a light crushed ice matrix (Barrette et al., 2002)

Figure 2.16: Total force from MTS load cell identifying five different failure modes under compressive failure of ice (Wells et al., 2010)

Figure 2.17: Thin-sections of indentation zones for tests conducted at $-5^{\circ} \mathrm{C}(\mathrm{a}-\mathrm{b})$ and $-15^{\circ} \mathrm{C}(\mathrm{c}-\mathrm{d})$. Side lighting is used to highlight fractures in (a) and (c); cross-polarized filters are used to highlight damage in (b) and (d). (e) plot of force-time traces for tests conducted with Compliant System 4 for warm ice $\left(-5^{\circ} \mathrm{C}\right)$ and cold ice $\left(-15^{\circ} \mathrm{C}\right)$ at an indentation rate of $4 \mathrm{~mm} / \mathrm{s}$. (Taylor, 2013) 
Figure 2.18: Ice strength plotted against crushing rate for ice samples for ice samples of different temperatures at $45 \mathrm{~mm}$ crushing depth (Dillenburg, 2012) ............................. 50 Figure 2.19: Progression of pressure profile from the results of double angle cone test (a) Initiation of loading (b) prior to the first major fracture (c) after the first major fracture (d) prior to the second major fracture (d) after the second major fracture (Reddy et al., 2012)

Figure 3.1: Geometry of ice specimen having taper angle $21^{\circ}$ (all dimensions are in $\mathrm{mm}$ )

Figure 3.2: MTS Load Frame 55

Figure 3.3 (a): Tekscan pressure sensor model 5101 with specification (collected from the official website: www.tekscan.com) (b): Tekscan sensor with holder ............................ 57

Figure 3.4: (a) Flat stainless steel indenter, (b) Spherical aluminum indenter ................ 59

Figure 3.5: (a) Larger grain size $\left(4-10^{+} \mathrm{mm}\right)(\mathrm{b})$ Smaller grain size $(0-4 \mathrm{~mm}) \ldots \ldots \ldots \ldots \ldots . . . . .60$ Figure 3.6: (a) Ice sample of $13^{\circ}$ taper angle (b) Ice sample of $21^{\circ}$ taper angle (c) Ice

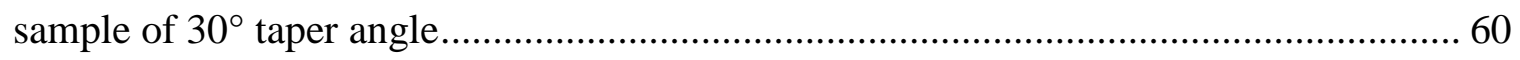

Figure 3.7: (a) Water distillation system (b) Water deionization system ....................... 62

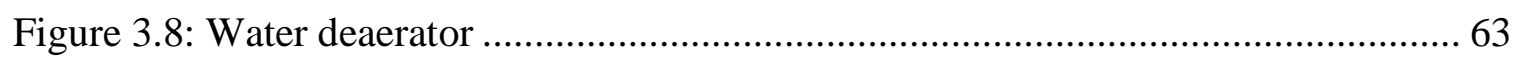

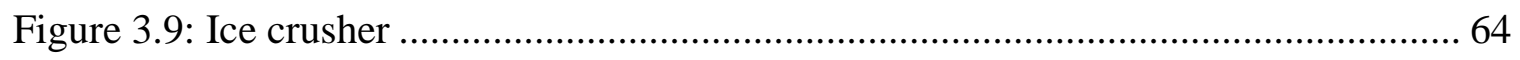

Figure 3.10: Water pouring method to the ice mould in the freezer ............................. 65

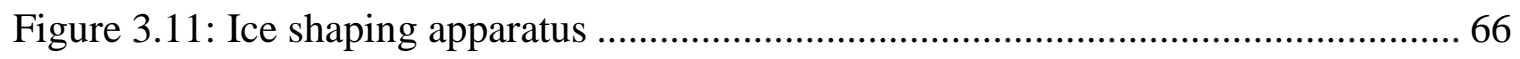

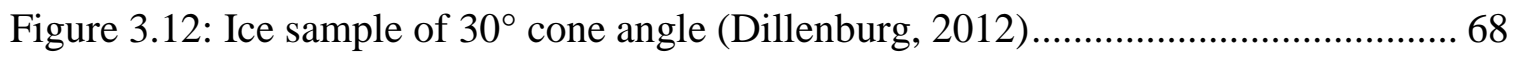


Figure 3.13: Drawings of the ice samples (a) $13^{\circ}$ (b) $21^{\circ}$ (c) $30^{\circ}$ 69

Figure 3.14: Arrangement of Tekscan sensor with MTS load frame .............................. 71

Figure 3.15: Photograph of the testing area after crushing test ....................................... 72

Figure 3.16: Ice spall sieving according to the spall size ……………........................... 73

Figure 3.17: (a) Vertical and (b) Horizontal cut of crushed ice sample ........................... 74

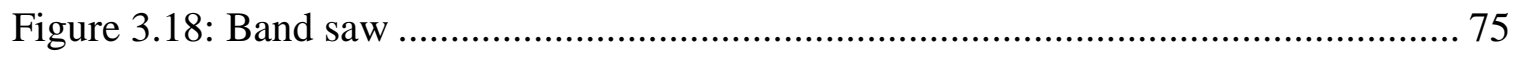

Figure 3.19 Vertical and Horizontal thick section ........................................................... 75

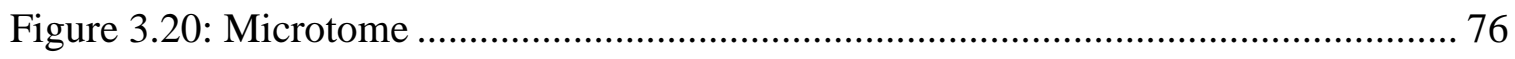

Figure 3.21: Cross polarizing and side lighting box ……………………..................... 77

Figure 4.1: Drawing of ice sample having $21^{\circ}$ taper angle (all dimensions are in $\mathrm{mm}$ ) .. 80

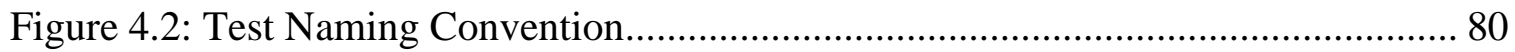

Figure 4.3: Force-time and Pressure-area plot for the test T17_0.1_13_1_10_F ............. 84

Figure 4.4: Pressure distribution images from tactile pressure sensor data corresponding to the load cell data for the test T17_0.1_13_1_10_F ...................................................... 84

Figure 4.5: After crushing image of the slow test T17_0.1_13_1_10_F .......................... 85

Figure 4.6: Spall distribution for the test T17_0.1_13_1_10_F......................................... 85

Figure 4.7: Vertical thin-section pictures using cross polarized light (colour) and side light (black and white) for the test T16_0.1_30_2_10_F .............................................. 86 Figure 4.8: Force-time and Pressure-area plot for the test T19_1_13_1_10_F................ 88 Figure 4.9: Pressure distribution images from tactile pressure sensor data corresponding to the load cell data for the test T19_1_13_1_10_F …………………………………..... 88 
Figure 4.10: After crushing image of the medium speed test T19_1_13_1_10_F 89

Figure 4.11: Spall distributions for the test T19_1_13_1_10_F. 89

Figure 4.12:Vertical thin-section pictures using cross polarized light (colour) and side light (black and white) for the test T13_1_30_1_10_F ............................................. 90

Figure 4.13: Force-time and Pressure-area plot for the test T21_10_13_1_10_F ........... 91

Figure 4.14: Pressure distribution images from tactile pressure sensor corresponding to the load cell data for the test T21_10_13_1_10_F....................................................... 92

Figure 4.15: After crushing image of the fast speed test T21_10_13_1_10_F................ 92

Figure 4.16: Spall distributions for the test T21_10_13_1_10_F ................................. 93

Figure 4.17: Vertical thin-section pictures using cross polarized light (colour) and side light (black and white) for the test T15_10_30_1_10_F ............................................ 94

Figure 4.18: Force-time and Pressure-area plot for the test T27_10_21_1_5_F ............. 95

Figure 4.19: Pressure distribution images from tactile pressure sensor data corresponding to the load cell data for the test T27_10_21_1_5_F .................................................... 96

Figure 4.20: After crushing image of the warm test T27_10_21_1_5_F ........................ 96

Figure 4.21: Spall distribution of test T27_10_21_1_5_F........................................... 97

Figure 4.22: Vertical thin-section pictures using cross polarized light (colour) and side light (black and white) for the test T27_10_21_1_5_F .............................................. 98

Figure 4.23: Force-time and Pressure-area plot for the test: T08_10_21_1_10_S........ 100 Figure 4.24: High-speed video snapshots corresponding to the load cell data from the test

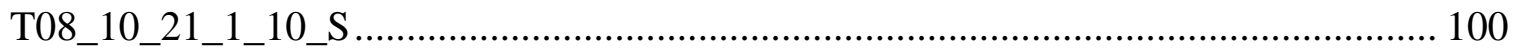

Figure 4.25: After crushing image of the test T08_10_21_1_10_S.............................. 101 
Figure 4.26: Spall distributions for the test T8_10_21_1_10_S 101

Figure 4.27: Vertical thin-section pictures using cross polarized light (colour) and side light (black and white) for the test T08_10_21_1_10_S

Figure 5.1: MTS load cell data identifying crushing failure for the test

T21_10_13_1_10_F 106

Figure 5.2: Pressure distribution images before and after crushing events from Tekscan sensor for the test T21_10_13_1_10_F 106

Figure 5.3: MTS load cell data identifying spalling event for the test T03_1_21_1_10_F 108

Figure 5.4: Snapshots of the spalling failure from video recording of the test T03_1_21_1_10_F 108

Figure 5.5: MTS load cell data for the three tests at different speeds: Slow speed test T02_0.1_21_2_10_F (blue); Medium speed test T05_1_21_2_10_F (green); Fast speed test T06_10_21_2_10_F (red) 109

Figure 5.6: Pressure distribution images for the three tests at different indentation speeds: Slow speed test T17_0.1_13_1_10_F, Medium speed test T19_1_13_1_10_F and Fast speed test T21_10_13_1_10_F 111

Figure 5.7: MTS load cell data corresponding to the pressure distribution images ....... 111 Figure 5.8: Vertical thin-section pictures showing the effect of indentation speed........ 112 Figure 5.9: During and after crushing images for the tests at different indentation speeds 113 
Figure 5.10: Total spall masses of ice crushing tests at different indentation speeds having different taper angle 114 Figure 5.11: MTS load cell data for two slow speed tests at different temperatures: Cold test T01_0.1_21_1_10_F (blue) and Warm test T25_0.1_21_1_5_F (green) 115 Figure 5.12: MTS load cell data for two fast speed tests at different temperatures: Cold test T06_10_21_2_10_F(blue) and Warm test T28_10_21_2_5_F 115 Figure 5.13: Vertical thin-section pictures using cross polarized light and side light (a)Cold test results $\left(-10^{\circ} \mathrm{C}\right)$ for the test T03_1_21_1_10_F (b) Warm test results $\left(-5^{\circ} \mathrm{C}\right)$ for the test T26_1_21_1_5_F 116 Figure 5.14: After crushing images for tests at two diferent temperatures. 117 Figure 5.15: Total spall mass comparison for four different cases of cold and warm tests

Figure 5.16: MTS load cell data for the tests using two different indenters: Flat indenter: T06_10_21_2_10_F (blue) and Spherical indenter: T07_10_21_2_10_S (green)........ 119 Figure 5.17: MTS load cell data for the previous tests for 0.1 second 119 Figure 5.18: Vertical thin-section pictures using cross polarized light (colour images) and side light (black and white images) for the tests using (a) Flat indenter:

T04_10_21_1_10_F (b) Spherical indenter: T08_10_21_1_10_S 120

Figure 5.19: After crushing ice sample pictures of fast speed tests using (a) Flat indenter for the test T04_10_21_1_10_F (b) Spherical indenter for the test T08_10_21_1_10_S 
Figure 5.20: Total spall mass comparison for six cases using flat and spherical indenter

Figure 5.21: Total spall mass comparison for fast speed tests using flat and spherical indenter with different taper angles

Figure 5.22: MTS Load cell data for two tests having different grain size distributions Test T03_1_21_1_10_F having grain size distribution of $4-10^{+} \mathrm{mm}$ (Blue line) and Test T05_1_21_2_10_F having grain size distribution of 0-4 mm (Green line)..... 123

Figure 5.23: Pressure distribution images for two tests having different grain size distributions. Test T27_10_21_1_5_F having grain size distribution of 4-10+mm (top) and Test T28_10_21_2_5_F having grain size distribution of 0-4 mm (bottom) 124 Figure 5.24: MTS load cell data corresponding to the pressure distribution images: Test T27_10_21_1_5_F having grain size distribution of 4-10 $\mathrm{mm}$ (Blue line) and Test T28_10_21_2_5_F having grain size distribution of 0-4 mm (Green line) 125

Figure 5.25: Horizontal thin-section pictures defining the grain distribution using ....... 126 Figure 5.26: Vertical thin-section pictures using cross polarized light (colour) and side light (black and white) for (a) Ice sample with bigger grain size (Test T13_1_30_1_10_F)

Figure 5.27: Total spall mass for the fast speed tests having bigger and smaller size grain distribution of ice seeds 127

Figure 5.28: Plots of force, area and pressure against time for three tests having taper angles (a) $13^{\circ}$ for the test T23_10_13_1_10_S (blue); (b) $21^{\circ}$ for the test T08_10_21_1_10_S (green); (c) 30 for the test T09_10_30_1_10_S (red)..... 129 
Figure 5.29: Vertical thin-section pictures using cross polarized light (colour) and side light (black and white) for ice samples having taper angles of (a) $13^{\circ}$ (Test

T21_10_13_1_10_F) (b) $21^{\circ}$ (Test T04_10_21_1_10_F) (c) $30^{\circ}$ (Test

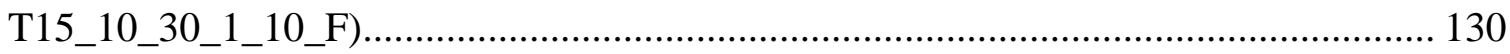

Figure 5.30: Total spall mass of three taper angles at different indentation rates .......... 131 


\section{List of Tables}

Table 3.1: Heights of the conical shape in ice specimens.......................................... 54

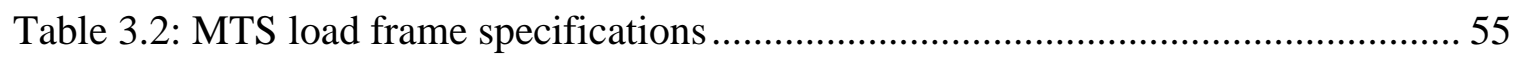

Table 3.3: Specification of the crushing plates .................................................. 56

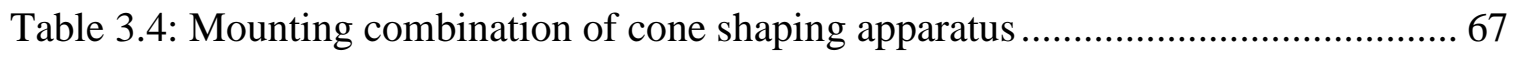

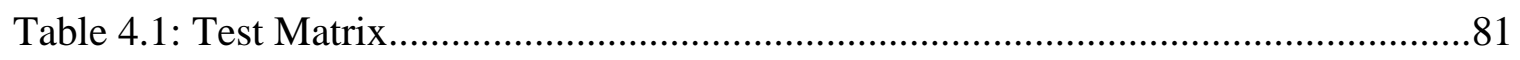




\section{List of Abbreviations and Symbols}

$\begin{array}{ll}E & \text { Young's modulus } \\ v & \text { Poisson's ratio } \\ \varepsilon^{e} & \text { elastic strain } \\ \varepsilon^{d} & \text { delayed-elastic creep strain } \\ \varepsilon^{v} & \text { viscous creep strain } \\ \varepsilon^{v} & \text { total strain } \\ \sigma & \text { axial stress } \\ \mu_{k} & \text { viscosity coefficient for a Kelvin unit } \\ \sigma_{d} & \text { stress in a Kelvin unit dashpot } \\ \varepsilon_{o}{ }^{v} & \text { reference strain rate } \\ \sigma_{o} & \text { constant unit stress (1 MPa) } \\ E_{k} & \text { Young's modulus of a Kelvin unit } \\ E_{0} & \text { Young's modulus of the virgin material } \\ & \text { shear stress } \\ & \end{array}$




$\begin{array}{ll}v & \text { activation volume } \\ \Delta H & \text { activation enthalpy } \\ B & \text { material constant } \\ R_{b} & \text { activation energy } \\ T_{B} & \text { Boltzman's constant } \\ \tau_{B} & \text { absolute tempperature } \\ T_{m} & \text { internally generated back stress } \\ V_{w} & \text { melting temperature } \\ A_{i} & \text { specific volume of water } \\ V_{3} & \text { cross-sectional area of damaged ice specimen } \\ S_{w} & \text { effective elastic strain } \\ S_{i} & \text { entropy of water } \\ f_{3} & \text { entropy of ice }\end{array}$




\begin{tabular}{|c|c|}
\hline$\sigma_{e f f}$ & effective stress \\
\hline$\varepsilon_{\text {eff }}$ & effective strain \\
\hline$D$ & damage variable \\
\hline$A_{T}$ & nominal interaction area \\
\hline$\sigma_{1}$ & maximum principle stress \\
\hline$\sigma_{2}$ & minimum principle stress \\
\hline$\beta$ & angle between the crack and $\sigma_{2}$ plane \\
\hline$S$ & damage state variable \\
\hline$G$ & energy release rate \\
\hline$G_{c}$ & critical energy release rate \\
\hline$K$ & stress intensity factor \\
\hline$K_{I C}$ & critical stress intensity factor for the Mode I fracture \\
\hline$K_{I}$ & stress intensity factor for mode I \\
\hline$K_{I I}$ & stress intensity factor for mode II \\
\hline$k_{I}$ & local stress intensity factor of the kinked crack tip for mode I \\
\hline$k_{I I}$ & local stress intensity factor of the kinked crack tip for mode II \\
\hline$C_{11}, C_{12}$ & geometric functions of crack kink angle \\
\hline$\alpha^{*}$ & crack kink angle where $\mathrm{G}$ is maximum \\
\hline$a$ & crack length \\
\hline$W$ & strain energy density \\
\hline$T_{s}$ & traction vector normal to ds \\
\hline
\end{tabular}




$\begin{array}{ll}d_{s} & \text { small increment along path } \\ u & \text { displacement vector } \\ C & \text { coefficient of power law for mean pressure } \\ D & \text { exponent of power law for mean pressure } \\ d & \text { top diameter } \\ x & \text { indentation depth } \\ R & \text { radius of the indentation surface } \\ \alpha & \text { taper angle } \\ P & \text { pressure } \\ F & \text { Force (from load cell) } \\ h p z & \text { high pressure zone } \\ \text { LEFM } & \text { Linear Elastic Fracture Mechanics } \\ \text { MUN } & \text { Memorial University of Newfoundland } \\ \text { JOIA } & \text { Japan Ocean Industries Association } \\ \text { MTS } & \text { Materials Testing System } \\ \text { STePS } & \text { Sustainable Technology for Polar Ships and Structures } \\ \text { NRC-IOT } & \text { National Research Council - Institute for Ocean Technology }\end{array}$




\section{Chapter 1: Introduction}

\subsection{Overview}

Exploration and development of arctic and subarctic oil and gas resources have generated great interest in recent years. Sea ice and icebergs pose a substantial challenge to the development of oil and gas production structures in northern regions. Offshore structures and vessels must be designed to withstand interaction with ice features which may fail in different modes such as compressive failure, flexural failure, spalling, bending, buckling, crushing or any combination of these failure modes. For vertical-walled structures compressive failure can result in high local loads and potentially excite dynamic response in the structures during the interaction with ice features. This may result in load amplifications and fatigue issues. Other issues such as resonance, durability and serviceability must also be considered in the design process. 
Understanding compressive ice failure processes is crucial for offshore structures. Improved knowledge of fracture and compressive failure will continue to improve confidence in the design of structures for ice conditions. Compressive ice failure is associated with primary load limiting mechanisms such as crushing and spalling. Crushing is associated with microstructural changes to the ice adjacent to the contact zone due to recrystallization, localized pressure melting and microfracture. This microstructural "damage" results in pressure softening of the ice, ultimately leading to the extrusion of crushed ice from the outer edge of the ice-structure interaction zones. This process produces load fluctuations and is associated with failure of the high pressure zones due to changes in pressure intensity without appreciable area change. Spalling fracture is associated with loss of the contact area at the ice-structure interface, which causes a sudden load drop and decrease in pressure. Spalling fracture is associated with presence of flaws in the ice such as grain boundaries and other defects, which serve as stress concentrators and promote the fracture process. The present research attempts to develop improved understanding of the effects of specimen geometry, crushing rate, temperature, indenter shape, grain distributions in failure processes. As well as, to improve the understanding of ice-structure interaction, local loading on ice, pressure distribution, fracture failure associated with crushing and spalling are also observed.

\subsection{Background}

High pressure zones (hpzs) are considered one of the most important elements of the interaction process in compressive ice failures (Jordaan, 2001). High pressure zones refer 
to the areas where an ice feature comes into contact against a vertical-walled structure and creates single or multiple localized areas of high pressure. According to Jordaan (2001) an $h p z$ is a localized region of ice contact through which the majority of loads are transmitted to the structure. While the state of stress is triaxial within hpzs (Wells et al., 2010), the formation of hpzs is associated with two processes (Jordaan et al., 2008). The first process involves spalling and fracture which cause concentration of stress in the area of hpzs (Mackey et al., 2007) and the second process is the formation of the damaged layer which is found in both small and medium scale tests (Jordaan et al., 2005b, 2008). A number of researchers have confirmed that the failure mechanisms of $h p z s$ are not associated exclusively with fractures (for example Meglis et al., 1999, Melanson et al., 1999, Xiao, 1997, Wells et al., 2010 etc.). Jordaan et al. (2008) focused on the softening processes that occur within this damaged layer. They described that within the layer, the processes vary with distance from the center. For example, microcracking and recrystallization occur near the outside, while local pressure melting can be observed along grain boundaries in the central part. They also noted that the crushing process involves the extrusion of the softened ice from the periphery of the $h p z$. In Figure 1.1, the behaviour of ice in the vicinity of a single $h p z$ is presented from Jordaan et al. (2008). As they explained, microcracking begins to take place when the ice comes into contact with the structure (A). Here, outside and within the $h p z$, the ice experience extensive microcracking. This forms a 'white zone' having entrained air and cracks which also causes fragmentation of the ice close to the edge of that zone. High confinement and pressure cause the pressure softening processes near the center of the $h p z$ due to the 
formation of fine-grained, recrystallized ice (B). A lower pressure is generated due to extrusion of the crushed and pressure softened ice (C). The layer again hardens because of the release of pressure (D). Cycles of pressure softening and hardening can result in the development of loading cycles that are associated with this crushing-extrusion process. Localized spalling causes the occasional breaking up of periods of load cycles and reduces the contact area near the $h p z$ (E). As mentioned by Jordaan et al. (2008), in small and medium scale tests, these spalls disrupt the cyclic loading until sufficient pressure builds to start the pressure softening and hardening again. However, in the case of full scale tests, the interactions involve many $h p z \mathrm{~s}$ and the interplay between spalling and crushing becomes more complex.

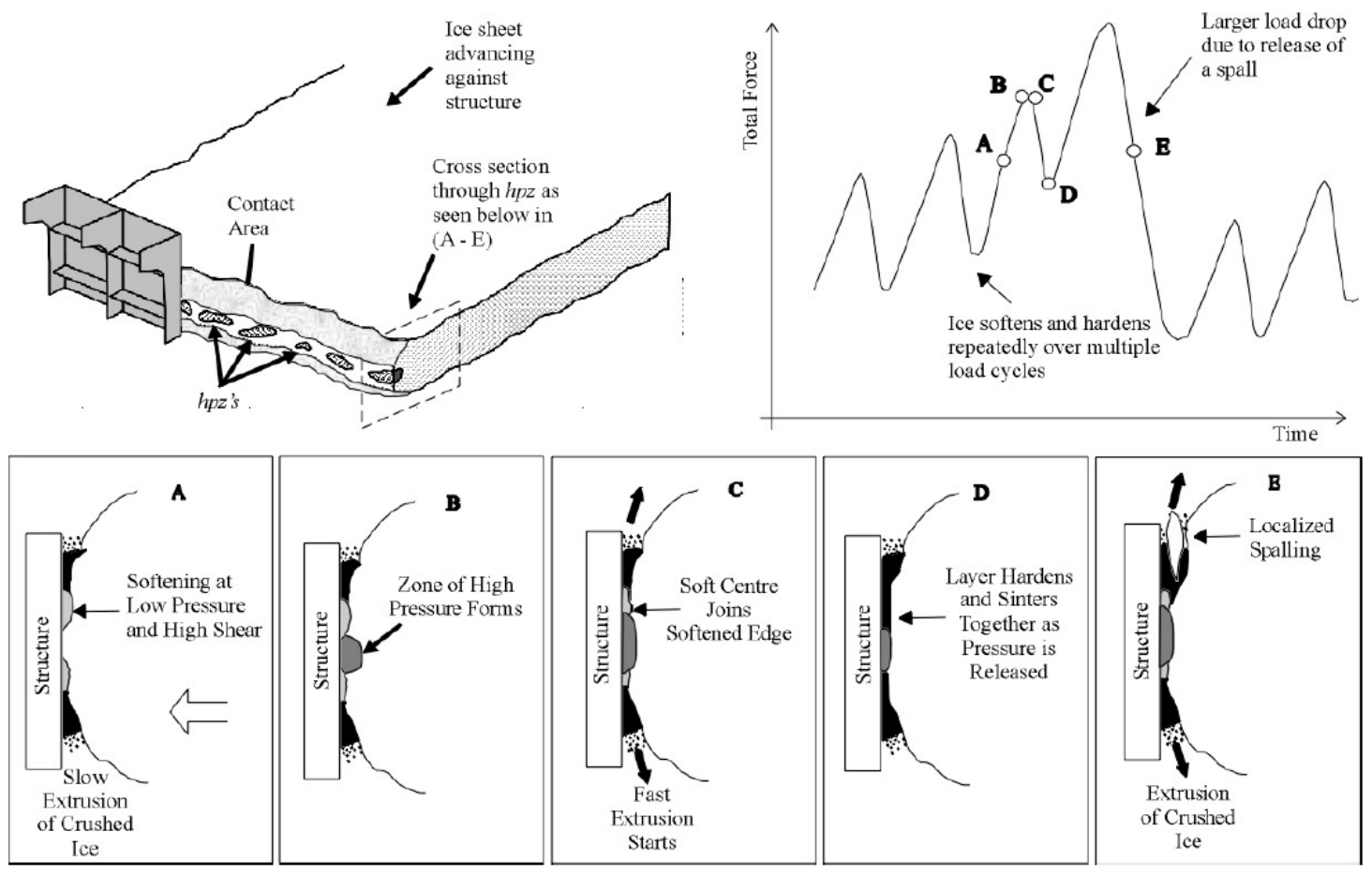

Figure 1. 1: Schematic representation of the link between load cycling and layer dynamics, after Jordaan, 2001 (Jordaan et al., 2008) 


\subsection{Objectives}

The main objectives of the research are to improve the present understanding of compressive ice failure associated with ice-structure interaction processes and explore the links among various factors that influence these failures. The goals of the thesis are to investigate the following factors:

- The behaviour of high pressure zones and the features that influence the formation and evolution of these zones.

- The processes that are responsible for limiting the ice force and pressure during ice-structure interaction.

- The effect of taper angle of the ice specimen.

- The effect of indentation rate on the ice-structure interaction process.

- The influence of temperature on ice compressive failure.

- The effect of using flat and spherical indenters in ice crushing process.

- The impact of using two different grain size distributions to make ice specimens.

\subsection{Scope and Outline of Research}

The research presented in this thesis is focused on the investigation of crushing and fracture during compressive ice failure. A series of small-scale laboratory experiments were conducted in which conical shaped samples with truncated tops made from freshwater polycrystalline ice were crushed using flat and spherical indenters. The experiments were performed in the cold room facilities of Memorial University of 
Newfoundland using a Materials Testing System. The aims of the work are to observe the crushing and spalling fractures and to understand the effects of indentation rate, temperature, different shaped indenters, ice specimen geometry and grain size of ice seeds.

This thesis has been categorized into six chapters. In Chapter 1, an introduction and outline of the research program is provided. Chapter 2 consists of a review of relevant literature on ice mechanics, damage mechanics and fracture mechanics, including mechanical properties and behaviour of ice and compressive ice failure processes. Previous indentation test programs relative to the present program are also reviewed. A detailed description of experimental methodology including the test set-up, procedures of water and ice preparation, testing and post-experimental works are provided in Chapter 3. In Chapter 4, the test matrix for this research program and sample test results are presented for both flat and spherical indenters. In Chapter 5, a detailed analysis of the results including observations of ice failure mechanisms and the effects of indentation rate, temperature, indenter type, grain size and ice specimen shape are provided. A summary of the main findings and results are discussed in Chapter 6 along with conclusions and recommendations for future work. 


\section{Chapter 2: Literature Review}

\subsection{Ice Mechanics}

In the design of offshore structures and ships for northern regions, the ice environment presents a significant challenge. To design for local and global pressures acting on the structures and estimate the design ice loads, detailed understanding of ice mechanics and compressive ice failure processes is required. In this chapter, a review of ice mechanics is summarized. Ice properties and behaviour are discussed including elastic and viscoelastic behaviour. The role of damage mechanics is included with a thorough description of the compressive ice failure process. In this section, behaviour of high pressure zones, microstructural damage, recrystallization and pressure melting are also reviewed. A brief description of local and global ice pressure along with the pressure-area scale effect are included. In the section on fracture mechanics, a brief review of relevant theoretical 
concepts related to fracture is discussed. In addition, five series of relevant indentation experiments are discussed.

\subsubsection{Properties and Behaviour of Ice}

Ice is a complex material as it is difficult to describe its material behaviour using a single fundamental theory. The behaviour of ice can be modelled using elasticity, viscoelasticity, damage mechanics or a combination of any of these. As Schulson and Duval (2009) described, ice displays a wide range of mechanical properties, including elasticity, viscoelasticity, creep rupture and brittle failure. At a low loading rate, the deformation of ice under stress may be modeled using viscoelastic theory and the irreversible (flow) term is extremely non-linear and time-dependent (Jordaan, 2001). According to Sanderson (1988) ice is a creeping, ductile material at low stresses and an extremely brittle material at high stresses and its behaviour is dependent on the rate of deformation and deformation history. Typically, two types of ice, glacial ice and sea ice exist in the ocean. Examples of glacial ice are icebergs or ice islands that are formed from perennial snow accumulation. Sea ice formation depends on the seasonal cooling and freezing of sea water. The modulus of elasticity of glacial ice ranges from 9.0 GPa to

9.5 $\mathrm{GPa}$ and for sea ice the range is as wide as $0.3 \mathrm{GPa}$ to $10 \mathrm{GPa}$ (Cammaert and Muggeridge, 1988). Glacial ice has statistically isotropic granular microstructure, which is similar to polycrystalline freshwater ice. The variety of ice behaviour presents challenges in the design for ice loads because of the high degree of variability in physical and mechanical properties of ice. 


\subsubsection{Elasticity}

For very fast loading, freshwater granular ice is normally treated as an isotropic elastic material (Schapery, 1997). Therefore, the elasticity of ice can be characterized by two constants: the Young modulus, $E$ and Poisson's ratio, $v$.

Elastic modulus of ice can be determined both statically and dynamically. Static tests generally include uniaxial tension, uniaxial compression and beam bending. The Young modulus of sea ice has been investigated by Mellor (1983), who reported that, the Young modulus using high frequency dynamic measurements varies from 9 to $9.5 \mathrm{MPa}$ in the temperature range of $-5^{\circ}$ to $-10^{\circ} \mathrm{C}$ and the Poisson's ratio varies from 0.3 to 0.33 for low porosity polycrystalline ice. Sinha (1989) showed that temperature does not have a significant effect on these constants as for the temperature range of $-50^{\circ}$ to $0^{\circ} \mathrm{C}, E$ varies from $9 \mathrm{GPa}$ to $10.16 \mathrm{GPa}$ and $v$ from 0.308 to 0.365 .

\subsubsection{Viscoelasticity and Creep Behaviour of Ice}

Viscoelasticity is the property of a material that shows both elastic and viscous characteristics while undergoing deformation. For these materials the relationship between stress and strain depends on time and the frequency of applied loading. They

display a time-dependent material response, where the response of stress depends on both the strain applied and the strain rate at which it was applied. Some interesting points about viscoelastic materials are (Lockett, 1972):

- Strain increases with time when the stress is constant (creep).

- Stress decreases with time when the strain is constant (relaxation). 
- The effective stiffness has dependency on the rate of applied load.

- Hysteresis occurs when cyclic loading is applied and the area in the hysteresis loop is a function of the loading rate.

- After an impact rebound of an object is less than $100 \%$.

Since ice is a viscoelastic material, it exhibits time-dependent strain for any level of stress. Furthermore, under fixed strain, it exhibits a time-dependent stress relaxation. The stress and strain behaviour of ice on time dependency is shown in Figure 2.1 and 2.2 respectively.

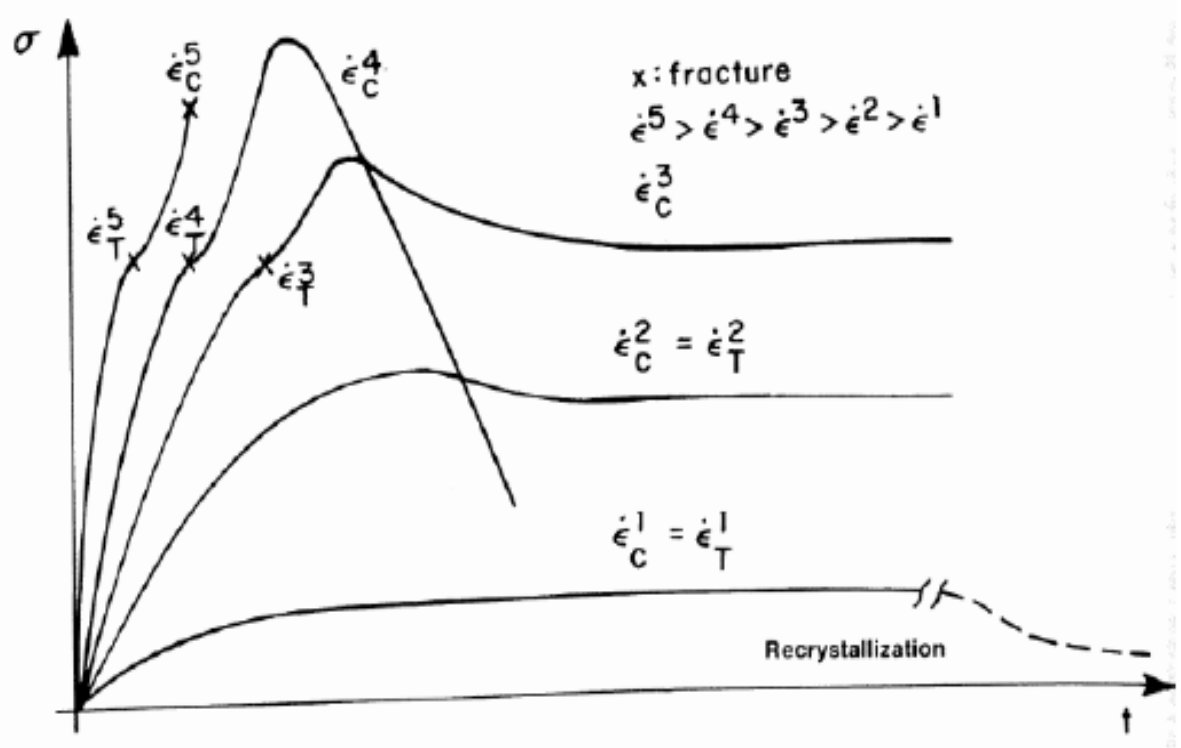

Figure 2.1: Typical constant strain rate creep curves for ice (Nadreau and Michel, 1984)

Figure 2.1 shows ice deformation under a constant strain rate. Increasing the strain rates increase the peak stresses and ultimately leads to a transition to a brittle failure mode. For 
low strain rates, the behaviour is pure creep and ice behaves similarly under tensile and compressive loadings. At intermediate strain rates, pressure softening is observed and the response during compressive failure starts to change from that observed during tensile mode.

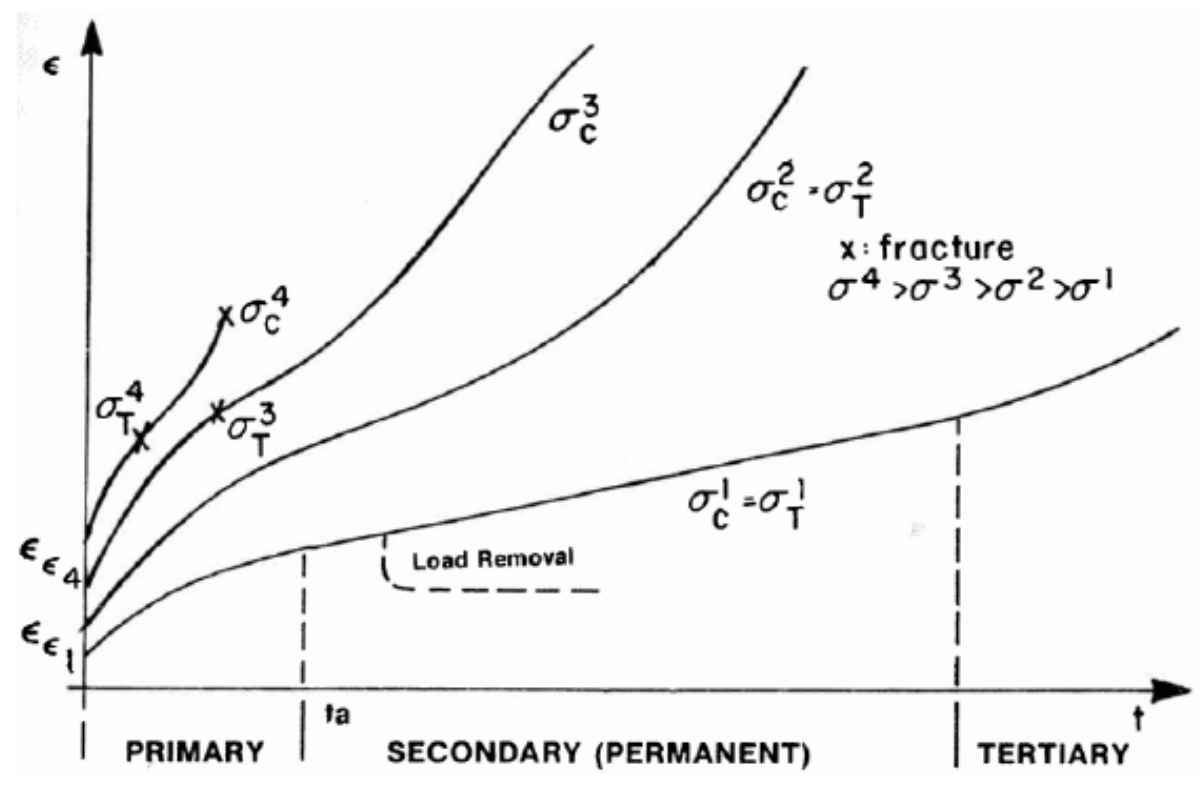

Figure 2.2: Typical creep curves for ice under constant stress (Nadreau and Michel, 1984)

Figure 2.2 shows behaviour of ice under constant stress. The creep behaviour is typically divided into three components: primary creep, secondary creep and tertiary creep. Ice that is subjected to constant stress deforms instantly as a result of instantaneous elastic strain, which is followed by time-dependent creep behaviour.

Primary creep is associated with time-dependent delayed elastic strain resulting from reversible grain boundary sliding due to shear stresses at grain boundaries. This creep 
component occurs in conjunction with elastic deformation of the grain. If compressive stresses are removed, the grain tries to recover its original shape (Sanderson, 1988). Secondary creep is the irrecoverable (flow) component of creep behaviour and is highly nonlinear and time-dependent, since ice creeps at all stresses and shows no yield point. Secondary or steady-state creep results in permanent deformation processes within the ice leading to irreversible rearrangement of the material. Tertiary creep results from damage processes, which soften the ice and accelerate strain-rates. The mechanisms typically associated with this behaviour are microcracking and dynamic recrystallization. Detailed descriptions of microcracking and dynamic recrystallization are included later in this chapter.

Viscoelastic behaviour is commonly modeled using a combination of springs and dashpots, where a spring represents elastic deformation and a dashpot represents viscous flow. A viscoelastic model can be constructed combining each component either in series or in parallel. Among various models, Maxwell and Kelvin-voigt spring-dashpot models are most common. In Figure 2.3, schematic diagrams of the Kelvin unit and Maxwell unit are given.

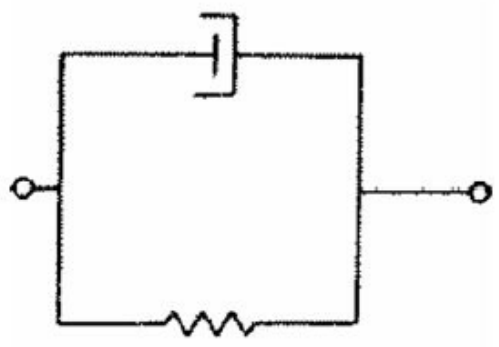

(a)

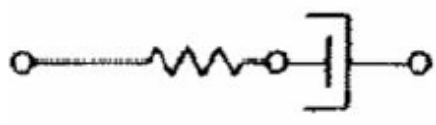

(b)

Figure 2.3: Schematics of (a) Kelvin unit (b) Maxwell unit 
Jordaan and McKenna (1988) used a Burgers model to represent the viscoelastic behaviour of ice. The Burgers model consists of a Maxwell unit and a Kelvin unit in series as shown in Figure 2.4.

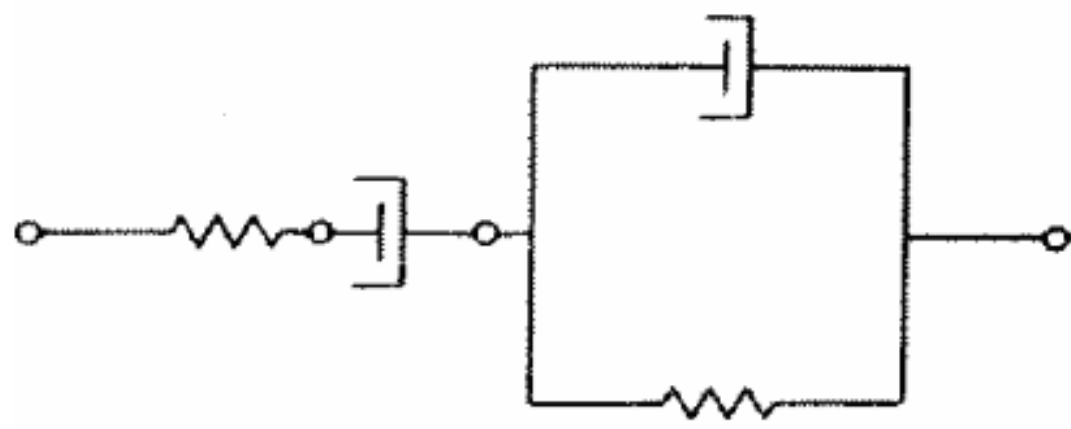

Figure 2.4: Burgers model consisting of a Maxwell unit and Kelvin Unit in series.

In this model, the instantaneous elastic strain is represented by the spring of the Maxwell unit, and the Kelvin unit represents the delayed elastic strain component. The viscous strain is modeled by the dashpot of the Maxwell unit. In this model, the total strain $\epsilon$ is the sum of the instantaneous elastic strain $\epsilon^{e}$, the delayed elastic strain $\epsilon^{d}$, and the viscous strain $\epsilon^{v}$, as given by

$\epsilon=\epsilon^{e}+\epsilon^{d}+\epsilon^{v}$

Works by Jordaan and Xiao (1992), Xiao (1997) and Li (2007) used this approach to model ice behaviour. Each of the components of the equation is discussed below. 
For an isotropic medium, elastic behaviour is expressed in Young's modulus, $E$ and Poisson's ratio, $v$. According to Hooke's law the instantaneous elastic strain $\epsilon^{e}$ can be expressed as:

$\epsilon^{e}=\frac{\sigma}{E}$

The second term is delayed elastic strain $\epsilon^{d}$. This is a completely recoverable portion of the strain, as it will recover after sufficient time when the specimen is unloaded. Sinha (1979) developed a model for $\epsilon^{d}$ based on viscoelasticity theory for constant, monotonic uniaxial loading, which depends on grain size and temperature. The expression for the delayed elastic strain given by Sinha (1979) is:

$$
\epsilon^{d}=\frac{c d_{1}}{d}\left(\frac{\sigma}{E}\right)^{2}\left[1-\exp \left(-\left(\alpha_{\mathrm{T}} t\right)^{b}\right)\right]
$$

Here, $d_{1}$ is unit size grain and $c, s, \alpha_{\mathrm{T}}$ and $b$ are constants.

A phenomenological model for delayed elastic strain proposed by Jordaan and McKenna (1988) was based on the assumption that the viscous coefficient of the dash pot of the Kelvin unit could be modeled as having a power law dependency on stress. Their equation for delayed elastic strain is

$\epsilon^{d}=\frac{\sigma}{E_{k}}\left[1-\exp \left\{-\int_{0}^{t} \frac{1}{\mu_{k} \sigma_{d}} d \tau\right\}\right]$

Here $\int_{0}^{t} \frac{1}{\mu_{k} \sigma_{d}} d \tau$ represents the reduced time or pseudo time. 
The viscous component of strain represented as permanent component of the total strain, where the mechanism depends on the movement of dislocation within ice grains. Glen (1955) modeled the viscous component of creep behaviour using the power-law creep equation known as Glen's law. It can be expressed as:

$\epsilon^{v}=A \sigma^{n}$

Here, $n$ is a material constant and $A$ is a shift factor of temperature given by

$A=B \exp \left(-\frac{Q}{R_{b} T}\right)$

where $B$ is a material constant, $Q$ is the activation energy, $R_{b}$ is Boltzman's constant ( $R=8.314 \mathrm{~J} \mathrm{~mol}^{-1} \mathrm{~K}^{-1}$ ) and $T$ is the absolute temperature. According to Sinha (1979) the equation is based on the thermorheologically simple principle, which means that when the behaviour of a material at a given temperature is known, the behaviour at other temperatures of interest can be known using a shift factor. Sinha used the following equation to relate viscous strain and stress at a given temperature.

$\epsilon^{v}=\epsilon_{0}^{v}\left(\frac{\sigma}{\sigma_{0}}\right)^{n}$

Here, $\epsilon_{0}^{v}$ is the reference strain rate and $\sigma_{0}$ is reference stress.

Jonas and Muller (1969) developed an expression based on dynamic recrystallization on strain rate

$\epsilon=\phi_{f} \exp \left(-\frac{\Delta H}{K T}\right) \exp \left(\frac{v\left(\tau-\tau_{B}\right)}{K T}\right)$

Here, $\phi_{f}$ is a structure factor, $v$ is the activation volume, $\tau$ is the shear stress, $\Delta H$ is the activation enthalpy in the presence of hydrostatic pressure and $\tau_{B}$ is the internally 
generated back stress. Results of Meglis et al. (1999) also supported their work, indicating that under higher confining pressures, dynamic recrystallization is an important factor resulting in the enhancement of creep rate. Work by Findley et al. (1976) and Schapery (1997) also prepared a similar viscoelastic model.

As described by Taylor (2010), delayed elastic and viscous strain components are less common for field loading at higher loading rates because these mechanisms have less time to become activated. Under this type of condition, the elastic component dominates, rather than the creep mechanism associated with lower loading rates. In these conditions it is reasonable to consider ice as a brittle elastic medium in the far field. However, in the case of the interaction interface near the field, the damaged layer that forms during crushing exhibits localized pressure softening behaviour. To understand the behaviour of local $h p z s$, ice has been modelled with a damaging nonlinear viscoelastic model (for example Xiao 1997).

\subsubsection{Damage Mechanics}

When engineering materials undergo different loadings and environmental conditions, it is common to cause microstructural changes, which decrease their strength and these changes damage the mechanical properties of these materials. Damage can be caused by microcracks, fractures, thermal effects and environmental degradation factors. As described by Jordaan et al. (2005b), damage mechanics refers to the softening of a material as a result of microstructural change within the material. Early work on damage was performed by Kachanov (1958), based on the original idea that the damage of a 
structure can be measured by a scalar factor equal to the ratio of the area of voids to the whole cross-sectional area, or the density of microcracks and voids, which would permanently affect either the elastic modulus, $E$ or shear modulus, $G$. Early work on damage mechanics, including application to ice, was based on this concept. For the case of uniaxial loading, considering the nominal stress in an undamaged cross section, the stress is defined as

$\sigma=\frac{P}{A_{0}}$

where $P$ is the uniaxial force and $A_{0}$ is the overall cross-sectional area. A scalar measure of isotropic damage, $D$ was introduced as the ratio of the area of the void spaces and microcracks, $A$ to the overall area $A_{0}$ (i.e. damaged area), as given by

$D=\frac{A}{A_{0}},(0 \leq D \leq 1)$

In this model, as the damages increase, the loads are carried over a progressively smaller intact area and the material is no longer as strong as it was originally. To include these effects, the effective stress $\sigma_{\text {eff }}$ is expressed as

$\sigma_{e f f}=\frac{P}{\left(A_{0}-A\right)}=\frac{P}{A_{0}(1-D)}=\frac{\sigma}{(1-D)}$

The strain experienced by the damaged material can be defined by the stress strain relationship. Thus,

$\epsilon_{e f f}=\frac{\sigma_{e f f}}{E_{0}}=\frac{\sigma}{E_{0}(1-D)}=\frac{\sigma}{E}$ 
where, $E_{0}$ is the Young's modulus of the virgin material. The parameter $E$ is the 'effective' modulus. This effective modulus accounts for the reduction of the modulus as a result of the effects of damage. This can be defined as

$E=E_{0}(1-D)$

Schapery $(1981,1984)$ focused on the links between the behaviours of materials and damage processes in his works and developed a constitutive model based on the behaviour of viscoelastic material, including the effects of distributed damage. Generalized J-integral theory is developed based on this concept for analysing fracture in damaging nonlinear viscoelastic materials. J-integral theory is further discussed in section 2.2.5.

\subsection{Fracture Mechanics}

Fracture mechanics is concerned with the study of the propagation of cracks in materials. This is the method of fracture analysis that can determine the stresses required to induce fracture for unstable crack growth in a structure containing cracks of known size and shape. It uses analytical solid mechanics to calculate the driving force to open a crack, and experimental solid mechanics to characterize a material's resistance to fracture. The study of fracture mechanics has reached a high level of sophistication and has gained wide industrial acceptance because many brittle failure problems can be solved with this theory. 


\subsubsection{Linear Elastic Fracture Mechanics}

Linear Elastic Fracture Mechanics (LEFM) is a basic theory of fracture that assumes a given material as linear elastic. The theory of elasticity is used to calculate the stress field near the crack tip based on the assumption and when the stresses around the crack tip exceed the material fracture toughness, a crack starts to propagate. LEFM is used when the plastic zone ahead of the crack is much smaller than the dimensions of the crack and of the specimen. Fracture toughness characterizes the resistance of a material to cracking, and may depend on a variety of factors such as temperature, environment and loading rate. For a given geometry, stress intensity factor is a term in fracture mechanics that use to predict the stress, strain and displacement near the crack tip as a result of remote loadings or residual stresses. For a given crack geometry, Venkatachalam et al. (2008) described that stress intensity factor does not characterize the crack tip conditions when the plastic zone near the crack tip is large or behaviour of the material is non-linear. So, calculation of stress intensity factor is limited to linear elastic fracture mechanics. For the other cases, crack tip parameters like the J-integral can be taken into account which considers a larger plastic zone near the crack tip and show non-linear behaviour.

\subsubsection{Modes of Fracture}

For cracked plates, three modes of fractures are common depending on the direction of the force applied to the plate. These force results in crack propagation as shown in Figure 2.5. A cracked body can experience one of these modes or a combination of any two or three (Anderson, 1995). 


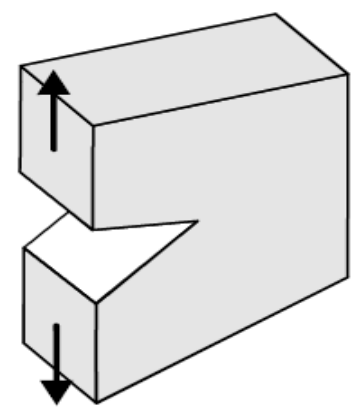

(a)

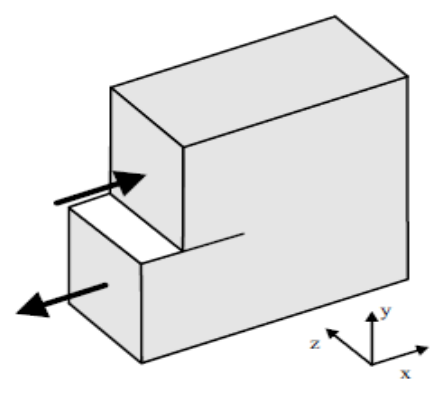

(b)

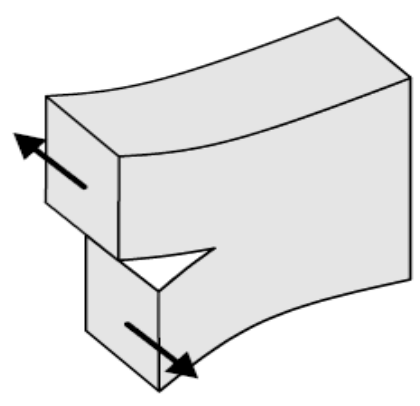

(c)

Figure 2.5: Modes of Fracture (a) Mode I, (b) Mode II and (c) Mode III (Anderson, 1995)

In mode I or opening mode the body is loaded by tensile forces, so the crack surfaces are pulled apart in the y-direction. The deformations are then symmetric with respect to the planes perpendicular to the $y$ axis and the $z$ axis.

In mode II, referred to as sliding mode, the body is loaded by shear forces parallel to the crack surfaces, which slide over each other in the x-direction. The deformations are then symmetrical with respect to the plane perpendicular to the $z$-axis and skewed symmetrically with respect to the plane perpendicular to the $y$-axis.

Finally, in mode III, the out-of-plane mode or tearing mode, the body is loaded by shear forces parallel to the crack and the crack surfaces slide over each other in the z-direction. The deformations are then skew-symmetrical with respect to the plane perpendicular to the $z$-axis and $y$-axis. 
From Anderson (1995), Figure 2.6 illustrates a cracked plate which is subjected to principal stresses $\sigma_{1}$ and $\sigma_{2}$, where $\sigma_{1}$ is greater than $\sigma_{2} ; \beta$ is the angle between the crack and the $\sigma_{1}$ plane.

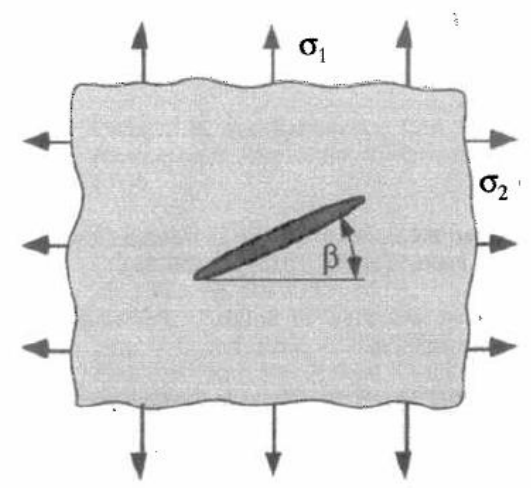

Figure 2.6: Cracked plane subjected to a biaxial stress state (Anderson, 1995)

\subsubsection{Energy-Release Rate}

Crack behaviour can be described by the energy-release rate $(G)$ or the stress intensity factor $(K)$. The energy release rate describes the global behaviour and stress intensity factor is a local parameter.

The energy-release rate is based on the energy criterion for fracture proposed by A. A. Griffith (1921) and further developed by Irwin (1957). In this approach, crack growth occurs when the energy available for crack growth is sufficient to overcome the resistance of the material. 
For linear elastic fracture mechanics, energy release rate $G$ is equivalent to J-integral, which is a measure of energy available for an increment of crack extension. It can be expressed as

$G=J=\int_{\Gamma}\left(w d y-t \frac{\partial d}{\partial x} d s\right)$

The energy release rate $G$ is also defined in elastic materials as the rate of change of potential energy released from a structure when a crack opens. For example, if a crack of length 2a is in a large elastic body with modulus $E$ subject to a tensile stress $(\sigma)$, the energy-release rate is given by

$G=\frac{\pi \sigma^{2} a}{E}$

At the moment when the crack becomes unstable or at the moment of fracture, $G$ is equal to the critical energy-release rate $G_{c}$.

As described by Erdogan, F. and Sih, G.C. (1963) in their original model, according to Griffth (energy) theory, the crack will grow in the direction along which the elastic energy release per unit crack extension will be maximum and the crack will start to grow when this energy reaches a critical value $G=G(\delta, \theta)$ and evaluation of $G(\delta, \theta)$ poses insurmountable mathematical difficulties.

If an infinite plate subjected to a uniform tensile stress, then the energy release rate, $G$ and stress intensity factor, $K$ can be relate as

$$
G=\frac{K_{1}^{2}}{E^{\prime}}
$$

where, $E^{\prime}=E$ for plane stress and $E^{\prime}=\frac{\mathrm{E}}{\left(1-\mathrm{v}^{2}\right)}$ for plane strain. 
$K_{1}$ is the stress intensity factor for mode I. When mode I and II are present, the energy release rate is given by:

$G=\frac{K_{1}^{2}}{E^{\prime}}+\frac{K_{11}^{2}}{E^{\prime}}$

\subsubsection{Critical Energy Release Rate}

As described by Anderson (2005), if the crack forms an infinitesimal kink at an angle $\alpha$ from the plane of the crack, the local stress intensity factors at the tip of this kink differ from the nominal $K$ values of the main crack. We define a local $x-y$ co-ordinate system at the tip of the kink and the following equations define the local stress field $k_{I}$ and $k_{I I}$ are the local stress intensity factors of the crack tip.

$k_{I}(\alpha)=\sigma \sqrt{\pi a}\left[\left(\cos ^{2} \beta+k \sin ^{2} \beta\right) C_{11}(\alpha)+\sin \beta \cdot \cos \beta(1-k) C_{12}(\alpha)\right]$

here, coefficients $C_{11}$ and $C_{12}$ are given by:

$$
\begin{aligned}
& C_{11}=\frac{3}{4} \cos \left(\frac{\alpha}{2}\right)+\frac{1}{4} \cos \left(\frac{3 \alpha}{2}\right) \\
& C_{12}=\frac{3}{4}\left[\sin \left(\frac{\alpha}{2}\right)+\frac{1}{4} \sin \left(\frac{3 \alpha}{2}\right)\right]
\end{aligned}
$$

The energy release rate for the kinked crack is given by:

$$
G(\alpha)=\left(\frac{k_{I}^{2}(\alpha)+k_{I I}^{2}(\alpha)}{E}\right) .
$$

The maximum energy release rate is

$$
G_{\max }(\alpha)=\frac{k_{I}^{2}(\alpha *)}{E}
$$

where $\alpha *$ is the angle at which both $\mathrm{G}$ and $k_{I}$ exhibit a maximum and $k_{I I}$ is 0 . 
Failure occurs when the local stress exceeds the material strength. A failure criterion for brittle materials was also developed by Irwin (1957) on the basis of critical stress intensity required to cause unstable crack propagation. It can be described as $K_{I}>K_{I C}$. Here, $K_{I C}$ is the critical stress intensity factor for the Mode I fracture (plane strain).

\subsubsection{J-Integral Theory}

For elastic and plastic cases, Rice (1968) introduced the J-integral theory which is used to describe the wide application of fracture mechanics based on the assumption that for non-decreasing stresses, nonlinear elasticity theory can be used to effectively model plastic behaviour. This expression is a path-independent integral taken around the curve $\Gamma$, given by

$J=\int_{\Gamma}\left(W d y-T_{S} \frac{\delta u}{\delta x}\right) d s$,

where $J=$ energy release rate, $W=$ strain energy density; $T_{s}=$ Traction vector normal to ds; $u=$ displacement vector; $d s=$ small increment along path $\Gamma$.

As with the other criteria, unstable failure occurs when energy release rate, $J$ exceeds the critical value $J_{c}$.

For linear elastic material, Rice (1968) defines the J-integral as equal to the energy release rate.

The J-integral around a crack tip is illustrated in Figure 2.7. 


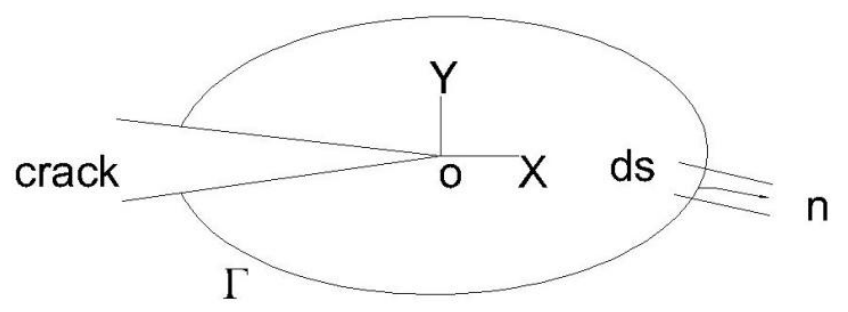

Figure 2.7: Illustration of J-integral around the crack tip

\subsection{Compressive Ice Failure}

When designing offshore structures for ice environment, one of the most important considerations is compressive ice failure. Ice behaviour depends on the rate of loading, and at low rates of loading, the deformation of ice is more likely to be viscoelastic. When the loadings increase, effects of fracture, damage and microstructural change become more prominent.

Ice compressive failure is a complex process. It may result ice induced vibrations in an offshore structure because of its cyclic nature (Jordaan et al. 2008). In the case of compressive failure, small zones of high pressure through which the majority of ice loads are transmitted are formed by spalls and splits. It has been observed that these high pressure zones $(\mathrm{hpzs})$ cover approximately only $10 \%$ of the global interaction area (Taylor et al., 2008). Spalling or crushing processes are associated with various mechanisms such as macrofracture, microfracture, recrystallization and pressure melting, which play different roles in the formation and development of hpzs (Jordaan et al. 2008; Jordaan, 2001). Crushing primarily has been associated with damage processes within 
$h p z s$, but spalling fracture is primarily associated with stress, geometry and flaws (Taylor et al., 2008).

Ice contains many natural internal flaws as a geophysical material which is a very important consideration for designing laboratory ice. Larger samples of ice have a higher probability of containing critical flaws, which cause the larger specimens to fracture at a lower stress level (Taylor, 2010).

As discussed by Sinha (1979) and Jordaan (2001), ice is a viscoelastic material with a strong time dependence of stress-strain behaviour. Variation in pressure is observed in the zones of high pressure even at low loading rates. The ice then undergoes microstructural change under compressive states and becomes prone to fracture. Failure initiates in localized areas where the contact is made. The global interaction area, defined as the projection of the structure onto the shape of the ice features increases as the interaction continues. This area includes spall area or high and low pressure zones as shown in Figure 2.8 (Jordaan et al. 2008). 

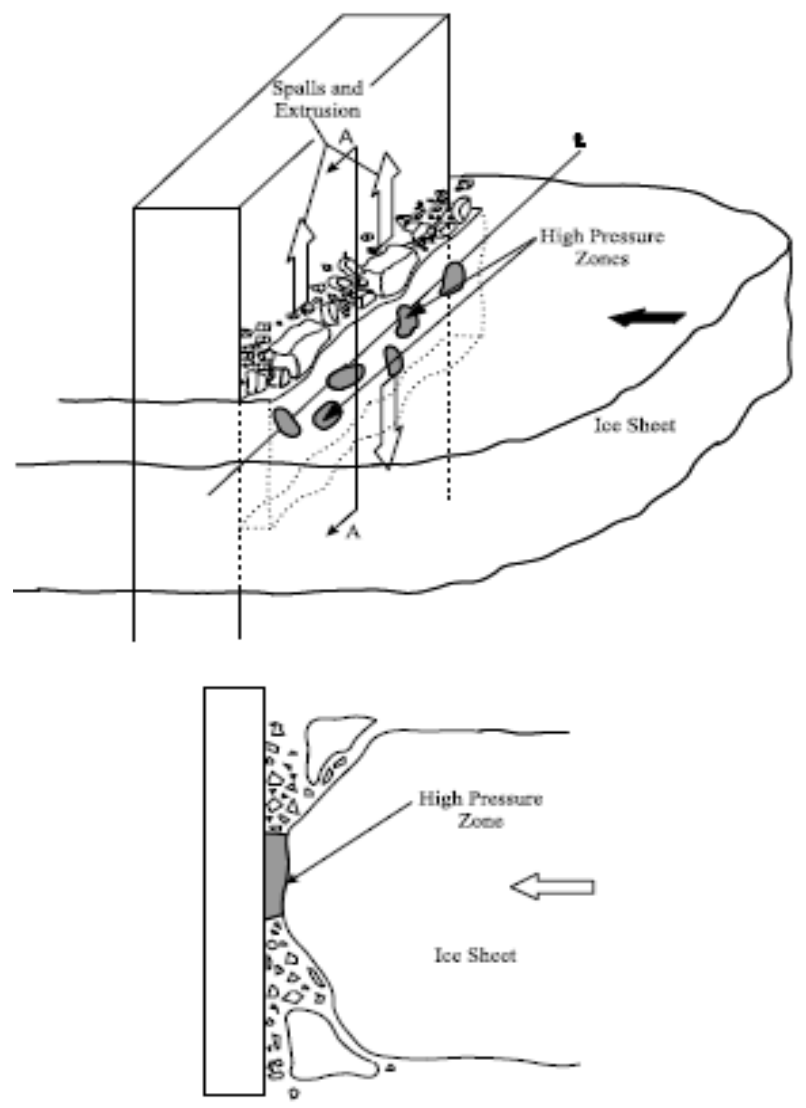

Section A-A

Figure 2.8: Schematic illustration of the main processes of spalling, extrusion and highpressure zone formation (Jordaan, 2001)

\subsubsection{Microstructural Damage in Ice}

During ice crushing tests, ice undergoes microstructural modification adjacent to the interface of ice and structure. This microstructural change is referred to as 'damage' when microstructural modifications change the original grain distributions and mechanical properties of ice. This process consists of irreversible components, which include microcracking and dynamic recrystallization, as well as pressure melting, which is a thermodynamically reversible process. 
The fracture process of ice depends on the indentation rate where the transition happens from creep and ductile mode to brittle mode as the indentation rate increases. The fracture behaviour and this transition also depend on other factors. As described by Sanderson (1988), the transition of deformation mechanics occurs as a result of complex interactions of the applied strain rate, temperature, load level and flaws in the ice.

Designing ice in the laboratory is always a challenge because in nature formation of ice is a geophysical process. In laboratory ice, the presence of irregularities and internal flaws is usual. So, to maintain the consistency for the quality of the ice specimens is very important. In the laboratory, ice can be produced in such a way that there are few or no internal flaws.

A fracture process can be either propagation-controlled or nucleation-controlled. According to Sanderson (1988), when a load is applied to the ice, it will deform, causing dislocations to glide within grains and pile up at grain boundaries resulting in local stress concentrations. Relieving of these stresses will cause microcracks along grain boundaries where the cracks will be under shear stress. This condition is referred as crack nucleation. A fracture process is called propagation-controlled when the applied load induces crack nucleation but the microcracks are too small to propagate and the load needs to increase until propagation (Sanderson, 1988). In both cases the cracks will cause the failure of ice. If the applied load is large enough to produce big microcracks that propagate the instant they form, this is called the nucleation-controlled fracture process (Sanderson, 1988). According to Schulson (1999), the brittle compressive failure of ice as a multi-step process is as described below: 
1) Crack nucleation at grain boundaries at a given applied stresses is approximately one quarter to one third of the failure stress.

2) Crack density increases progressively and uniformly as load rises.

3) Sudden formation of one or more macroscopic shear failures can result in complete failure of ice.

Schulson (1999) also mentioned that shear cracks nucleate along grain boundaries followed by the formation of wing cracks as a result of frictional sliding of the shear cracks. As described by Taylor (2010), for low confining pressures failure was observed to occur by axial splitting as it is typical for uniaxial compression tests, while shear fracture was observed to occur for increased confinement (Rist et al., 1988; Weiss and Schulson, 1995). Boundary conditions have a significant effect on the observed mode of fracture as failure of cylindrical specimens under confinement was observed to result from the linkage of microcracks along the direction of maximum shear stress. Previously, Raj and Ashby (1971) first modeled the local tensile and compressive stresses generated by shear traction during grain boundary sliding. Jordaan and McKenna (1988) modeled two possible mechanisms of shear crack formation in ice subjected to compressive loading due to the grain boundary sliding as represented in Figure 2.9. 


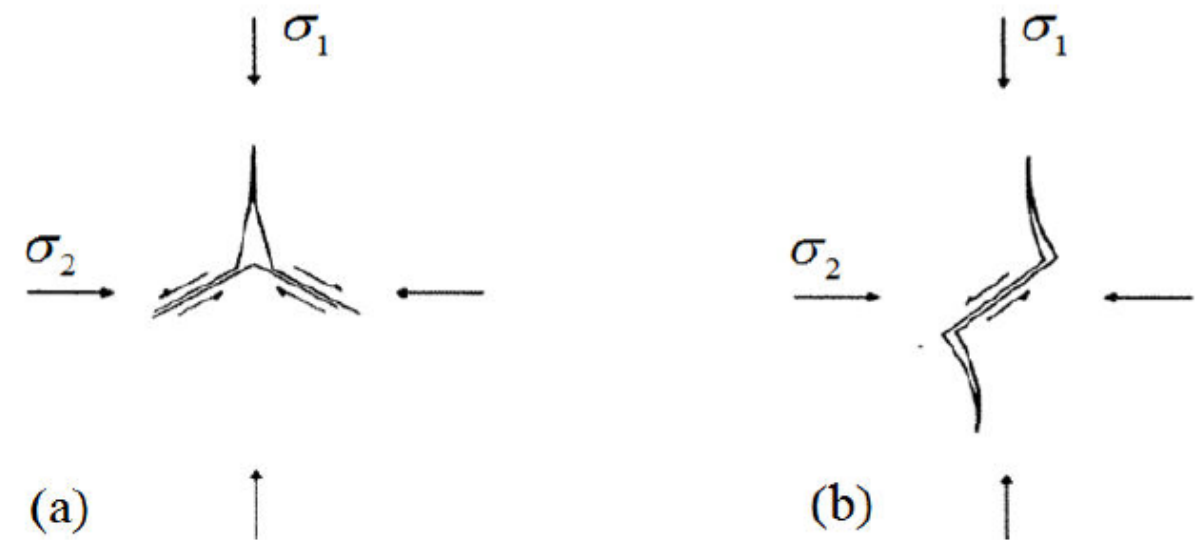

Figure 2.9: Crack formation due to grain boundary sliding for (a) triple point junction of the grains and (b) 'wing' cracks between two grain boundaries (Jordaan and McKenna, 1988)

For higher confinement pressure of more than $20 \mathrm{MPa}$, shear fracture is inhibited and the failure is dominated by viscoelastic flow (Murrel et al., 1991). Melanson (1998) and Li et al. (2005) reported that between the confinement pressures of $30 \mathrm{MPa}$ and $50 \mathrm{MPa}$, little microfractural effect is evident.

The indentation rate during ice-structure interaction has a strong effect on the failure process. During a test with an indentation rate of $0.3 \mathrm{~mm} / \mathrm{s}$, a permanent depression with evidence of substantial damage in the vicinity of the indenter was observed in Hobson's Choice Ice Island medium-scale experiments (Frederking et al., 1990) as shown in Figure 2.10. The experiment was done with a rigid spherical indenter of $0.8 \mathrm{~m}^{2}$ area, radius of curvature of $1.28 \mathrm{~m}$ and overall diameter of $1 \mathrm{~m}$. In this experiment localized spalling and ejection of ice were not evident but a large spall and creep deformation were observed. 


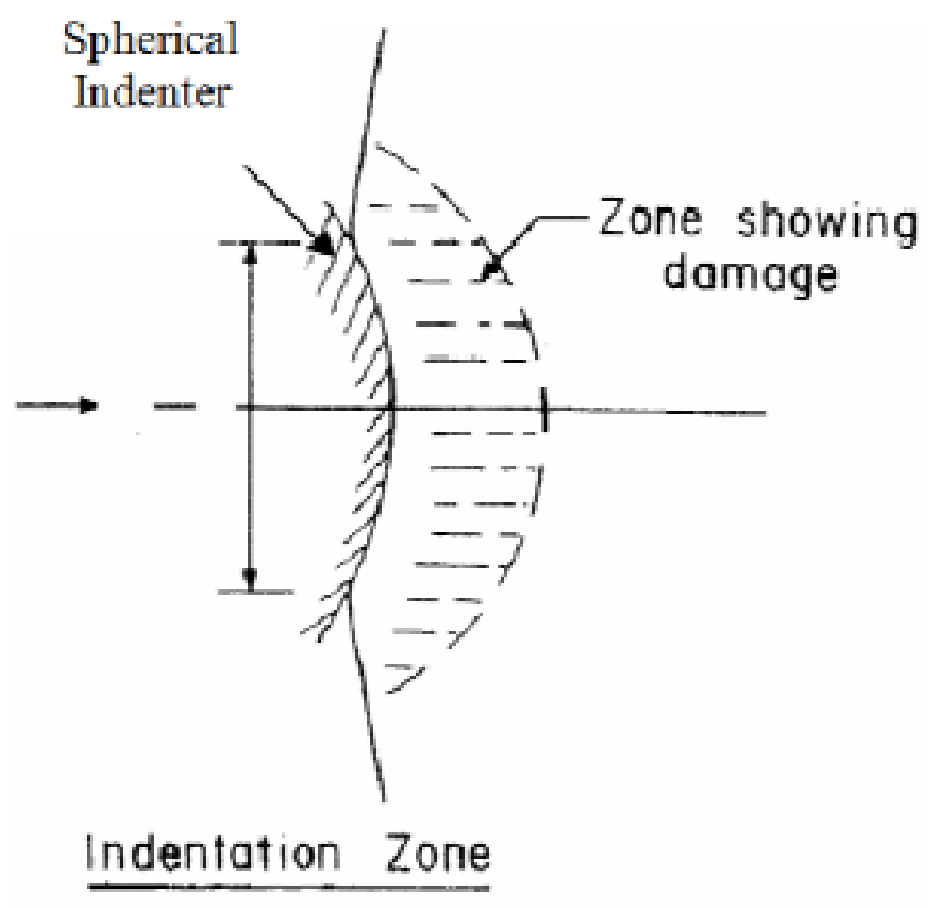

Figure 2.10: Test results from Hobson's Choice Ice Island experiments (Frederking et al., 1990, modified from Taylor, 2010)

Thin-sectioning results show significant recrystallization without any distinct layer in the indentation region. The report of Masterson et al. (1999) shows similar ductile failures for indentation speeds of $0.03 \mathrm{~cm} / \mathrm{s}$. A permanent depression with some localized radial cracking near the indentation zone was observed without any evidence of crushing and spalling, as shown in Figure 2.11. 


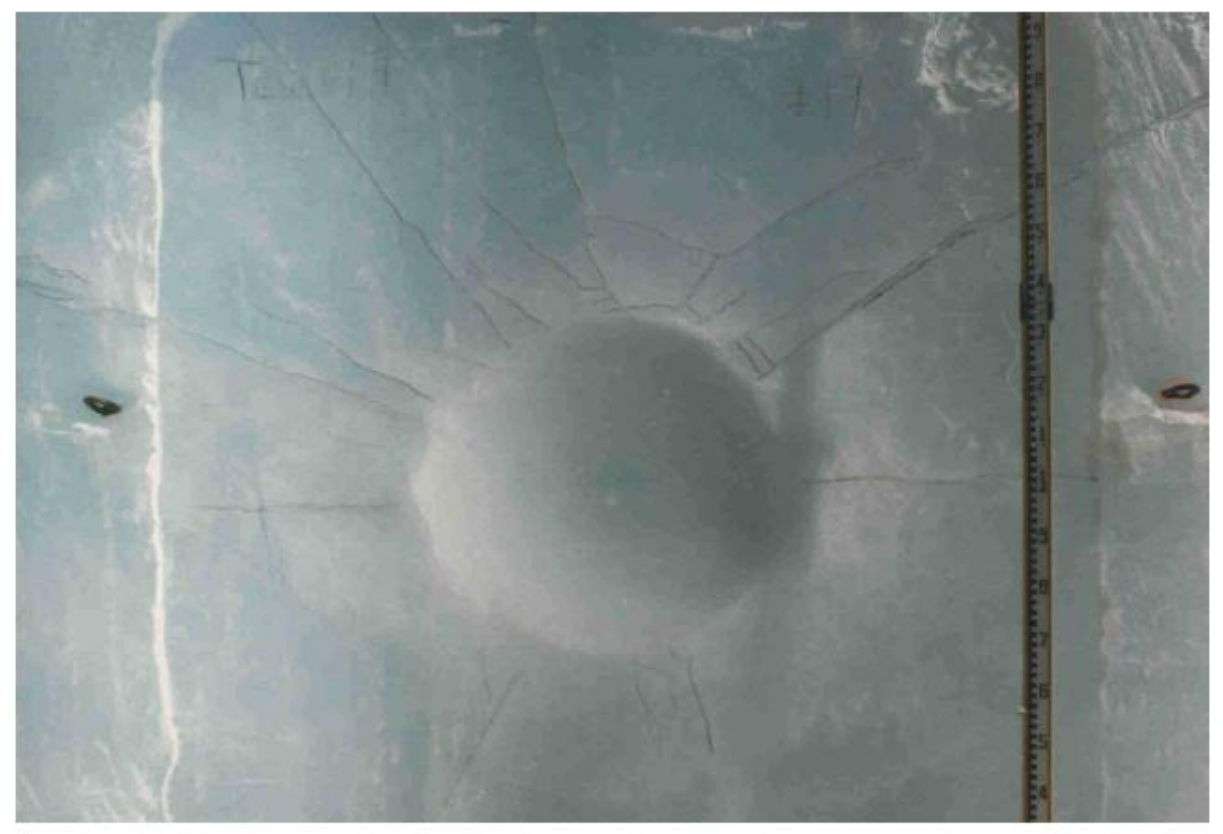

Figure 2.11: Ductile failure observed for slow test ( $\mathrm{v}=0.03 \mathrm{~cm} / \mathrm{s})$ during Rae Point experiments with $1 \mathrm{~m}^{2}$ spherical indenter (Masterson et al., 1999)

Failure of ice changes from the ductile to the brittle range as the indentation rate increases, resulting in the development of a highly damaged layer with crushing and extrusion of pulverized ice. Kheisin and Cherepanov (1976) first reported this mechanism by conducting a drop test of a $300 \mathrm{~kg}$ steel ball on an ice sheet and observed microcracking along the basal planes. From Hobson's Choice medium scale experiments (Frederking et al., 1990) an extensive white layer of crushed material with occasional 'blue' recrystallized zones was observed (Jordaan, 2001). During full scale iceberg impact tests conducted on Grappling Island, Labrador (Crocker et al., 1997) a layer of fine grained and microcracked ice in the thin-sections of ice samples was observed (Muggeridge and Jordaan, 1999). They also reported that the "crack like" layer boundary 
was similar to an extrusion plane and grains within the zone are forced toward the free surface.

Figure 2.11 shows the result of a medium-scale field test at $1 \mathrm{~cm} / \mathrm{s}$ during Rae Point experiments (Masterson et al., 1999). In fast tests, the damaged layer becomes prominent in the area adjacent to the indenter. Spalling and extensive microcracking are prevalent in the outer areas of the indentation zone where the ice is subjected to high shear and low confining pressures. In the interior regions, microcracking and recrystallization accompanied by pressure softening are prominent.

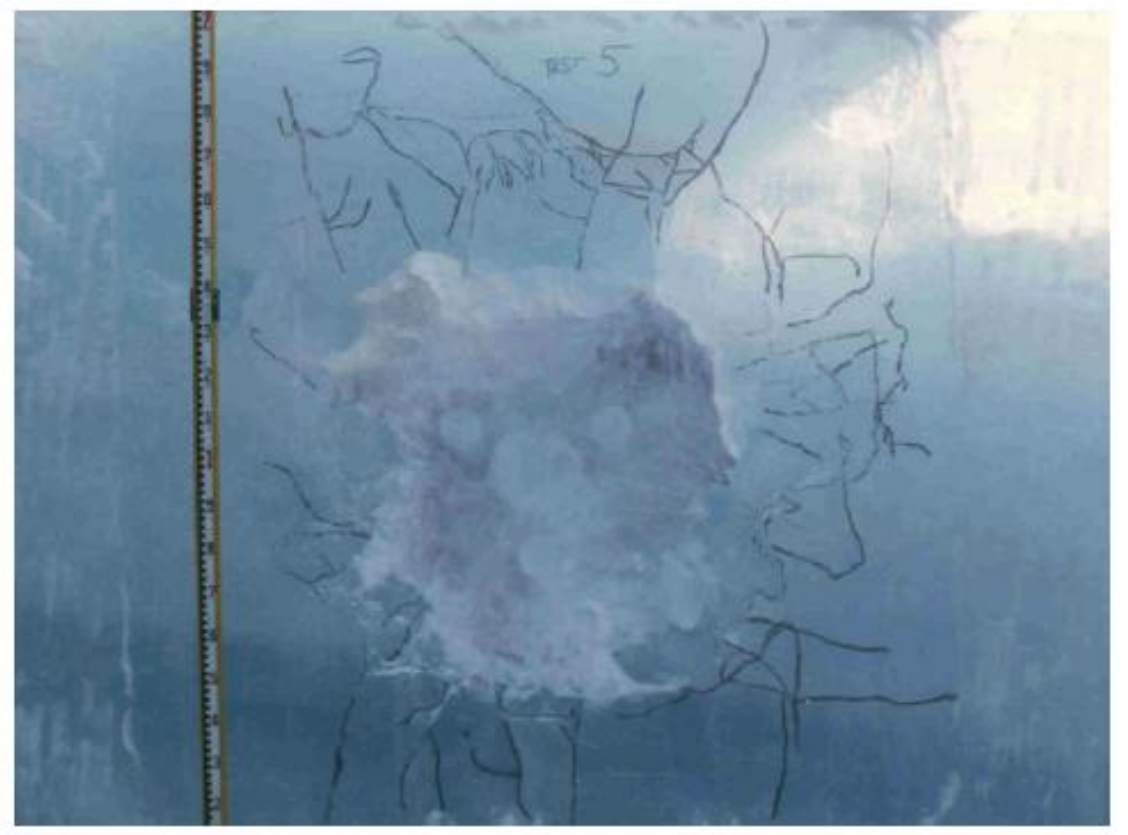

Figure 2.12: Brittle failure observed for fast test $(\mathrm{v}=1.0 \mathrm{~cm} / \mathrm{s})$ during Rae Point experiments with $1 \mathrm{~m}^{2}$ spherical indenter (Masterson et al., 1999)

Investigation of brittle failure is more challenging than ductile failure due to the complexity of internal flaws, crushing behaviour and random pressure distributions. 
Jordaan and Xiao (1992), Xiao (1997) and Li (2007) have done extensive research on the interplay between damage and fracture.

\subsubsection{Dynamic Recrystallization}

Poirier and Guillope (1979) described dynamic recrystallization as change of the grain size, shape and orientation with little or no chemical change, due to deformation-induced reworking. This is the process that involves formation or migration of grain boundaries (Vernon, 1981). The term dynamic recrystallization refers to the process of recrystallization that is associated with the progress of deformation; otherwise it is referred to as static recrystallization (Xiao, 1997). Urai et al. (1986) defined dynamic recrystallization as changes in grain size, grain boundary structure and dislocation density and sub-structure.

Dynamic recrystallization serves to increase material softening and enhance creep behaviour. It results a change in the grain boundaries by increasing or decreasing grain size. Poirier (1985) proposed an expression to define a new generated grain size, $D$ as a result of recrystallization that only depends on the applied stress.

$\frac{D}{b}=K\left(\frac{\sigma}{\mu}\right)^{-r}$

where, $b, K, \mu$ and $r$ are constants. Duval et al. (1983) stated that dynamic recrystallization does not initiate under low stresses and is periodic under intermediate stresses. He also reported that with increasing strain rate a continuous dynamic recrystallization process is observed and results in more distinct softening of ice. Urai et 
al. (1986) found that dynamic recrystallization of polycrystalline material under a triaxial load depends on hydrostatic pressure. He suggested that the driving force behind dynamic recrystallization has four main contributors. They are: internal energy sources, surface energy of grain boundaries, intragranular lattice defect energy and elastic energy due to external loading. Meglis et al. (1999), Passchier and Trouw (2005) and Wei and Dempsey (1991) have further investigated the influence of dynamic recrystallization with the influence of the creeping effect, pressure melting and kinking mechanisms.

\subsubsection{Pressure Melting}

Like dynamic recrystallization, pressure melting is another mechanism of interest for ice that is subjected to high confining pressure. The melting point of ice decreases with the increase of pressure (Nordell,1990). Early work by Hobbs (1974) defined pressure melting based on the thermodynamic equation as

$\frac{d T_{m}}{d p}=\frac{\left(V_{w}-V_{i}\right)}{\left(S_{w}-S_{i}\right)}$

where $T_{m}$ is the melting temperature of ice in Kelvin, $p$ is pressure, $V_{w}$ and $V_{i}$ are the specific volumes of water and ice and $S_{w}$ and $S_{i}$ are the entropy of water and ice.

According to the second law of thermodynamics, the denominator of the right hand term of the equation should be positive. Also, the density of ice is less than the density of water so the numerator of the equation is negative. Therefore, the melting point of ice is inversely proportional to the pressure. Using Clausius-Clapeyron's equation, the equation can be given as:

$d T_{m}=-A d p$ 
Here, $A$ is a constant that depends on temperature. For ice at $0^{\circ} \mathrm{C}, A=0.0742^{\circ} \mathrm{C} / \mathrm{MPa}$ and at $-10^{\circ} \mathrm{C}, A=0.0833^{\circ} \mathrm{C} / \mathrm{MPa}$.

The experimental work on the relationship between pressure melting and the temperature of ice is studied by a number of authors. Some of the results are presented in Figure 2.13. From the graph it can be seen that at $-2.7^{\circ} \mathrm{C}$, the pressure to cause melting is $35 \mathrm{MPa}$. Although, when the temperature is low as $-10^{\circ} \mathrm{C}$, the pressure melting increases to 120 MPa.

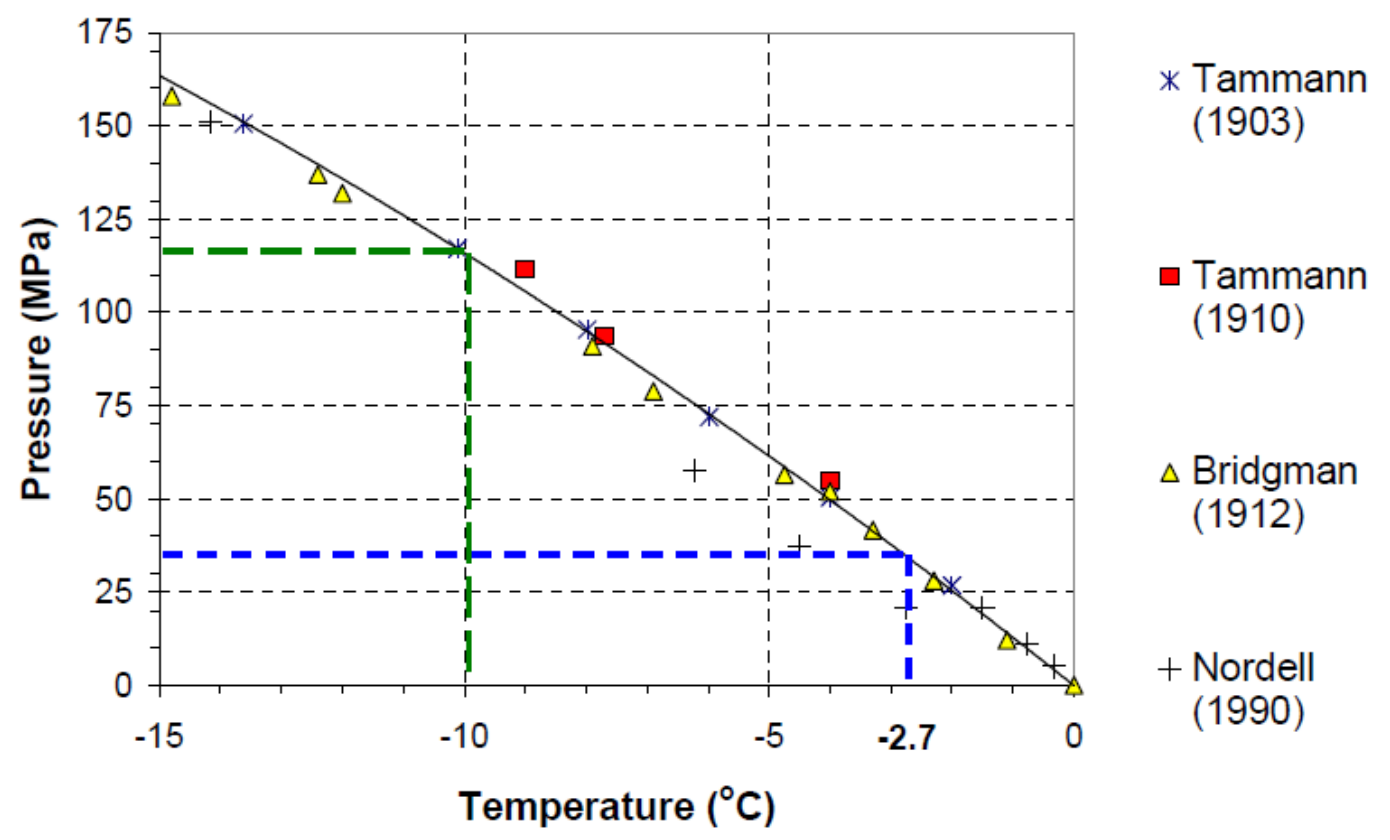

Figure 2.13: Plot of pressure melting data for ice (Taylor, 2010) 


\subsubsection{Local and Global Ice Pressure}

As defined by Jordaan et al. (2005a), when the structure is projected onto the original shape and size of the ice feature, and the area reduction caused by spalling and fracture during interaction is not considered, it is referred to as the global area or nominal interaction area. It contains the area with little or no pressure and also the high pressure zones. The local design area is a smaller part of the global area that subjected to higher local pressure than average global pressure and used to design the shell, panel or the stiffening elements of a structure. Figure 2.14 defines the global and local area as given by Jordaan et al. (2005a).

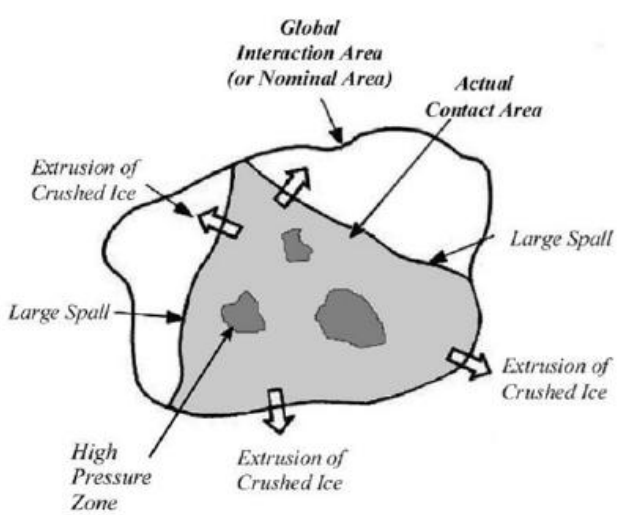

(a)

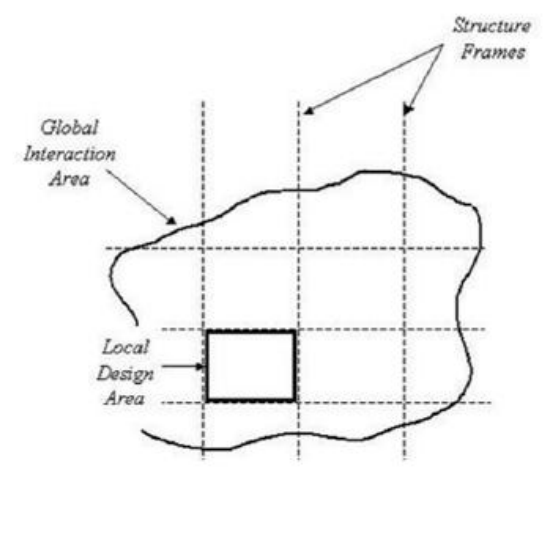

(b)

Figure 2.14: Illustrations of (a) global interaction area and (b) local design area (Jordaan et al., 2005a)

Typically, global pressures are determined for areas from ten square meters to hundreds of square meters and local pressures are associated with areas from $0.6 \mathrm{~m}^{2}$ to $10 \mathrm{~m}^{2}$ (Jordaan et al., 2005a). Designing for local pressure is an important factor in ice mechanics because the global loads are the sum of local loads. 
As described by Taylor (2010), local pressure data were used to produce estimations of global pressure based on three different approaches. They are (1) standard correlation probabilistic averaging; (2) composite correlation probabilistic averaging, (3) linear extrapolation of local pressures.

\subsubsection{Pressure-Area Scale Effect}

The definition of the scale effect in ice-structure interaction is that average pressure decreases with increasing contact area (Sanderson, 1988). While there is no scale effect in the classical theories of elasticity, viscoelasticity or plasticity, in the case of ice compressive failure, there is evidence of scale effect. There are mainly two factors which are dominant in this situation. The first factor is that ice is prone to fracture, so it reduces the volume of material to be crushed and aids in the formation of hpzs. The second factor is probabilistic averaging, which results from the transition from local pressure on small areas to global pressure, where the effect is averaged (Jordaan et al. 2006).

When a ship strikes a large ice feature, the failure process induces spalling events and extrusion of the ice. This is due to the fracture related to flaws in the ice mass and the nature of the crushing process. The occurrence of fractures during interaction is random. Accordingly, the load reduction and scale effect in an arbitrary impact are random and the crushing strength of different ice floes is also random. As it is known that the average global pressure decreases with increasing nominal contact area (Sanderson 1988), Jordaan et al. (1996) developed alternative model of the pressure-area relationship to 
represent ice strength as a whole including these failure effects. The pressure-area power law relationship can be described as

$P=C A^{-D}$

where the area is treated as being normalized, i.e., divided by a reference area, say $1 \mathrm{~m}^{2}$. Then $A$ is dimensionless, and $C$ has units of pressure. Typically $C$ is lognormally distributed with a mean of 3 and standard deviation of 1.5 , and $D$ is normally distributed with the mean of 0.4 and standard deviation of 0.2 .

If the nominal interaction area is $A_{T}$ and the area free of pressure $A$, the effective stress on the remaining area is

$\sigma_{e}=\frac{F}{\left(A_{T}-A\right)}=\frac{F}{A_{T}(1-D)}$

Here, $F$ is the load and $D=\frac{A}{A_{T}}$ is a measure of the damage caused by fracture. The decrease of average pressure with the global interaction area constitutes a scale effect. In the 'classical' analysis of indentation using rigid indenters against elastic or plastic materials, the failure pressure will not depend on the area. As an example, the von Mises plane strain solution for the applied pressure $P$ at failure is

$\frac{P}{\sigma_{y}}=2.97$

Here, $\sigma_{y}$ is the yield stress of ice. As can be seen, there is no dependence of force on area in this equation. Similar results can be found for elastic and viscoelastic materials. There is considerable evidence of scale effect, where average pressure decreases with the global interaction area because of the fracture of ice and probabilistic averaging. 
Pressure-area scale effect also implies that a panel of constant width should experience decreasing pressure as a function of increasing ice thickness (Taylor, 2010). The relationship between pressures and ice thickness has been studied with full scale data for panels of approximately unit width from the datasets of Molikpaq, STRICE, JOIA and Cook Inlet. Results indicate that a trend of decreasing pressure with increasing thickness is observed as expected from the pressure-area effect (Taylor, 2010).

In summary, present work is an attempt to understand the fracture processes, compressive ice failure, microstructural changes, recrystallizations, force and pressure effects on various factors that have been considered for the test program.

\subsection{Review of Previous Indentation Test Programs}

Full, medium and small-scale indentation tests have been conducted to investigate material properties, crushing behaviour, pressure distributions and mechanisms of failure. In this section relevant small-scale indentation tests that have been conducted recently will be discussed. The tests are primarily focused on the investigation of failure processes, behaviour of high pressure zones and microstructural modifications at various indentation rates, temperatures and ice specimen structures.

Small-scale ice indentation tests done by Barrette et al. (2002) were based on the interaction between a small spherical indenter and freshwater, polycrystalline ice having a grain size between 3.35 and $4.75 \mathrm{~mm}$. The ice specimens they used were confined in a cylindrical steel mould having $150 \mathrm{~mm}$ internal diameter and 60-130 $\mathrm{mm}$ height. They 
used a spherical steel indenter with a diameter of $20 \mathrm{~mm}$ and a radius of curvature of 25.6 $\mathrm{mm}$. This indenter is scaled down by the reduction factor 50 from the spherical indenter used in Hobson's Choice Ice Island medium-scale tests (Frederking et al. 1990). They investigated the effect of temperature and displacement rate on the response of a single high pressure zone under these circumstances. They also studied the physical characteristics of ice damage and layer formation with the changed morphology of the ice near the ice-indenter interface. All the tests were done with a Materials Testing System (MTS) test frame in a cold room, the indentation rate ranging from 0.1 to 10 $\mathrm{mm} / \mathrm{s}$ at temperatures of $-2^{\circ} \mathrm{C},-10^{\circ} \mathrm{C}$ and $-20^{\circ} \mathrm{C}$ up to the penetration depth of $2.6 \mathrm{~mm}$. To produce thin-sections of the indented area and to study the modified damaged layer, they used the double-microtome method developed by Sinha (1977) following each indentation test.

Figure 2.15(a) presented below is from an indentation test done by Barrette et al. (2002) at $-10^{\circ} \mathrm{C}$ with a displacement rate of $4 \mathrm{~mm} / \mathrm{s}$. The force-time data follows a cyclic loading with peak frequencies in the order of $50-100 \mathrm{~Hz}$ as shown in the graph. In Figure 2.5(b) the indented ice surface was defined by a dark zone having an irregular shape and a surface area surrounded by lighter areas of crushed ice. From the thin-section pictures these darker areas indicate areas of recrystallization and the lighter areas indicate that the region is heavily dominated by microfracture as defined by Jordaan (2001). 


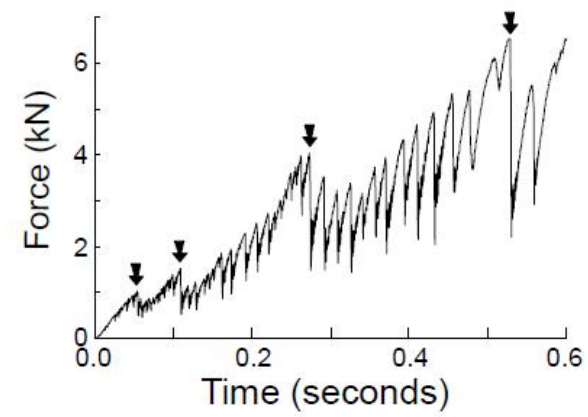

(a)

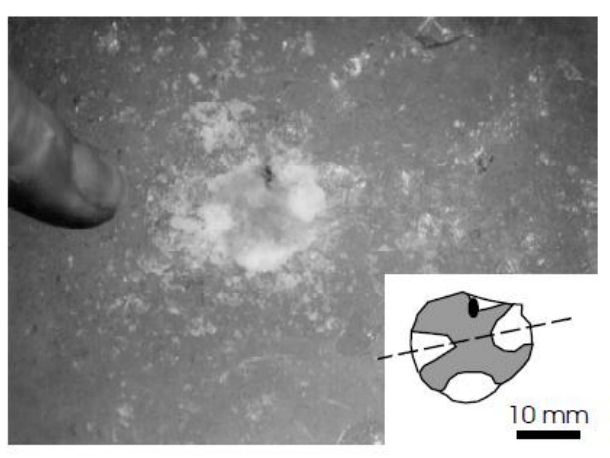

(b)

Figure 2.15: (a) Load trace data; arrows indicate large load drop. (b) Ice surface showing a dark grey, cross-shaped zone at the center in a light crushed ice matrix (Barrette et al., 2002)

They found that the damage layer produced by the interaction between the indenter and granular ice extended to a maximum depth of $4-5 \mathrm{~mm}$ below the surface of the indentation. Thin-sections of the high temperature test $\left(-2^{\circ} \mathrm{C}\right)$ show fine grained ice with little evidence of microcracking, and the low temperature test $\left(-20^{\circ} \mathrm{C}\right)$ is heavily dominated by microcracking. The test conducted at $-10^{\circ} \mathrm{C}$ follows a central recrystallized zone with a dense area of microcracks on both sides.

Wells et al. (2010) did similar indentation tests as Barrette et al. (2002) using the same type of $20 \mathrm{~mm}$ spherical indenter to create a single high pressure zone during icestructure interaction. The dimensions of their ice specimen were $20 \times 20 \times 10 \mathrm{~cm}$ and were made of polycrystalline, freshwater ice having a grain size of $\sim 4 \mathrm{~mm}$. The differences between the two programs are that Wells used an unconfined ice specimen and bubbles in the ice were removed using a vacuum mould. Tactile pressure sensor data and high-speed video recordings were also used to improve the understanding of local 
pressure distributions, the physical process of ice failure and the dynamic activity of the high pressure zone at the ice-structure interface. The tests were conducted at an indentation rate ranging from 0.2 to $10 \mathrm{~mm} / \mathrm{s}$ at $-10^{\circ} \mathrm{C}$. The indentation depth was from 3 to $20 \mathrm{~mm}$. They found the failure process to be highly dependent on indentation rate. They reported that for the slow speed tests $(0.2 \mathrm{~mm} / \mathrm{s})$ failure was mostly ductile without showing any evidence of spalling, crushing or cyclic loading. In medium ranges (2-5 $\mathrm{mm} / \mathrm{s}$ ) the behaviour of ice failure was unpredictable and showed a mix of ductile and crushing failure. In this range some localized spalling, isolated crushing and cyclic loading events were observed. In fast indentation tests $(8-10 \mathrm{~mm} / \mathrm{s})$ spalling became more dominant and showed an inclination to brittle failure with mixed mode behaviour. Wells et al. (2010) classified the failure behaviour of ice into five categories as shown in Figure 2.16. They described ductile failure as a steady rise of total force with time, with little or no localized spalling or crushing. When the indentation rate is increased to more than $2 \mathrm{~mm} / \mathrm{s}$, the ductile behaviour is interrupted by localized spalls, mixed mode or isolated crushing events. Breaking a single flake of ice with a sharp load drop from the periphery of the damaged layer is indicated as a localized spall. In this case a high pressure zone is created prior to the spalling, and just after the removal of spall a drop of force and reduction of pressure are observed due to the loss of contact area. 


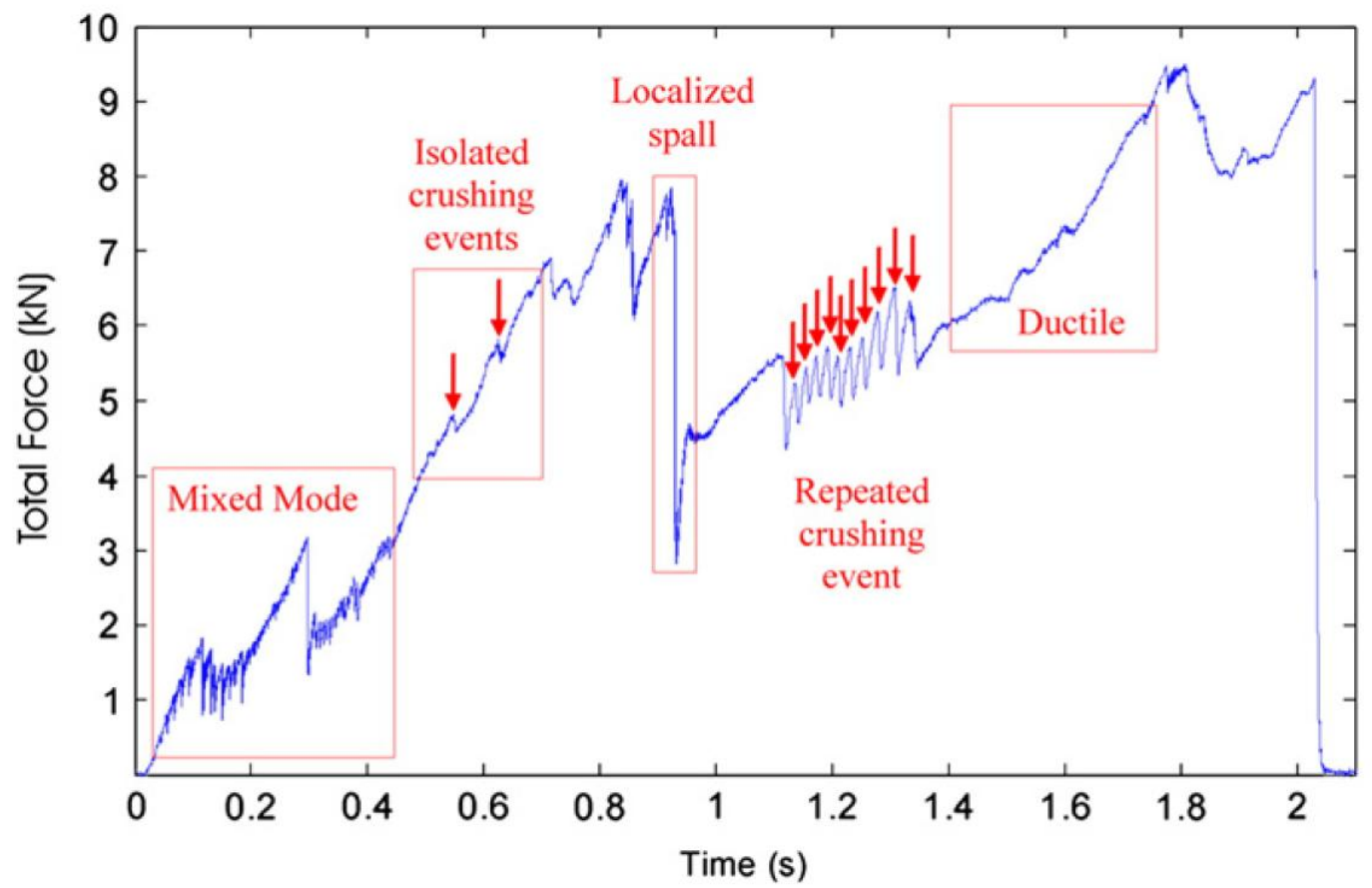

Figure 2.16: Total force from MTS load cell identifying five different failure modes under compressive failure of ice (Wells et al., 2010)

Wells classified crushing failure as either an isolated crushing event or a repeated crushing event. An isolated crushing event is defined as a load drop with decrease of pressure from the center of the $h p z$ with little change in the contact area. This event is accompanied by the extrusion of crushed ice particles from the periphery of the $h p z$ due to the sudden failure of hpzs. Repeated crushing events are described as dynamic activity with an increasing trend of force data without interruption by any other failure processes. This failure behaviour was observed mostly in the tests that were done at $2-5 \mathrm{~mm} / \mathrm{s}$. The mixed mode failure refers to a combination of crushing and small localized spalls having irregular force and pressure distribution with multiple load drops. Mixed mode behaviour 
was observed in the tests with indentation rates above $2 \mathrm{~mm} / \mathrm{s}$ and as the indentation rate increases the failure also shows an increasing trend.

Experiments done by Browne (2012) were an extension of the research done by Barrette et al. (2002) and Wells et al. (2010). A single high pressure zone was successfully modeled using small, spherical, steel indenters having diameters of 20 and $40 \mathrm{~mm}$ with radii of curvatures of 25.6 and $51.2 \mathrm{~mm}$ to indent ice specimens and confined in steel moulds. Freshwater, polycrystalline ice samples having 150 and $300 \mathrm{~mm}$ in diameter were made of $2.00-3.35 \mathrm{~mm}$ or $3.35-4.75 \mathrm{~mm}$ ice seeds. All the tests were done using a Materials Testing System with indentation rate varying from $2 \mathrm{~mm} / \mathrm{s}$ to $10 \mathrm{~mm} / \mathrm{s}$ and temperature from $-5^{\circ} \mathrm{C}$ to $-15^{\circ} \mathrm{C}$. Six different indentation systems consisting of four (4) compliant systems and two (2) rigid systems were used for the experiments. The compliant systems consisted of simply-supported steel beams of varying compliance, while the rigid systems were essentially as stiff as the MTS. To study the results, Browne used load and displacement data, high-speed video recordings and tactile pressure-sensor data. Following each test, thin-sections were prepared and photographed.

Analyzing the effect of temperature, Browne stated that a warm test $\left(-5^{\circ} \mathrm{C}\right)$ follows a ductile failure resulting in a steady increase in load without any cyclic pattern, but that cold tests $\left(-15^{\circ} \mathrm{C}\right)$ are dominated by crushing and spalling with a regular cyclic loading pattern. He also found similar kinds of microstructural modification in the thin-sections as found by Barrette et al.(2002) and Wells et al. (2010). 
Among the six indentation systems, Browne (2012) observed that structural feedback has significant effects on the frequency and amplitude of the cyclic loading pattern. The compliant structure deflects under the load, which results in a decrease of relative loading rate, and continues to deflect until the strength of the ice is exceeded by the structural resistance. Another effect observed by Browne was that the frequency of cyclic loading has a positive, linear relationship with the indentation rate and the stiffness of the structure. Rigid indentation tests resulted in smaller load drops than compliant indentation tests.

Fracture and damage processes observed during the tests done by Browne (2012) were further discussed by Taylor et al. (2013). In Figure 2.17, thin section pictures and force trace plots for two different temperatures are given. From the thin section pictures it can be seen that the damaged layer of warmer ice is heavily dominated by a fine-grained, recrystallized microstructure, but colder tests show that only the central portion of the layer is recrystallized and that the outer edges are highly microfractured. From the forcetime plots, it can be observed that tests done at $-5^{\circ} \mathrm{C}$ follows a ductile failure, where as the test done at $-15^{\circ} \mathrm{C}$ has a number of cyclic loadings. The tests were conducted at 4 mm/s using Compliant System 4. 

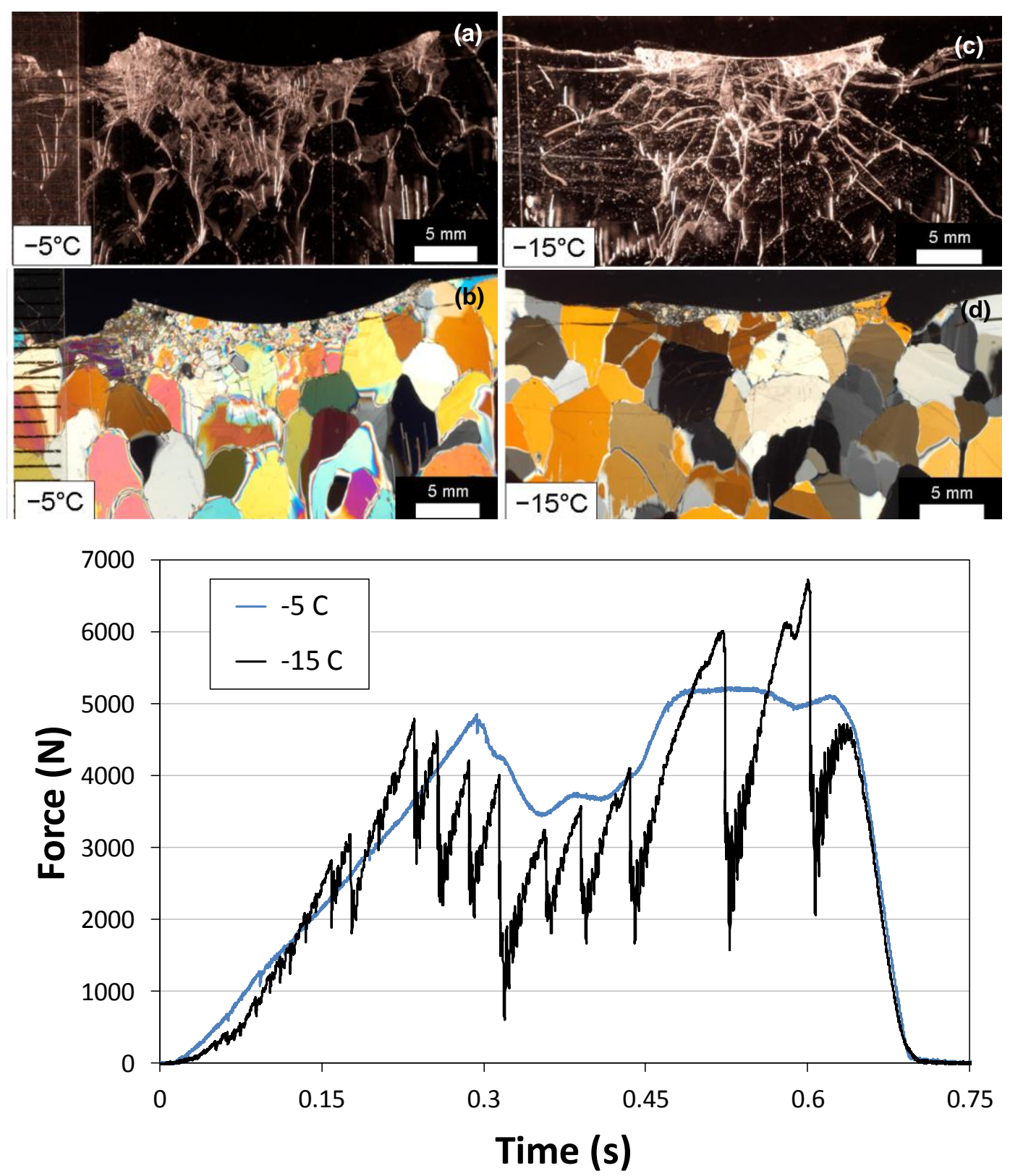

(e)

Figure 2.17: Thin-sections of indentation zones for tests conducted at $-5^{\circ} \mathrm{C}(\mathrm{a}-\mathrm{b})$ and $-15^{\circ} \mathrm{C}(\mathrm{c}-\mathrm{d})$. Side lighting is used to highlight fractures in (a) and (c); cross-polarized filters are used to highlight damage in (b) and (d). (e) plot of force-time traces for tests conducted with Compliant System 4 for warm ice $\left(-5^{\circ} \mathrm{C}\right)$ and cold ice $\left(-15^{\circ} \mathrm{C}\right)$ at an indentation rate of $4 \mathrm{~mm} / \mathrm{s}$. (Taylor, 2013) 
Small-scale indentation tests done by Dillenburg (2012) focused on indentation rate, temperature and crushing behaviour with conical shaped ice specimens having a $30^{\circ}$ cone angle. A total of seventy-two (72) experiments were done using a range of $0.01 \mathrm{~mm} / \mathrm{s}$ to $100 \mathrm{~mm} / \mathrm{s}$ crushing rates and testing temperatures of $-10^{\circ} \mathrm{C}$ and $-5^{\circ} \mathrm{C}$ with the same test setup and constant control parameters. Ice samples were made using 4-10 mm ice seeds and distilled, de-ionized and deaerated water chilled to $0^{\circ} \mathrm{C}$, used to fill the cylindrical ice mould. The ice sample was then shaped to the desired angle by the same ice shaping apparatus that was used for the current research program. Two (2) experiments were done without shaping the ice samples, and four (4) conical ice samples were used without the support of the ice holder. A cylindrical ice specimen was also used for testing to compare loads with the conical ice specimen. The MTS load frame located in the cold room at Memorial University of Newfoundland was used to record the force data against time and displacement. The tests were recorded with a high-speed video camera for fast speed tests and a regular camera with a time-lapse function for slow speed tests. Sampling rate ranges for the recordings were 0.2 to 2000 frames per second. The indenter modified by Dillenburg for the experiments had two components: a crushing plate and a backing plate, both having a diameter of $254 \mathrm{~mm}$. The crushing plate was made of stainless steel of $19.05 \mathrm{~mm}$ thickness and the backing plate was made of structural steel with $31.75 \mathrm{~mm}$ thickness. The contact surface of the crushing plate was circularly sanded and coarse roughness of the surface was 6.3-12.5 $\mu \mathrm{m}$. Every ice sample was weighed prior to and after the experiments and the spalls that were on the surface of the crushed 
ice samples, considered for weighing. After each test, thin-sections were done for horizontal and vertical sections of the ice sample using cross-polarized light.

Dillenburg (2012) processed all the experimental data with a Python software tool. After processing the data, nominal ice crushing pressure, energy, ice strength, maximum crushing force, nominal pressure at peak force and true crushing velocity were calculated. Dillenburg identified three ranges of ice deformation behaviour to investigate: ductile range $(0.01-0.1 \mathrm{~mm} / \mathrm{s})$, ductile to brittle range $(0.3-3 \mathrm{~mm} / \mathrm{s})$ and brittle range $(10-$ $100 \mathrm{~mm} / \mathrm{s}$ ). The spalls collected for ductile ranges had a weight of $13-16 \mathrm{~g}$ for both temperatures $-5^{\circ} \mathrm{C}$ and $-10^{\circ} \mathrm{C}$. At the indentation rate of $0.1 \mathrm{~mm} / \mathrm{s}$, the warmer test $\left(-5^{\circ} \mathrm{C}\right)$ shows spalls of $30 \mathrm{~g}$, but the colder test shows spalls of about $85 \mathrm{~g}$. At an indentation rate of more than $3 \mathrm{~mm} / \mathrm{s}$, the warmer ice shows a greater tendency to spall, with $15-60 \mathrm{~g}$ more ice mass than the colder ice samples.

Analyzing ice crushing strength, peak force and nominal pressure, Dillenburg found that the transition from ductile to brittle ice failure during warm tests seems to cause a shift to higher crushing rates compared to cold tests when observing ice crushing strength, peak force and nominal pressure at peak force. Figure 2.18 illustrates the results of crushing energy more clearly. It was also indicated that the test specimen temperature is more important in ductile ice deformation than it is in the ductile-to-brittle or brittle regimes.

Dillenburg (2012) observed that the influence of structural constraint around the specimen does not have a strong effect but helps to maintain the original shape. The support of the ice samples especially at a low crushing rate reduces the nominal pressure 
variation and brings it to a constant level. However, in the case of fast crushing rate, load increasing is interrupted by recurring load drops. She also compared the cylindrical shaped and conical shaped ice samples and found that the shape of the conical specimen allows a controlled increase in load compared to the cylindrical sample, which seems to deform rather unpredictably with sudden pulverizations.

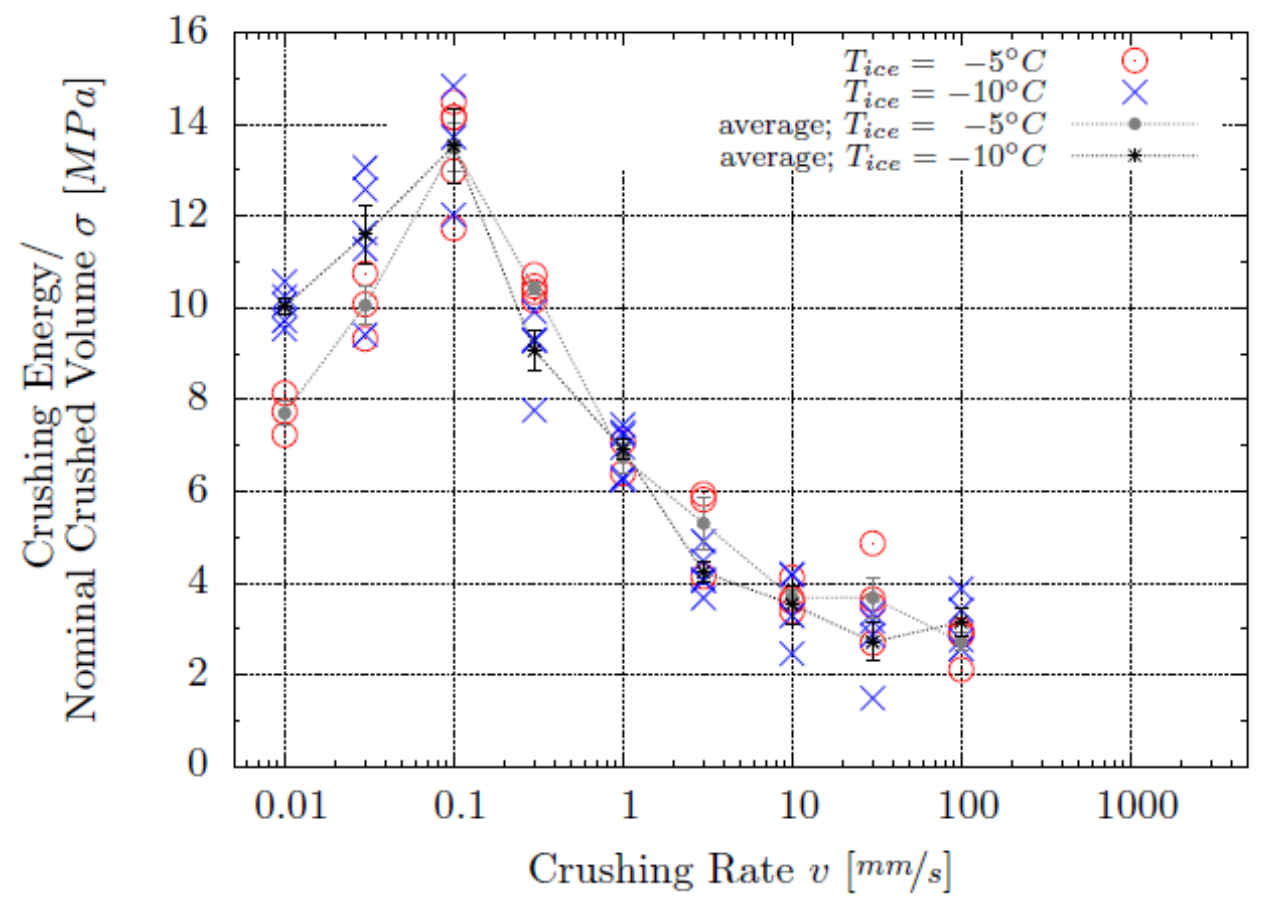

Figure 2.18: Ice strength plotted against crushing rate for ice samples for ice samples of different temperatures at $45 \mathrm{~mm}$ crushing depth (Dillenburg, 2012)

As part of the STePS ${ }^{2}$ research project at Memorial University of Newfoundland, Reddy et al. (2012) conducted some experiments by crushing $1 / 4 \mathrm{~m}$ and $1 \mathrm{~m}$ diameter conical ice specimens against a rigid high-resolution pressure panel in quasi-static conditions (provided by NRC-IOT). The pressure panel consisted of an array of strips of deformable 
polymer that responded to the local pressure and using a high-speed camera it was viewed from the bottom of the module. The following pictures in Figure 2.19 show the progression of internal pressure profile from the double angle cone experiment. The double angle cone had a $30^{\circ}$ cone and the top of the cone was converted to $10^{\circ}$ cone angle. From the images it can be seen that a considerable amount of change of cross sectional area is observed following each major fracture. Reddy et al. (2012) also developed a procedure to calculate nominal pressure by analyzing the frames.

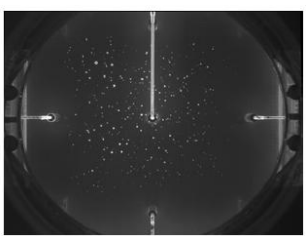

(a)

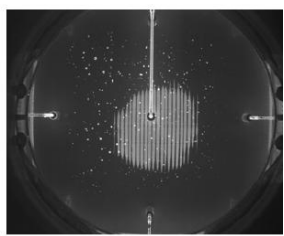

(b)

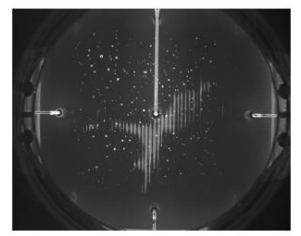

(c)

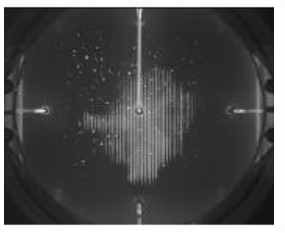

(d)

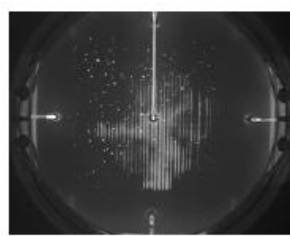

(e)

Figure 2.19: Progression of pressure profile from the results of double angle cone test (a) Initiation of loading (b) prior to the first major fracture (c) after the first major fracture (d) prior to the second major fracture (d) after the second major fracture (Reddy et al., 2012)

Similar types of experiments by crushing an ice cone with variable control parameters were previously done by a number of researchers (Bruneau et al. 2011, 2012, 2013, Dillenburg, 2012, Sullivan and Pilling, 2011, Dragt and Bruneau, 2013). In the present program, laboratory experiments have been done as a cost-effective means to study the influence of indentation rate, temperature and grain size on fracture and damage processes during the indentation of conical ice specimens using flat and curved indenters. 


\section{Chapter 3: Experimental Methodology}

\subsection{Overview}

In the present study, the experiments were designed to improve the understanding of crushing and failure of ice under compressive loading. In all the tests the ice samples were made of polycrystalline freshwater ice. A Materials Testing System (MTS) load frame was used to conduct the experiments and collect the force data against time and indentation depth. To study the pressure variations within the contact area, tactile pressure-sensor technology was used in selected tests. The failure behaviour of ice samples is observed from the recorded videos. The objectives of the present research are to study the behaviours of compressive ice failure and investigate the effects of indentation rate, temperature, ice sample geometry and grain size during compressive ice failure tests by using two types of indenters. In each test the parameters studied were ice specimen strength, maximum force from the data collected from the MTS data 
acquisition system, pressure distribution, the nature of loading, spall distributions and thin section picture analysis.

All the tests, water preparation, sample preparation and post experimental works were conducted in the cold room of the Thermal lab at Memorial University of Newfoundland.

\subsection{Design of Experiment}

Natural ice features such as icebergs will have irregular surface and local protrusions that will contact a structure during an interaction. In the present study a conical ice specimen has been used to represent idealized shape for those local ice features. Spalling fracture is an important aspect of the compressive ice failure process. For sharp cones, the onset of crushing begins from first contact, making it more difficult to isolate individual fracture events. Using truncated cones allows for a build up at elastic stresses after contact, making it easier to isolate the initial spalls and associated local drops. An area of about $10 \%$ of total was used for the initial contact area based on $h p z$ data from the JOIA program (Frederking, 2004, Taylor et al., 2008).

As illustrated in Figure 3.1, conical ice specimens were used and had an average diameter of $268.8 \mathrm{~mm}$ at the bottom and $25.4 \mathrm{~mm}$ at the top. The indentation for each test was done from the top surface of the ice sample to the depth of $70 \mathrm{~mm}$. However, for the analysis in this research the data were considered only up to the conical shape of the ice sample. 


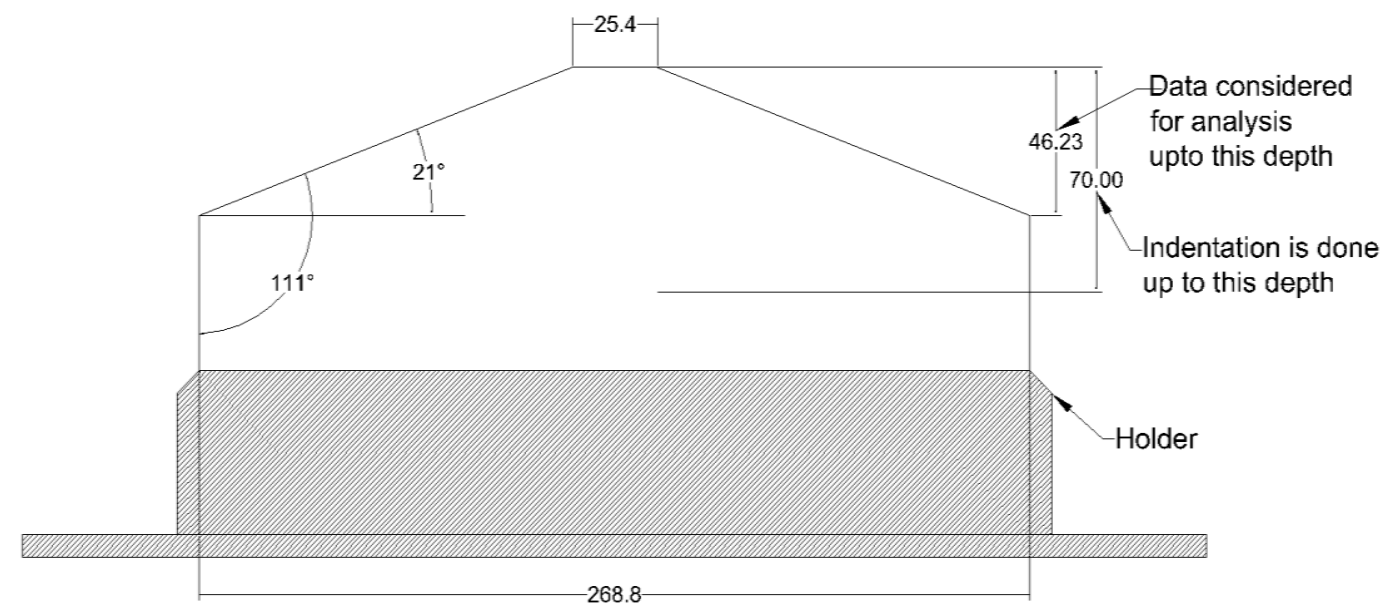

Figure 3.1: Geometry of ice specimen having taper angle $21^{\circ}$ (all dimensions are in $\mathrm{mm}$ )

Table 3.1 shows the heights of the conical shape of the ice samples and corresponding taper angles that have been considered for analysis.

Table 3.1: Heights of the conical shape in ice specimens

\begin{tabular}{|c|c|}
\hline Taper angle of ice specimen & Height of conical shape(from top) \\
\hline $13^{\circ}$ & $25.38 \mathrm{~mm}$ \\
\hline $21^{\circ}$ & $46.23 \mathrm{~mm}$ \\
\hline $30^{\circ}$ & $67.48 \mathrm{~mm}$ \\
\hline
\end{tabular}

The load frame that was used to conduct the experiments is a MTS model 311.21 located at the cold room of MUN. Specifications of the machine are given in Table 3.2. This model is specially designed for axial compression tests and stiffness of the frame is very high with a resistance to deformation of $1 \times 10^{6} \mathrm{kN} / \mathrm{m}$. The MTS load frame can be seen in Figure 3.2. 
Table 3.2: MTS load frame specifications

\begin{tabular}{|c|c|}
\hline Component & Details \\
\hline Actuator & Hydraulic, maximum stroke: $152.4 \mathrm{~mm}$ \\
\hline Load cell & Strain gauge, Force capacity: $500 \mathrm{kN}$ \\
\hline LVDT & Provides the displacement signal for position feedback. \\
\hline Data acquisition system & Sampling rate: up to $6 \mathrm{kHz}$. \\
\hline Base plate & Structural steel, diameter: $355.6 \mathrm{~mm}$. Connected to actuator. \\
\hline
\end{tabular}

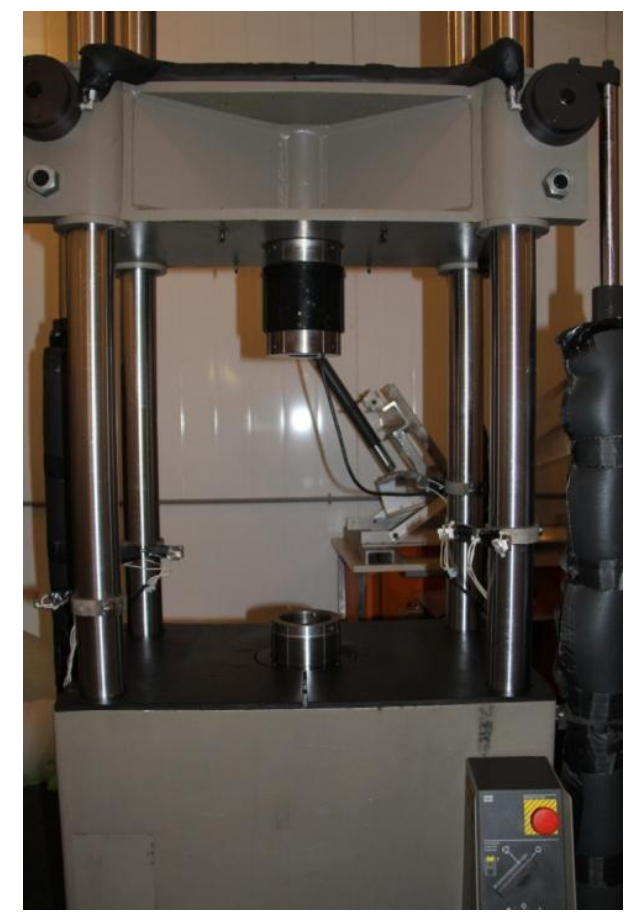

Figure 3.2: MTS Load Frame

To study the ice-structure interaction processes a flat crushing plate and spherical indenter have been used, mounted directly to the MTS load frame. The specifications of the two different crushing plates are given in Table 3.3. 
Table 3.3: Specification of the crushing plates

\begin{tabular}{|c|c|}
\hline Flat Indenter & Spherical Indenter \\
\hline Material: Stainless Steel & Material: Aluminum \\
\hline Diameter: $254 \mathrm{~mm}$ & Diameter: $254 \mathrm{~mm}$ \\
\hline Thickness: $19.05 \mathrm{~mm}$ & Radius of Curvature: $325.12 \mathrm{~mm}$ \\
\hline
\end{tabular}

The Tekscan I-Scan pressure measurement system was used to determine pressure distribution and high pressure zones at the ice-indenter interface. This system consists of a handle and tactile pressure sensor. Pressure data from the sensor are transmitted to an external computer, where the I-Scan software was installed, through a Tekscan USB handle at a rate of $100 \mathrm{~Hz}$. The pressure sensor is a thin flexible sheet consisting of a matrix of individual sensing elements, called 'sensels'. Static and dynamic interface pressure distributions are recorded by each sensel over the whole area that the sensor covers. Sensor model number 5101 was used for the tests. The sensor has a pressure rating of $5000 \mathrm{psi}$ and resolution of 100 sensels $/ \mathrm{in}^{2}$. To see the pressure distribution and variation of high pressure zones, the recorded data were saved as a video file throughout the indentation test. The handle of the Tekscan sensor was shielded by a plastic holder to protect the handle from the ice spalls during the tests. The handle along with the sensor were mounted on the MTS frame by clamps in such a way that the sensor stayed stationary and attached to the indenter plate. To improve the accuracy of the Tekscan sensor measurements the data were post-calibrated based on the MTS load cell data. The calibration procedure and results are included in Appendix B. In Figure 3.3, (a) shows 
pressure sensor model 5101 with specifications and (b) shows the sensor attached to the handle.

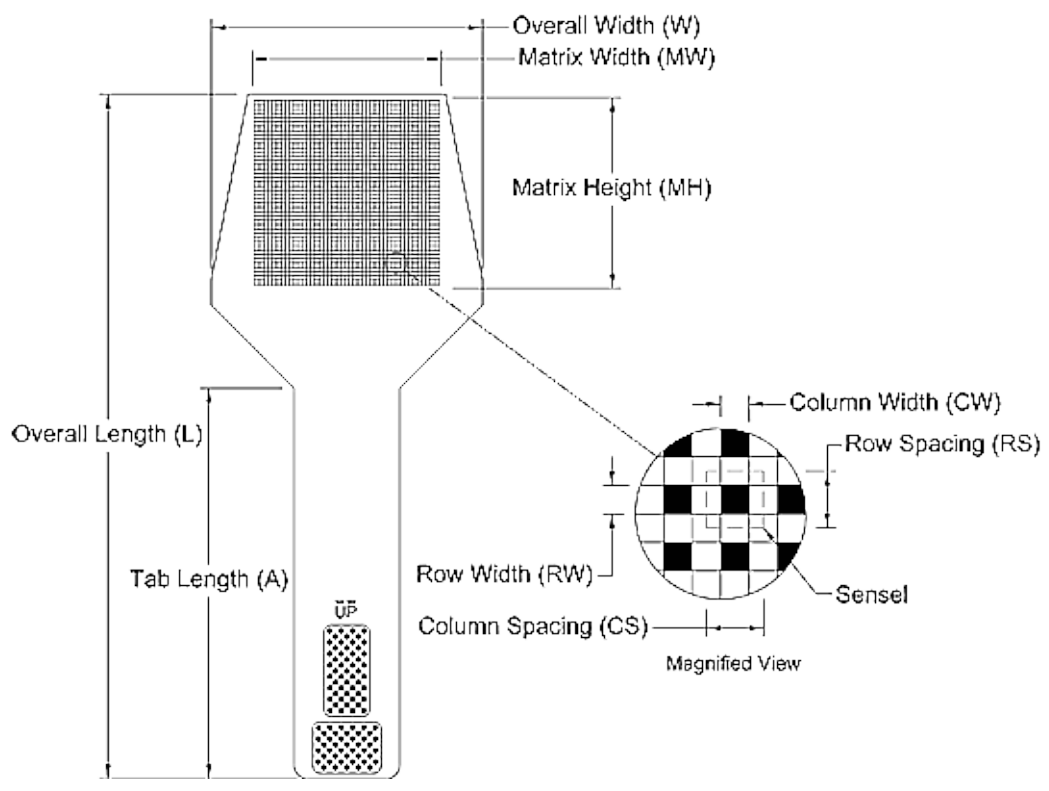

\begin{tabular}{|c|c|c|c|c|c|c|c|c|c|c|c|c|}
\hline \multirow{2}{*}{$\begin{array}{c}\text { Overall } \\
\text { Length } \\
\text { L }\end{array}$} & \multirow{2}{*}{$\begin{array}{c}\text { Overall } \\
\text { Width } \\
\text { W }\end{array}$} & \multirow{2}{*}{$\begin{array}{c}\text { Tab } \\
\text { Length } \\
\text { A }\end{array}$} & \multirow{2}{*}{$\begin{array}{c}\text { Matrix } \\
\text { Width } \\
\text { MW }\end{array}$} & \multirow{2}{*}{$\begin{array}{c}\text { Matrix } \\
\text { Height } \\
\text { MH }\end{array}$} & \multicolumn{3}{|c|}{ Columns } & \multicolumn{3}{|c|}{ Rows } & \multirow{2}{*}{$\begin{array}{c}\text { Total } \\
\text { No. of } \\
\text { Sensels }\end{array}$} & \multirow{2}{*}{$\begin{array}{c}\text { Resolution } \\
\text { Sensel Density }\end{array}$} \\
\hline & & & & & $\mathrm{CW}$ & $\begin{array}{c}\text { Pitch } \\
\text { CS } \\
\end{array}$ & Qty. & RW & $\begin{array}{c}\text { Pitch } \\
\text { RS }\end{array}$ & Qty. & & \\
\hline $\begin{array}{c}\text { (in.) } \\
13.39\end{array}$ & $\begin{array}{l}\text { (in.) } \\
5.86\end{array}$ & $\begin{array}{l}\text { (in.) } \\
6.59\end{array}$ & $\begin{array}{l}\text { (in.) } \\
4.40\end{array}$ & $\begin{array}{l}\text { (in.) } \\
4.40\end{array}$ & \begin{tabular}{|l} 
(in.) \\
0.050
\end{tabular} & $\begin{array}{l}\text { (in.) } \\
0.100\end{array}$ & 44 & $\begin{array}{l}\text { (in.) } \\
0.050\end{array}$ & $\begin{array}{l}\text { (in.) } \\
0.100\end{array}$ & 44 & 1936 & $\begin{array}{c}\text { (sensel per in.2) } \\
100.0\end{array}$ \\
\hline $\begin{array}{l}(\mathrm{mm}) \\
340.1\end{array}$ & $\begin{array}{l}(\mathrm{mm}) \\
148.8\end{array}$ & $\begin{array}{l}(\mathrm{mm}) \\
167.4\end{array}$ & $\begin{array}{l}(\mathrm{mm}) \\
111.8\end{array}$ & $\begin{array}{l}(\mathrm{mm}) \\
111.8\end{array}$ & $\begin{array}{c}(\mathrm{mm}) \\
1.3\end{array}$ & $\begin{array}{c}(\mathrm{mm}) \\
2.5\end{array}$ & 44 & $\begin{array}{c}(\mathrm{mm}) \\
1.3\end{array}$ & $\begin{array}{c}(\mathrm{mm}) \\
2.5\end{array}$ & 44 & 1936 & $\begin{array}{c}\text { (sensel per cm²) } \\
15.5\end{array}$ \\
\hline
\end{tabular}

PRESSURE RANGES

\begin{tabular}{|c|c|c|c|c|c|c|c|c|}
\hline $\mathbf{p s i}$ & 6 & 10 & 50 & 150 & 350 & 500 & 3,000 & 5,000 \\
\hline $\mathbf{k P a}$ & 41 & 69 & 345 & 1,034 & 2,413 & 3,448 & 20,685 & 34,475 \\
\hline
\end{tabular}

(a)

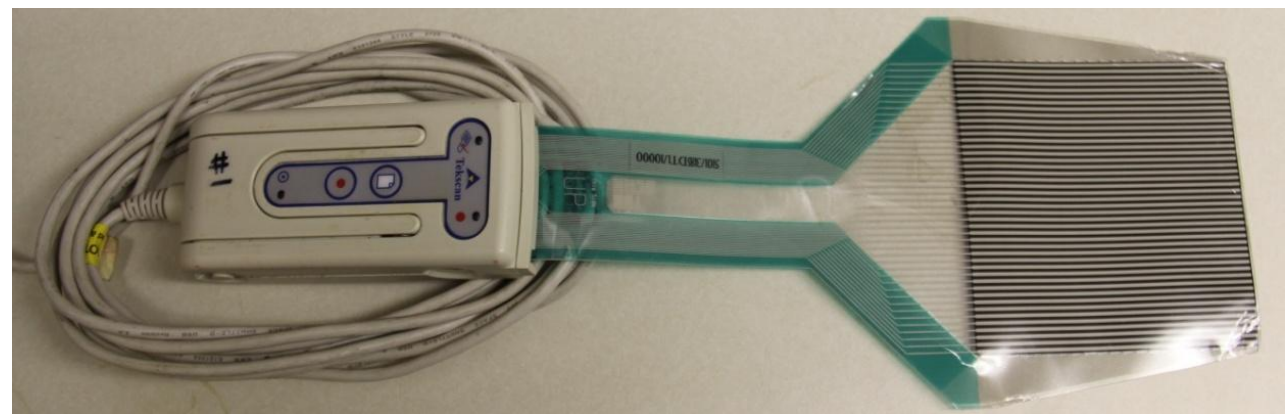

(b)

Figure 3.3 (a): Tekscan pressure sensor model 5101 with specification (collected from the official website: www.tekscan.com) (b): Tekscan sensor with holder 
To record the video of each test, a high-speed camera or a Canon 60D digital SLR camera were used. The slow tests were recorded by SLR and the medium and fast tests were recorded by high-speed camera. High-speed videos allow study of the failure process, crushing and spalling very closely and clearly during the indentation tests.

\subsubsection{Test Plan Design}

To design small-scale experiments there are a number of factors which are considered as a challenge when compared to the real scenario. For example, the types and shapes of multiyear ice or iceberg that comes into direct contact with offshore vertical structures and environmental parameters like ice velocity and temperature.

In the design of the experimental program, some factors are controlled during the experiments, while other parameters are held constant throughout the test. The input controlled parameters considered in the current research are discussed below.

Ice Crushing Rate: Ice interacting with a structure can occur over a wide range of speeds depending on the drift speed and size of the ice. To cover a range of practical situations, indentation speeds from $0.1 \mathrm{~mm} / \mathrm{s}$ to $10 \mathrm{~mm} / \mathrm{s}$ were utilized in the experiments.

Ice Temperature: The experiments were done at two different temperatures. Twentyfour (24) tests were done at $-10^{\circ} \mathrm{C}$ and four (4) tests were done at $-5^{\circ} \mathrm{C}$. The reason for choosing these temperatures was to compare the crushing test in a warmer and cooler environment. Both temperatures are practical for an ice-covered region, as described by 
Jones et al. (2003), iceberg temperatures vary from $0^{\circ} \mathrm{C}$ to $-10^{\circ} \mathrm{C}$ from the surface of the ice to a depth of 1-2 meters.

Indenter Shape: Two types of indenters were used in these experiments to study the effect of indenter geometry on the behaviour of ice failures and high pressure zones. Twenty-two (22) tests were done using a flat plate and six (6) tests were done using a spherical indenter. The specifications of the indenters are given in Table 3.3 and the photographs of the indenters are given in Figure 3.4.

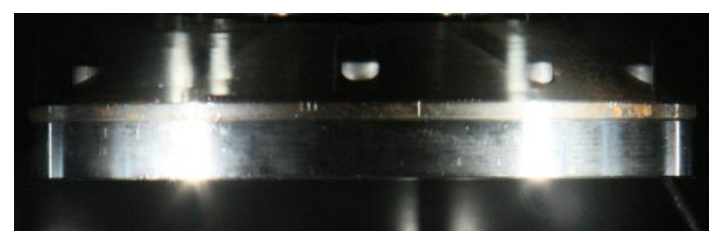

(a)

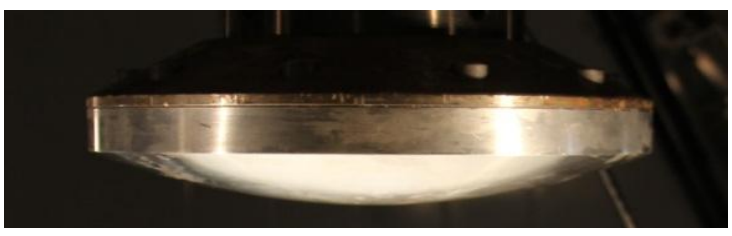

(b)

Figure 3.4: (a) Flat stainless steel indenter, (b) Spherical aluminum indenter

Grain Size: There are different types of ice that may be encountered by offshore structures in Arctic oceans. Sea ice is formed by the cooling and freezing of sea water and glacial ice is formed due to the compression and consolidation of snow. Schulson and Duval (2009) reported that it takes thousands of years to develop the orientation of grain distribution of glacial ice under the effects of rotation of the lattice by dislocation glide and static recrystallization. Schulson and Duval (2009) also stated that glacial ice is composed of equiaxed, randomly oriented grains and can be considered mechanically isotropic. Grain size of glacial ice typically ranges from 1 to $10 \mathrm{~mm}$, while sea ice that develops over a period of weeks to months has a grain size distribution of 5-10 mm. However, according to Cammaert and Muggeridge (1988) the diameter of columnar 
grains is about $20-40 \mathrm{~mm}$ near the bottom of a sea ice cover. In this thesis freshwater, granular ice samples similar to glacial ice were considered with larger $\left(4-10^{+} \mathrm{mm}\right)$ and smaller (0-4 mm) grain size as shown in Figure 3.5.

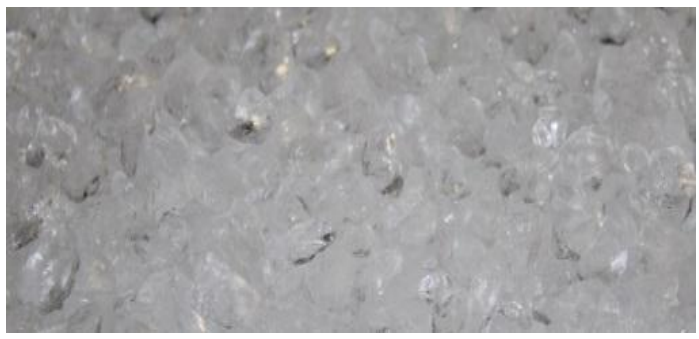

(a)

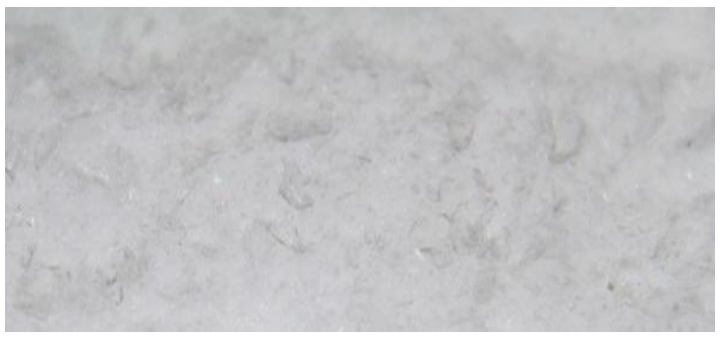

(b)

Figure 3.5: (a) Larger grain size $\left(4-10^{+} \mathrm{mm}\right)$ (b) Smaller grain size $(0-4 \mathrm{~mm})$

Ice Specimen Shape: Ice specimens having three different taper angles ranging from $13^{\circ}$ to $30^{\circ}$ were investigated throughout the experiment to study the variation of maximum force, pressure, loading pattern and associated failure processes with the change of the taper angle. In Figure 3.6 the ice samples having different taper angles are shown.

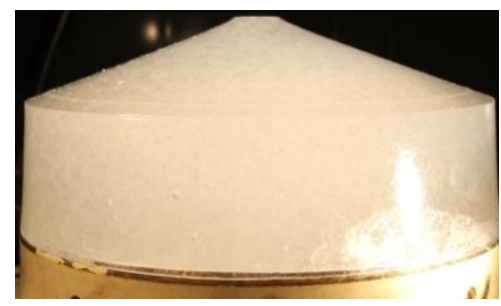

(a)

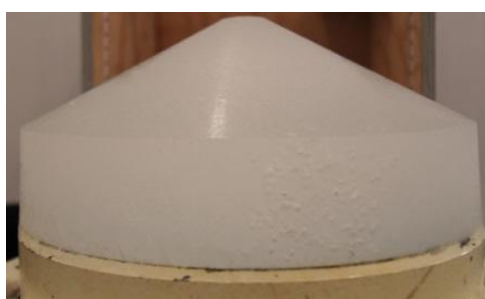

(b)

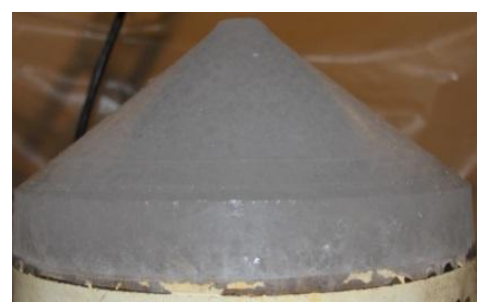

(c)

Figure 3.6: (a) Ice sample of $13^{\circ}$ taper angle (b) Ice sample of $21^{\circ}$ taper angle (c) Ice sample of $30^{\circ}$ taper angle

In addition to the above parameters, there were few factors which might have an effect on experimental results but could not be completely eliminated because of the restrictions 
of the laboratory and limitations of time. One factor is that the ice holders and ice moulds were limited in numbers and vary slightly in dimensions and weights. As well, there may be some dissimilarities of the ice specimen geometry due to variation during shaping of the sample. Another potential source of variability is the presence of air bubbles in the ice specimens. To minimize entrapped air, careful attention was paid to pour the deaerated, chilled water very slowly into the ice moulds and efforts were made to be consistent to ensure the same quality of ice was achieved for all specimens.

\subsection{Water Preparation Procedure}

Water preparation for ice samples consists of three standard processes as described by Bruneau et al. (2011). Detailed description of the processes including the major steps (1) water distillation, (2) water deionization and (3) water deaeration is given below.

\subsubsection{Distillation and deionization}

The distillation system in the Thermal lab at MUN is used to produce distilled water from tap water, which is stored in a large glass container and connected via a hose pipe to the deionizer system. Once the distillation process has started, the 'operate' light comes on, and the 'reset' switch is needed to trigger the start of the distillation process. There are two valves in the connecting hose, which need to be open, and the red power button needs to be pressed to start the operation of deionization. The green valve (on the deionizer's right hand side) is opened to collect the water in a storage container. Figure 
3.7(a) shows the water distillation system and Figure 3.7 (b) shows the water deionization system.

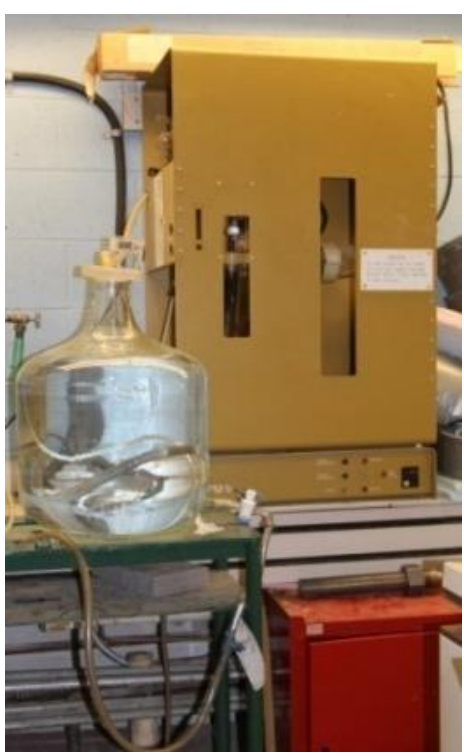

(a)

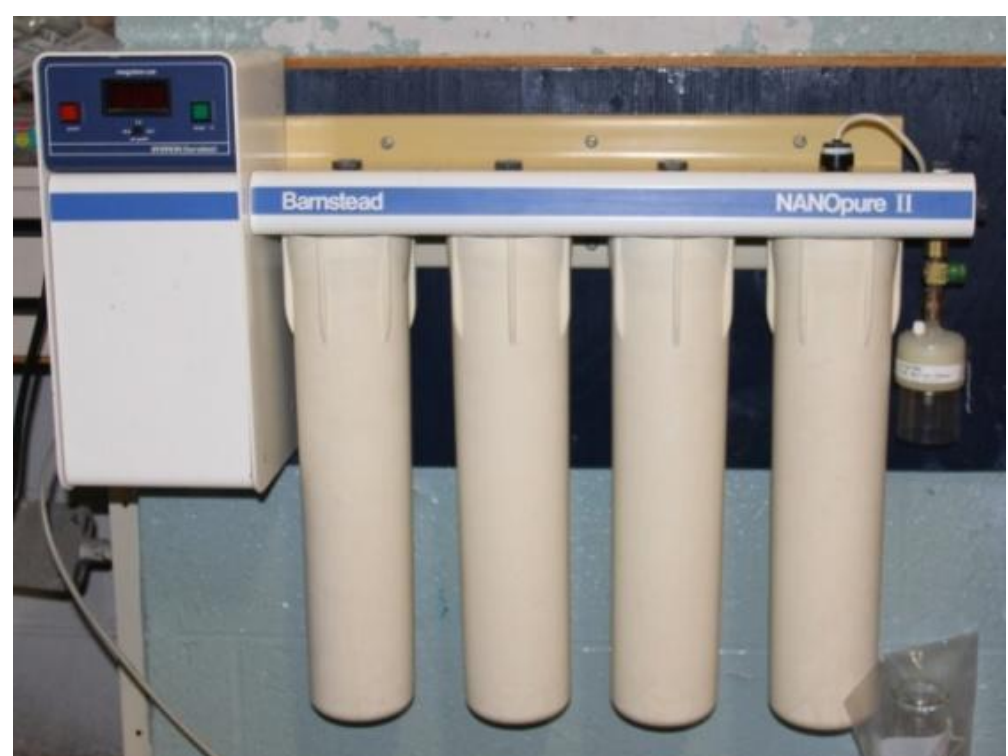

(b)

Figure 3.7: (a) Water distillation system (b) Water deionization system

\subsubsection{Degasification}

Distilled and deaerated water from the storage container is sucked into the deaerator cylinder by a vacuum placed on the deaeration chamber. To fill the deaerator cylinder, the isolation valve of the pump needs to be closed and the gas ballast valve needs to be open by 1.5 turns. As well, the tap of the switching valve has to be in a closed position before the pump is switched on. The isolation valve has to open to connect the pump with the deaerator by turning the switching valve to the downward position.

When the pressure of the deaerator was starting to fall by approximately 15 inches of mercury the valves were closed in the opposite direction before switching off the pump. 
This created a lower pressure and sucked the water from the laboratory container into the deaeration chamber. To start the degasification process, the ballast valve on the vacuum pump was closed to create a deeper vacuum. Figure 3.8 shows the deaerator in operation.

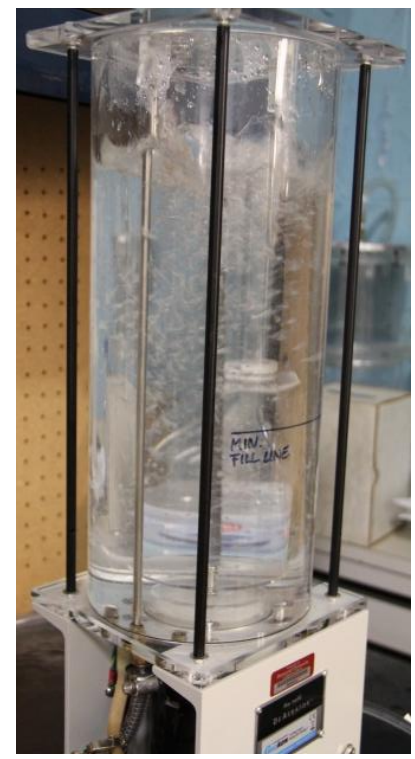

Figure 3.8: Water deaerator

After the degassing process, the deaerator and vacuum pump were shut down by closing the isolation valve and opening the gas ballast valve. The switching valve was turned open carefully to the atmosphere when the water movement slowed down and there were no visible bubbles around the deaerator impeller. The vacuum in the pump had to be released before draining the deaerated water. To collect the water, the pinch clamp was opened and the water was poured into a storage container. The collected water was then stored in a freezer for at least two days to cool just above $0^{\circ} \mathrm{C}$ before using it to make ice samples. 


\subsection{Ice Specimen Preparation}

As described by Bruneau et al. (2012), there are some important factors like crystal size, orientation, ice density and homogeneity that is essential to make ice samples in the laboratory for ice crushing test. To produce the ice sample for the experiment, commercially purchased freshwater, polycrystalline ice was used. The ice blocks were stored in the cold room at $-10^{\circ} \mathrm{C}$ for at least 2-3 days. The ice cubes were crushed into uniform pebbles using a commercial ice crusher (Clawson Machine, 2011). The ice particles were then sieved using $4 \mathrm{~mm}$ opening laboratory sieves. Two sized ice seeds were separated to produce compact ice specimens. The first type of specimen was made from ice seeds of $4 \mathrm{~mm}$ to $10 \mathrm{~mm}$ in diameter with negligible amount of ice seeds with diameter of more than $10 \mathrm{~mm}$ and the second type of specimen was made from ice seeds of 0-4 mm diameter. Figure 3.9 shows a picture of the ice crusher.

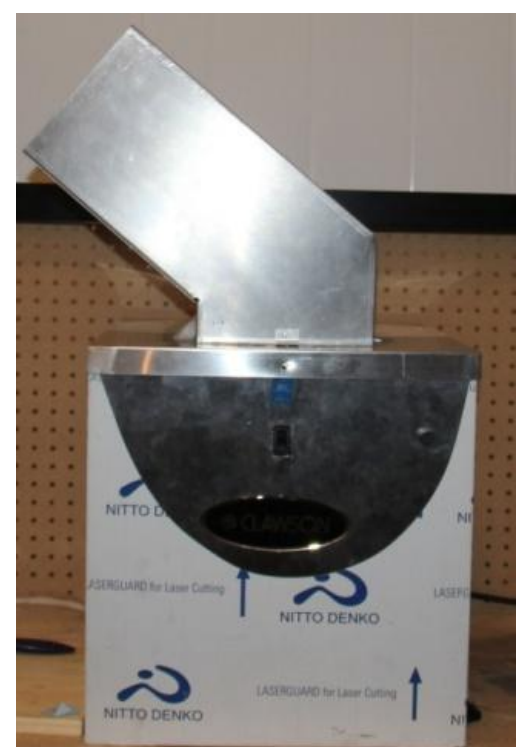

Figure 3.9: Ice crusher 
Ice samples were produced based on the method reported by Bruneau et al. (2012) and Dillenburg (2012). Moulds and ice holders were fitted together, filled with ice seeds and thoroughly shaken to reduce the air gaps among the ice seeds and maximize the ice volume before being flooded with water. The holders were then set in a modified chest freezer that allows vertical suspension of cylindrical moulds into the cold space. Being equipped with removable covers allows expansion of ice as it freezes. Also the sides of the moulds are wrapped with insulated materials to enable directional freezing (Bruneau et al., 2012). Based in the ice sample fabrication techniques described by Cole (1979), the cold, distilled, deionized and deaerated water was then poured into the ice mould filled with various sized ice seeds. For current experiments, the water was poured directly through a plastic pipe to flood the ice from the bottom and it was done very slowly to allow the air bubbles to escape as shown in Figure 3.10.

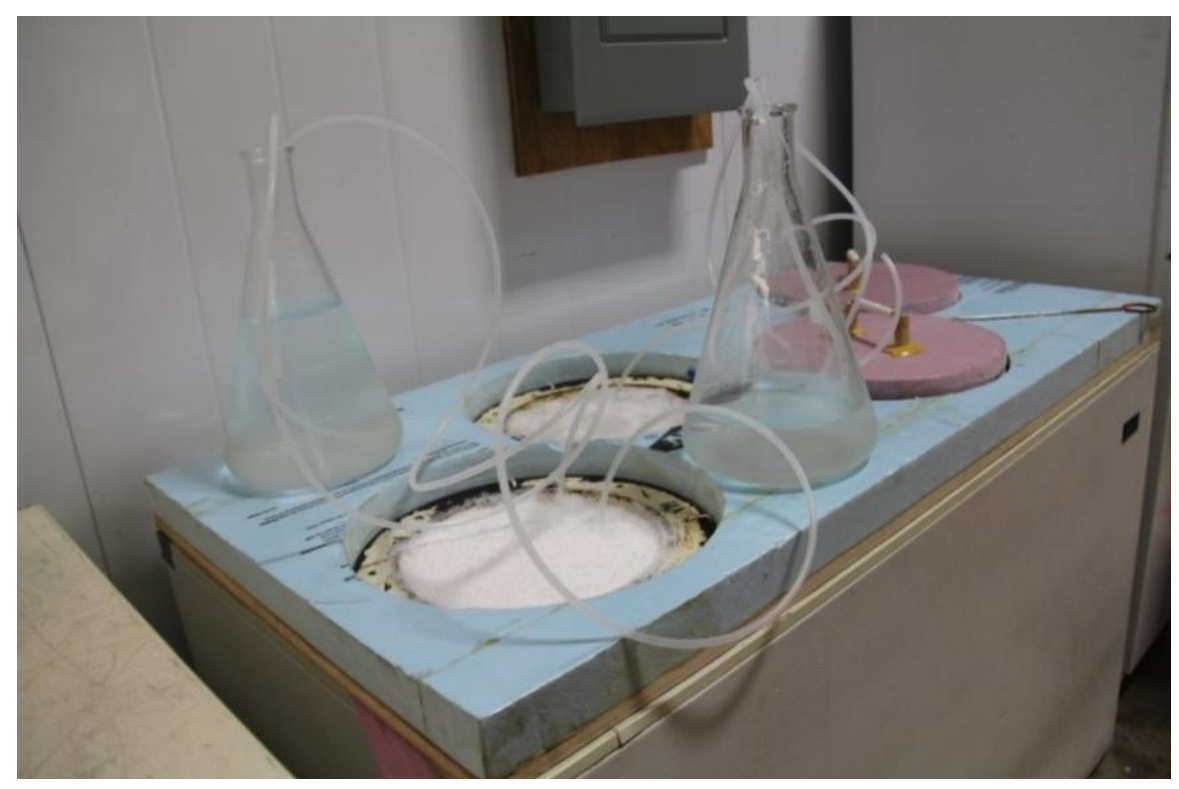

Figure 3.10: Water pouring method to the ice mould in the freezer 
It should be noted that the ice specimens were not completely bubble free, but the volume of air bubbles was quite low and consistent for all the test specimens. When the moulds were completely filled with water, the tops were enclosed with insulated covers. The compacted ice was then ready to freeze and the temperature was lowered during freezing to almost $-30^{\circ} \mathrm{C}$.

\subsubsection{Ice specimen shaping}

Following two days of freezing, the ice samples were taken out of the freezer and the insulations and covers were removed. To ensure a smooth fit with the contact surface of the shaping apparatus wheel and the load frame base, the ice holder base was melted to flatten the contact surface using an aluminum plate at room temperature. To shape the ice samples an ice shaping apparatus was used, which is shown in Figure 3.11.

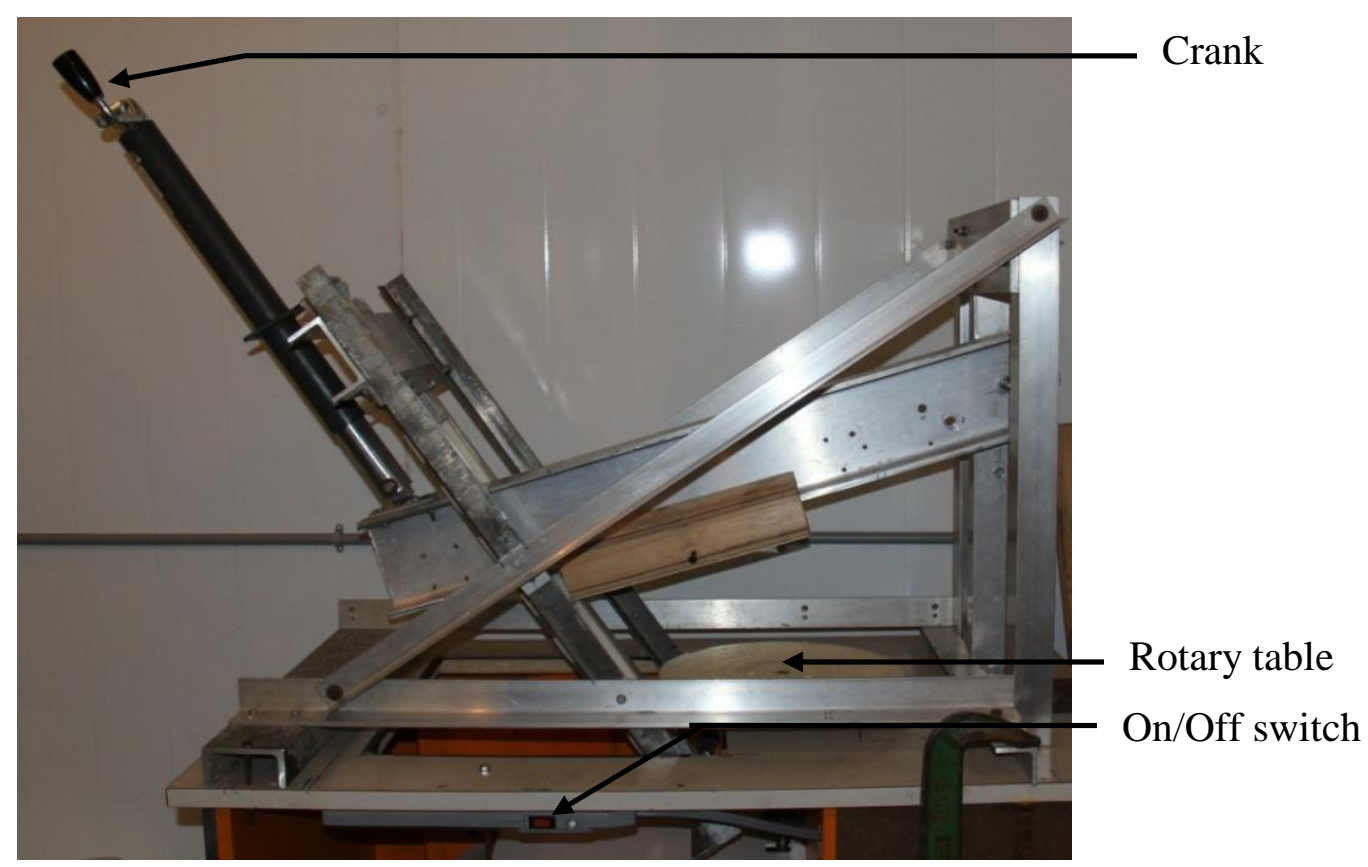

Figure 3.11: Ice shaping apparatus 
The cone shaping apparatus contains a sharp planer blade to shape the ice. Ice holders were mounted on a nylon base plate in the shaping apparatus which rotates at approximately $300 \mathrm{rpm}$. As described by Lieu and Mote (1984), cutting force does not depend on the rotational speed and if the planner blade is sharp enough, then it will not affect the internal structure of the ice sample. There is an arm and preset pivot points which allow cutting at multiple angles by dissembling the arm and changing the pivot points. The arrangements of the pivot-point bore holes are named as A, B, C, D, E and 1, 2. Table 3.4 is the combination of pivot-point mounting and corresponding cone angles.

Table 3.4: Mounting combination of cone shaping apparatus

\begin{tabular}{|c|c|}
\hline Mounting Combination & Corresponding cone angle \\
\hline E2 & $20^{\circ}$ \\
\hline D2 & $25^{\circ}$ \\
\hline B1 & $30^{\circ}$ \\
\hline A1 & $35^{\circ}$ \\
\hline
\end{tabular}

In this research, combinations D2, B1 and A1 were used to get the desired cone angles. The final cone angle can be reached when the blade reaches at the end of the ice cone just prior to touching the steel holder. Figure 3.12 shows an ice cone with a $30^{\circ}$ cone angle (Dillenburg, 2012). 


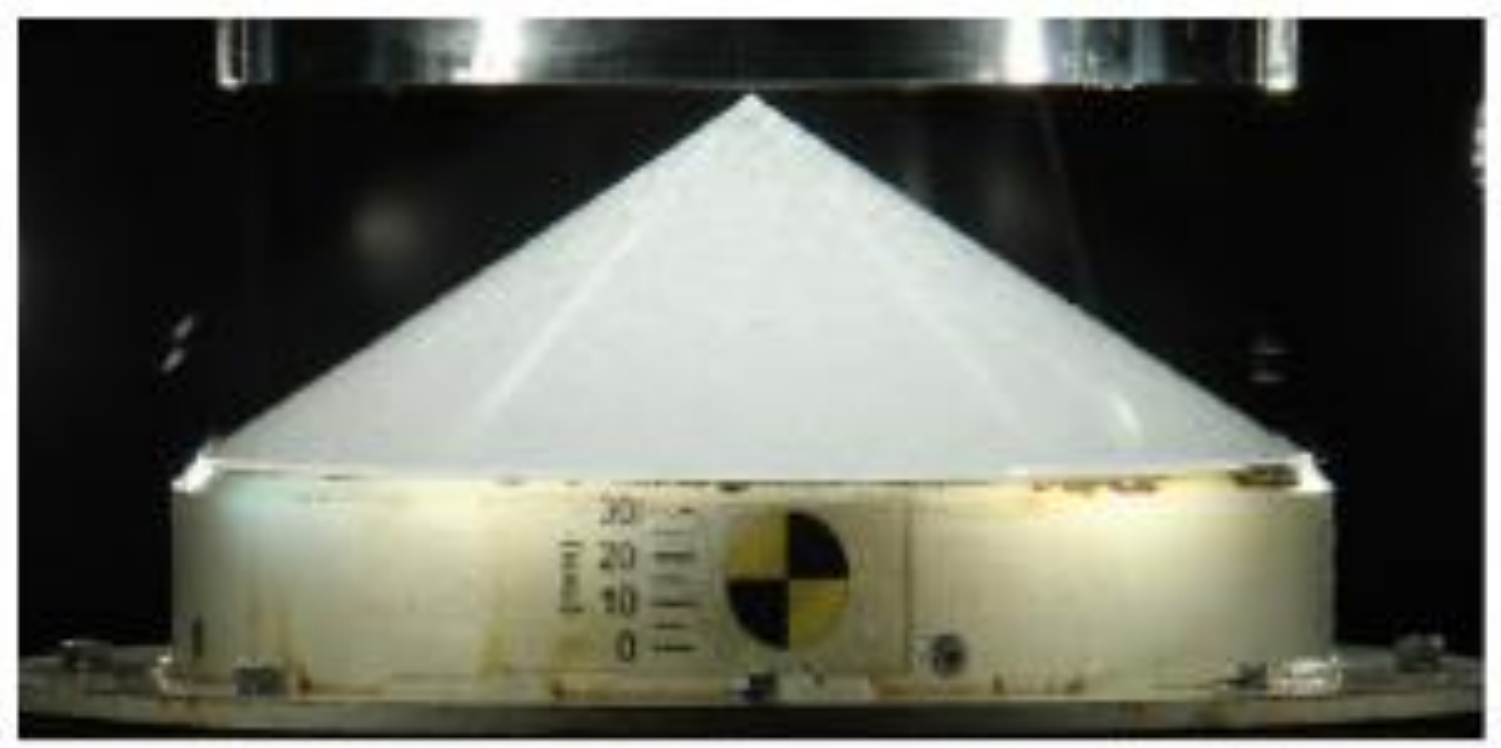

Figure 3.12: Ice sample of $30^{\circ}$ cone angle (Dillenburg, 2012)

In the current work, the shaping was stopped when the top of the ice samples reached $25.4 \mathrm{~mm}$ (1 inch) in diameter, which was about $10 \%$ of the base diameter of the ice samples. The actual shaping angle was less than the theoretical amount since the blade had to be stopped before it could reach the final angle. For this reason, the final angles for the models were measured, calculated and then drawn in commercial package AutoCAD.

Ice specimens with three different geometries and cone angle were used in the experiments. The drawings of the ice samples are shown in Figure 3.13. The blade angles and the corresponding taper angles of the ice samples are also given. 


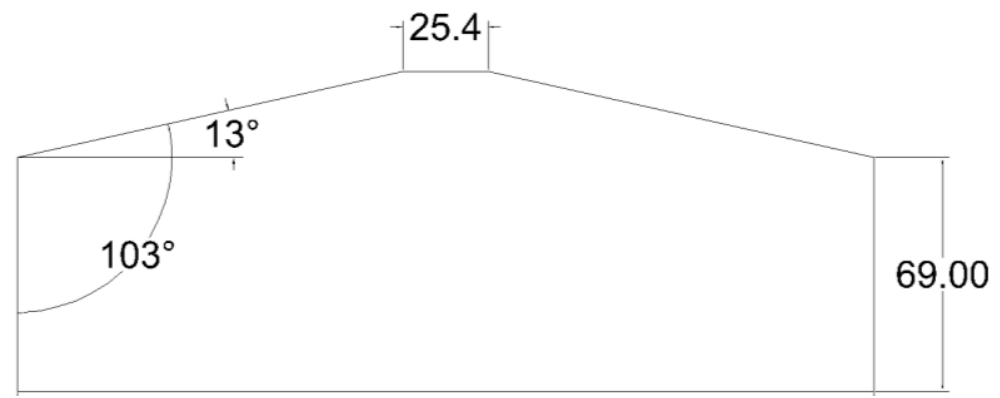

\begin{tabular}{|c|c|}
\hline Blade Angle & $25^{\circ}$ \\
\hline Taper angle & $13^{\circ}$ \\
\hline
\end{tabular}

(a)

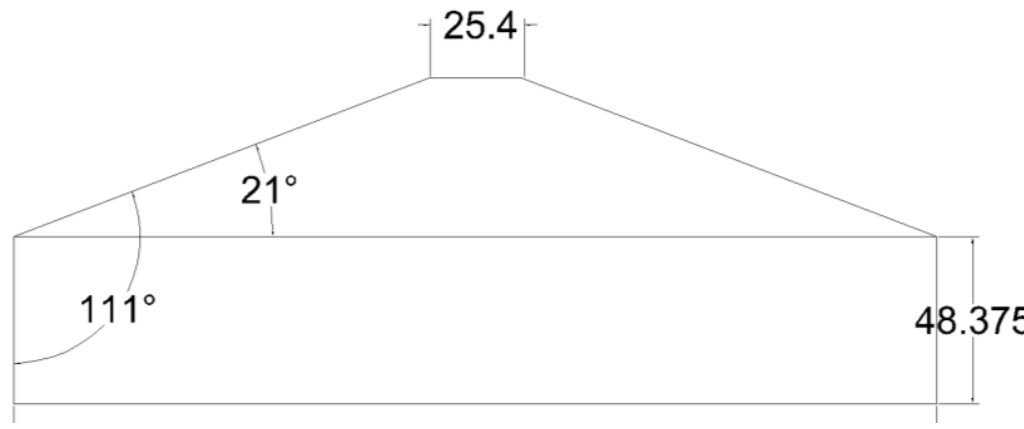

\begin{tabular}{|c|c|}
\hline Blade Angle & $30^{\circ}$ \\
\hline Taper angle & $21^{\circ}$ \\
\hline
\end{tabular}

(b)

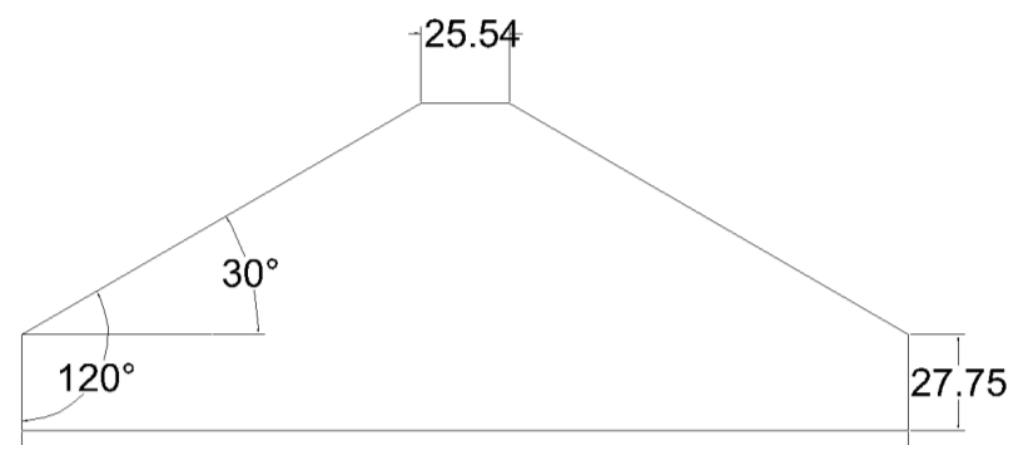

\begin{tabular}{|c|c|}
\hline Blade Angle & $35^{\circ}$ \\
\hline Taper angle & $30^{\circ}$ \\
\hline
\end{tabular}

(c)

Figure 3.13: Drawings of the ice samples (a) $13^{\circ}$ (b) $21^{\circ}$ (c) $30^{\circ}$ (all dimensions are in $\mathrm{mm}$ )

After shaping, the ice specimens were covered and stored in the cold room for at least twenty-four (24) hours to adjust it to the desired temperature (Jones et al., 2003). 


\subsection{Testing Procedure}

All compressive ice tests were conducted in the cold room at Memorial University of Newfoundland. The tests were done at temperatures of $-5^{\circ} \mathrm{C}$ or $-10^{\circ} \mathrm{C}$ and indentation speeds ranged from $0.1 \mathrm{~mm} / \mathrm{s}$ to $10 \mathrm{~mm} / \mathrm{s}$. The load frame is an MTS machine of the model number 311.21. Specifications of the machine are given earlier in Table 3.2. The crushing apparatus, modified by Dillenburg (2012) consists of two plates. The crushing plate that contacts the ice specimen is bolted to a back plate and exchangeable. The reinforcing backing plate has direct surface contact with the load cell and is rigid enough to avoid vibrations in the system. The flat indenter used in the current program, has a surface roughness of $0.13 \mu \mathrm{m}$, and was previously used by Dragt and Bruneau (2013). Part of the work of current research program was to design a spherical indenter to differentiate the pattern of loading and crushing failure from the flat indenter. The specifications of the two different crushing plates are given in Table 3.3.

For testing, the specimens are bolted to the base plate, firmly attached to the hydraulic ram. The tests are controlled by the MTS control system and data collections are controlled by the data acquisition system. The hydraulic ram is used to control vertical displacement and the indentation rate of the ice specimen while the MTS crosshead remains fixed. Before a test is conducted the ice specimen is brought as close as possible to the indenter plate by the hydraulic ram. A Tekscan tactile pressure sensor was used for selected tests. The sensor and handle were mounted on the MTS machine with clamps so the sensor remained straight and fixed along the indenter plate as the ice specimen moved 
upward. Figure 3.14 shows the arrangement of Tekscan sensor mounted against the indenter surface.

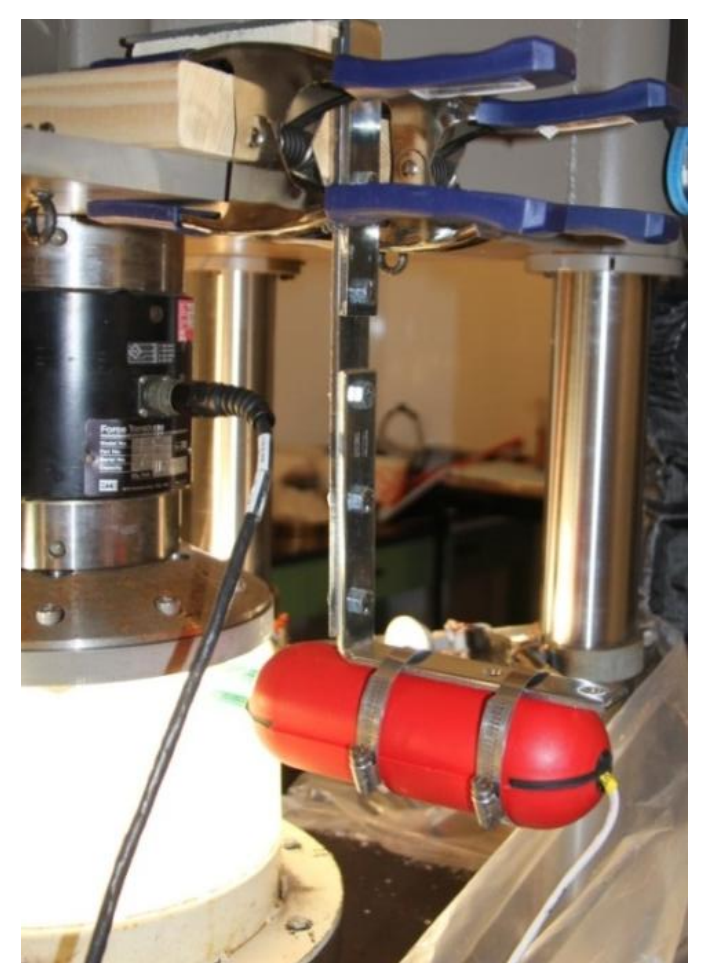

Figure 3.14: Arrangement of Tekscan sensor with MTS load frame

After few millimetres of indentation during the tests, the area of the tactile pressure sensor was fully covered by the contact area of the ice sample. To maintain the consistency, the test was continued until it reached the final indentation depth. When analyzing the sensor data, only up to the data which had full contact area, fully inside the sensor was considered.

The testing area was uniformly illuminated in order to produce high quality video recordings using four 50W halogen bulbs and four 4W LED spot lights. The slow tests 
(indentation rate of $0.1 \mathrm{~mm} / \mathrm{s}$ ) were recorded by a Canon 60D Digital SLR camera and the medium and fast tests were recorded by a high-speed video camera. After each test, the hydraulic ram was lowered, the crushed ice specimen with the holder was detached from the base plate, and the spalls were collected for weighing. The spall collection and weighing procedure is described in Section 3.6. Then the crushed ice specimen was weighed and removed from the holder for the thin-sectioning process. A detailed description of the thin-section procedure is described in Section 3.7.

\subsection{Spall Collection and Weighing}

To collect the spalls, the testing area was surrounded with a plastic sheet to hold the spalls in the testing area. After each test all the spalls were collected from the sides of the MTS machine, the face of the indenter and the ice holder. The photograph following the crushing test is given in Figure 3.15.

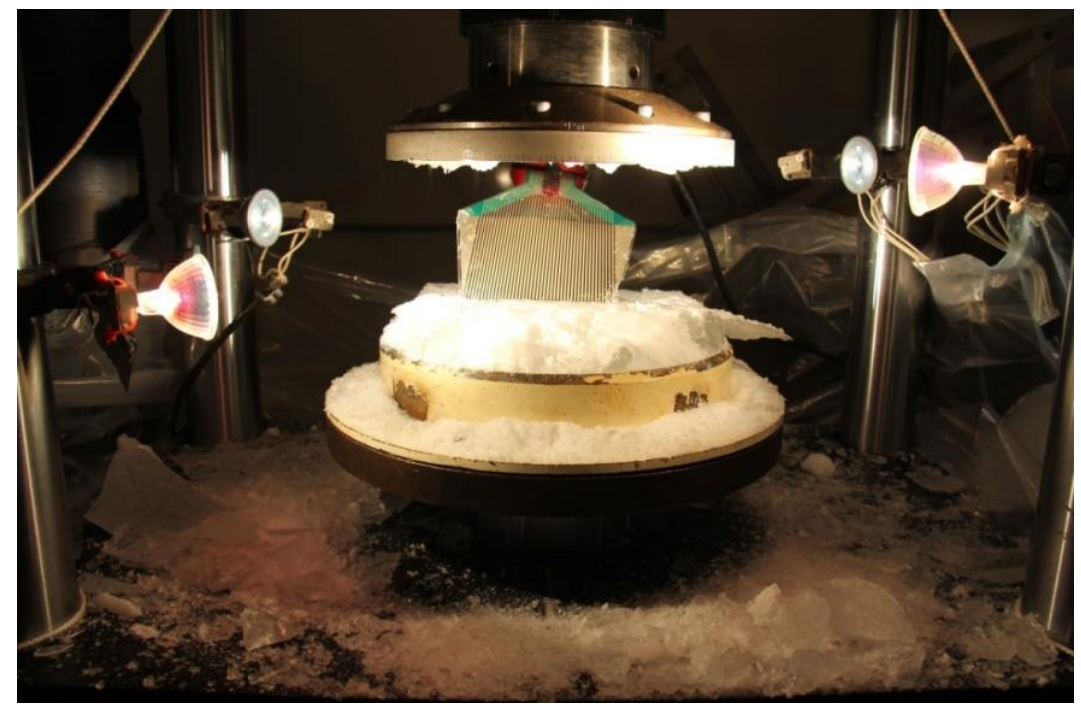

Figure 3.15: Photograph of the testing area after crushing test 
Then all the ice spalls were sieved and weighed using seven different sized laboratory sieves. The openings of the sieves are as follows:

(a) $>100 \mathrm{~mm}$ (b) $50 \mathrm{~mm}$ (c) $38.1 \mathrm{~mm}$ (d) $25 \mathrm{~mm}$ (e) $10 \mathrm{~mm}$ (f) $4.75 \mathrm{~mm}$ (g) $<4.75 \mathrm{~mm}$

Examples of these ice spalls are given in Figure 3.16.
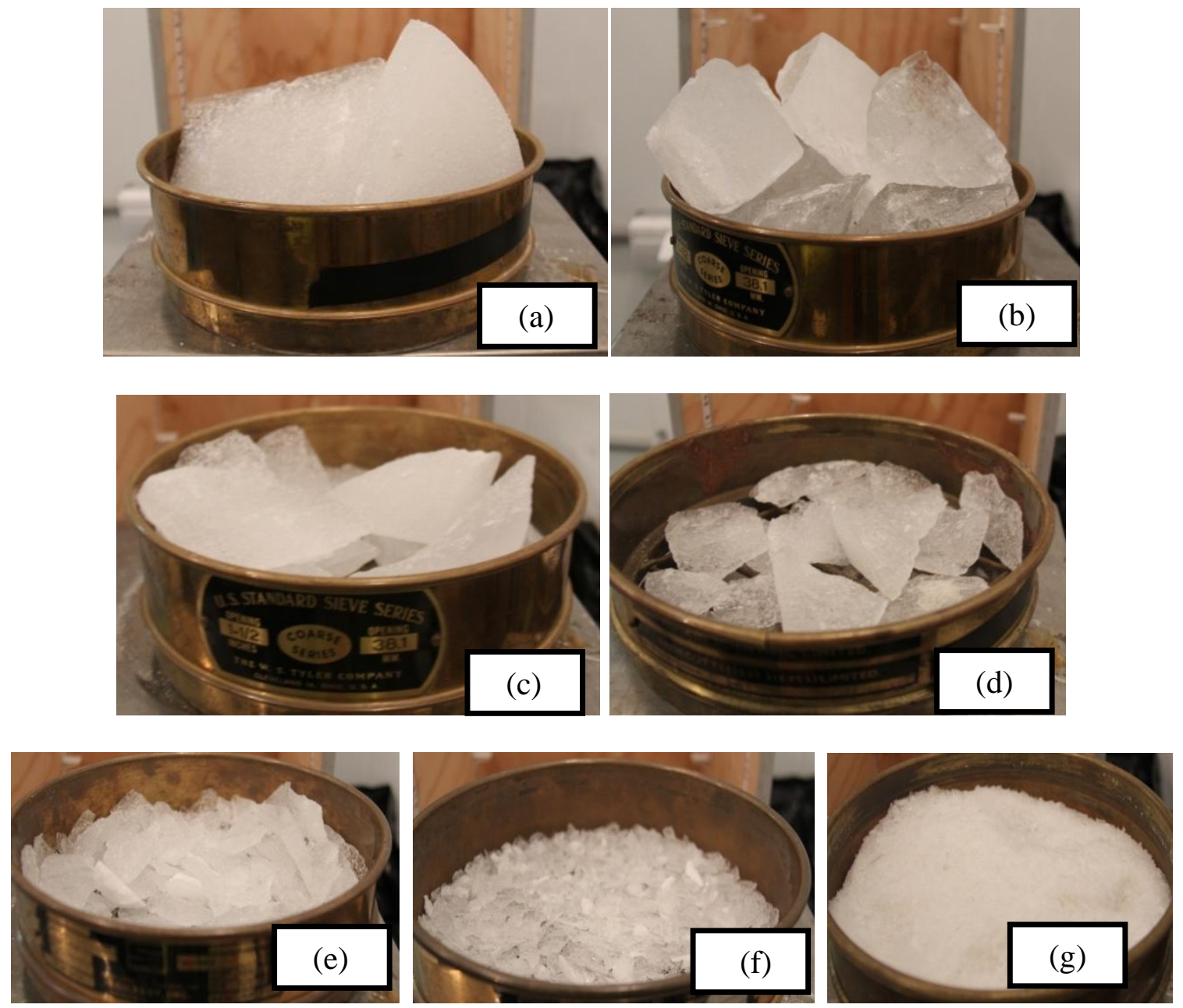

Figure 3.16: Ice spall sieving according to the spall size 
The mass of the ice spalls for each case was determined by subtracting the empty sieve mass from the ice and sieve mass. The whole process was done with an extra caution to minimize the ice particle loss to $10 \%$ or less.

\subsection{Thin-sectioning Procedure}

The crushed ice samples were thin-sectioned to study the microstructural changes of the damaged surface and changes in crystal size. Thin-sectioning was done using a microtome machine with the 'double microtome' method developed by Sinha (1977).

After each test, the ice sample was removed from the ice holder using a heating band. With a bandsaw the ice samples were vertically cut in half and a sample of a vertical thick section was taken and then cut horizontally to take a sample of the horizontal thick section. The photographs of vertical and horizontal sections are shown in Figure 3.17 and the picture of the band saw is shown in Figure 3.18.

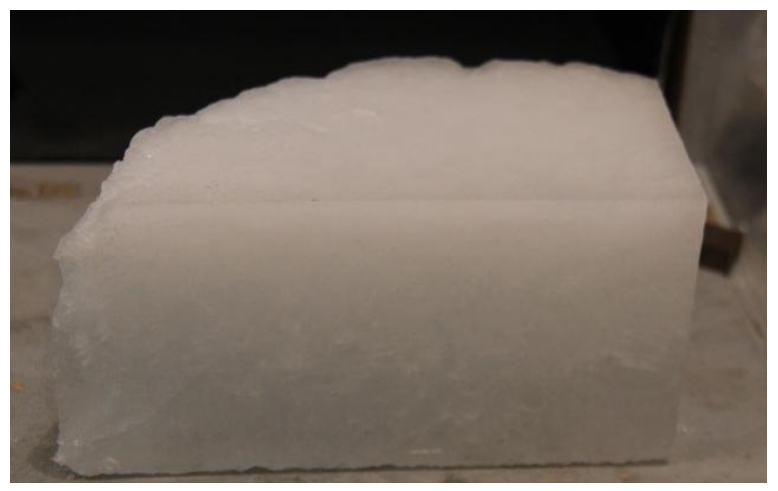

(a)

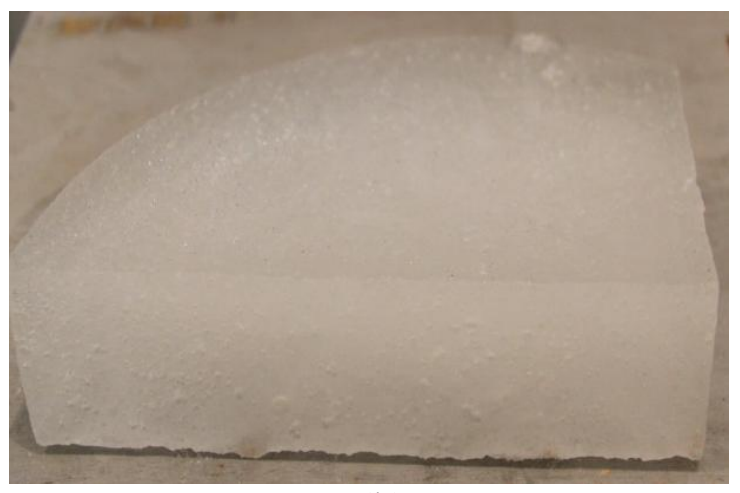

(b)

Figure 3.17: (a) Vertical and (b) Horizontal cut of crushed ice sample 


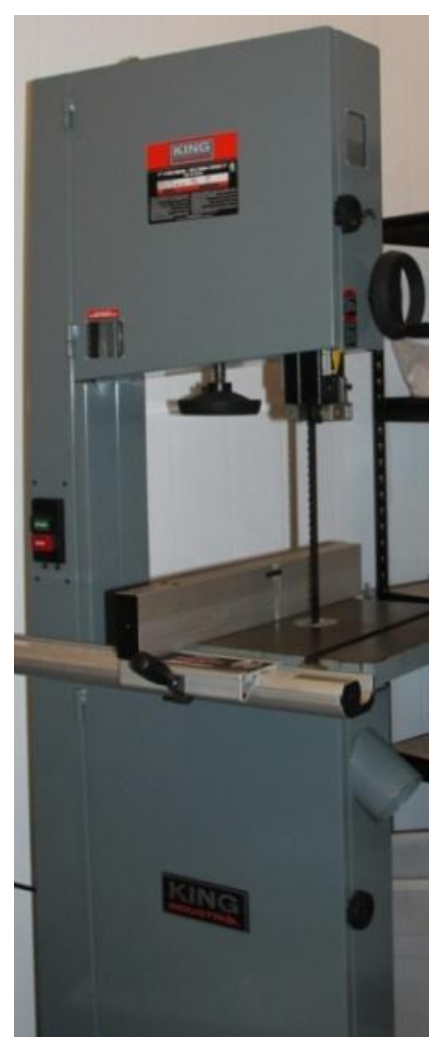

Figure 3.18: Band saw

The vertical thick sections with a thickness of around $10 \mathrm{~mm}$ were cut from the center of the ice samples and the horizontal thick sections with the same thickness were cut from the central cylindrical area of the ice samples as shown in Figure 3.19.

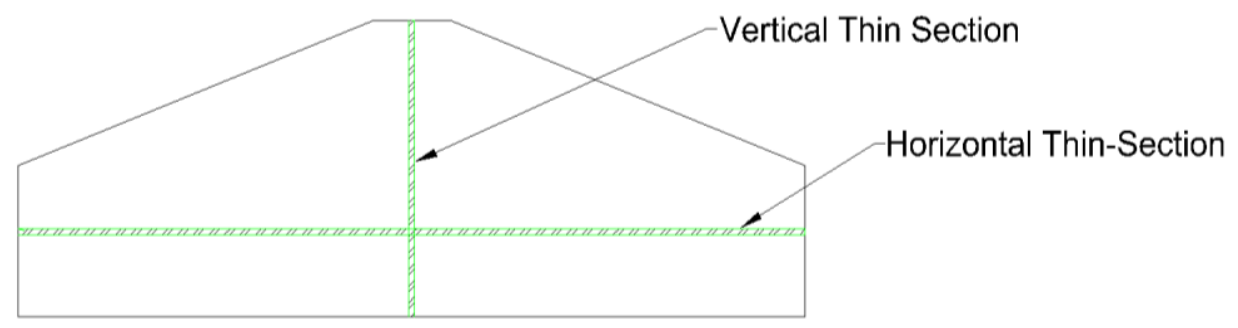

Figure 3.19 Vertical and Horizontal thick section 
To make the thin-section the piece of ice section was freeze-welded to a glass slide using drops of cold water. It was then placed on the microtome in a fixed position and shaved very slowly each time until it reached a thickness of about $5 \mathrm{~mm}$. Then the ice sample was removed from the glass, flipped and then welded to a new, clear class slide with the shaved surface now against the glass. This time the section was welded with cold water only on three sides, leaving the indented crushed side untouched. The new surface was shaved until it reached the thickness of less than $0.5 \mathrm{~mm}$ to make the final thin-section. Figure 3.20 shows the microtome machine used in these experiments.

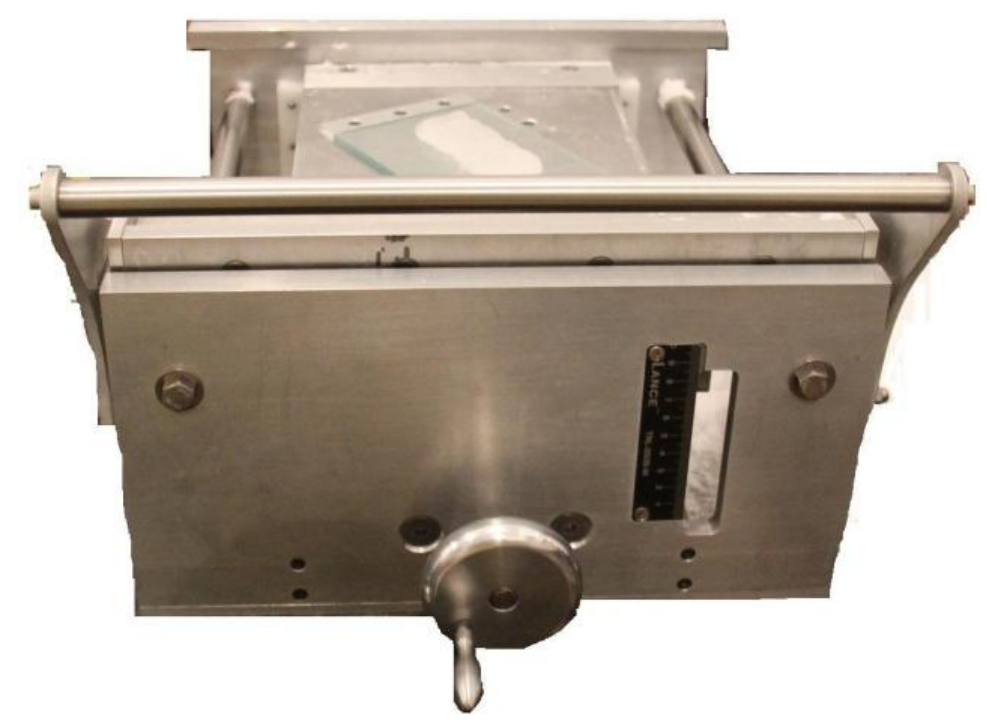

Figure 3.20: Microtome

Each test had two thin-sections, horizontal and vertical. Both of them were photographed using a Canon 60D Digital-SLR camera. Each thin-section was photographed with two different lighting techniques. One used cross polarized light transmitted through the section and the other one used a regular light reflected from the side. Cross polarized light was used to view the crystal structure and change of microstructure. Two polarized 
filters at a right angle were used to block the light waves completely because a polarized filter only allows light waves of the same orientation to pass through. Under the cross polarized filters the section shows different colours depending on the randomly orientated crystals in the ice sheet. With the help of a transparent scale, grain size distribution and size of the ice particles was also observed. Normal light was used as side lighting oriented at approximately $45^{\circ}$ pointed at the sample to view the grain boundaries and microfractures within the thin-sections. For minimum light reflections the photography box was covered with black paper on all sides as shown in Figure 3.21.

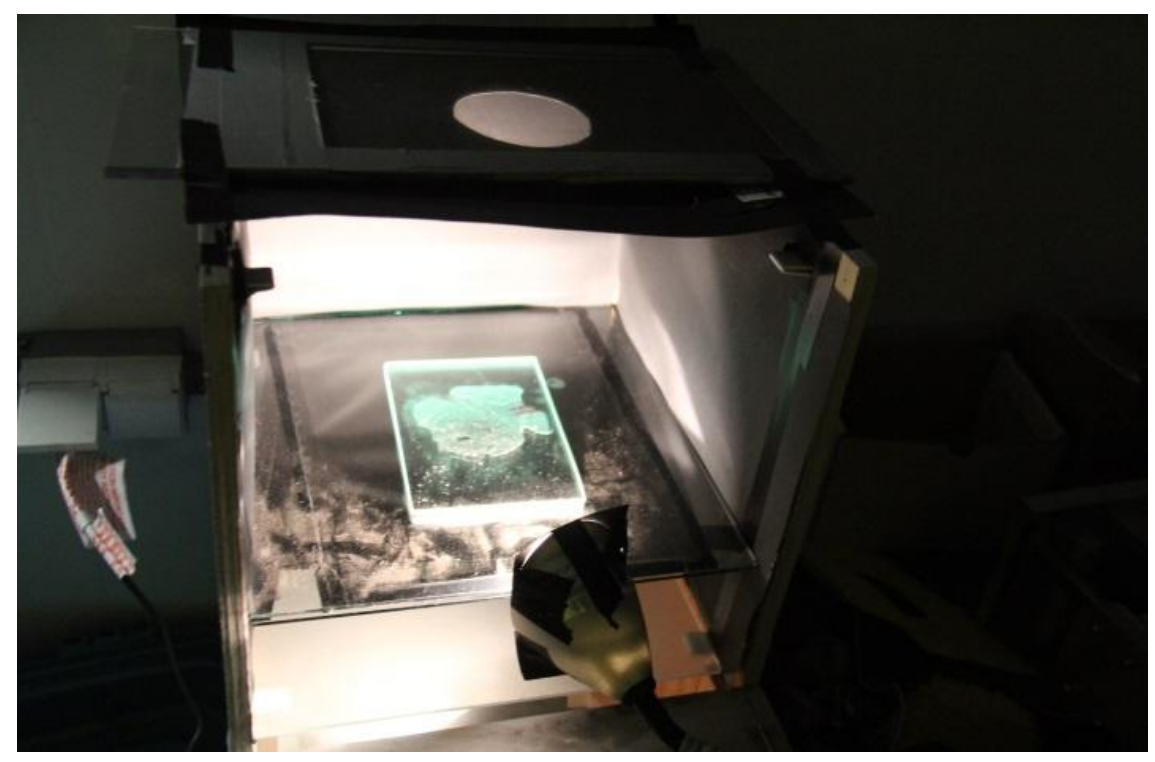

Figure 3.21: Cross polarizing and side lighting box 


\section{Chapter 4: Experimental Results}

\subsection{Overview}

A total of twenty-eight (28) tests were completed for this research program. Each test considered a unique combination of different test parameters including indentation rate, temperature, indenter shape, taper angle of ice sample and grain size distribution. To study the effect of the indentation rate more clearly, three cases of different indentation rates using the flat indenter are presented. A warm test $\left(-5^{\circ} \mathrm{C}\right)$ using the flat indenter and a cold test using spherical indenter are also discussed. Sample results are presented in this chapter for these cases. Throughout the thesis one of the main concerns is to investigate the force, pressure and cyclic loading patterns observed during ice compressive tests from the data collected from the MTS load cell. Tactile pressure sensor data provided an efficient way to observe the formation and evolution of high pressure zones. It was noted previously that even though the Tekscan sensor was used until it 
reached final depth of the crushing test, results considered only up to the contact area of the ice that was fully inside the sensor. The reason is when the area of the ice sample crossed the sensor area, there were many high pressure zones and pressure distributions which were not recorded. Therefore, during the later stages of the test, the area recorded by the sensor did not represent the overall observations.

To study the damage and cracking pattern in the vicinity of the indentation zones, thinsections were produced from both vertical and horizontal sections of crushed ice. In addition, the ice pieces were collected from the testing area and weighed to help quantify the amount of crushed and spalled ice produced. High-speed and regular video recordings of ice crushing tests were observed closely to see the patterns of crushing, extrusion and pulverization. In this chapter, representative sample results are included. Test results for all experiments are presented in Appendix A.

The area and pressure are calculated from the relationship of indentation depth and taper angle measurements as given below

$$
\begin{aligned}
& R=\frac{d}{2}+\mathrm{x} * \tan (90-\alpha) \\
& A=\pi R^{2} \\
& P=\frac{F}{A}
\end{aligned}
$$

where, $d$ is top diameter; $x$ is indentation depth; $\alpha$ is taper angle; $R$ is radius of the indentation surface; $A$ is surface area; $P$ is pressure; $F$ is Force (from load cell). 
As shown in Figure 4.1 the area in yellow colour indicates the area considered for the force data analysis. The drawing is given for the sample having taper angle of $21^{\circ}$.

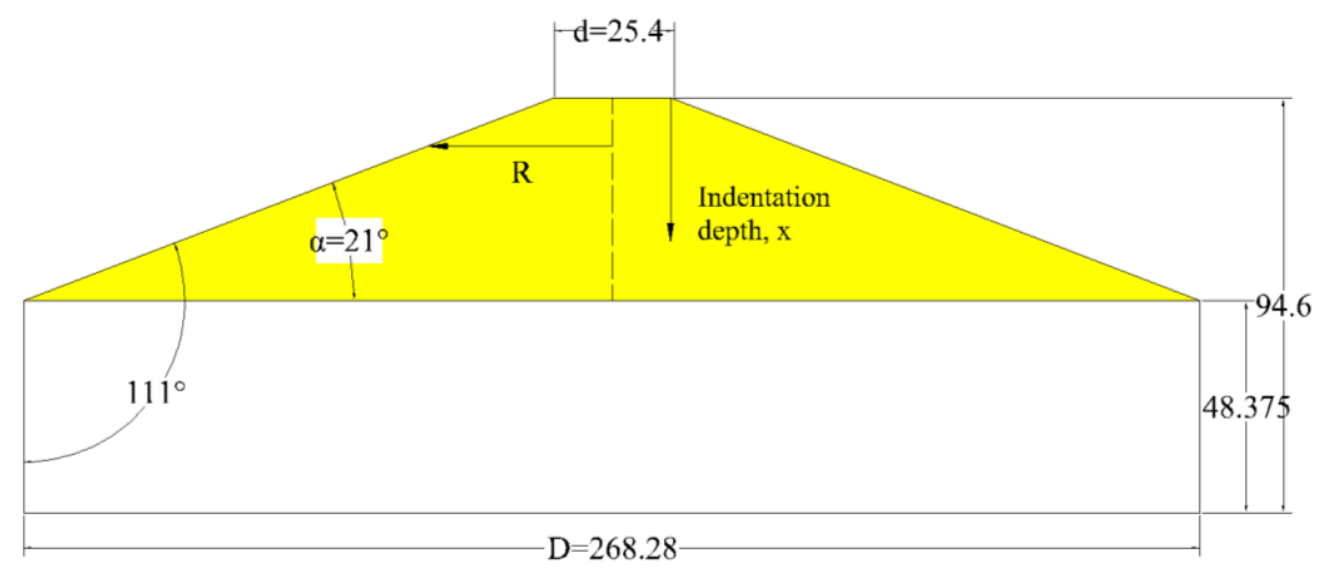

Figure 4.1: Drawing of ice sample having $21^{\circ}$ taper angle (all dimensions are in $\mathrm{mm}$ )

The test naming convention has been prepared in a way so that the parameters used for a particular test can be identified easily. An example of the test naming convention is given below.

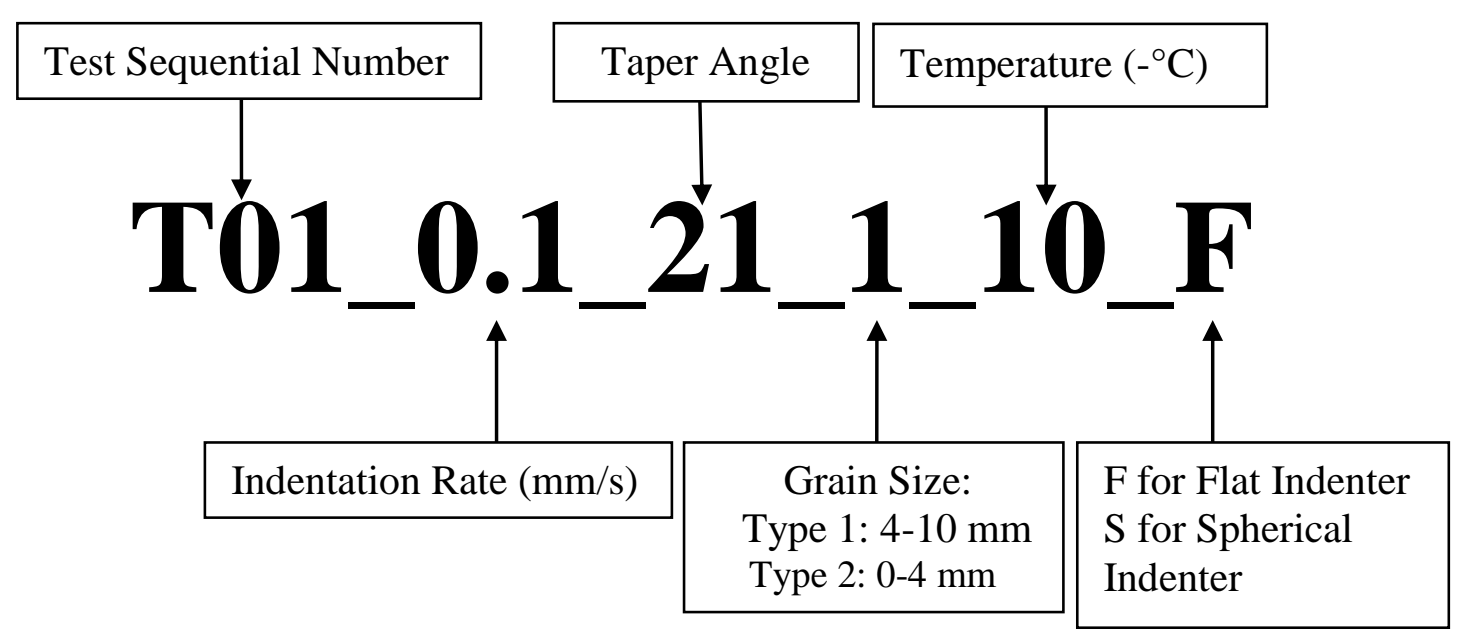

Figure 4.2: Test Naming Convention 
The test matrix of the experiments is given below in Table 4.1.

Table 4.1: Test Matrix

\begin{tabular}{|c|c|c|c|c|c|}
\hline Test ID & $\begin{array}{c}\text { Indentation } \\
\text { Rate } \\
(\mathrm{mm} / \mathrm{s}) \\
\end{array}$ & $\begin{array}{l}\text { Taper } \\
\text { Angle }\end{array}$ & $\begin{array}{c}\text { Grain Size } \\
(\mathrm{mm})\end{array}$ & Temperature & Indenter \\
\hline T01_0.1_21_1_10_F & 0.1 & 21 & $4-10^{+}$ & -10 & Flat \\
\hline T02_0.1_21_2_10_F & 0.1 & 21 & $0-4$ & -10 & Flat \\
\hline T03_1_21_1_10_F & 1 & 21 & $4-10^{+}$ & -10 & Flat \\
\hline T04_10_21_1_10_F & 10 & 21 & $4-10^{+}$ & -10 & Flat \\
\hline T05_1_21_2_10_F & 1 & 21 & $0-4$ & -10 & Flat \\
\hline T06_10_21_2_10_F & 10 & 21 & $0-4$ & -10 & Flat \\
\hline T07_10_21_2_10_S & 10 & 21 & $0-4$ & -10 & Spherical \\
\hline T08_10_21_1_10_S & 10 & 21 & $4-10^{+}$ & -10 & Spherical \\
\hline T09_10_30_1_10_S & 10 & 30 & $4-10^{+}$ & -10 & Spherical \\
\hline T10_10_30_2_10_S & 10 & 30 & $0-4$ & -10 & Spherical \\
\hline T11_0.1_30_1_10_F & 0.10 & 30 & $4-10^{+}$ & -10 & Flat \\
\hline T12_10_30_2_10_F & 10 & 30 & $0-4$ & -10 & Flat \\
\hline T13_1_30_1_10_F & 1 & 30 & $4-10^{+}$ & -10 & Flat \\
\hline T14_1_30_2_10_F & 1 & 30 & $0-4$ & -10 & Flat \\
\hline T15_10_30_1_10_F & 10 & 30 & $4-10^{+}$ & -10 & Flat \\
\hline T16_0.1_30_2_10_F & 0.1 & 30 & $0-4$ & -10 & Flat \\
\hline T17_0.1_13_1_10_F & 0.1 & 13 & $4-10^{+}$ & -10 & Flat \\
\hline T18_0.1_13_2_10_F & 0.1 & 13 & $0-4$ & -10 & Flat \\
\hline T19_1_13_1_10_F & 1 & 13 & $4-10^{+}$ & -10 & Flat \\
\hline T20_1_13_2_10_F & 1 & 13 & $0-4$ & -10 & Flat \\
\hline T21_10_13_1_10_F & 10 & 13 & $4-10^{+}$ & -10 & Flat \\
\hline T22_10_13_2_10_F & 10 & 13 & $0-4$ & -10 & Flat \\
\hline T23_10_13_1_10_S & 10 & 13 & $4-10^{+}$ & -10 & Spherical \\
\hline T24_1_13_1_10_S & 1 & 13 & $4-10^{+}$ & -10 & Spherical \\
\hline T25_0.1_21_1_5_F & 0.1 & 21 & $4-10^{+}$ & -5 & Flat \\
\hline T26_1_21_1_5_F & 1 & 21 & $4-10^{+}$ & -5 & Flat \\
\hline T27_10_21_1_5_F & 10 & 21 & $4-10^{+}$ & -5 & Flat \\
\hline T28_10_21_2_5_F & 10 & 21 & $0-4$ & -5 & Flat \\
\hline
\end{tabular}




\subsection{Results using Flat Indenter}

A total of twenty-two (22) tests were done using the flat indenter. Among them, eighteen tests were done at $-10^{\circ} \mathrm{C}$ and four tests were done at $-5^{\circ} \mathrm{C}$. The flat indenter is made of stainless steel having a diameter of $254 \mathrm{~mm}$ and a thickness of $19.05 \mathrm{~mm}$. Each test was done up to the indentation depth of $70 \mathrm{~mm}$. In this section three tests are discussed having three different indentation speeds: $0.1 \mathrm{~mm} / \mathrm{s}, 1 \mathrm{~mm} / \mathrm{s}$ and $10 \mathrm{~mm} / \mathrm{s}$. A warm test result at $-5^{\circ} \mathrm{C}$ is also discussed.

\subsubsection{Slow Speed Test $(0.1 \mathrm{~mm} / \mathrm{s})$}

The slow speed tests mainly show ductile failure throughout the experiment. To study the behaviour of compressive ice failure only the first 60 seconds were analyzed for this case. Force-time data collected from the MTS load cell and pressure-area data calculated from Equations 4.1-4.3 are presented in Figure 4.3. The maximum force for this ice sample was recorded as $355 \mathrm{kN}$ and maximum pressure was $32.88 \mathrm{MPa}$ up to the indentation depth of $6 \mathrm{~mm}$.

Tactile pressure sensor data provides an overview of pressure distributions throughout the indentation. The load increases and decreases correspond to the pressure fluctuations on the contact surface of the ice sample and indenter. Figure 4.4 represents the pressure distribution images which are compared to the data from Figure 4.3. In Figure 4.3, event (a) and (b) show an increasing trend of load and pressure with time and area. Similar results can be observed in Tekscan images in Figure 4.4 as from event (a) to (b) pressure distributions have increased with significant increase of contact area. At event (c) a peak 
load and pressure are observed in force-time and pressure-area plots. At that event, some high pressure zones are observed in the tactile pressure sensor image with a consistent round contact surface, that represents no spalling events but a damage enhanced creep and slow crushing failure. There is a load drop and decrease of high pressure zones at event (d) and another sharp load drop is observed at event (e) at the load cell data. Correspondingly, decrease the intensity of high pressure zones without any significant change of contact area are observed from pressure distribution images (c) to (d) and (d) to (e). A slow increase of the load can be seen after 17 seconds at event (f). Decreasing intensity of high pressures in tactile image (f) corresponds the decreasing pressure at point (f) in the pressure-area plot. The load cell data and tactile pressure sensor data show good agreement in the results during the compressive tests. Throughout the slow test, the contact area has maintained a constant circular shape with the evidence of damaged enhanced creep and no large spalling events from the periphery of the indenter. The after crushing image in Figure 4.5 shows that following the test, the sample has similar round shaped final surface with smooth finish. From the image it is clear that the failure is ductile and has a final surface representing slow continuous crushing and damage enhanced creep. 

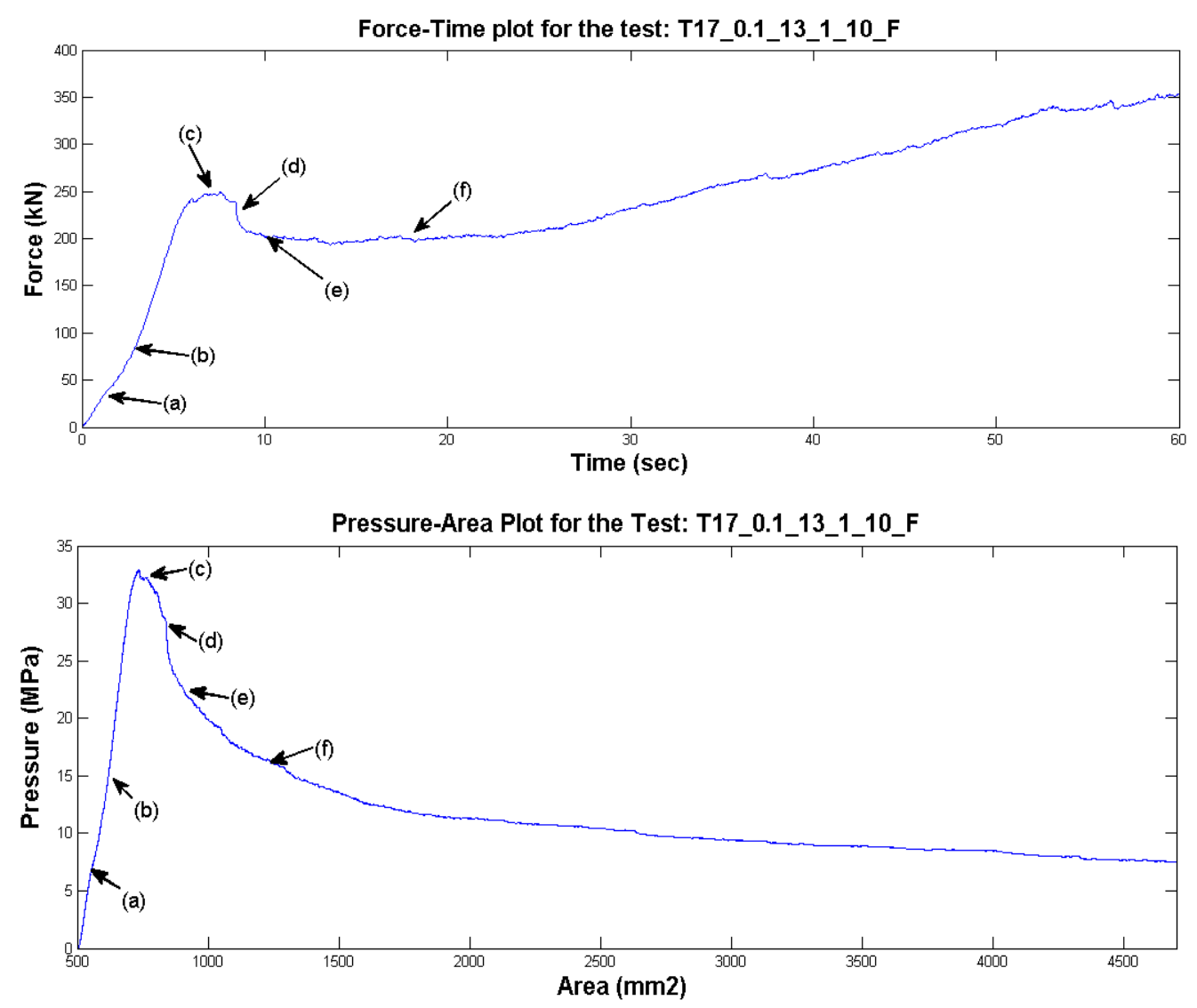

Figure 4.3: Force-time and Pressure-area plot for the test T17_0.1_13_1_10_F

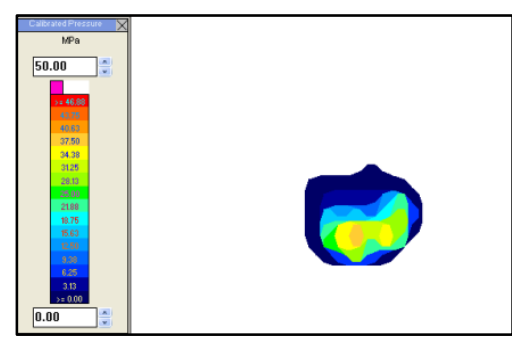

(a)

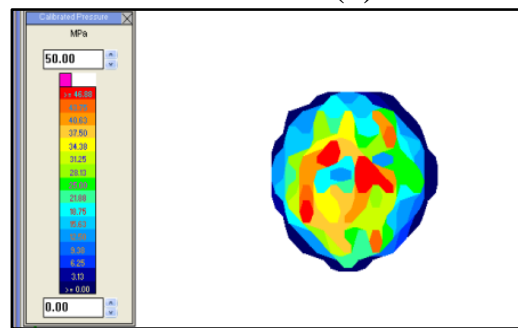

(d)

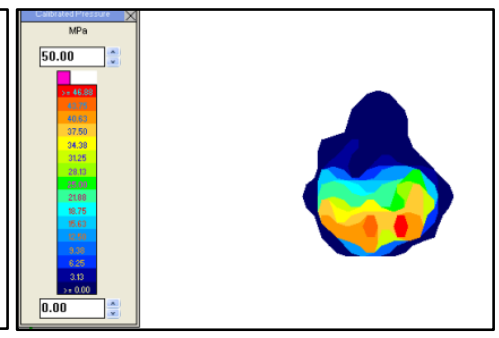

(b)

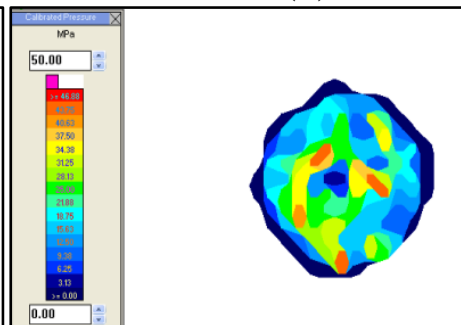

(e)

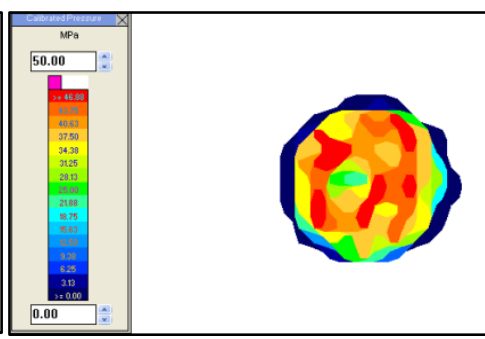

(c)

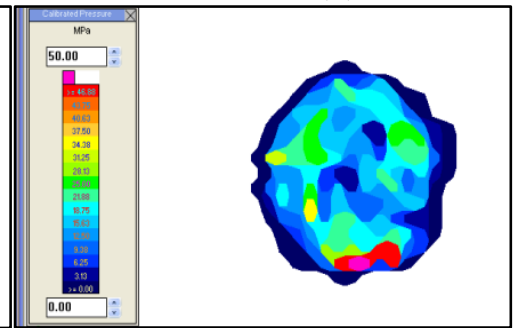

(f)

Figure 4.4: Pressure distribution images from tactile pressure sensor data corresponding to the load cell data for the test T17_0.1_13_1_10_F 


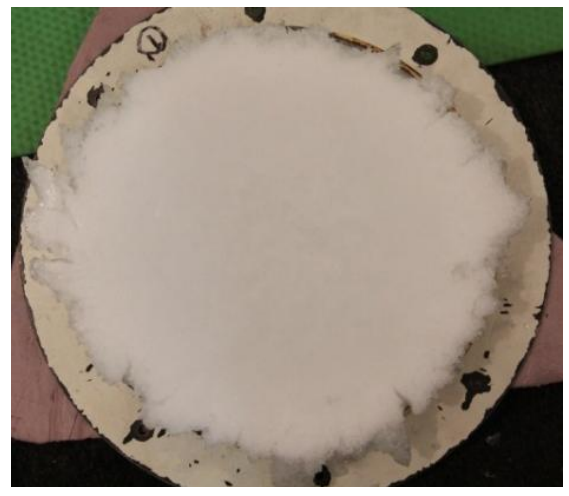

Figure 4.5: After crushing image of the slow test T17_0.1_13_1_10_F

The spall mass distribution from the same test is given below in Figure 4.6, which shows that there was small amount of spalling, dominated by a few large spalls. In this test, total spalls collected were $2.4 \mathrm{~kg}$. From the distribution, it can be seen that the mass of the large sized spalls is considerable, even though the amount of the medium and small sized spalls are significantly lower. A large sized spall may be the reason for the load drop at the beginning of the test as can be seen from the Figure 4.3. This is indication of large time-dependent fractures at slower rates.

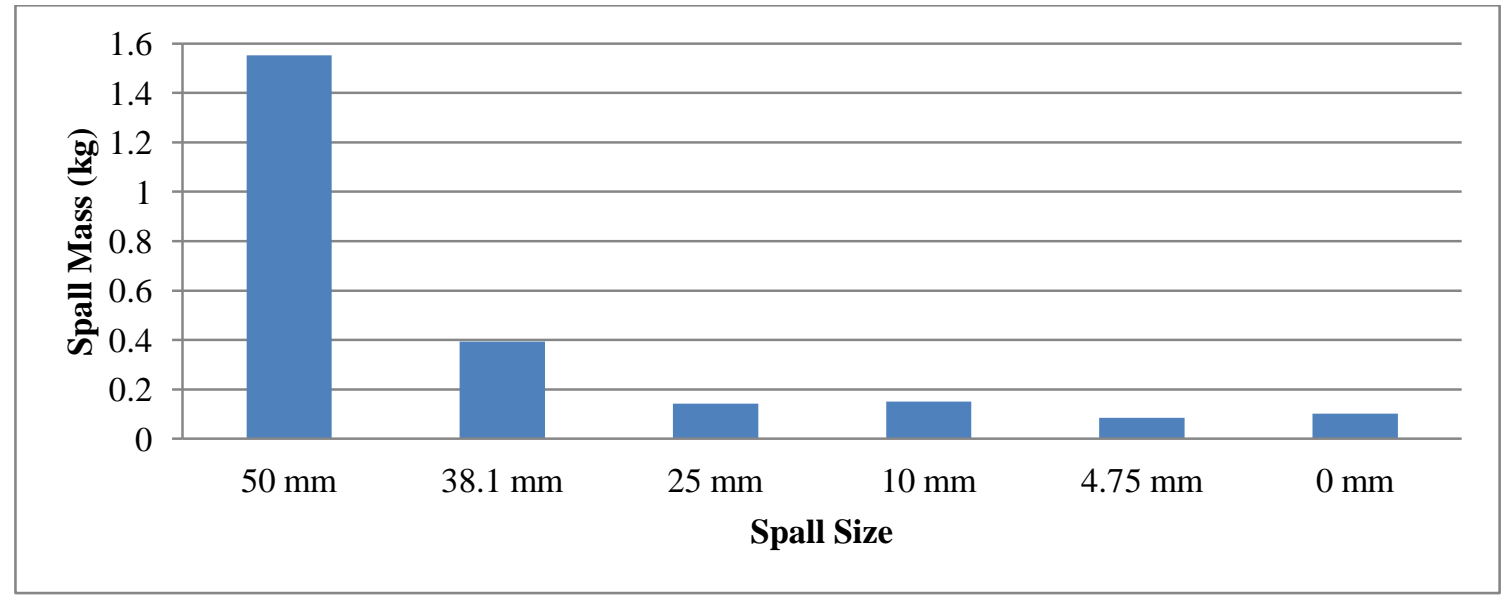

Figure 4.6: Spall distribution for the test T17_0.1_13_1_10_F 
The thin-section pictures of the vertical section having a height of $40 \mathrm{~mm}$ presented in Figure 4.7 are from the test T16_0.1_30_2_10_F. The first picture was taken through cross polarized light and the second picture was taken using the side lighting technique. From the cross polarized light image a damaged layer up to $30 \mathrm{~mm}$ in depth is observed, while from the side light picture large area containing microfractures can be seen. In the case of slow speed tests, the damage is observed to be highest compared to medium and fast speed tests.
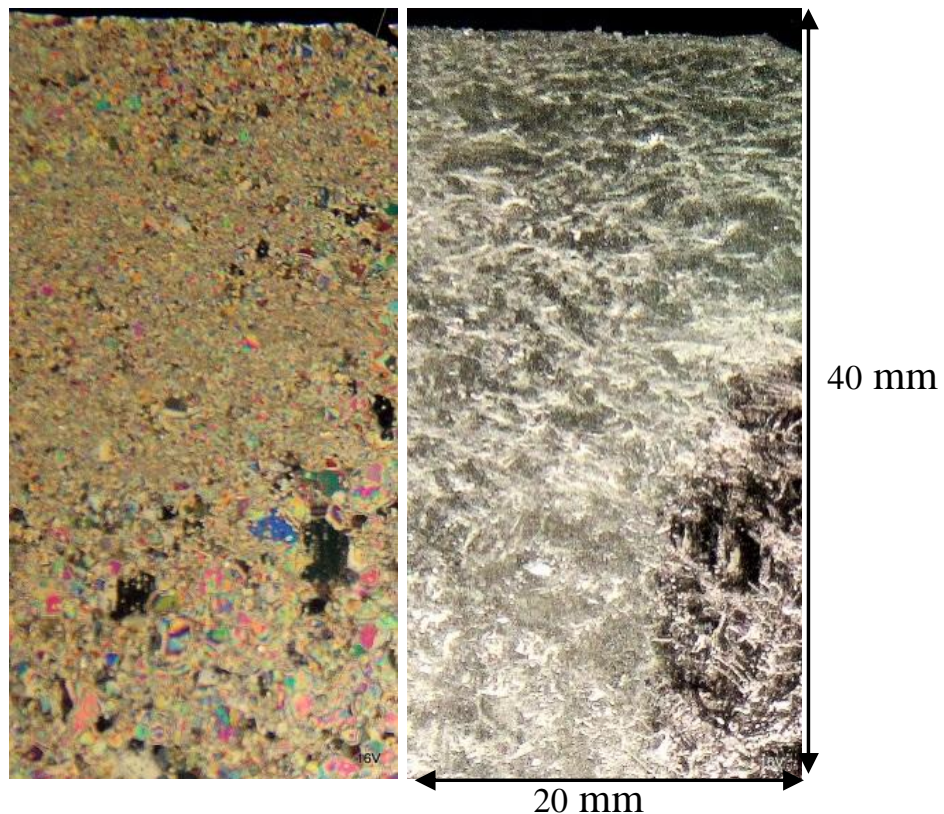

Figure 4.7: Vertical thin-section pictures using cross polarized light (colour) and side light (black and white) for the test T16_0.1_30_2_10_F 


\subsubsection{Medium Speed Test $(1 \mathrm{~mm} / \mathrm{s})$}

A medium speed test is presented in this section to discuss the results. The force-time and pressure-area plots are given in Figure 4.8 and the corresponding pressure distribution images from tactile pressure sensor are given in Figure 4.9. For this test, the maximum force was recorded as $40 \mathrm{kN}$ and maximum pressure was $33 \mathrm{MPa}$, up to the indentation depth of $6 \mathrm{~mm}$. In the force-time plot, large fluctuation of loads can be seen at point (a), (b) and (c). By observing the corresponding pressure distribution images from Figure 4.9 it can be seen that the contact area has been increased from event (a) to (b). Afterwards, a decrease in area and the intensity of high pressure zones at event (c) indicates a spalling event. A sharp drop of force at load cell data is the result of high pressure zone failure without an applicable change in time and position. At event (d) a peak force and a high pressure zone is observed under a bigger area of contact. Subsequently, another load increment can be seen at point (e) with a bigger area of contact with pressure distributions in tactile pressure image (e). Later, point (f) shows a drop of load within a fraction of a second without changing the position. In the images of the pressure distributions the contact areas of (e) and (f) are almost same, but there is a decrease in intensity of high pressures. 

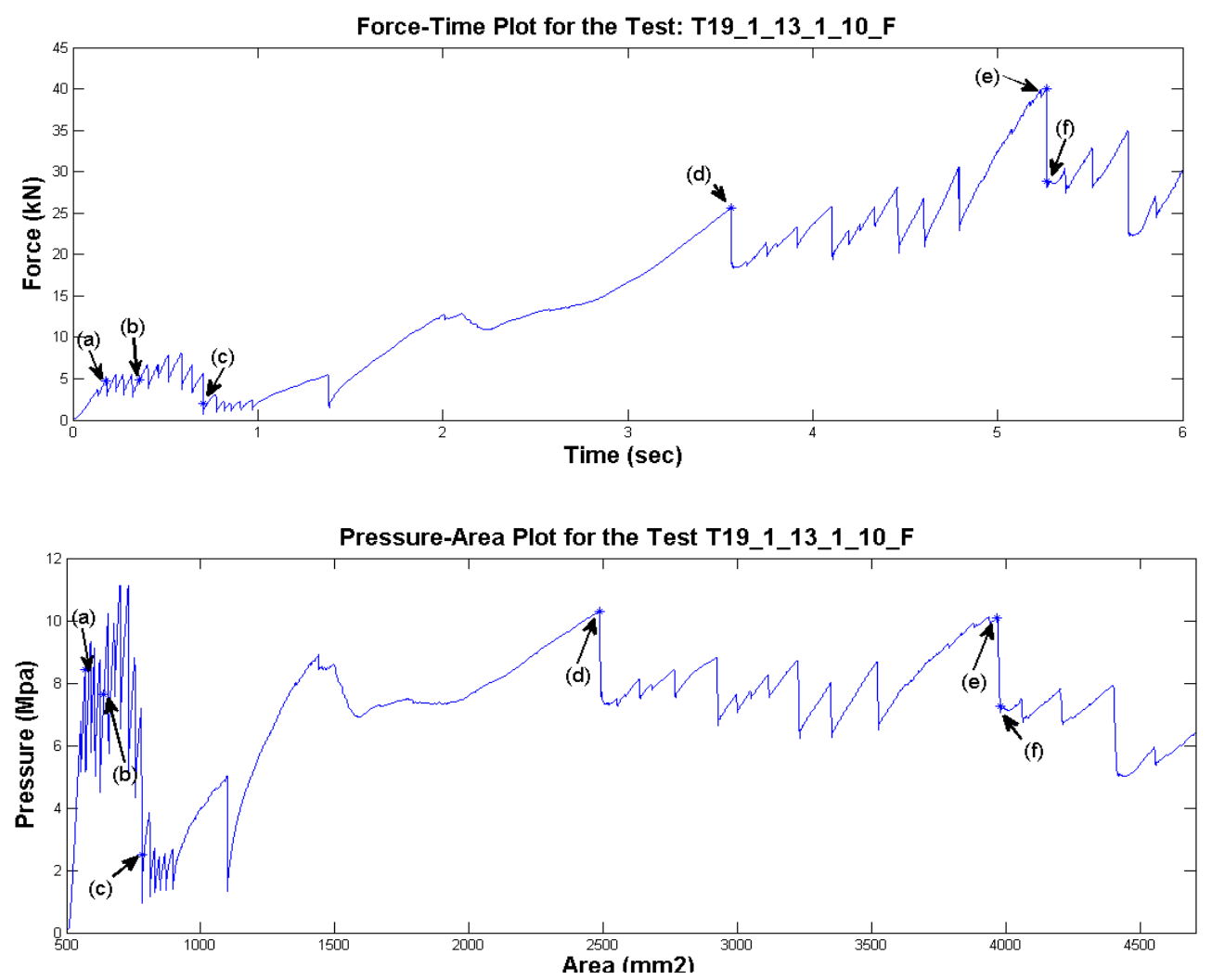

Figure 4.8: Force-time and Pressure-area plot for the test T19_1_13_1_10_F

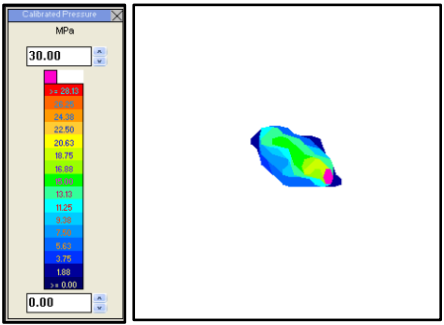

(a)

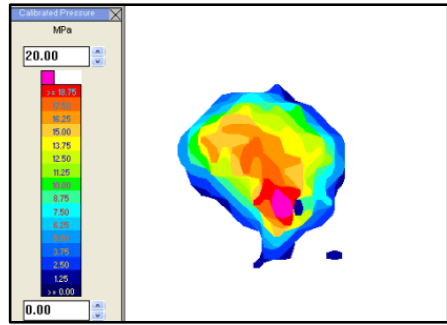

(d)

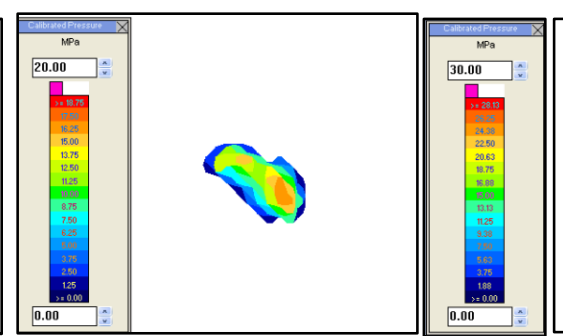

(b)

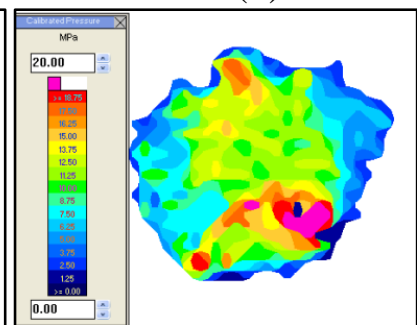

(e)

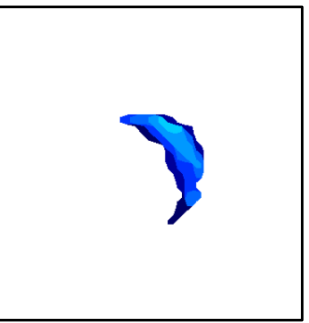

(c)

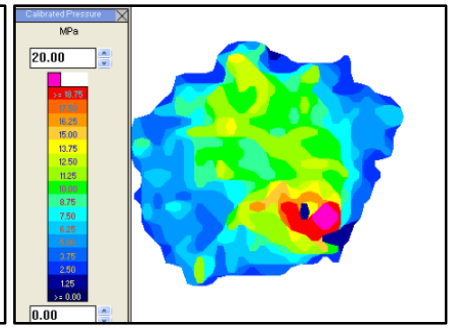

(f)

Figure 4.9: Pressure distribution images from tactile pressure sensor data corresponding to the load cell data for the test T19_1_13_1_10_F 
The photograph taken after crushing for the same test shows a mixture of ductile and brittle failure in Figure 4.10. In the center a smooth surface can be seen but at the edge there are random spalls.

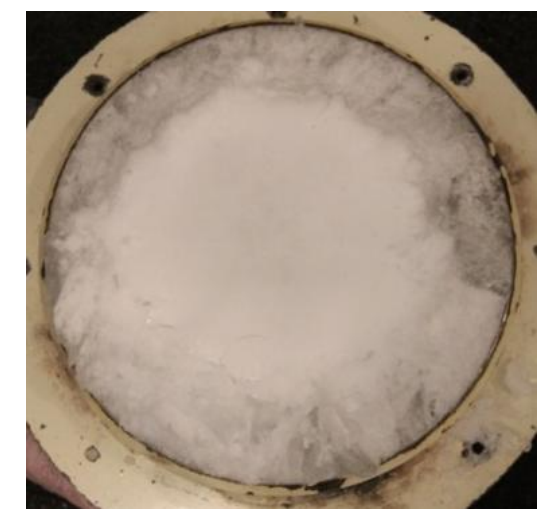

Figure 4.10: After crushing image of the medium speed test T19_1_13_1_10_F

From Figure 4.11 the collected spall data is given from the same test. The spall distribution show that it has more large sized spalls compared to medium and small sized spalls. The total spall weight for this test was $2.7 \mathrm{~kg}$.

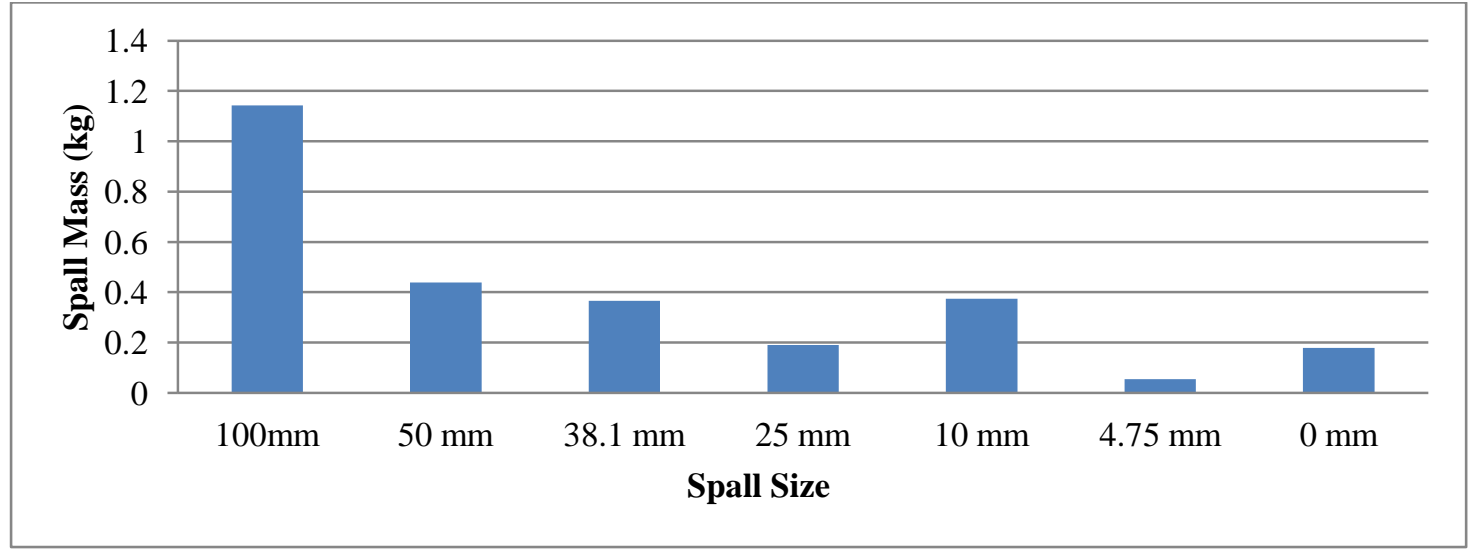

Figure 4.11: Spall distributions for the test T19_1_13_1_10_F 
Thin-section pictures for a medium speed test are given in Figure 4.12. The first image, taken through the cross polarized light, shows a clear view of the damage zone. The second image was taken using side lighting technique which shows the extensive microfracture in this region. Both images show a layer of damage having almost $20 \mathrm{~mm}$ of depth.
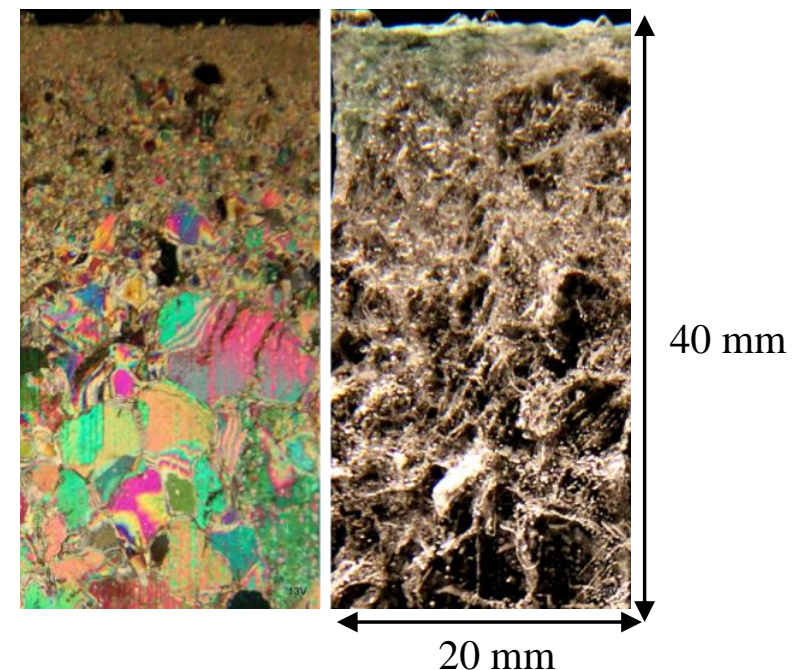

Figure 4.12:Vertical thin-section pictures using cross polarized light (colour) and side light (black and white) for the test T13_1_30_1_10_F

\subsubsection{Fast Speed Test $(10 \mathrm{~mm} / \mathrm{s})$}

Force-time data from the MTS load cell and tactile pressure sensor data from Tekscan instrumentation are given here from test T21_10_13_1_10_F. The force-time and pressure -area plots are given in Figure 4.13. The maximum force of this test was recorded as $36 \mathrm{kN}$ and the maximum pressure observed was $7 \mathrm{MPa}$ up to the indentation depth of $10 \mathrm{~mm}$. Events (a), (b) and (d) show peak loads at those points and corresponding high pressure zones are observed in pressure distribution images in Figure 
4.14. Events (c) and (e) show load drops with decreasing the intensity of high pressure zones without an appreciable change in the contact area and time. Another load drop can be seen at point (f) which is a result of decreasing high pressure zones. As well, a decrease in the contact area in pressure distribution image at event (f) is the evidence of spalling event.
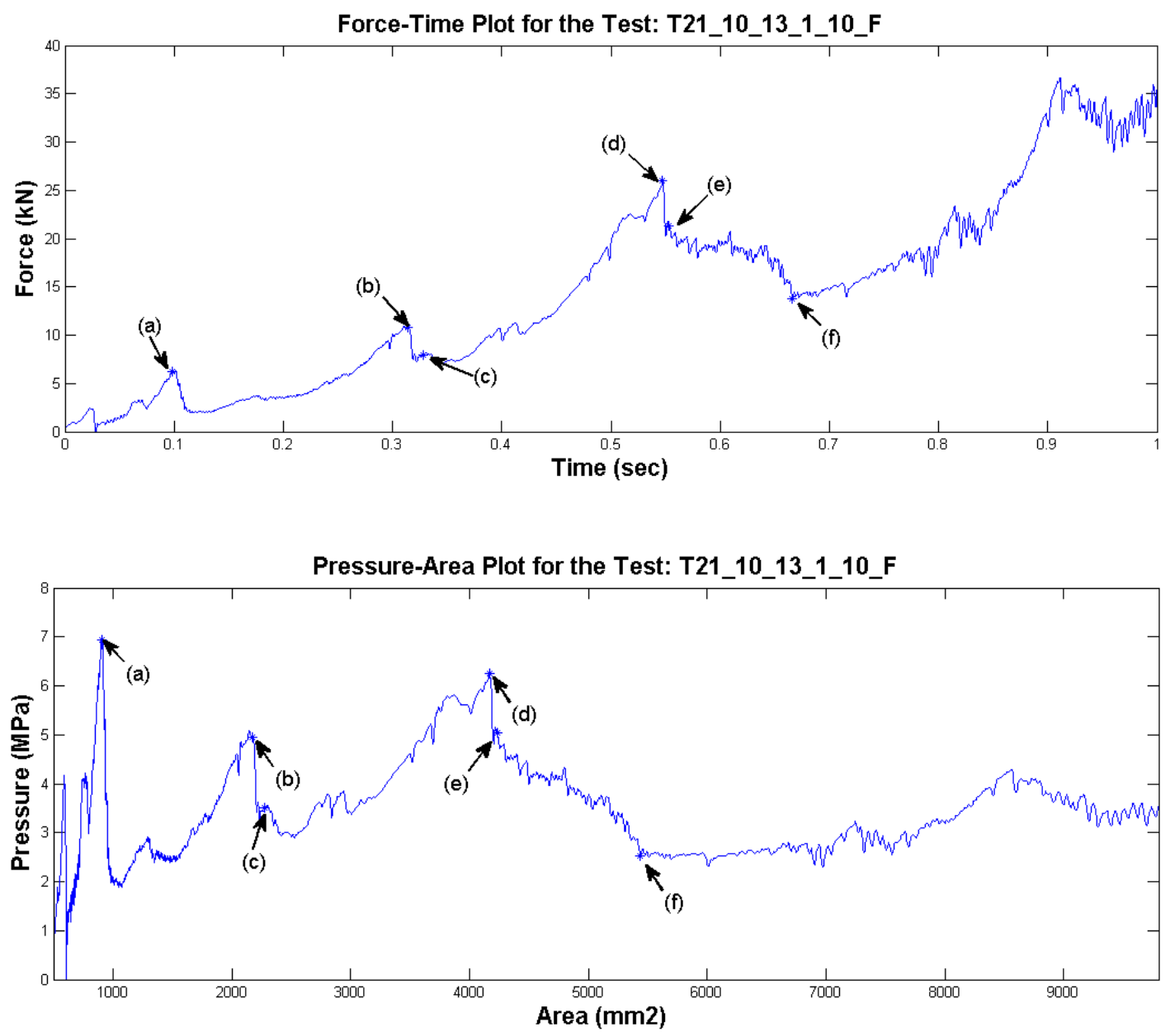

Figure 4.13: Force-time and Pressure-area plot for the test T21_10_13_1_10_F 


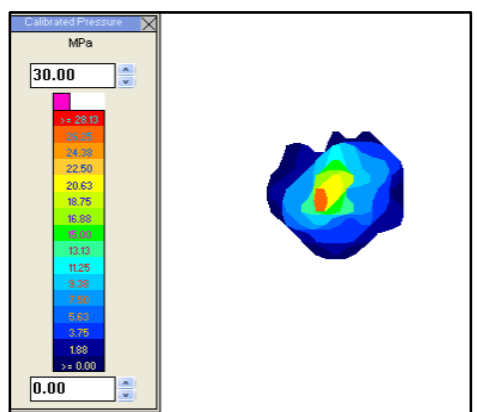

(a)

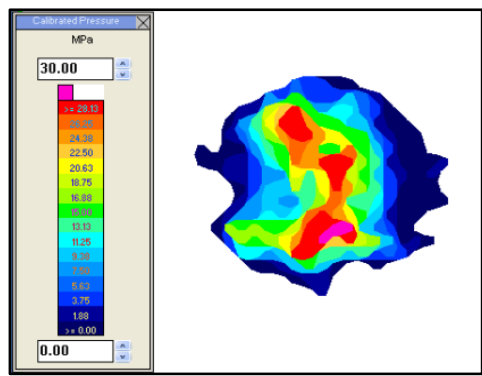

(d)

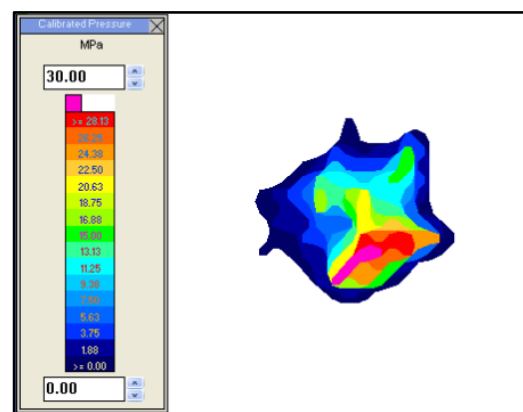

(b)

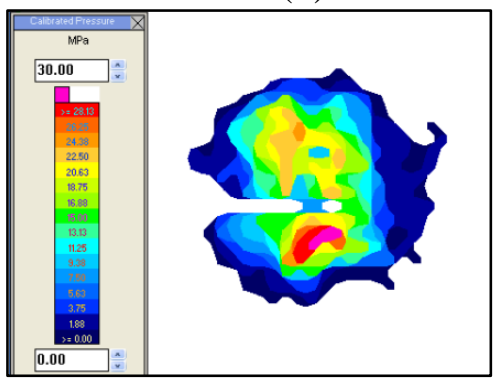

(e)

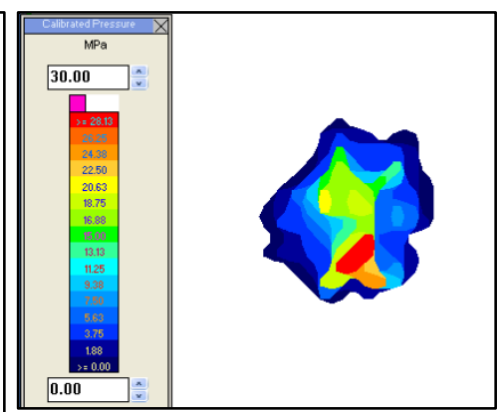

(c)

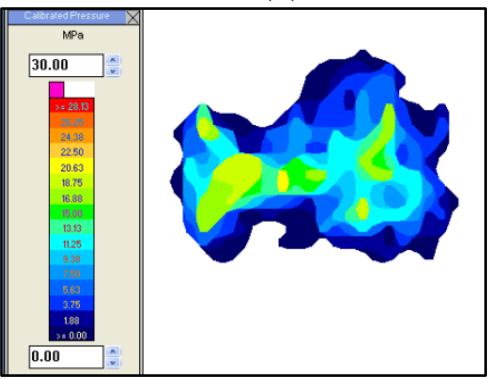

(f)

Figure 4.14: Pressure distribution images from tactile pressure sensor corresponding to the load cell data for the test T21_10_13_1_10_F

The following Figure 4.15 is the photograph taken after crushing for the same test. From the image it can be seen that the failure was brittle in nature and extensive random spalling was observed.

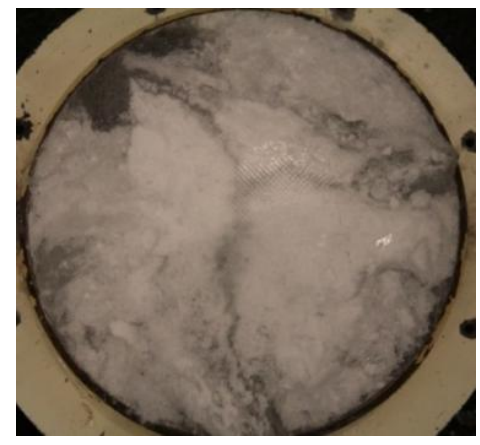

Figure 4.15: After crushing image of the fast speed test T21_10_13_1_10_F 
The spall distributions for the test discussed above is presented in Figure 4.16. The spall effects were totally random in case of this fast speed tests with a total spall mass of 2.88 $\mathrm{kg}$. The distribution shows that there are big sized spalls and also significant amount of medium and small sized spalls are present.

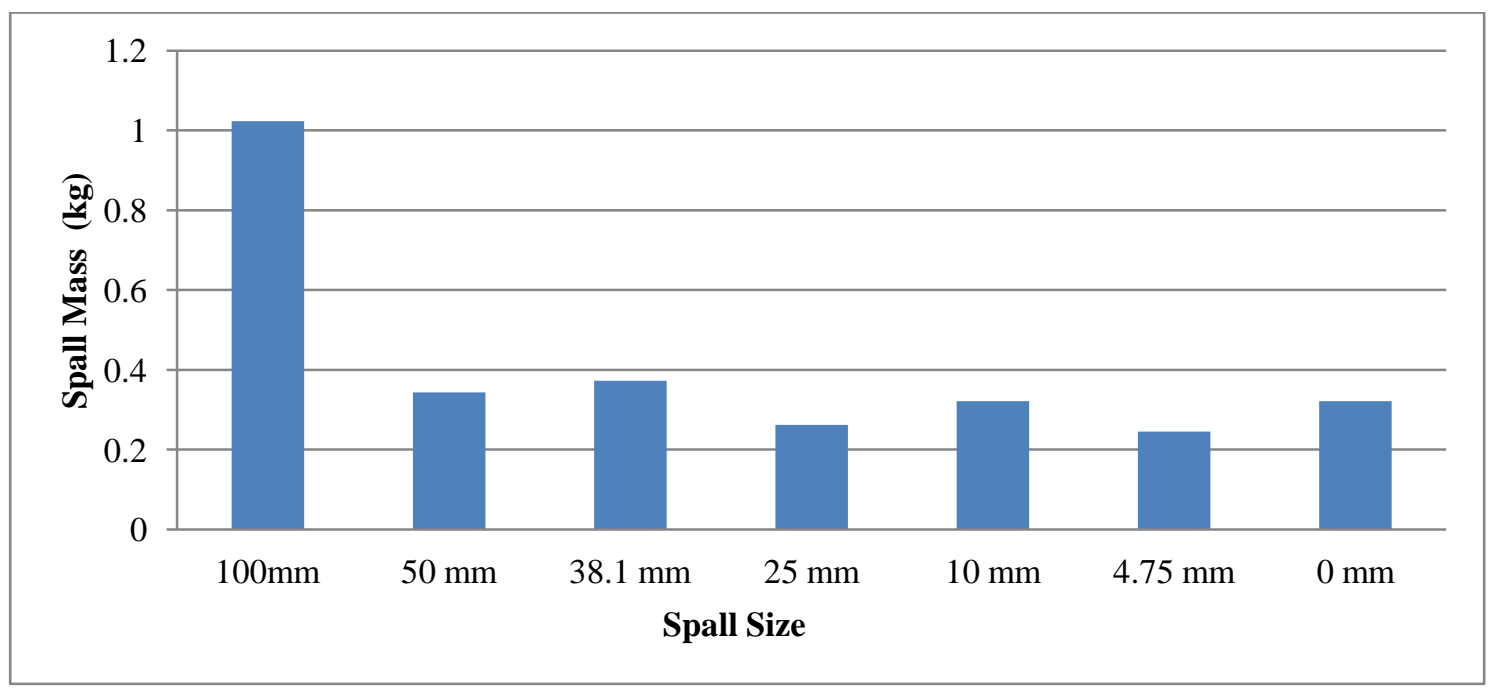

Figure 4.16: Spall distributions for the test T21_10_13_1_10_F

Thin-section pictures of the vertical section are presented in Figure 4.17 from a fast indentation test. The first picture was taken through the cross polarized lighting shows that the recrystallization is more prominent. Microfracture and cracks dominate the damaged layer appearing as the white and opaque zone in the second image which was taken using the side lighting technique. During this fast indentation test, the depth of damaged layer is only a few millimetres. 

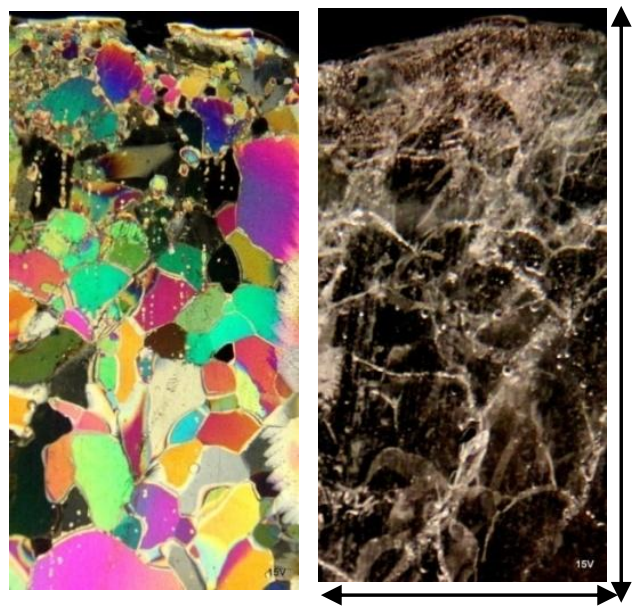

$40 \mathrm{~mm}$

$20 \mathrm{~mm}$

Figure 4.17: Vertical thin-section pictures using cross polarized light (colour) and side light (black and white) for the test T15_10_30_1_10_F

\subsubsection{Warm Ice Test $\left(-5^{\circ} \mathrm{C}\right)$}

The warm ice tests were done at a temperature of $-5^{\circ} \mathrm{C}$ using the flat indenter. A sample test results are given here to discuss the force-time effect with tactile pressure sensor data. The maximum force observed was $26 \mathrm{kN}$ and maximum pressure was $7 \mathrm{MPa}$ up to the indentation depth of $10 \mathrm{~mm}$ in this fast test. The force-time and pressure-area curves are provided in Figure 4.18 and the corresponding images of the tactile pressure sensor are given in Figure 4.19. It can be seen that from point (a) the load under indentation has started to increase up to point (b). After the peak load at point (b), a load drop is noticed at point (c) within a fraction of second. The tactile pressure sensor data also show corresponding pressure distributions with the loss of some contact area but experiencing higher pressure. This is because of the extrusion of spalls from the ice sample. At point (d) a peak load is observed in force-time data and some high pressure zones are located at event (d) in pressure distribution image. Again, at point (e) another peak load is observed 
at 0.75 seconds and point (f) shows a load drop event in an interval of 0.2 seconds. From the pressure distribution images, event (e) shows a full contact area with the sensor and pressure distributions all over the contact surface, slightly higher in the center. Later, at event (f) decrease in pressures and contact area can be seen in tactile pressure data because of the spalling incidents. From the force-time data in Figure 4.18 it can be inferred that the failure process in warmer test shows an inclination to ductile failure, even though this was a fast speed test.
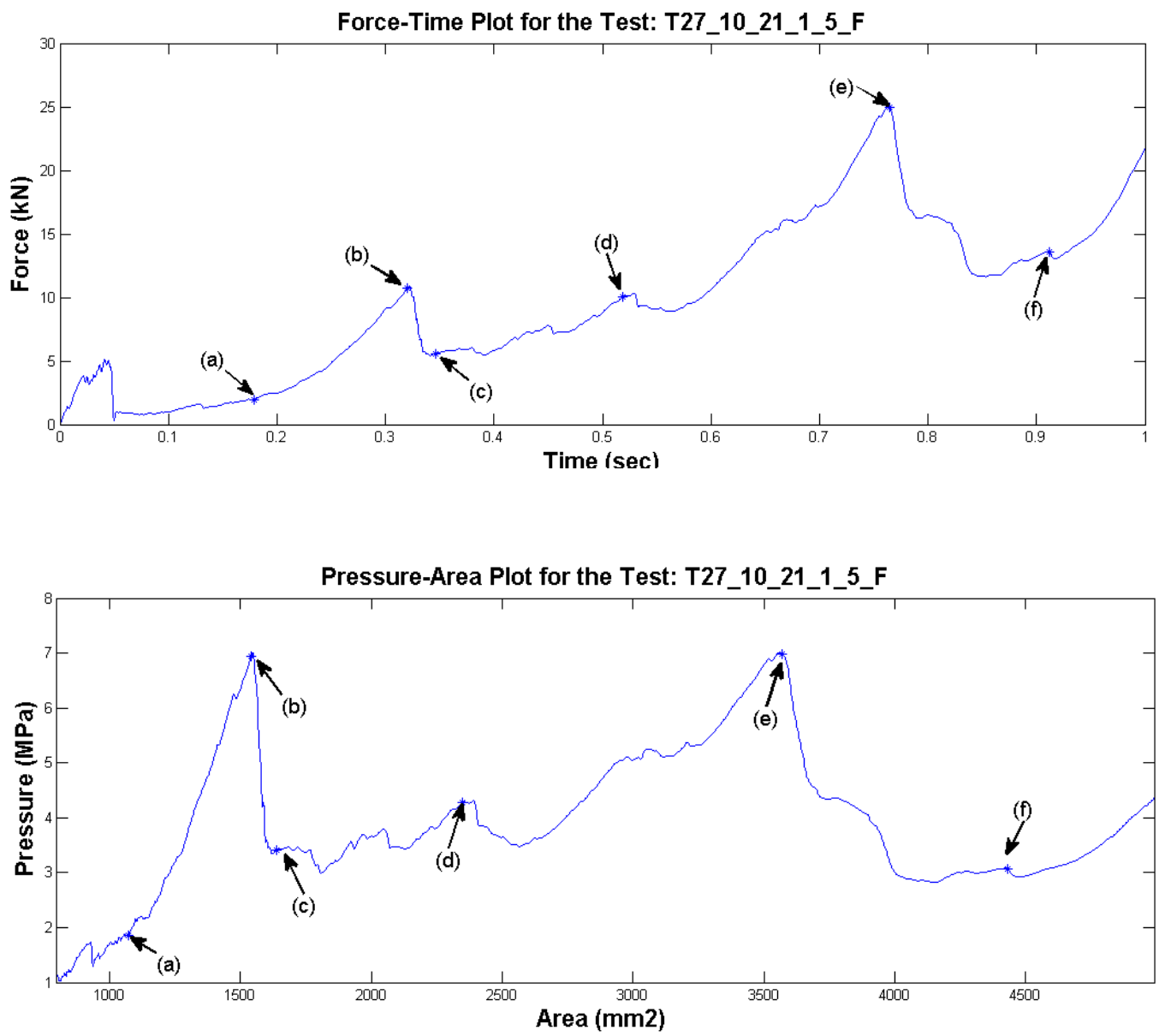

Figure 4.18: Force-time and Pressure-area plot for the test T27_10_21_1_5_F 


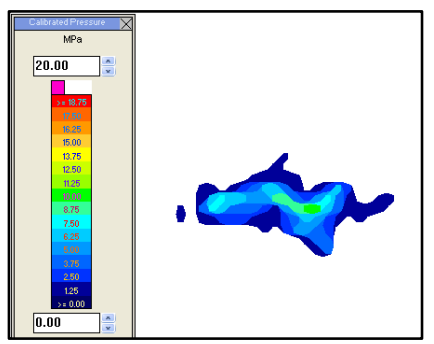

(a)

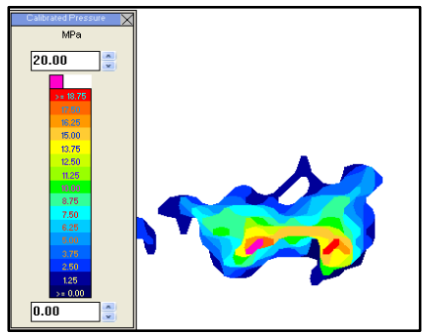

(d)

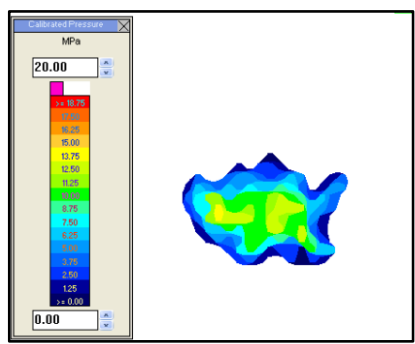

(b)

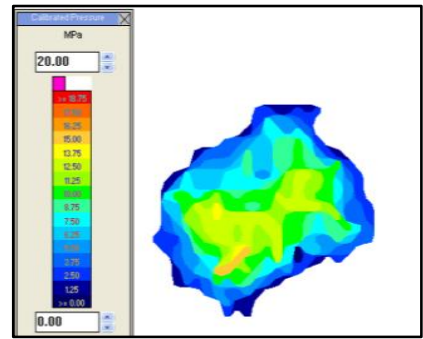

(e)

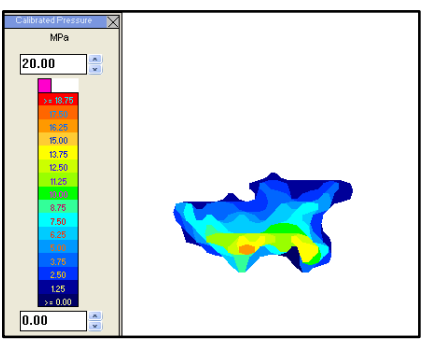

(c)

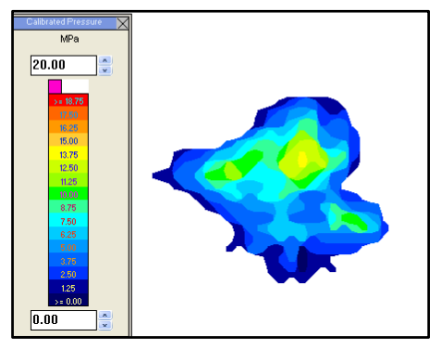

(f)

Figure 4.19: Pressure distribution images from tactile pressure sensor data corresponding to the load cell data for the test T27_10_21_1_5_F

The image of the ice sample after crushing shows the randomness of failure consistent with other fast speed tests. From Figure 4.20 it can be seen that more spalling happened at the edges and even though it was a fast speed test, the indentation zone was found to have smooth slippery finish at the center of the sample indicating the test was done at a warmer temperature.

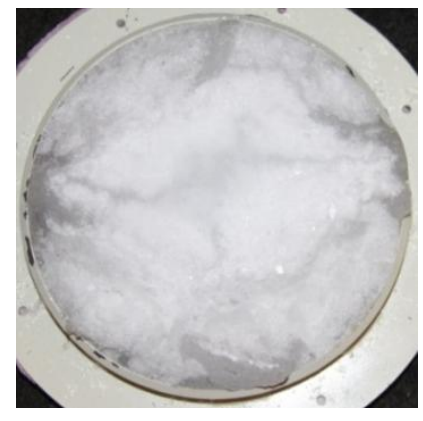

Figure 4.20: After crushing image of the warm test T27_10_21_1_5_F 
The spall distribution for the same test is given below with a total spall mass of $1.8 \mathrm{~kg}$. Figure 4.21 shows that the big sized spalls are more compared to medium and small sized ice spalls.

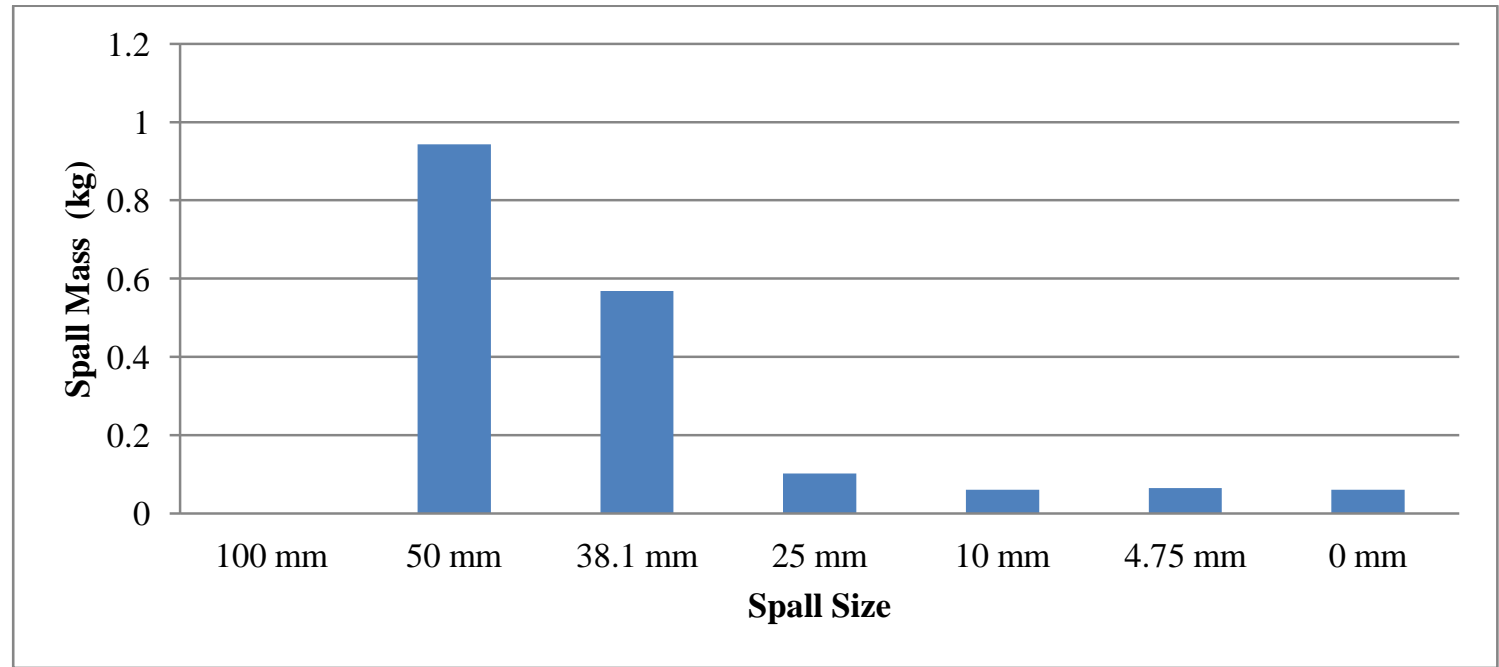

Figure 4.21: Spall distribution of test T27_10_21_1_5_F

The pictures of a vertical thin-section of $20 \mathrm{~mm}$ in height given in Figure 4.22 are from the same test. From the thin-section pictures, the damaged layer and effect of microfracture can be seen clearly. The first image of cross polarized lighting shows the heavily dominated recrystallization layer up to the middle of the thin-section image. The second image of side lighting reveals the microfractured zone near the edge having a visible white and opaque area up to the depth of few $\mathrm{mm}$. 

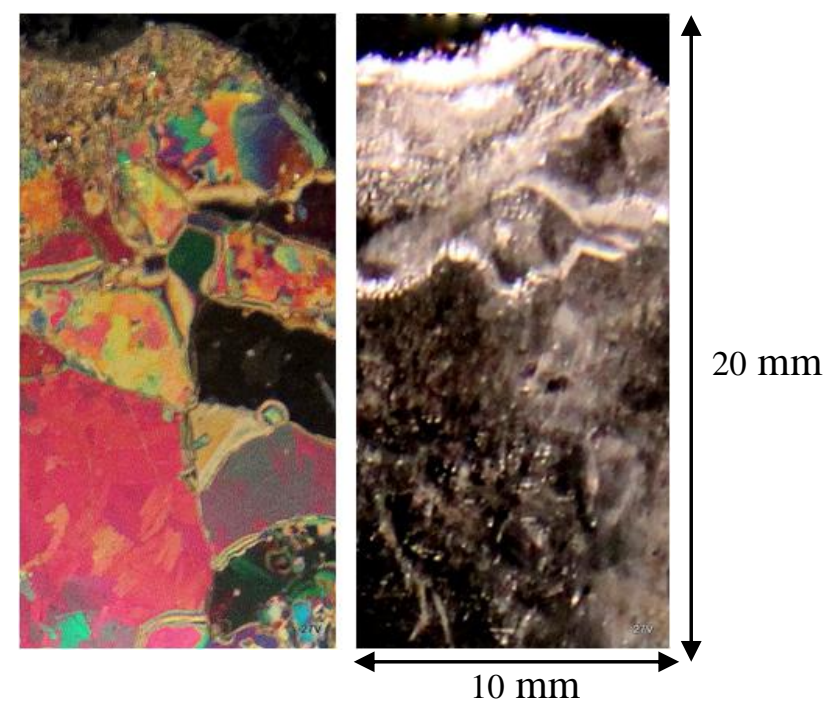

Figure 4.22: Vertical thin-section pictures using cross polarized light (colour) and side light (black and white) for the test T27_10_21_1_5_F

\subsection{Result Using Spherical Indenter}

The spherical indenter used in this research is made of aluminum and has a radius of curvature of $325.12 \mathrm{~mm}$. In total six (6) tests were done using this indenter with ice samples having three different taper angles. The force-time data presented here are generated from the MTS load cell data acquisition system and the area and pressure are calculated as previous. In the case of spherical indenter, Tekscan sensor was not used because of the curved shape of the indenter, the area under the sensor would not fully cover the ice sample. Therefore, the pressure distributions would not be possible to record for the entire contact area. Instead, snapshots from high-speed video recording were observed corresponding to forces from the load cell data. Photographs of ice samples immediately after crushing tests were also taken for all the cases as a post 
experimental analysis. The spalling effect and thin section pictures were also analyzed in the case of the spherical indenter using same method.

The results are given from a ice crushing test that used spherical indenter. The force-time and pressure-area plots for the test are given in Figure 4.23 and snapshots of the test are given in Figure 4.24. The fluctuations of forces and pressures in Figure 4.23 indicate that it was a fast speed test. The maximum force noted was $30 \mathrm{kN}$ and maximum pressure was 5.5 MPa up to the indentation depth of $45 \mathrm{~mm}$ in this test.

Loads at points (a) to (f) in force-time data correspond to the snapshots of events (a) to (f). A peak load at point (a) is observed from load cell data and from the snapshots it is seen that there are some small spalling events going on in a smaller contact area. At point (b) a sharp load drop is observed which is because of a large spall failure as shown in Figure 4.24(b) with a red indicator. At point (c) an increase of overall load is observed in load cell data with a continuous cyclic loading pattern because of the continuous crushing events as can be seen from the snapshot 4.24(c). In Figure 4.24, event (d) and (e) show some large spall events (as indicated) which are the cause for the load drops at points (d) and (e) in Figure 4.23. Loading at point (f) show similar pattern like point (c) as the load increases with cyclic loadings and no significant spalling is observed at event (f). 

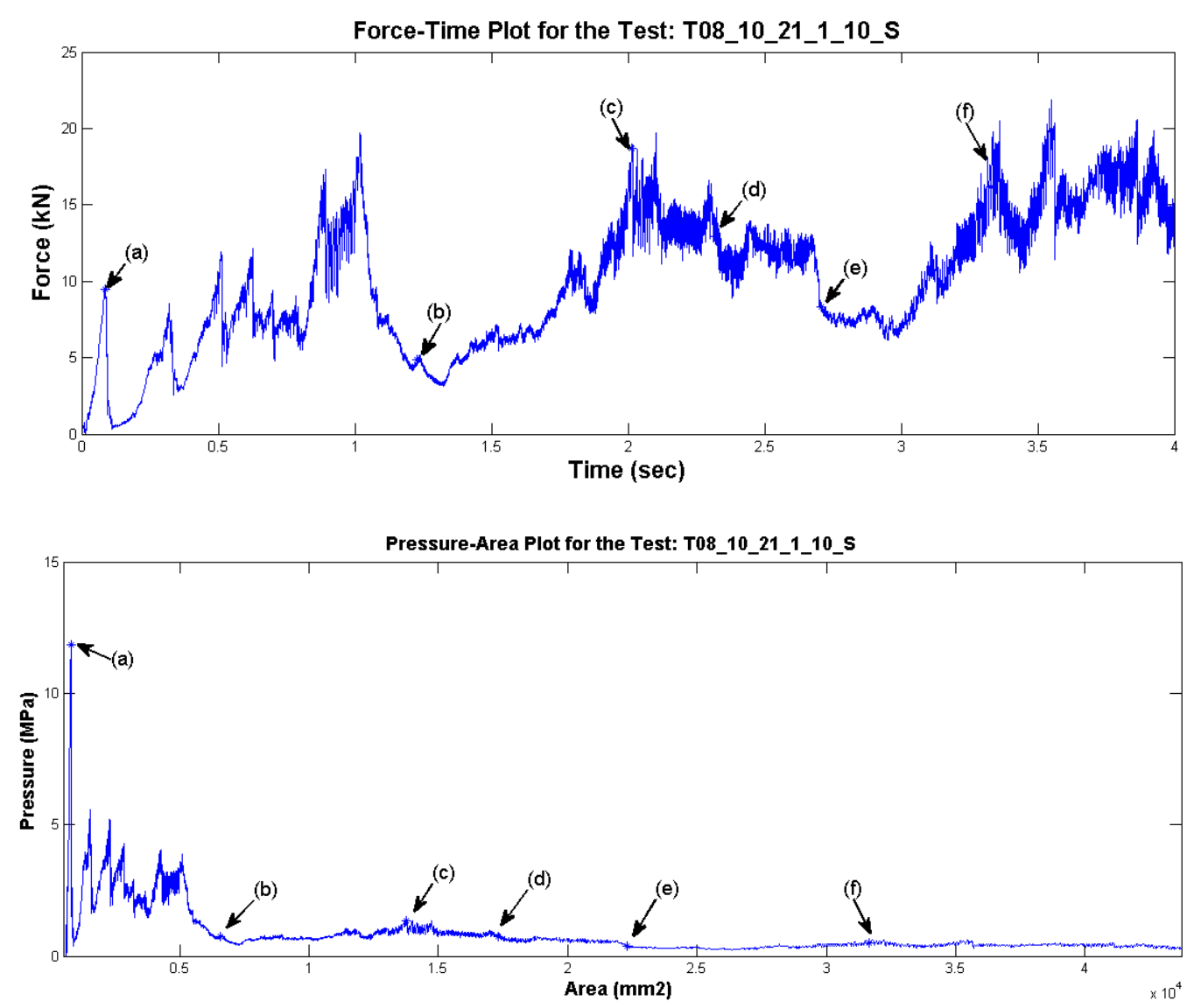

Figure 4.23: Force-time and Pressure-area plot for the test: T08_10_21_1_10_S

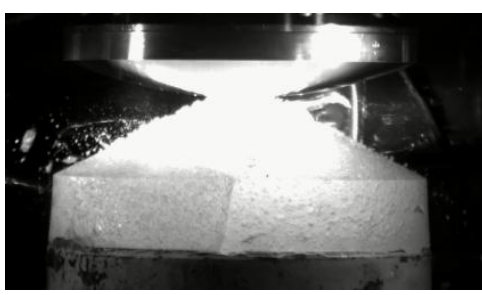

(a)

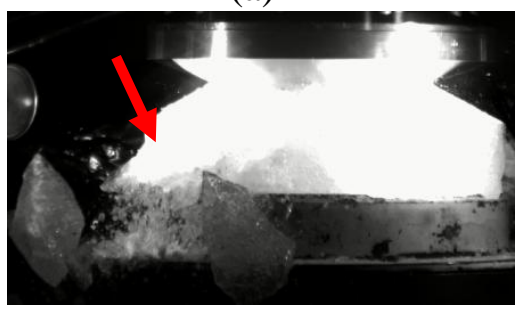

(d)

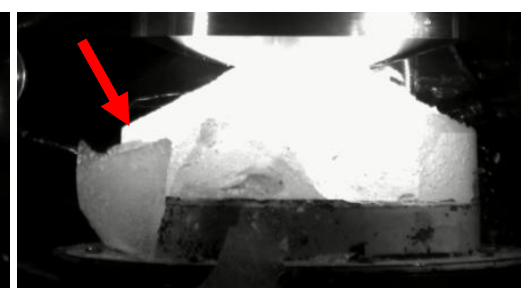

(b)

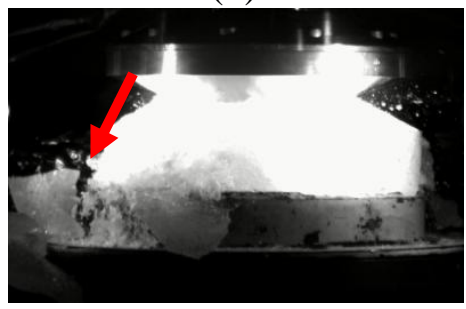

(e)

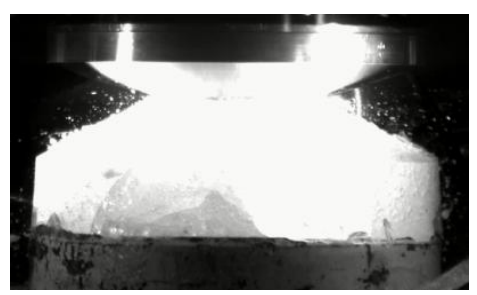

(c)

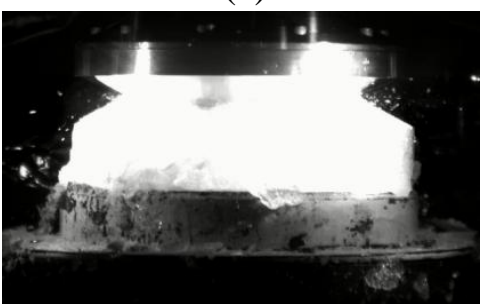

(f)

Figure 4.24: High-speed video snapshots corresponding to the load cell data from the test T08_10_21_1_10_S 
Figure 4.25 shows an image of an ice sample after the crushing test. The image shows clear evidence that the indentation has been done using a spherical indenter. The depth in the middle is more than at the edges, and also the edges show more ice than the center because of the higher height of the indenter in the center.

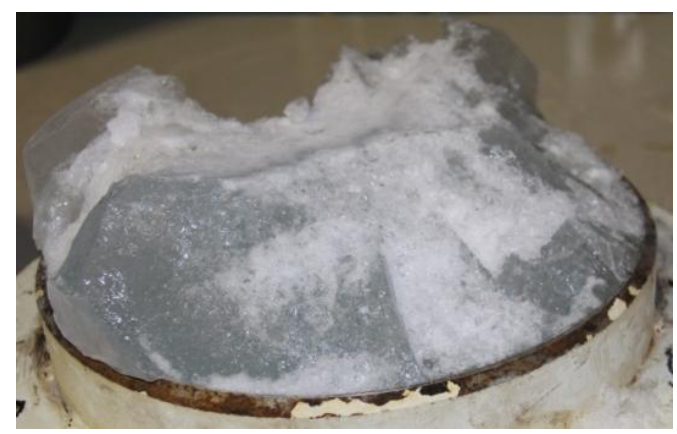

Figure 4.25: After crushing image of the test T08_10_21_1_10_S

The spall mass distribution for the same test is given in Figure 4.26. The spalling effect shows randomness in their mass distributions. As well, it can be seen that the bigger and small sized spalls weigh more than medium sized spalls.

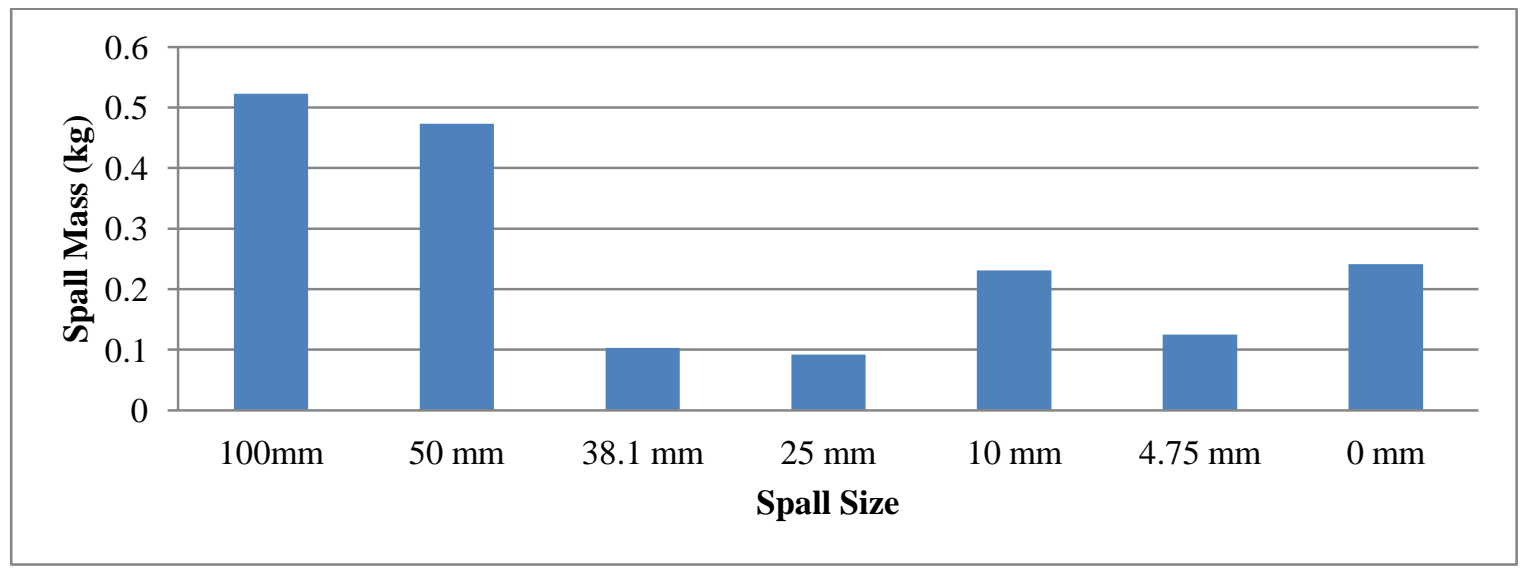

Figure 4.26: Spall distributions for the test T8_10_21_1_10_S 
Vertical thin-section pictures of the same test are presented in Figure 4.27. The first image was taken through cross polarized light and shows a small recrystallized area near the edge. The second image was taken using a side lighting technique and shows that the microfractures are dominant in this case. From this image, the crack propagation and damaged layer can be observed very clearly. A curvature and depression in the center of the thin section images indicate that the sample used a spherical indenter, resulting in greater amounts of recrystallizations and microfracture in the center of contact.
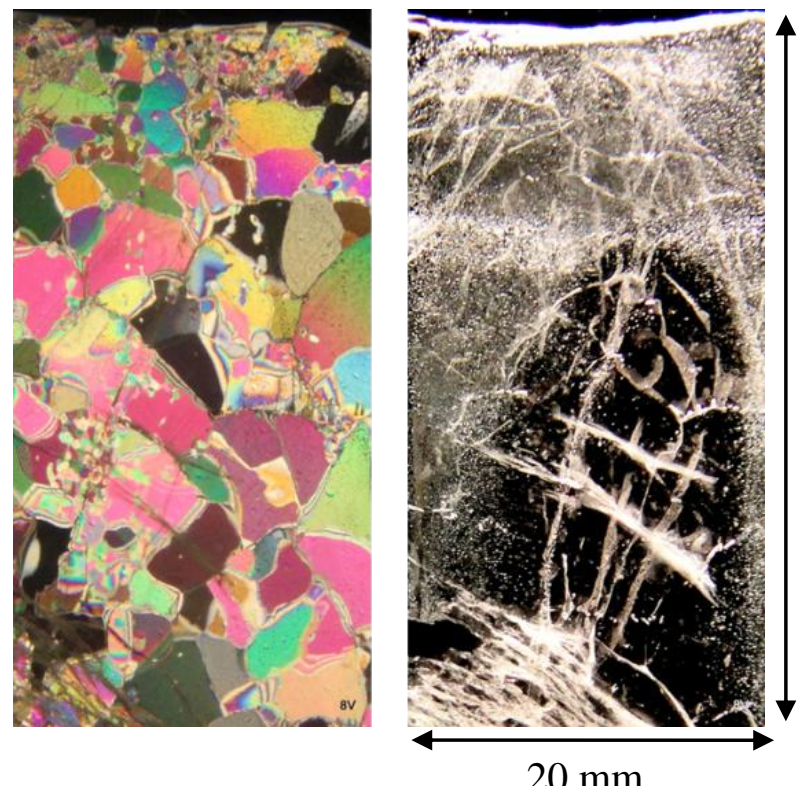

$40 \mathrm{~mm}$

Figure 4.27: Vertical thin-section pictures using cross polarized light (colour) and side light (black and white) for the test T08_10_21_1_10_S

\subsection{Summary}

Five selected representative test events have been discussed in this chapter. Four of them used the flat indenter and one of them used the spherical indenter for crushing tests. From 
the load cell data cyclic loading patterns are observed which are directly dominated by the indentation rate. The slow speed test has the lowest and the fast speed test has the highest cyclic loading pattern. The warm test result also shows a similar result with less cyclic loadings and a greater inclination to ductile failure (slow crushing and damage enhanced creep).

Results from tactile pressure sensor data show an agreement with the load cell data collected from the MTS machine. Formation and evolution of high pressure zones and the behaviour of crushing patterns with change in the contact area throughout the indentation were observed. The effect of spalling fracture can also be determined with the tactile pressure sensor data. The videos from the regular and high-speed camera provide an efficient way to understand the spalling and loading effects.

Thin-section images from both vertical and horizontal sections provide an excellent way to study the damage mechanisms at the indentation zones. From the cross polarized light, the amount of recrystallization can be observed and the side lighting technique has been used to see the amount and nature of microfractures.

The photographs taken after crushing reveal the types of loadings. By studying the images, failure types can be determined. Besides, the spall mass distributions are observed to study the patterns of spalling for different factors that used in the tests. 


\section{Chapter 5: Analysis}

\subsection{Overview}

In this chapter, results and data collected from the small-scale indentation tests are discussed and compared. Test results have been grouped to allow for the comparisons of tests having same factors, except for the parameter of interest. The influence of the factors such as indentation rate, temperature, indenter shape, ice specimen shape and grain size of ice seeds were considered. To improve the understanding of the compressive failure mechanisms and high pressure zones, special attention was paid to the load limiting factors and the test conditions that drive these mechanisms.

To provide a further assessment of the influence of the factors that were used during the ice compressive tests a qualitative evaluation of the ice failure mechanisms, thin-sections and spall distributions are also presented in this chapter. 


\subsection{General Test Observations}

All tests have been examined individually to study the behaviour of the ice failures and the nature of high pressure zones at the contact interface. One of the main focuses of this thesis is an examination of force data against displacement and time, as well as pressure distribution against area. High-speed and regular video recordings were observed and synchronized with the load cell data to determine the cause of ice failures and associated load drops. Tactile pressure sensor data were also synchronized with the videos and load cell data to see the pressure distributions with continuous change of contact area. Additional focus was placed on the load limiting failure mechanisms that occurred during cyclic loading events. A detailed description of observed failure processes associated with crushing and spalling for representative sample events is provided below.

\subsubsection{Crushing}

As Jordaan et al., (2008) described, crushing refers to the pulverization and extrusion of softened ice from the periphery of high-pressure zones. Crushing is one of the main load limiting mechanisms which produces a cyclic loading and also associated with load fluctuations and failure of the high pressure zones.

From the analysis of the regular and high-speed videos crushing has been observed to be a continuous extrusion of fine grained material from the periphery of the indenter. A sample test is discussed below to study the behaviour of crushing failure where tactile pressure distribution data from a Tekscan sensor synchronized with force-time data from the MTS load cell. This test was a fast speed test $(10 \mathrm{~mm} / \mathrm{s})$ done at $-10^{\circ} \mathrm{C}$ with a flat 
indenter. A period of loading in the force- time plot is shown in Figure 5.1 for the test, where three rapid load drop events occurring from (a) to (b), (c) to (d) and (e) to (f) are observed. From the images of tactile pressure sensor data, corresponding crushing events with the failure of high pressure zones are presented in Figure 5.2. Events (b), (d) and (f) show that the intensities of high pressures have been decreased because of the loss of some contact area from (a), (c) and (e) respectively.

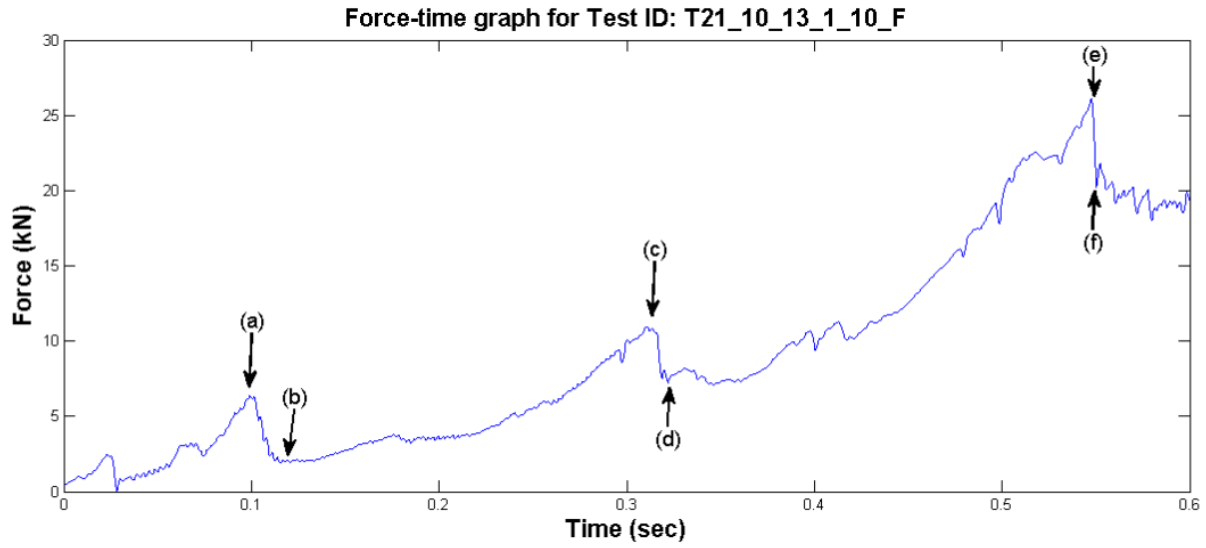

Figure 5.1: MTS load cell data identifying crushing failure for the test T21_10_13_1_10_F

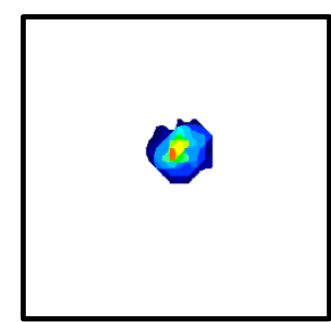

(a)

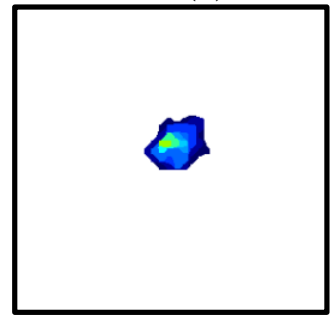

(b)

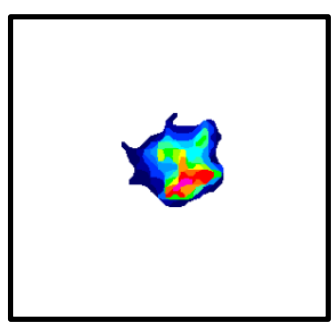

(c)

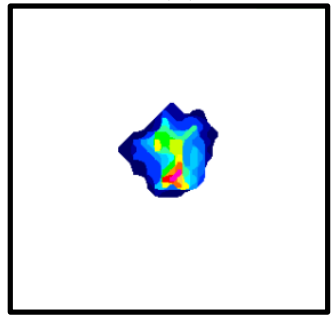

(d)

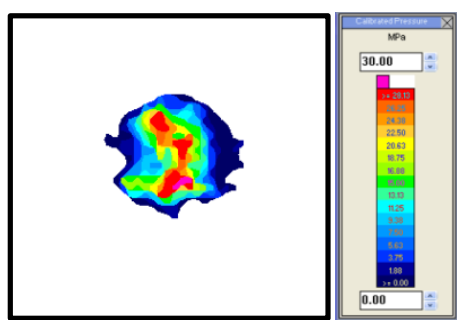

(e)

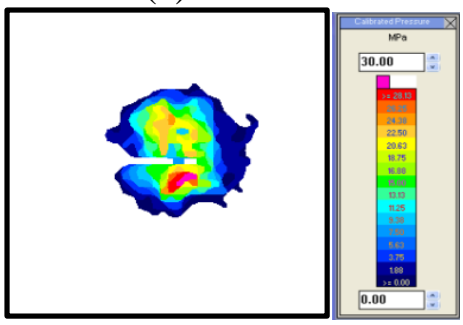

(f)

Figure 5.2: Pressure distribution images before and after crushing events from Tekscan sensor for the test T21_10_13_1_10_F 


\subsubsection{Spalling}

According to Jordaan (2001), spall is localized fractures that initiates from crack near the high pressure zones. Spalling was observed to be the most common load limiting failure mechanism during these experiments. It was not observed to occur as regularly and steadily as crushing, but rather was inconsistent and irregular in nature. Flaws present in the ice such as grain boundaries promoted this process. Spalling was also observed to depend on the rate of indentation, particularly for indentation rates greater than $1.0 \mathrm{~mm} / \mathrm{s}$.

Based on careful examinations of each test to detect the reasons for failure, it has been determined that there are significant numbers of spalls that dominate the failure of ice. Loss of a large contact area in the ice-structure interface is indicative of spalling failure. In such cases a sharp load drop is observed to occur in a fraction of second at the same indentation depth which corresponds with the loss of the contact area. Tactile pressure sensor images provide a valuable tool to investigate the amount of contact area loss. From the high-speed and regular videos the size of the spalls which broke away from the specimens can be observed. During an ice crushing test at a given indentation rate it was observed that continuous cyclic loading may be interrupted by spalling fracture. As an example, in Figure 5.3 the force-time data for the medium speed test identifying the load drops due to spalling have been indicated as (a), (b), (c) and (d). In Figure 5.4 snapshots of the events (a) to (d) of spalling failure from the regular video of the same test are presented. The spalls are specified with a red indicator at the time of the event. High pressure zones in the ice-structure interface also depend on the contact area. Variation of 
high pressure zones with time and position due to spalling increases with increasing indentation rate.

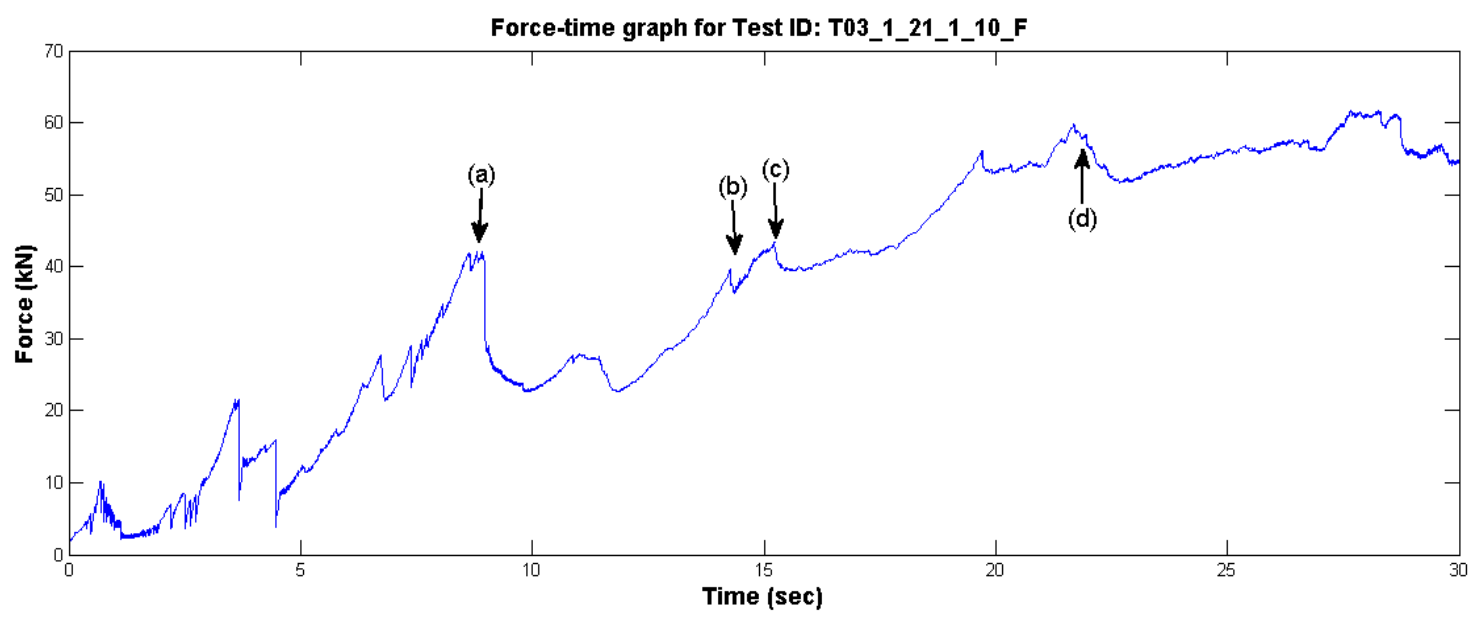

Figure 5.3: MTS load cell data identifying spalling event for the test T03_1_21_1_10_F

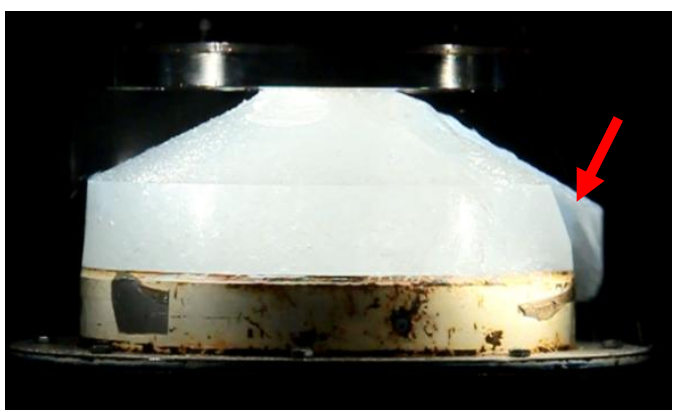

(a) $\mathrm{t}=9.06 \mathrm{sec}$

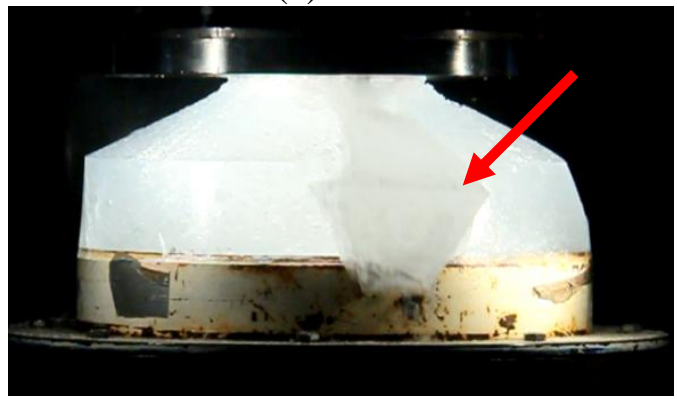

(c) $t=15.3 \mathrm{sec}$

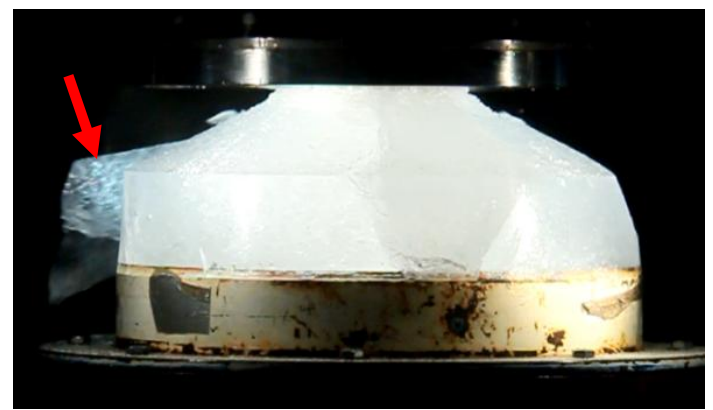

(b) $t=14.7 \mathrm{sec}$

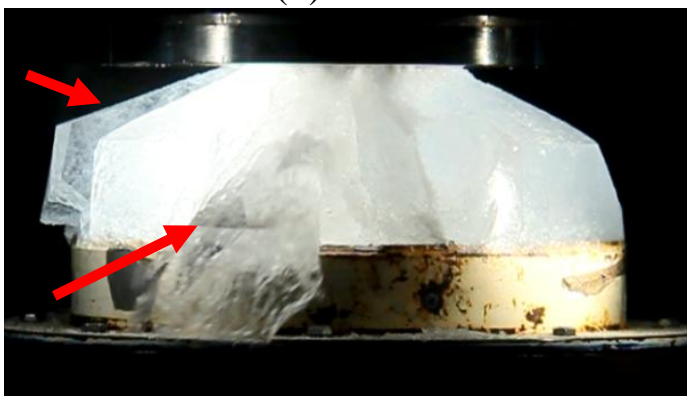

(d) $\mathrm{t}=22.53 \mathrm{sec}$

Figure 5.4: Snapshots of the spalling failure from video recording of the test T03_1_21_1_10_F 


\subsection{Effect of Indentation Rate}

Indentation rate has a significant effect on failure processes and crushing behaviours. The slow tests $(0.1 \mathrm{~mm} / \mathrm{s})$ tend to exhibit slow continuous crushing failure throughout the tests. The fast tests $(10 \mathrm{~mm} / \mathrm{s})$ experience brittle failure from the very start. The medium speed tests $(1 \mathrm{~mm} / \mathrm{s})$ have a combination of ductile and brittle failure. In Figure 5.5 forcedisplacement plots are given for three tests having different indentation rates up to the indentation depth of $10 \mathrm{~mm}$. These tests were done at $-10^{\circ} \mathrm{C}$ with a flat indenter. From the curve of the slow test $(0.1 \mathrm{~mm} / \mathrm{s})$ it can be seen that the sample has experienced ductile failure with a continuously increasing trend of load. In the medium speed test $(1 \mathrm{~mm} / \mathrm{s})$ the ice has experienced a brittle failure with a large amount of cyclic loading during the first few seconds, and then it has started to experience a ductile failure with steadily increasing load. In the fast speed test $(10 \mathrm{~mm} / \mathrm{s})$ the initial load builds up at the same slope as in the slow test, and then the load trace is flat until $3 \mathrm{~mm}$, after which regular patterns of cyclic loadings are observed.

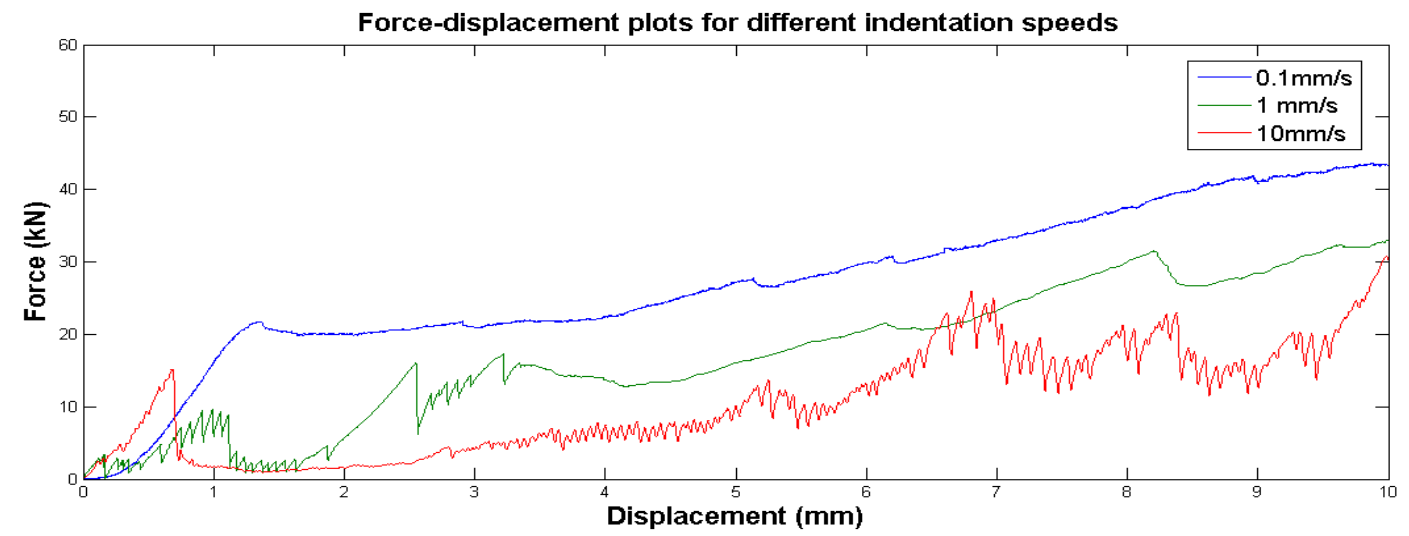

Figure 5.5: MTS load cell data for the three tests at different speeds: Slow speed test T02_0.1_21_2_10_F (blue); Medium speed test T05_1_21_2_10_F (green); Fast speed test T06_10_21_2_10_F (red) 
Images from tactile pressure sensor data are shown in Figure 5.6 to compare the force and pressure distribution at different speeds. Corresponding load cell data can be observed in Figure 5.7. The tests were done at $-10^{\circ} \mathrm{C}$ using an ice specimen made with ice seeds of $4-10^{+} \mathrm{mm}$ in diameter and having a taper angle of $13^{\circ}$.

From the results of the slow test, it can be observed that the high pressures of tactile sensor data and forces of load cell data increase steadily with contact area and displacement. From event (a) to event (d) high pressure zones are observed within the contact area, and the contact area grows steadily with a consistent symmetric circular shape indicating little spalling occurs.

In case of the medium speed test, it is evident that a spall has occurred due to a highly asymmetric contact area at event (a). As the area under the sensor increases, pressures also increase and continuous change of the positions of high pressure zones is observed from event (b) to event (d). Similar results can be observed from the load cell data that the forces increase with displacements steadily.

From the images of the fast speed test, it can be seen that a local high pressure zone has built up at event (a), which continues to increase the intensity at event (b) and event (c) though the contact area is irregular indicating small spalls from around the periphery of the contact area. A significant decrease in contact area occurs at event (d) resulting in a drop of load in the load cell data, which indicates a large spalling event. 
Slow Speed Test $(0.1 \mathrm{~mm} / \mathrm{s})$
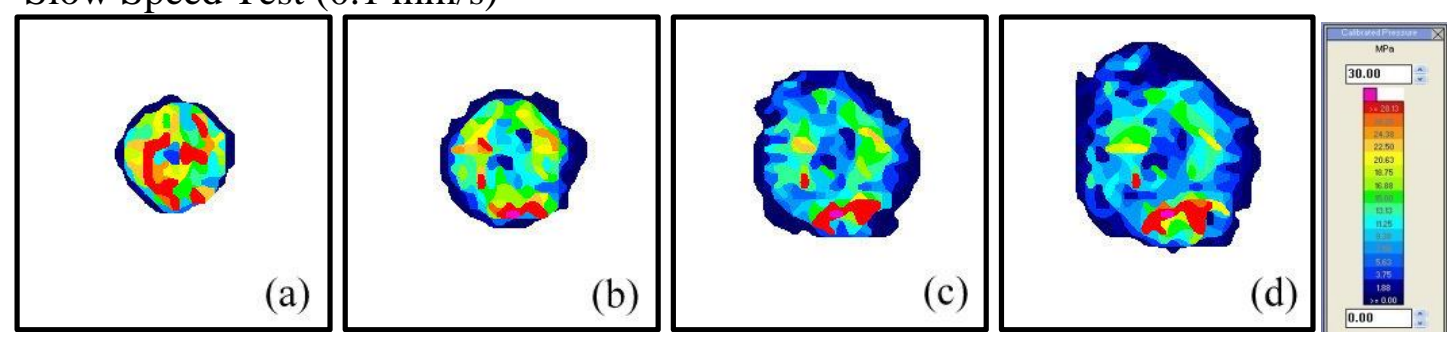

Medium Speed Test (1 mm.s)
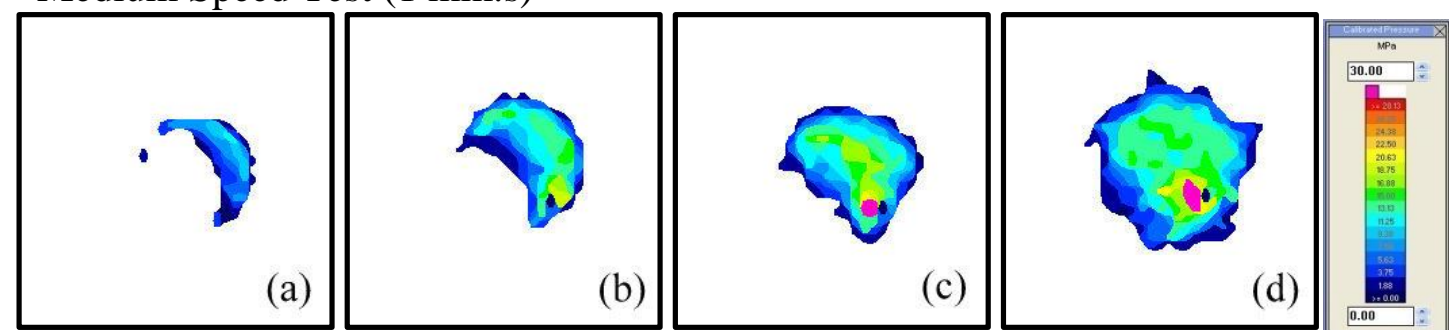

Fast Speed Test $(10 \mathrm{~mm} / \mathrm{s})$
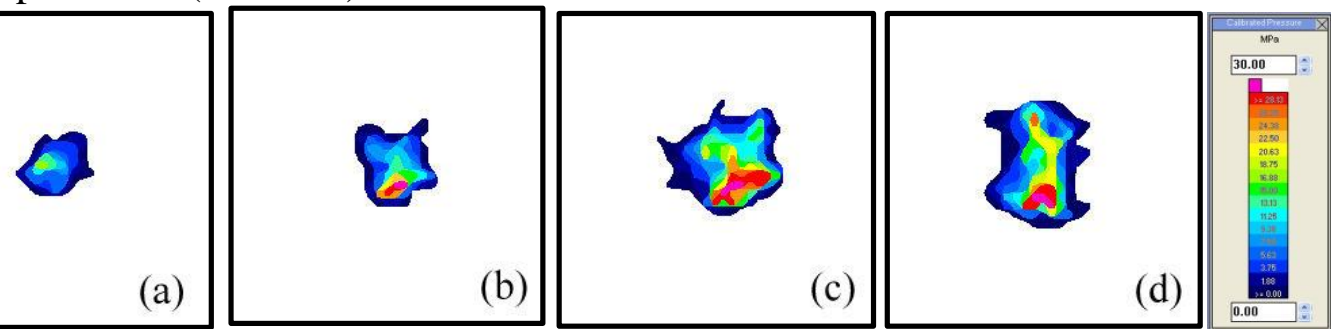

Figure 5.6: Pressure distribution images for the three tests at different indentation speeds: Slow speed test T17_0.1_13_1_10_F, Medium speed test T19_1_13_1_10_F and Fast speed test T21_10_13_1_10_F

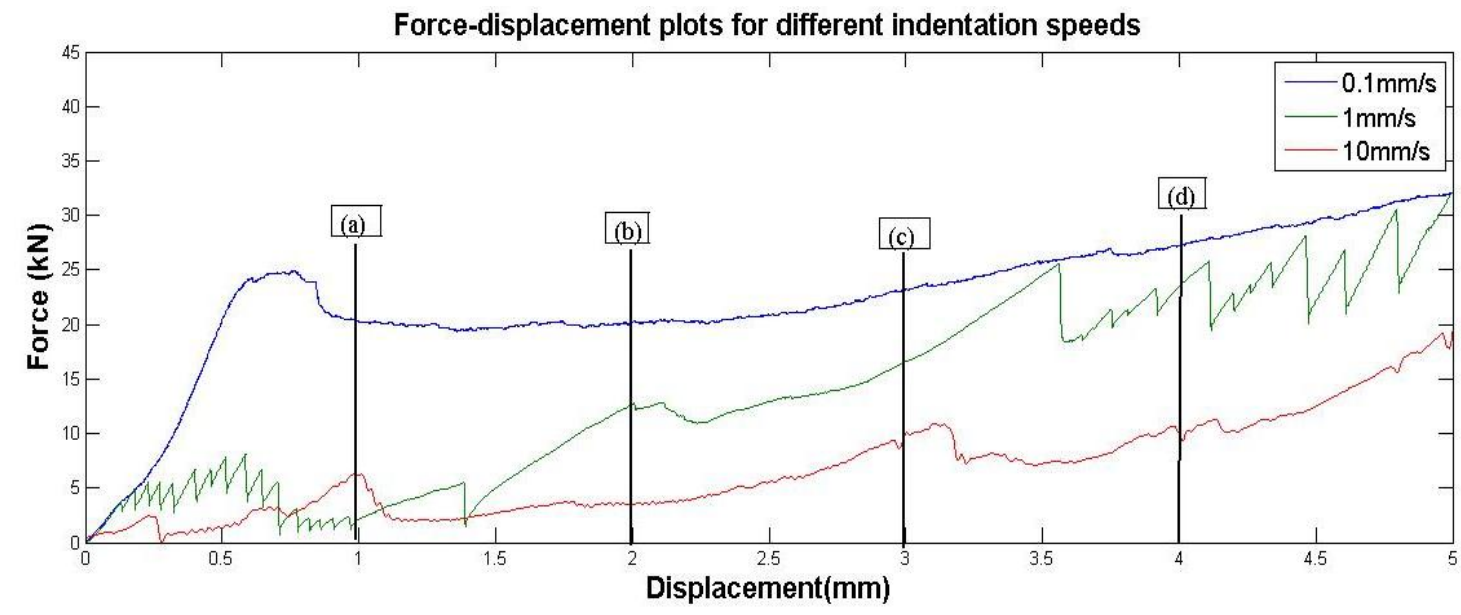

Figure 5.7: MTS load cell data corresponding to the pressure distribution images 
Vertical thin-section pictures in Figure 5.8 present details of the microstructure at three different indentation speeds. Each section is $40 \mathrm{~mm}$ in height and $20 \mathrm{~mm}$ in width. The thin-sections in Figure 5.8 (a) for the slow test show that the sample is heavily dominated by recrystallization and microfracture having the depth of the damaged area about $35 \mathrm{~mm}$ in length. In the case of the medium speed test in Figure 5.8 (b), both recrystallization and microfracture have an effect to a depth of about 15-20 mm. The fast test in Figure 5.8(c) shows a different result. Recrystallization and microfracture are concentrated in the immediate crushing zone, and only extend to a depth of about $10 \mathrm{~mm}$.

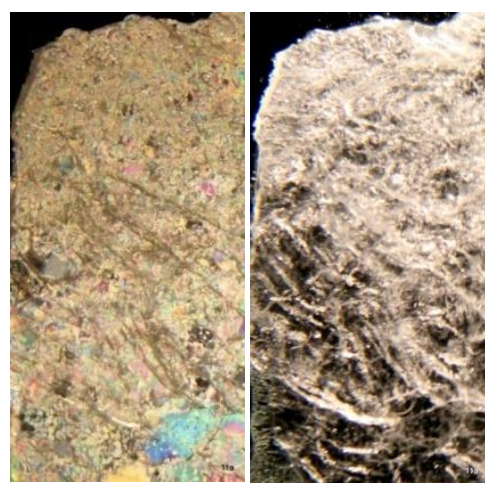

(a) Ind. rate $=0.1 \mathrm{~mm} / \mathrm{s}$

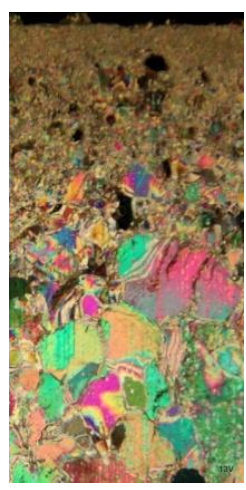

(b) Ind. rate $=1 \mathrm{~mm} / \mathrm{s}$

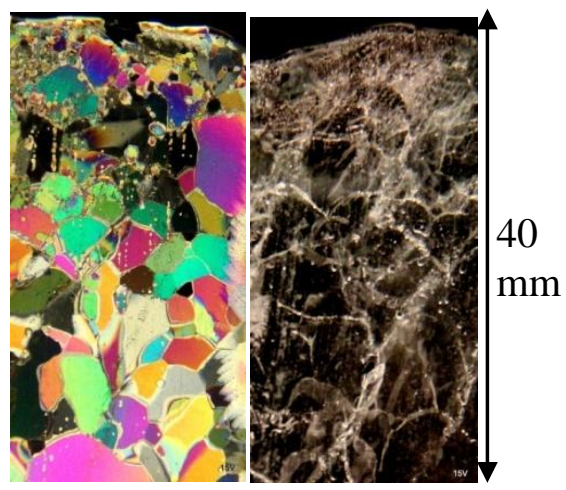

(c) Ind. rate $=10 \mathrm{~mm} / \mathrm{s}$

Figure 5.8: Vertical thin-section pictures showing the effect of indentation speed using cross polarized light (colour) and side light (black and white) (a) Slow speed test

(T11_0.1_30_1_10_F) (b) Medium speed test (T13_1_30_1_10_F)

(c) Fast speed test (Test T15_10_30_1_10_F)

Figure 5.9 show photographs of the specimens during the tests and immediately after crushing for the same three tests. In case of the slow speed test, the image during crushing in Figure 5.9(a) shows clear evidence of slow crushing and damage enhanced creep. From the after crushing image of the slow speed test it can be seen that there is a very smooth finish with no spall on the top of the ice sample. The medium speed test 
images taken during the test and after the test in Figure 5.9(b) show both damage and fracture failure. In the center of the crushed sample, there is clear evidence of extreme and highly damaged failure of ice which has a smooth surface, but the spalled areas near the edges have much rougher and more irregular surfaces. From Figure 5.9 (c), the image taken during the fast test shows crushing and spalling occuring simultaneously. The after crushing image of the fast speed test shows brittle failure and has rough and irregular surface at the final stage of the test with a smaller zone of localized damage. This sample contained many small spalls and fractured ice pieces.

Photographs taken during the tests:

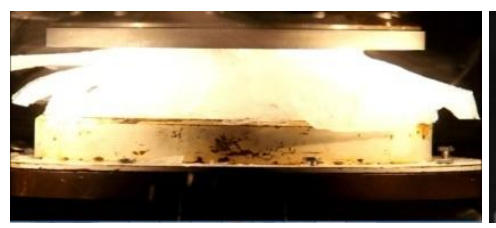

(a) Ind. rate: $0.1 \mathrm{~mm} / \mathrm{s}$

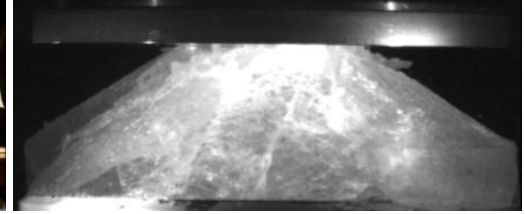

(b) Ind. rate: $1 \mathrm{~mm} / \mathrm{s}$

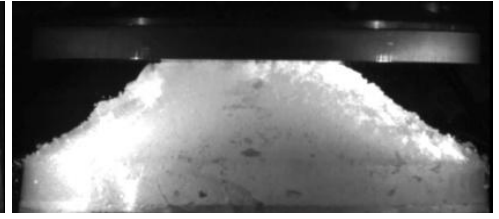

(c) Ind. rate: $10 \mathrm{~mm} / \mathrm{s}$

Photographs taken after the crushing tests:

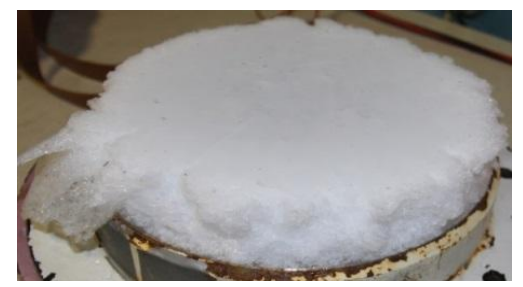

(a) Ind. rate: $0.1 \mathrm{~mm} / \mathrm{s}$

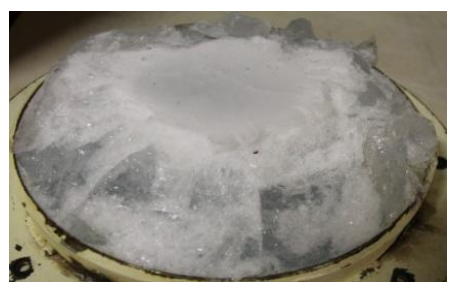

(b) Ind. rate: $1 \mathrm{~mm} / \mathrm{s}$

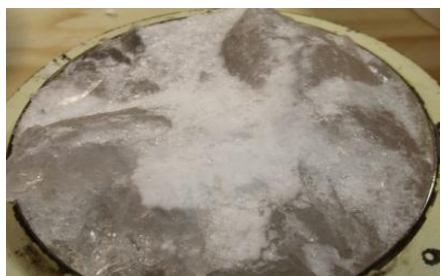

(c)Ind. rate: $10 \mathrm{~mm} / \mathrm{s}$

Figure 5.9: During and after crushing images for the tests at different indentation speeds

The total spall masses as a function of indentation speeds is presented in Figure 5.10 for different taper angles. The spall masses given here are the total masses of all spalls collected after each test. From the plot, fewer ice spalls are observed from the specimens 
of the slow tests than the fast tests. This is believed to result because the ice has enough time to dissipate energy through damage-enhanced creep and these crushing and damage mechanisms dissipate adequate energy so as to prevent local fractures. By comparision, the medium and fast tests have more spall mass than the slow tests, but the difference between them is very small. Taper angle is also observed to have an effect based on indentation speeds, as is explained further in section 5.7.

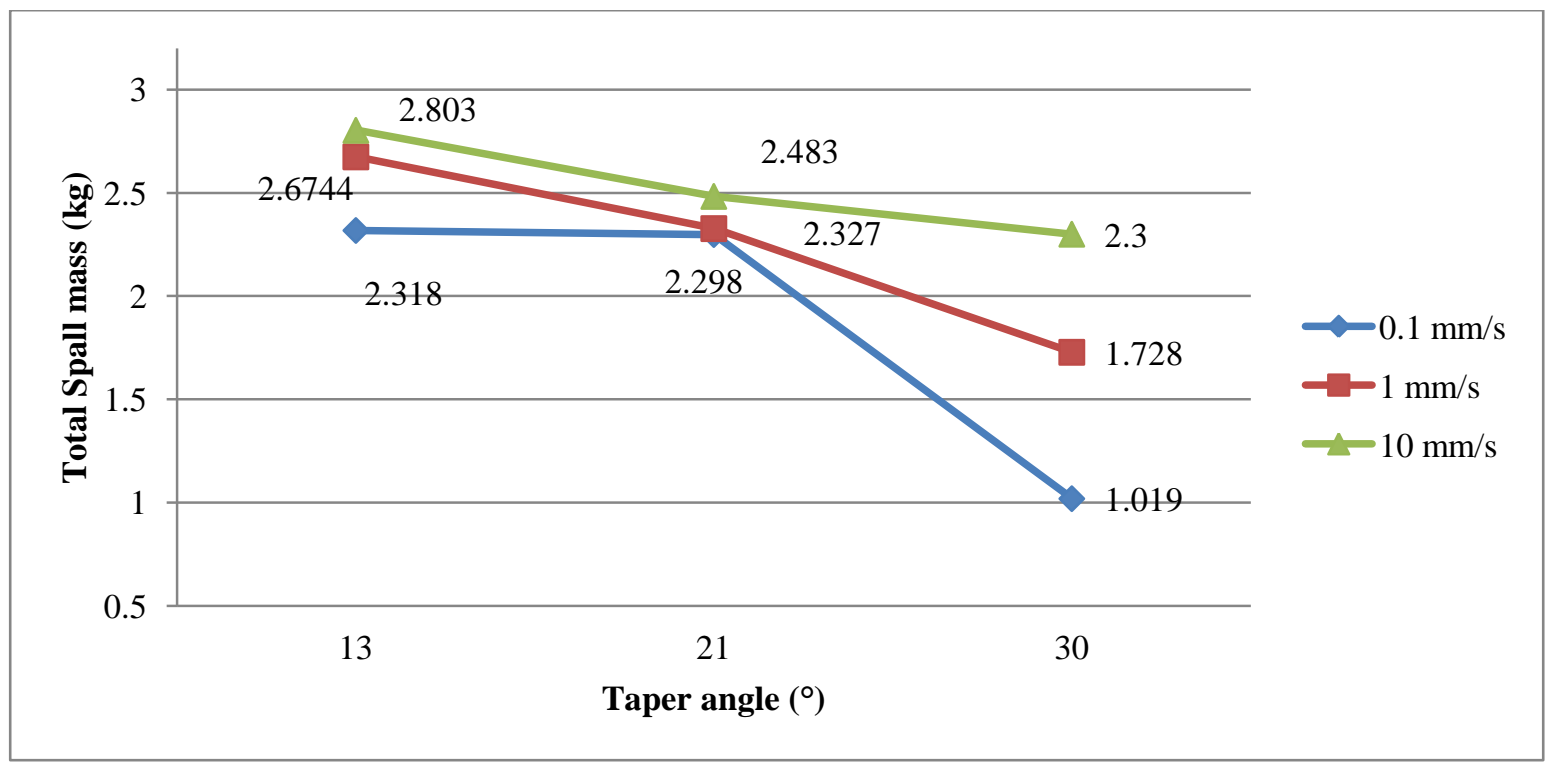

Figure 5.10: Total spall masses of ice crushing tests at different indentation speeds having different taper angle

\subsection{Effect of Temperature}

The effect of temperature can be observed from the force-displacement plots of Figure 5.11. The plots are from the slow tests, where in general the force at $-5^{\circ} \mathrm{C}$ tends to be slightly higher than the force at $-10^{\circ} \mathrm{C}$. From the plots of fast indentation tests given in 
Figure 5.12, it is observed that the warmer test exhibits more ductile failure, dominated by damage, but the cold test experiences more brittle failure. It should be noted that the large load drops still occur in the warm tests due to spalling events, but there are fewer cyclic loadings in this case.

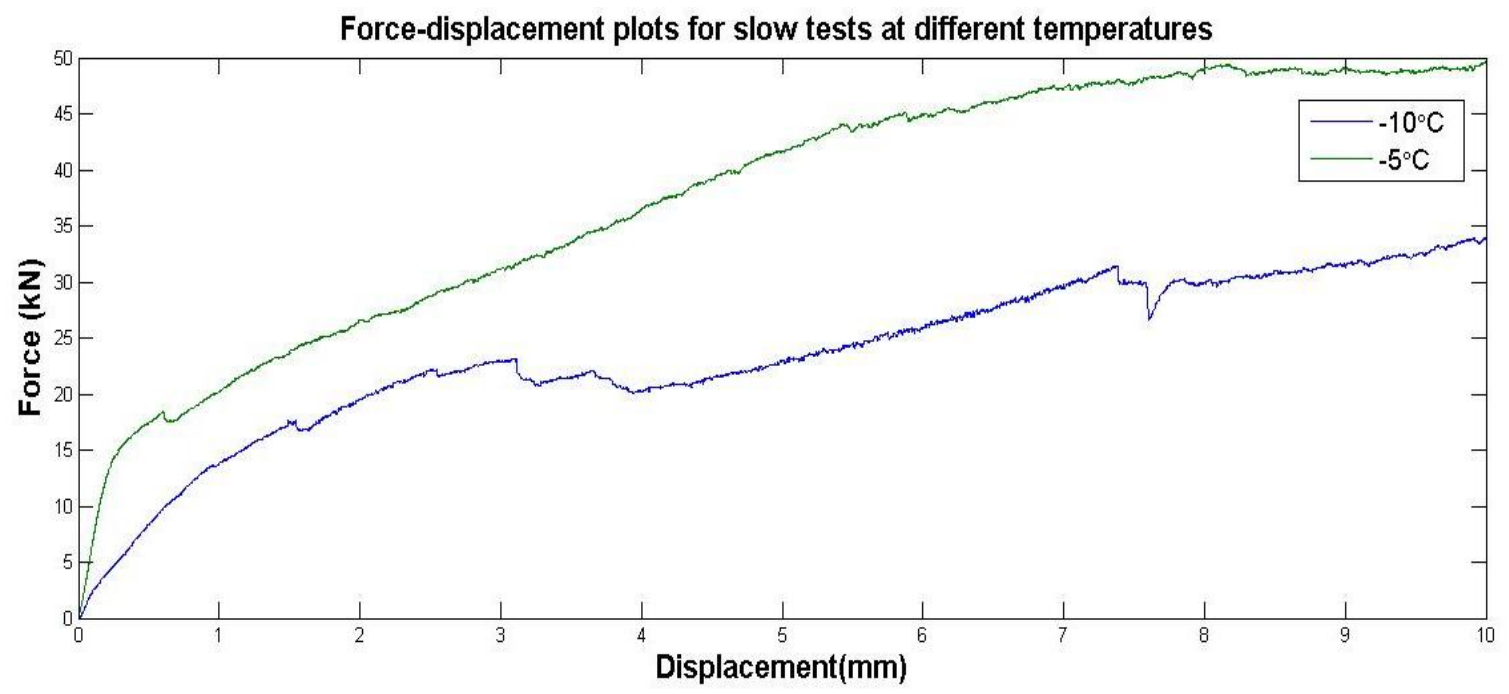

Figure 5.11: MTS load cell data for two slow speed tests at different temperatures: Cold test T01_0.1_21_1_10_F (blue) and Warm test T25_0.1_21_1_5_F (green)

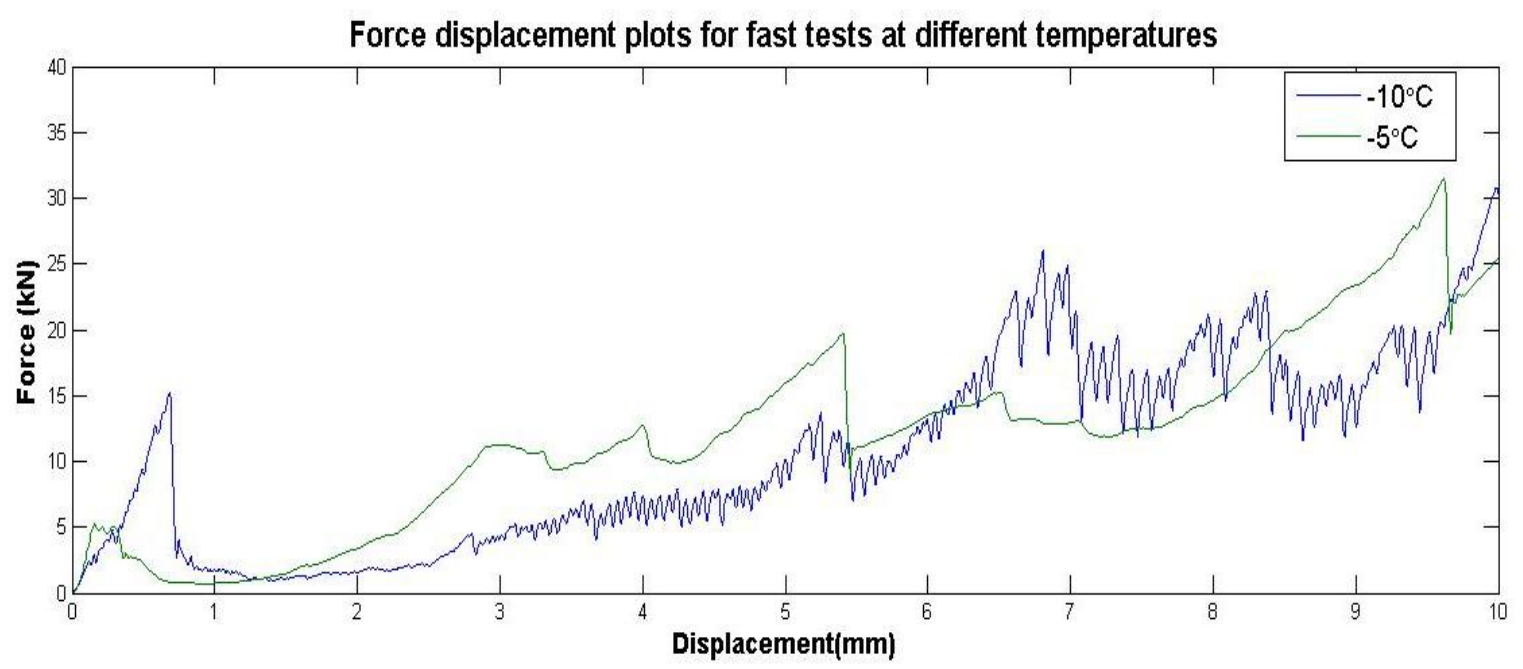

Figure 5.12: MTS load cell data for two fast speed tests at different temperatures: Cold test T06_10_21_2_10_F(blue) and Warm test T28_10_21_2_5_F 
Vertical thin-section pictures in Figure 5.13 show clear evidence of the effect of temperature. Thin-section pictures taken using cross polarized light show that in warmer ice $\left(-5^{\circ} \mathrm{C}\right)$ the damaged layer is more heavily dominated by recrystallization as compared to colder $\left(-10^{\circ} \mathrm{C}\right)$ ice. From the pictures taken using a side lighting technique, it is observed that cold tests are dominated by zones of microfracture. The warm test also has microfracture in the central zone, but the depth of the damaged layer appears to be larger in the case of the cold ice test than in the warm ice test. Barrette et al. (2002), Li et al. (2004) and Browne (2012) found similar observations in their research.
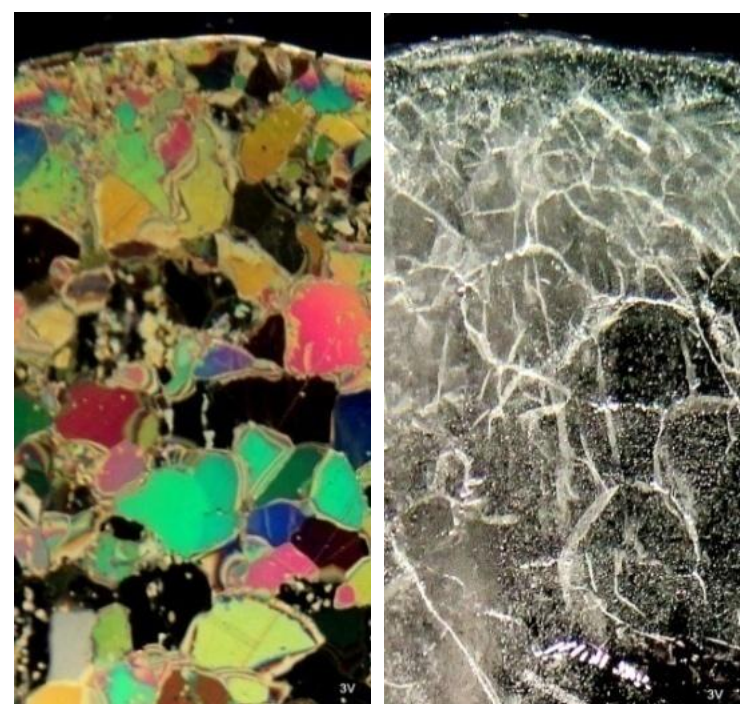

(a)

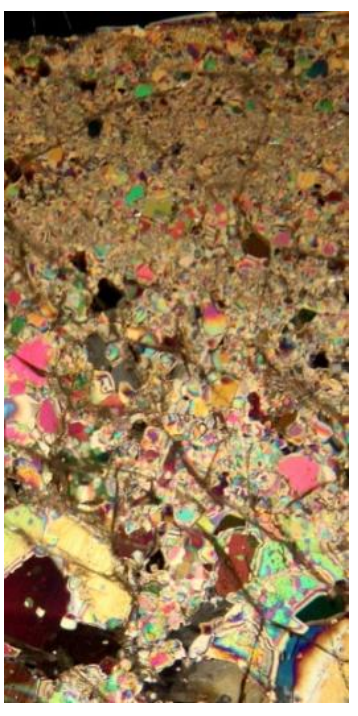

(b)

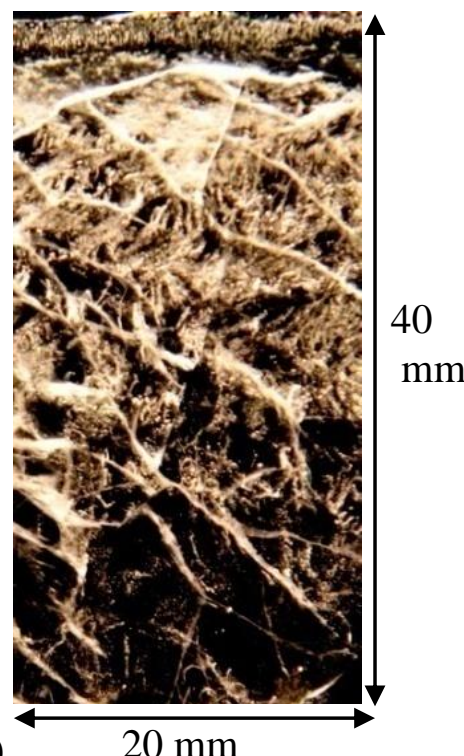

$20 \mathrm{~mm}$

Figure 5.13: Vertical thin-section pictures using cross polarized light and side light (a)Cold test results $\left(-10^{\circ} \mathrm{C}\right)$ for the test T03_1_21_1_10_F (b) Warm test results $\left(-5^{\circ} \mathrm{C}\right)$ for the test T26_1_21_1_5_F 
After crushing images of the cold and warm tests are given in Figure 5.14. The ice sample of cold test has a rough and very dry surface with some small spalls on the top of the sample. The ice sample of warm test has a smooth and slippery surface with higher damage.

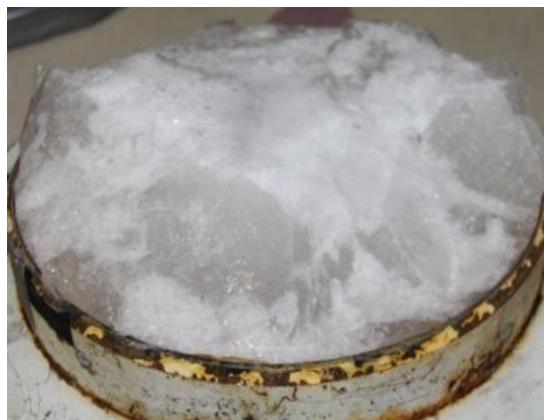

(a)

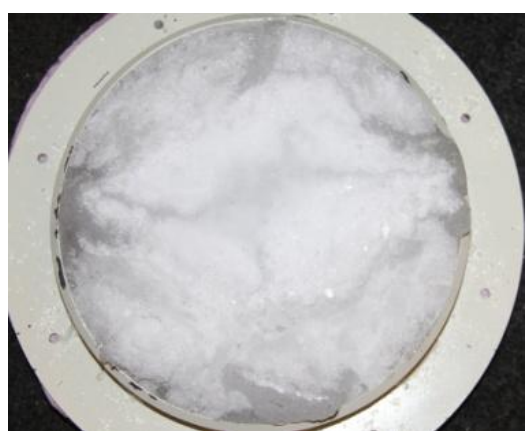

(b)

Figure 5.14: After crushing images for tests at two diferent temperatures (a) Cold test T06_10_21_2_10_F (b) Warm test T28_10_21_2_10_F

In this work, four (4) cases were considered for warm tests. To compare the total spall masses as a function of temperature, these results along with the corresponding cold tests have been plotted in Figure 5.15. From this chart, it is observed that only in the slow test there are more spalls measured at $-5^{\circ} \mathrm{C}$. In the other three cases, cold tests consistently have more total spall masses than warm tests though these differences are small. As mentioned previously, since the warm tests tend to exhibit more ductile failure and the faster tests have more brittle failure, it is expected that the faster events would be more sensitive to temperature effects. As very small number of large fracture events is observed in slow speed tests, it seems that slow tests result in fewer but larger spall events. 


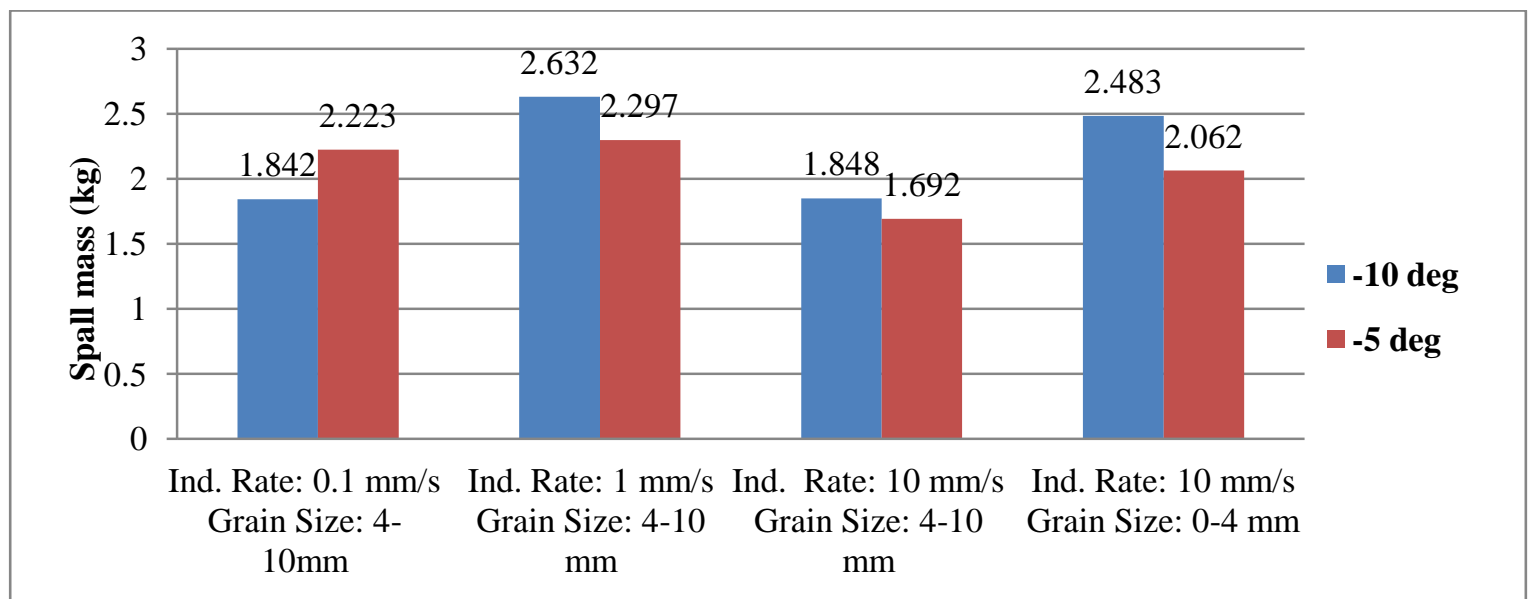

Figure 5.15: Total spall mass comparison for four different cases of cold and warm tests

\subsection{Effect of Indenter Shape}

Using different shaped indenters for ice samples having same parameters introduced a new dimension to consider. Specifications and pictures of the two indenters that were used in this thesis have been presented in Chapter 3.

In Figure 5.16 force-time plots for comparing results of flat and spherical indenters are given. The tests were carried out at $-10^{\circ} \mathrm{C}$ and at a rate of $10 \mathrm{~mm} / \mathrm{s}$. The ice samples used here were made of smaller ice seeds $(0-4 \mathrm{~mm})$ and had a taper angle of $21^{\circ}$. From the graphs, it is apparent that the force produced by the spherical indenter is much less than for the flat indenter. The failure behaviour is observed to be a combination of ductile and brittle, in the case of the spherical indenter, while in other case, the failure is completely brittle. 
To more closely examine the cyclic crushing force-time plots for the time window shown in Figure 5.16 is given in Figure 5.17. The cyclic loading pattern in the case of the flat indenter is significantly higher than the spherical indenter.

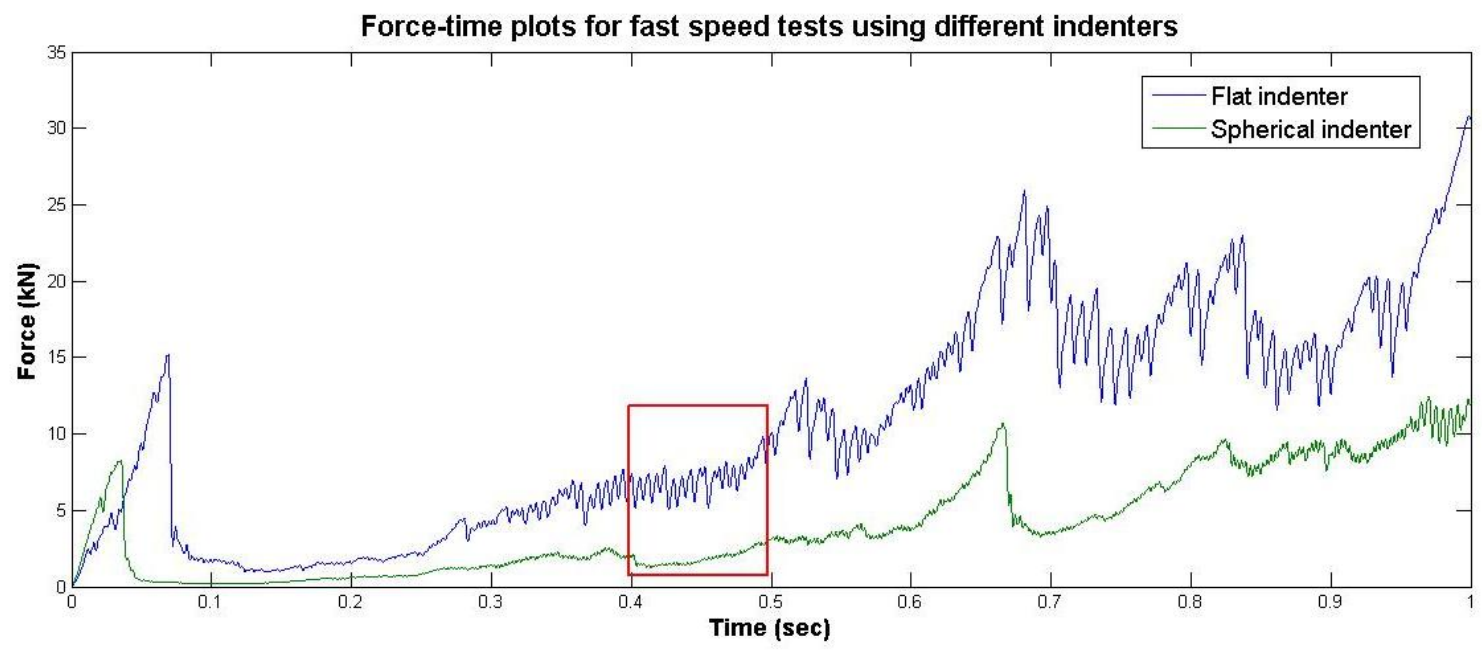

Figure 5.16: MTS load cell data for the tests using two different indenters: Flat indenter: T06_10_21_2_10_F (blue) and Spherical indenter: T07_10_21_2_10_S (green)

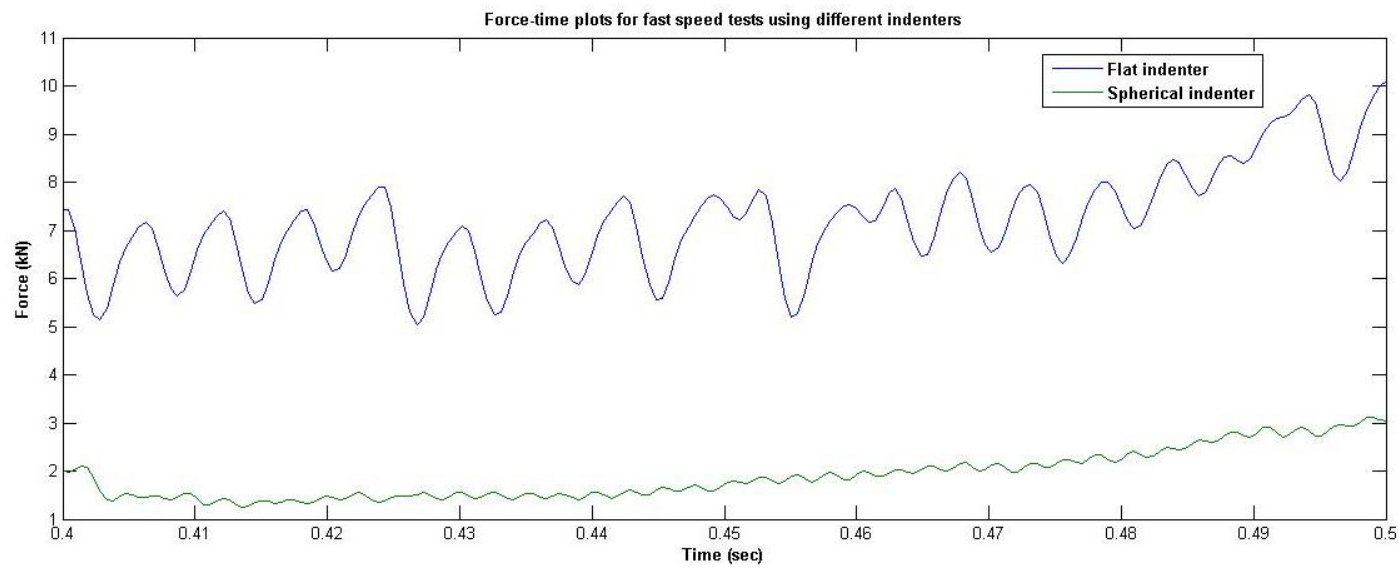

Figure 5.17: MTS load cell data for the previous tests for 0.1 second 
To observe the microstructural modification of the damaged layer, vertical thin-section photographs are given in Figure 5.18. Thin-section photographs using both cross polarized light and side light have been included for the flat indenter at (a) and thinsection pictures for the ice sample having the same testing parameters and the same ice geometry but using the spherical indenter are given at (b). Pictures using cross polarized light indicate that the results for the flat indenter are heavily dominated by recrystallization while the results for the spherical indenter show a smaller recrystallized area near the center. The thin-section pictures taken using the side lighting technique show that the results for the spherical indenter are dominated by microfracture near the contact surface of the ice and the indenter has produced a concave surface in the center.
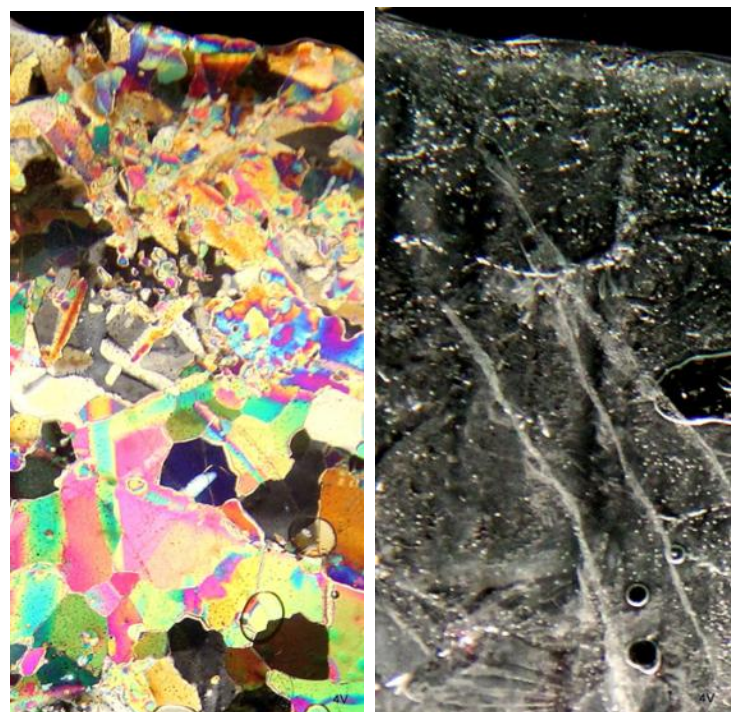

(a)

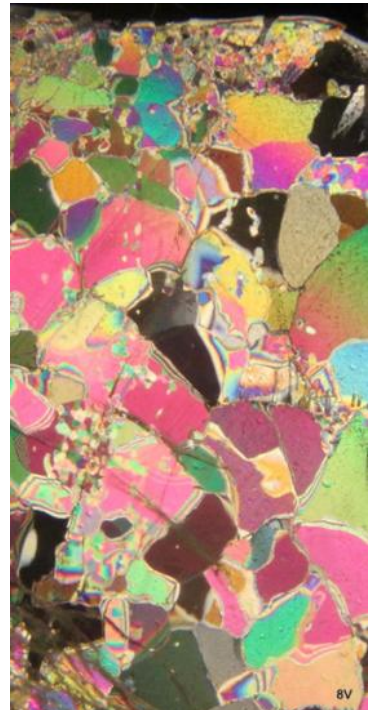

(b)

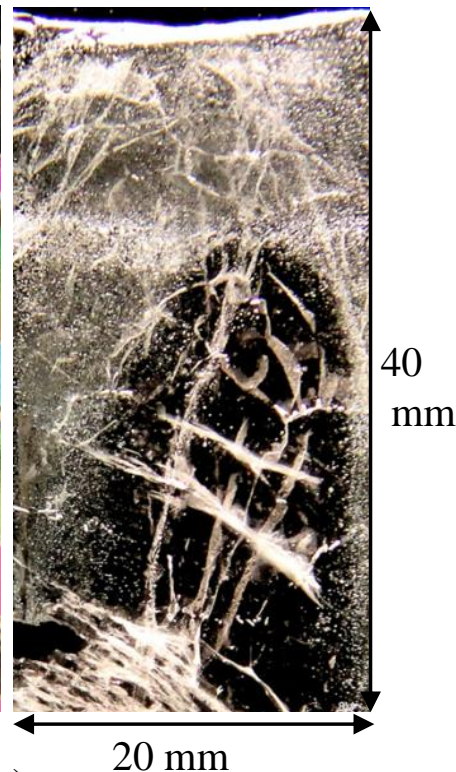

$20 \mathrm{~mm}$

Figure 5.18: Vertical thin-section pictures using cross polarized light (colour images) and side light (black and white images) for the tests using (a) Flat indenter:

T04_10_21_1_10_F (b) Spherical indenter: T08_10_21_1_10_S 
Specimen pictures taken at the end of the each test have been given in Figure 5.19 for the corresponding tests considered in Figure 5.18. These post-testing pictures exhibit some attributes which depend on which indenter has been used for the test. In the case of the flat indenter, the indentation surface tends to be rough but with a flat profile. The test samples indented using the spherical indenter tend to have a depression in the center, with more localized spalling, which result in less accumulation of crushed ice near the edges of the indentation zone.

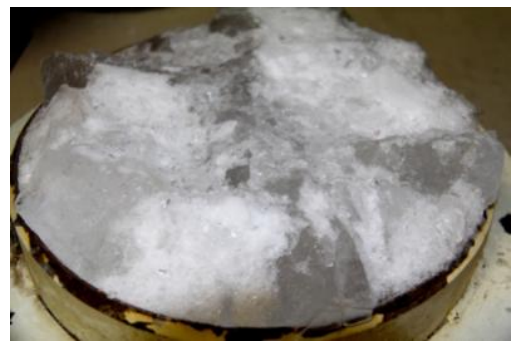

(a)

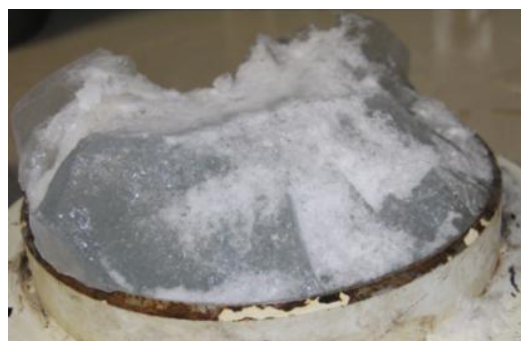

(b)

Figure 5.19: After crushing ice sample pictures of fast speed tests using (a) Flat indenter for the test T04_10_21_1_10_F (b) Spherical indenter for the test T08_10_21_1_10_S

Total spall masses for all six cases using the spherical indenter are given below in Figure 5.20 and are compared to tests having the same test parameters but conducted using the flat indenter. In all the cases, results for the spherical indenter have less spalled mass than the flat indenter. The indenters have penetrated to the same indentation depth at the end of the test, but for the convex surface of the spherical indenter, it has touched the final depth only in the central area. This may be one possible reason that the final mass of spalls is somewhat lower for the cases of the spherical indenter.

Another possible explanation is that the flat indenters tend to provide higher contact pressure near the edges of the contact zone, as compared to the spherical indenter, 
resulting in a more edge spalling for tests using the flat indenter. The total spall masses for the indenters with different taper angles at the indentation rate of $10 \mathrm{~mm} / \mathrm{s}$ are presented in Figure 5.21. The results show that the mass of generated spalls using the flat indenter are slightly higher than for the spherical indenter, with the greatest difference being observed for the steeper taper angle.

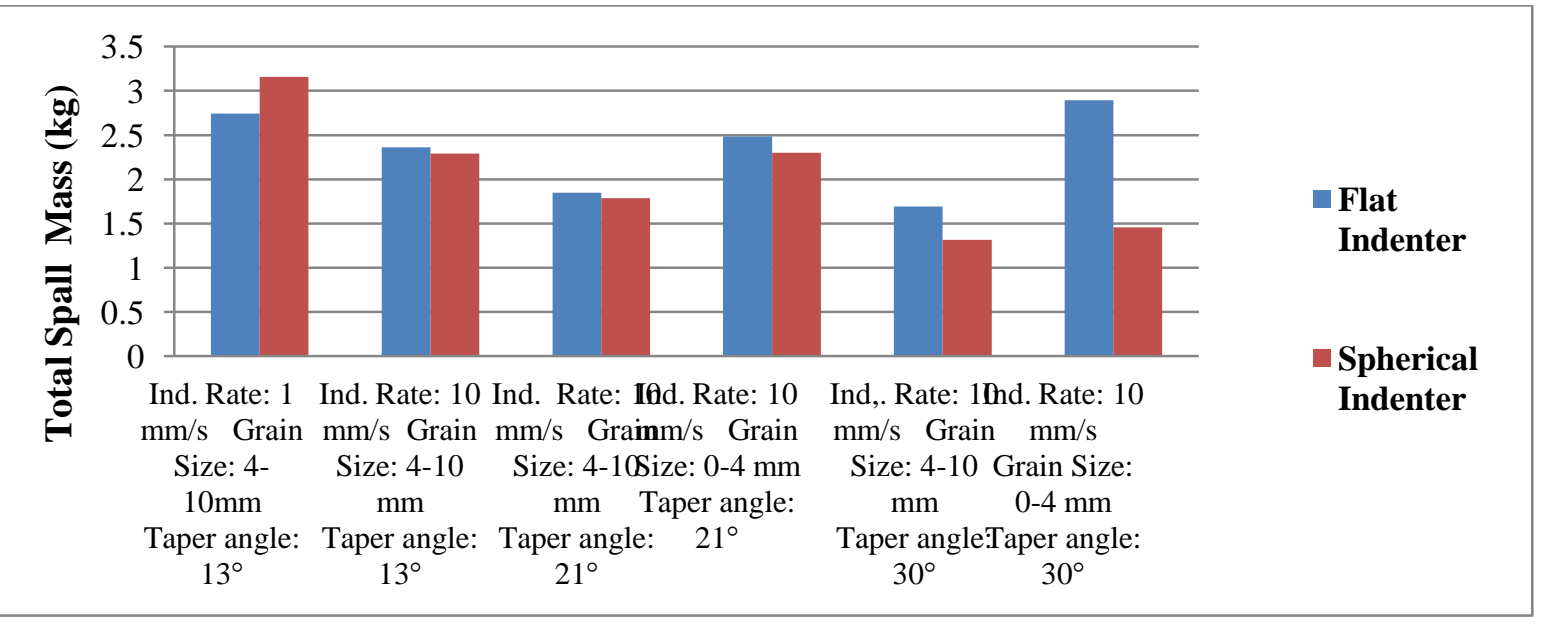

Figure 5.20: Total spall mass comparison for six cases using flat and spherical indenter

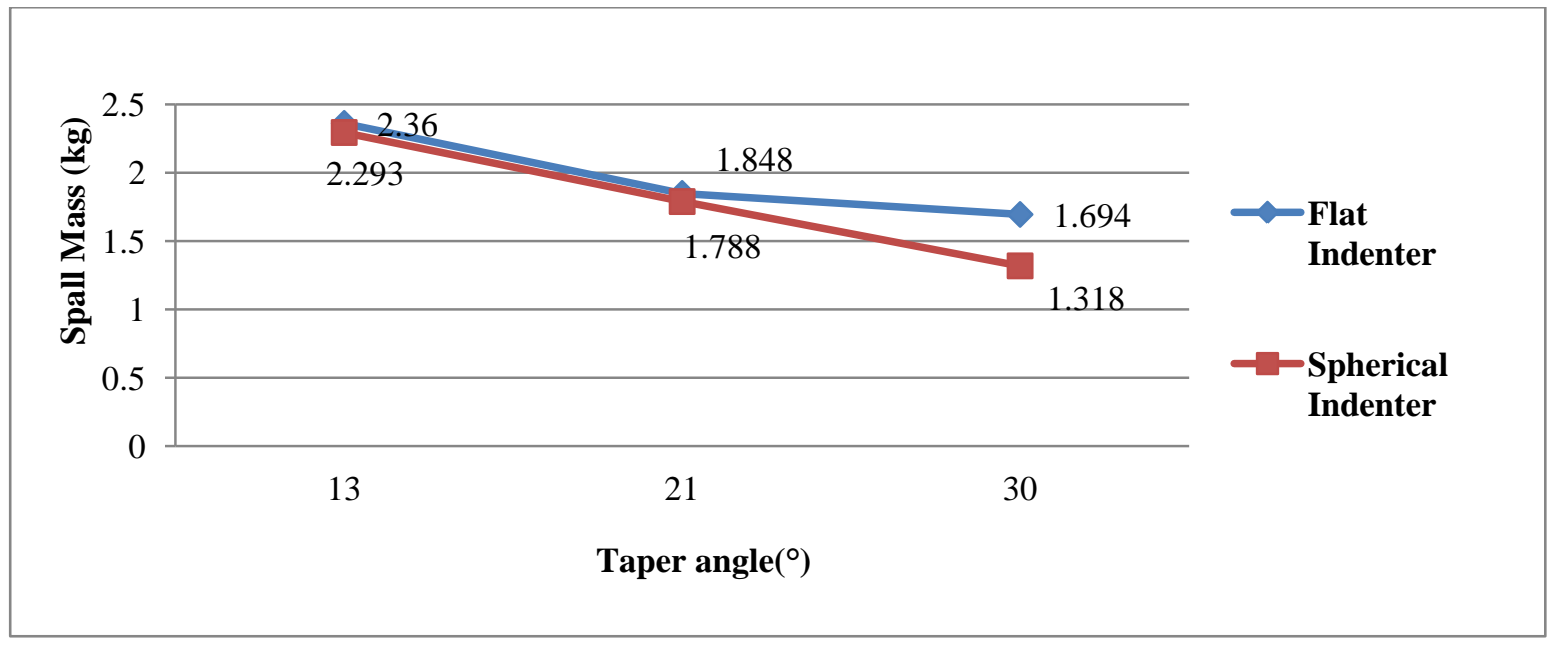

Figure 5.21: Total spall mass comparison for fast speed tests using flat and spherical indenter with different taper angles 


\subsection{Effect of Grain Size}

It is observed that grain size has a strong effect on crushing behaviour. The forcedisplacement plots given below in Figure 5.22 are for the ice samples with two different grain size distributions. The samples had an indentation rate of $1 \mathrm{~mm} / \mathrm{s}$ with a taper angle of $21^{\circ}$. For the ice sample made of smaller ice seeds $(0-4 \mathrm{~mm})$ less spalling fractures are observed as compared to the specimen made of bigger ice seeds $\left(4-10^{+} \mathrm{mm}\right)$. From these plots it can be seen that both tests exhibit cyclic loading for the first few millimetres of indentation depth, but after about $3 \mathrm{~mm}$, the sample with the bigger grain size experiences large failures with higher force. However, the ice sample with smaller grain size continues to exhibit a continuous crushing with occasional spalling events.

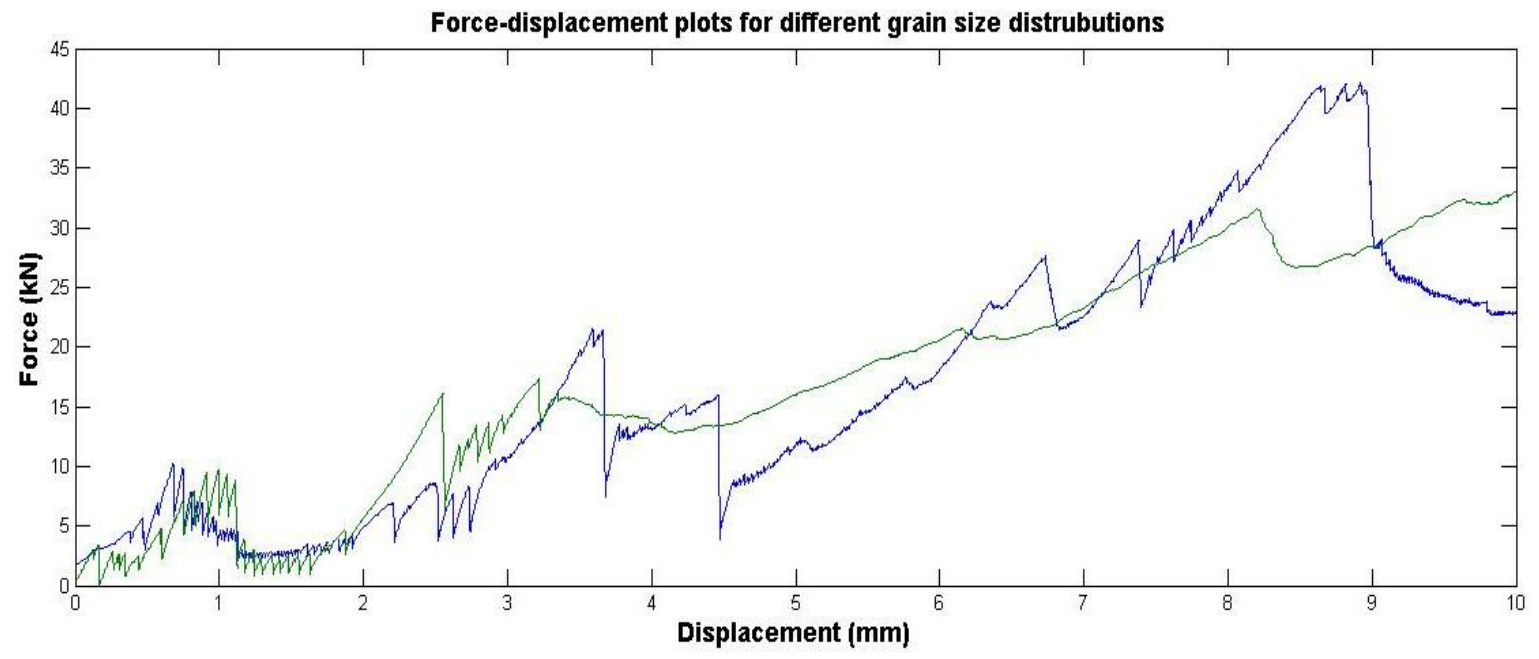

Figure 5.22: MTS Load cell data for two tests having different grain size distributions Test T03_1_21_1_10_F having grain size distribution of $4-10^{+} \mathrm{mm}$ (Blue line) and Test T05_1_21_2_10_F having grain size distribution of 0-4 mm (Green line) 
The tactile pressure sensor data are given below in Figure 5.23 and corresponding forcetime data is given in Figure 5.24 for the tests with two different grain sizes. These were 1 $\mathrm{mm} / \mathrm{s}$ tests done at $-5^{\circ} \mathrm{C}$ with the samples having taper angle of $21^{\circ}$.

In Figure 5.23, the tactile pressure sensor images from event (a) to (e) show significantly higher pressures in case of smaller grain size ice sample than the bigger grain size ice sample with continuous change of contact area. Correspondingly, from the load cell data in Figure 5.24, the force in smaller grain size ice samples is observed to be slightly higher except at event (c). Because of a spalling event at 0.57 second, event (c) of smaller grain size ice sample shows lower force and loss of some contact area.

Grain size distributions: $4-10^{+} \mathrm{mm}$

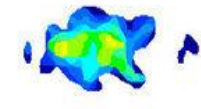

(a)

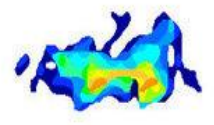

(b)

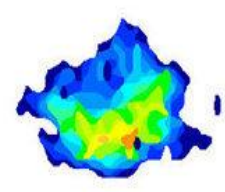

(c)

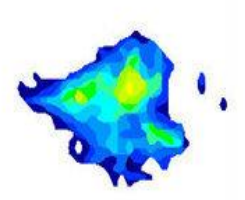

(d)

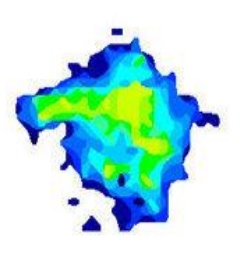

(e)
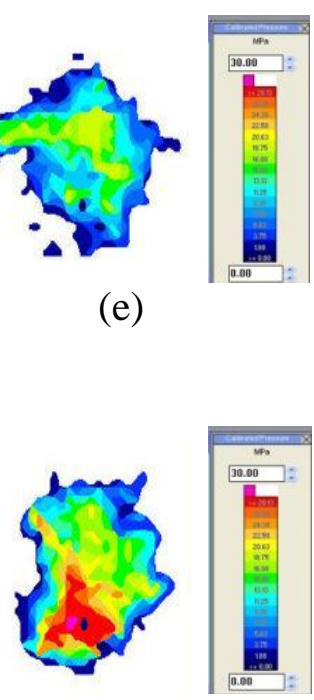

(e)

Figure 5.23: Pressure distribution images for two tests having different grain size distributions. Test T27_10_21_1_5_F having grain size distribution of 4-10mm (top) and Test T28_10_21_2_5_F having grain size distribution of 0-4 mm (bottom) 


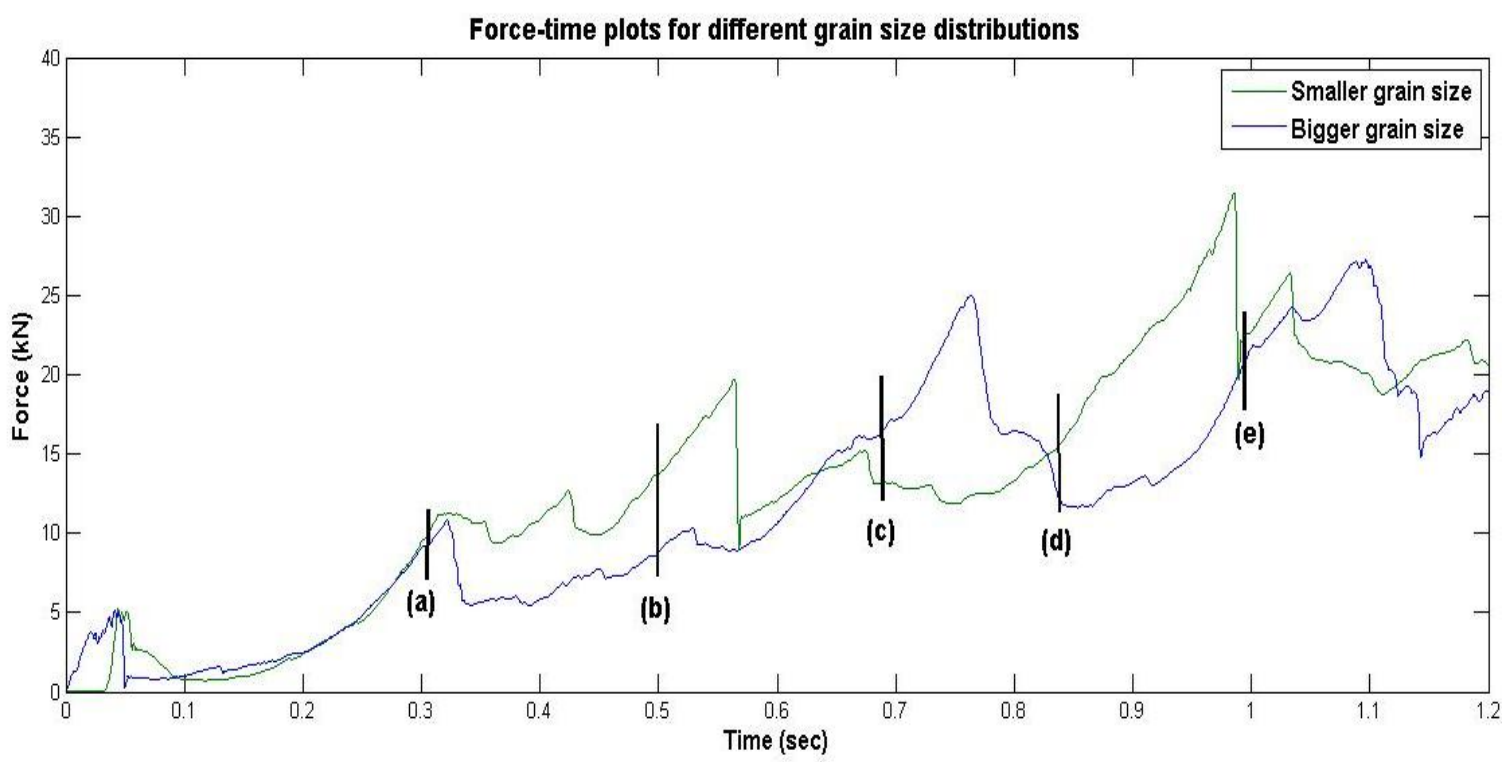

Figure 5.24: MTS load cell data corresponding to the pressure distribution images: Test T27_10_21_1_5_F having grain size distribution of $4-10^{+} \mathrm{mm}$ (Blue line) and Test T28_10_21_2_5_F having grain size distribution of 0-4 $\mathrm{mm}$ (Green line)

In Figures 5.25 and 5.26 the thin-section pictures for horizontal and vertical sections are presented using cross polarized light and side lighting technique. From the horizontal thin-section pictures in Figure 5.25, the grain distribution of bigger and smaller grain sized ice samples can be seen. Different colour grains indicate that the crystallographic orientations of ice grains are different from each other. The vertical thin-section pictures in Figure 5.26 are from the medium speed tests done at $-10^{\circ} \mathrm{C}$ show that the recrystallization effect is stronger in smaller grain sized ice samples than in ice samples with larger grain size for the tests having same other test parameters. 


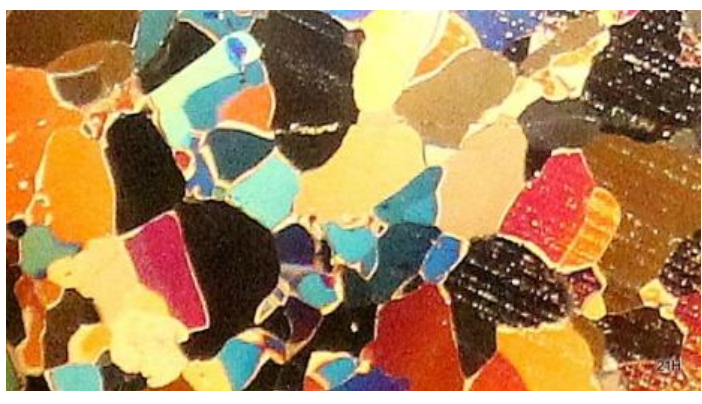

(a)

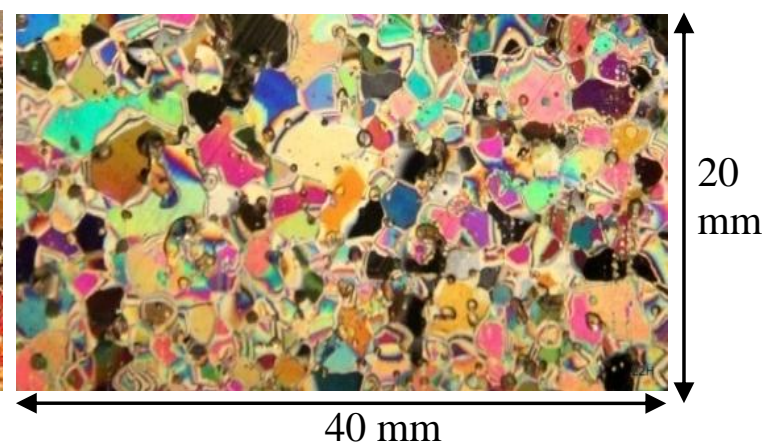

(b)

Figure 5.25: Horizontal thin-section pictures defining the grain distribution using cross polarized light for grain size (a) $4-10^{+} \mathrm{mm}$ (b) $0-4 \mathrm{~mm}$
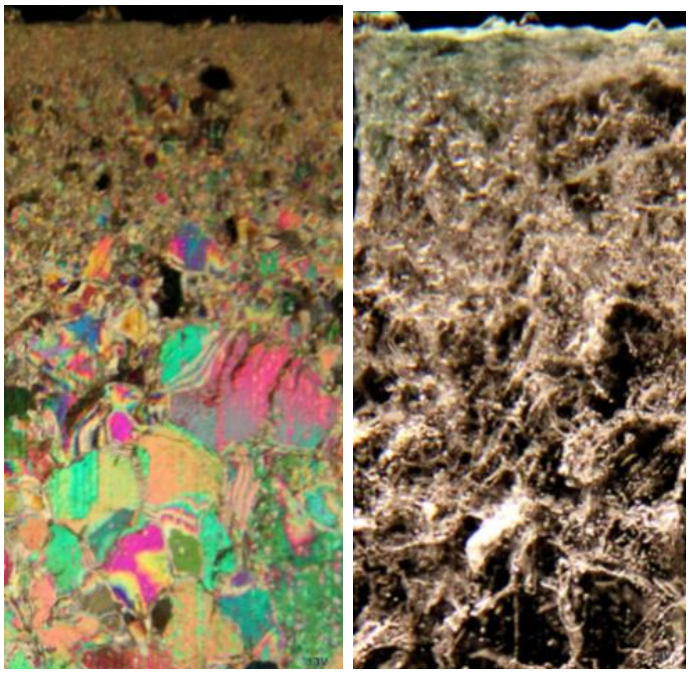

(a)

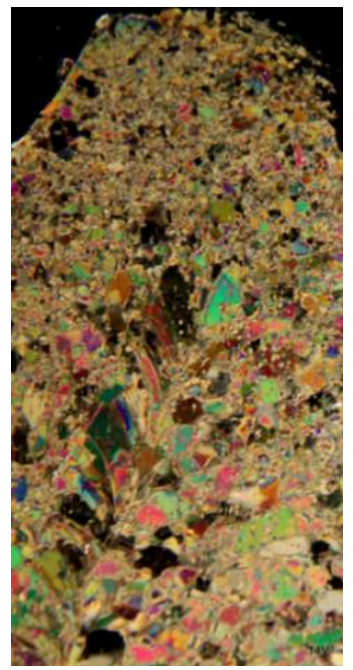

(b)

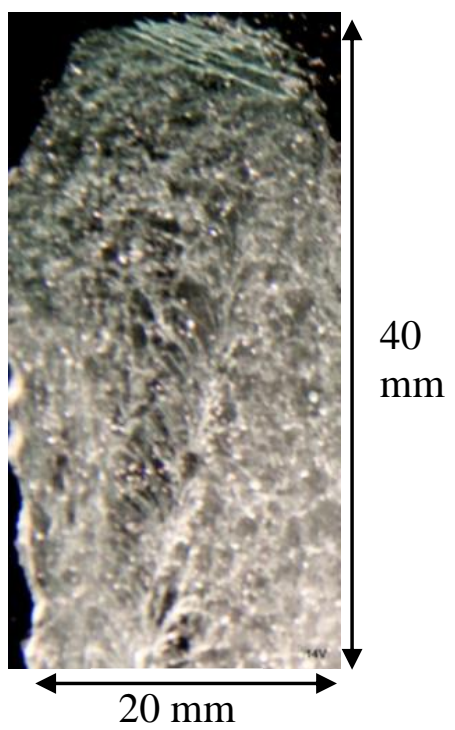

Figure 5.26: Vertical thin-section pictures using cross polarized light (colour) and side light (black and white) for (a) Ice sample with bigger grain size (Test T13_1_30_1_10_F)

(b) Ice sample with smaller grain size (Test T14_1_30_2_10_F)

The total spall mass values for the fast tests are presented in Figure 5.27 for different grain size distributions. From the plot it can be seen that the ice samples made from smaller ice seeds have more spalling mass than ice samples made from bigger ice seeds. These results are observed for all of the different angles considered. These results indicate that there is more failed volume (mass) of ice, but the bigger grain size ice (4- 
$\left.10^{+} \mathrm{mm}\right)$ has more large spalls while the fine grained ice $(0-4 \mathrm{~mm})$ results in more fine grained crushed ice.

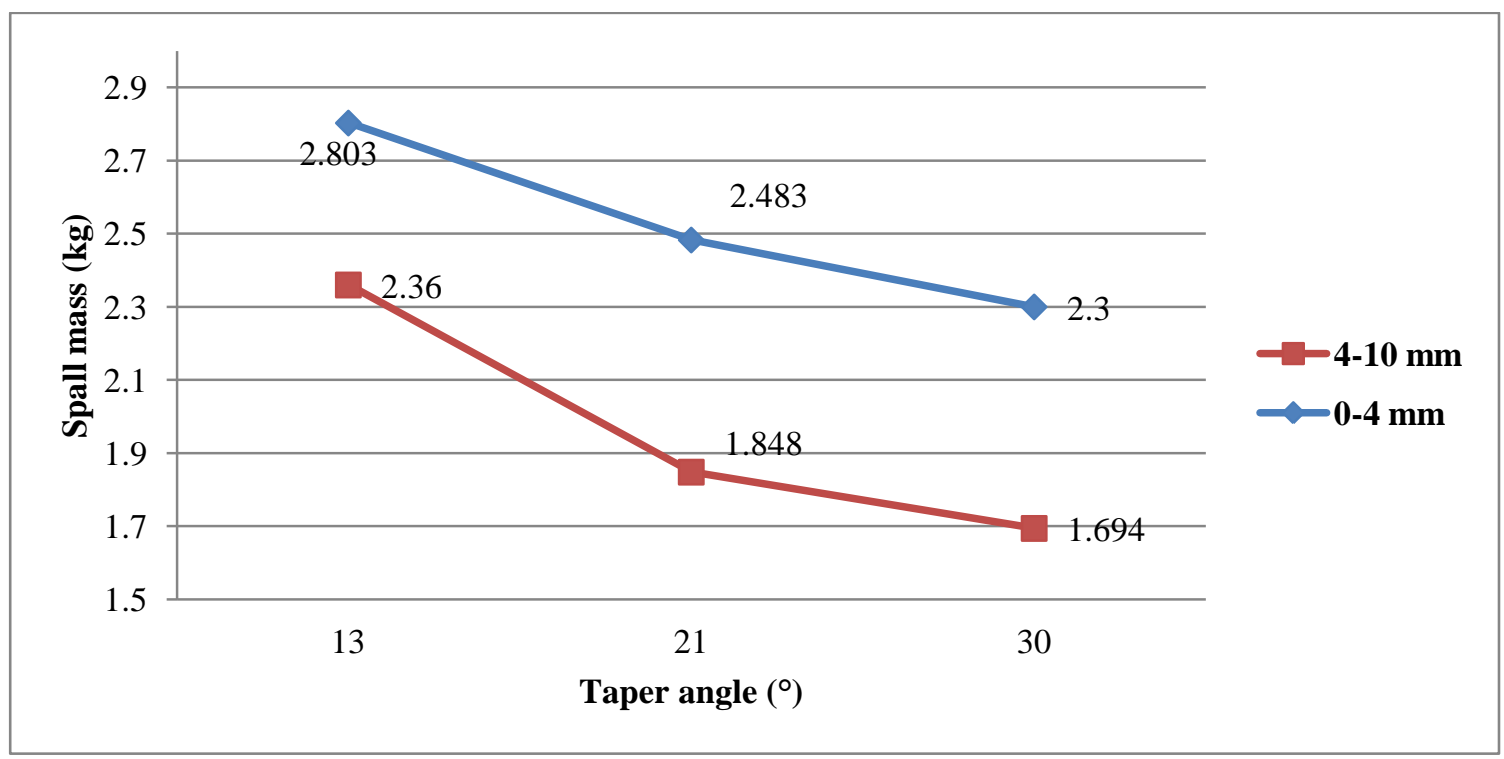

Figure 5.27: Total spall mass for the fast speed tests having bigger and smaller size grain distribution of ice seeds

Total spall mass values show some random results in case of medium and slow speed tests. Some tests show the domination of spalls are more in smaller grain sized ice samples and other tests show different results. The main reason for this can be illustrated by analyzing the nature of fracture. An initial big spall can dominate the total spall mass, even if no significant spall mass is observed for the rest of the indentation. So, further investigation is needed to determine the relationship of spalls having different grain sizes. 


\subsection{Effect of the Taper Angle of Ice Sample}

The shape of the ice sample was observed to have a strong influence on failure and crushing behaviour. Steeper ice specimens have less volume than flatter ice. Given that the flatter ice needs more pressure to fail than steeper ice, they also show dissimilarity in crushing behaviour and failure properties.

The plots of force, area and pressure againt time given in Figure 5.28 are for the three tests having different shaped ice samples. The tests investigated here were done at an indentation rate of $10 \mathrm{~mm} / \mathrm{s}$ at a temperature of $-10^{\circ} \mathrm{C}$ and used the spherical indenter. From Figure 5.28(a) it can be seen that the $13^{\circ}$ ice sample shows higher force than the $21^{\circ}$ and $30^{\circ}$ ice samples. The amount of fluctuations of forces have an effect on the shape of the ice samples. The cyclic loadings seem to minimize as the ice samples become more steep, indicating less random spall failures and more continous crushing failures. Therefore, an ice sample with $13^{\circ}$ taper angle experiences more random spalling and cyclic loading than ice samples with $30^{\circ}$ taper angle. In Figure 5.28(b) comparision of contact area against time is plotted. At any point, the contact area of $13^{\circ}$ taper angle ice sample is higher than $21^{\circ}$ and $30^{\circ}$ ice samples. From the curves of pressure vs time in Figure 5.28(c), it can be seen that pressures of ice samples with different taper angles do not have significant differences, rather show randomness against time. 


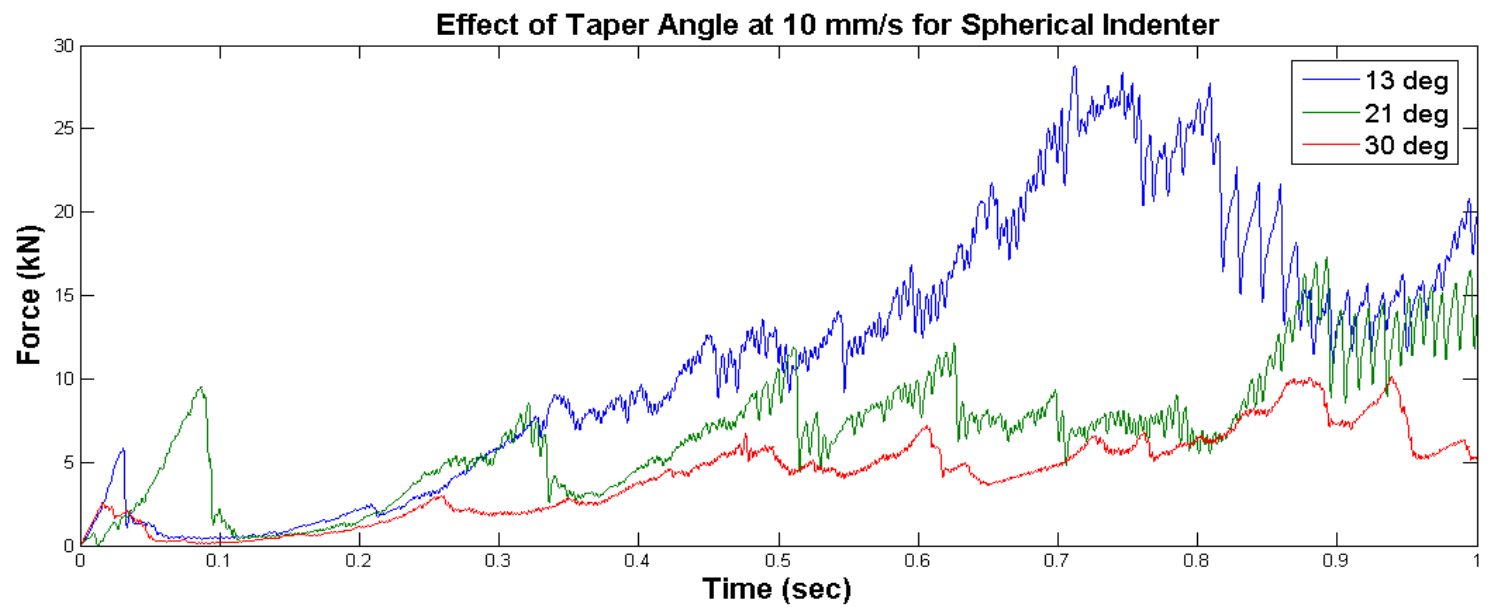

(a)

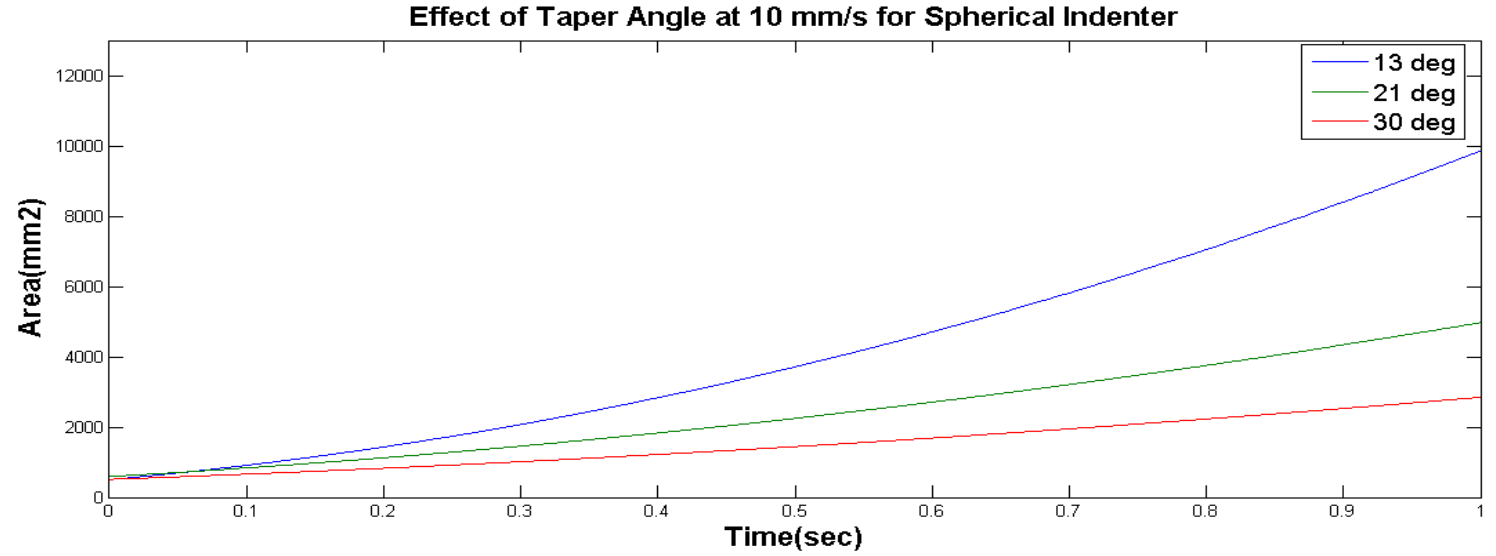

(b)

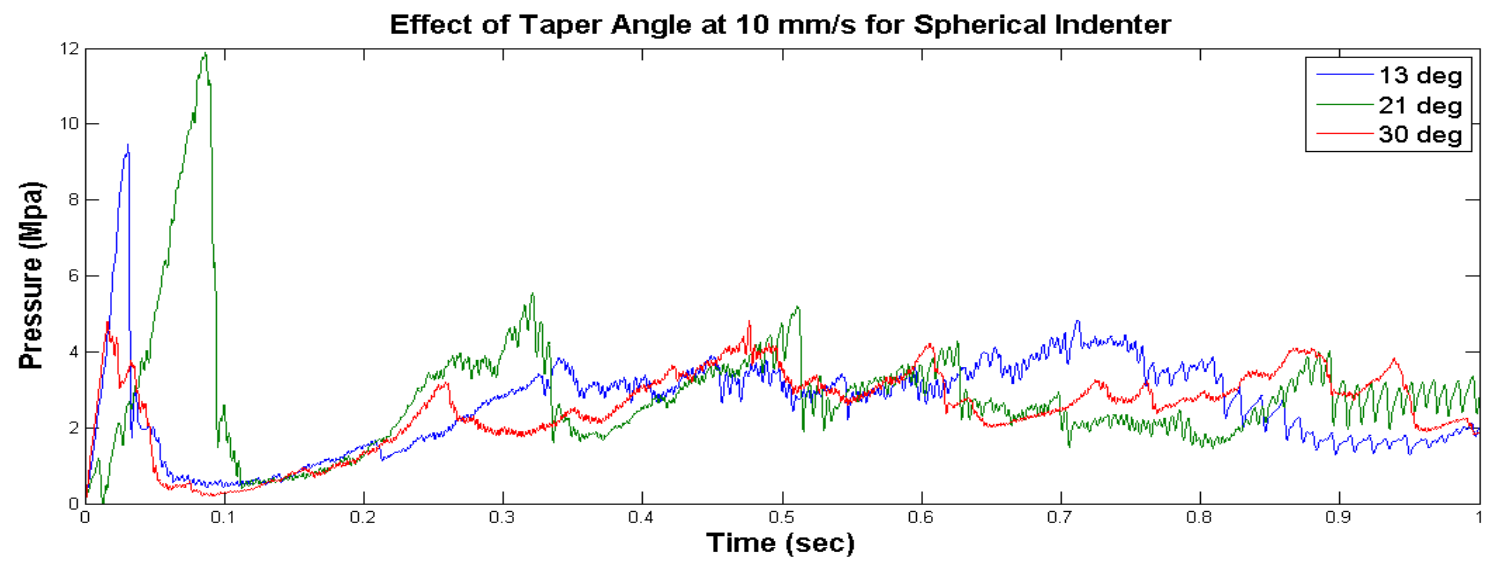

(c)

Figure 5.28: Plots of force, area and pressure against time for three tests having taper angles (a) $13^{\circ}$ for the test T23_10_13_1_10_S (blue); (b) $21^{\circ}$ for the test T08_10_21_1_10_S (green); (c) 30 ${ }^{\circ}$ for the test T09_10_30_1_10_S (red) 
Thin-section pictures using cross polarized light and side light for ice samples with different taper angles are given in Figure 5.29. The tests were done at the indentation rate of $10 \mathrm{~mm} / \mathrm{s}$ using flat indenter at $-10^{\circ} \mathrm{C}$. The pictures show that the $13^{\circ}$ tapered ice specimen is more dominated by recrystallization than the other samples and the $30^{\circ}$ tapered ice sample has lesser amount of recrystallized microstructure in the center. The side lighting pictures show microfractures in the interface of ice samples and indenter where $13^{\circ}$ taper angled ice sample has more microfracture than $30^{\circ}$ taper angled ice sample.

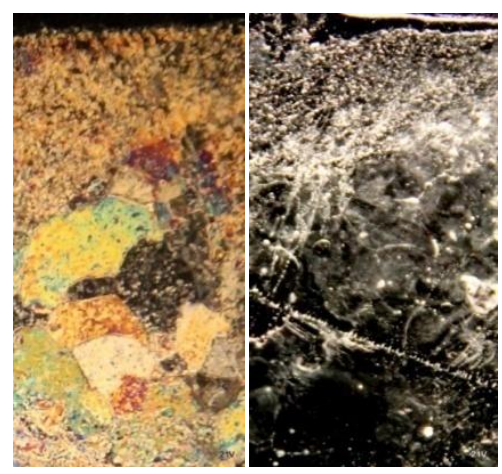

(a)

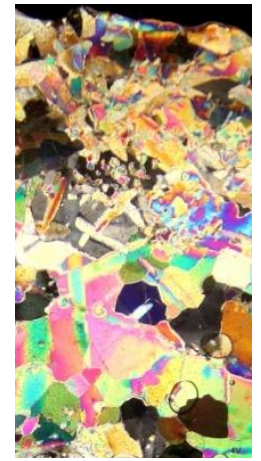

(b)

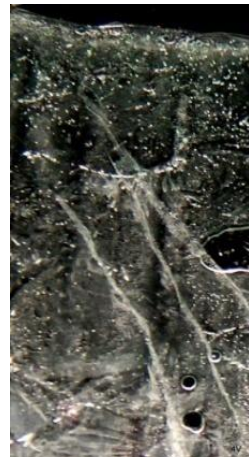

)

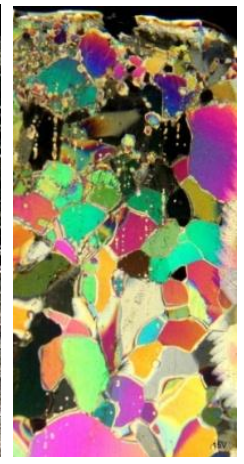

(c)

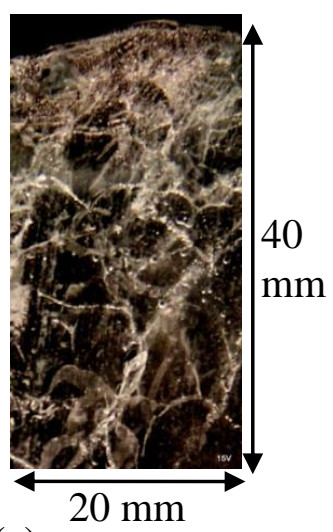

)

Figure 5.29: Vertical thin-section pictures using cross polarized light (colour) and side light (black and white) for ice samples having taper angles of (a) $13^{\circ}$ (Test T21_10_13_1_10_F) (b) $21^{\circ}$ (Test T04_10_21_1_10_F) (c) $30^{\circ}$ (Test T15_10_30_1_10_F)

Total masses of spalls give very distinguished results in the cases of different taper angles. The graphs given in Figure 5.30 are the total mass of spalls having different shaped ice samples with respect to the different indentation speeds. The results show that the $13^{\circ}$ taper angle ice samples have more spalls than those of $21^{\circ}$ and $30^{\circ}$ taper angle ice 
samples have fewer spalls than those of $21^{\circ}$. Part of the reason for these results can be predicted as $13^{\circ}$ ice samples had more initial mass than the other two and the $30^{\circ}$ ice samples had removed a lot more ice while shaping it into a steeper structure. Initially the average mass of the undeformed specimen was $11.44 \mathrm{~kg}$ for $13^{\circ}, 9.8 \mathrm{~kg}$ for $21^{\circ}$ and 9.1 $\mathrm{kg}$ for $30^{\circ}$ ice sample.

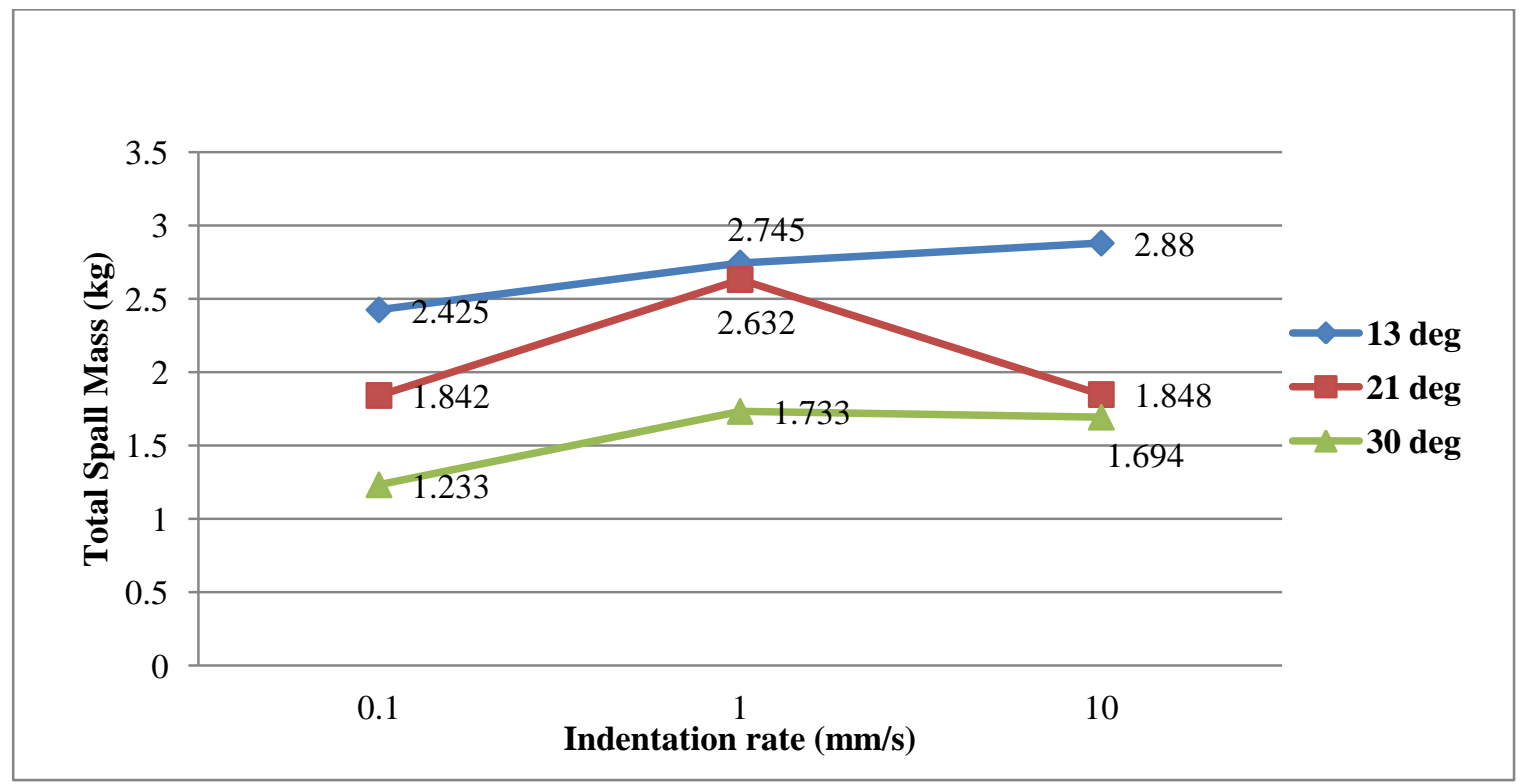

Figure 5.30: Total spall mass of three taper angles at different indentation rates

\subsection{Summary}

Total twenty-eight (28) tests have been done to investigate the behaviour of crushing, spalling, high pressure zones and the effect of various factors used for the ice compressive tests. Crushing and spalling events have been observed from the regular and high-speed videos, synchronized with tactile pressure sensor data and load cell data. 
Crushing and spalling are identified as the most common load limiting factors for compressive ice tests. Indentation speeds have very prominent effects in failure behaviour. Slow enhanced creep failure is present in slow speed tests and brittle and irregular failure in fast speed tests, while in medium speed tests the failure is random and shows a complex mixture of ductile and brittle failure. In thin-section pictures the amount of damage (recrystallization and microfracture) decrease as the indentation rate increases. The warm tests show more of an inclination to ductile failure than cold tests. Studying the thin-section pictures it has been concluded that in the case of warm tests the damaged layer is more heavily dominated by recrystallization and that the cold tests have more microfractural effects. Tests that used the spherical indenter show lower forces than the tests that used the flat indenter and also have less cyclic loadings. The damaged layer in thin-section pictures reveals that domination of recrystallization is more prevalent in the case of the flat indenter, likely due to the larger contact zone as compared with the spherical indenter. Ice samples made of smaller ice seeds require higher loads to trigger failure as compared with larger ice seeds. Moreover, the loading is mostly ductile with very little evidence of cyclic loading in the cases of ice samples with smaller ice seeds. The damaged layer of ice samples with smaller ice seeds shows more recrystallization effects than ice samples with bigger ice seeds. As the taper angle increases the ice sample becomes steeper and the number of cyclic failure events and force decreases. The thinsection pictures of flatter ice samples show that they have more recrystallization effects than steeper ice samples and spall data reveal that flatter ice samples have more spalls, than for steeper ice specimens. 


\section{Chapter 6: Discussion and Conclusion}

\subsection{Summary and Conclusion}

Small-scale indentation tests have been conducted to improve the understanding of ice compressive failure during ice-structure interaction. Main objectives of this research were to investigate the nature of high pressure zones and load limiting features such as crushing and spalling and using two different shaped indenters to study the effect of indentation rate, temperature, grain size and ice specimen shape.

To produce the ice specimens commercially purchased freshwater, polycrystalline ice was crushed into ice seed using an industrial ice crusher and sieved using $4 \mathrm{~mm}$ opening laboratory sieves to have two different sized ice seeds. Water for ice samples was prepared by distillation, deionization and deaeration processes. The compacted water and ice mixture were set in the freezer and after two days of freezing the samples were 
shaped using an ice shaping apparatus. Ice specimens were confined in a steel ring holder to remain fixed during the ice crushing tests. For each test the final indentation depth was up to $70 \mathrm{~mm}$. The indentation was done throughout the conical area and then halfway through the cylindrical area. The data collected from the MTS load cell were the force against indentation depth and time. To understand the nature and variation of high pressure zones within the contact area, tactile pressure sensor data proved to be an excellent technology. Regular and high-speed video recordings were used to observe the crushing behaviours and failure mechanisms during the indentation tests. Spall effects and distributions were observed as a post-experimental analysis as well as thin-sections were done to study the internal structures and grain distributions of ice. Thin sections were also helped to understand the characteristics of the damaged layer and spalling failure.

Throughout the program a total of twenty-eight (28) tests were done. Because of the limitation of the ice holders and freezers, only eight tests per week were performed. These small-scale laboratory tests allow to study and understand the compressive failure process during ice-structure interaction in a cost effective and efficient way. The main observations and findings from these experiments are given below.

\subsubsection{Crushing and Spalling}

From the regular and high-speed video recordings it has been observed that crushing failure is the load limiting failure mechanism and responsible for the cyclic loading pattern in load cell data, and is directly related to the variation of high pressure zones. 
Spalling is also a very important load limiting factor but it does not occur as regularly and frequently as crushing. It occurs occasionally and failure of the high pressure zone in this case is directly linked to the decrease in the contact area due to removal of spall from the edge of the indenter.

\subsubsection{Effect of Indentation speed}

Three indentation speeds were considered for the experiments. They are categorized as slow speed test $(0.1 \mathrm{~mm} / \mathrm{s})$, medium speed test $(1 \mathrm{~mm} / \mathrm{s})$ and fast speed test $(10 \mathrm{~mm} / \mathrm{s})$. The slow tests have shown continuous crushing and damage enhanced creep (ductile) failure throughout the indentation while the fast tests have shown brittle failure often with cyclic loading. The medium speed tests have shown a failure which is a combination of ductile and brittle behaviour. The indentation rate has an effect on the microstructural change in the damaged layer. As the indentation rate increases the amount of recrystallization and microfracture decreases and the slow speed tests showed significantly more recrystallization and microfracture than in the medium and fast speed tests. The spall distributions have indicated a variation in the case of different indentation speeds. In the slow speed tests fewer spalls were recorded than the other cases because continuous damage enhanced creep allow enough time to dissipate energy and prevent local fracture. During slow tests, when spalls did occur they tended to be quite large due to the dependent growth of large fractures. 


\subsubsection{Effect of Temperature}

The compressive tests were done at two different temperatures. Twenty-four (24) tests were done at cold temperature $\left(-10^{\circ} \mathrm{C}\right)$ and four (4) tests were done at warm temperature $\left(-5^{\circ} \mathrm{C}\right)$. Warm tests have shown an inclination to ductile failure, and have no significant cyclic loading pattern. However, the cold tests that were done at the same indentation speed have shown a continuous cyclic loading pattern with large load drops. Thin-section pictures of warm tests have shown damaged layers near the indentation edge are heavily dominated by recrystallization. However, in the cold tests, the layers are observed to be dominated more by microfractures. As warm tests follow ductile failures, the spall masses are slightly less than for the tests done at $-10^{\circ} \mathrm{C}$.

\subsubsection{Effect of Indenter Shape}

Two types of indenters, a flat plate and spherical shaped indenter were used in this research program. From the load cell data it is observed that the samples that used the flat indenter for testing show higher force during the indentation, which has been attributed to larger contact areas during a indentation. The samples that used the spherical indenter show low force throughout the test and the frequency of cyclic loading are less than the flat indenter case. The indenters also show an effect on microstructural modification. Analyzing thin-section pictures it is observed that samples of the flat indenter are heavily dominated by recrystallization than the spherical indenter and also the samples of the spherical indenter have more domination of microfracture. The thin-section pictures show a depression in the top center of the sections in the cases of the spherical indenter 
as a projection of the convex indenter shape. Also, the spall masses are observed to be slightly less in the case of the spherical indenter.

\subsubsection{Effect of Grain Size}

Ice specimens made of fine or smaller grain ice seeds $(0-4 \mathrm{~mm})$ seem to be stronger than samples made of coarse or bigger grain ice seeds $\left(4-10^{+} \mathrm{mm}\right)$. However, they contained more flaws than bigger grain sized ice samples. The average force and pressure are slightly higher in the case of ice samples made of smaller ice seeds. In the thin-section pictures, the recrystallization effects are observed to be more in the cases of ice samples with smaller ice seeds. Spall distributions data show that the fast indentation tests are observed to have more spalls in the case of smaller grain sized ice samples.

\subsubsection{Effect of Taper Angle}

The shape of the ice sample has a direct effect on the measured load data. The force and pressure are observed to decrease as the taper angle of the ice samples become steeper. Therefore, ice samples with $13^{\circ}$ taper angle seem to be stronger than ice samples with $21^{\circ}$ and $30^{\circ}$ taper angle. The numbers of cyclic loading events also decrease as the taper angles become steeper. By observing the thin-section pictures it is concluded that the flatter or low angle ice samples show the highest amount of recrystallization due to higher confinements, while the steeper ice samples show the lower amounts of recrystallization. Also the spall masses are larger in the case of the flatter ice sample and decrease as the taper angle become steeper, indicating that more spalling will occur for low angle ice specimens. 


\subsection{Recommendation for Future Work}

Ice compressive failure is a complex process and needs much more attention and research to fully understand the behaviour. Due to the limitations of the laboratory equipment and time, several recommendations have come forward through the experimental program. A small-scale indentation test is undoubtedly a good and cost efficient way to study this subject, but at the same time medium scale and full scale tests are strongly recommended to better understand the real scenario of ice-structure interactions. Results from this work are representative of pressures expected to be present during full scale interactions, but loads from laboratory scale cannot be directly applied in design. Scale effects are an important part of ongoing research and should be considered further.

From the testing program, a number of recommendations can be made. First of all, increase the range of temperatures and indentation speeds. In the practical cases of the ice environments, the temperature can change from $-40^{\circ} \mathrm{C}$ to $0^{\circ} \mathrm{C}$. Therefore, the use of a wide range of temperatures is recommended for further work. The range of crushing in a real scenario varies from a very slow rate to a very high rate. Therefore, study of a broad range of indentation speeds from $0.001 \mathrm{~mm} / \mathrm{s}$ to $100 \mathrm{~mm} / \mathrm{s}$ is recommended for further small-scale investigations.

The use of different shaped indenters yielded different results. Therefore, the use of some additional shapes of the indenters is recommended for further investigations. An indenter having a sharp notch can be used to create a single high pressure zone in flat ice samples. 
Only two sizes of ice seeds were considered to make the samples for the thesis. Another recommendation is to use more tightly bonded grain size ranges such as 2-4 $\mathrm{mm}$ or 4-6 $\mathrm{mm}$. Use of a real sample of iceberg ice would also be helpful to provide an observation to differentiate natural ice from laboratory ice. In future experiments, using a slow and efficient method to pour the water into the ice seeds is also recommended to help minimizing the air content of the ice.

Only three different angles were used to shape the ice samples due to the limitation of the shaping blade. Therefore, in future tests, it would be of interest to make ice samples having angles flatter that $13^{\circ}$ and steeper than $30^{\circ}$. Furthermore, to increase the size of ice sample is recommended to study force and pressure distribution in the far field ice, so the influence of loading conditions can be assumed. To see the alignment sensitivity, slightly tilted specimens at a certain angle will provide a new observation.

Due to the size of the tactile pressure sensor only a small area of the ice sample that was covered by the sensor were considered for analysis. Therefore, it was not possible to view the nature of high pressure zones and pressure distributions till the end of the test. Use of a bigger Tekscan sensor is recommended for further work. Also, use of a temperature sensor would provide a new investigating tool to study the variation of temperatures and thermal changes at the moment of crushing.

The results from the experiments illustrate good agreements with fracture and damage mechanics theories and show a similar outcome with small-scale compressive tests that have been done previously. The results of this thesis are an effort to improve the 
understanding of the compressive ice failure process and provide a guideline for smallscale laboratory experiments. This attempt has been made to provide insight into failure processes and to determine the effects of various parameters on crushing processes to improve the design of offshore structures in ice environments.

Through such experiments to our understanding fundamental aspects of ice failure processes, greater confidence in modelling ice loads for design can be achieved. 


\section{Bibliography}

Anderson, T.L. (2005). Fracture mechanics: fundamentals and applications, 3rd edition. CRC Press. Boca Raton, FL.

Barrette, P., Pond, J., and Jordaan, I. (2002). Ice damage and layer formation in small scale indentation experiments. Ice in the Environment, Proceedings of the 16th international Symposium on Ice, IAHR, Dunedin, New Zealand, vol. 3:246-253.

Browne, T. (2012). Analysis of Compressive Ice Failure during Ice-structure Interaction. M. Eng Thesis. Memorial University of Newfoundland. St. John's, Canada .

Bruneau, S., Dillenburg, A., Ritter, S. (April, 2011). Ice Specimen Fabrication Techniques and Indentation Experiments, A StePS ${ }^{2}$ Pilot Laboratory Investigation of IceStructure Interaction. STePS ${ }^{2}$-RP001-2011. Memorial University of Newfoundland, Sustainable Technology for Polar Ships and Structures, St. John's, NL, Canada, P. 139.

Bruneau, S., Dillenburg, A., Ritter, S. (June 2012). Ice Sample Production Techniques and Indentation Tests for Laboratory Experiments Simulating Ship Collisions with Ice. ISOPE 2012. Rhodos, Greece. 
Bruneau,S., Colbourne,B., Dragt,R., Dillenburg,A., Ritter,S., Pilling,M., Sullivan,A. (June, 2013). Laboratory Indentation Tests Simulating Ice-Structure Interactions Using Cone-Shaped Ice Samples and Steel Plates. POAC. Espoo, Finland.

Cammaert, A. B. and Muggeridge, D. B. (1988). Ice interaction with offshore structures. Van Nostrand Reinhold.

Clawson Machine; Clawson Hail Queen Ice Cube Crusher. (2011).

Cole, D.M. (1979). Preparation of polycrystalline ice specimens for laboratory experiments. Cold Regions Science and Technology, vol. 1, p. 153-159.

Crocker, G.B., Croasdale, K.R., McKenna, R.F., English, G.M., Guzzwell, J. and Bruneau,S.J. (1997). C-CORE iceberg impact experiment-phase 2, final report. C-CORE, Memorial University of Newfoundland, Newfoundland.

Dillenburg, A. K. (2012). Rate dependency in conical ice indenter failure. Master's Thesis, University of Duisburg-Essen, Institute of Ship Technology, Ocean Engineering and Transport Systems .

Dragt, R.C., Bruneau, S.E. (June, 2013). The Collision of Cone Shape Ice Samples against Steel Plates of Varying Surface Roughness. POAC'13. Espoo, Finland.

Duval P., Ashby, M.F., Anderman, I. (1983). Rate-controlling processes in the creep of polycrystalline ice. J Phys. Chem. 87, 4066-4074. 
Erdogan, F. and Sih, G.C. (1963). On the crack extension in plates under plane loading and transverse shear. Journal of Basic Engineering 85, 519-527.

Findley, W.N., Lai, J.S. and Onaran, K. (1976). Creep and relaxation of nonlinear viscoelastic. North-Holland Publishing Company.

Frederking, R.M.W., Jordaan, I.J., and McCallum, J.S. (1990). Field tests of ice indentation at medium scale: Hobson's Choice ice island 1989. Proceedings of 10th International Symposium on Ice, IAHR' 90, Espoo, Finland, Vol. 2, pp. 931-944.

Glen, J.W. (1955). The creep of polycrystalline ice. Proceedings Royal Society of London, Piccadilly, London, W., Ser. A, Vol. 228, pp.519-538.

Glen, J.W. (1975). The mechanics of ice: cold regions science and engineering monograph II-C2b. Cold Regions Research and Engineering Laboratory, Hanover, New Hampshire , pp. 29-33.

Griffith, A.A. (1920). Phenomena of rupture and flow in solids. Philos. Trans. R. Soc. London, Ser. A221, pp. 163-198.

Hobbs, P. (1974). Ice physics. Clarendon Pres, Oxford.

Irwin, G.R. (1957). Fracture. Handbuch der physic. 6. Springer-Verlag, Berlin.

Jonas, J.J. and Muller, F. (1969). Deformation of ice under high internal shear stresses. Canadian Journal of Earth Sciences, Vol. 6, pp.963-968. 
Jones. S. J.,Gagnon, R.E., Derradji, A., and Bugden,A. (2003). Compressive strength of iceberg ice. Canadian Journal of Physics.

Jordaan ,I., Li,C, Sudom, D. Stuckey, P., and Ralph, F. (2005a). Principles for local and global ice design using pressure area relationships. POAC, Procedeedings, Vol 1, pp 375385. Potsdam, N.Y.

Jordaan, I. and McKenna, R. (1988). Constitutive relations for creep of ice. Proceedings of 9th IAHR International Symposium on Ice, Vol. 3, Sapporo, Japan , pp. 47-58.

Jordaan, I. J. and Xiao, J. (1992). Interplay between damage and fracture in ice-structure interaction. Proceedings of the 11th International Symposium on Ice, IAHR, Banff, Alberta,1992, Vol 3: 1448 - 1467.

Jordaan, I., Frederking, R. and Li, C. (2006). Mechanics of ice compressive failure, probabilistic averaging and design load estimation. Proceedings of 18th International Symposium on Ice, IAHR, Sapporo, Japan, Vol. 2, pp.223-230.

Jordaan, I., Fuglem, M., and Matskevitch, D. (1996). Pressure-area relationship and the calculation of global forces. Proceedings of 13th International Symposium on Ice, Volume 1, Beijing, China, pp. 166-175.

Jordaan, I..J., Wells, J., Xiao, J., Derradji-Aouat, A. (2008). Ice crushing and cyclic loading in compression. Proceedings 19th IAHR Symposium on Ice, Vancouver, British Columbia, Canada. 
Jordaan, I.J. (2001). Mechanics of ice-structure interaction. Engineering Fracture Mechanics; 68, pp.1923-1960.

Jordaan, I.J., Li., C, Barrette, P., Duval, P. and Meyssonnier, J. (2005b). Mechanisms of ice softening under high pressure and shear. Proc. 18th Int. POAC, Vol. 1, pp. 249-260. Kachanov, L. (1958). On the creep rupture time. Izv. An SSSR, Ofd. Tekhn. Nauk. (8), $26-31$.

Kheisin, D.E. and Cherepanov, N.V. (1976). Change of ice structure in the zone of impact of a solid body against the ice cover surface. Problems of the Arctic and Antarctic, Edited by Treshnikov, A.F., Issue 33-35, pp. 239-245.

Li, C. (2002). Finite element analysis of ice-structure interaction with a viscoelastic model coupled with damage mechanics. M.Eng Thesis, Memorial University, St. John's, NL, Canada.

Li, C. (2007). Probability and fracture mechanics applied to ice load estimation and associated mechanics. Ph.D Thesis, Memorial University of Newfoundland, St. John's, NL, Canada.

Li, C., Jordaan, I.J., Barrette, P. (2005). Strain localization and fracture of cylindrical ice specimens under confining pressure. Proceedings of 18th POAC. Potsdam, New York, Vol. 1, pp. 213-224. 
Li., C., Barrette, P., Jordaan, I.J. (2004). High Pressure Zones at Different Scales During Ice-Structure Indentation. Proceedings of 23rd International Conference on Offshore Mechanics and Arctic Engineering.

Lieu, D.K. and Mote, C.D. Jr. (1984). Experiments in the machining of ice at negative rake angles. Journal of Glaciology, vol. 30.

Lockett, F. J. (1972). Nonlinear Viscoelastic Solids. Academic Press, London and New York.

Mackey, T. (2005). Laboratory indentation of polycrystalline ice: an investigation of fracture. M.Eng Thesis, Memorial University, St. John's, NL, Canada .

Mackey, T., Wells., J., Jordaan, I.J., Derradji-Aouat, A. (2007). Experiments on the fracture of polycrystalline ice. Proceedings of POAC 2007. Dalian, China .

Masterson, D. M., Spencer, P. A., Nevel, D. E. and Nordgren, R. P. (1999). Velocity effects from multiyear ice tests. Proceedings 18th OMAE Conference. S. John's, Canada, 1999.

Meglis, I., Melanson, P., and Jordaan, I. (1999). Microstructural change in ice: II. creep behavior under triaxial stress conditions. Journal of Glaciology 45 (151), 438-448.

Melanson, P.M. (1998). Damage and microstructural change in laboratory grown ice under high pressure zone conditions. M. Eng. Thesis, Memorial University, St. John's, NL, Canada . 
Melanson, P.M., Meglis, I.L., Jordaan, I.J. and Stone, B.M. (1999). Microstructural change in ice: I. Constant-deformation-rate tests under triaxial stress conditions. Journal of Glaciology, Vol. 45, No.151, pp. 417-422.

Mellor, M. (1983). Mechical behaviour of sea ice. U.S. Army Cold Region Research and Engineering Lab, Honover, CRREL Report 83-1.

Muggeridge, K. and Jordaan, I. (1999). Microstructural change in ice: III. observations from an iceberg impact zone. Journal of Glaciology 45 (151), 449-455.

Murrell, S.A.F., Sammonds, P.R. and Rist, M.A. (1991). Strength and failure modes of pure ice and multi-year sea ice under triaxial loading. Proceedings of Symposium on Ice Structure Interaction, IUTAM / IAHR, St. John's, NF, Canada, pp. 339-361.

Nadreau, J.P., Michel, B. (1984). Ice properties in relation to ice forces. Proceedings of 2nd State-of-the-Art IAHR Working Group on Ice Forces, Hamburg.

Nordell, B., (1990). Measurement of a P-T coexistence curve for ice-water mixture. Cold Regions Science and Technology, 19: 83-88.

Passchier, C.W., Trouw, R.A.J. (2005). Microtectonics. Springer-Verlag. Berlin.

Poirier, J. P., Guillope, M. (1979). Dynamic recrystallization during creep of singlecrystalline halite: An experimental study. Journal of Geophysical Research , 84, 55575567.

Poirier, J.P. (1985). Creep of crystals. Cambridge University Press, London. 
Raj, R., Ashby, M.F. (1971). On grain boundary sliding and diffusion creep. Metall Trans. 2. , 1113-1127.

Reddy, P.S., Colbourne, B., Daley, C. Bruneau, S.E., Gagnon, R. (2012). Strength and Pressure Profiles from Conical Ice Crushing Experiments. ICETECH Paper No. ICETECH12-132-RF.

Rice, J. (1968). A path-independent integral and the approximate analysis of strain concentration by notches and cracks. concentration by notches and cracks. Journal of Applied Mechanics, 191-309.

Rist, M.A., Murrel, S.A.F. and Sammonds, P.R. (1988). Experimental results on the failure of polycrystalline ice under triaxial stress conditions. Proceedings of 9th IAHR Conference, (pp. 118-127). Sapporo.

Sanderson, T. (1988). Ice mechanics: risks to offshore structures. Graham \& Trotman, London.

Schapery, R.A. . (1997). Nonlinear viscoelastic and viscoplastic constitutive equations based on thermodynamics. Mechanics of Time-Dependent Materials, Vol. 1 , pp. 209240.

Schapery, R.A. (1984). Correspondence principles and a generalized J integral for large deformation and fracture analysis in viscoelastic media. International Journal of Fracture, 25, pp. 195-223. 
Schapery, R.A. (1981). On viscoelastic deformation and failure behaviour of composite materials with distributed flaws. Advances in Aerospace Structures and Materials, The American Society of Mechanical Engineers, pp.5-20.

Schulson, E.M. (1999). The structure and mechanical behavior of ice. JOM Vol. 51, No. 2 , pp. 21-27.

Schulson, E.M., and Duval, P. (2009). Creep and fracture of ice. Cambridge University Press, UK.

Sinha, N. (1989). Elasticity of natural types of polycrystalline ice. Cold Regions Science and Technology 17, pp. 127-135.

Sinha, N.K. (1979). Grain boundary sliding in polycrystalline materials. Phil. Mag. A, Vol. 40 , pp. 825-842.

Sinha, N.K. (1977). Technique for studying the structure of sea ice. Journal of Glaciology 18 , pp. 315-323.

Sullivan, A. Pilling, M. (Aug 2011). Influence of Ice Shapes on Indentation Loads. Work Term Report for STePS2 program, Memorial University Division Of Cooperative Engineering.

Taylor, R.S. (2010). Analysis of Scale Effect in Compressive Ice Failure and Implications for Design. Ph.D Thesis, Memorial University, St. John's, NL, Canada. 
Taylor, R.S., Browne, T., Jordaan, I., Gürtner, A. (June, 2013). Fracture and Damage during Dynamic Interactions between Ice and Compliant Structures at Laboratory Scale. roceedings of Offshore Mechanics and Arctic Engineering Conference, Nantes, France .

Taylor, R.S., Frederking, R, and Jordaan, I. J. (2008). The nature of high pressure zones in compressive ice failure. Proceedings 19th IAHR Symposium on Ice,Vancouver, British Columbia, Canada .

Urai, J.L., Means, W.D.M. and Lister, G.S. (1986). Dynamic recrystallization of minerals. American Geophysical Union, Geophysical Monograph 36, pp. 161-199.

Venkatachalam, G., Harichandran, R., Rajakumar, C., Dharmaraja, C. and Pandivelan, C. . (2008). Determination of J -integral and stress intensity factor using the commercial FE software ABAQUS in austenitic stainless steel (AISI 304) plates. The International Journal of Advanced Manufacturing Technology , DOI: 10.1007/s00170-008-1872-z.

Vernon. R.H. (1981). Optical microstructure of partly recrystallized calcite in some naturally deformed marbles. Tectonophysics, Vol. 78, pp. 601-612.

Wei, Y., Dempsey, J.P. (1991). Fractographic examinations of fracture in polycrystalline S2 ice. Journal of Materials Science. 26. (1991), pp. 5733-5740.

Weiss, J. and Schulson, E.M. (1995). The failure of fresh-water granular ice under multiaxial compressive loading. Acta Metallurgica et Materialia, Vol. 43 , pp. 23032315. 
Wells, J., Jordaan, I., Derradji-Aouat, A. and Taylor, R. (2010). Small-scale laboratory experiments on the indentation failure of polycrystalline ice in compression: Main results and pressure distribution. Cold Regions Science and Technology, Vol. 65, No. 3, pp. 314-325.

Xiao, J. (1997). Damage and fracture of brittle viscoelastic solids with application to ice load models. Ph.D Thesis, Memorial University, St. John's, NL, Canada .

Xiao, J. (1991). Finite element modeling of damage processes in ice-structure interaction. M.Eng Thesis. Memorial University of Newfoundland. St. John's, NL, Canada. 


\section{APPENDIX A - Test Results: Total Load and Pressure, After Crushing Pictures, Spall Distributions and Thin-section Photographs}



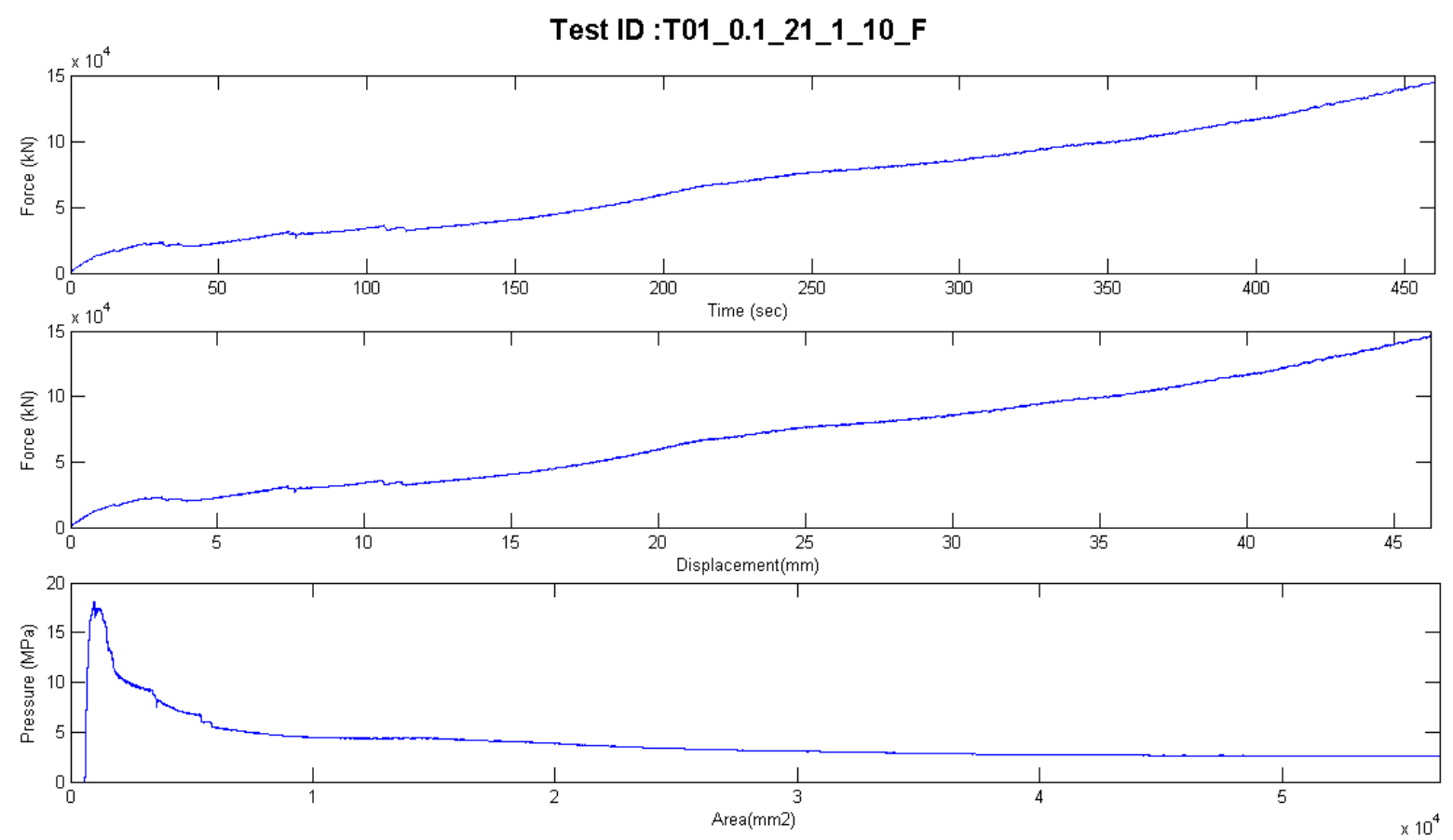

T01_0.1_21_1_10_F: Force and pressure traces

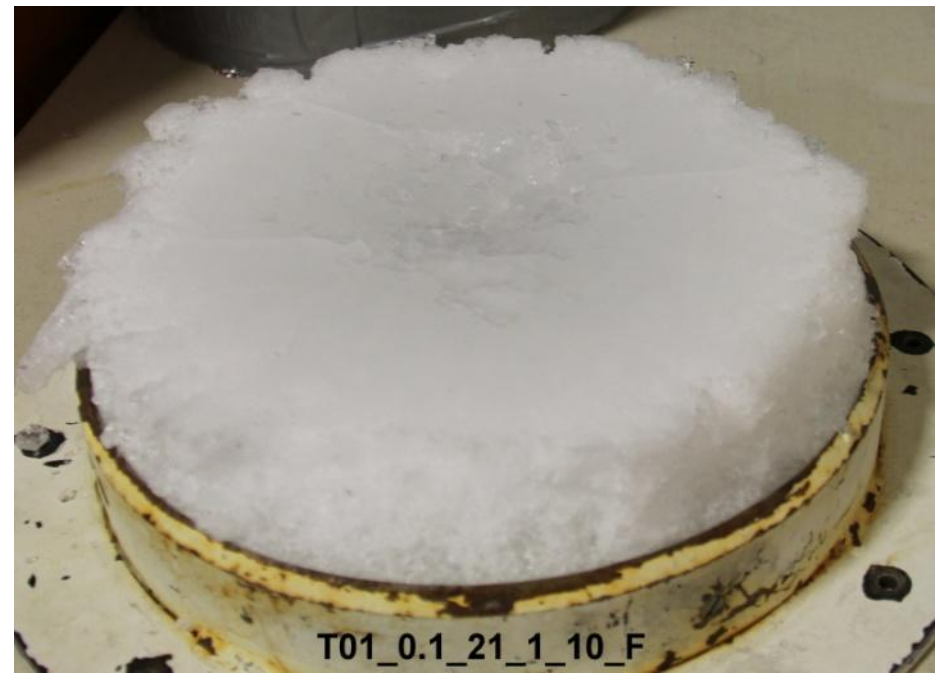

T01_0.1_21_1_10_F: After crushing picture 


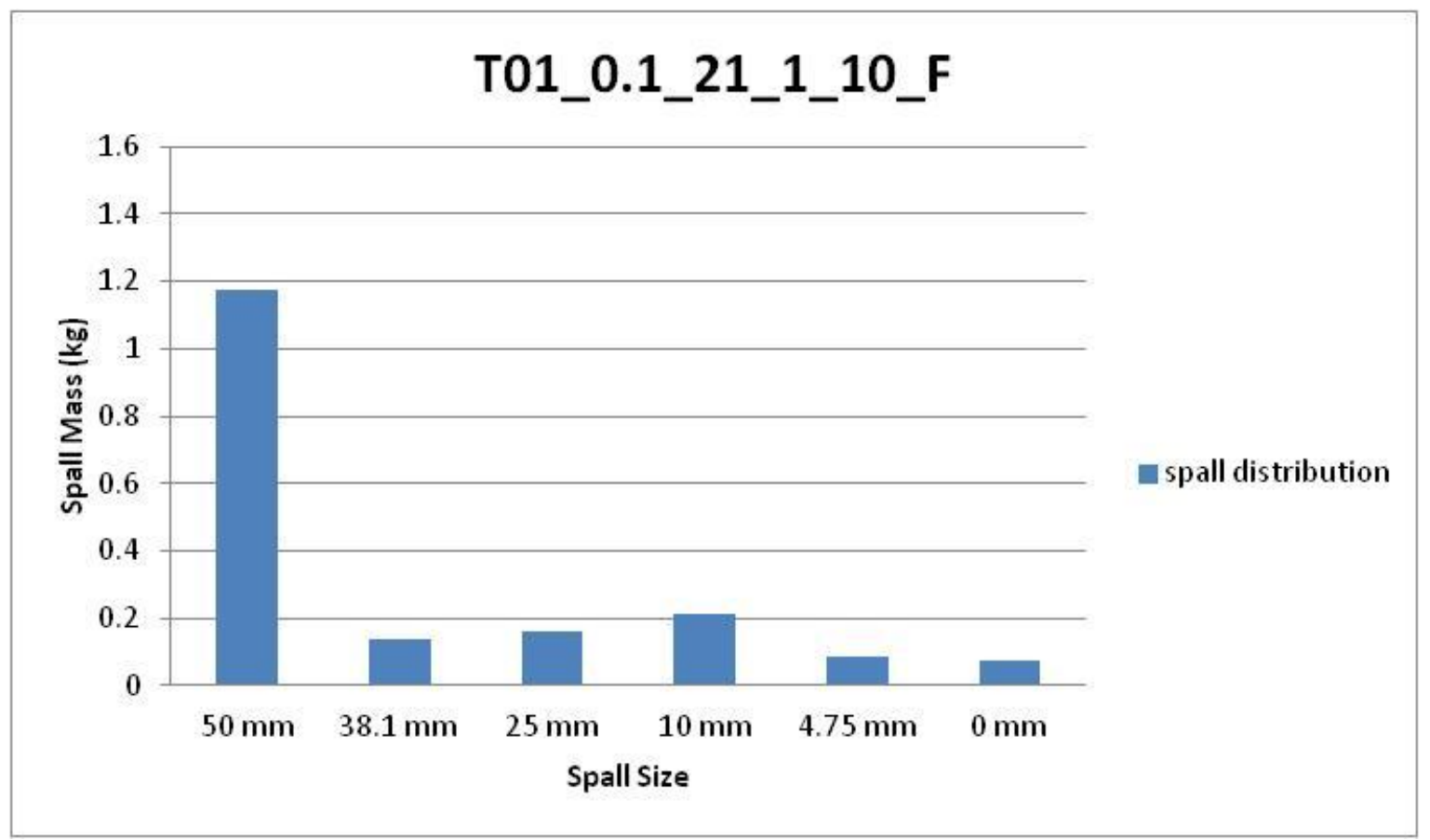

T01_0.1_21_1_10_F: Spall mass distributions
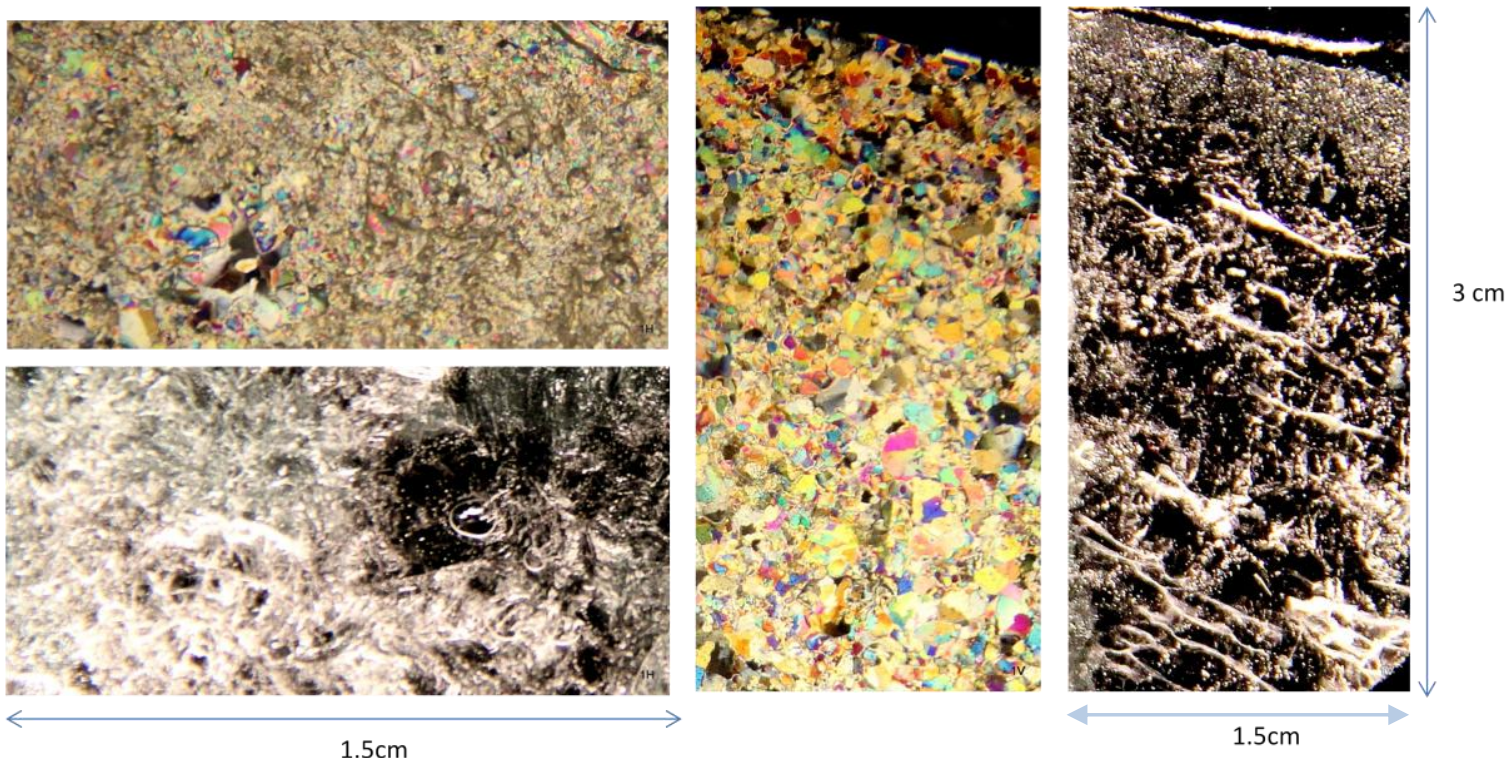

T01_0.1_21_1_10_F: Thin section pictures Horizontal (left) and Vertical (right) 

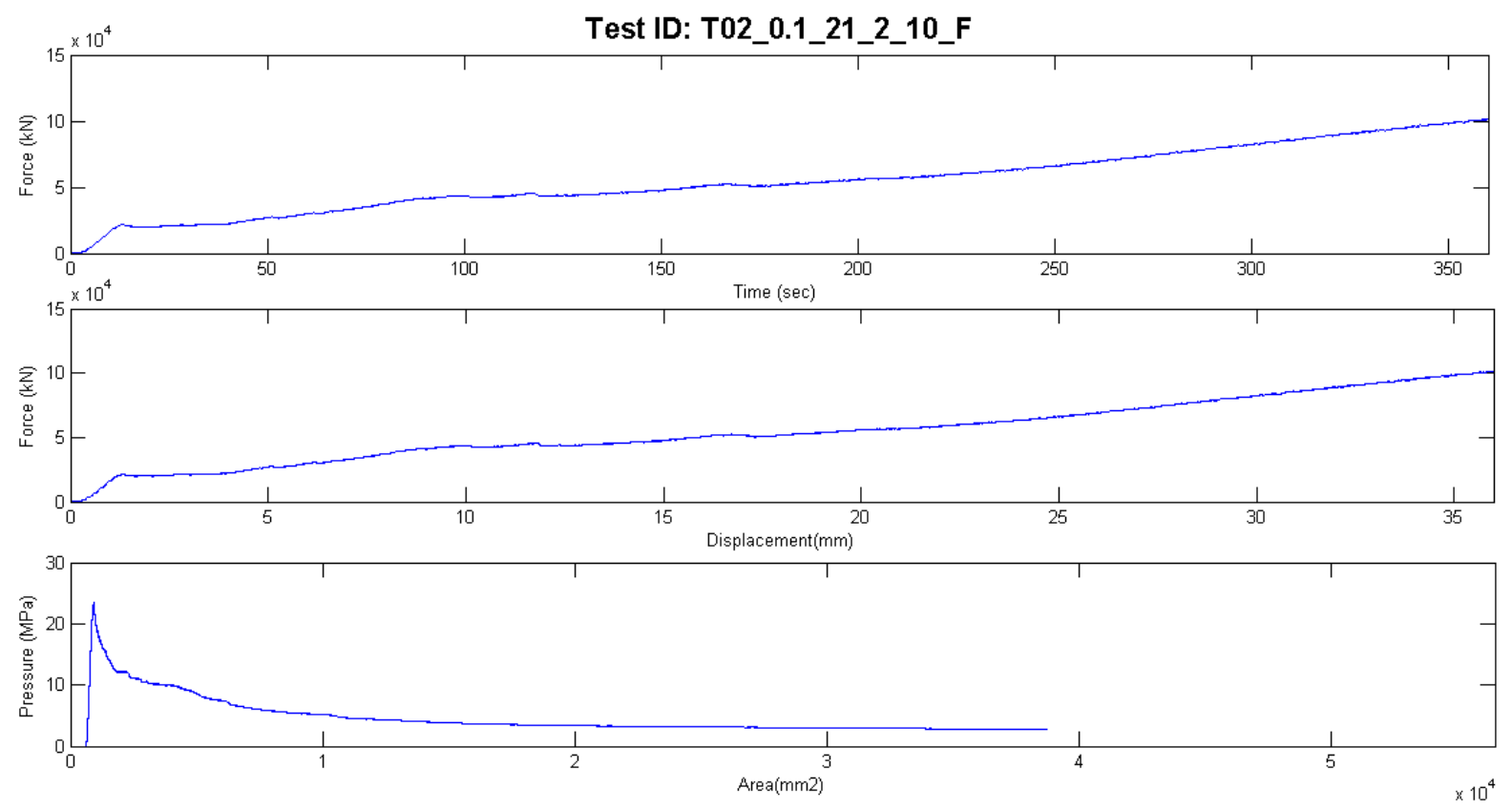

T02_0.1_21_2_10_F: Force and pressure traces

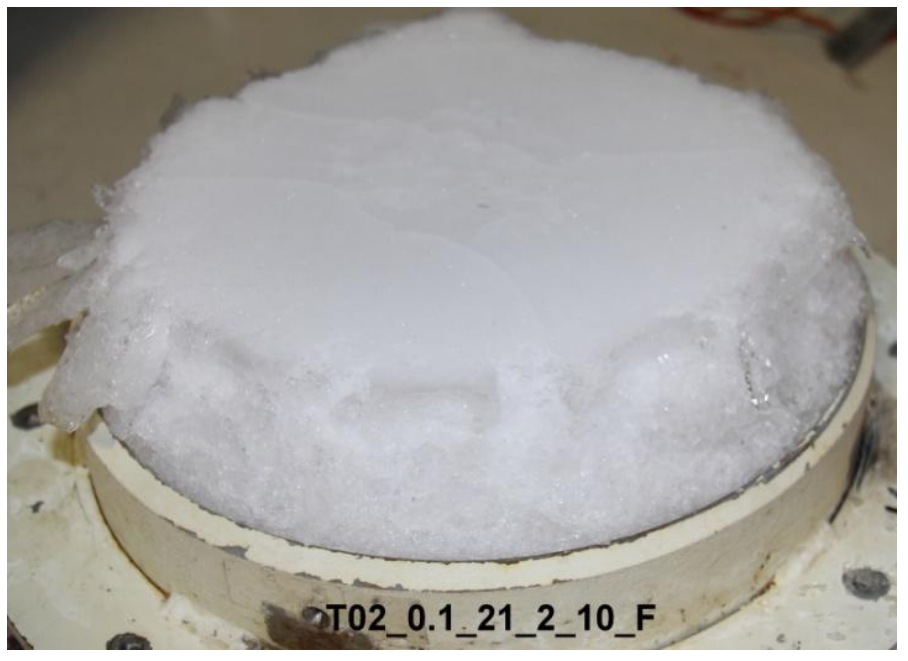

T02_0.1_21_2_10_F: After crushing picture 


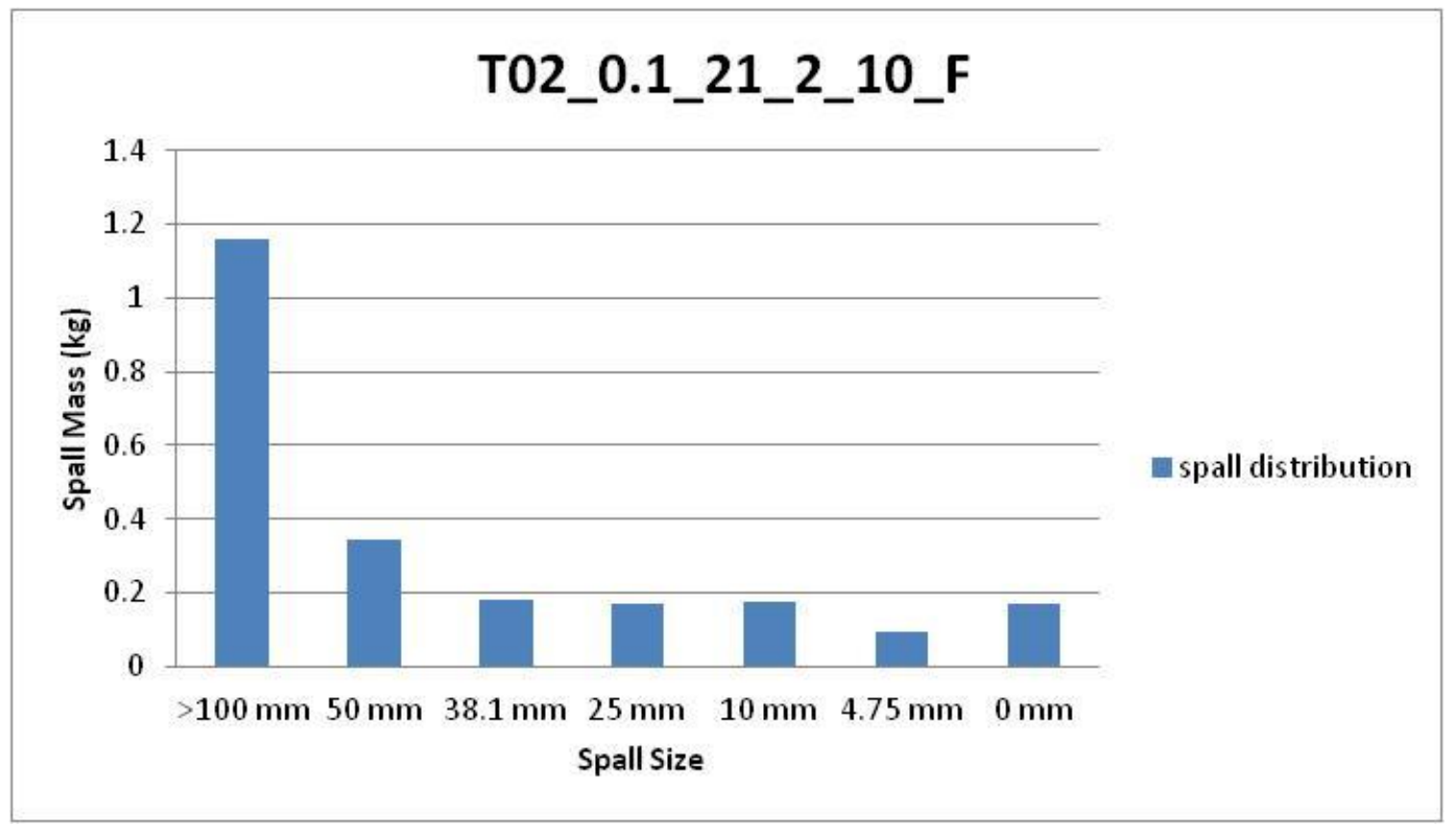

T02_0.1_21_2_10_F: Spall mass distributions
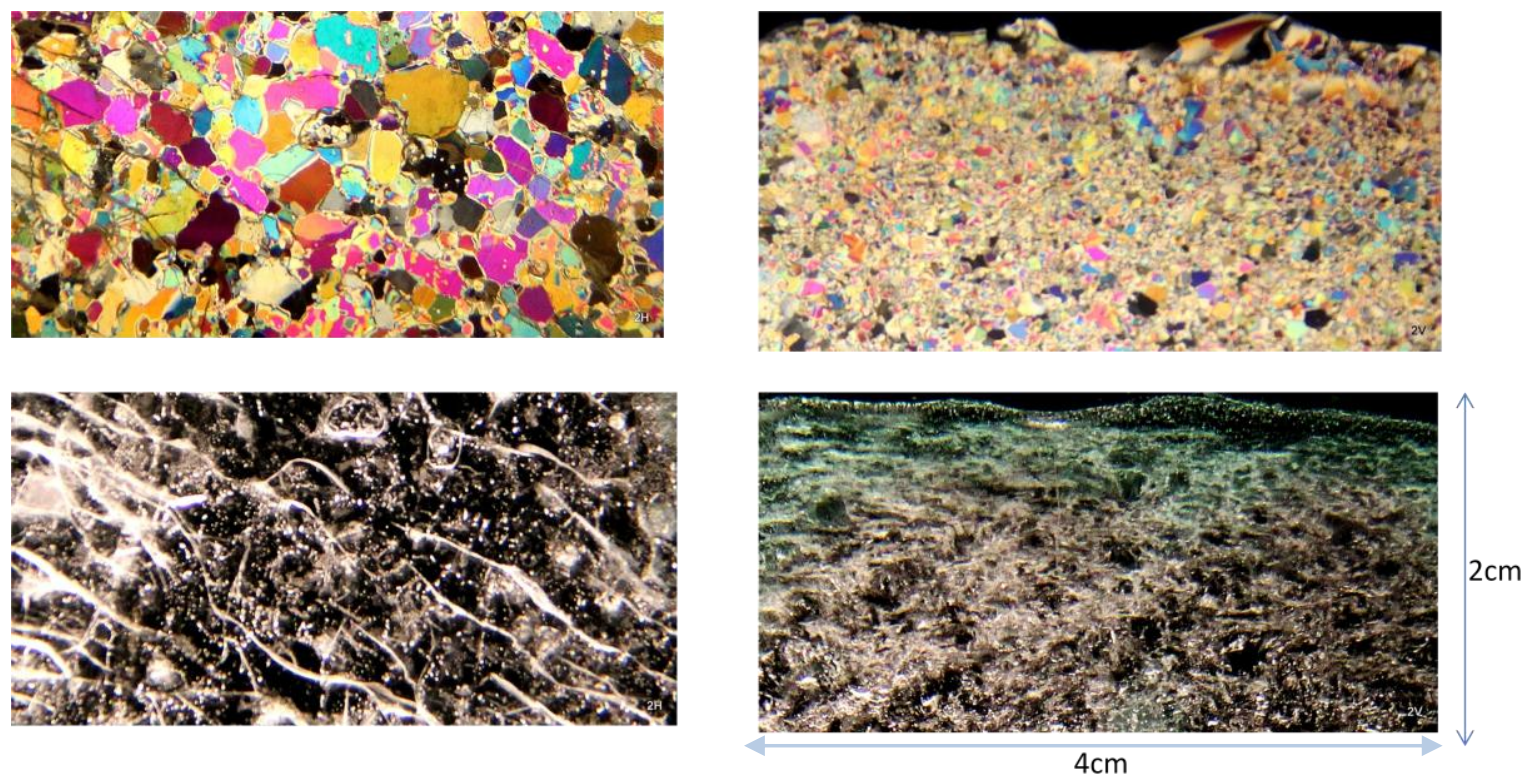

T02_0.1_21_2_10_F: Thin section pictures Horizontal (left) and Vertical (right) 

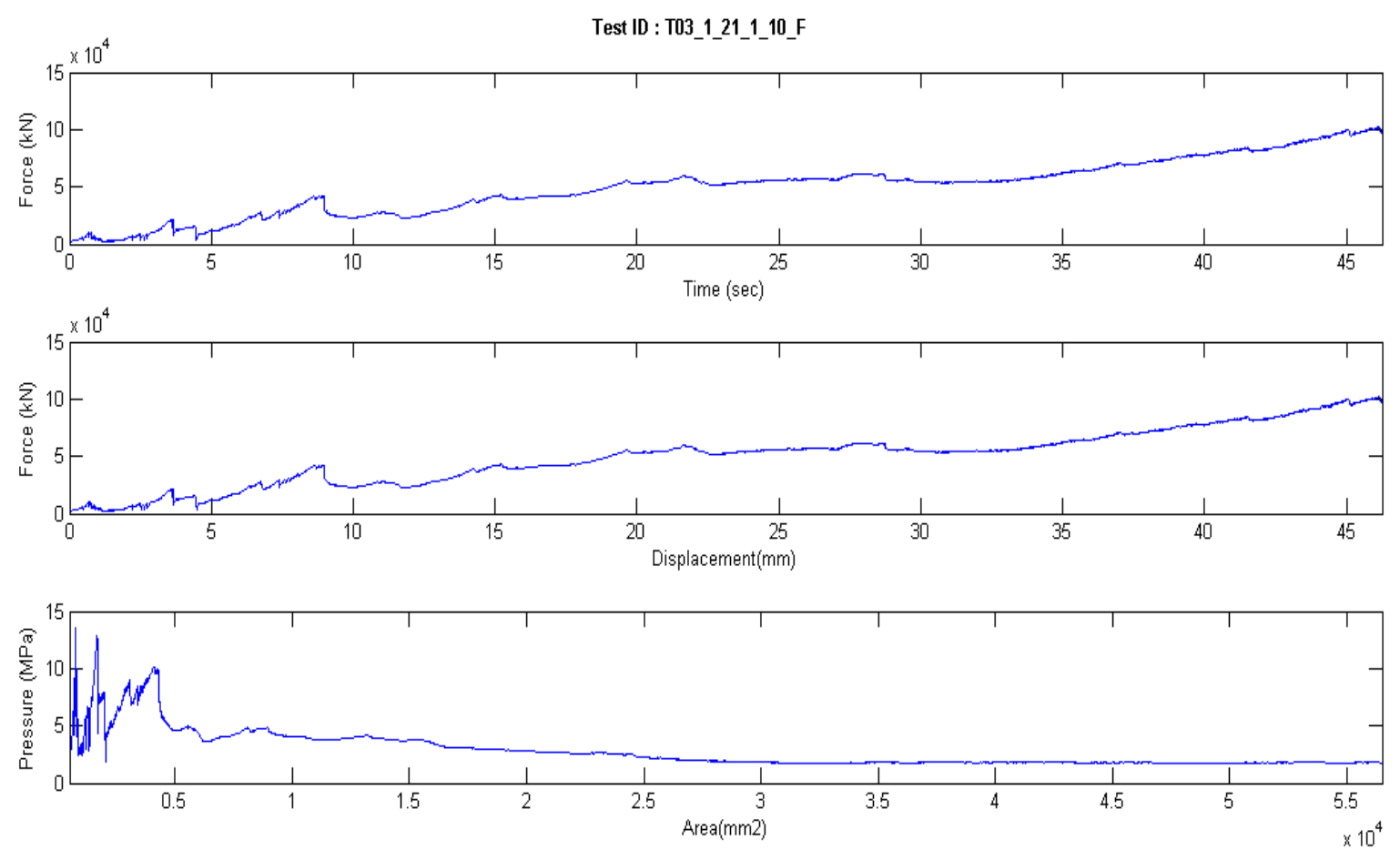

T03_1_21_1_10_F: Force and pressure traces

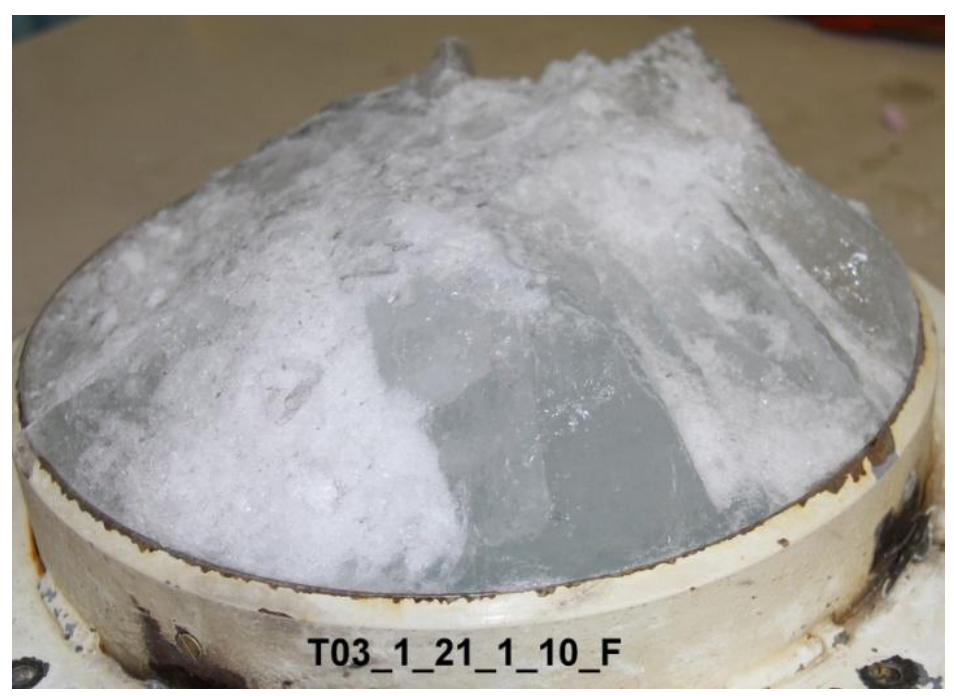

T03_1_21_1_10_F: After crushing picture 


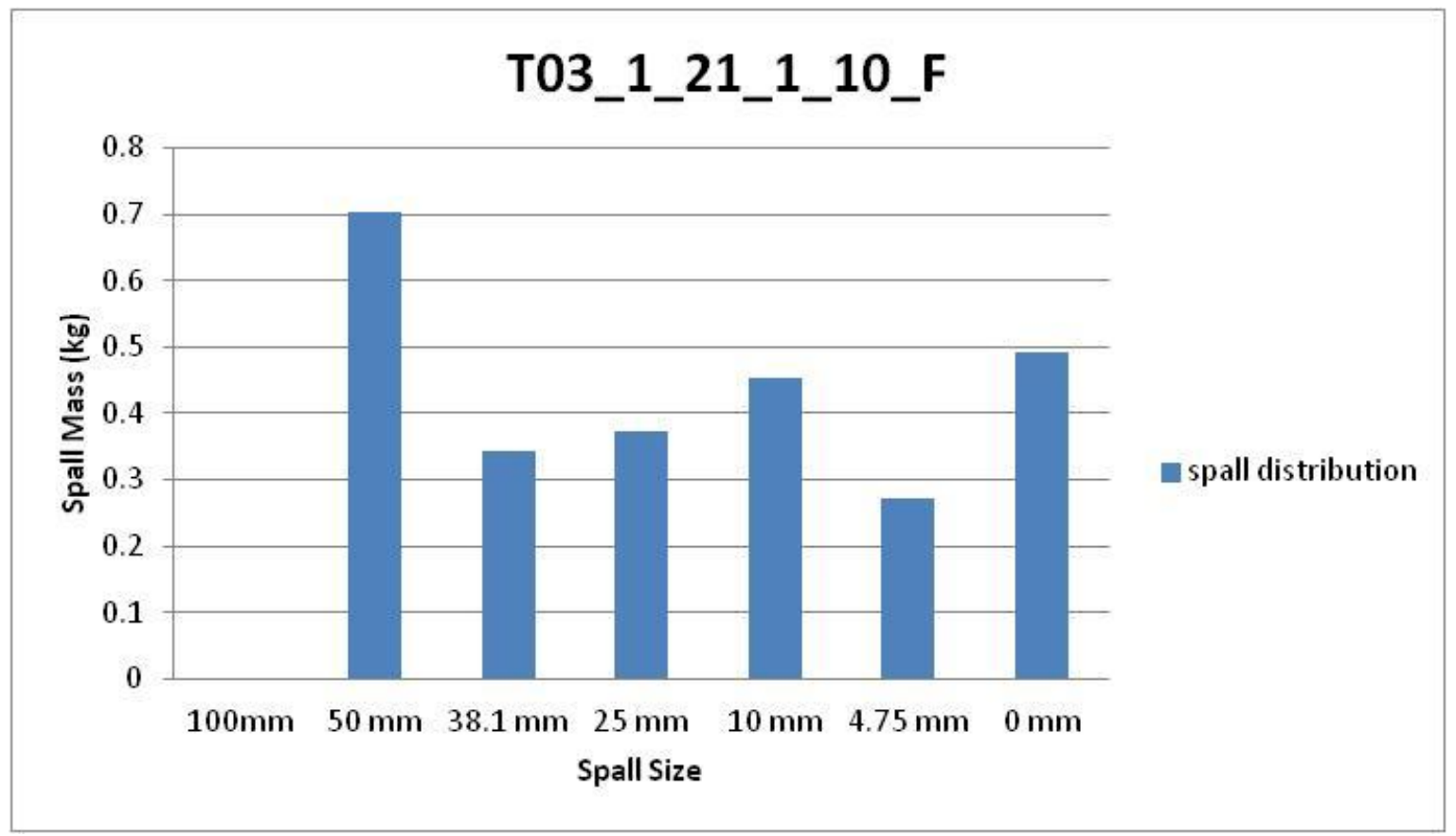

T03_1_21_1_10_F: Spall mass distributions
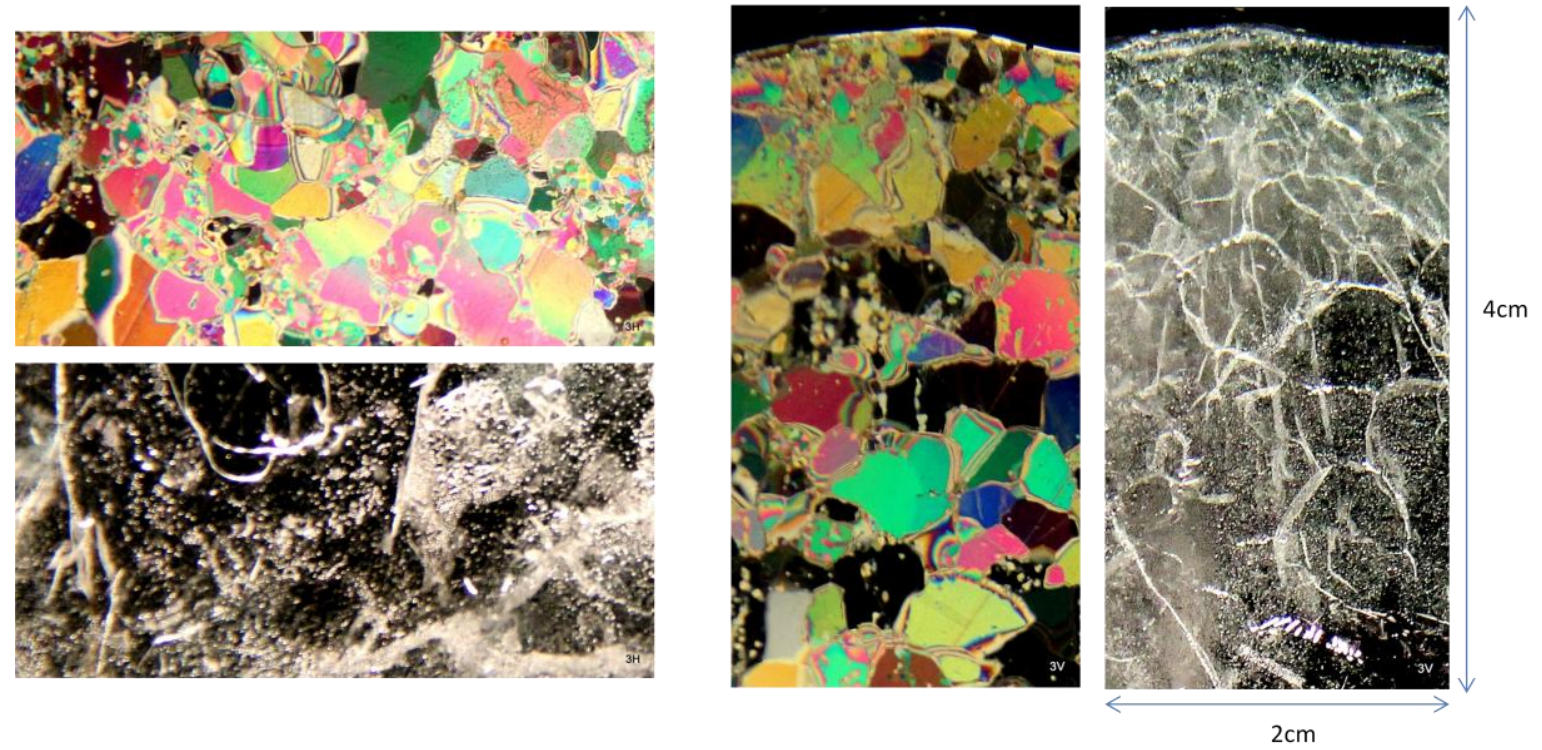

T03_1_21_1_10_F: Thin section pictures: Horizontal (left) and Vertical (right) 
Test ID : T04_10_21_1_10_F
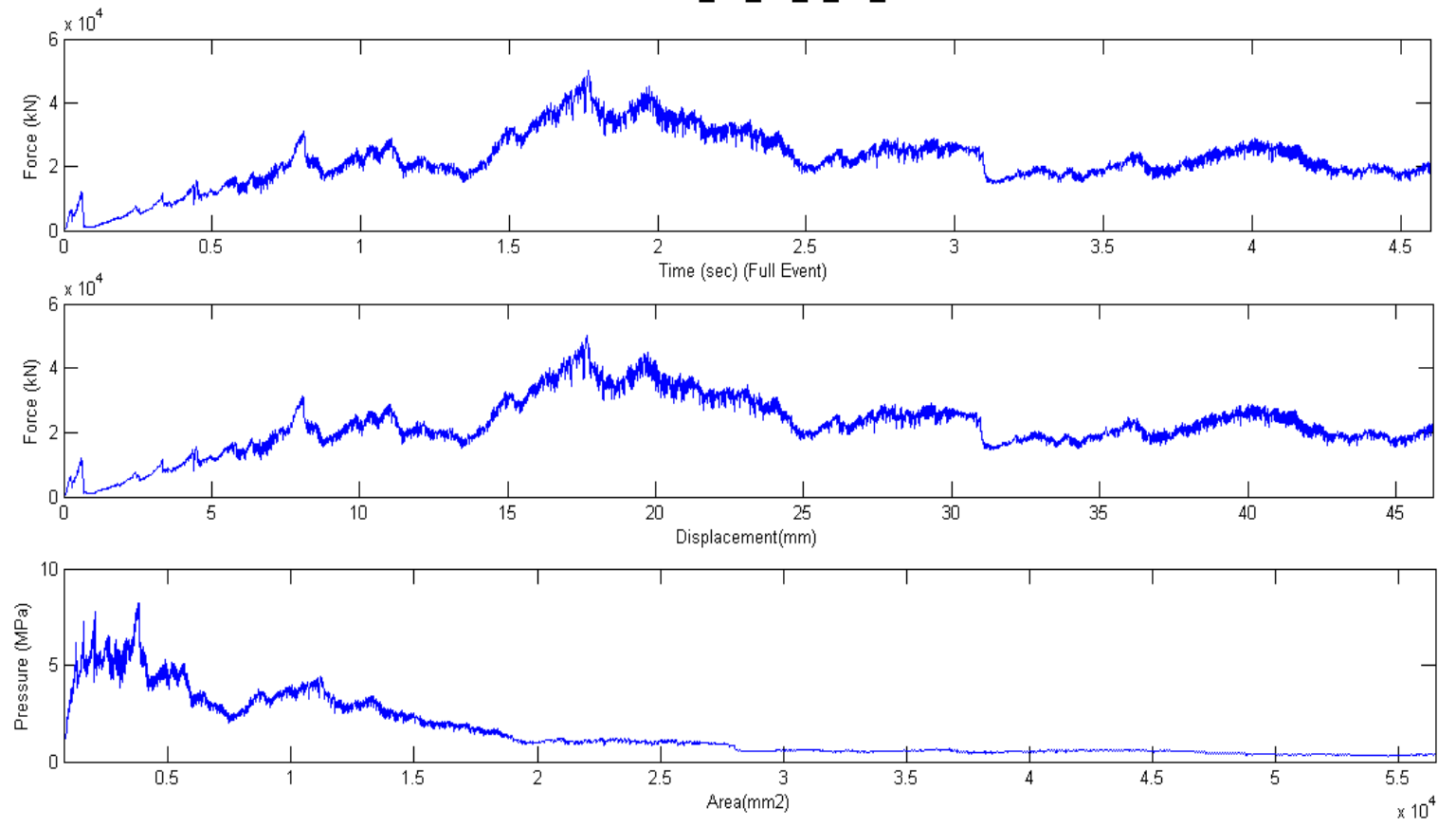

T04_10_21_1_10_F: Force and pressure traces

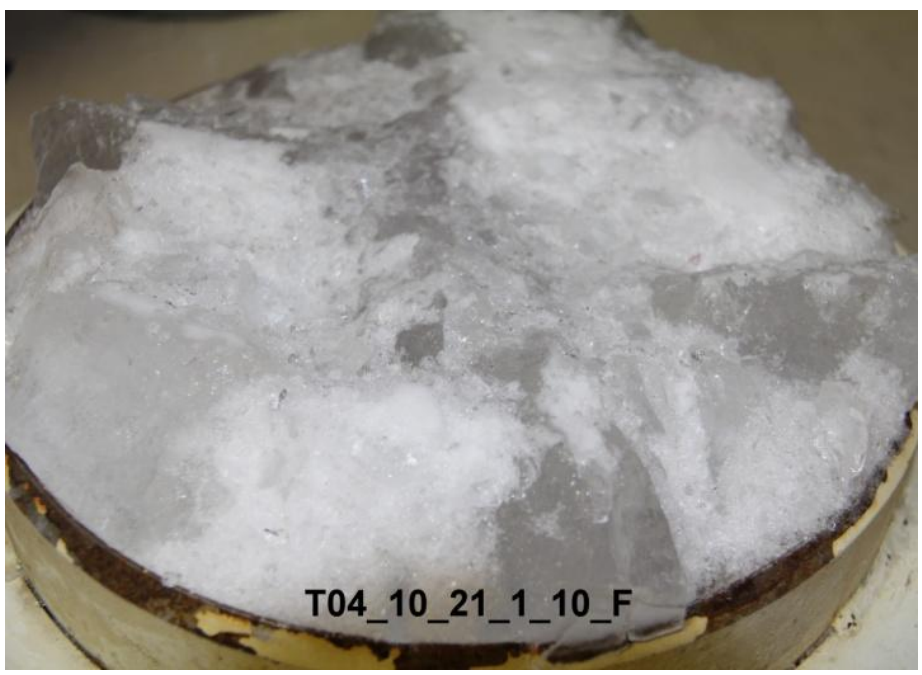

T04_10_21_1_10_F: After crushing picture 


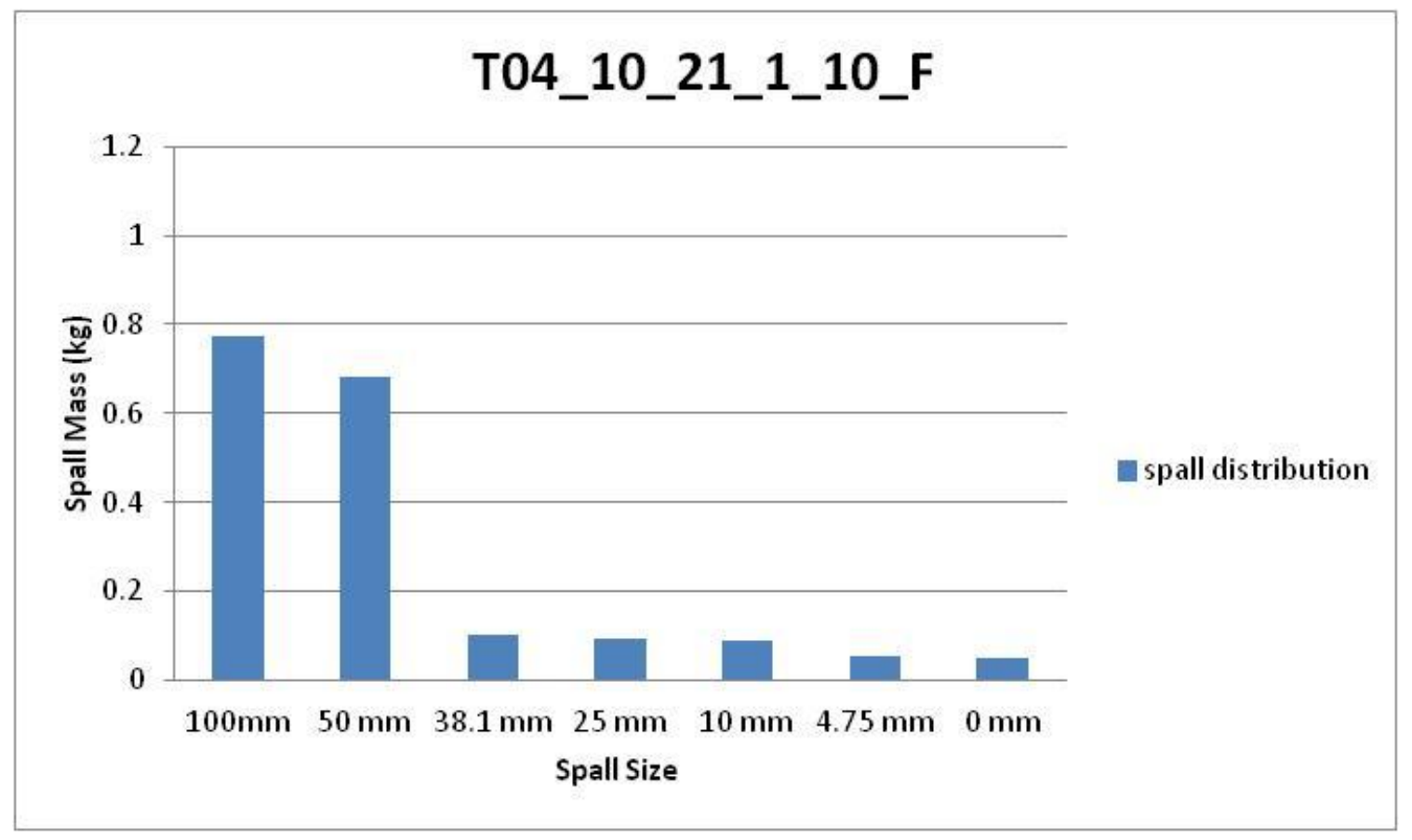

T04_10_21_1_10_F: Spall mass distributions
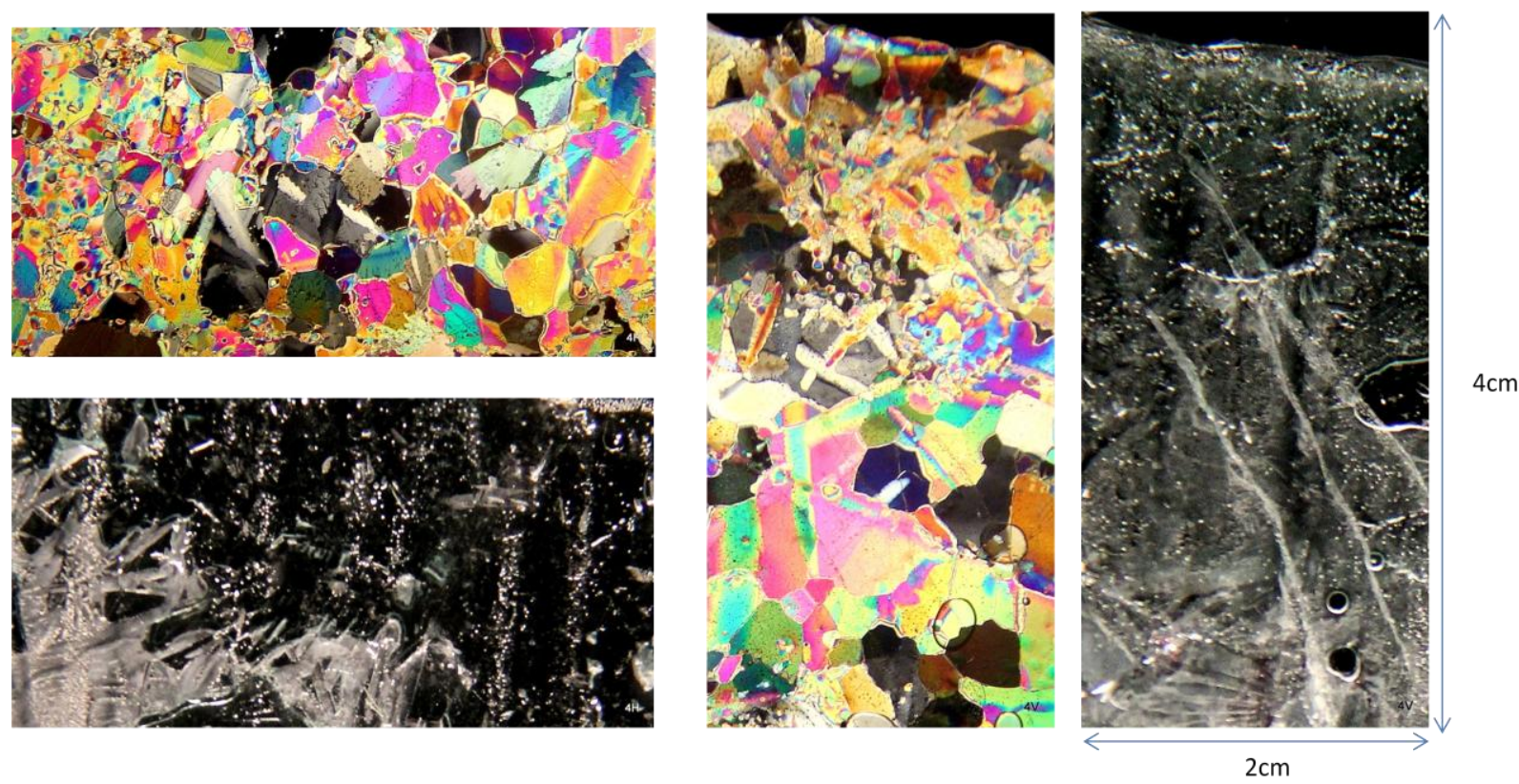

T04_10_21_1_10_F: Thin section pictures: Horizontal (left) and Vertical (right) 

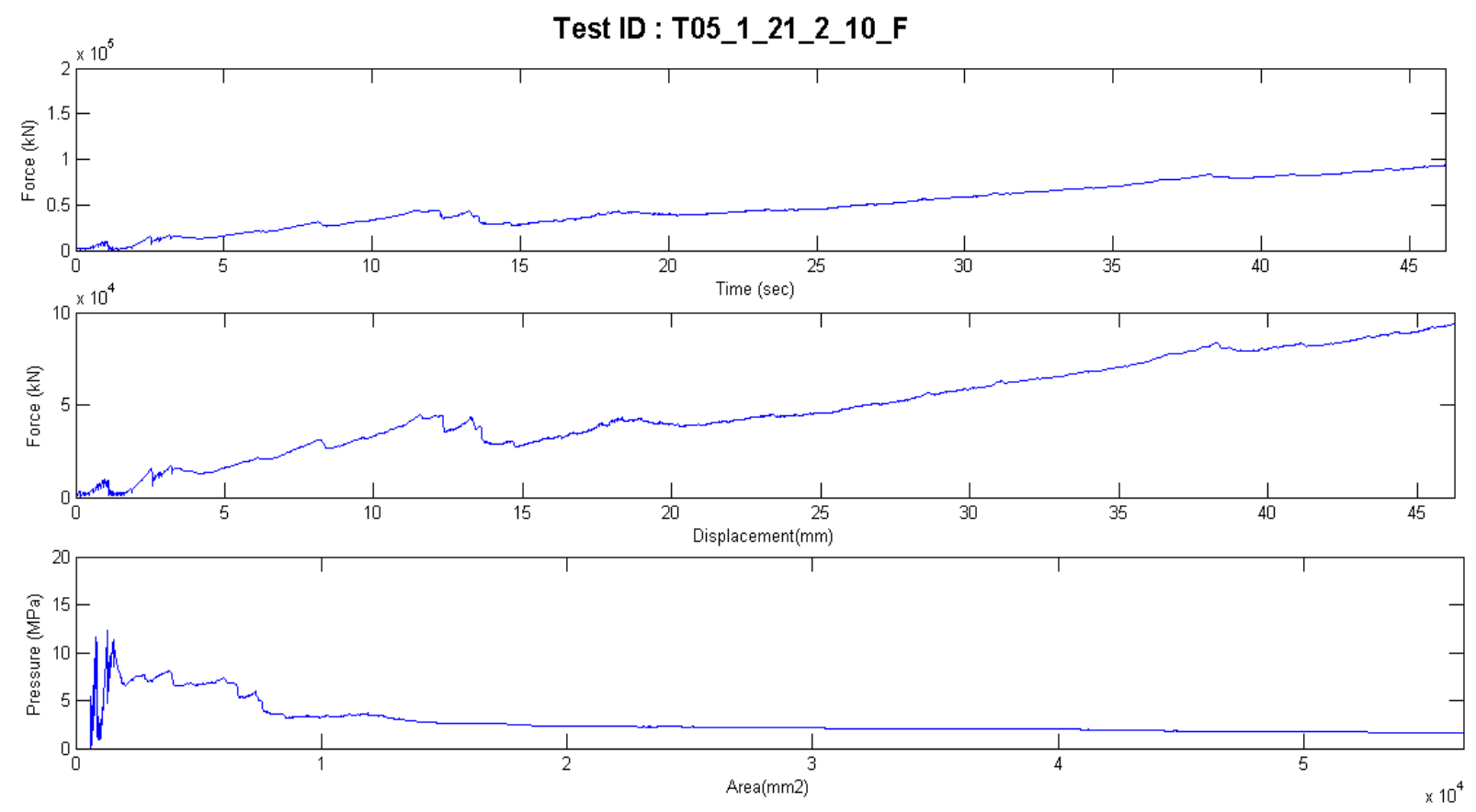

T05_1_21_2_10_F: Force and pressure traces

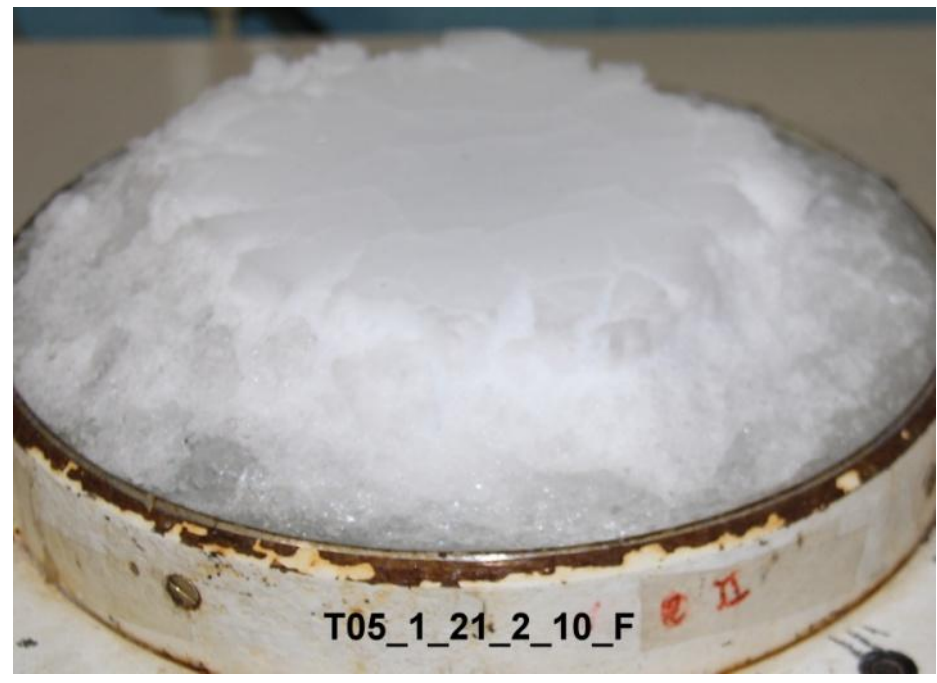

T05_1_21_2_10_F: After crushing picture 


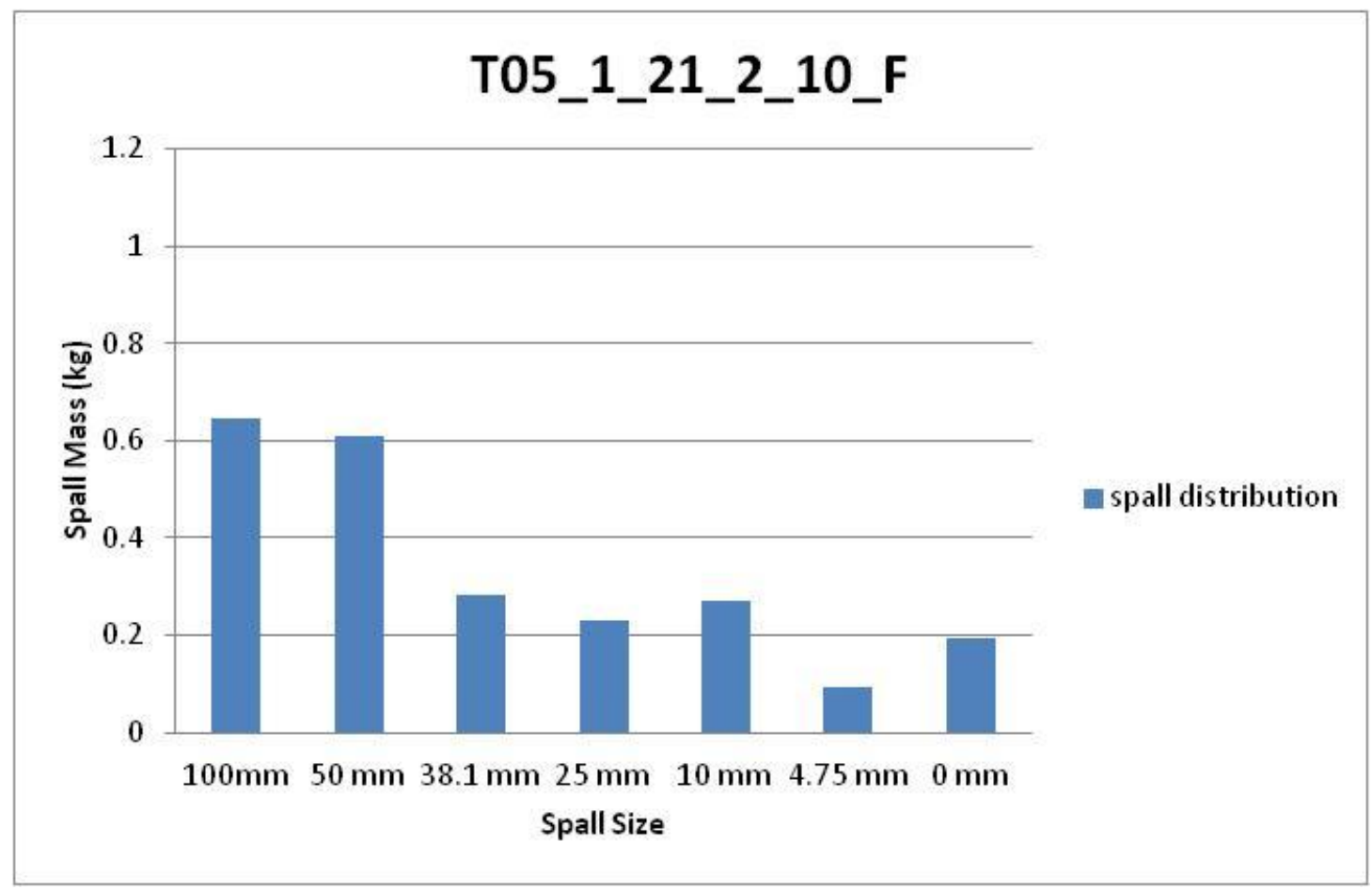

T05_1_21_2_10_F: Spall mass distributions
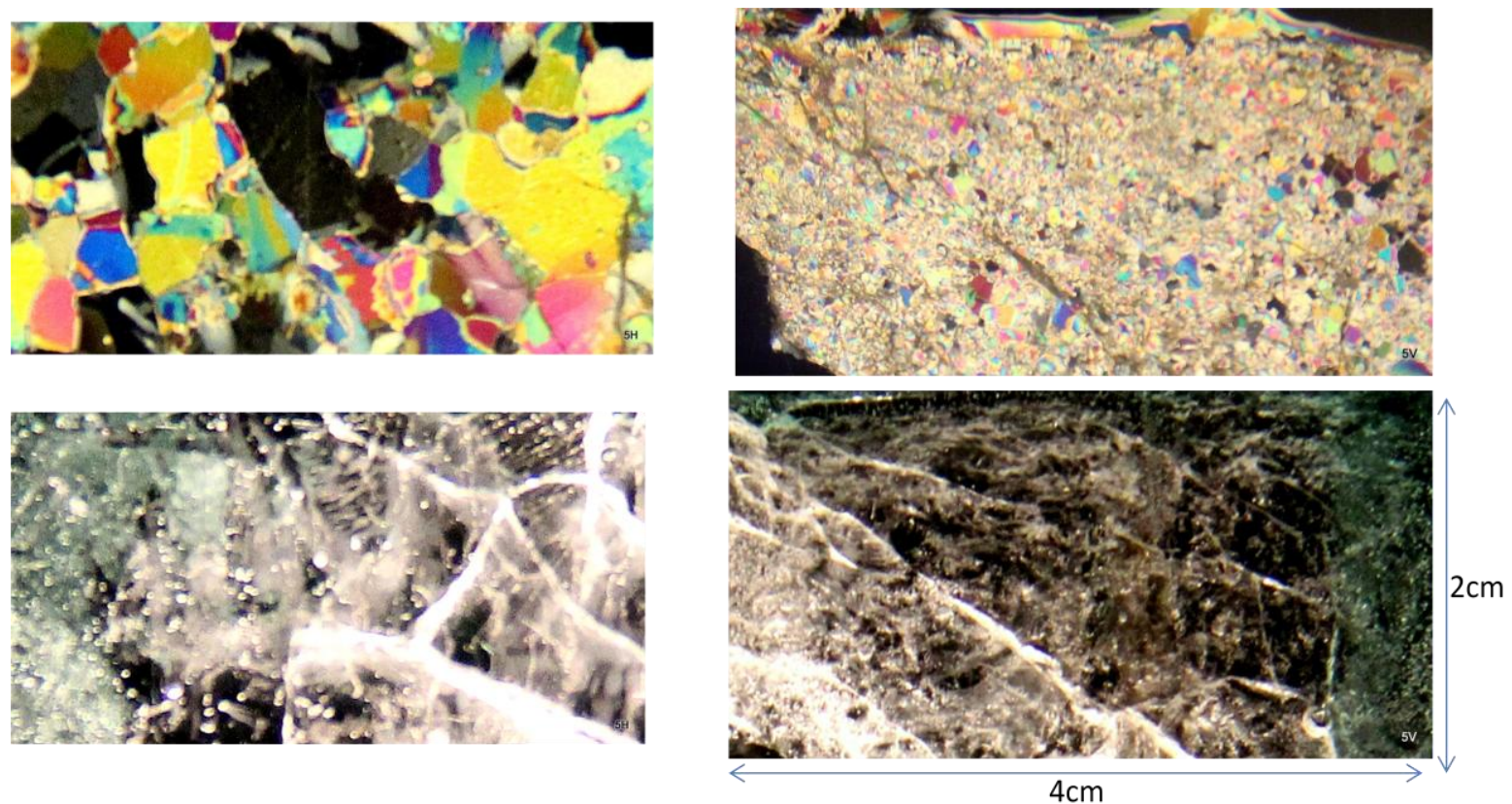

T05_1_21_2_10_F: Thin section pictures: Horizontal (left) and Vertical (right) 

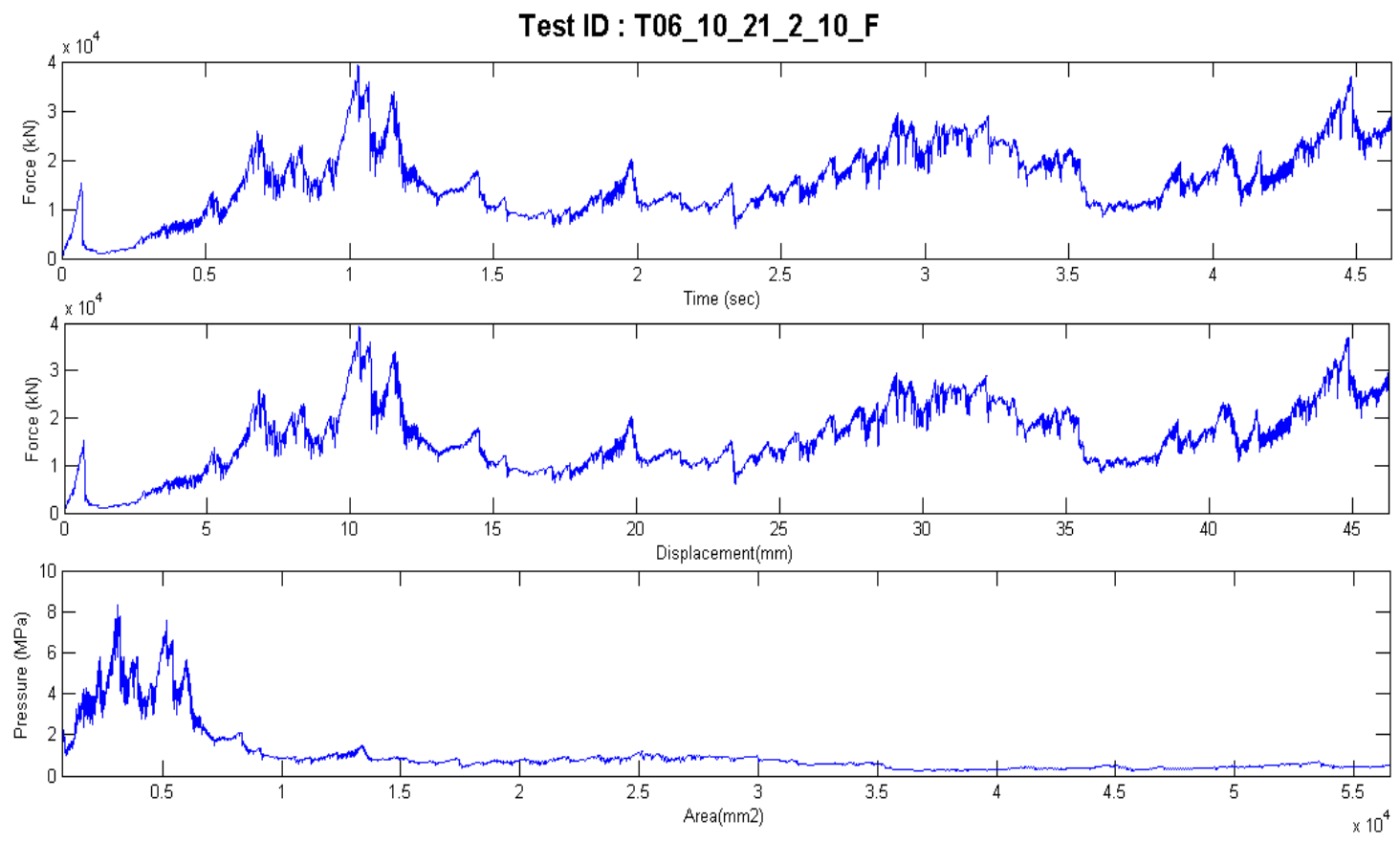

T06_10_21_2_10_F: Force and pressure traces

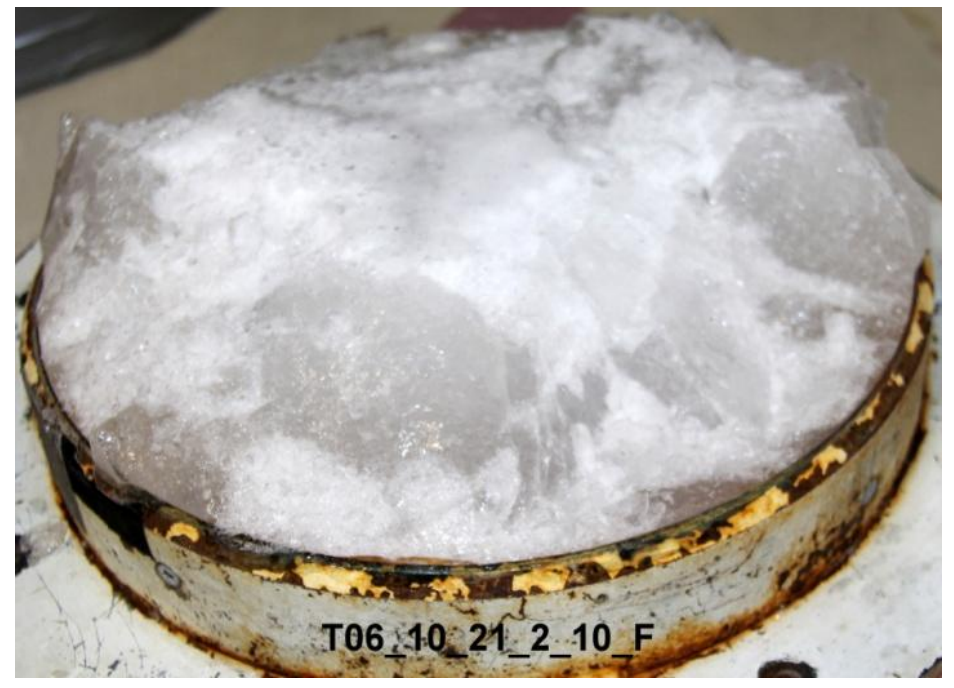

T06_10_21_2_10_F: After crushing picture 


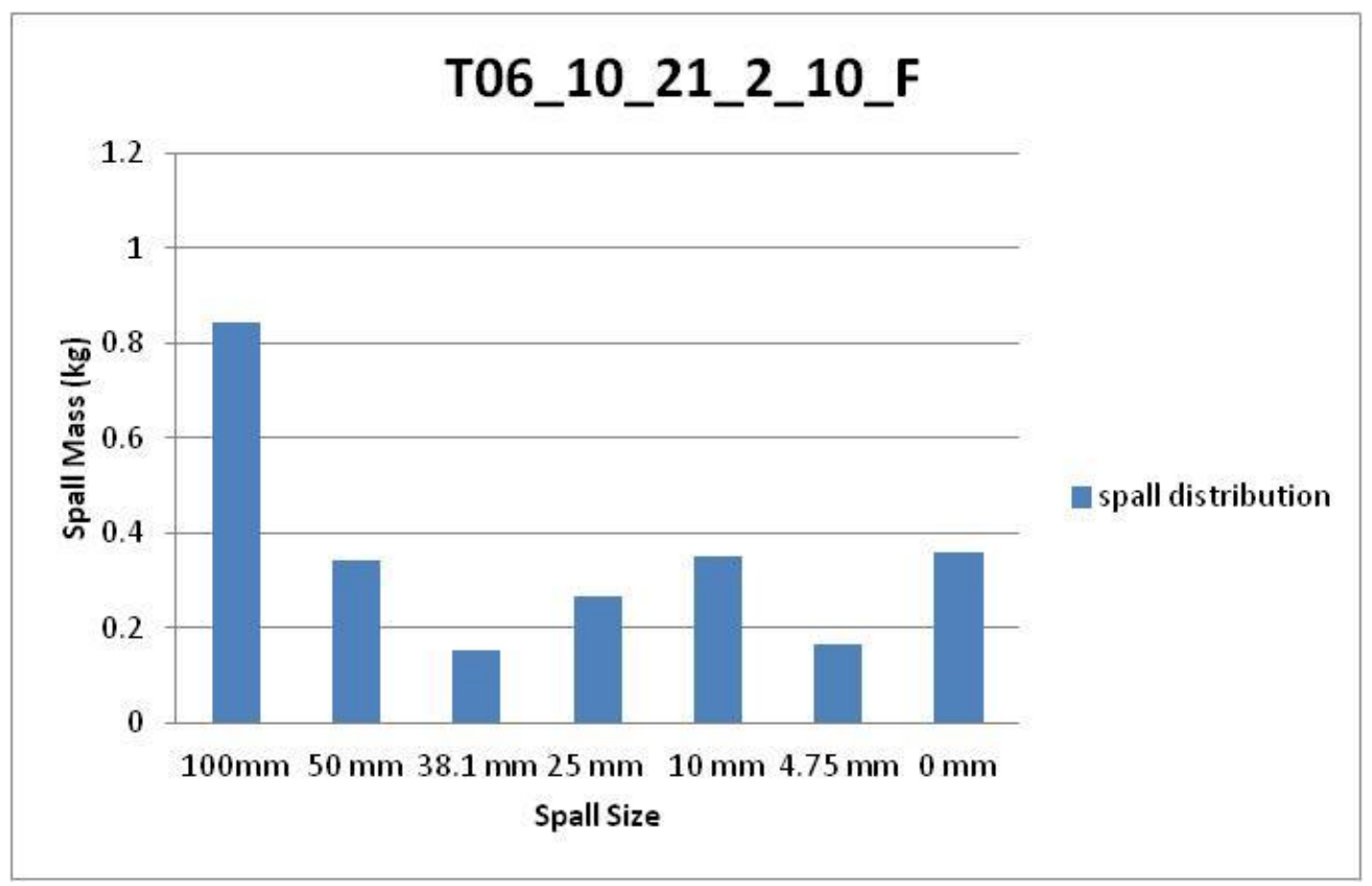

T06_10_21_2_10_F: Spall mass distributions
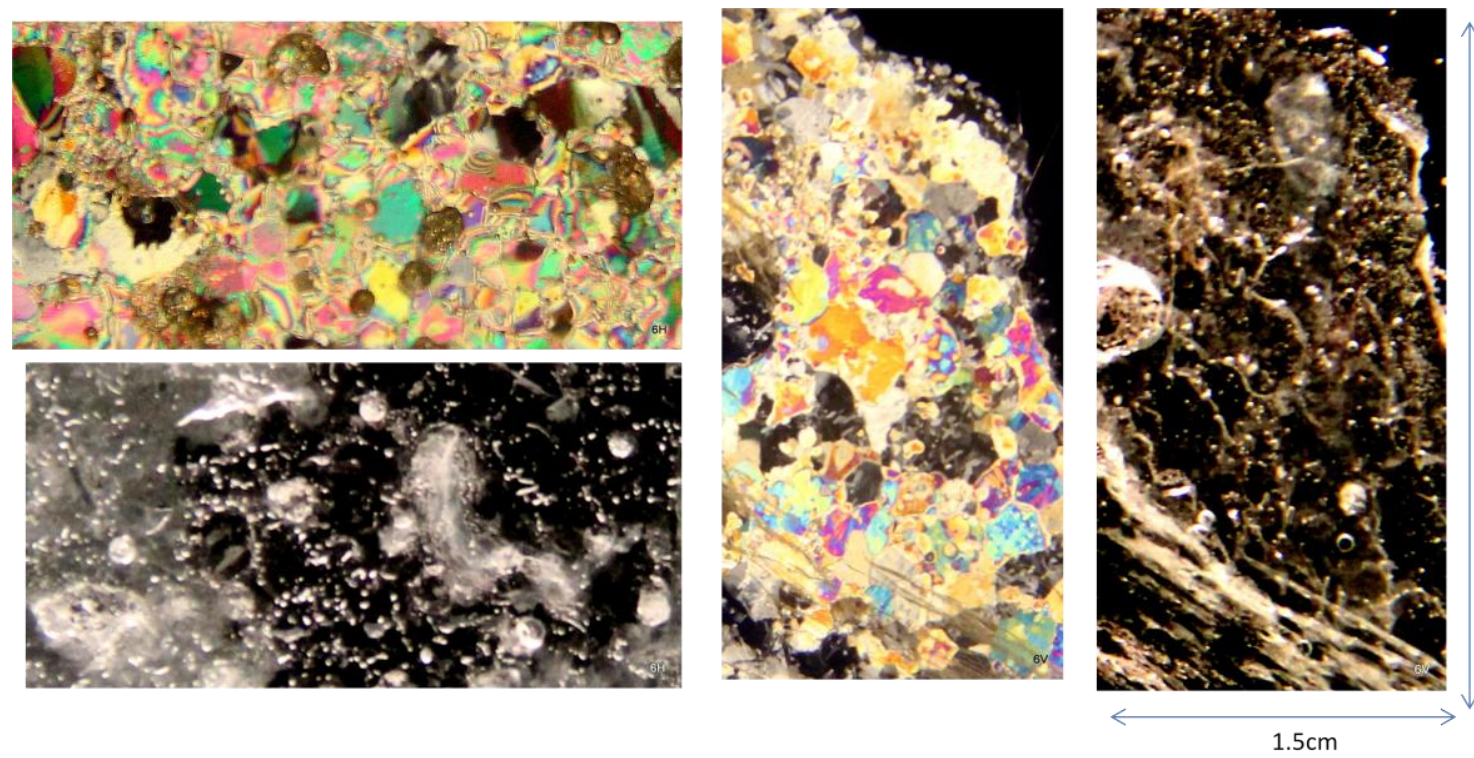

T06_10_21_2_10_F: Thin section pictures: Horizontal (left) and Vertical (right) 
Test ID : T07_10_21_2_10_S
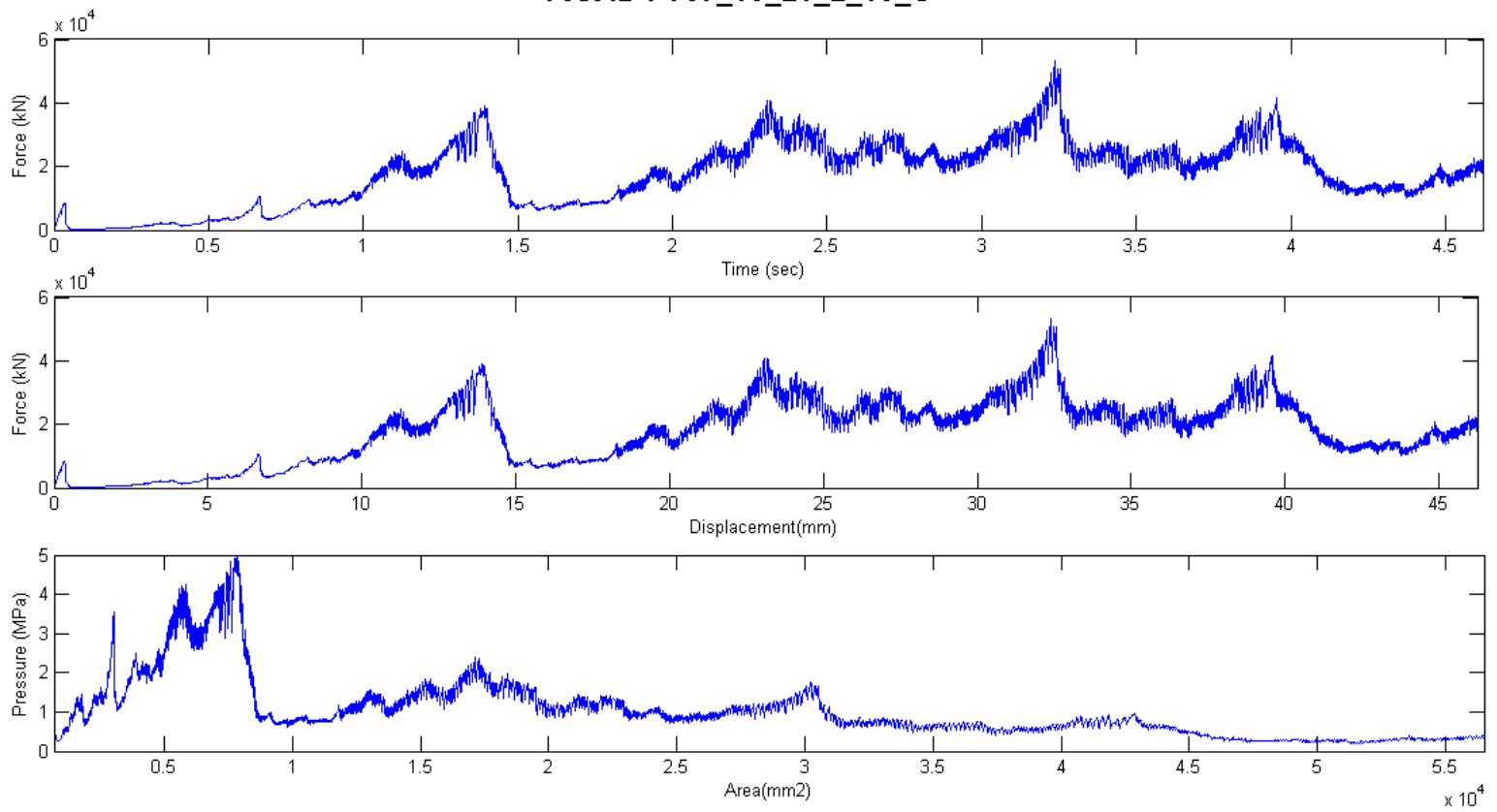

T07_10_21_2_10_S: Force and pressure traces

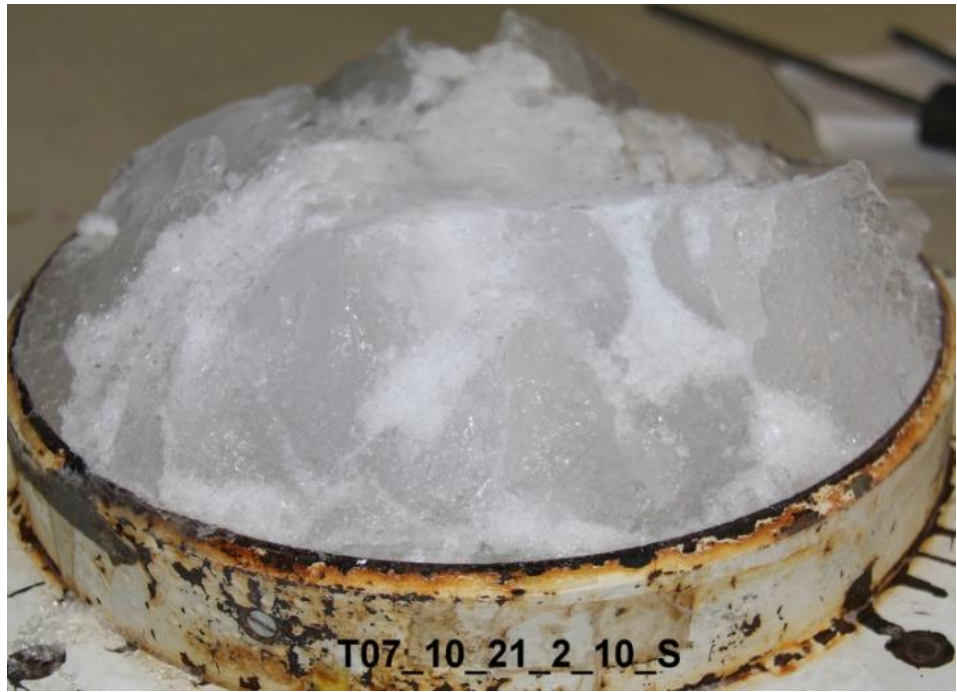

T07_10_21_2_10_S: After crushing picture 


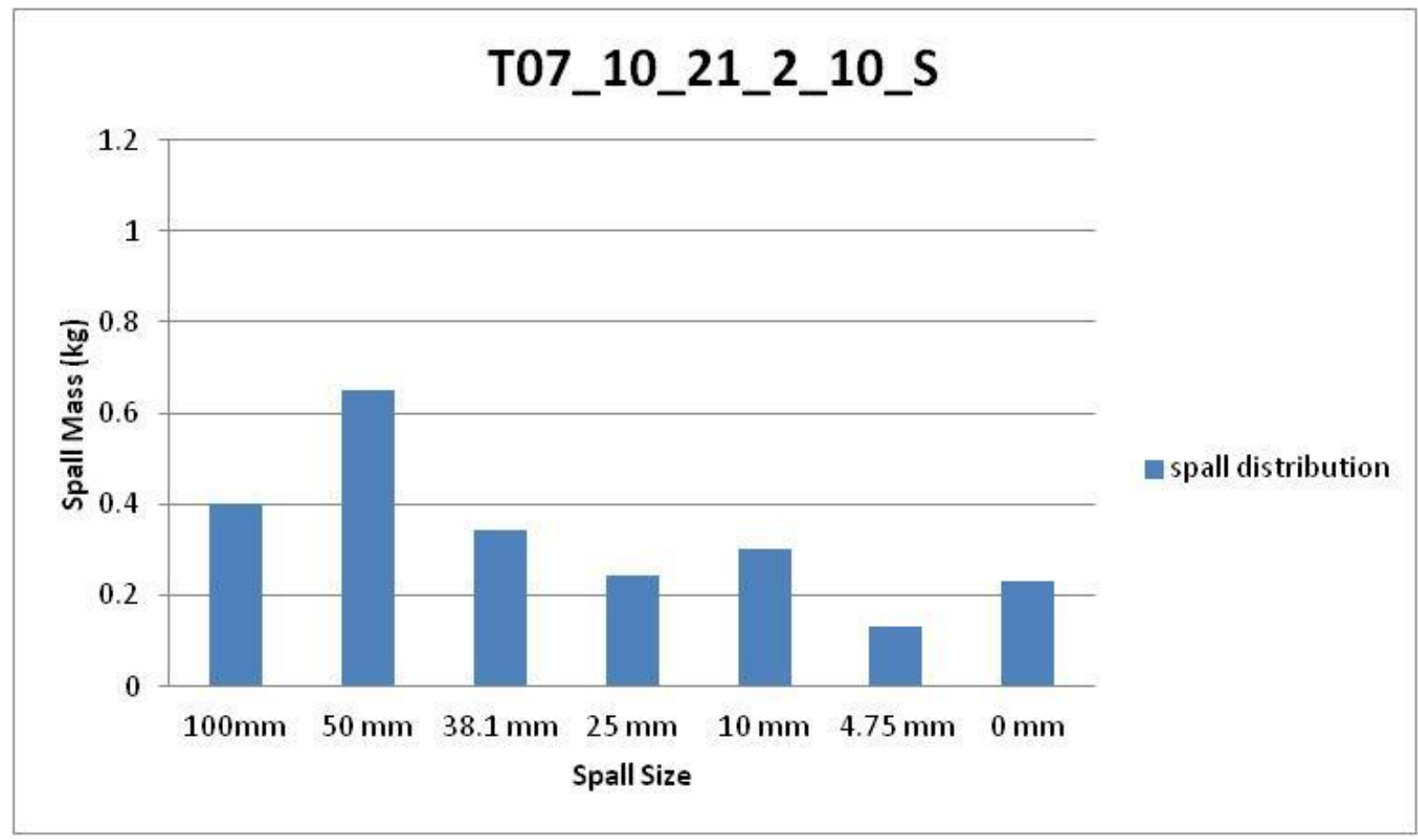

T07_10_21_2_10_S: Spall mass distributions
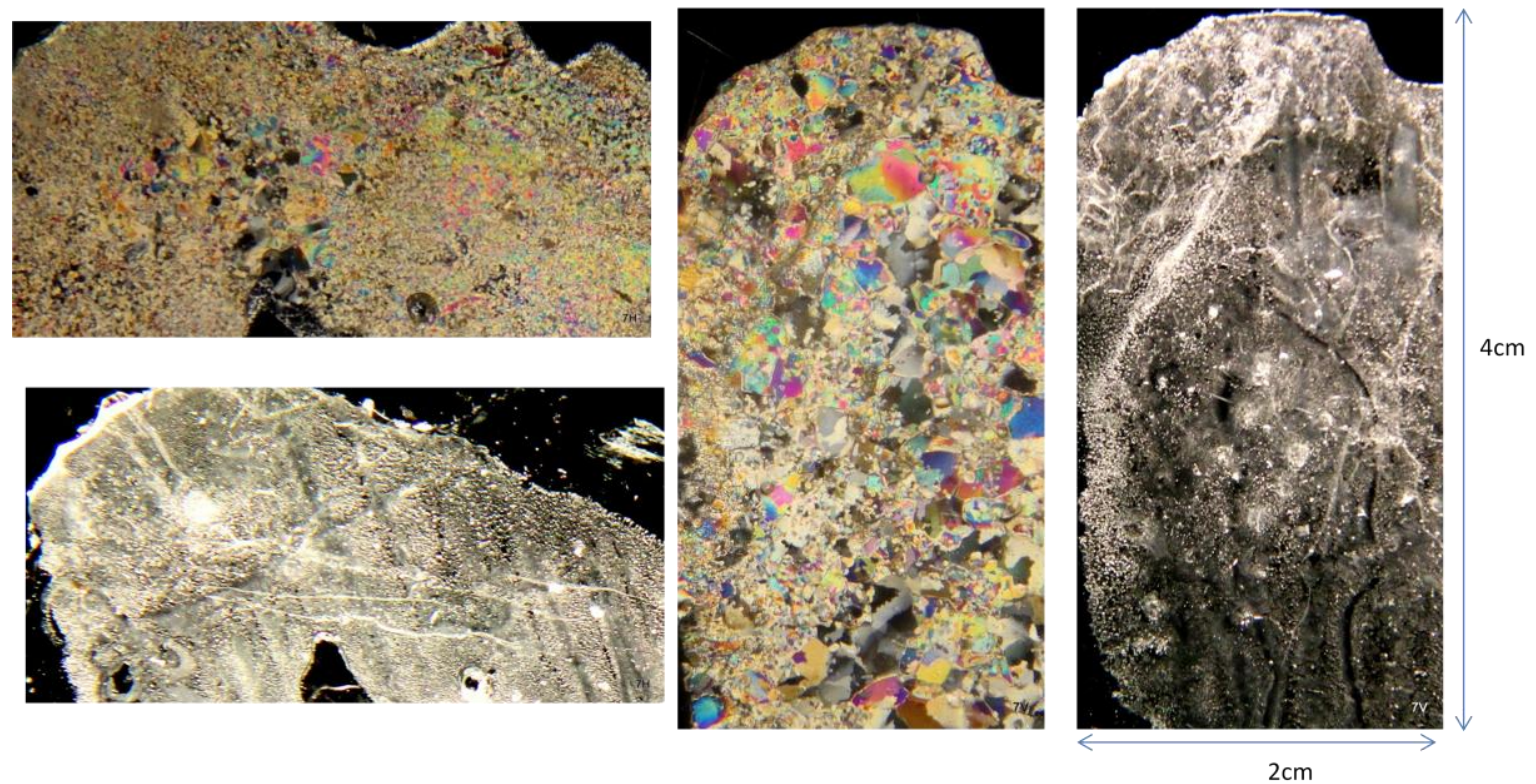

T07_10_21_2_10_S: Thin section pictures: Horizontal (left) and Vertical (right) 
Test ID : T08_10_21_1_10_S
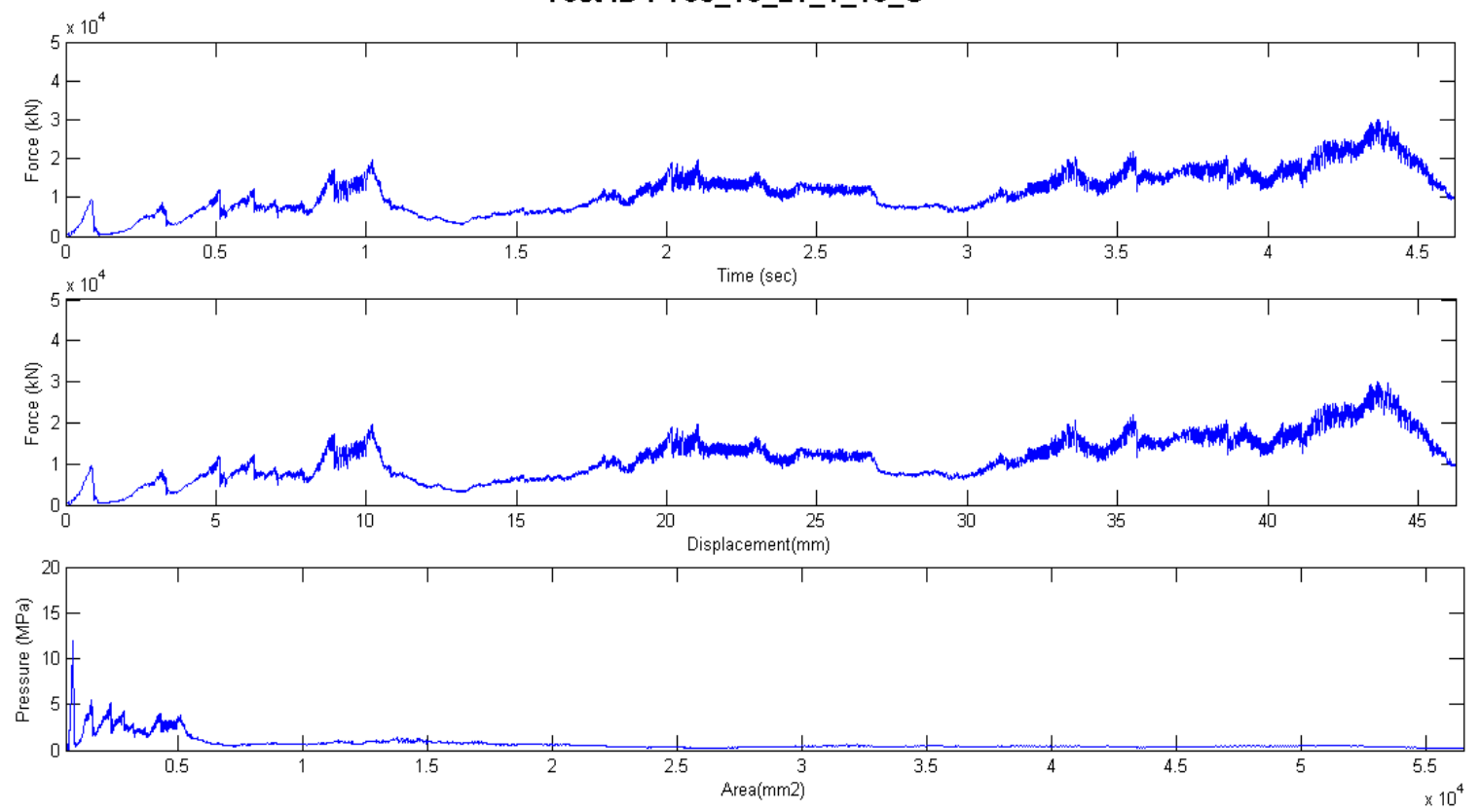

T08_10_21_1_10_S: Force and pressure traces

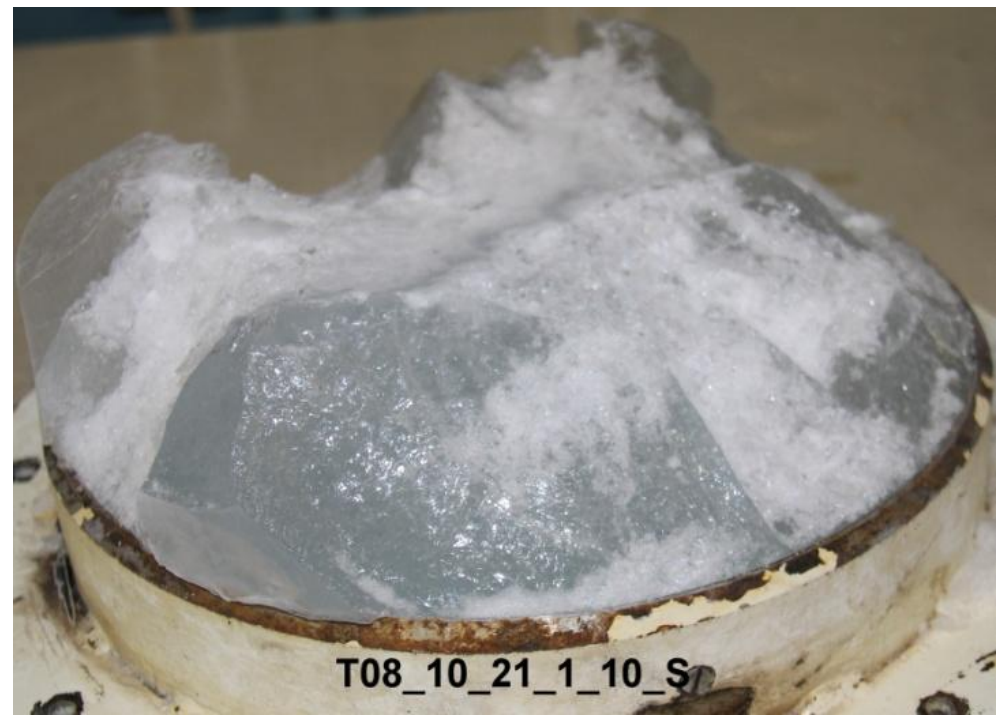

T08_10_21_1_10_S: After crushing picture 


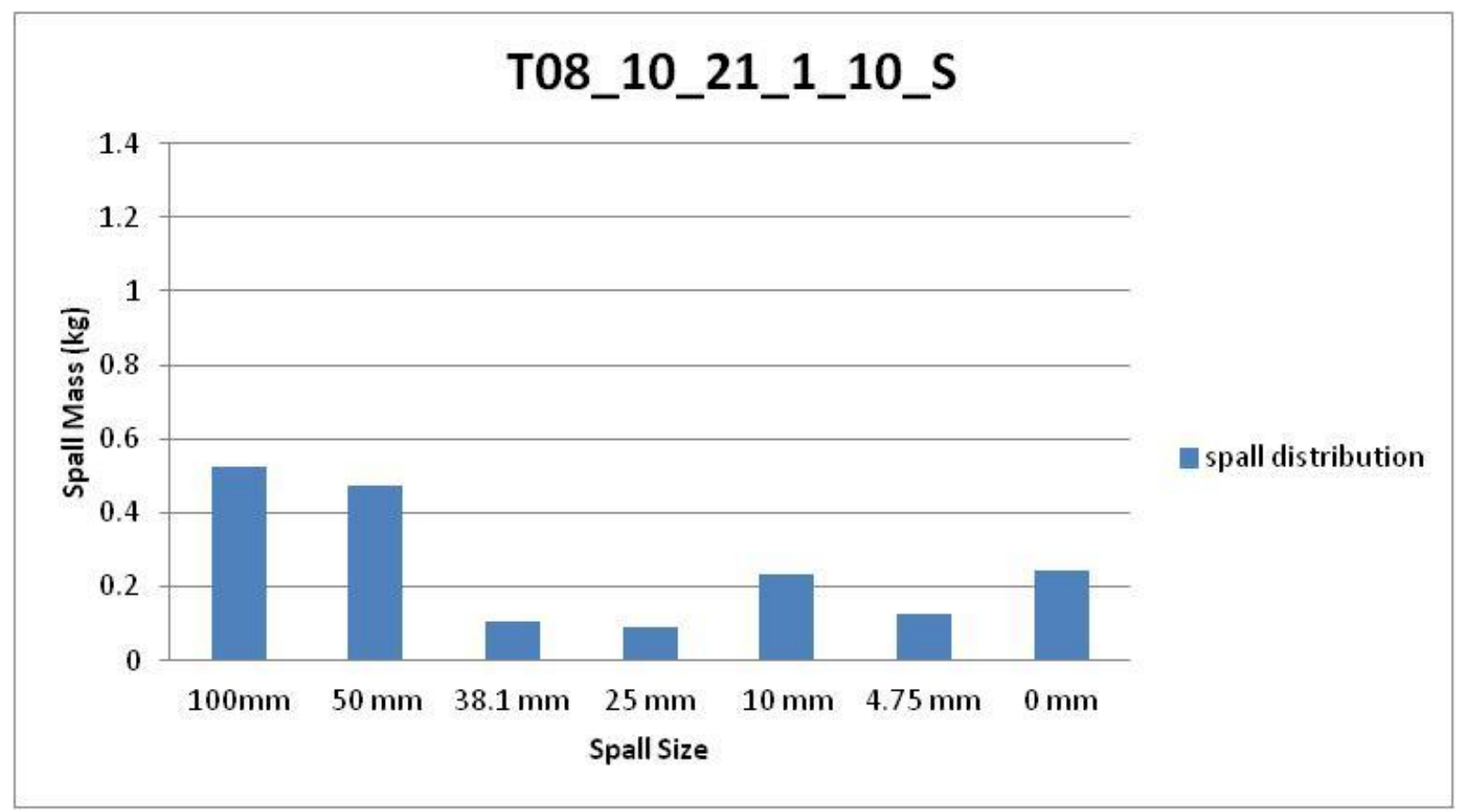

T08_10_21_1_10_S: Spall mass distributions
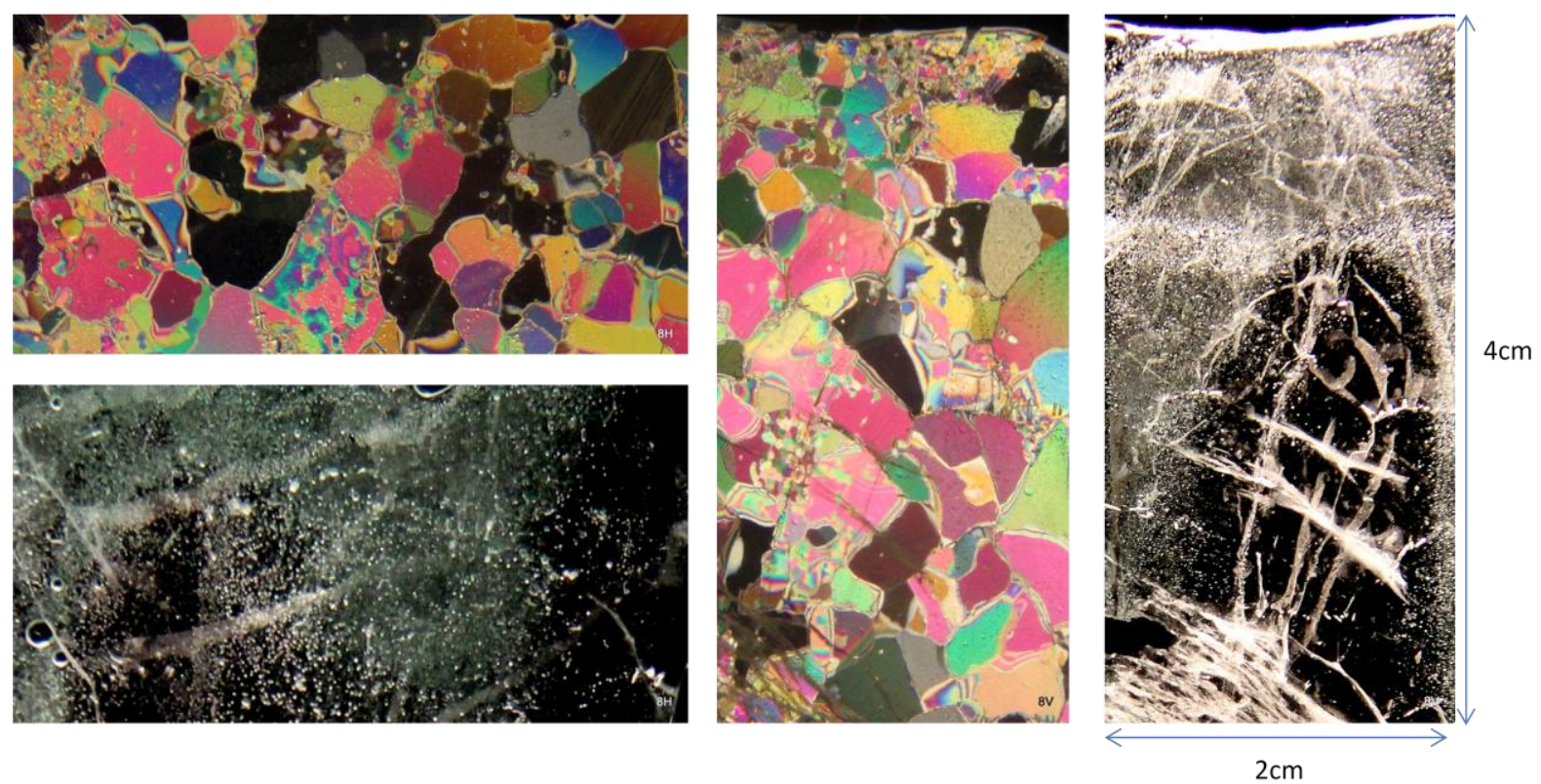

T08_10_21_1_10_S: Thin section pictures: Horizontal (left) and Vertical (right) 
Test ID : T09_10_30_1_10_S
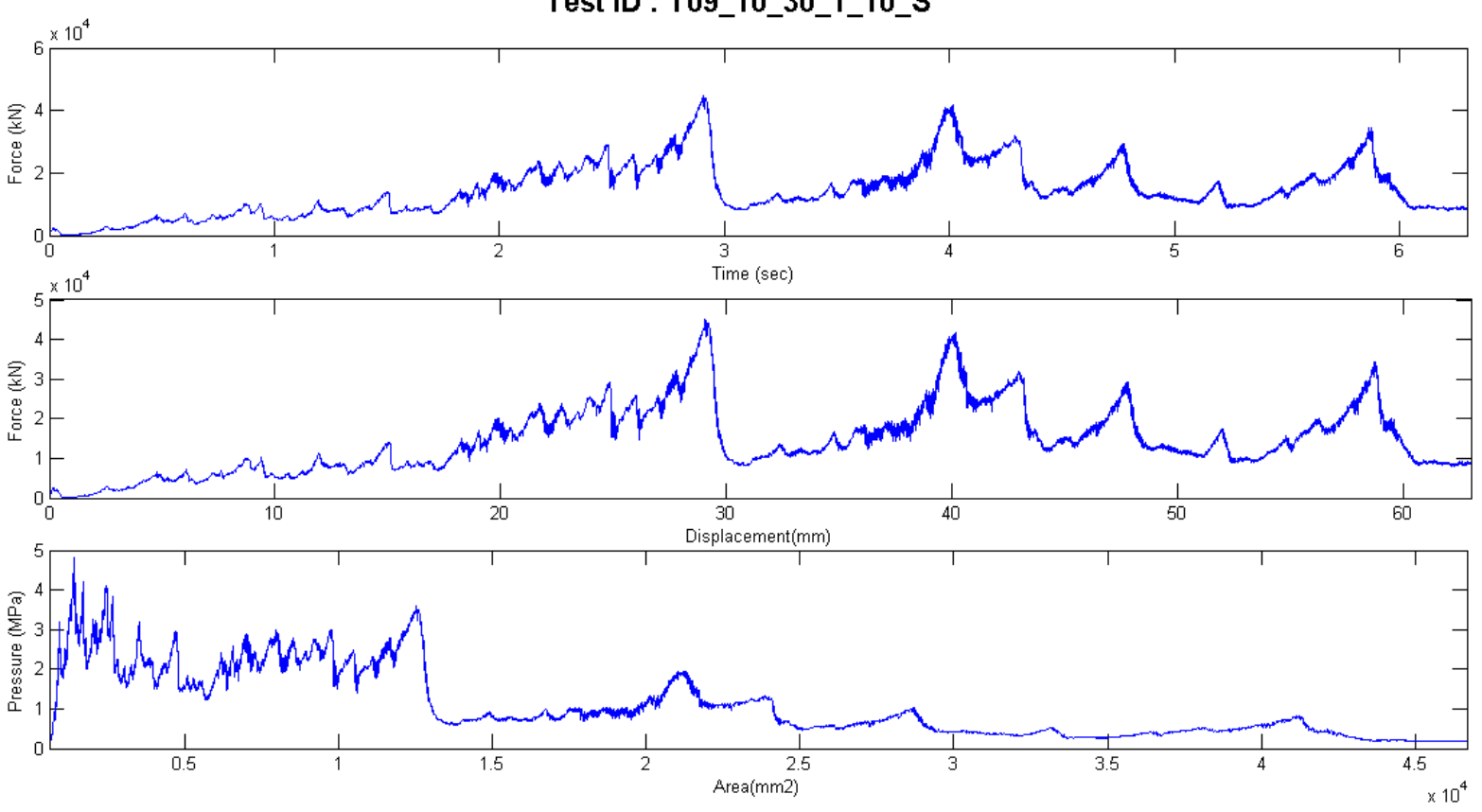

T09_10_30_1_10_S: Force and pressure traces

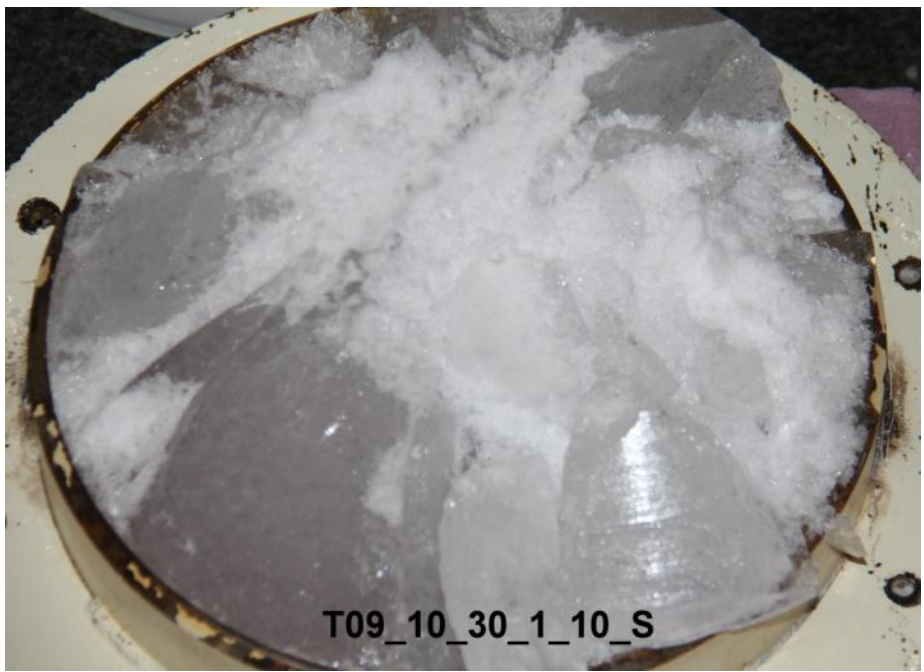

T09_10_30_1_10_S: After crushing picture 


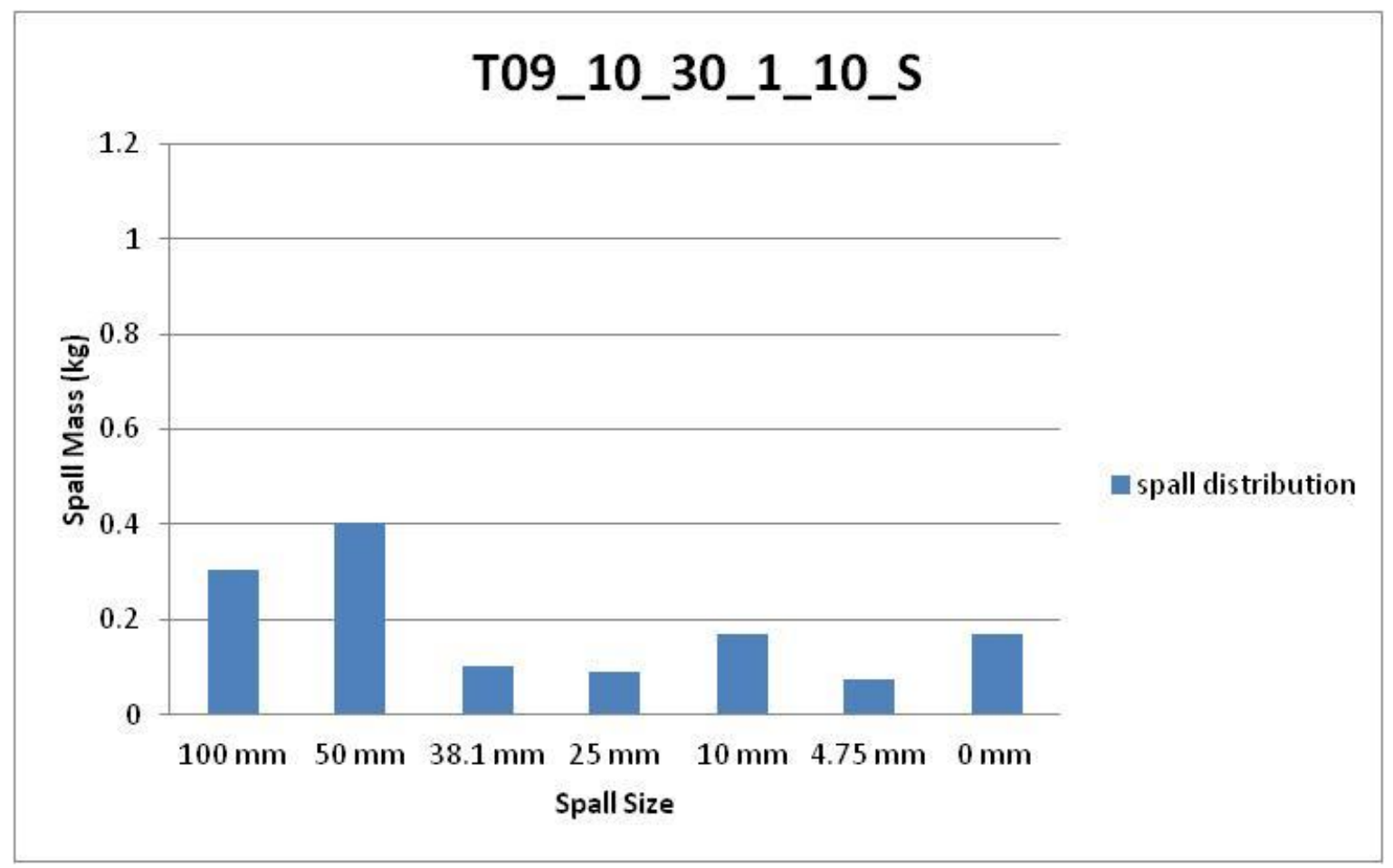

T09_10_30_1_10_S: Spall mass distributions
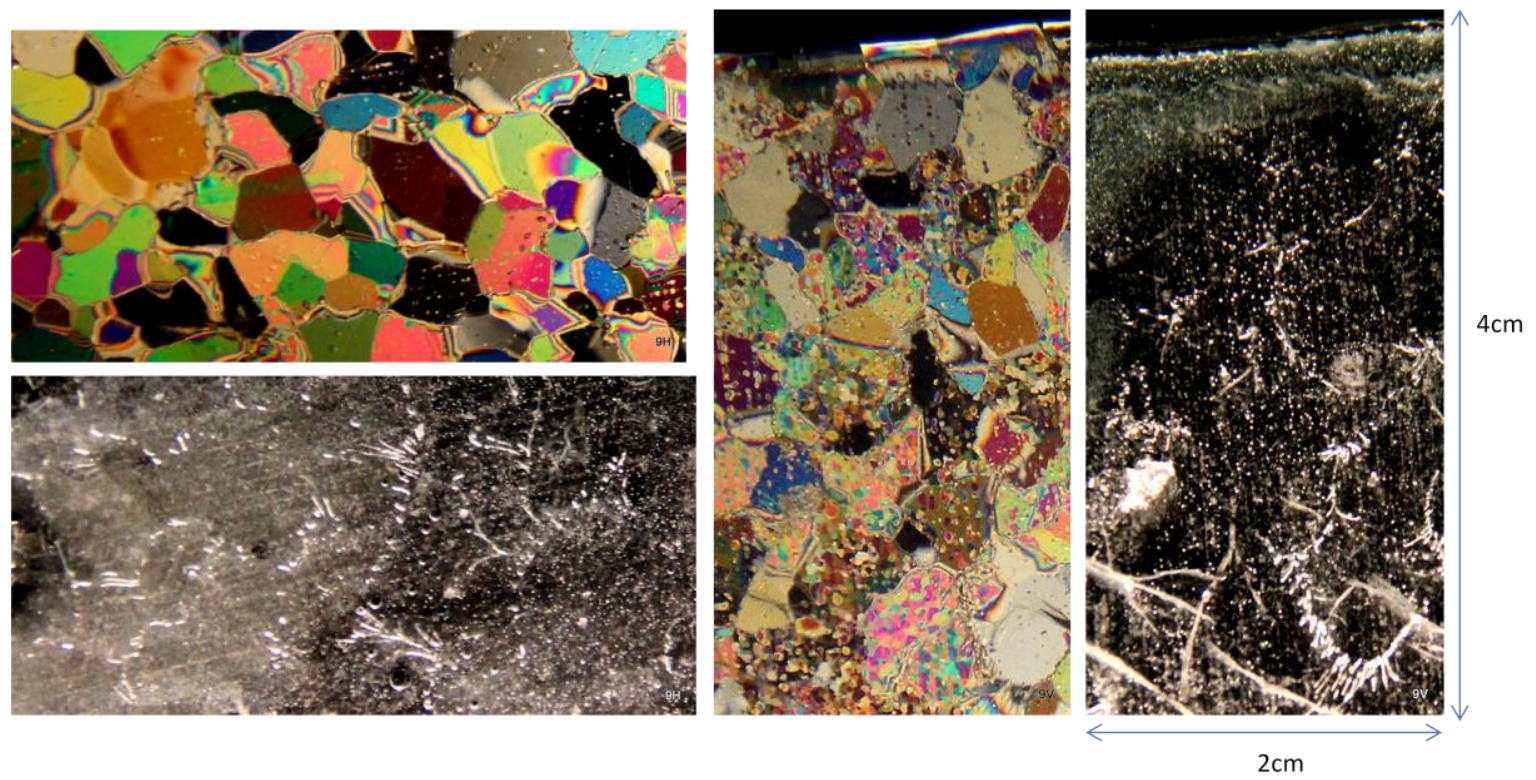

T09_10_30_1_10_S: Thin section pictures: Horizontal (left) and Vertical (right) 
Test ID : T10_10_30_2_10_S
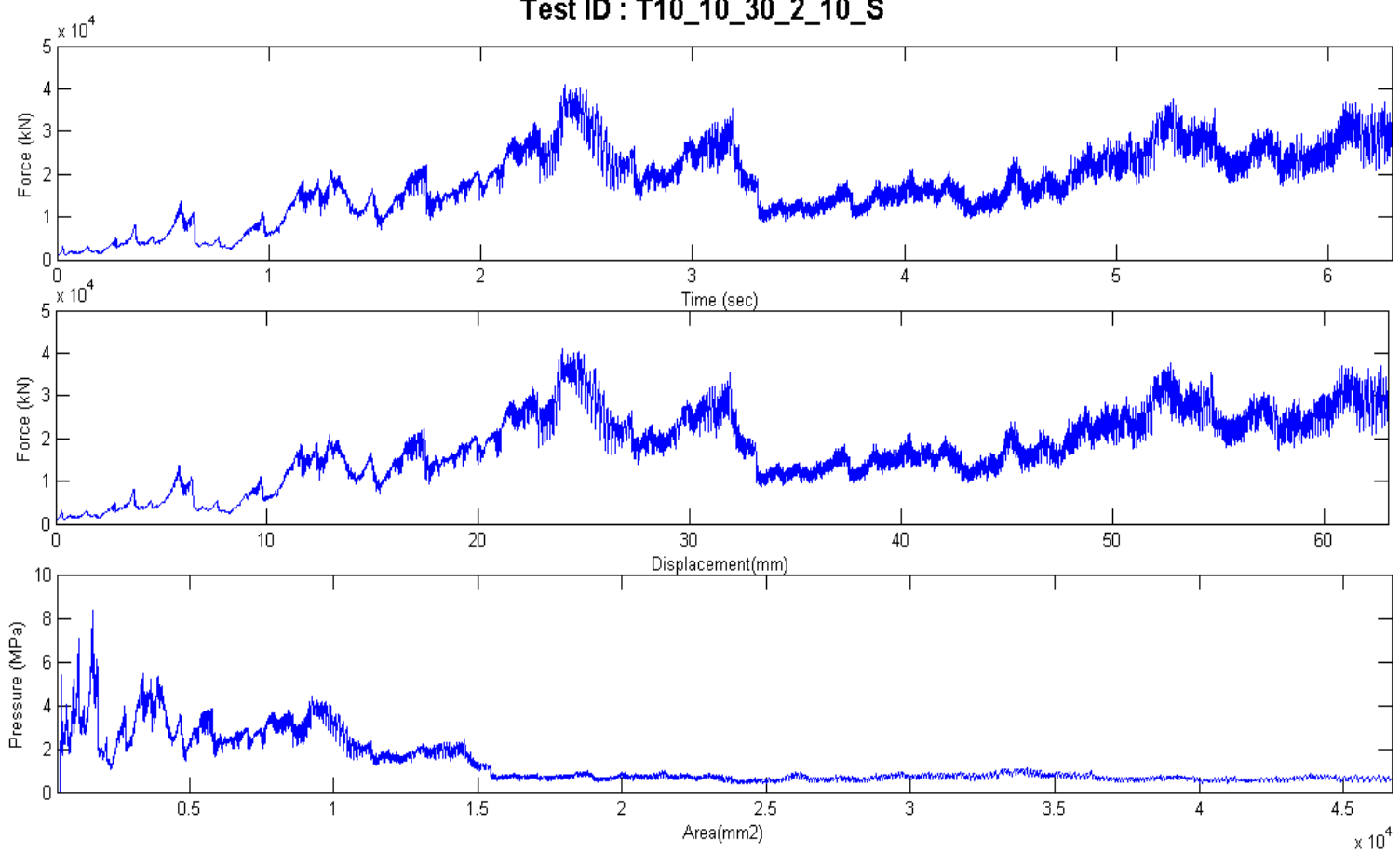

T10_10_30_2_10_S: Force and pressure traces

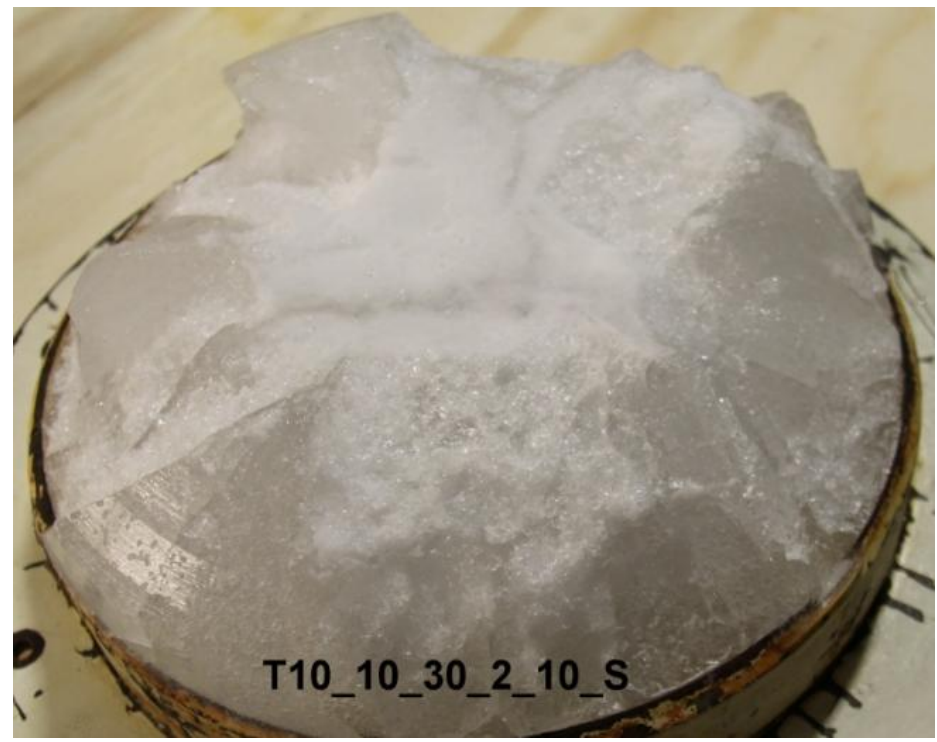

T10_10_30_2_10_S: After crushing picture 


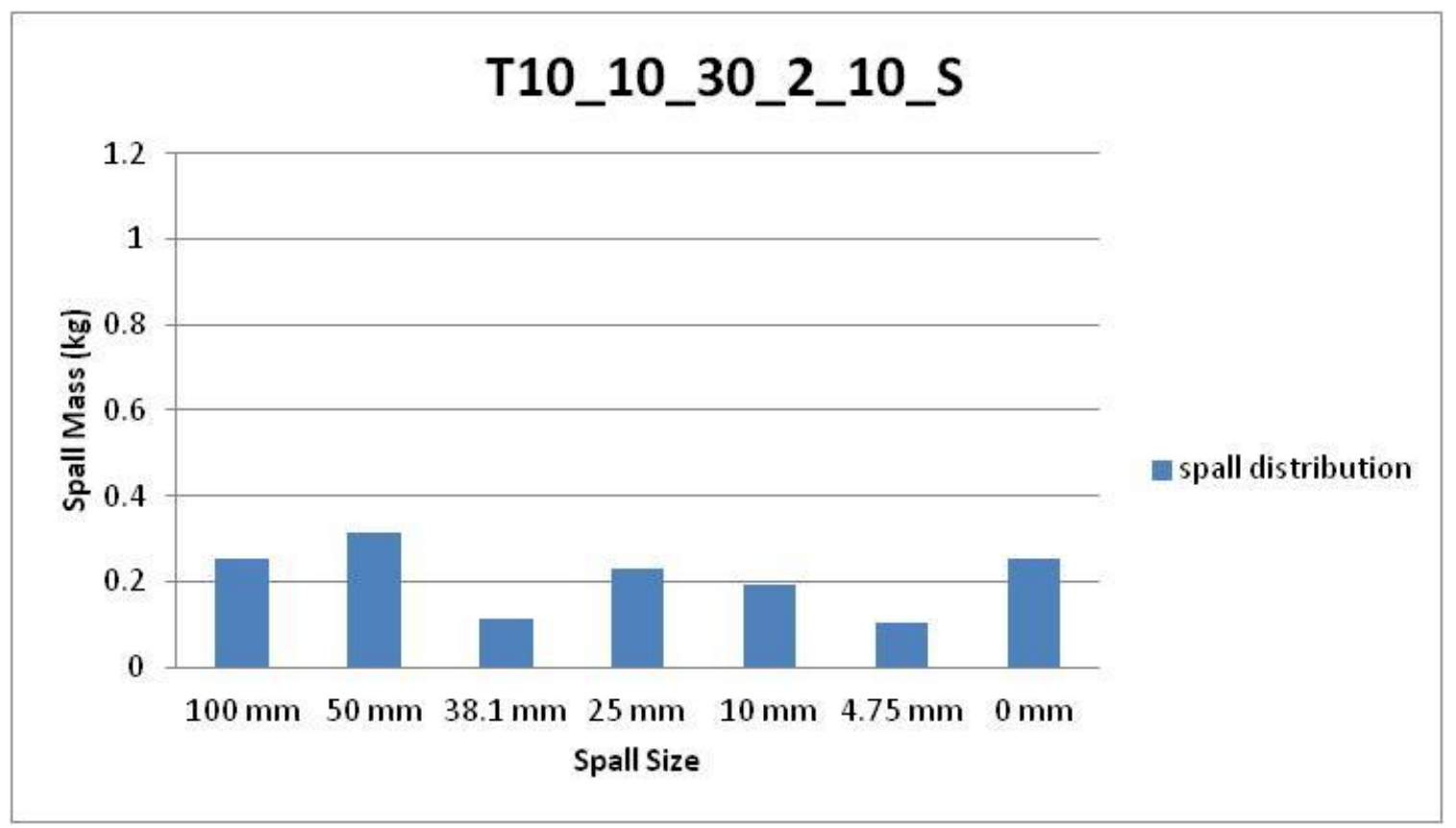

T10_10_30_2_10_S: Spall mass distributions
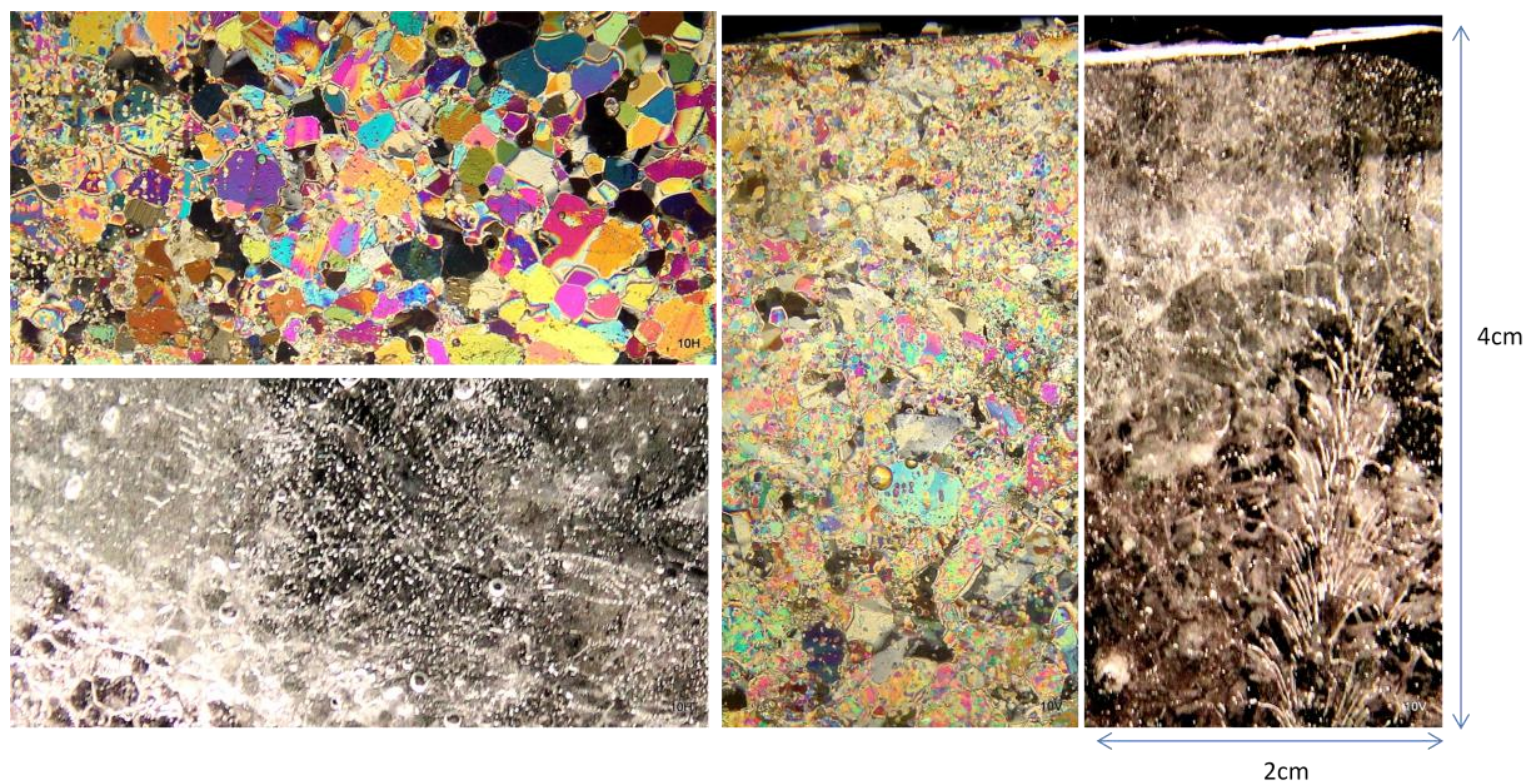

T10_10_30_2_10_S: Thin section pictures: Horizontal (left) and Vertical (right) 

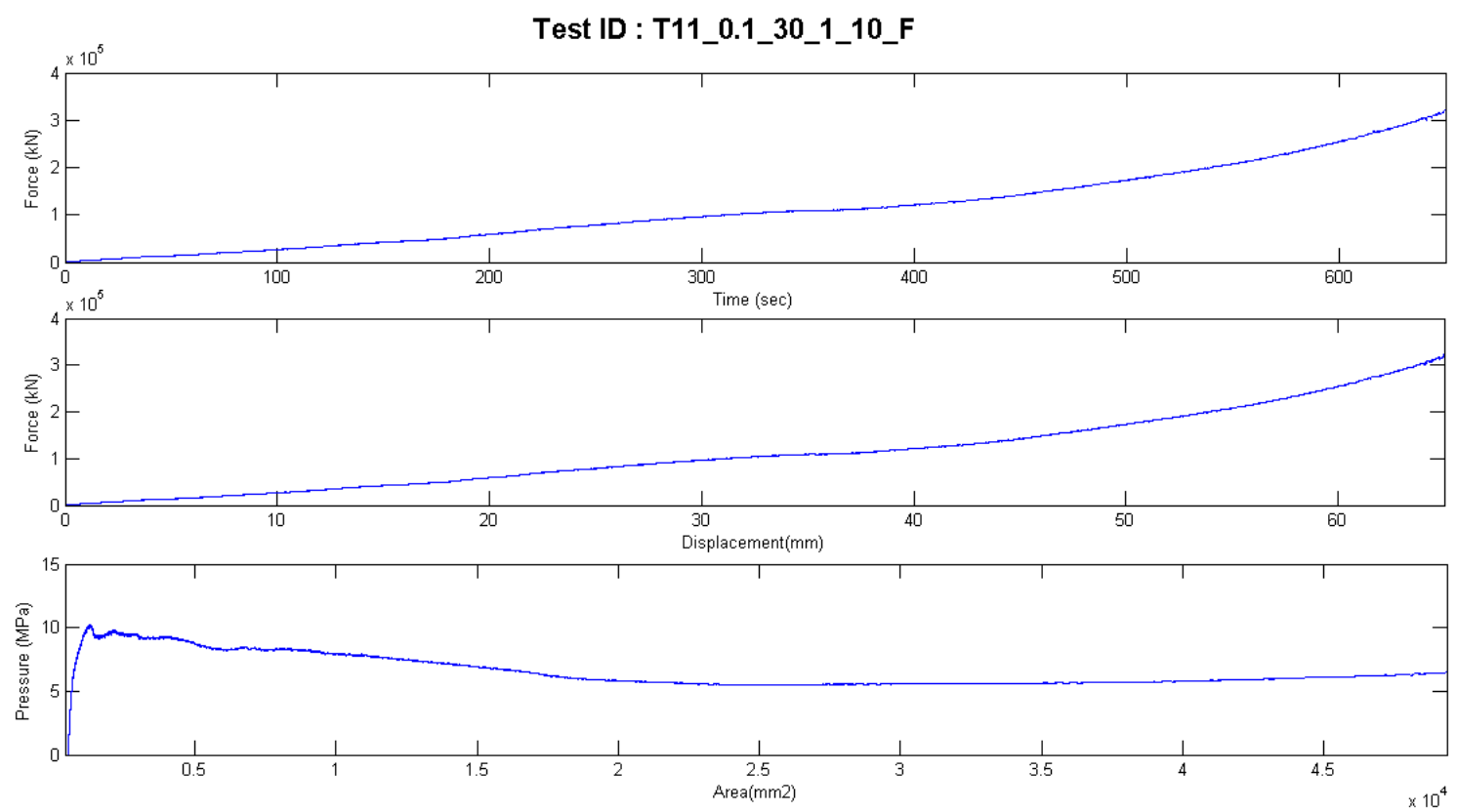

T11_0.1_30_1_10_F: Force and pressure traces

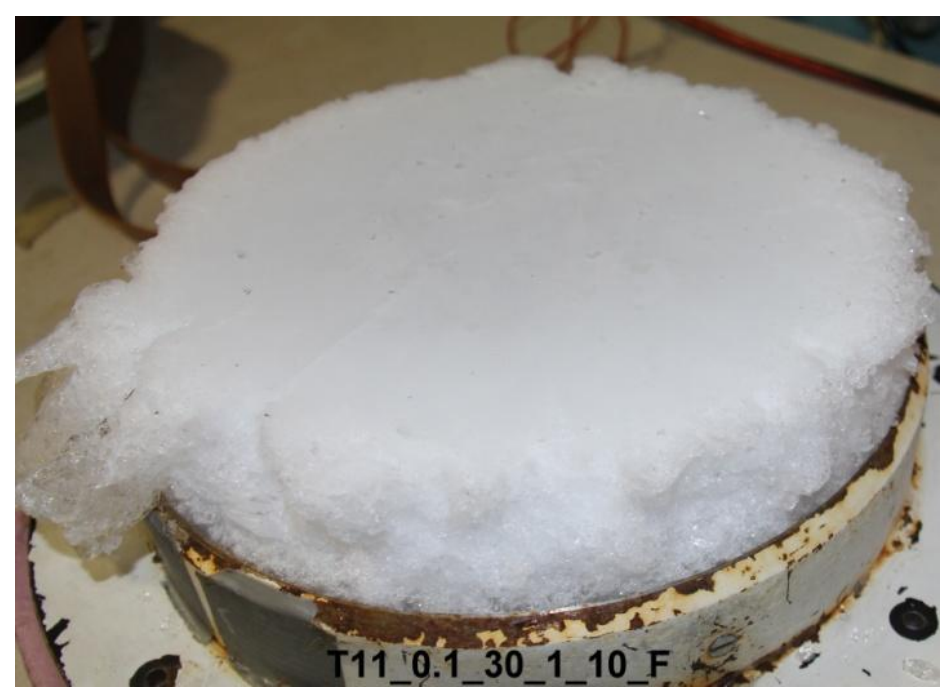

T11_0.1_30_1_10_F: After crushing picture 


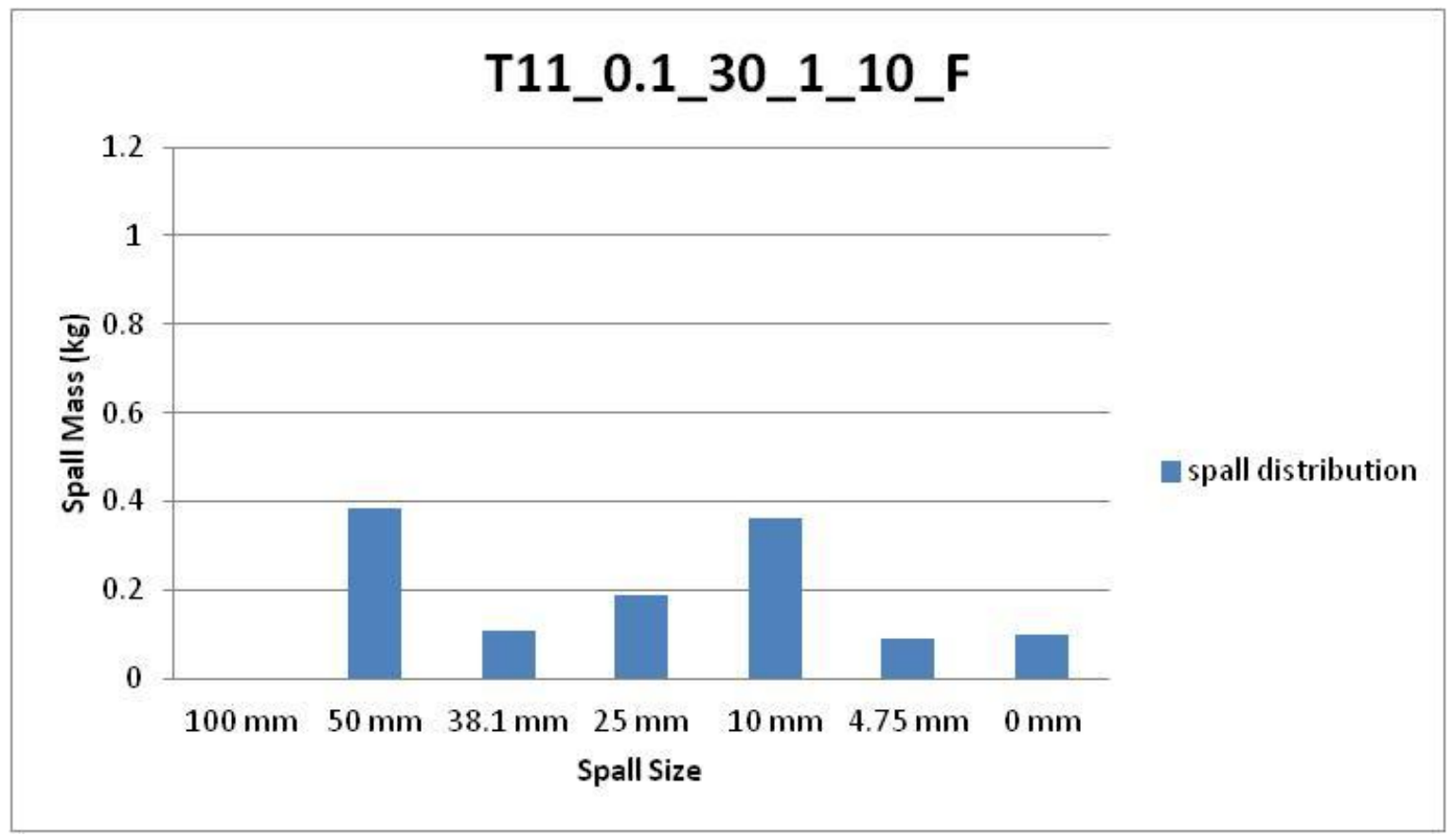

T11_0.1_30_1_10_F: Spall mass distributions
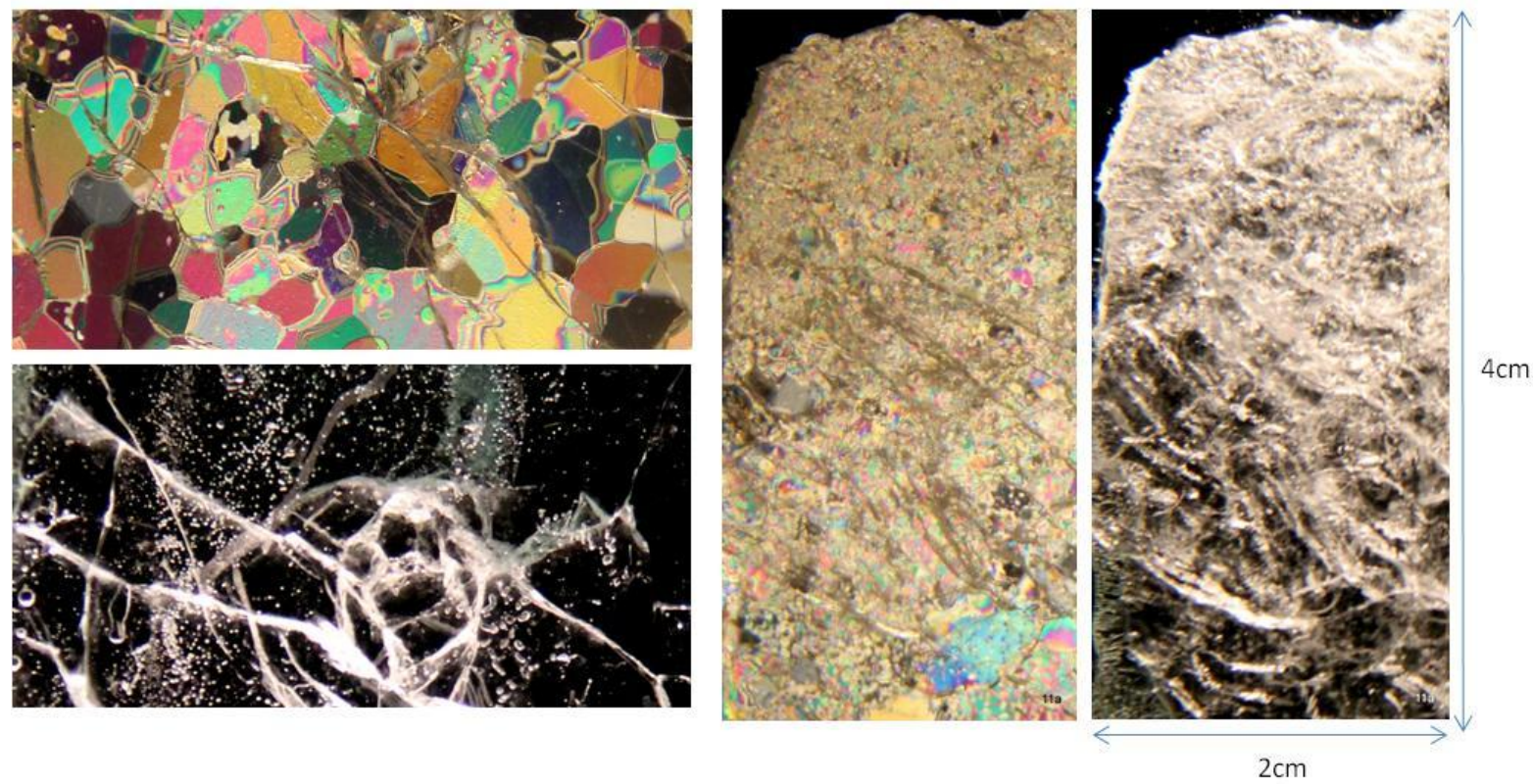

T11_0.1_30_1_10_F: Thin section pictures: Horizontal (left) and Vertical (right) 

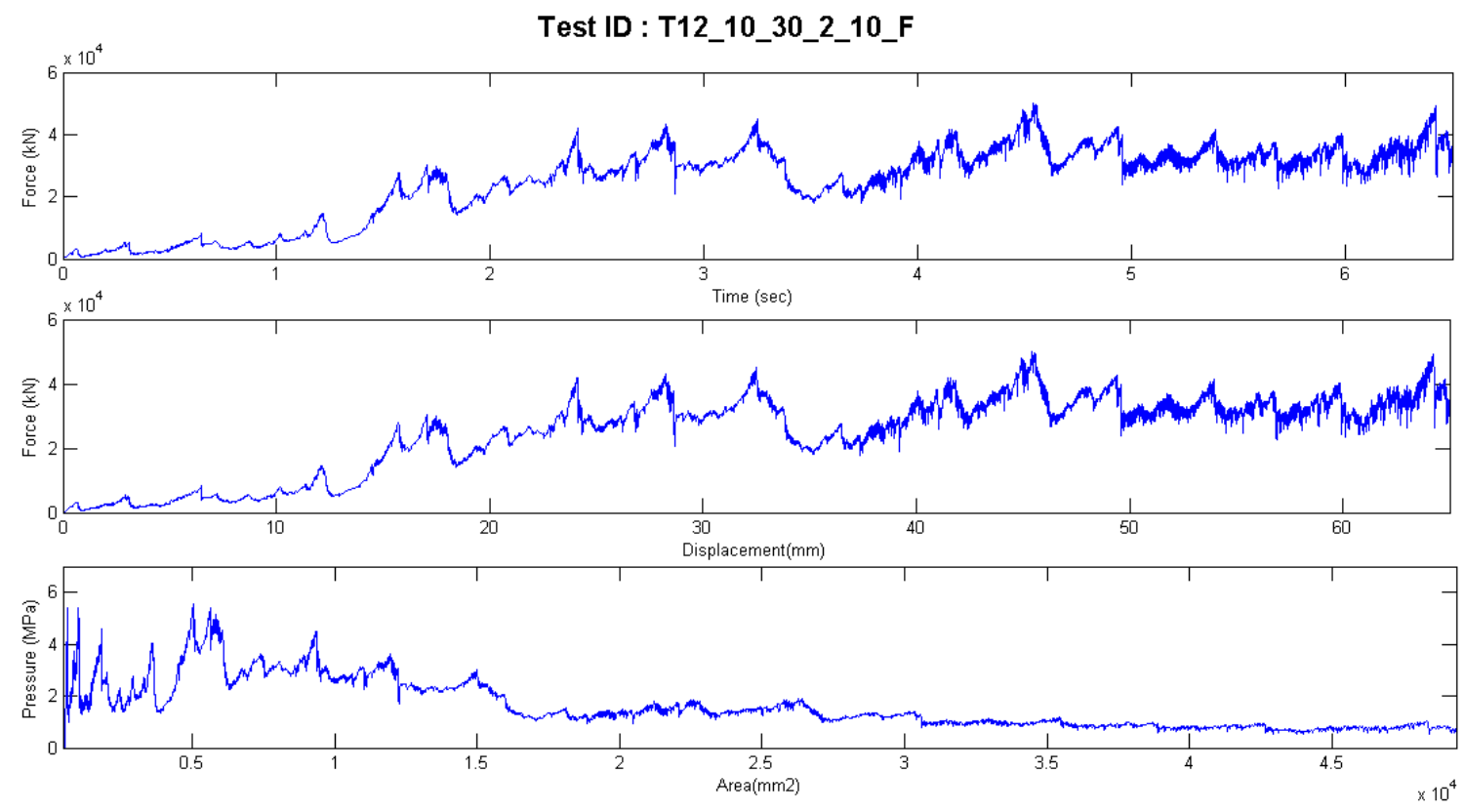

T12_10_30_2_10_F: Force and pressure traces

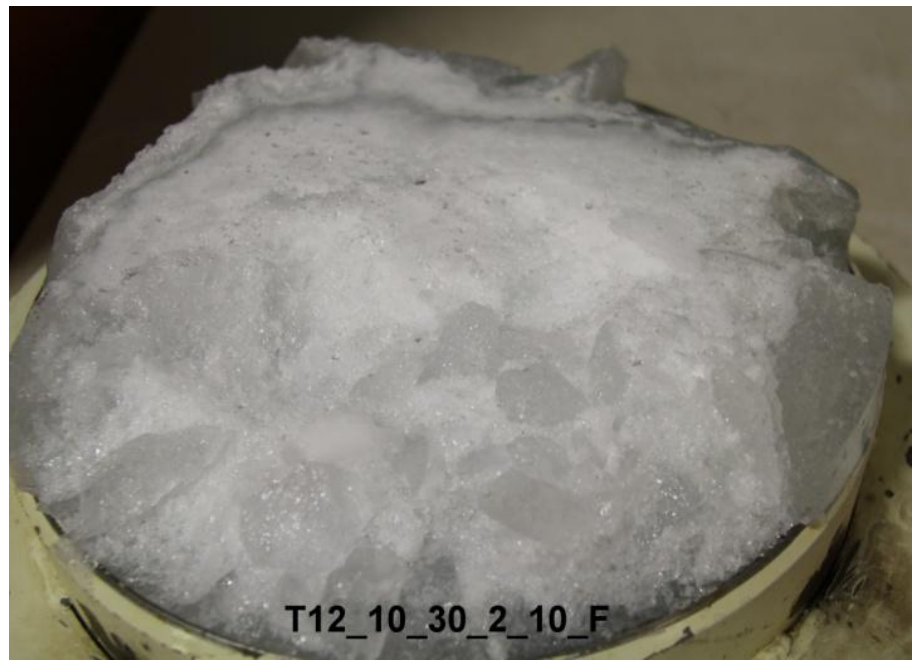

T12_10_30_2_10_F: After crushing picture 


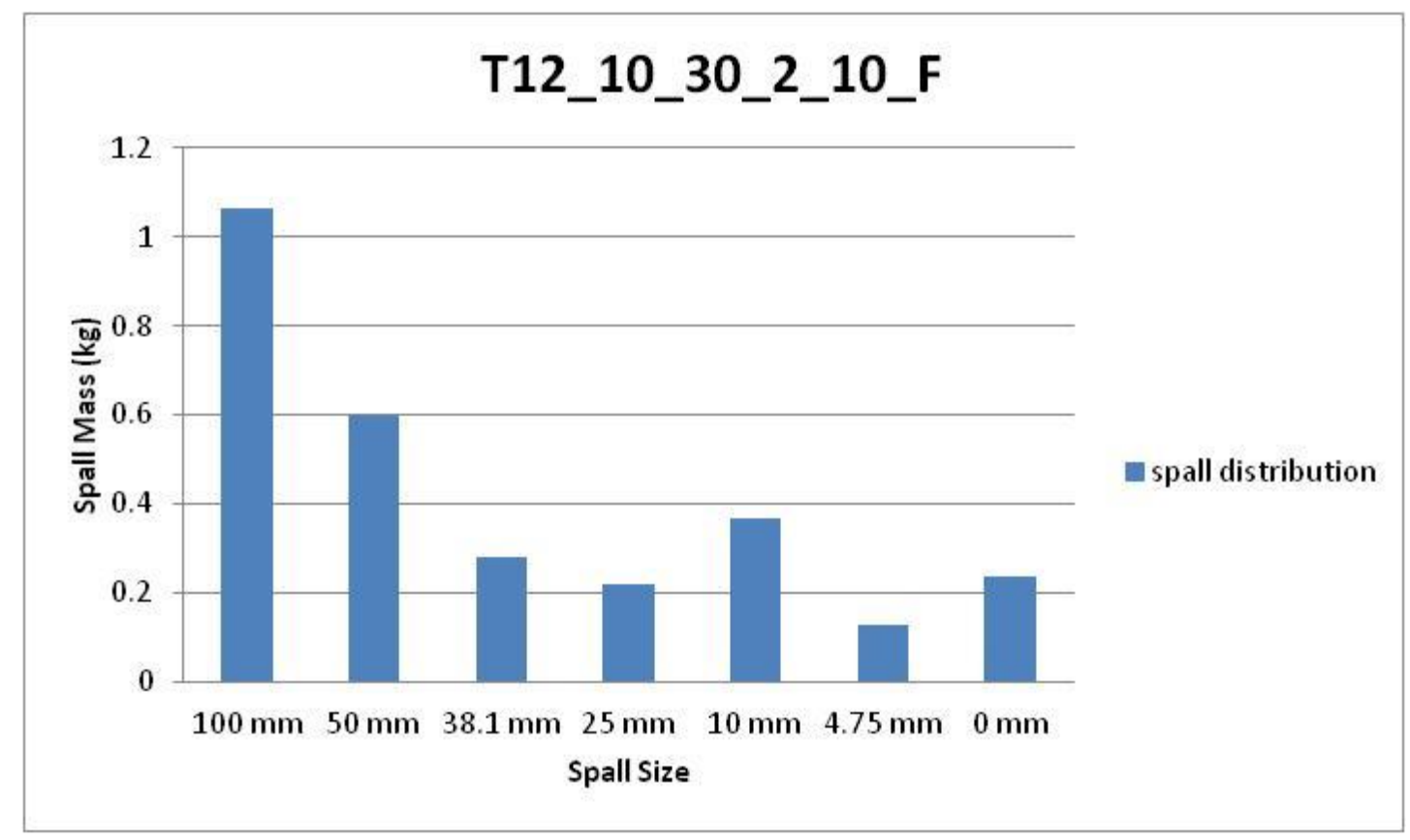

T12_10_30_2_10_F: Spall mass distributions
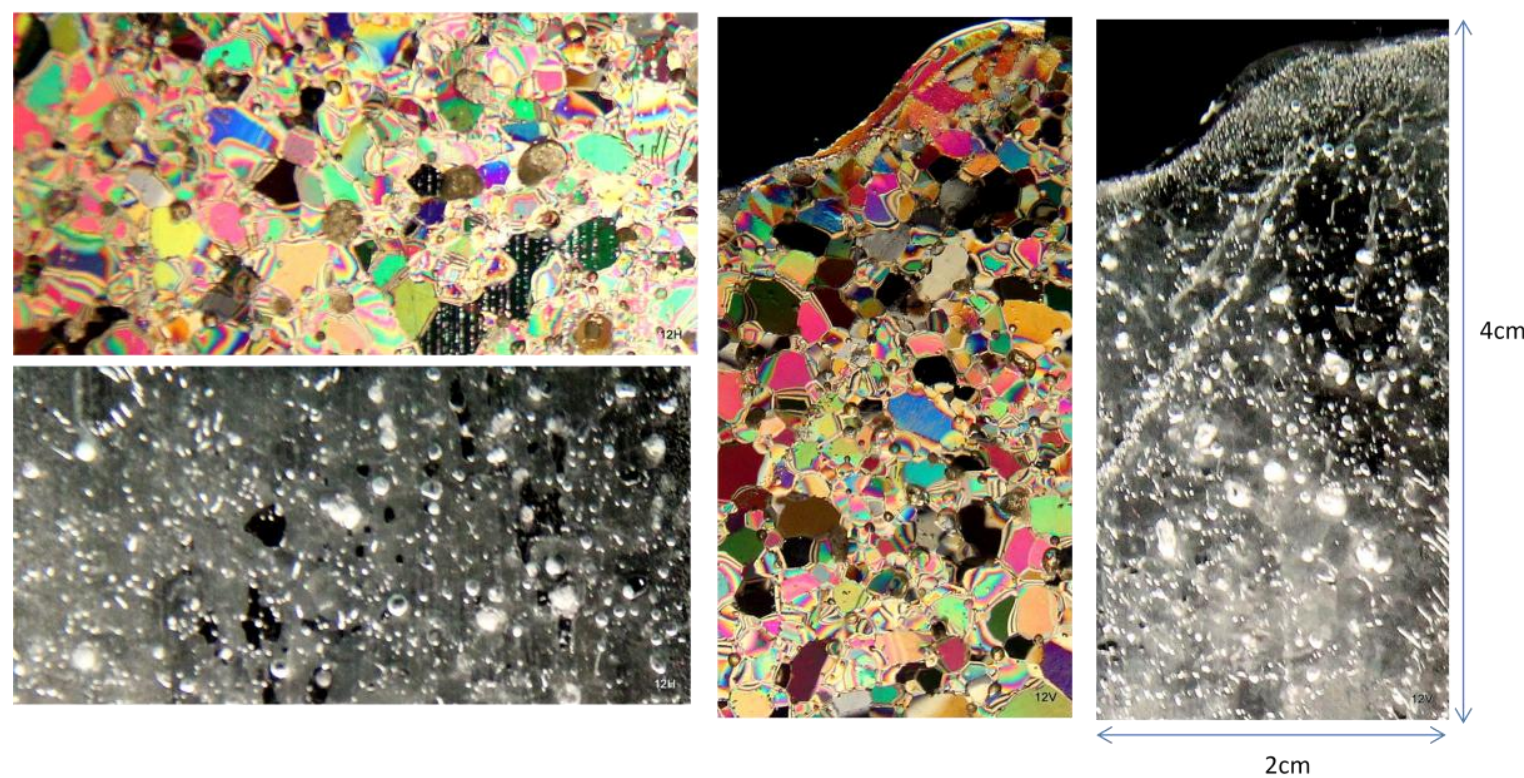

T12_10_30_2_10_F: Thin section pictures: Horizontal (left) and Vertical (right) 
Test ID : T13_1_30_1_10_F
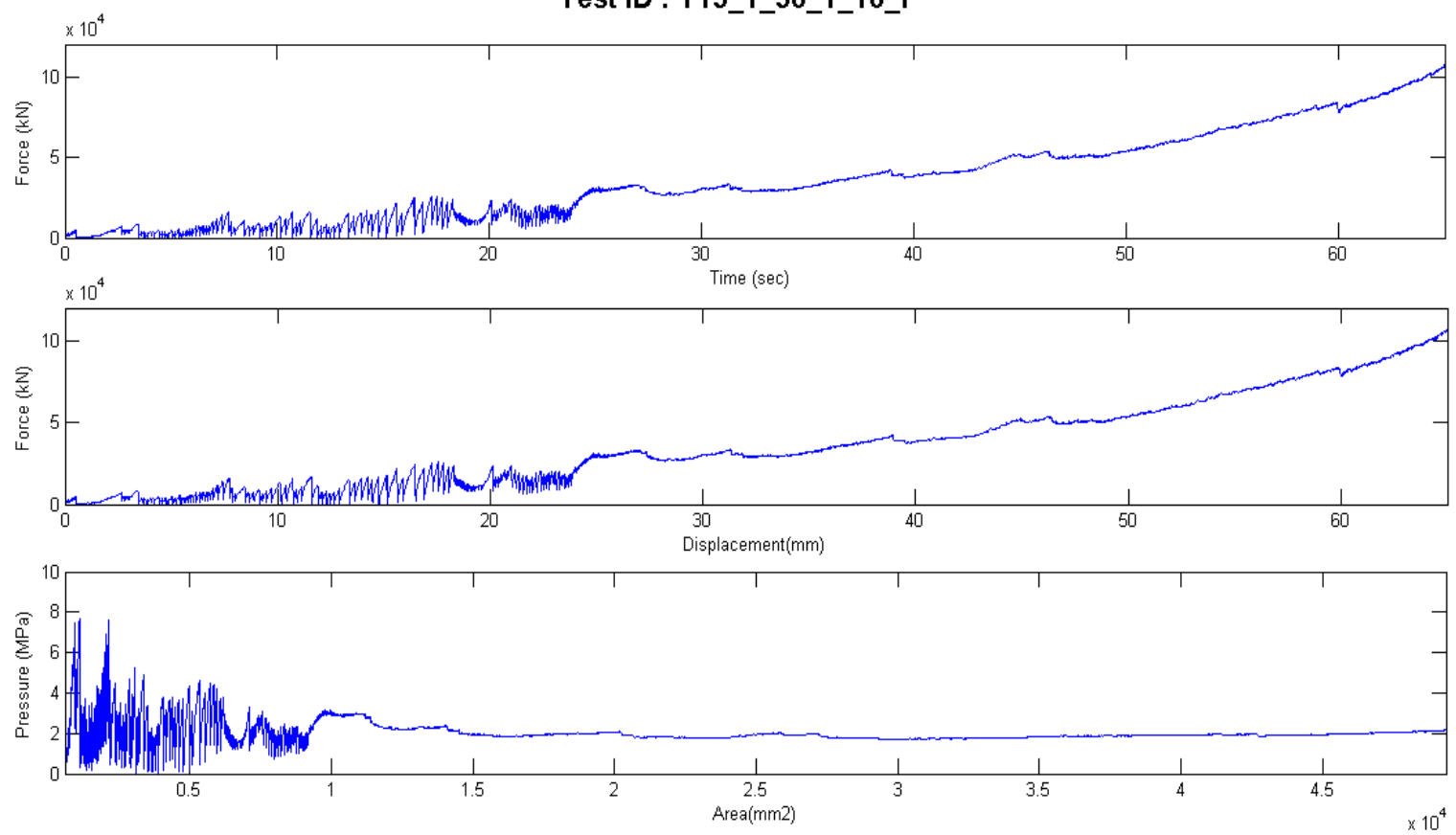

T13_1_30_1_10_F: Force and pressure traces

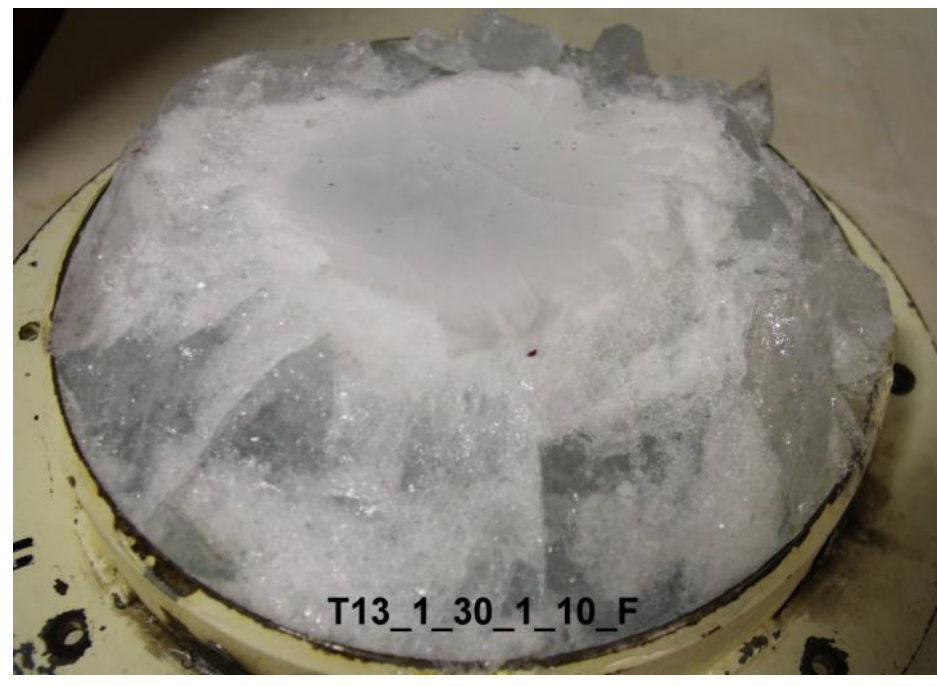

T13_1_30_1_10_F: After crushing picture 


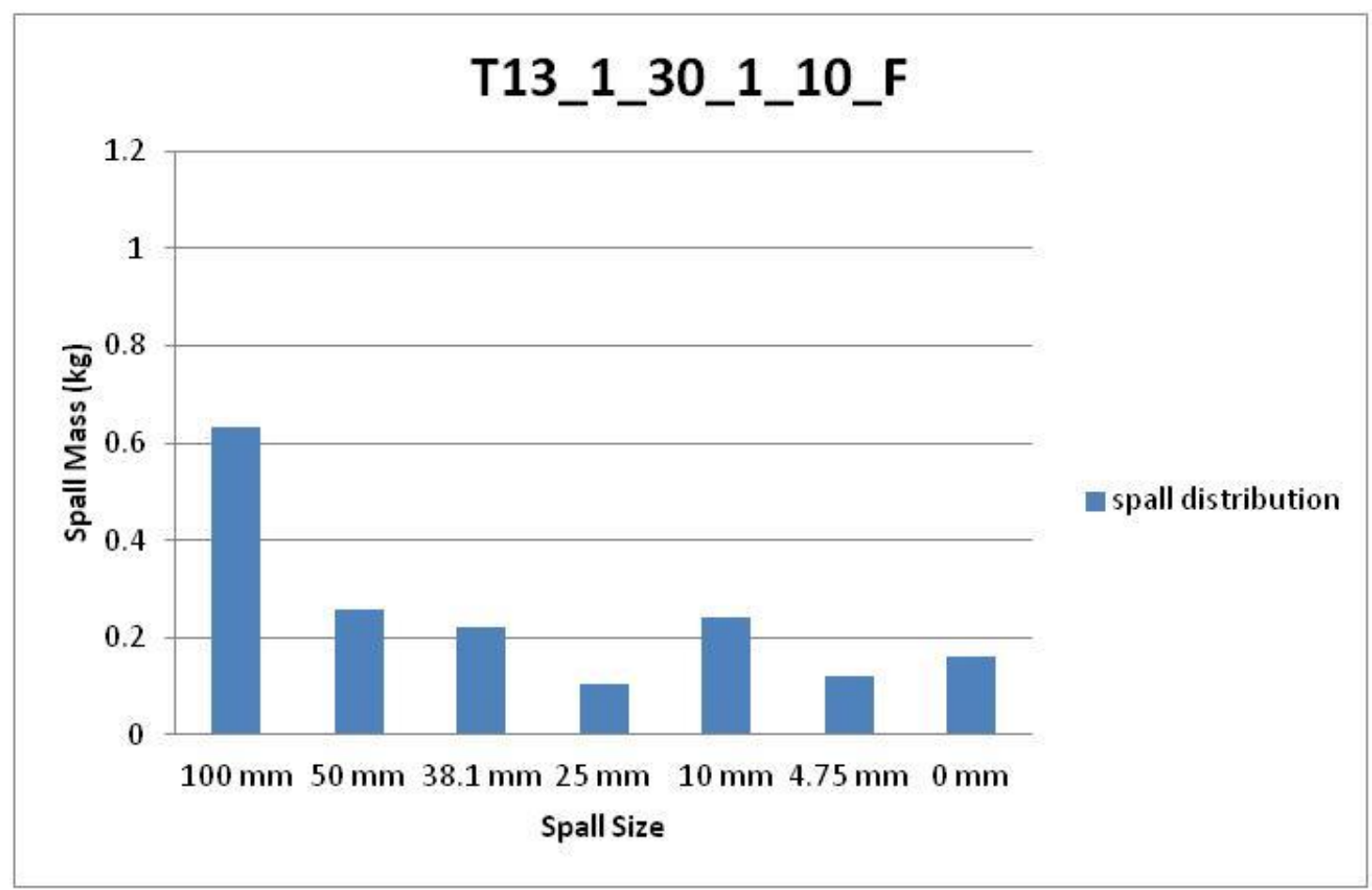

T13_1_30_1_10_F: Spall mass distributions
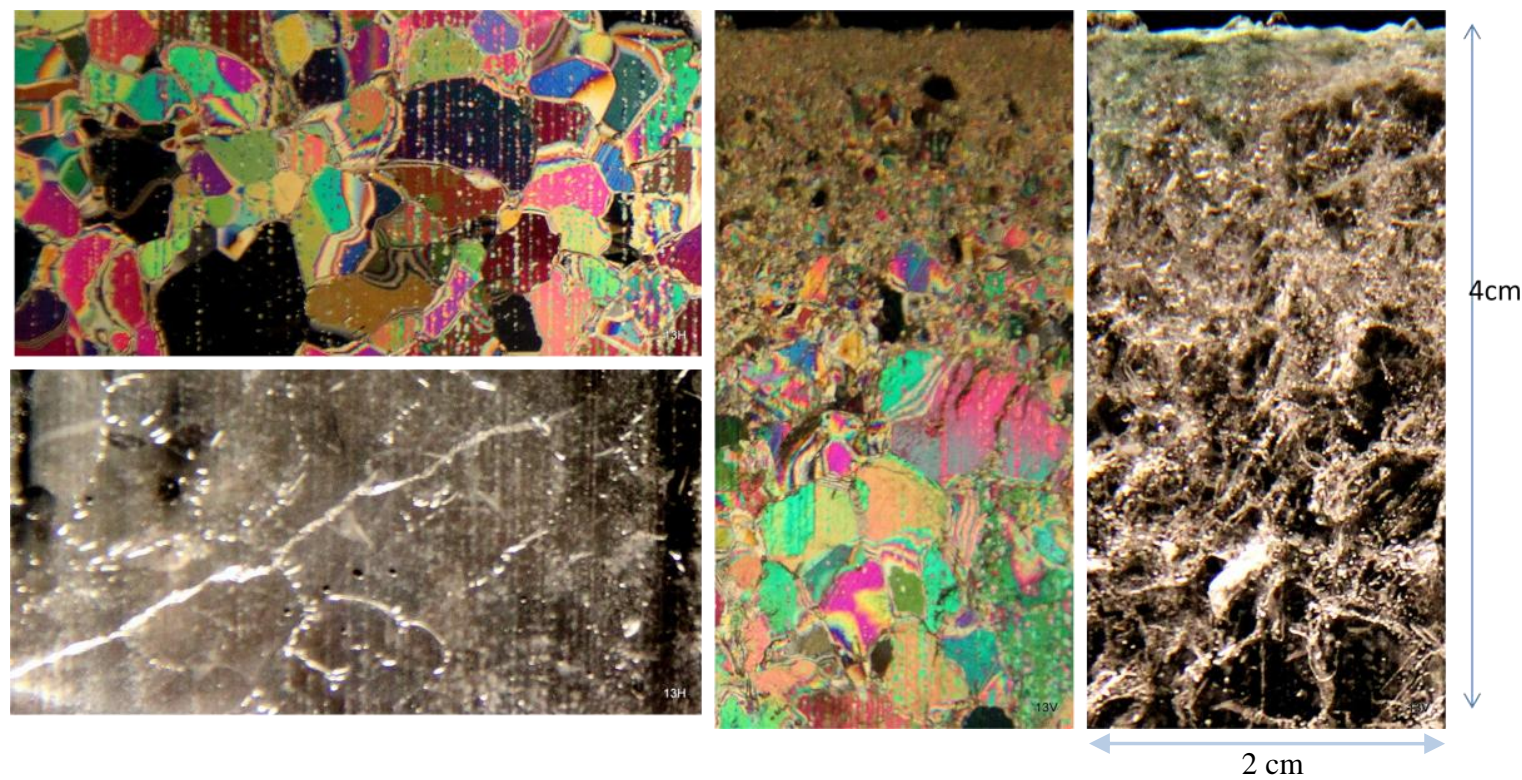

T13_1_30_1_10_F: Thin section pictures: Horizontal (left) and Vertical (right) 

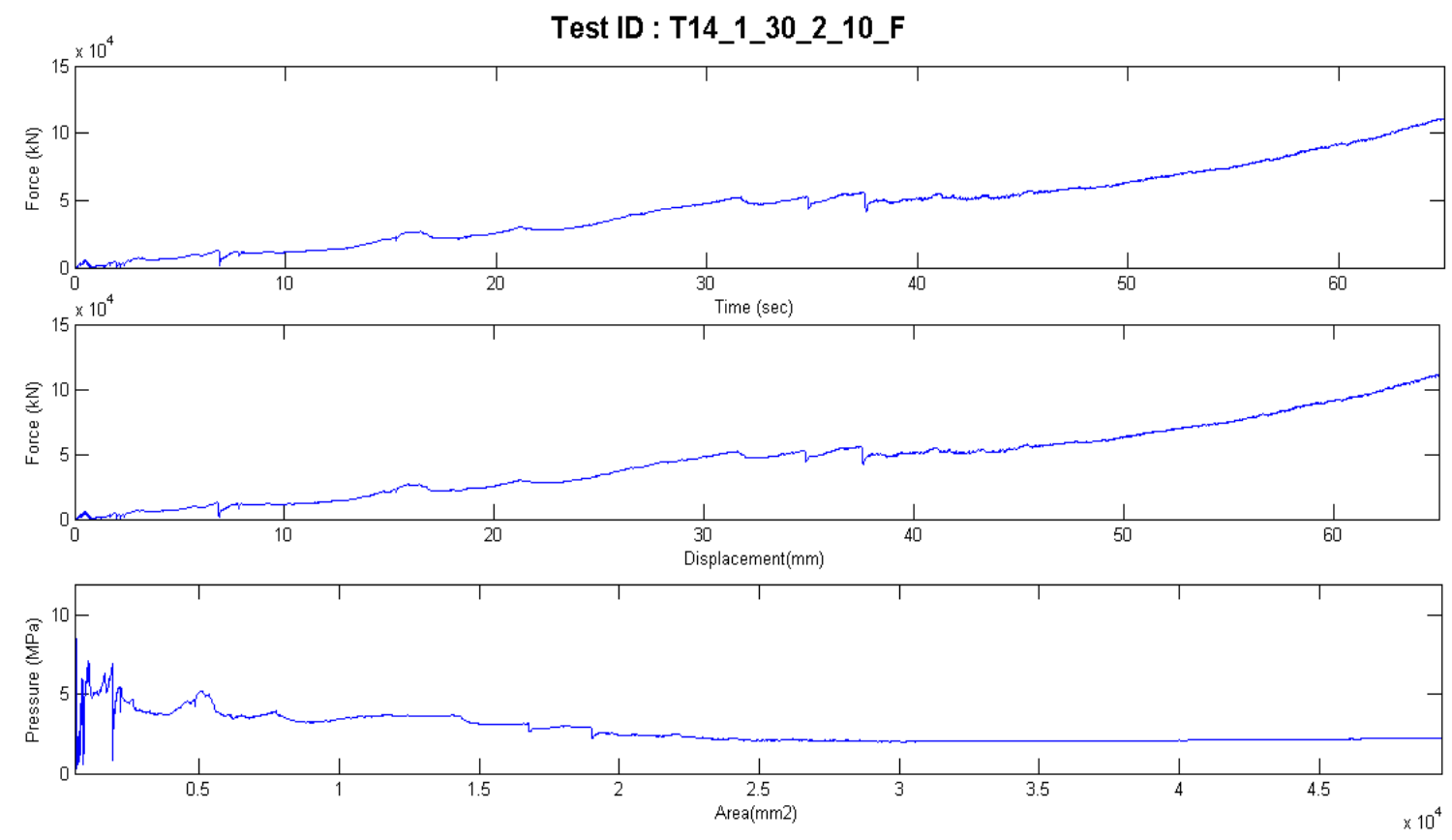

T14_1_30_2_10_F: Force and pressure traces

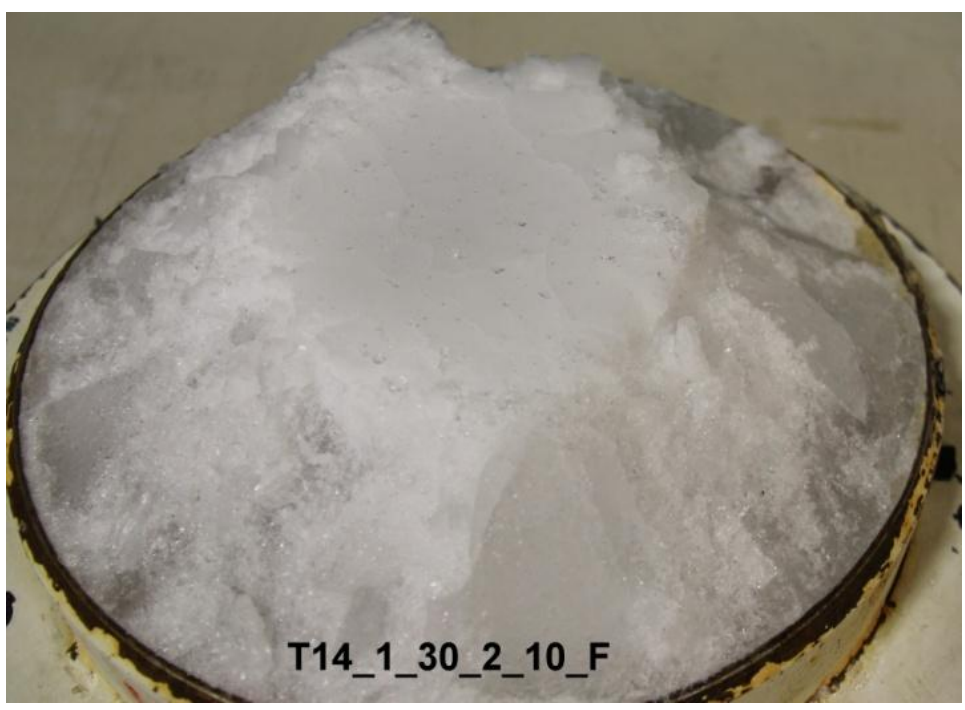

T14_1_30_2_10_F: After crushing picture 


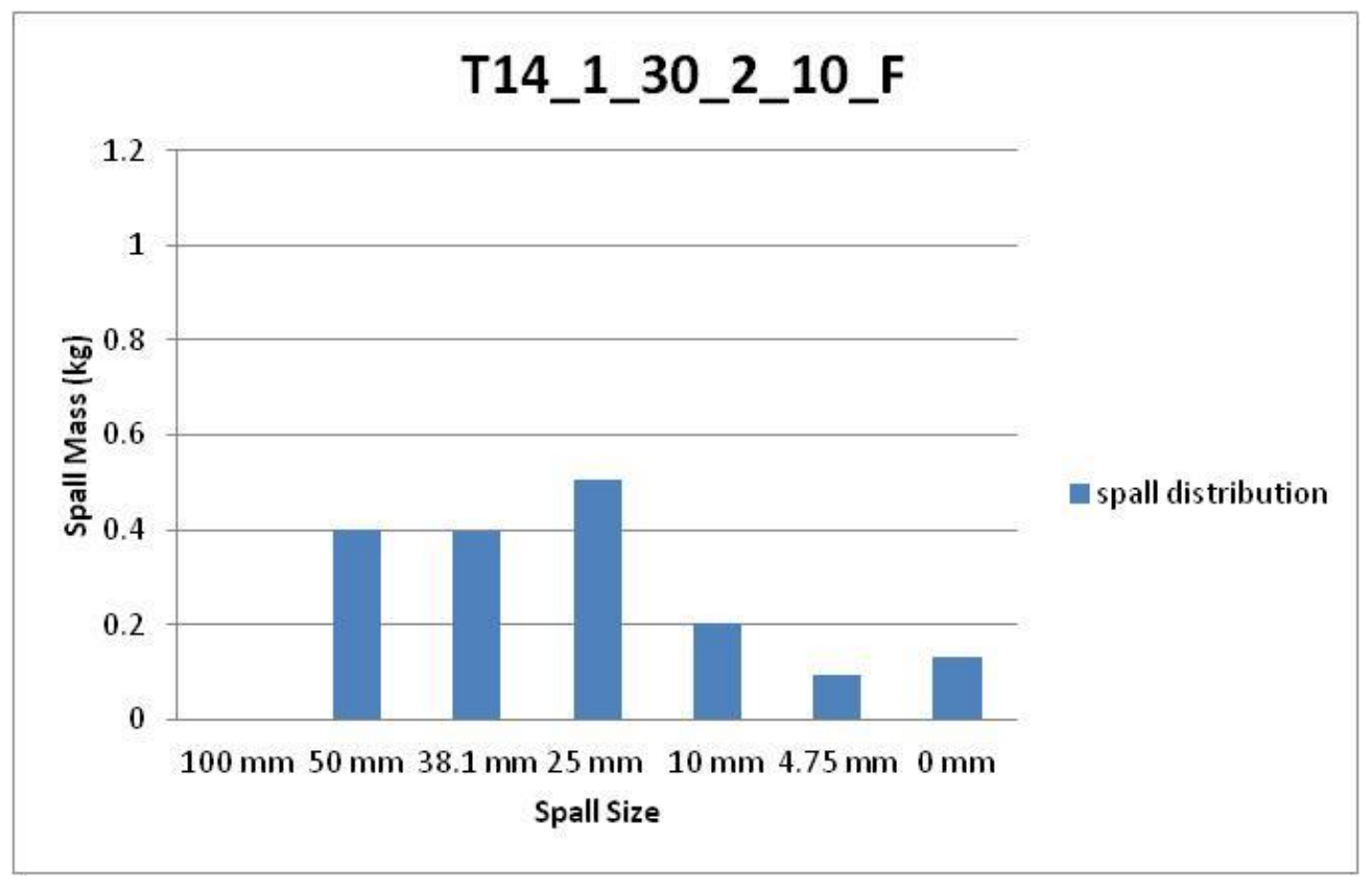

T14_1_30_2_10_F: Spall mass distributions
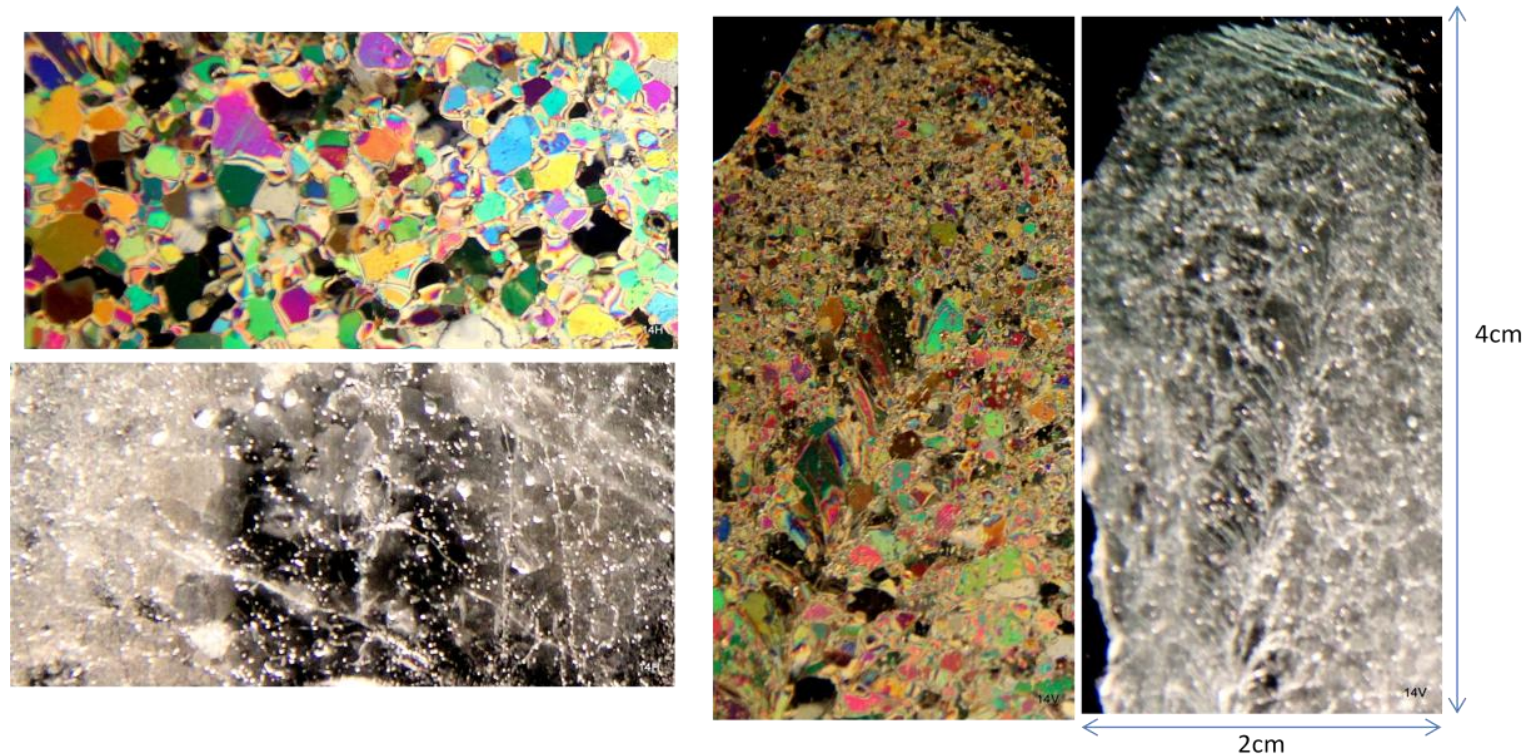

T14_1_30_2_10_F: Thin section pictures: Horizontal (left) and Vertical (right) 

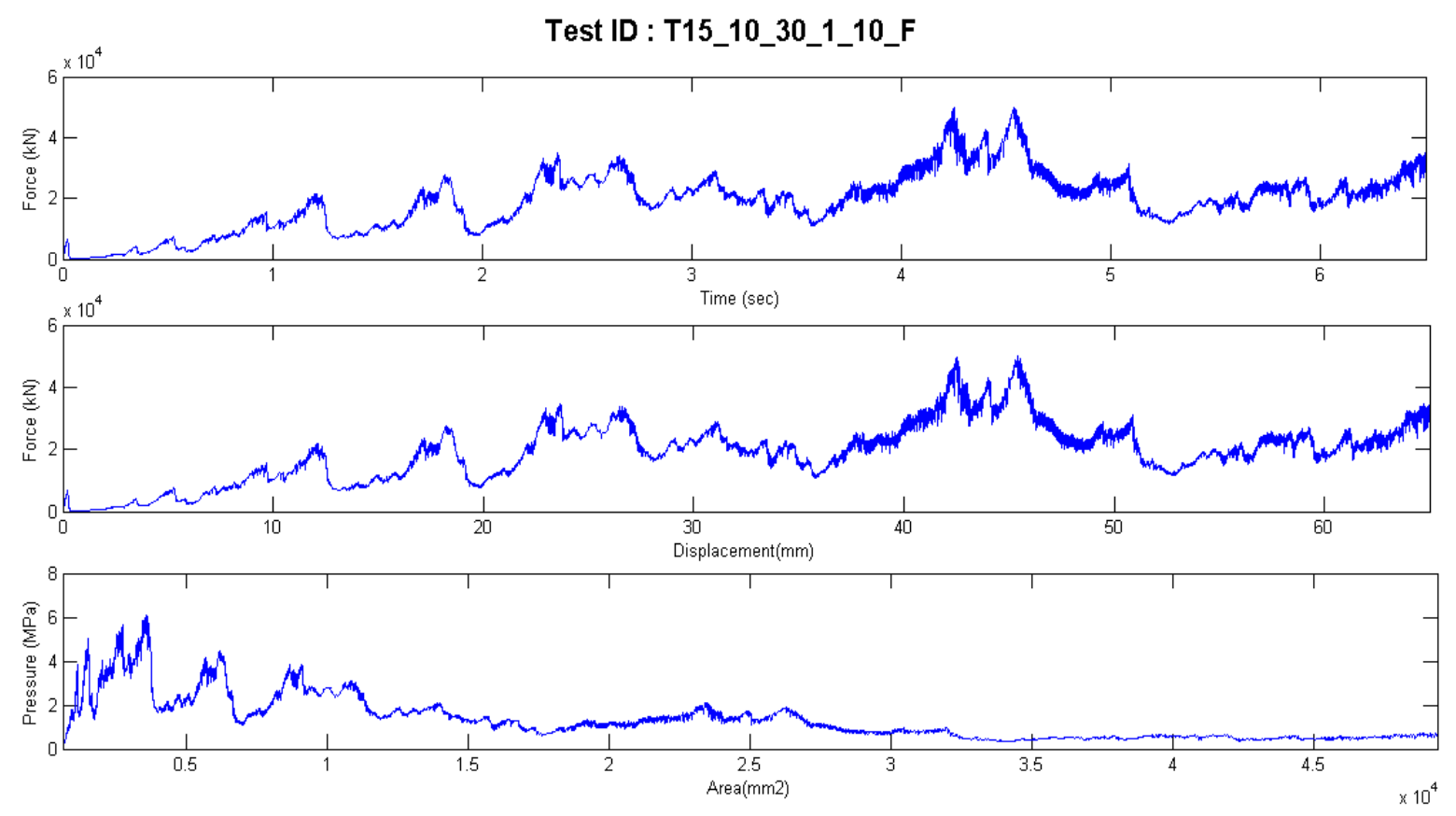

T15_10_30_1_10_F: Force and pressure traces

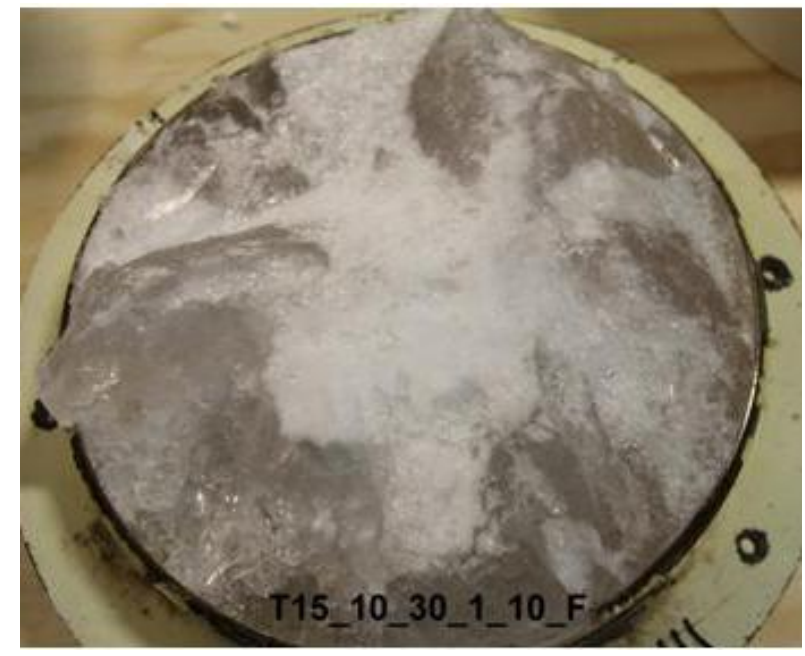

T15_10_30_1_10_F: After crushing picture 


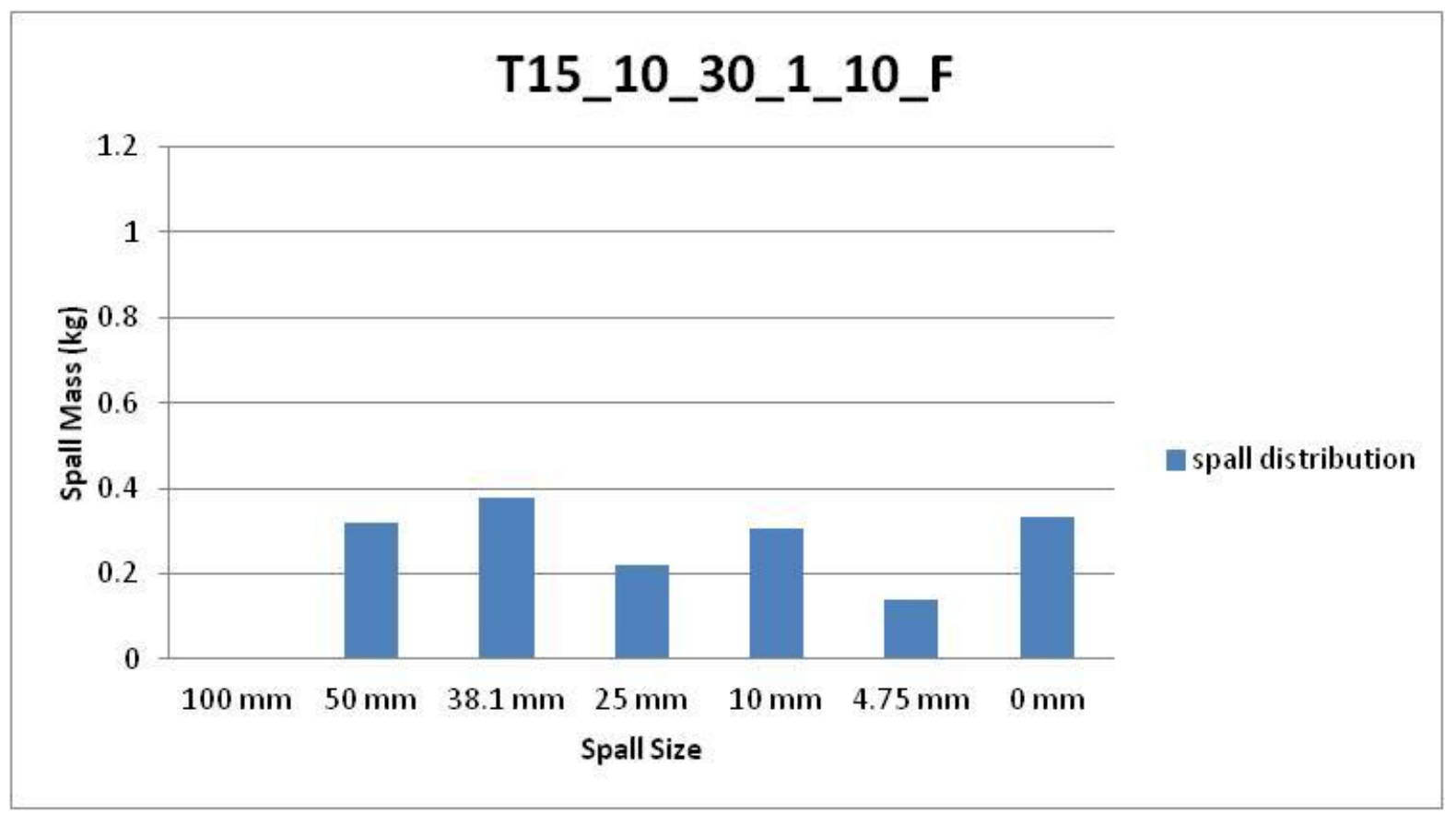

T15_10_30_1_10_F: Spall mass distributions
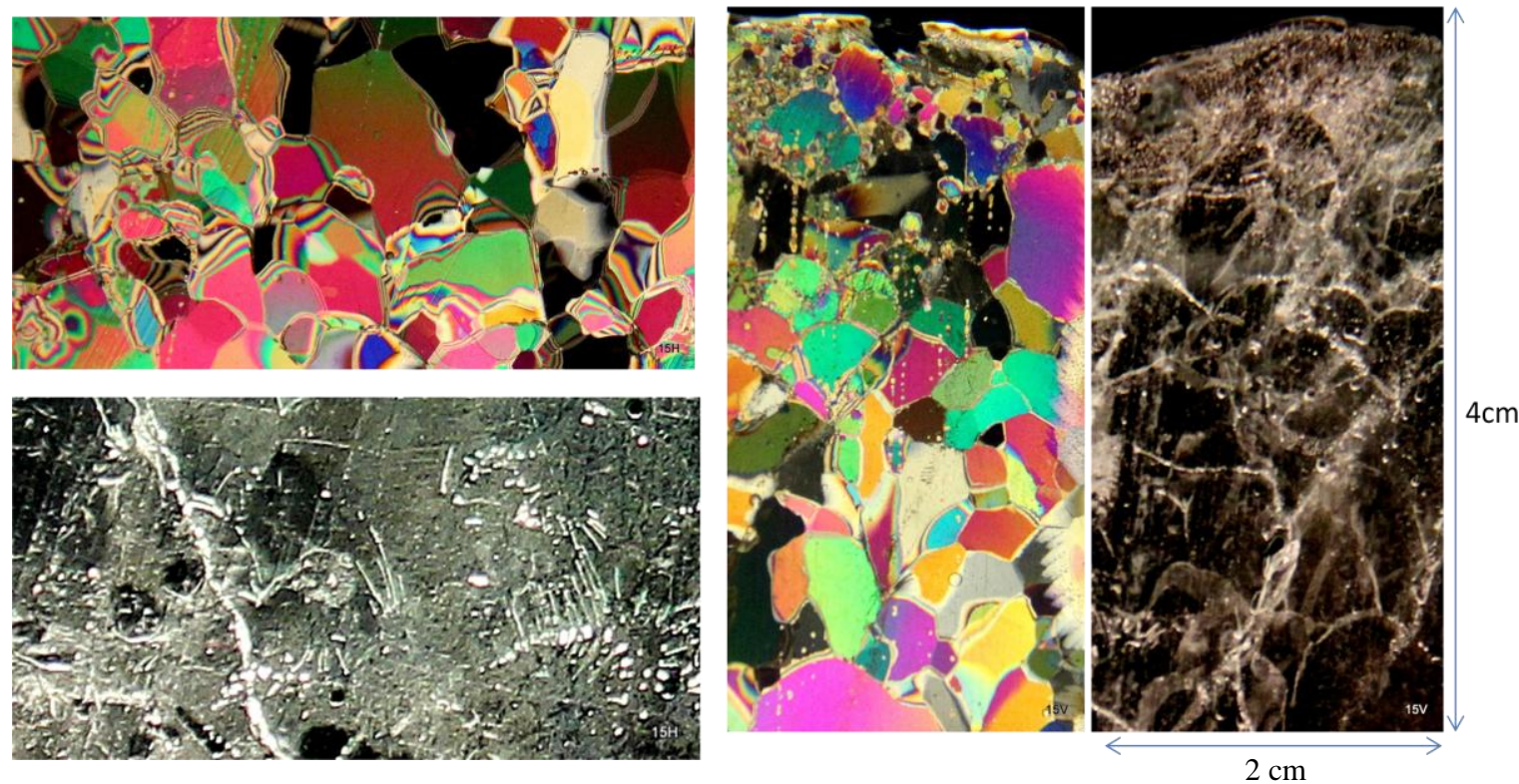

T15_10_30_1_10_F: Thin section pictures: Horizontal (left) and Vertical (right) 

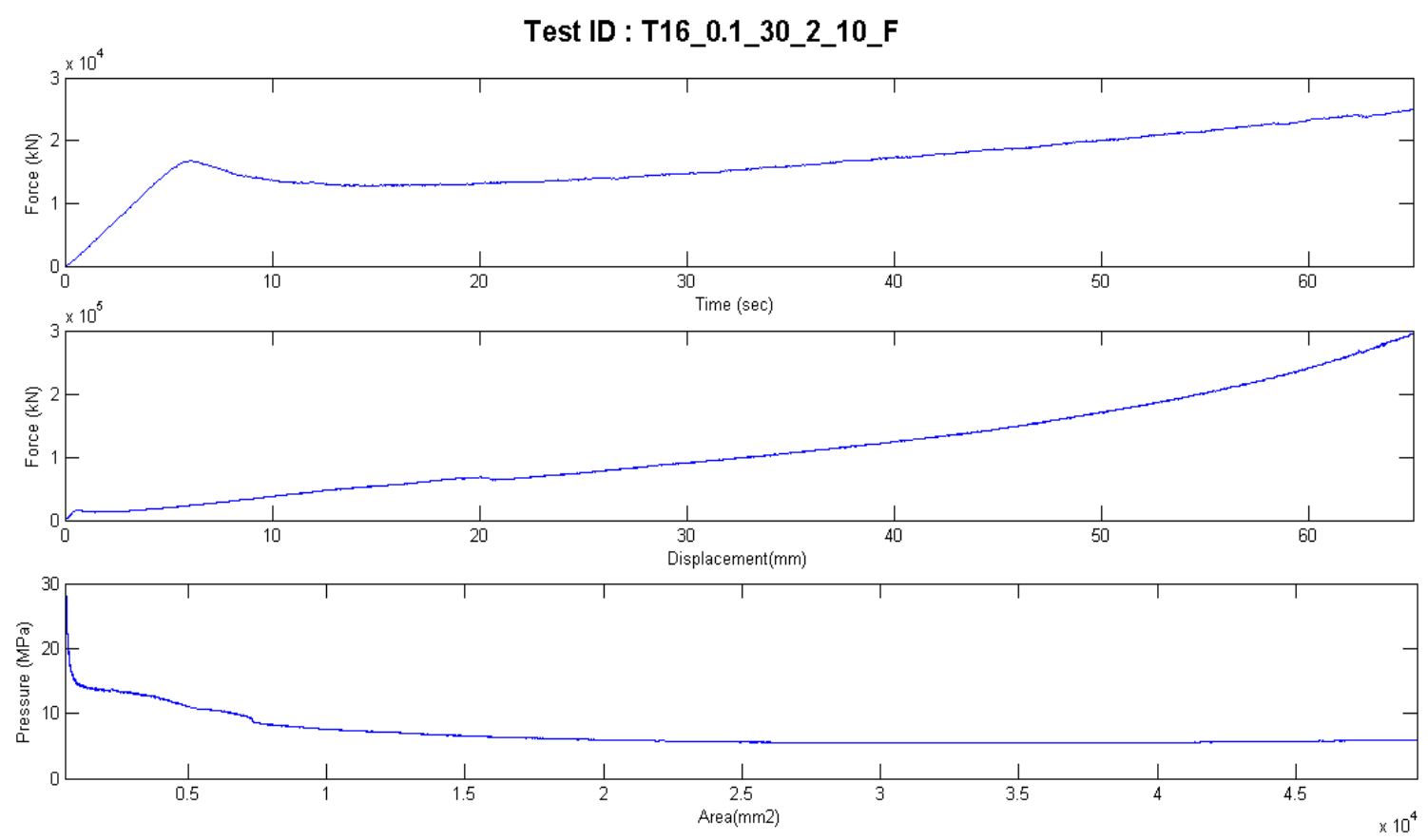

T16_0.1_30_2_10_F: Force and pressure traces

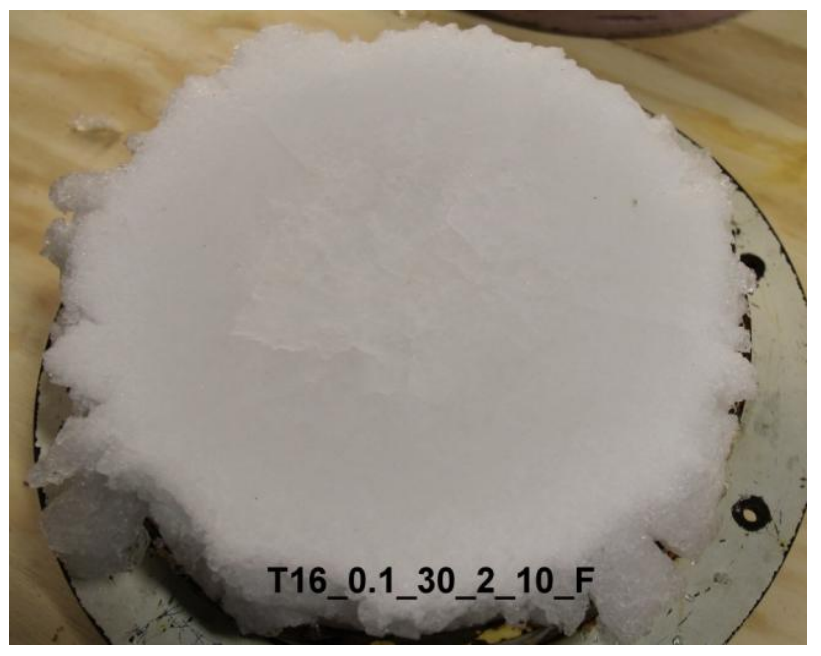

T16_0.1_30_2_10_F: After crushing picture 


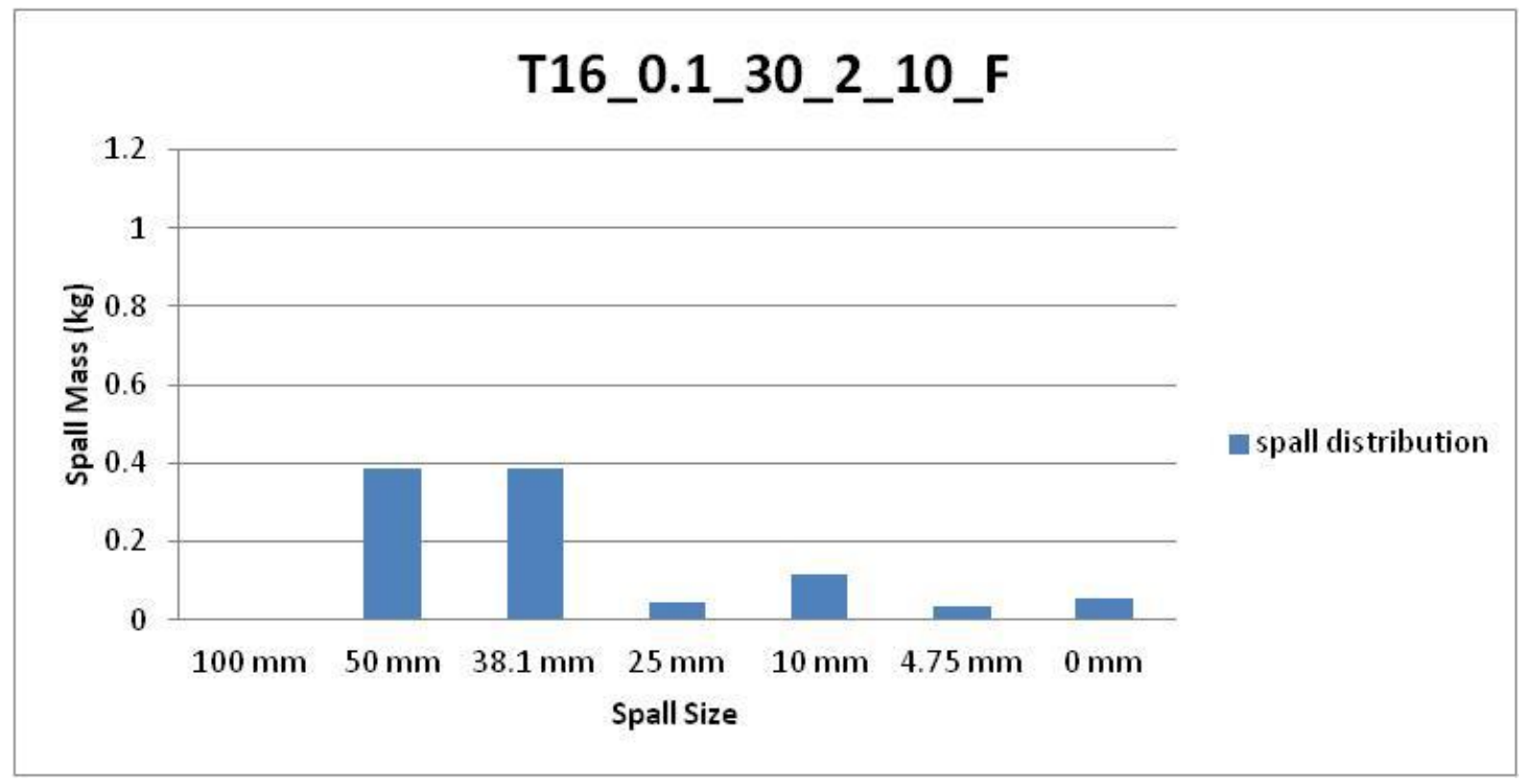

T16_0.1_30_2_10_F: Spall mass distributions
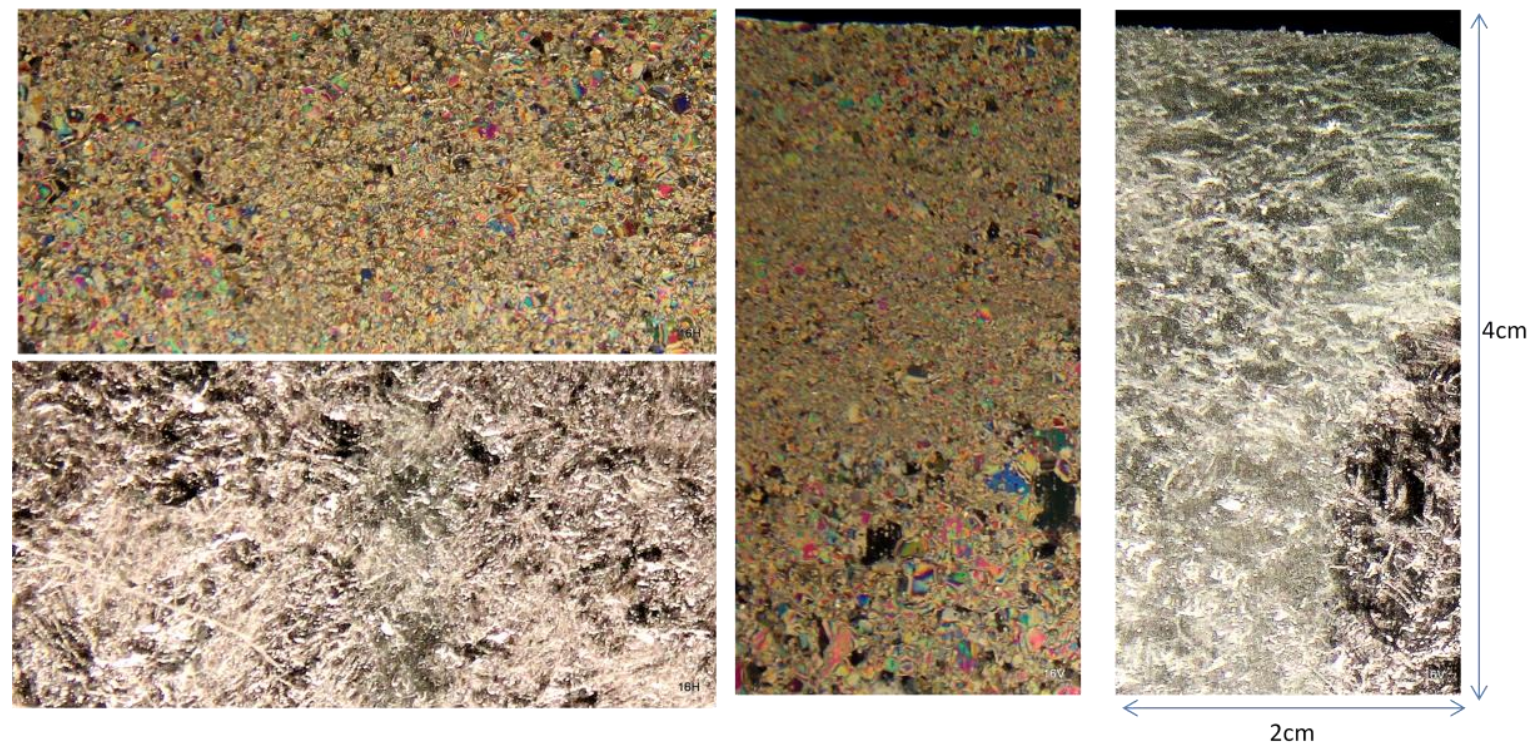

T16_0.1_30_2_10_F: Thin section pictures: Horizontal (left) and Vertical (right) 
Test ID : T17_0.1_13_1_10_F
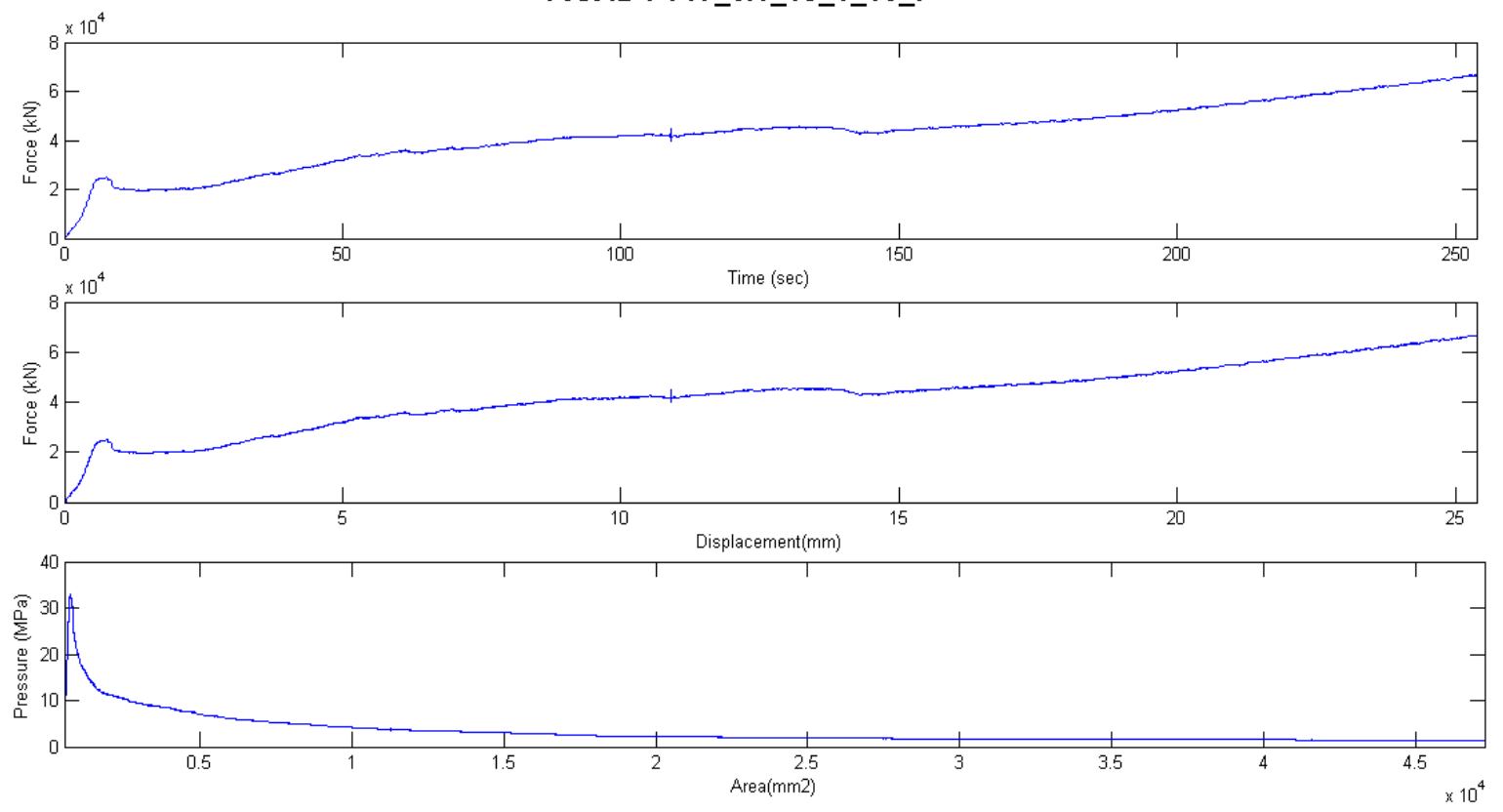

T17_0.1_13_1_10_F: Force and pressure traces

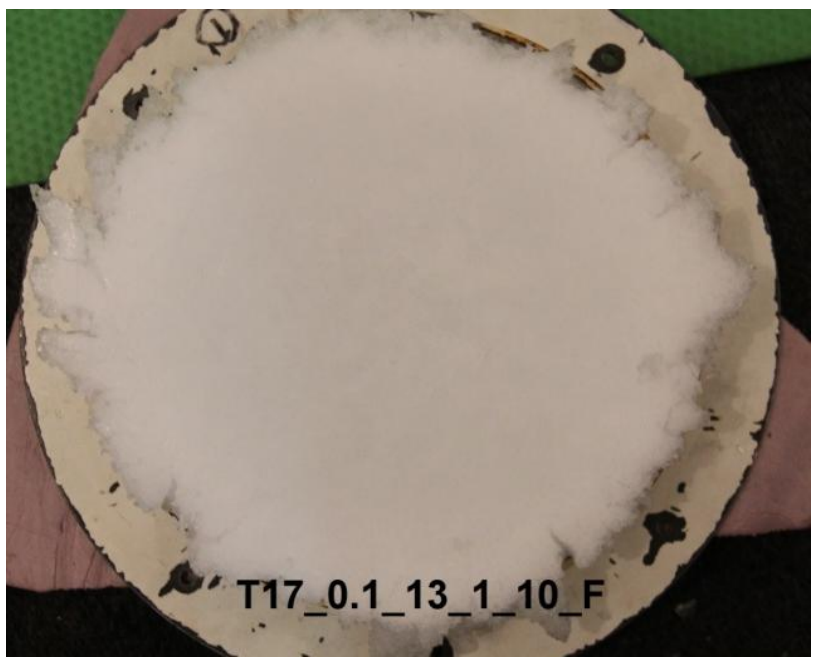

T17_0.1_13_1_10_F: After crushing picture 


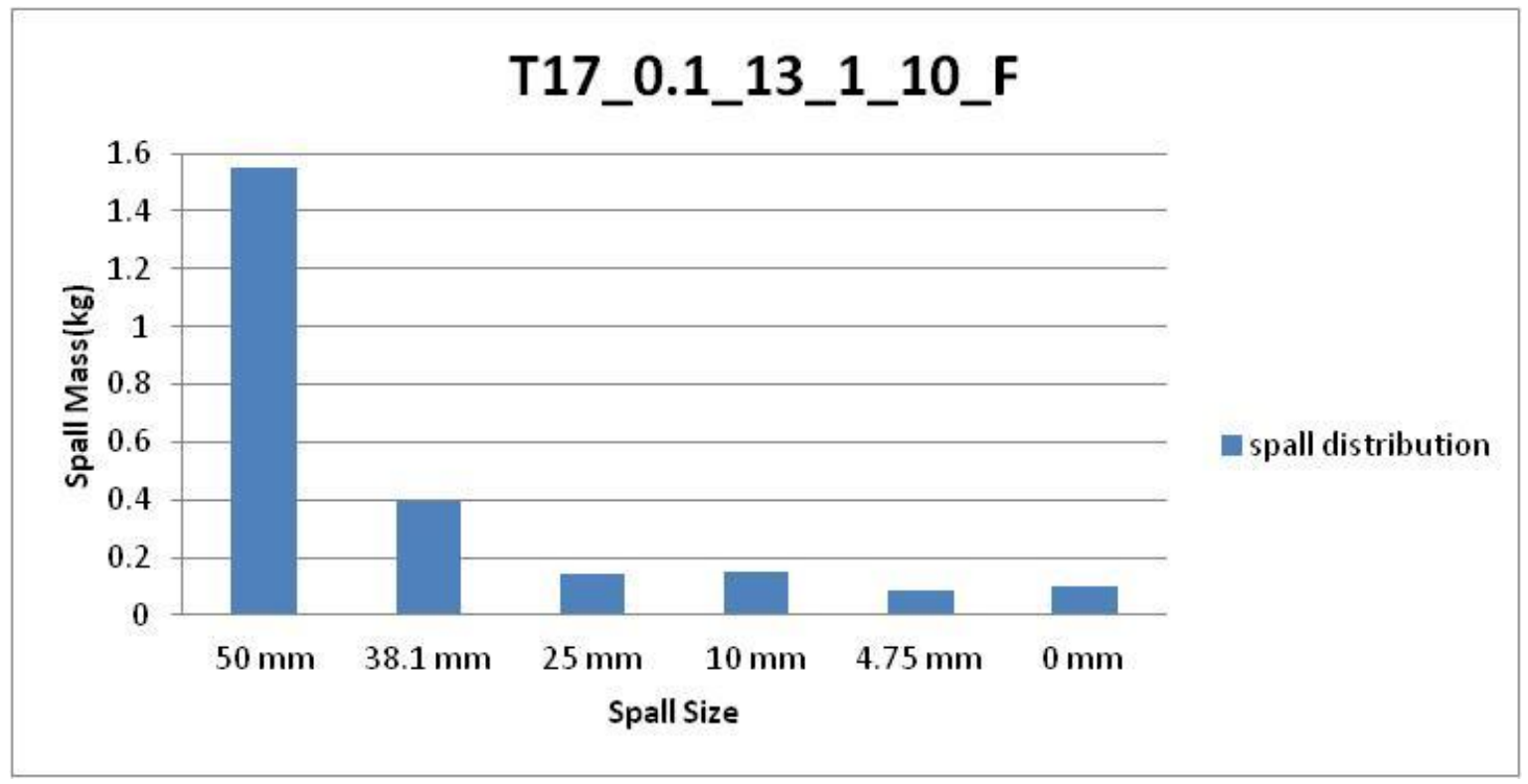

T17_0.1_13_1_10_F: Spall mass distributions
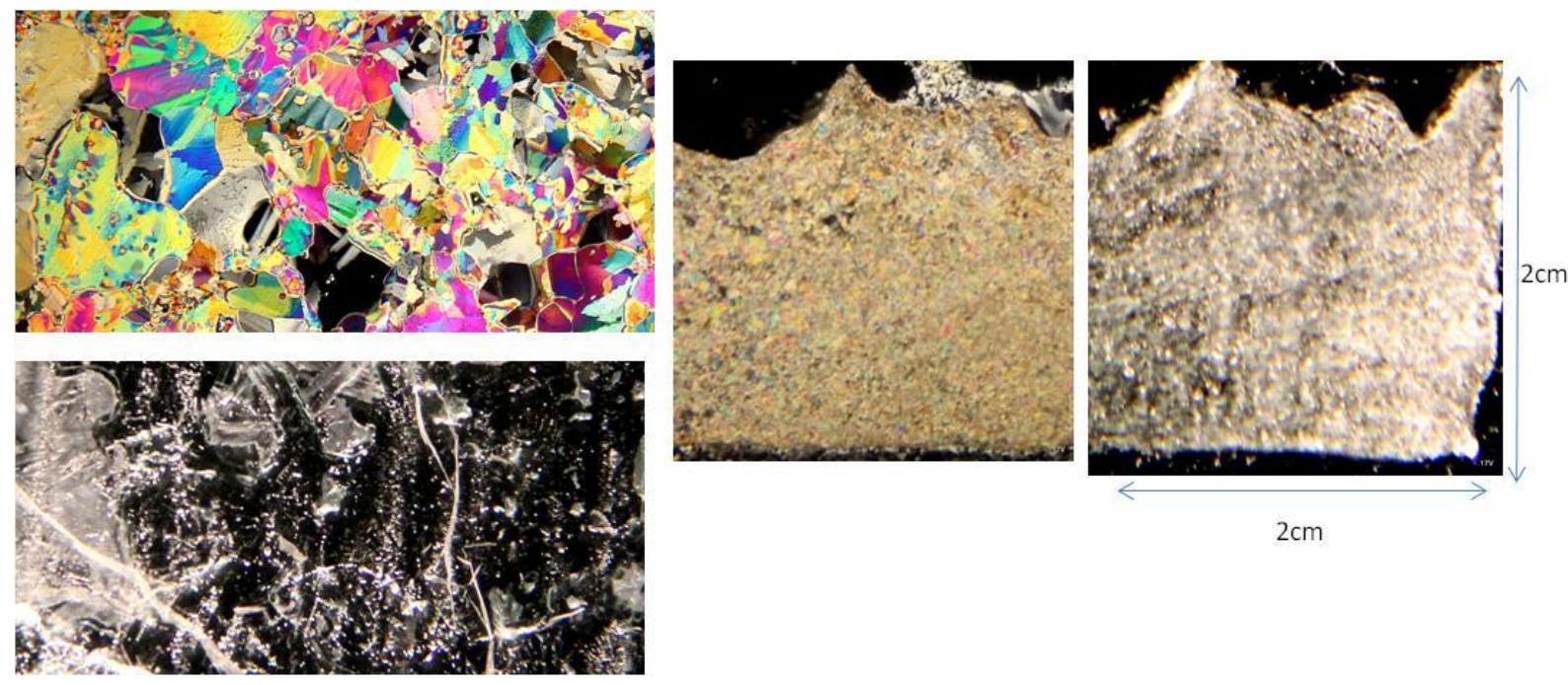

T17_0.1_13_1_10_F: Thin section pictures: Horizontal (left) and Vertical (right) 

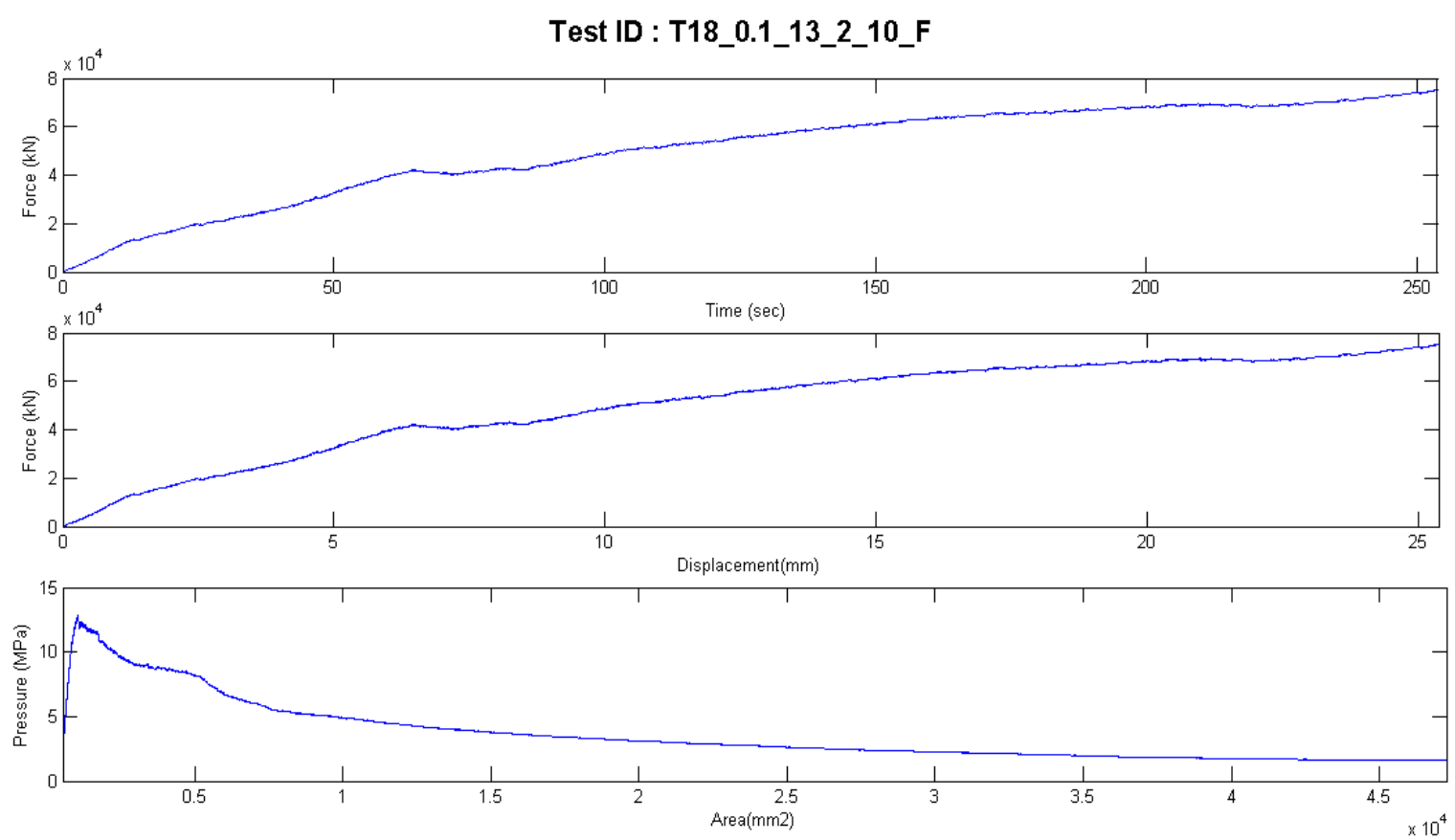

T18_0.1_13_2_10_F: Force and pressure traces

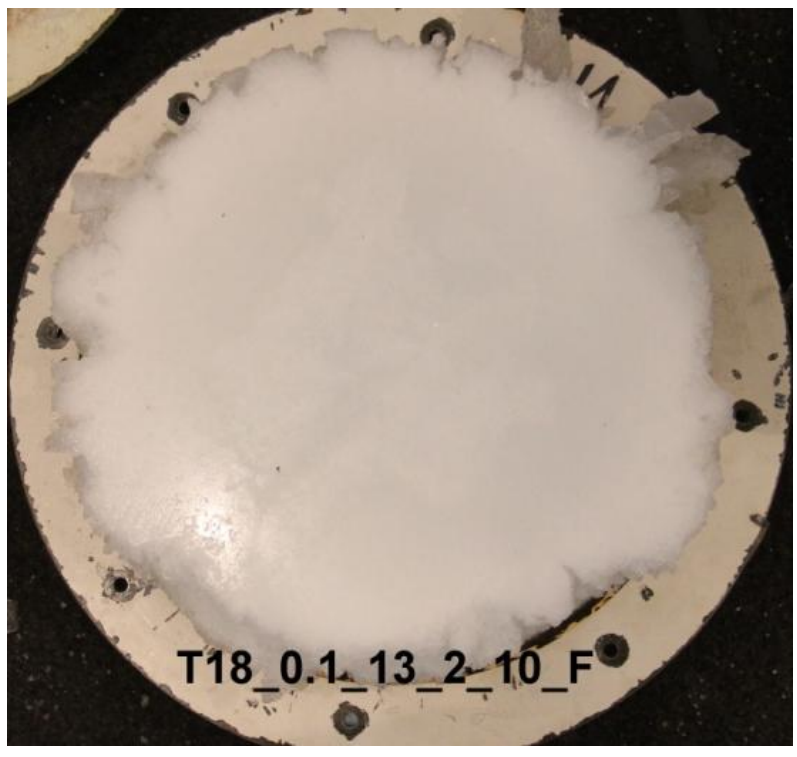

T18_0.1_13_2_10_F: After crushing picture 


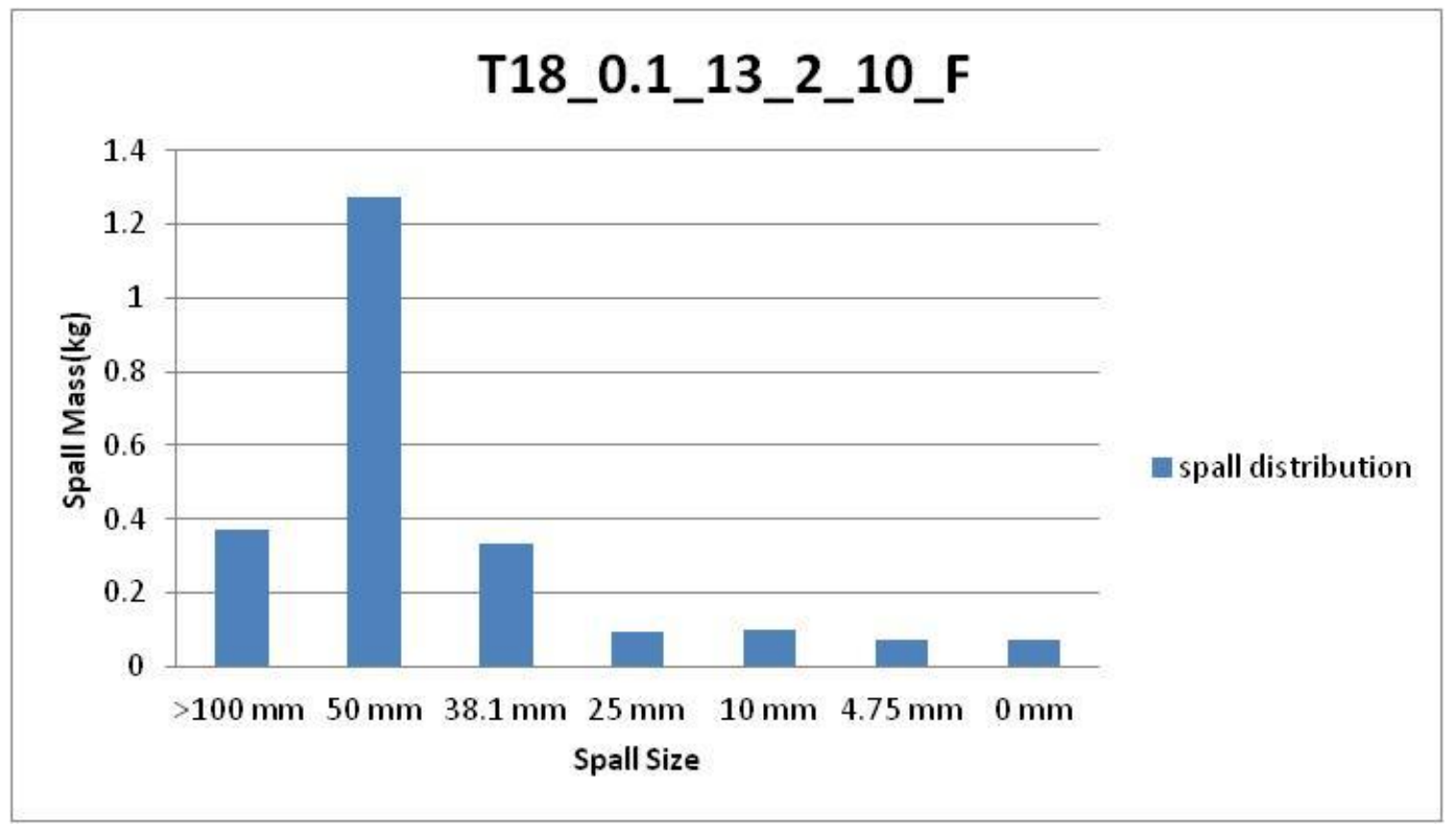

T18_0.1_13_2_10_F: Spall mass distributions
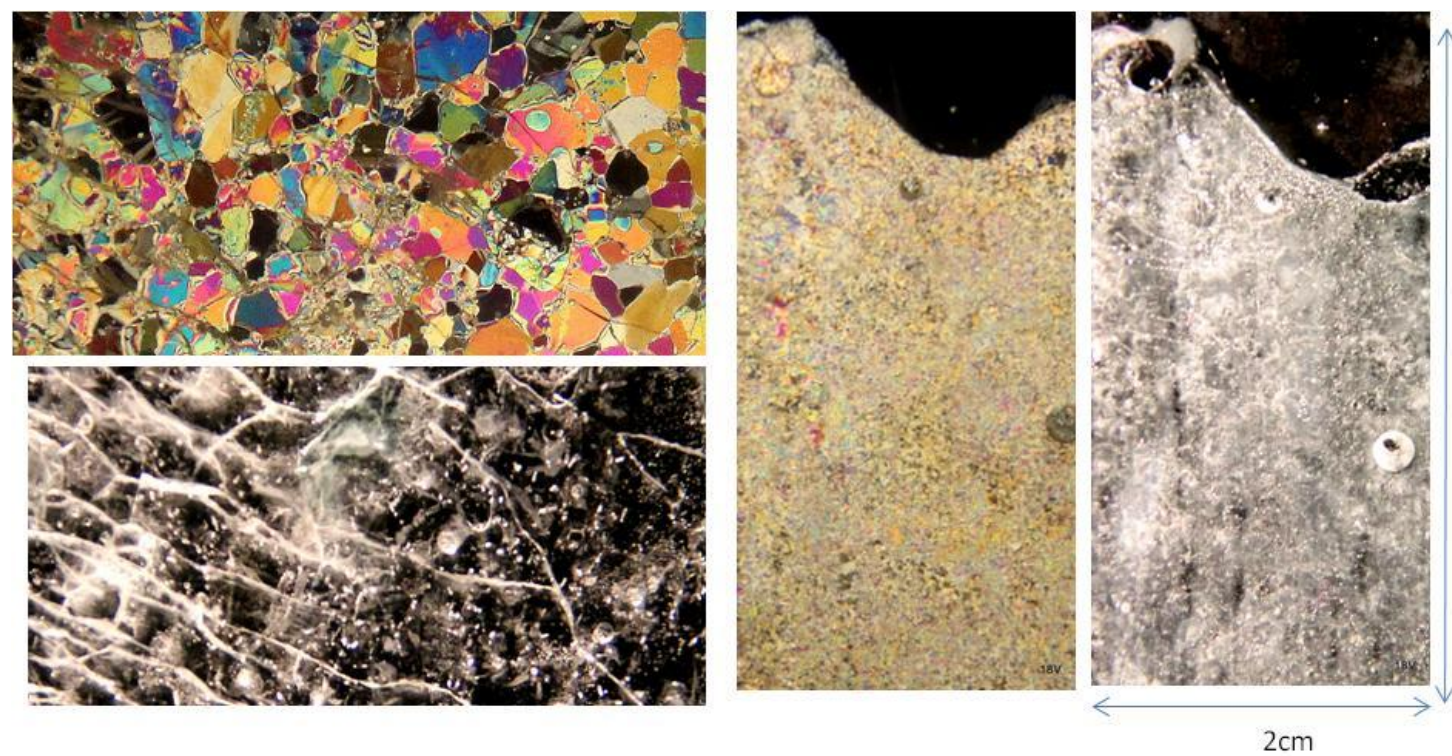

$4 \mathrm{~cm}$

T18_0.1_13_2_10_F: Thin section pictures: Horizontal (left) and Vertical (right) 
Test ID : T19_1_13_1_10_F
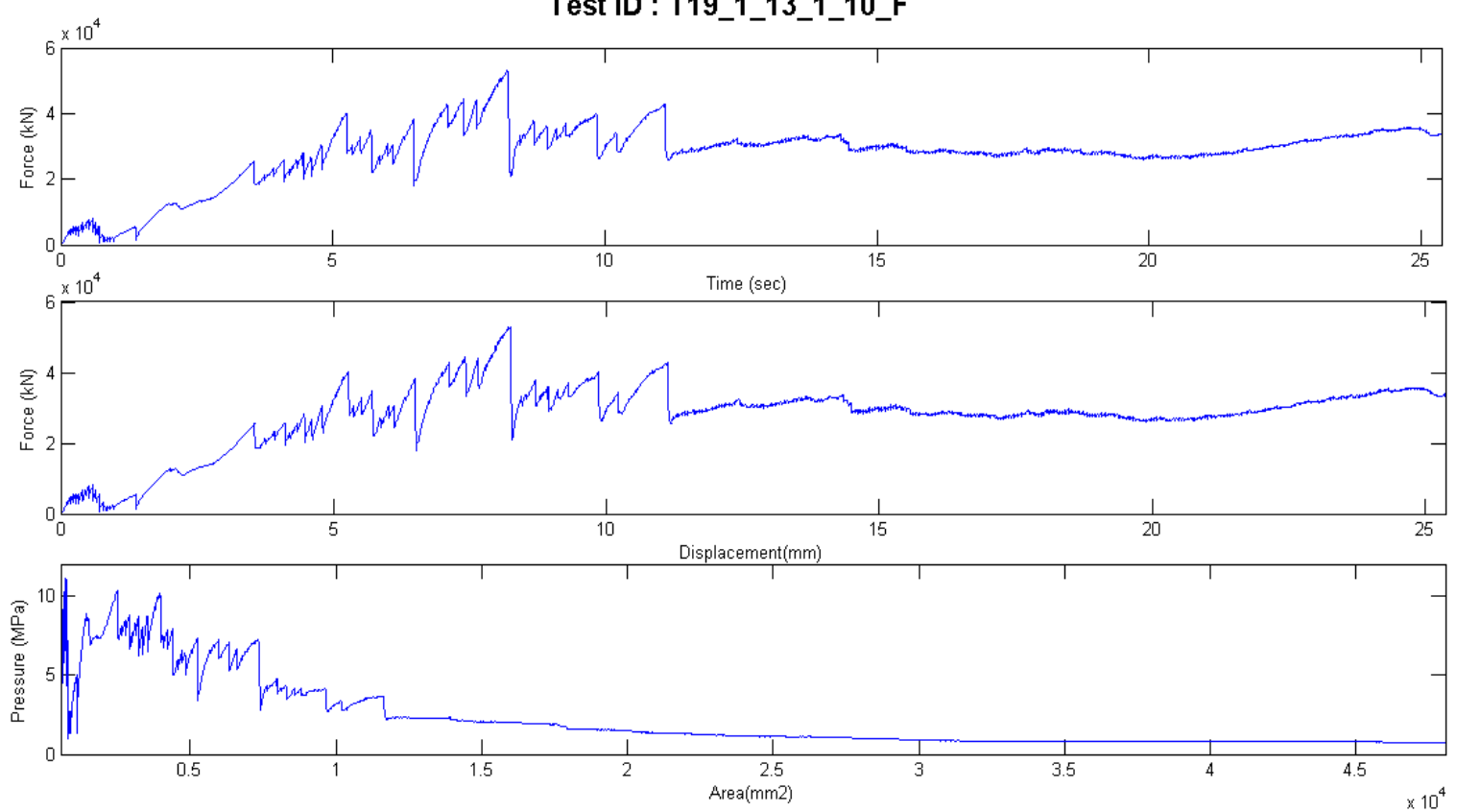

T19_1_13_1_10_F: Force and pressure traces

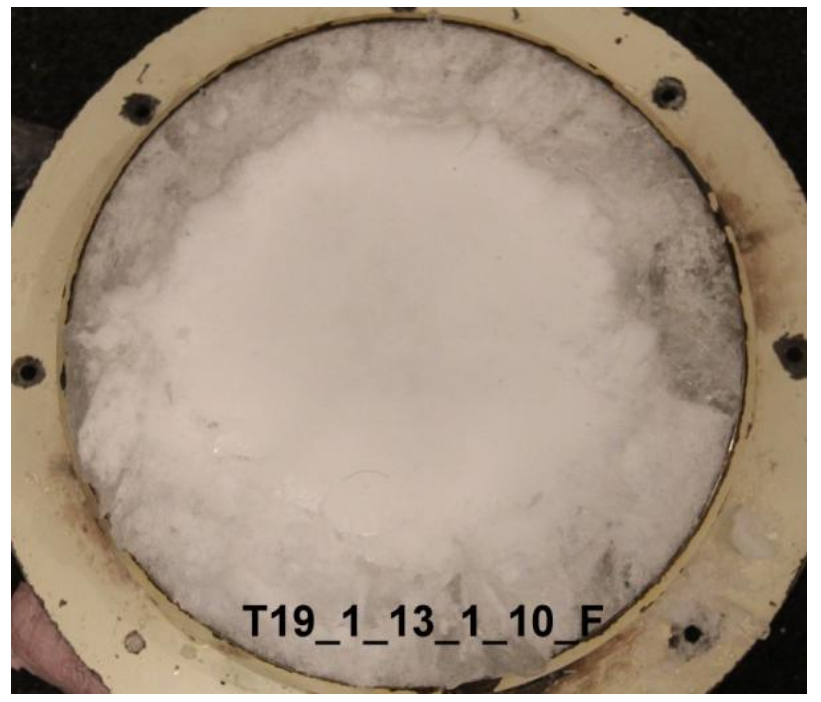

T19_1_13_1_10_F: After crushing picture 


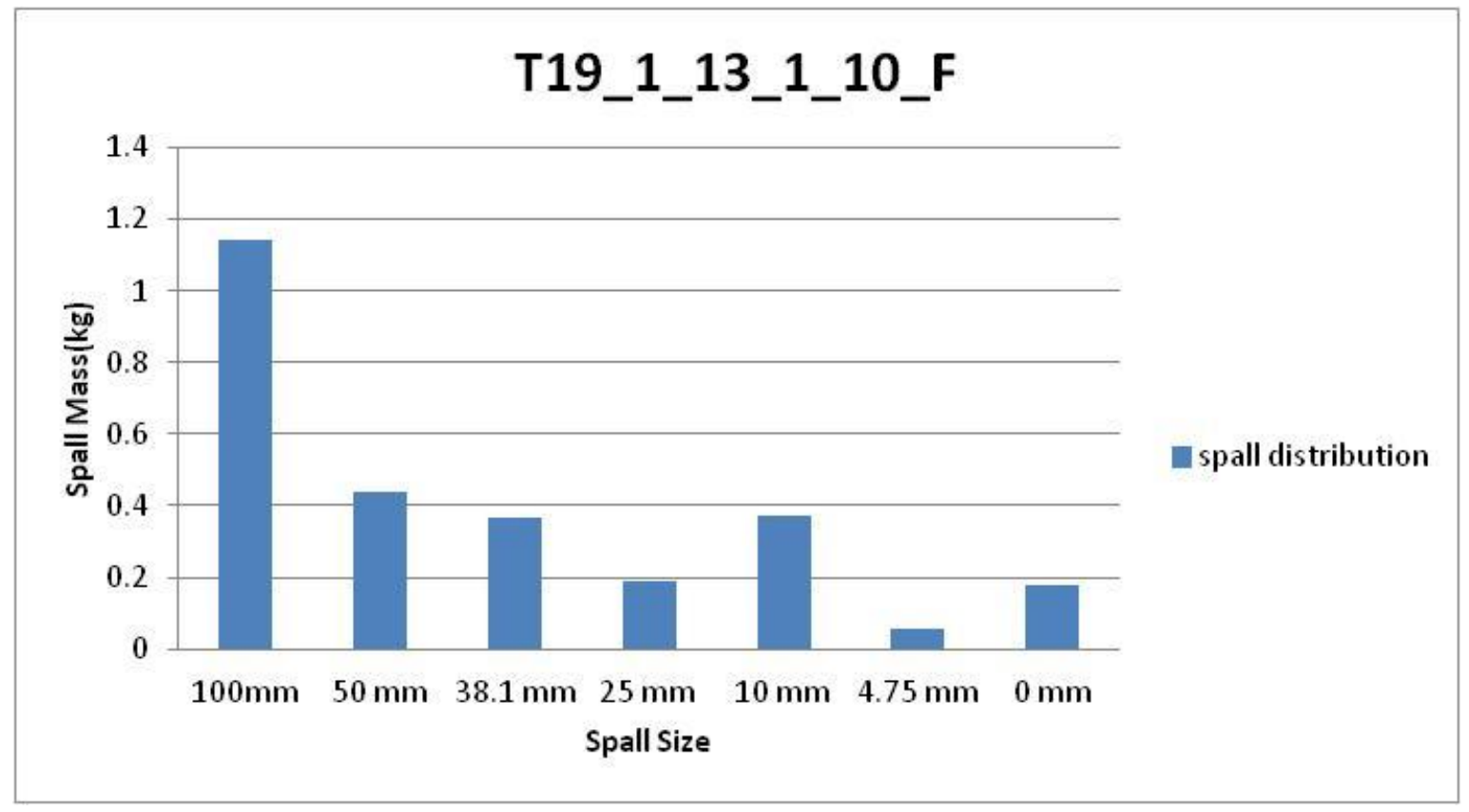

T19_1_13_1_10_F: Spall mass distributions
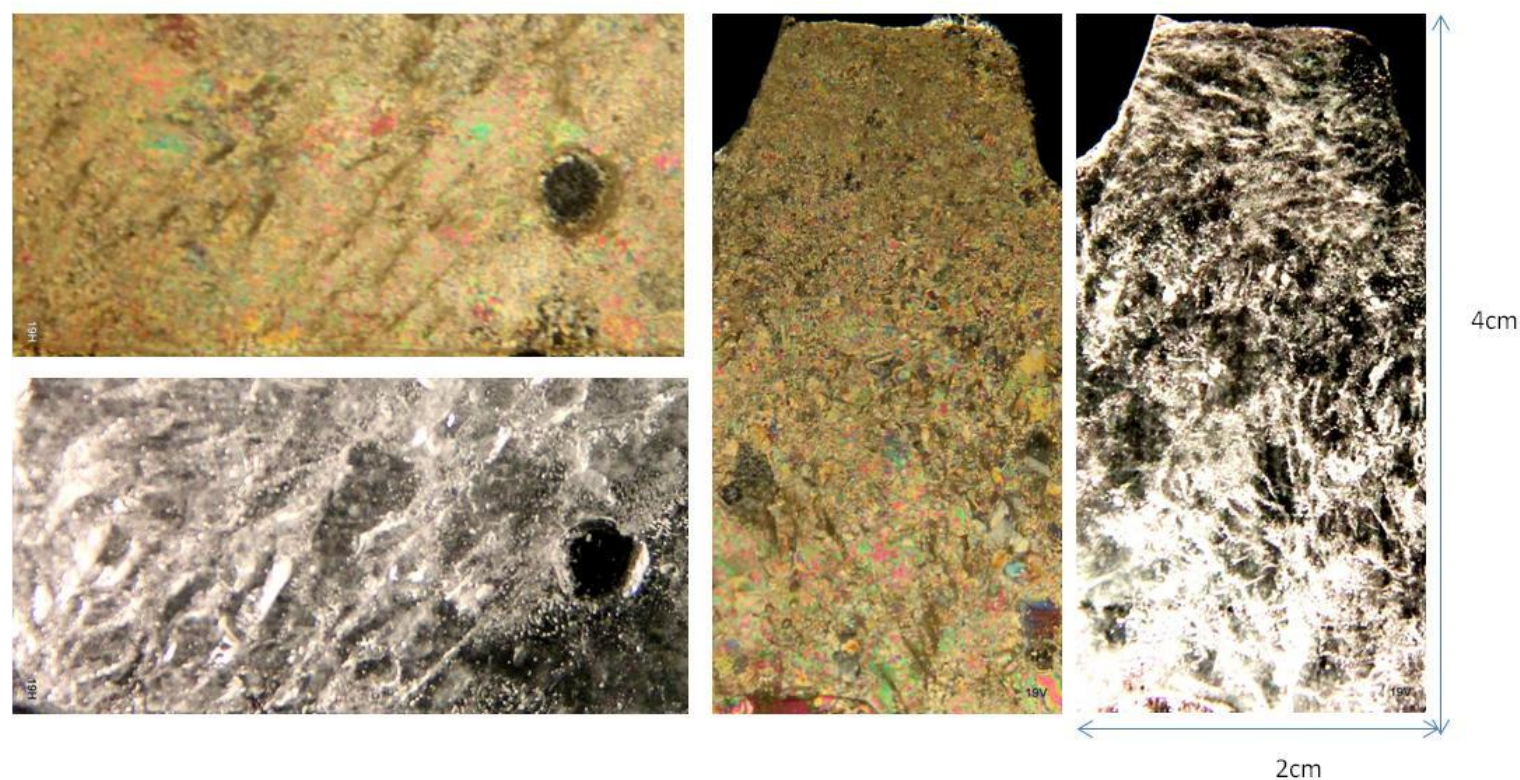

T19_1_13_1_10_F: Thin section pictures: Horizontal (left) and Vertical (right) 


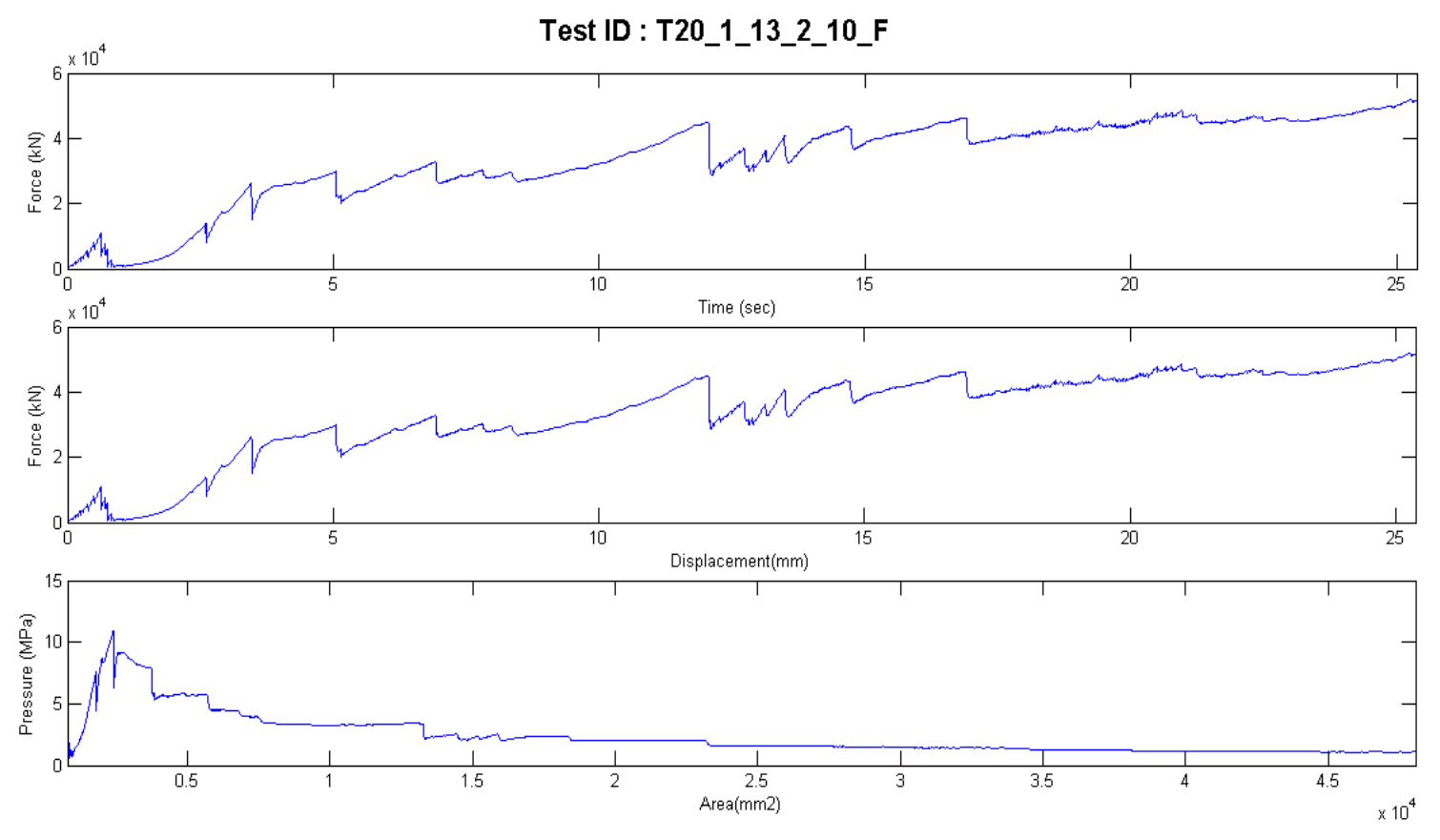

T20_1_13_2_10_F: Force and pressure traces

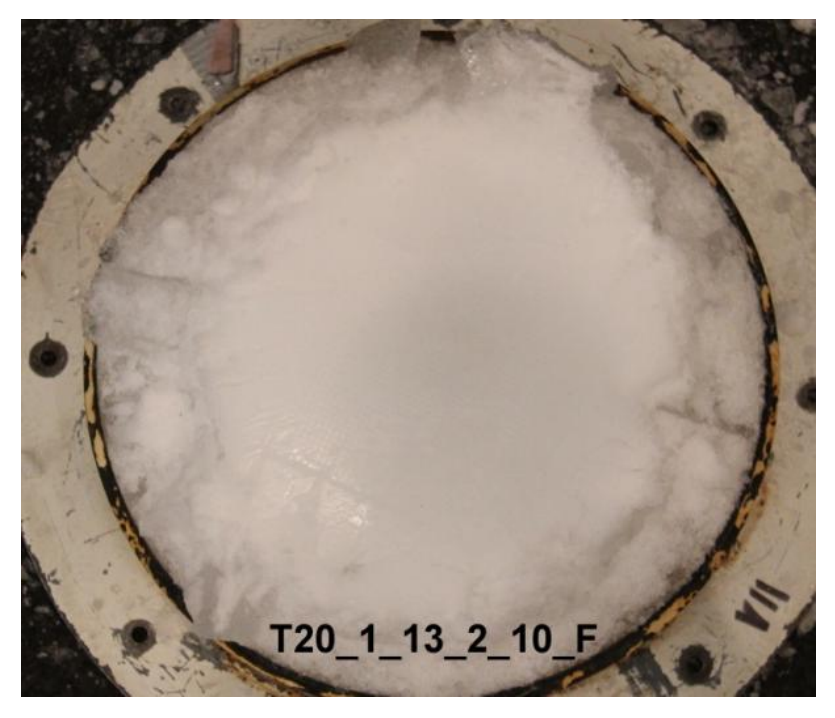

T20_1_13_2_10_F: After crushing picture 


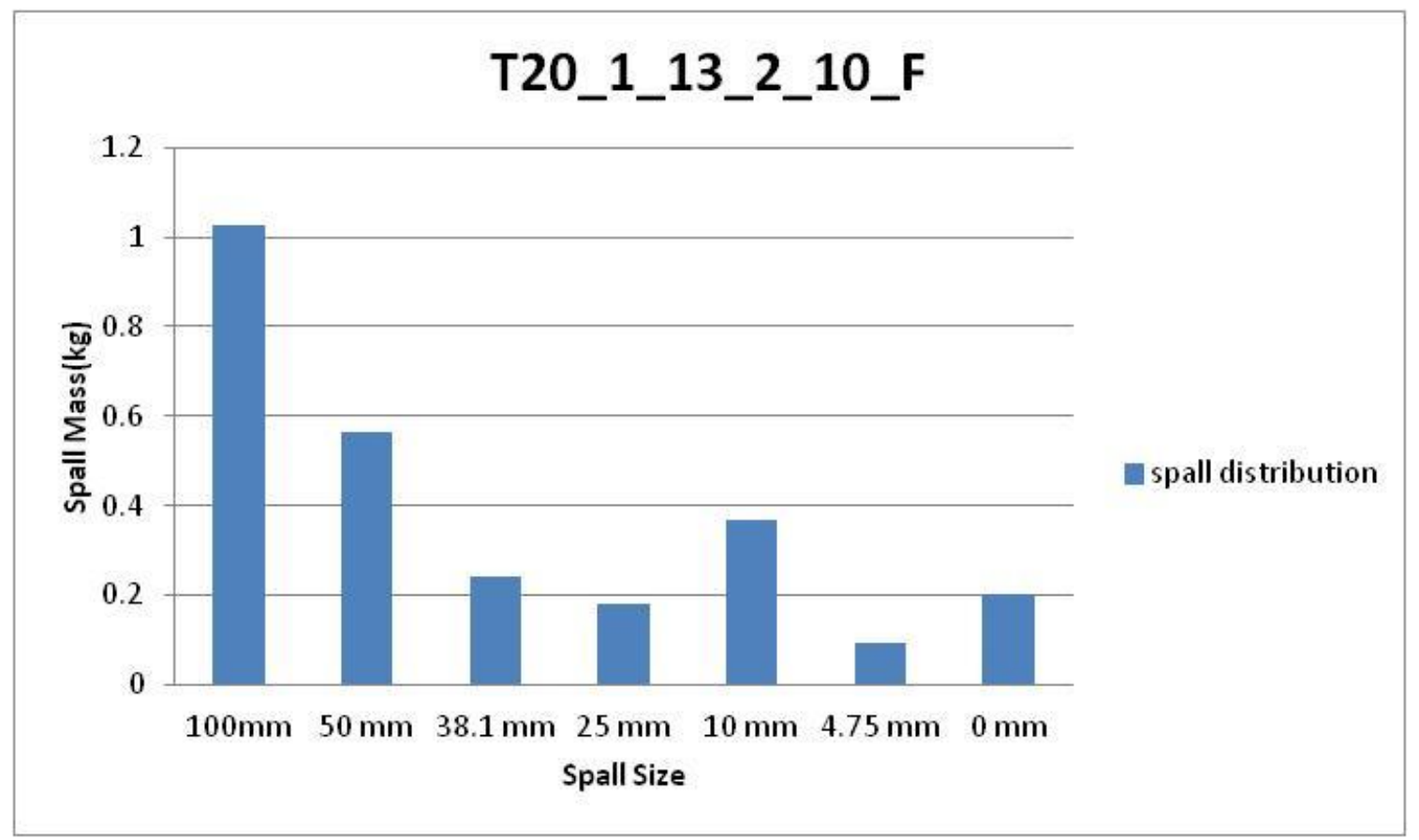

T20_1_13_2_10_F: Spall mass distributions
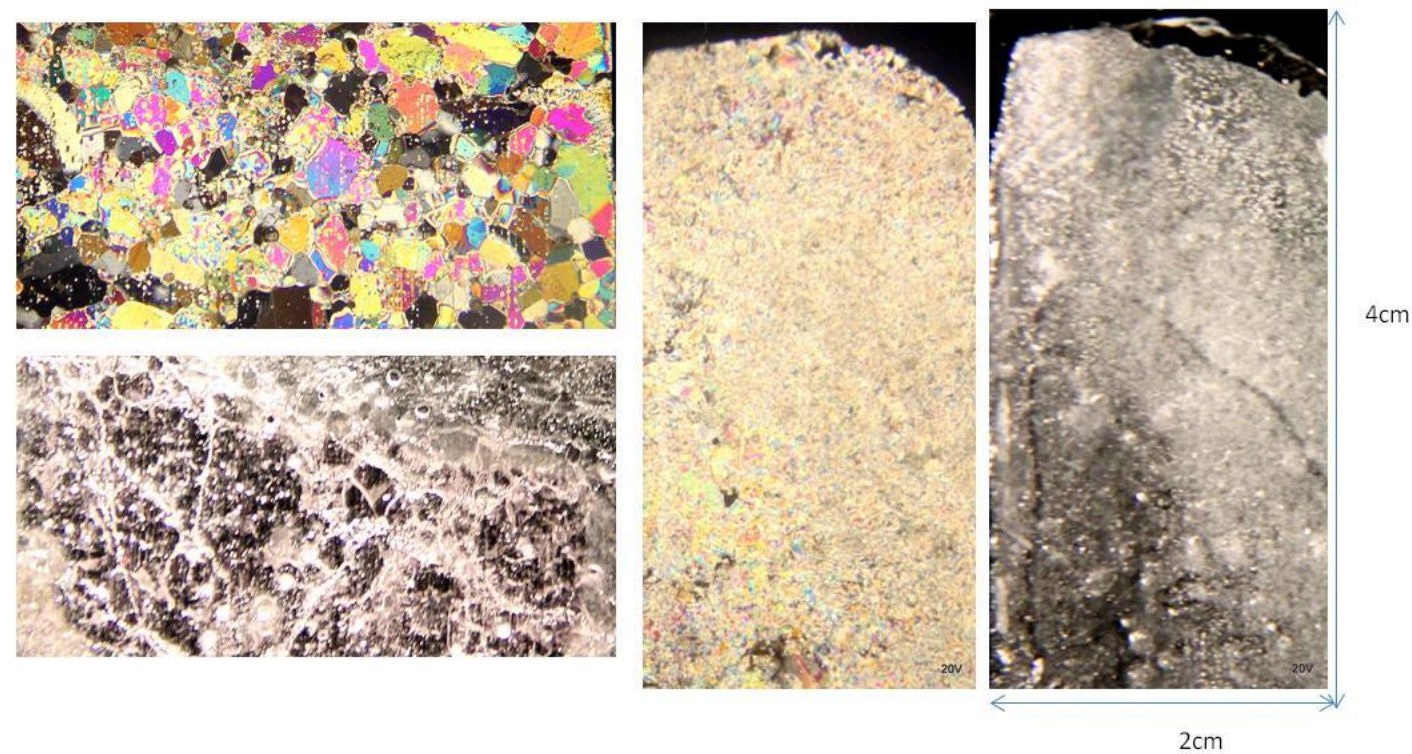

T20_1_13_2_10_F: Thin section pictures: Horizontal (left) and Vertical (right) 
Test ID : T21_10_13_1_10_F
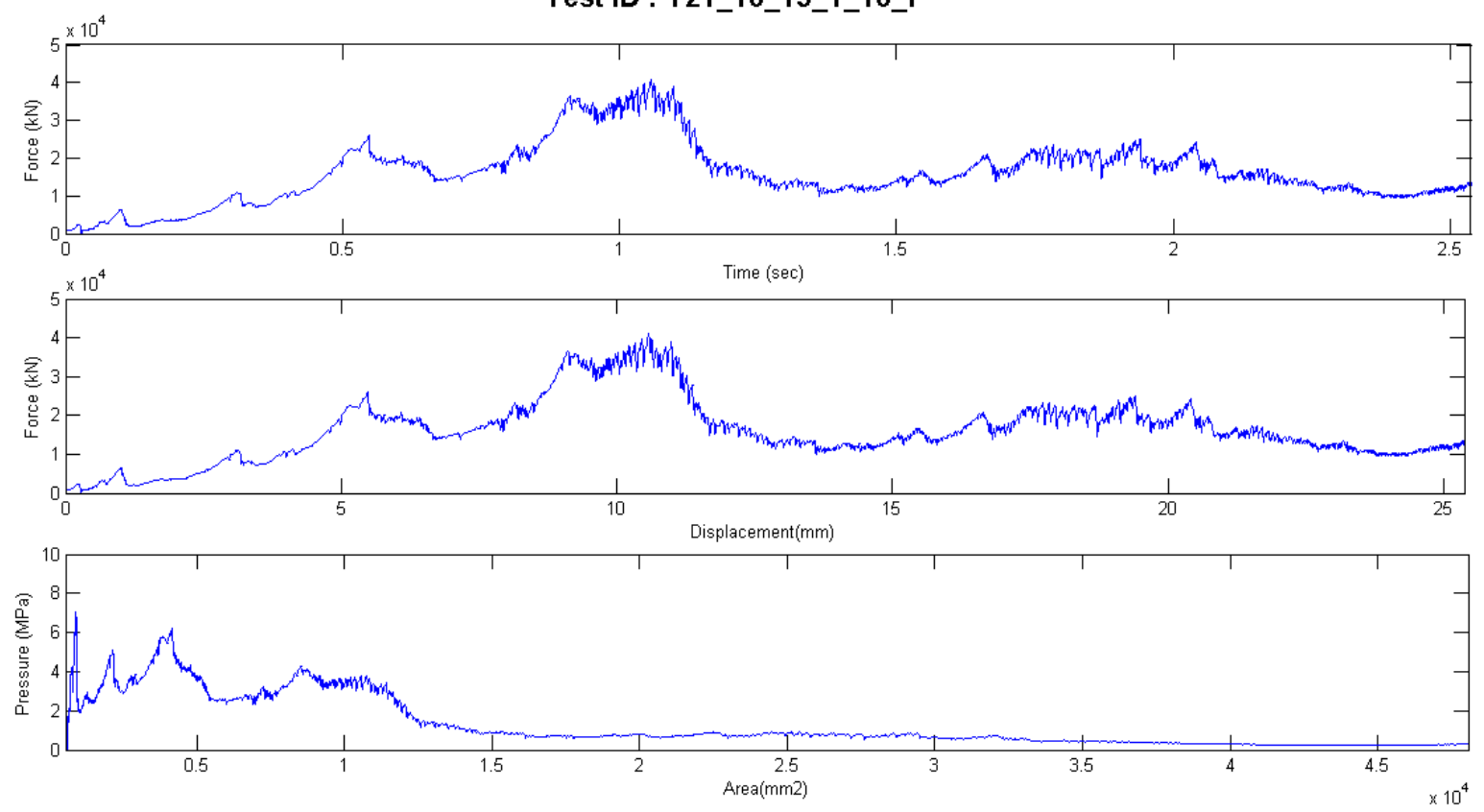

T21_10_13_1_10_F: Force and pressure traces

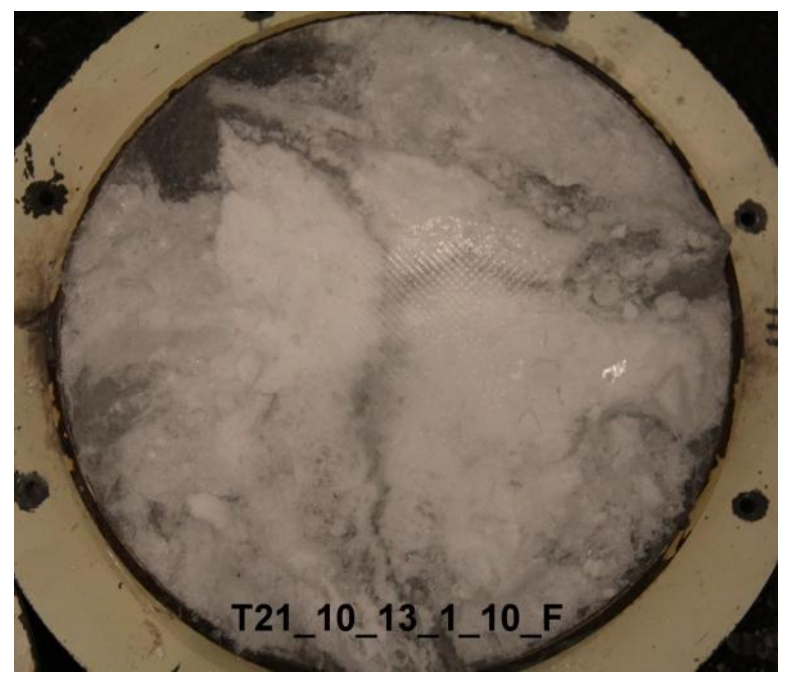

T21_10_13_1_10_F: After crushing picture 


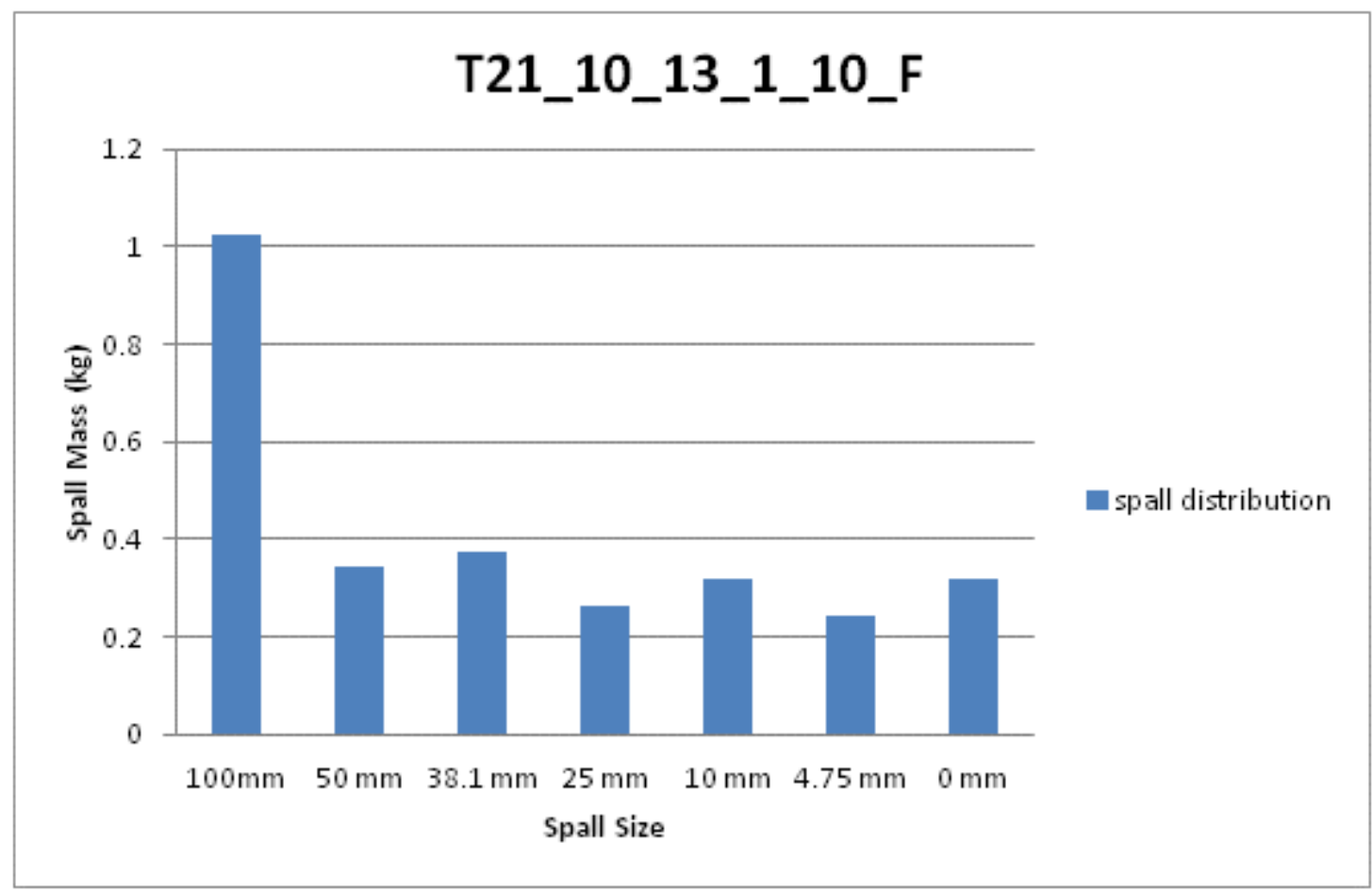

T21_10_13_1_10_F: Spall mass distributions
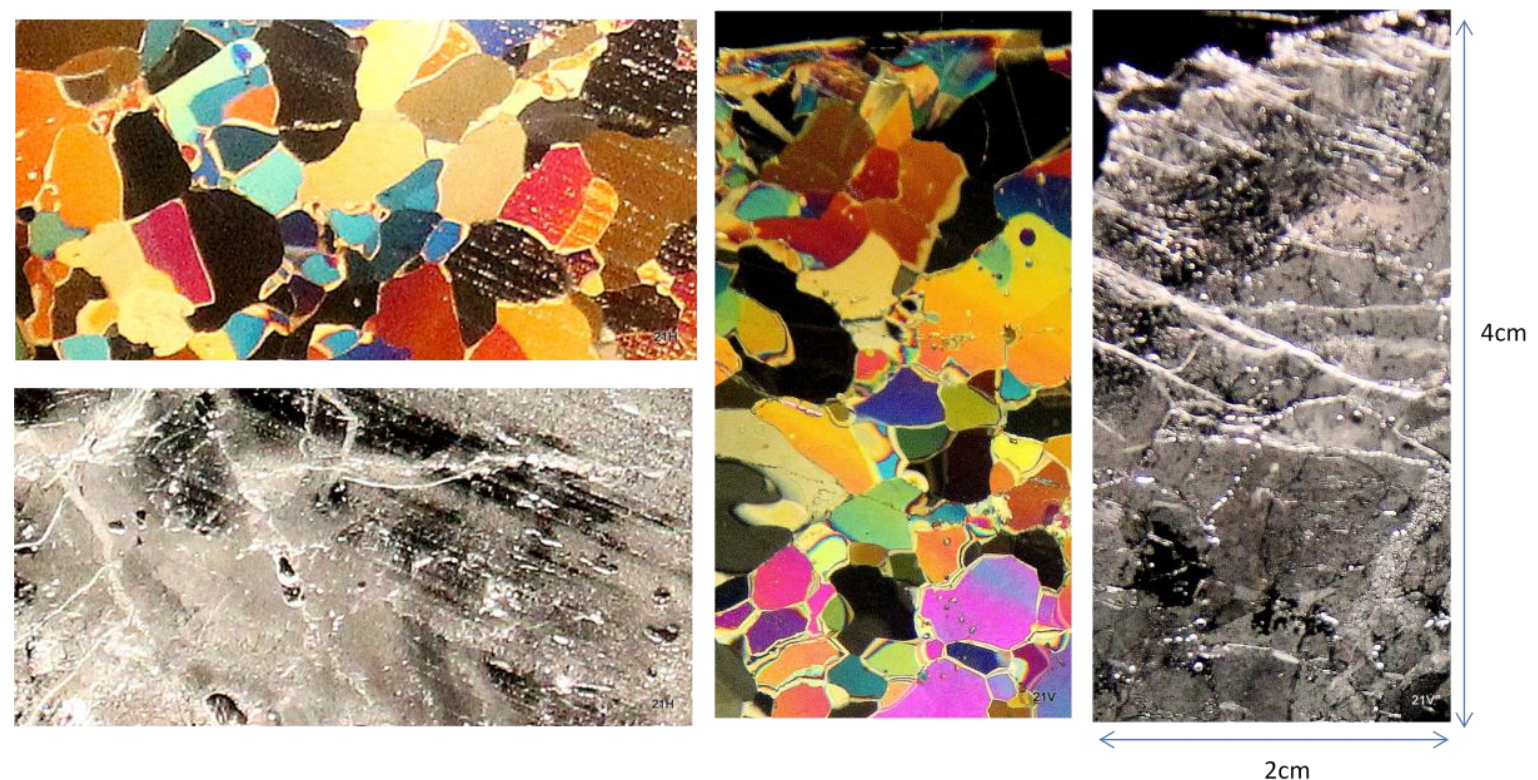

T21_10_13_1_10_F: Thin section pictures: Horizontal (left) and Vertical (right) 

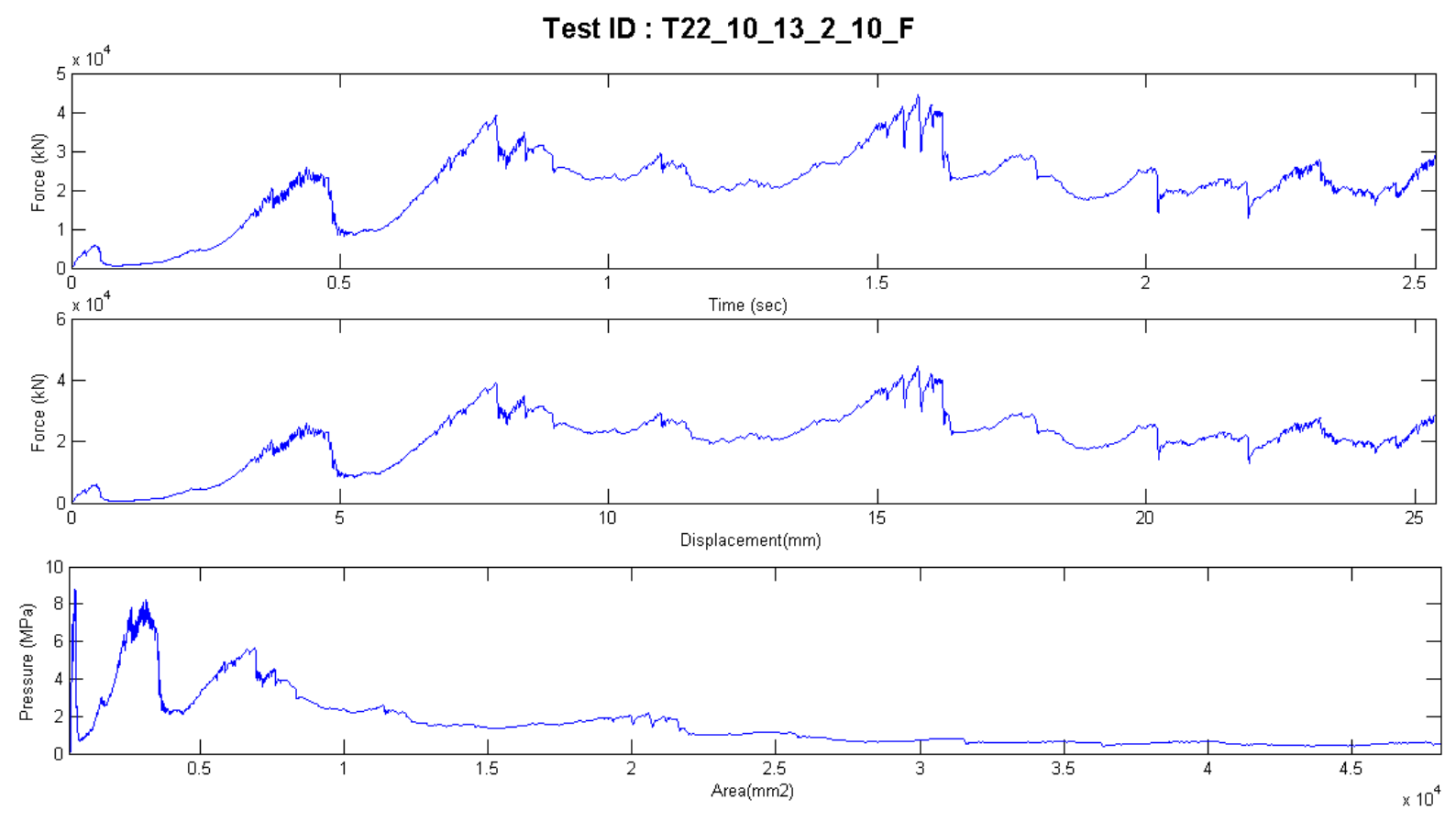

T22_10_13_2_10_F: Force and pressure traces

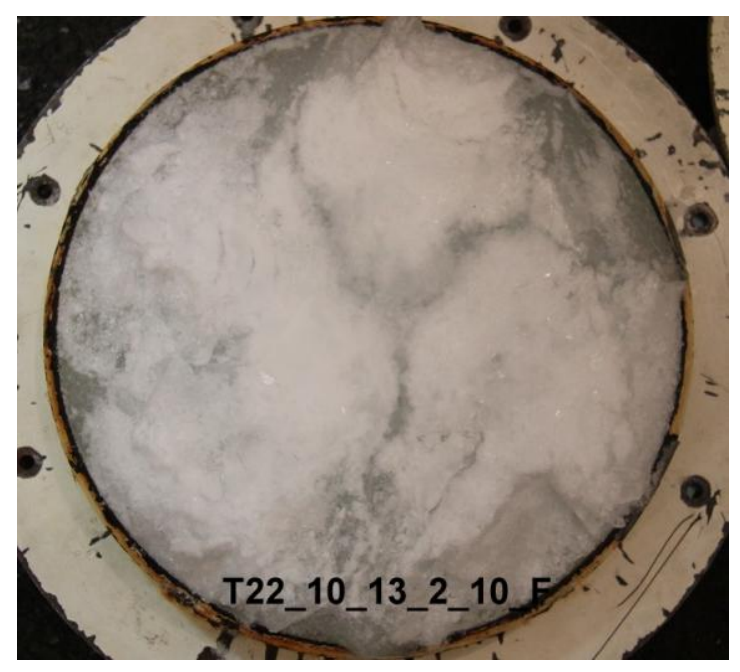

T22_10_13_2_10_F: After crushing picture 


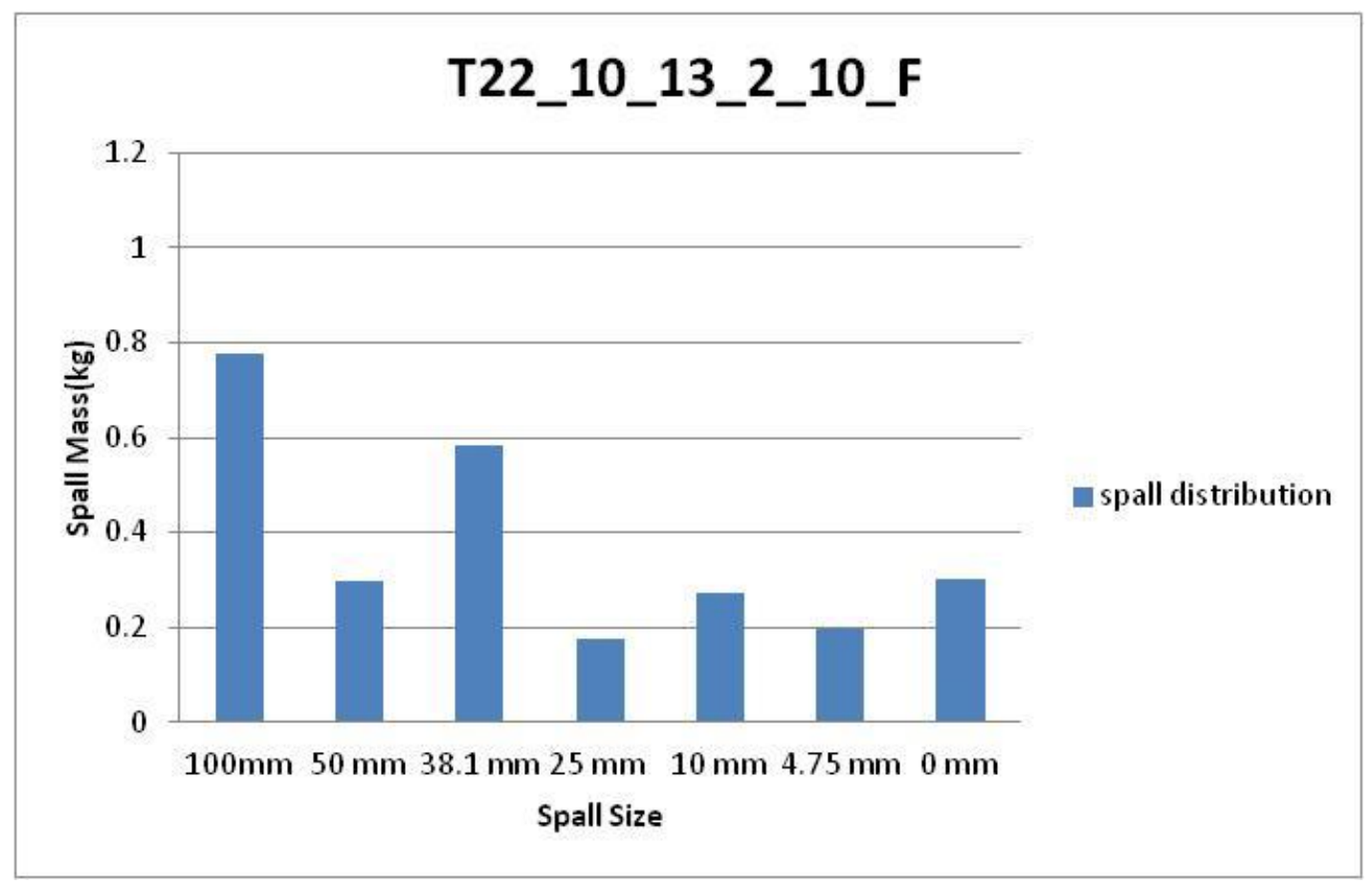

T22_10_13_2_10_F: Spall mass distributions
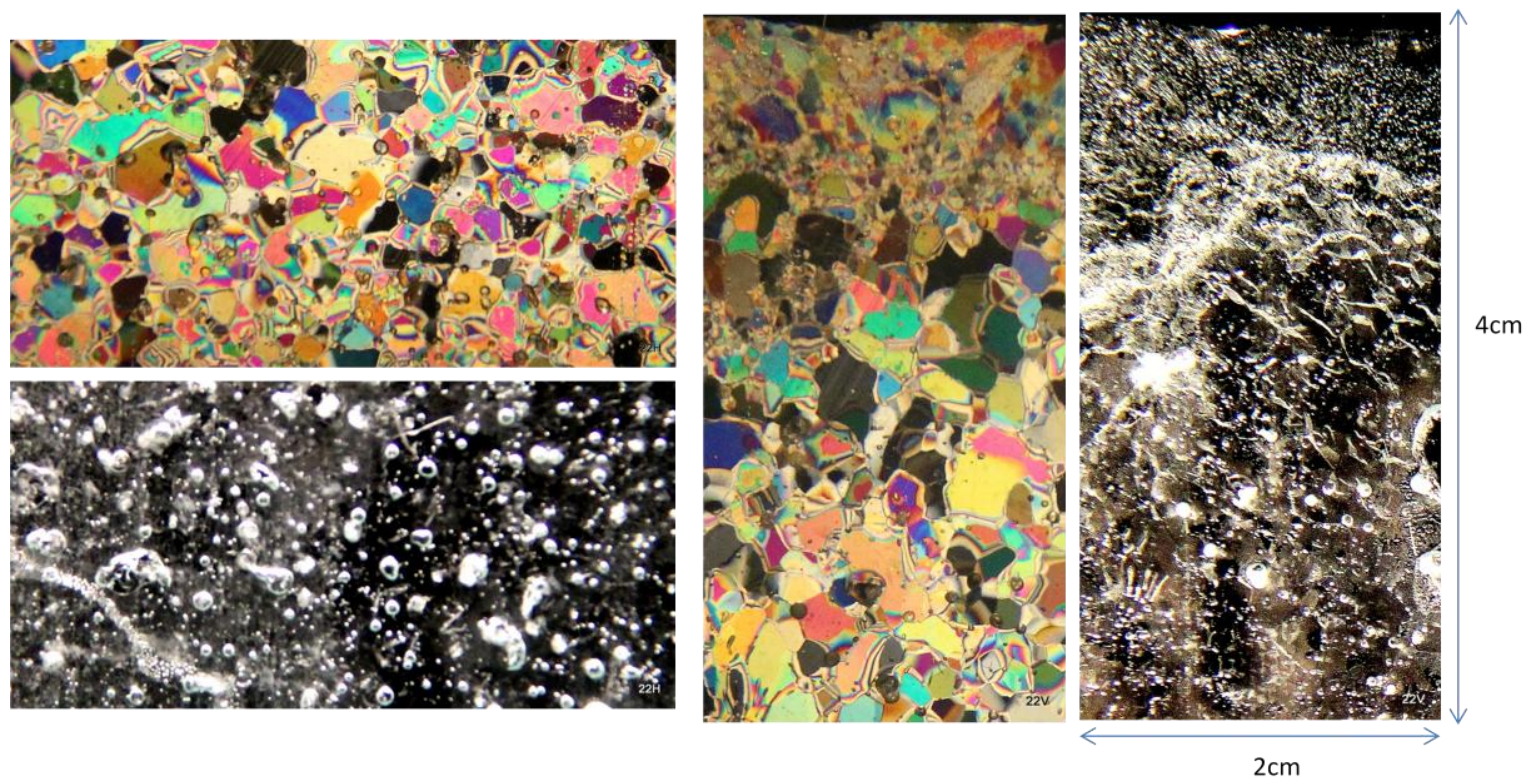

T22_10_13_2_10_F: Thin section pictures: Horizontal (left) and Vertical (right) 
Test ID : T23_10_13_1_10_S
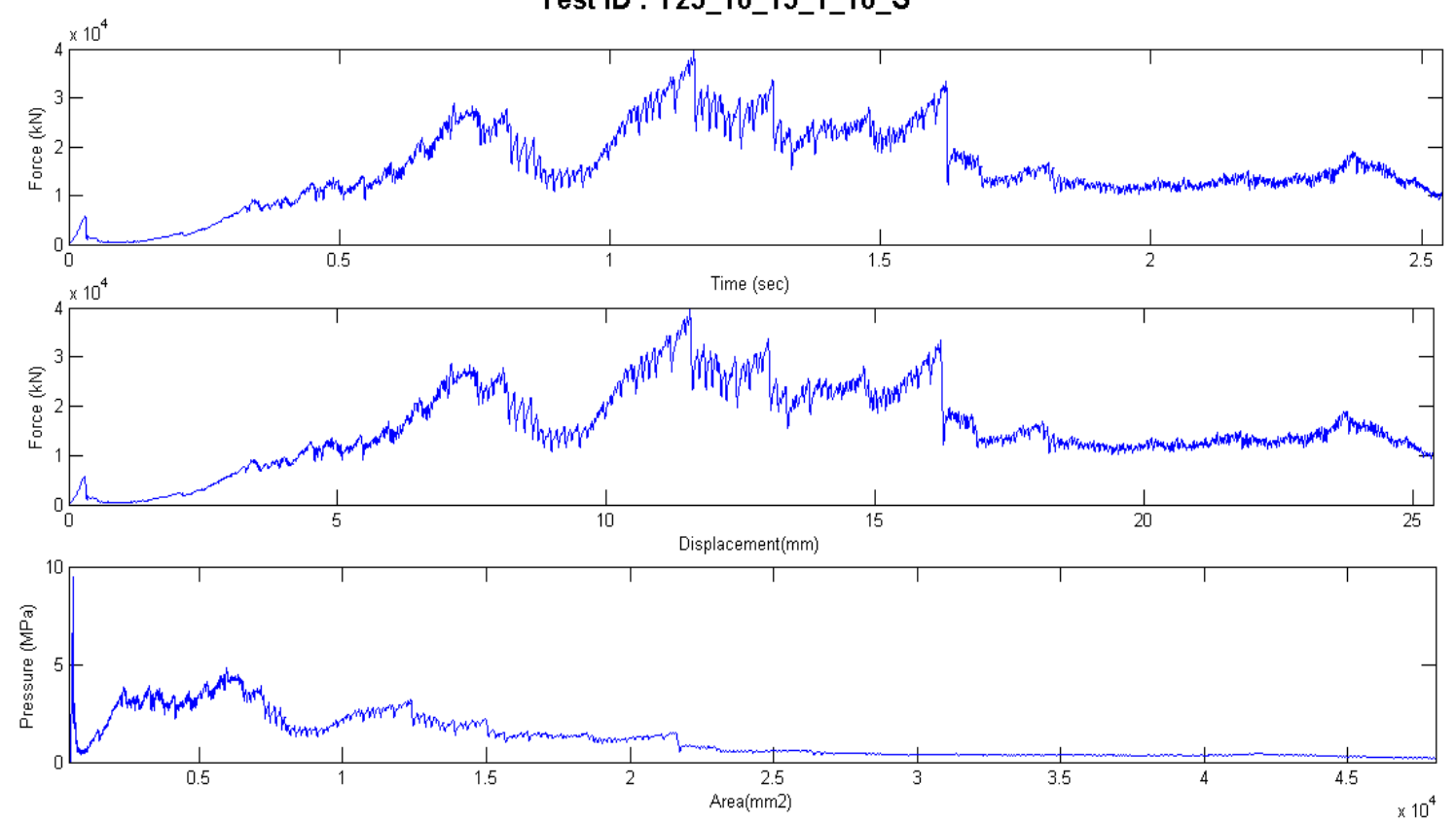

T23_10_13_1_10_S: Spall mass distributions: Force and pressure traces

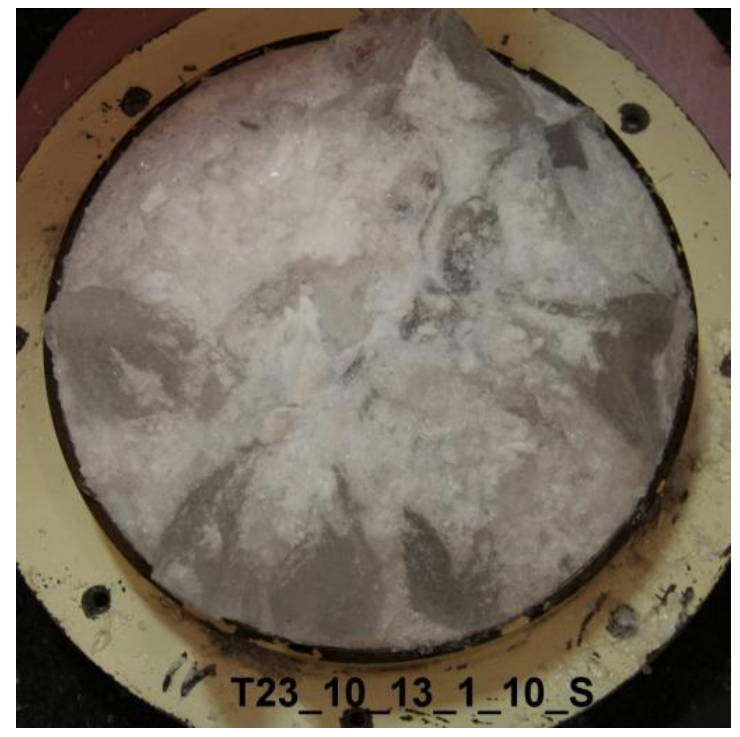

T23_10_13_1_10_S: After crushing picture 


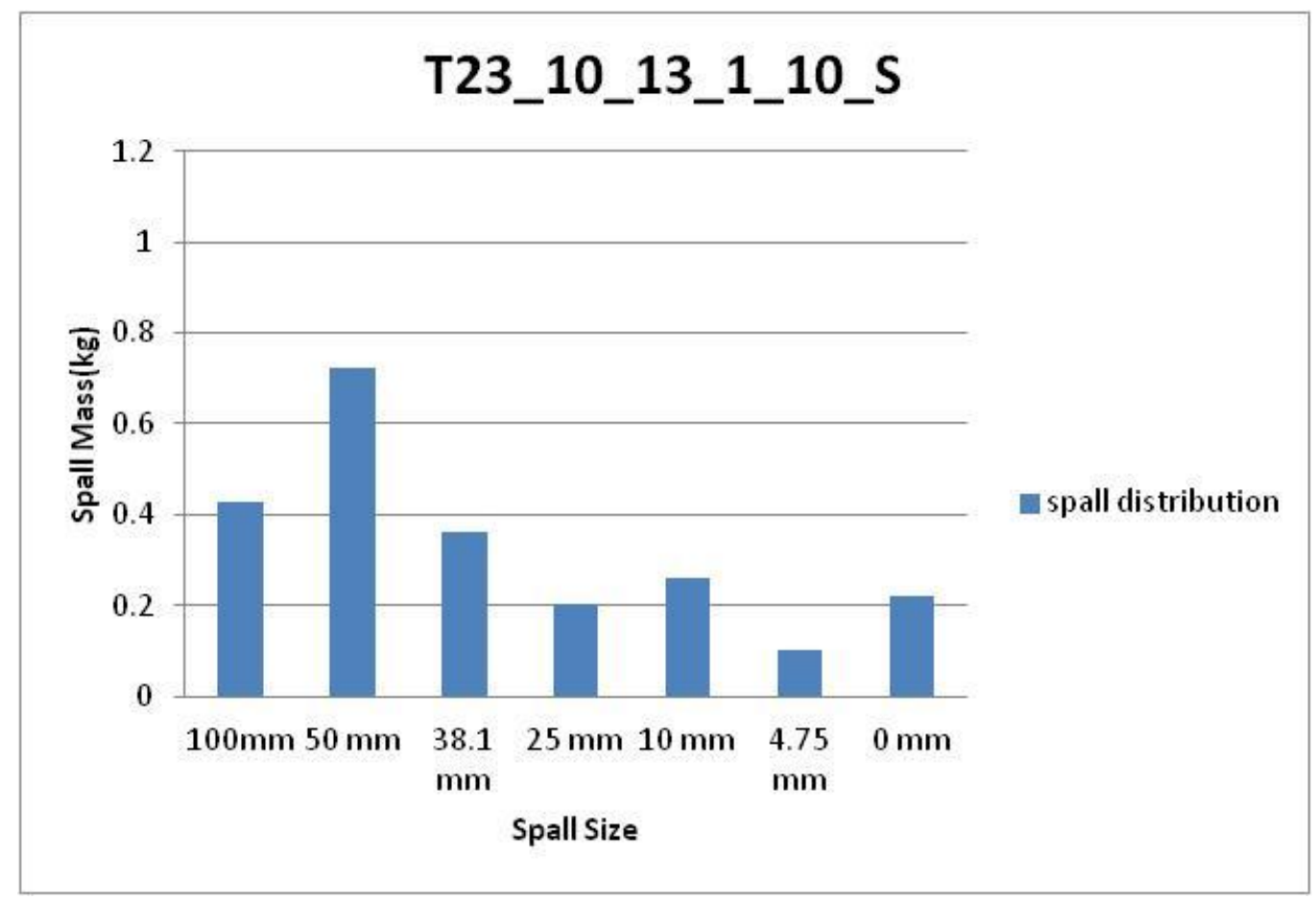

T23_10_13_1_10_S: Spall mass distributions
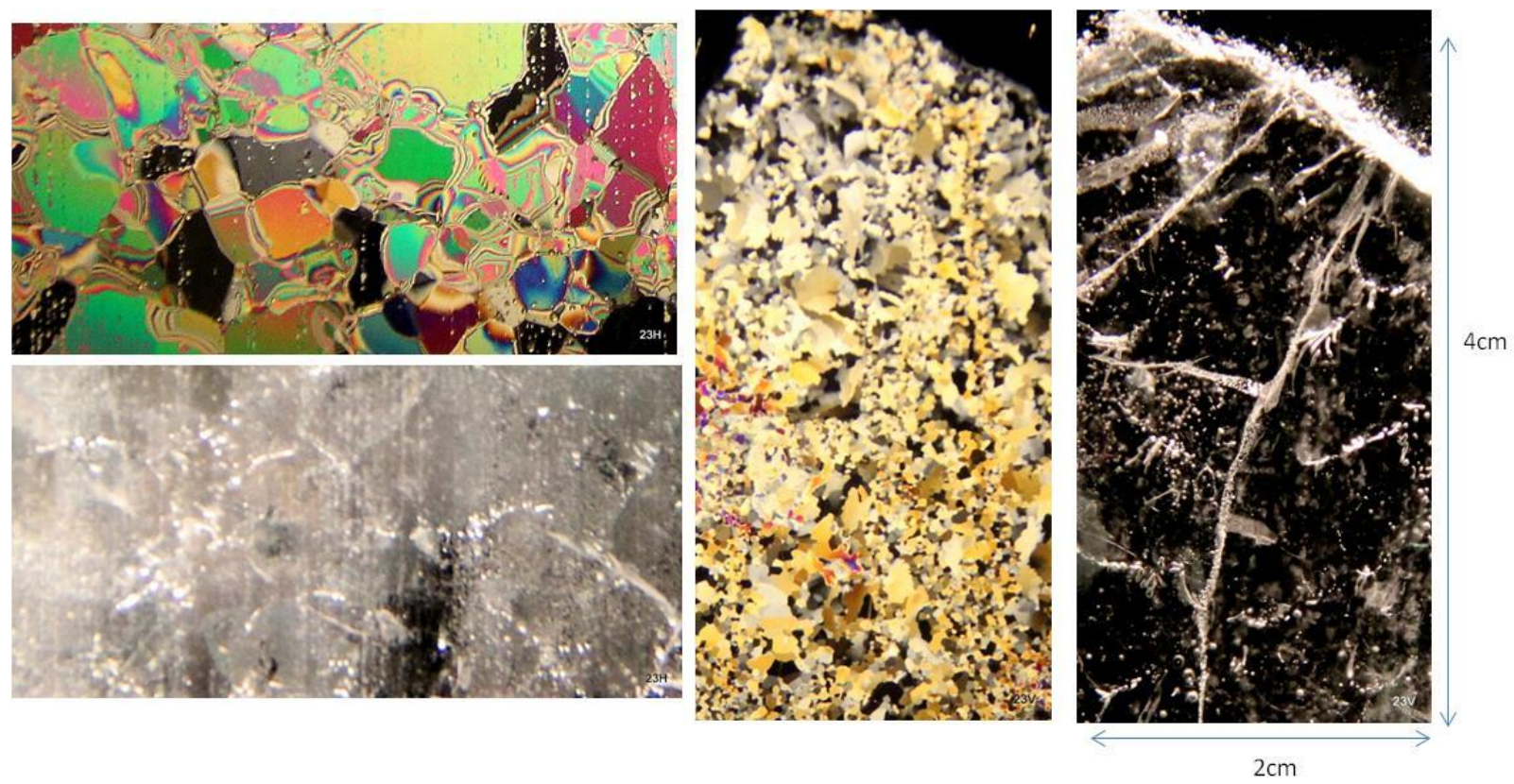

T23_10_13_1_10_S: Thin section pictures: Horizontal (left) and Vertical (right) 
Test ID : T24_1_13_1_10_S
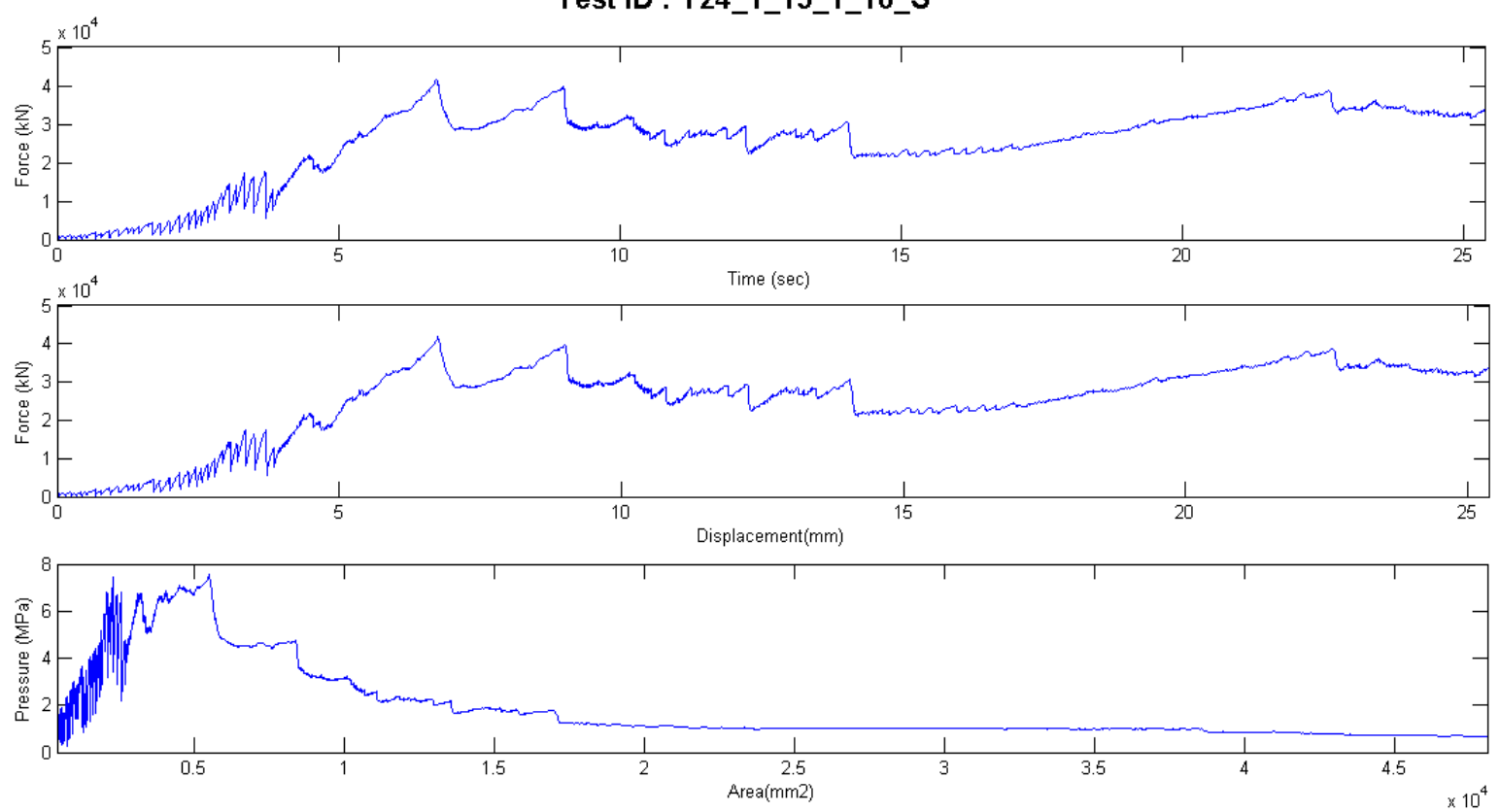

T24_1_13_1_10_S: Force and pressure traces

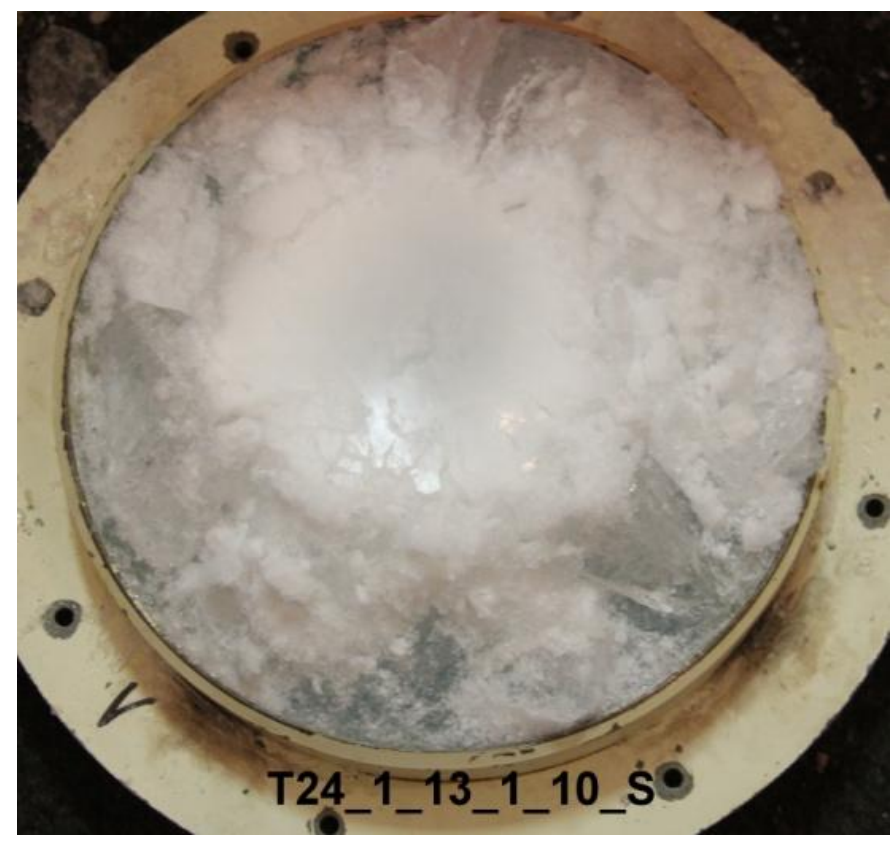

T24_1_13_1_10_S: After crushing picture 


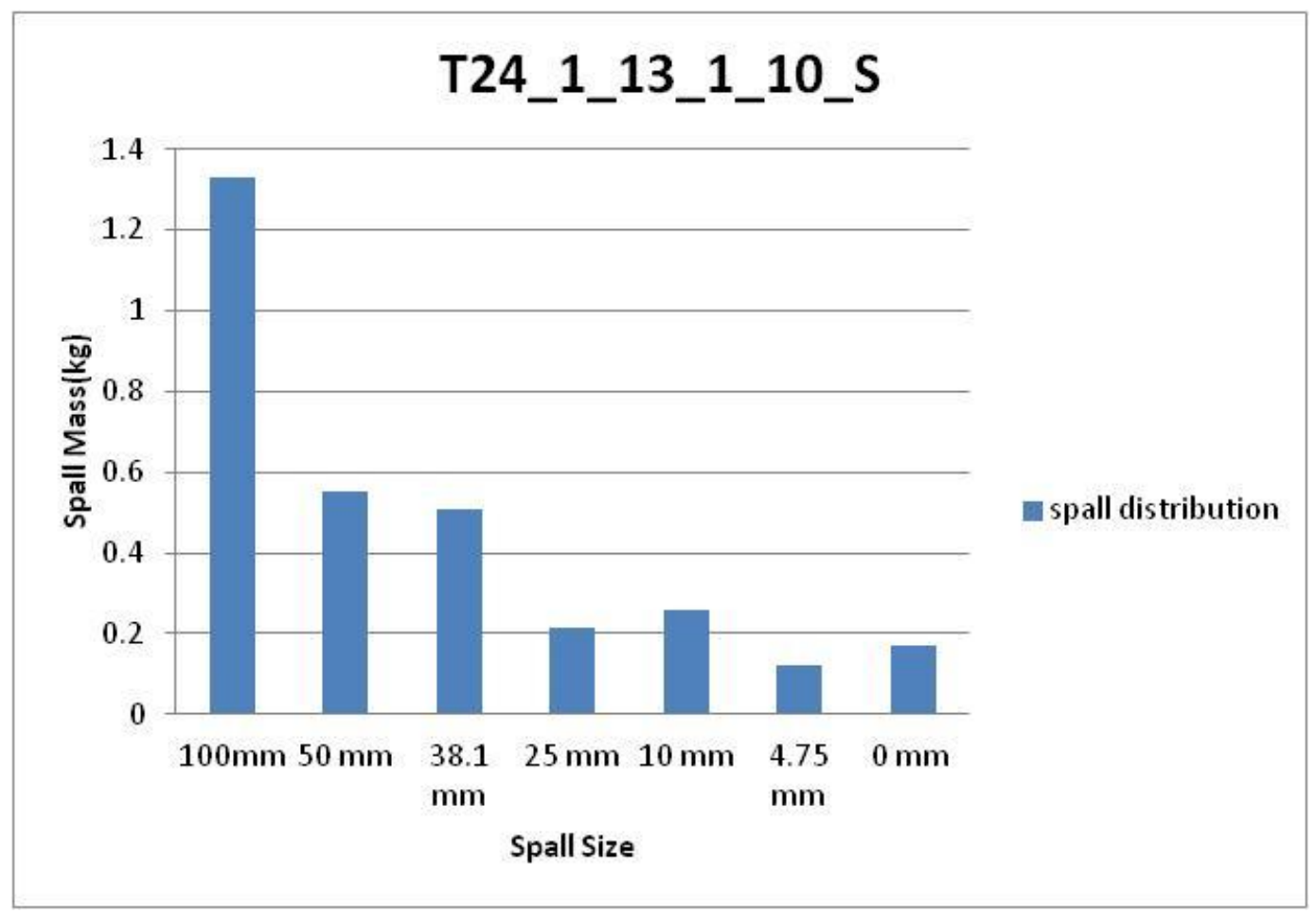

T24_1_13_1_10_S: Spall mass distributions
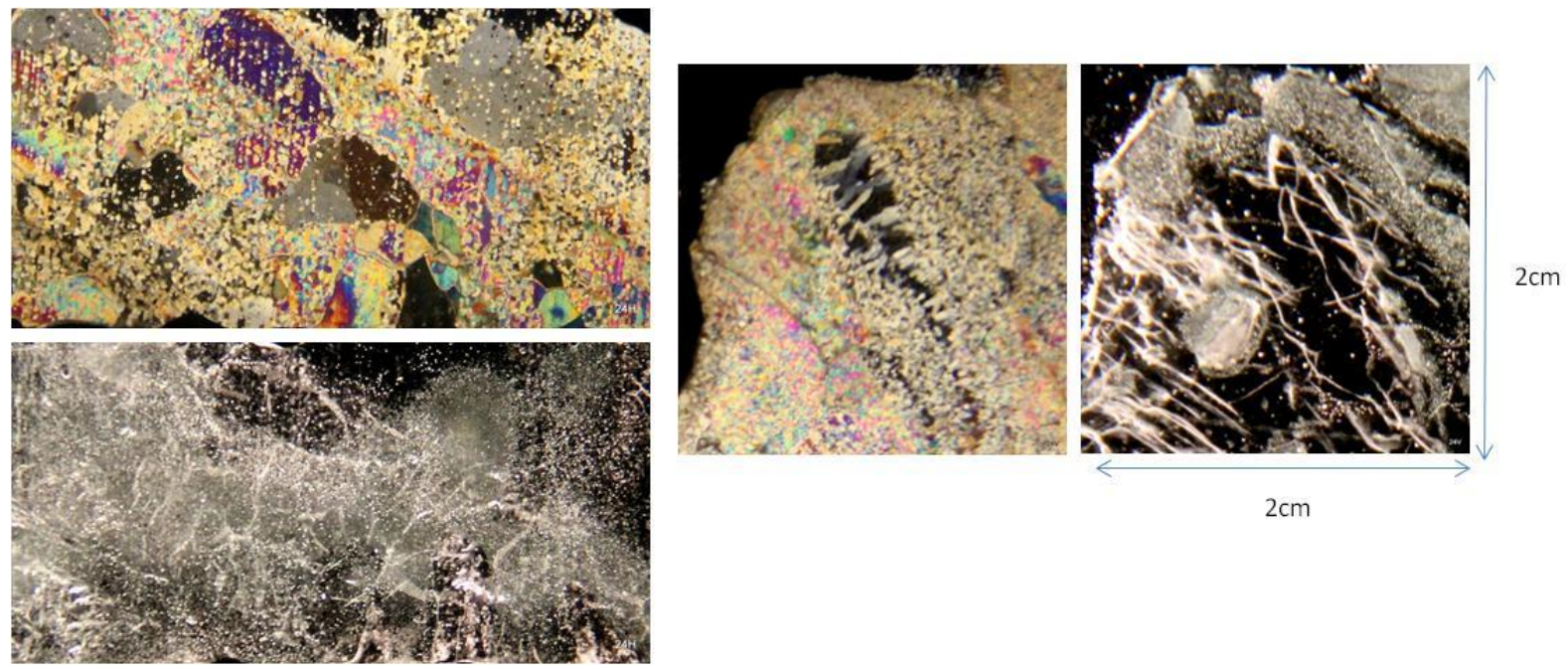

T24_1_13_1_10_S: Thin section pictures: Horizontal (left) and Vertical (right) 

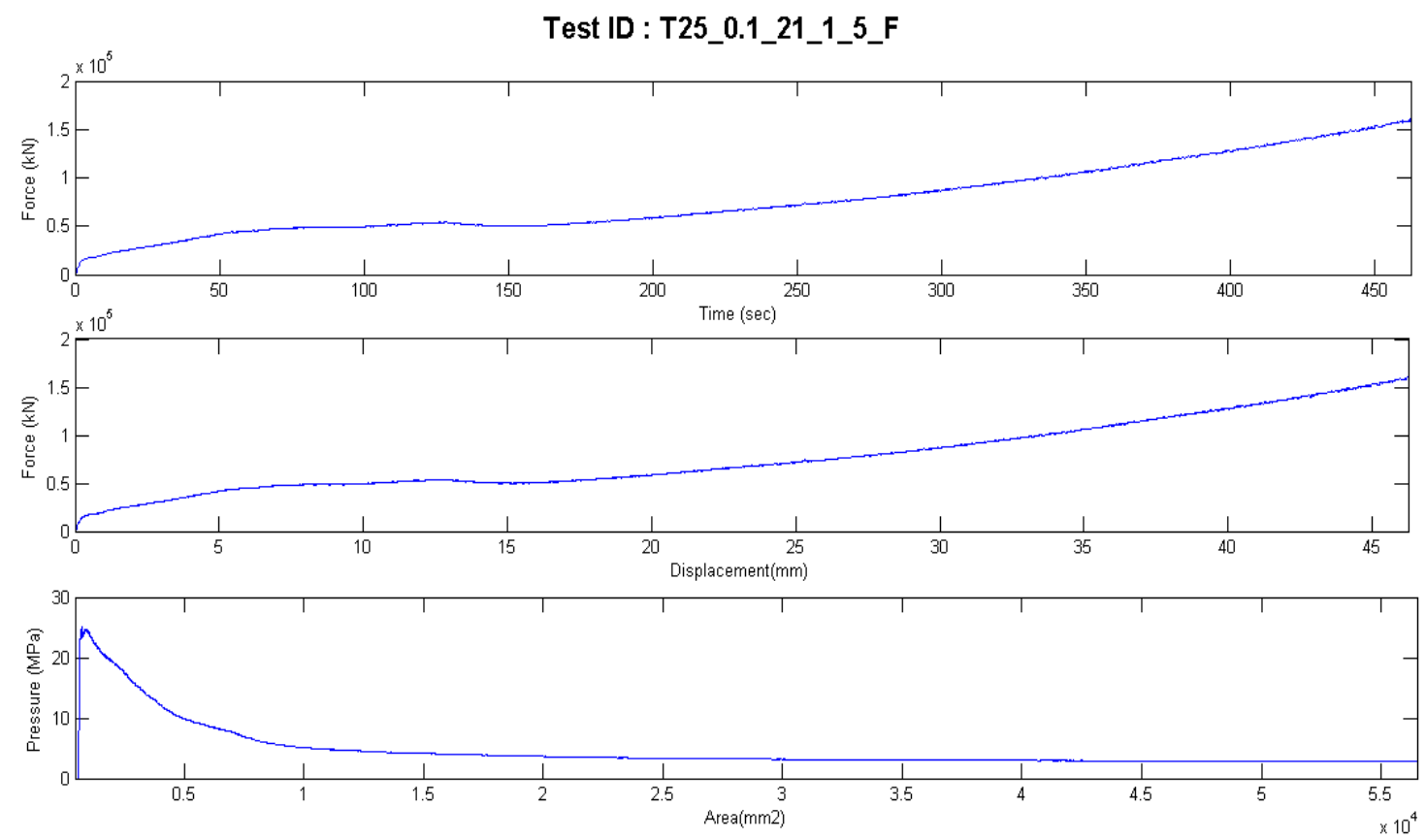

T25_0.1_21_1_5_F: Force and pressure traces

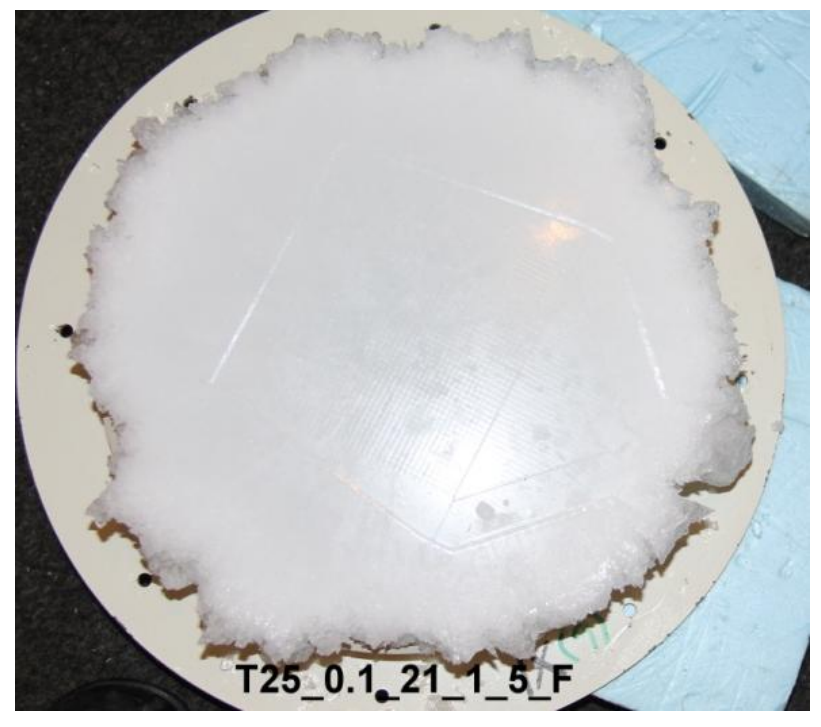

T25_0.1_21_1_5_F: After crushing picture 


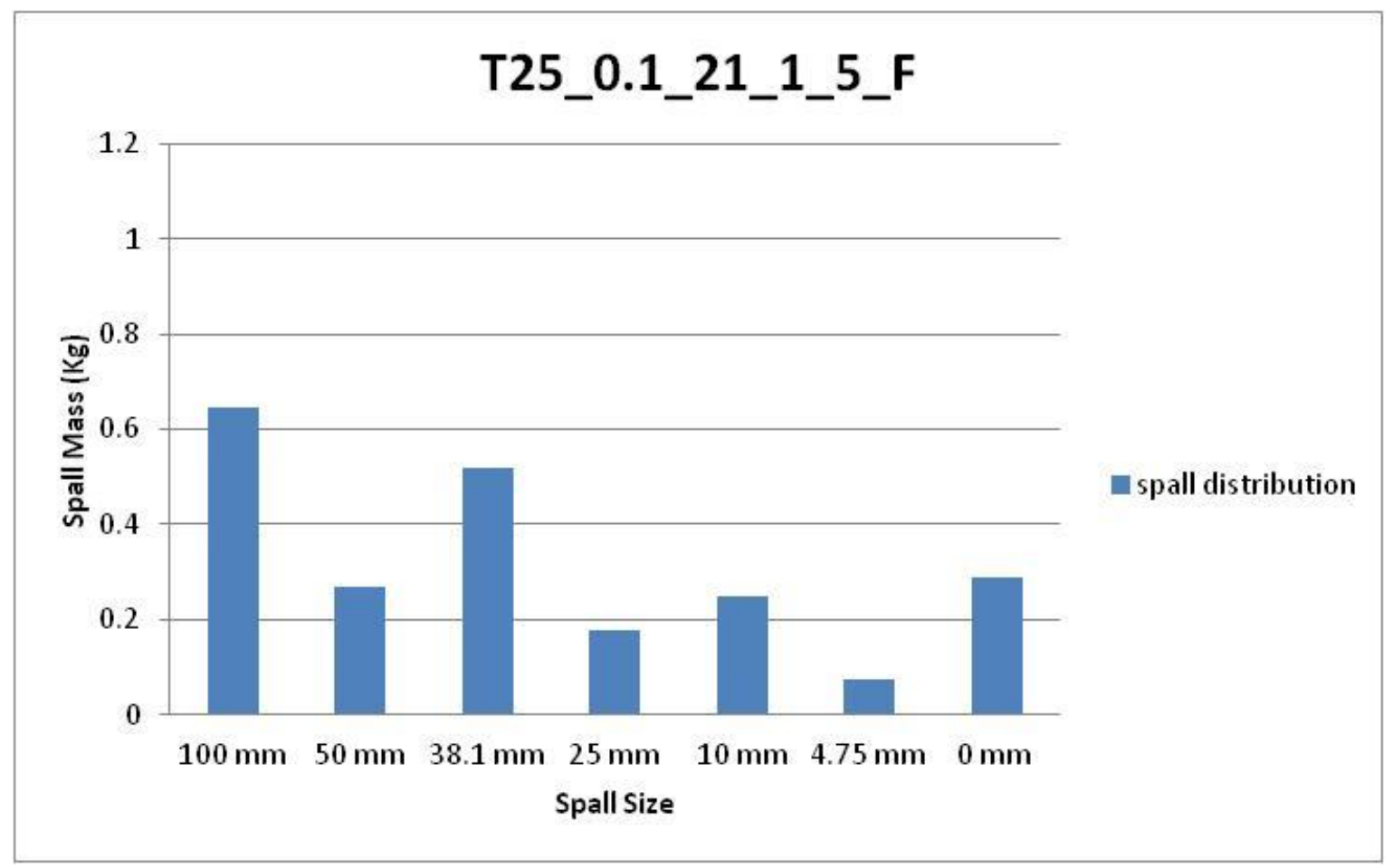

T25_0.1_21_1_5_F: Spall mass distributions
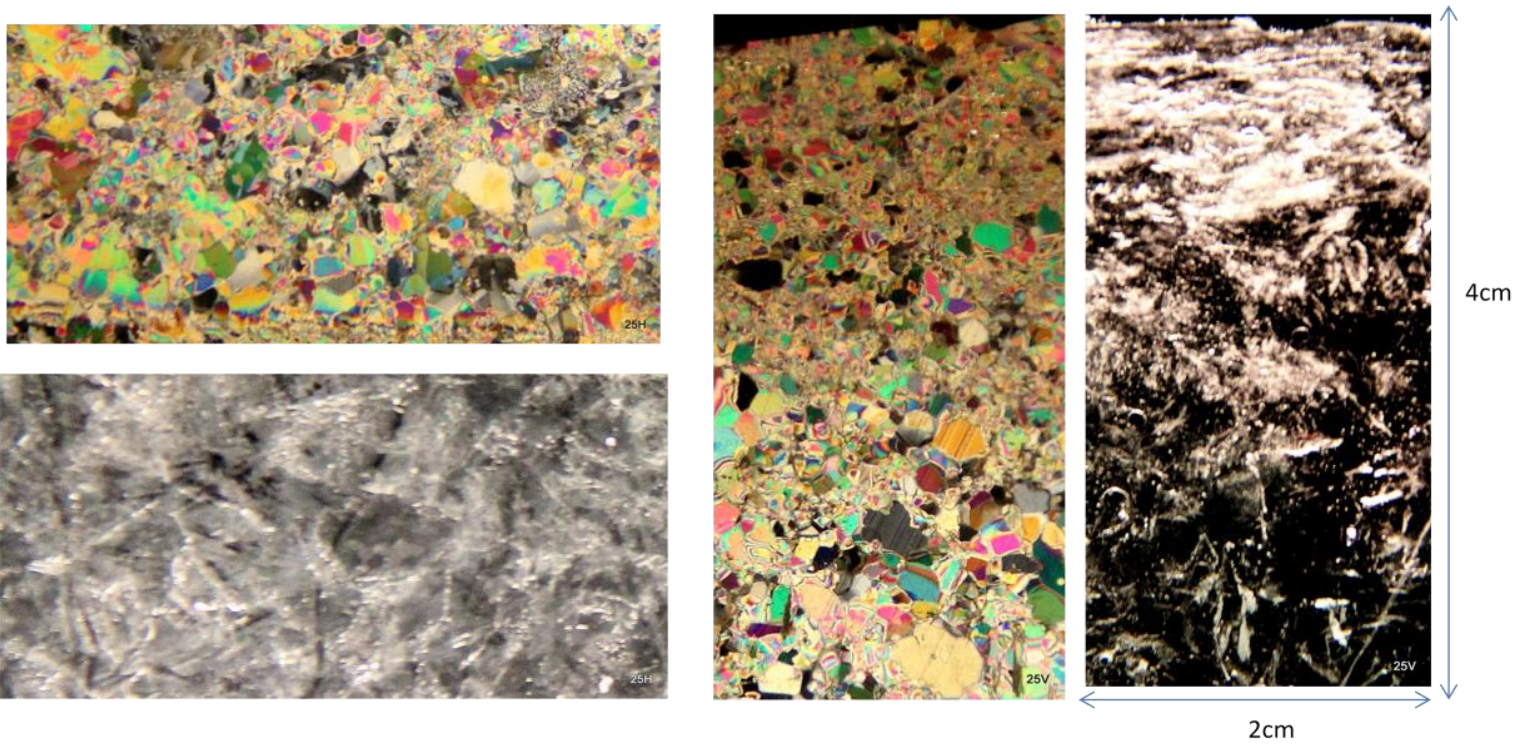

T25_0.1_21_1_5_F: Thin section pictures: Horizontal (left) and Vertical (right) 

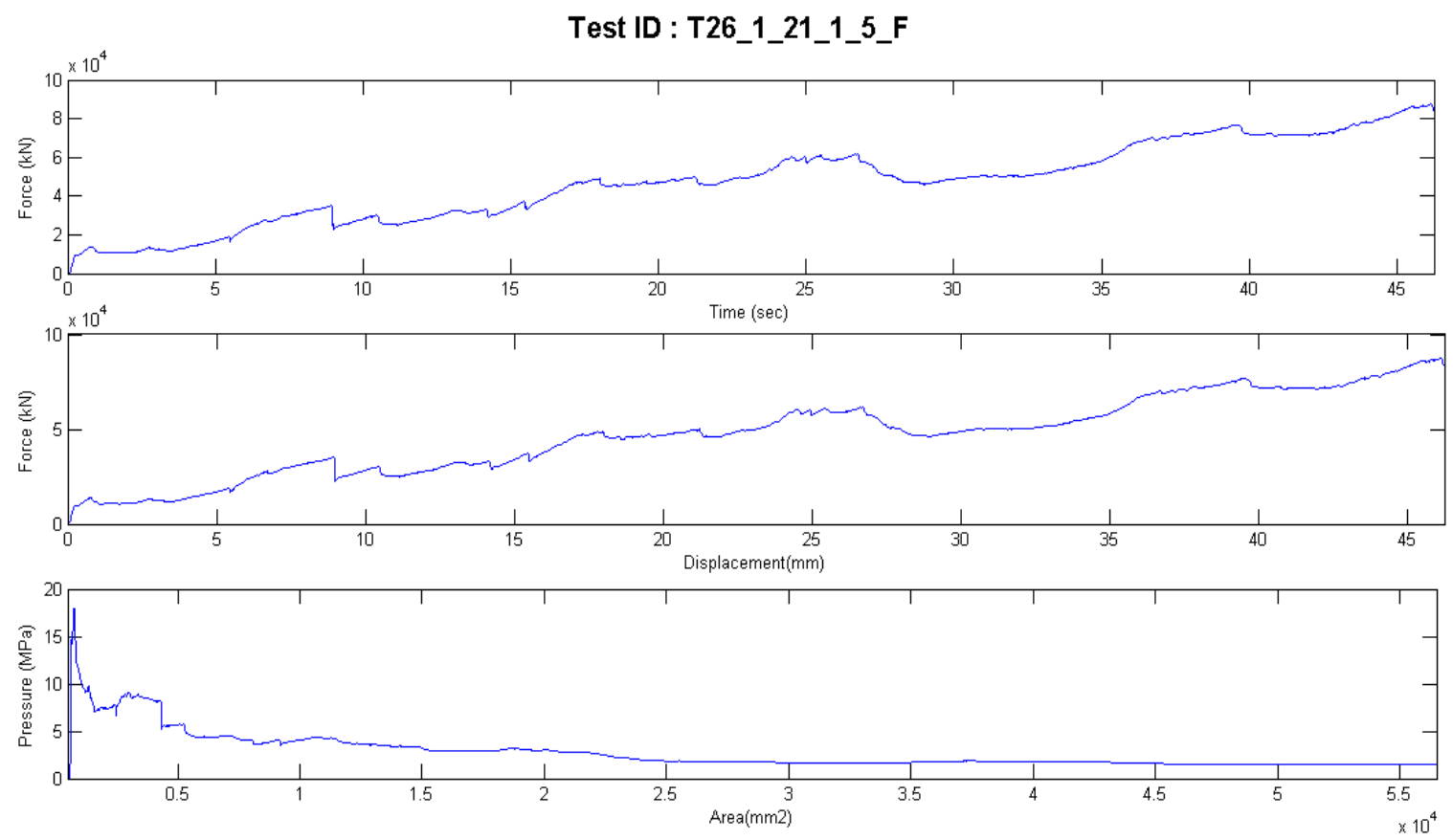

T26_1_21_1_5_F: Force and pressure traces

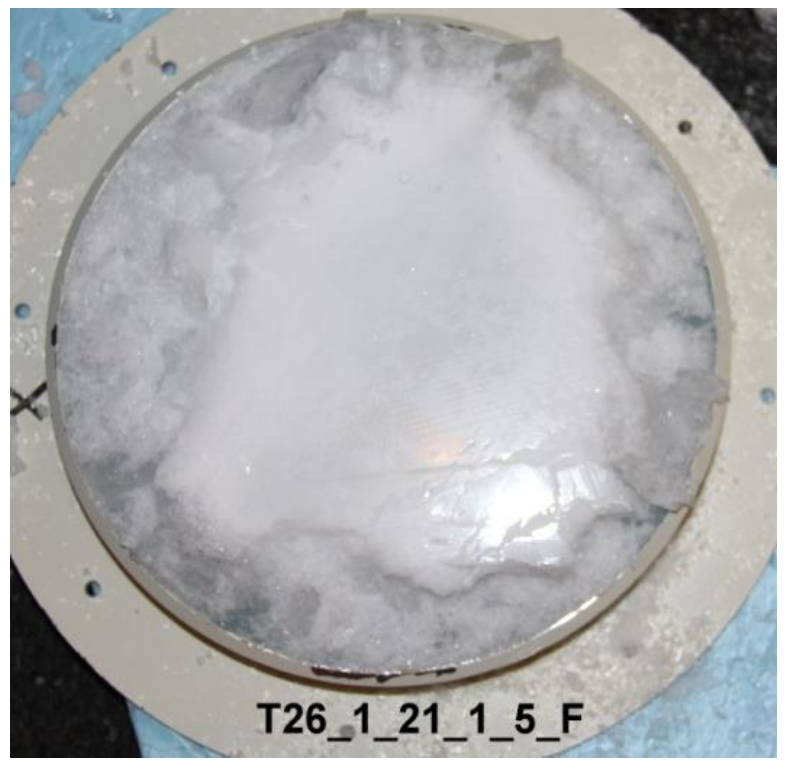

T26_1_21_1_5_F: After crushing picture 


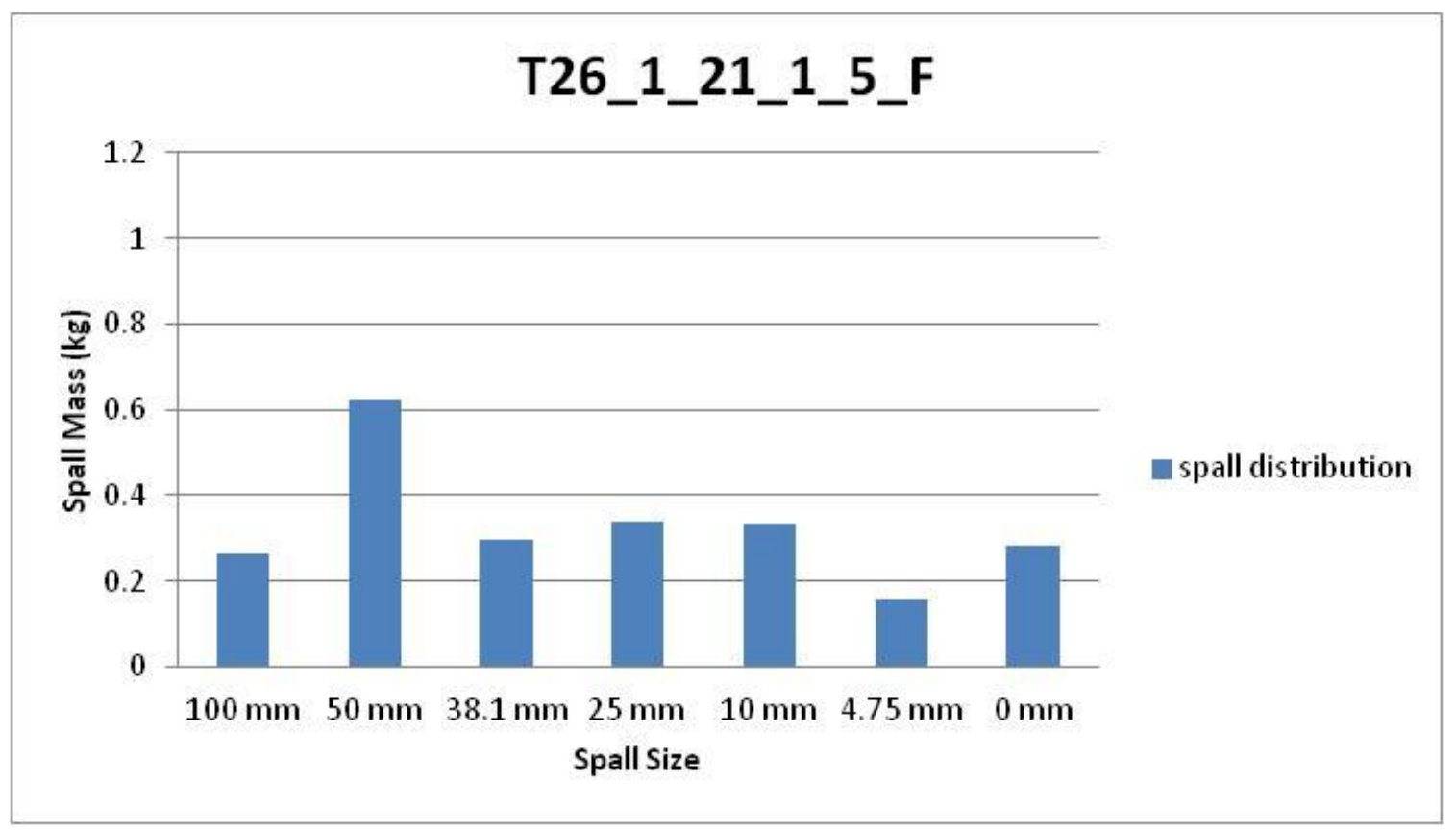

T26_1_21_1_5_F: Spall mass distributions
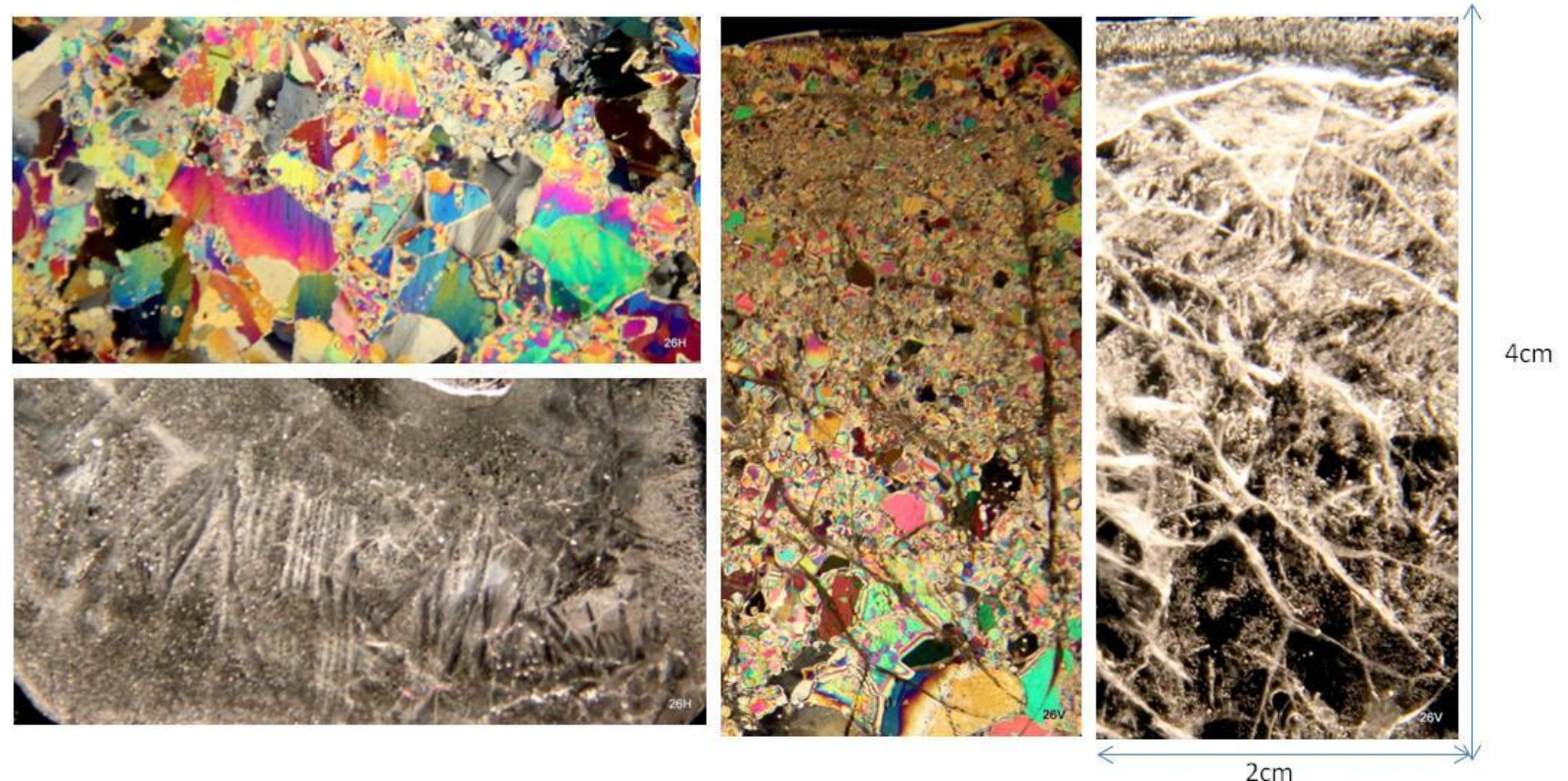

T26_1_21_1_5_F: Thin section pictures: Horizontal (left) and Vertical (right) 

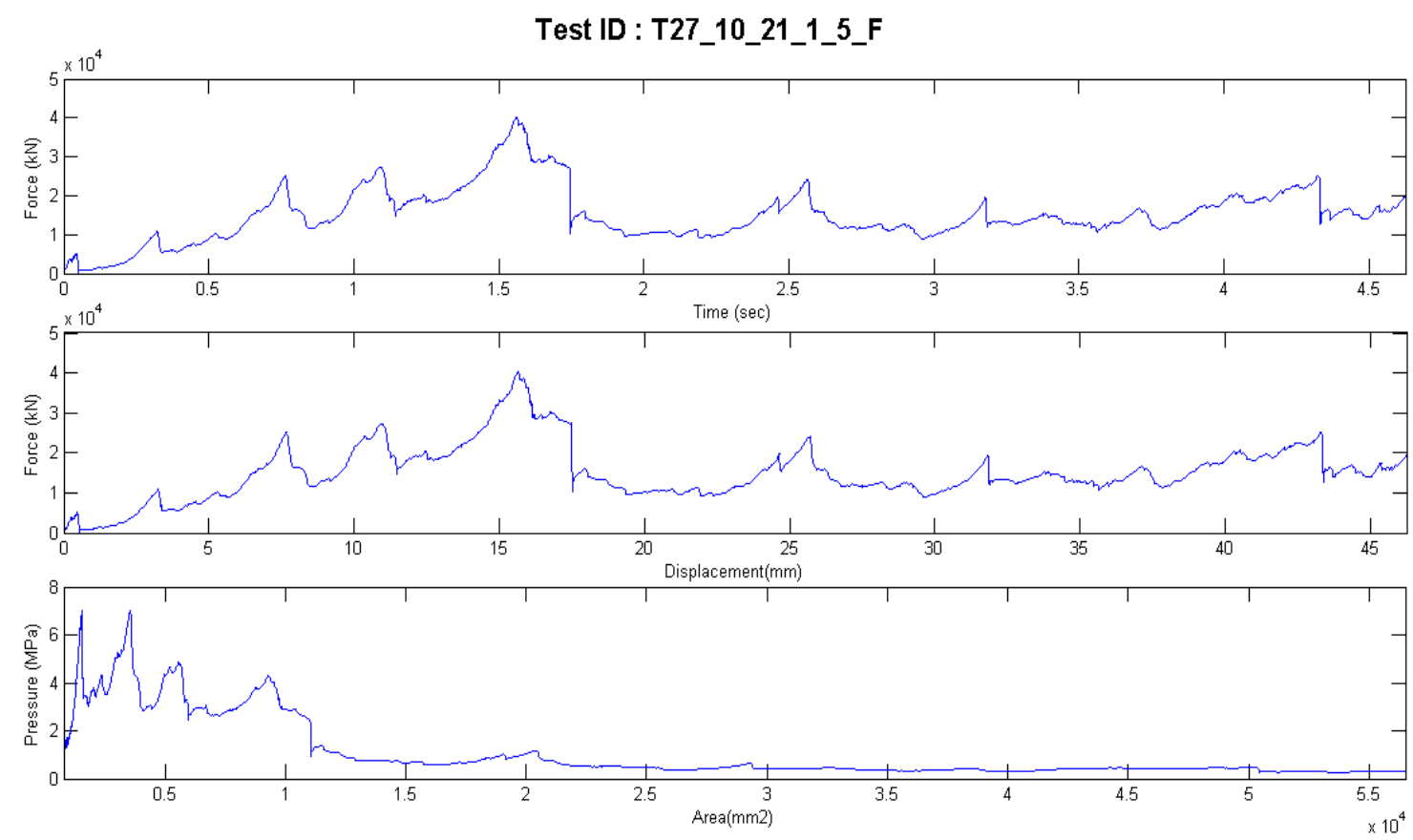

T27_10_21_1_5_F: Force and pressure traces

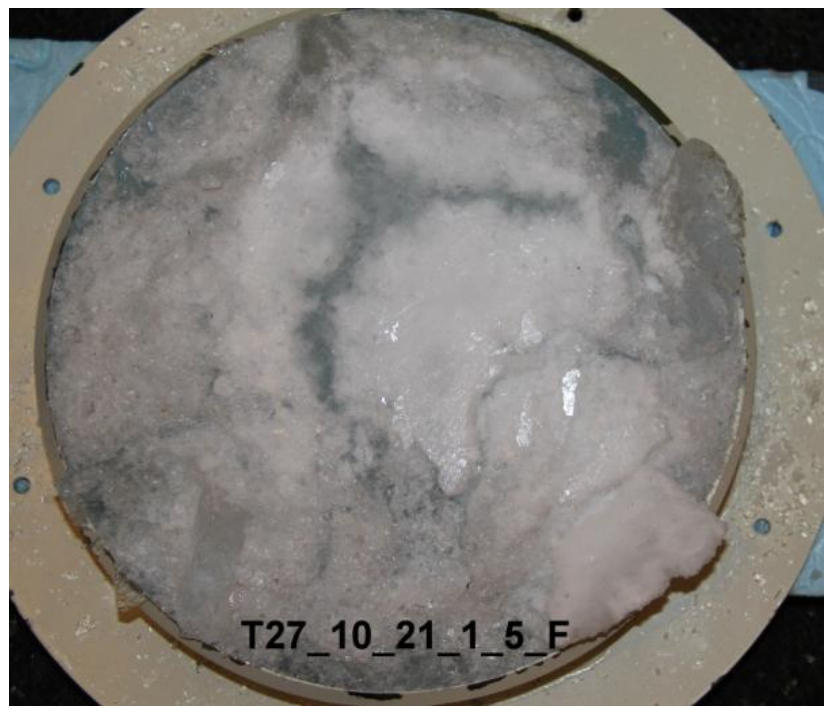

T27_10_21_1_5_F: After crushing picture 


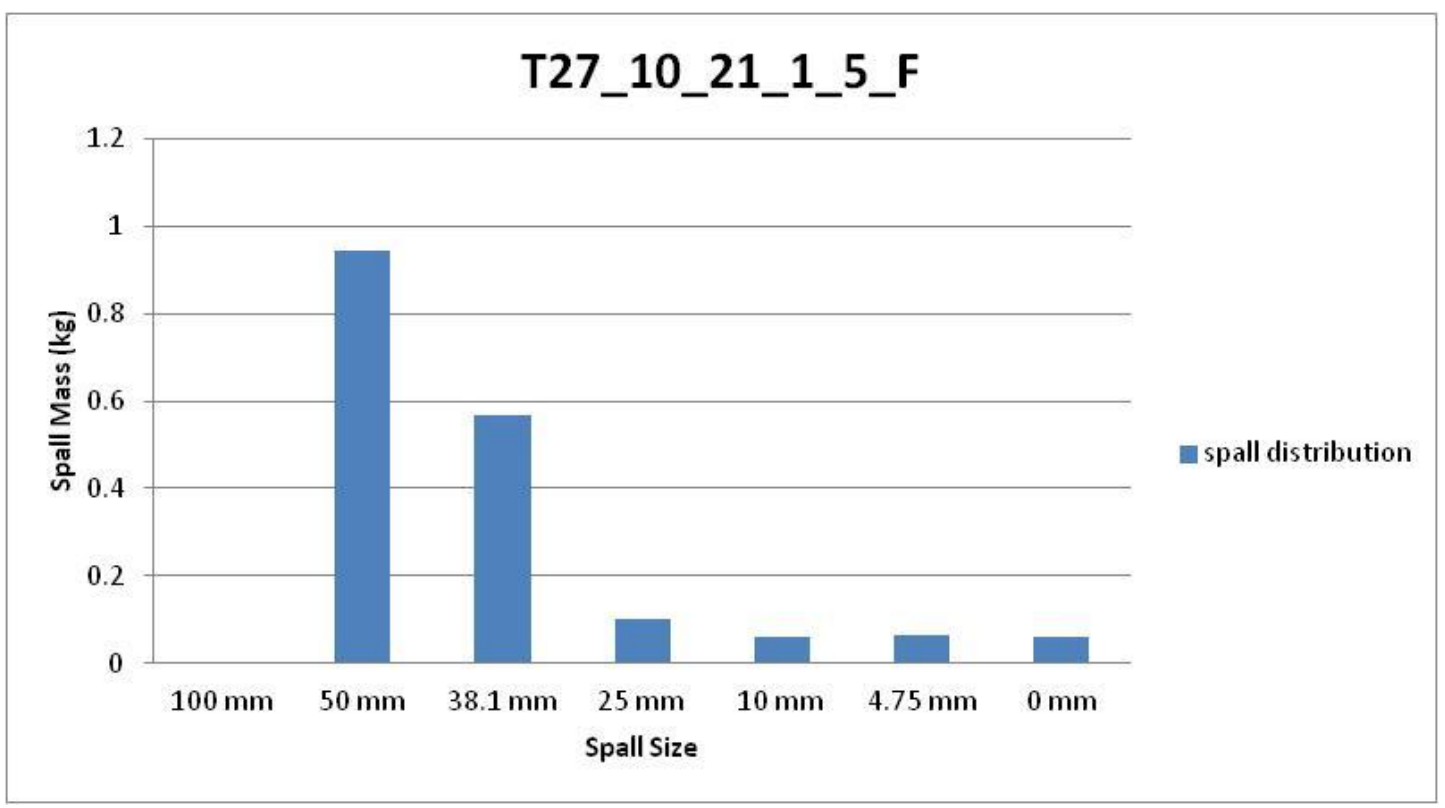

T27_10_21_1_5_F: Spall mass distributions
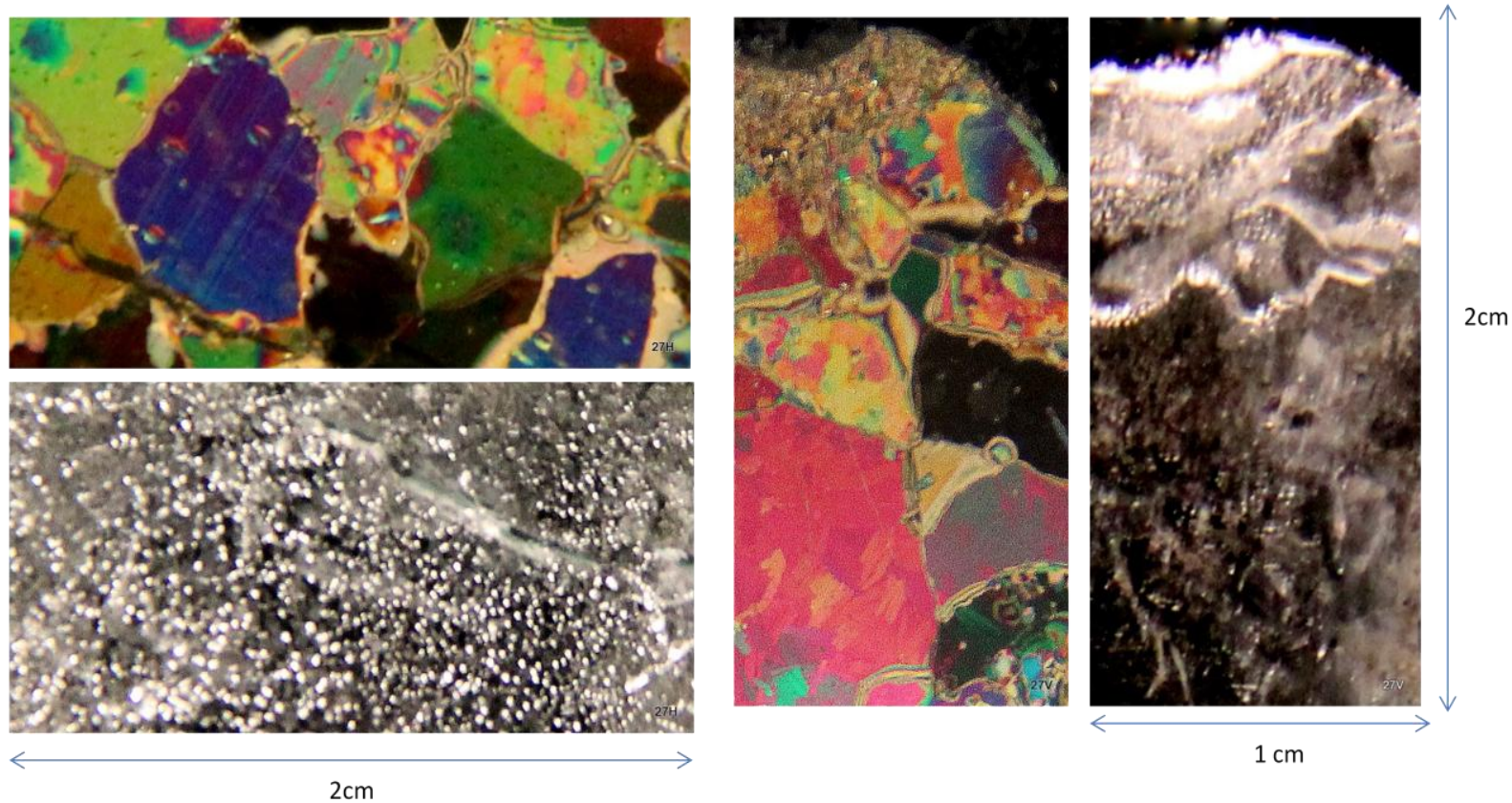

T27_10_21_1_5_F: Thin section pictures: Horizontal (left) and Vertical (right) 
Test ID : T28_10_21_2_5_F
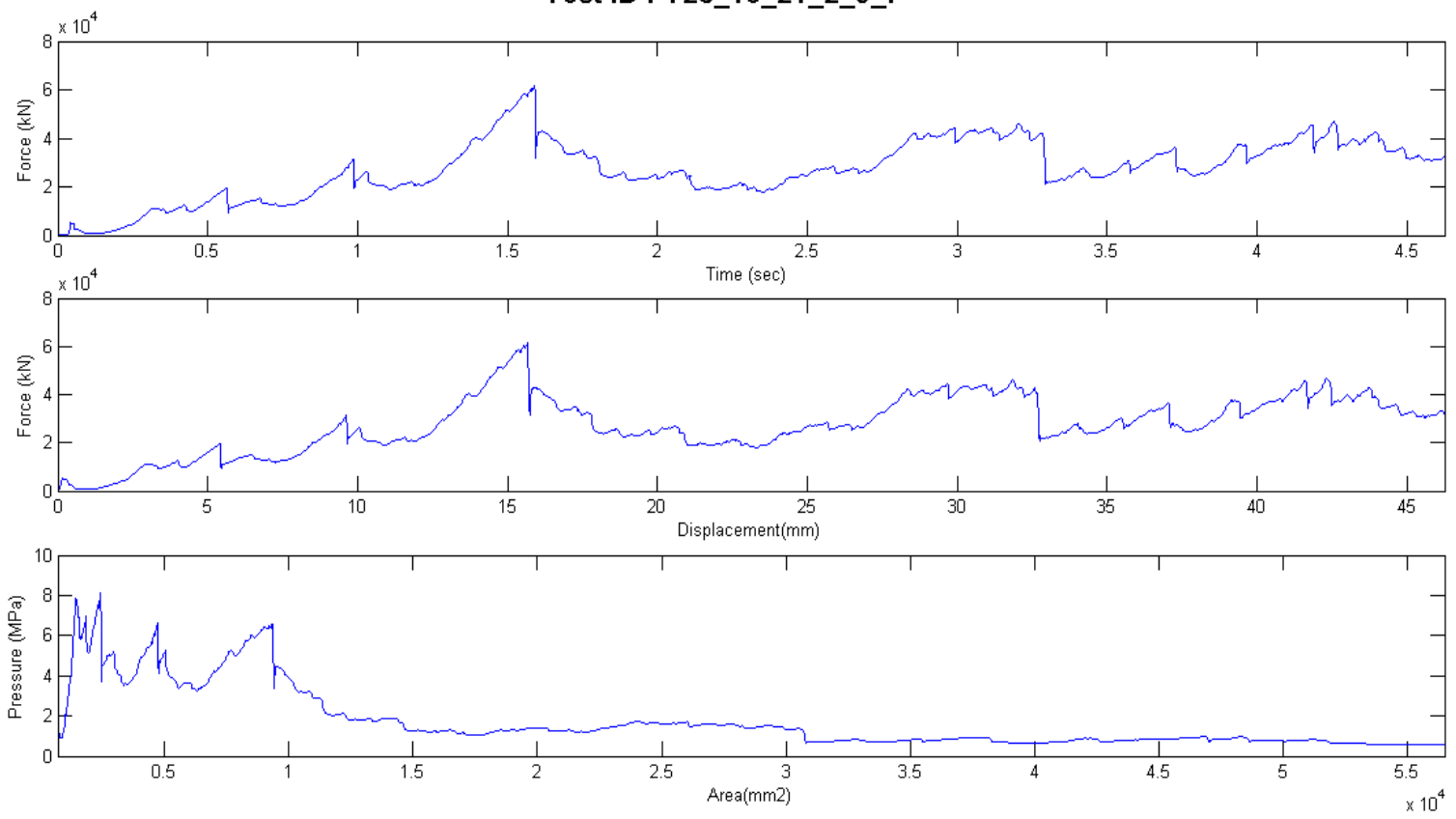

T28_10_21_2_5_F: Force and pressure traces

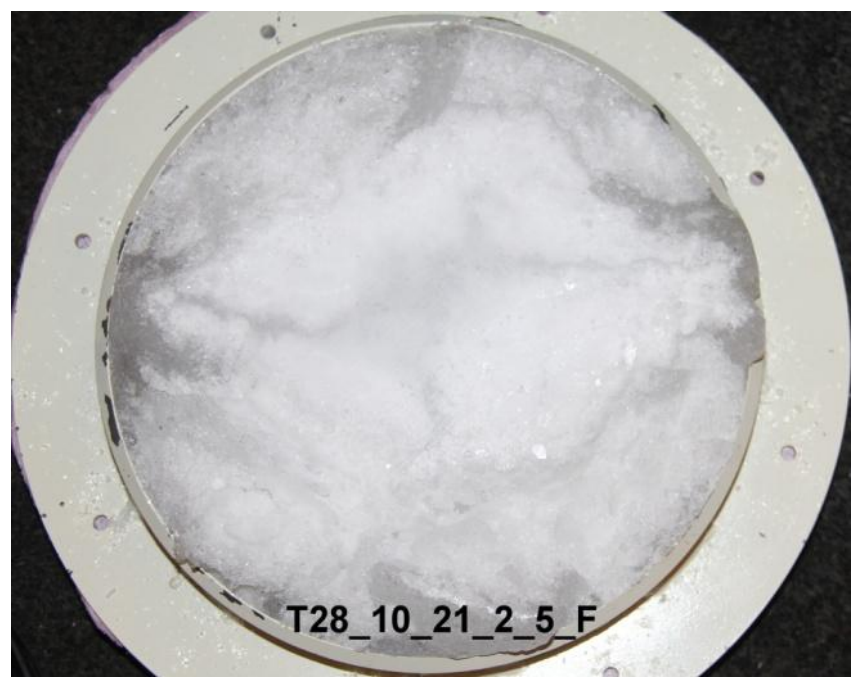

T28_10_21_2_5_F: After crushing picture 


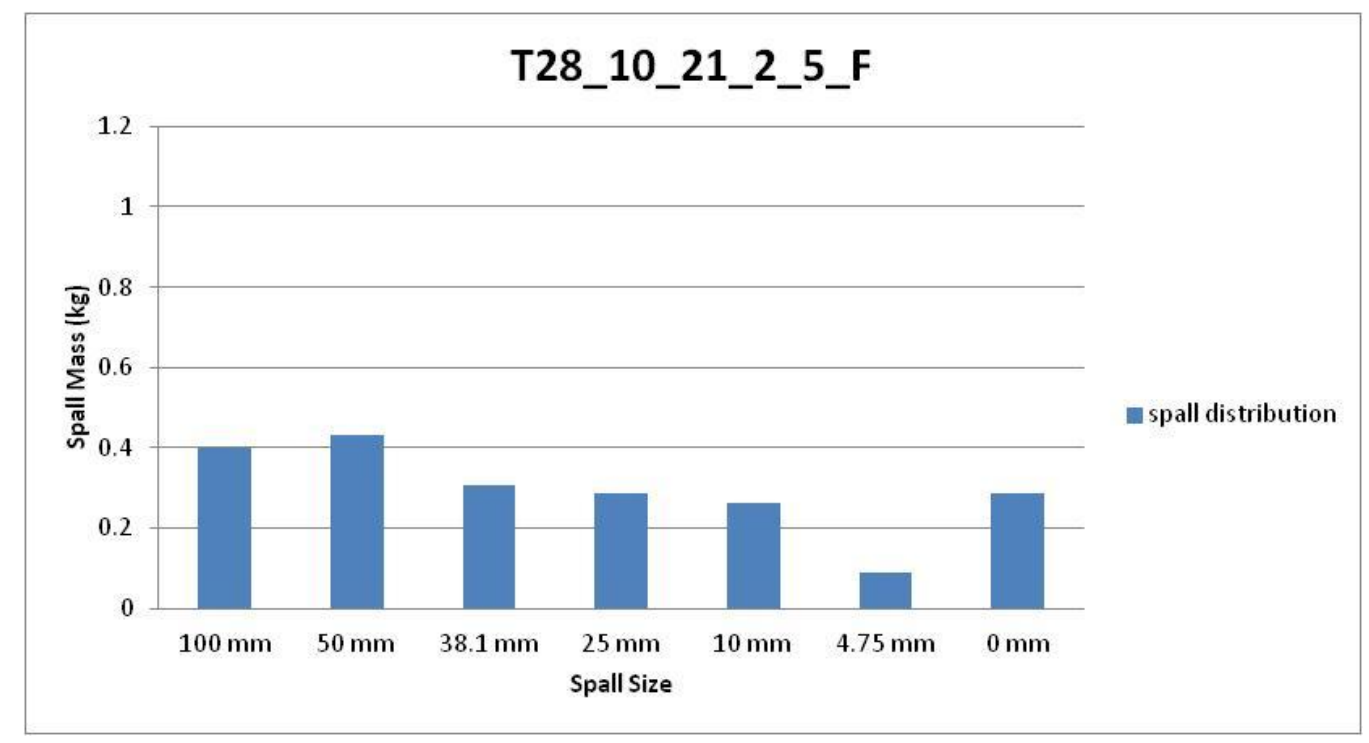

T28_10_21_2_5_F: Spall mass distributions
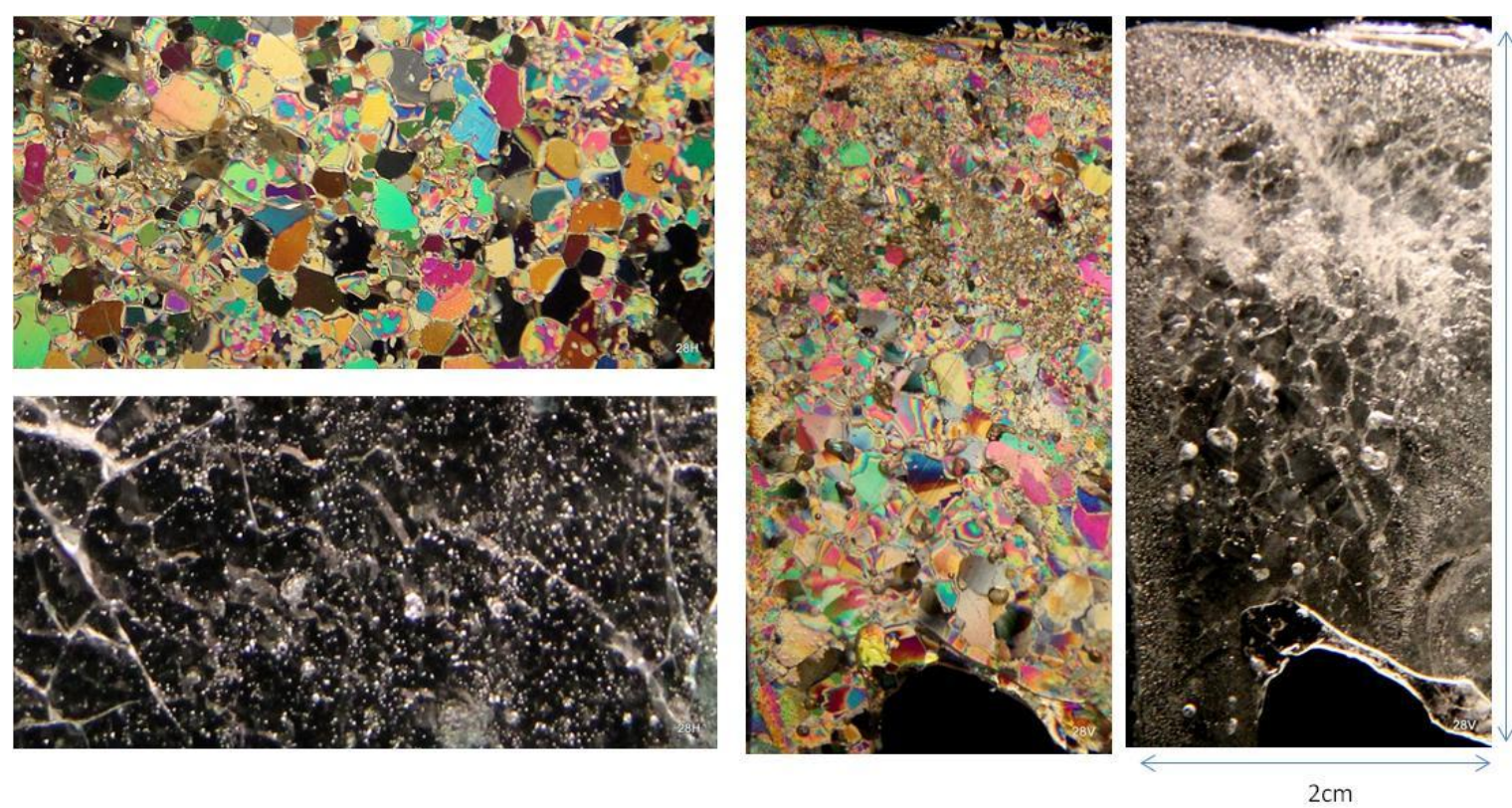

T28_10_21_2_5_F: Thin section pictures: Horizontal (left) and Vertical (right) 


\section{APPENDIX B - Calibrations}




\section{Tekscan I-Scan system}

The Tekscan I-Scan system requires three processes before the testing. The procedures consist of preconditioning, equilibration and calibration. The processes are discussed below.

Preconditioning: New sensors need to precondition to minimize the effects of hysteresis and drift and to improve the repeatability during the experiment. The sensor was uniformly loaded at least $20 \%$ greater than the anticipated load for the tests at the testing temperature. To achieve uniform load over the sensor, it was covered fully by soft cloths on the both sides.

Equilibration: Equilibration is done before the calibration process. It is done applying a uniform pressure to the entire active area of the sensor to compensate the differences within the sensels of a sensor. In the experiments multi load equilibration is used to assure the uniform sensor behaviour over a larger range of pressures. In this process, the sensor was loaded from $50 \mathrm{kN}$ to $450 \mathrm{kN}$ with $50 \mathrm{kN}$ increment each time. The data was recorded and saved.

Calibration: Each sensel of a sensor is an individual force sensor. When the force is applied to the sensors, the impedances of the loaded sensels change. The impedance of the sensel changes from $10 \mathrm{M} \Omega$ with no load to less than $20 \mathrm{~K} \Omega$ with full load. This process correlates the digital output from the sensels to engineering units like force and pressure. The procedure consists of a series of single load calibrations. Single load calibration procedure assumes that with zero applied loads, the sensor has zero output. A 
known force from load cell data is applied for calibration to an area of the sensor. The digital output value and corresponding load value is saved in a calibration file. The graph of digital output of the sensor from varying load is a curve that follows a power law relationship. So, near the calibration point the curve and the straight line is close but the difference increases when it moves to the extremes.

Calibration and tactile sensor information is given below for the selected tests. 
T17 $0.1 \quad 13 \quad 1 \quad 10$ F.fsx Frame 6500 of $6500(11 / 28 / 2012$ 10:47:35.792AM)

Tekscan Pressure Measurement System 7.00-101

SENSOR TYPE 5101

ROWS 44

COLS 44

ROW SPACING 2.54 millimeters

COL SPACING 2.54 millimeters

SENSEL AREA $6.4516 \mathrm{~mm} 2$

NOISE THRESHOLD 3

SECONDS PER FRAME 0.01

MICRO SECOND D

UNITS $\overline{M P a}$

TIME 11/28/2012 10:46:30.829AM

SCALE FACTOR $0.000128467 \mathrm{MPa} / \mathrm{raw}$

EXPONENT 2.48353

SATURATION PRESSURE $121.76 \mathrm{MPa}$ (Exponential Extrapolation)

CALIBRATION_POINT_1 33.7845 (KNewtons) 39071 (Raw Sum) 516 (Number of Loaded Cells)

CALIBRATION MODE 1 Point

CALIBRATION_POINT22 20.51 (KNewtons) 13447 (Raw Sum) 98 (Number of Loaded Cells)

CALIBRATION MODE 2 Point

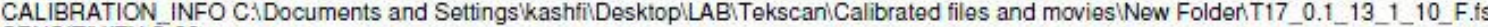
SENSITIVITY S-30

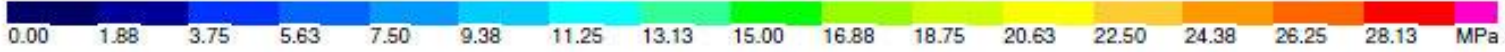

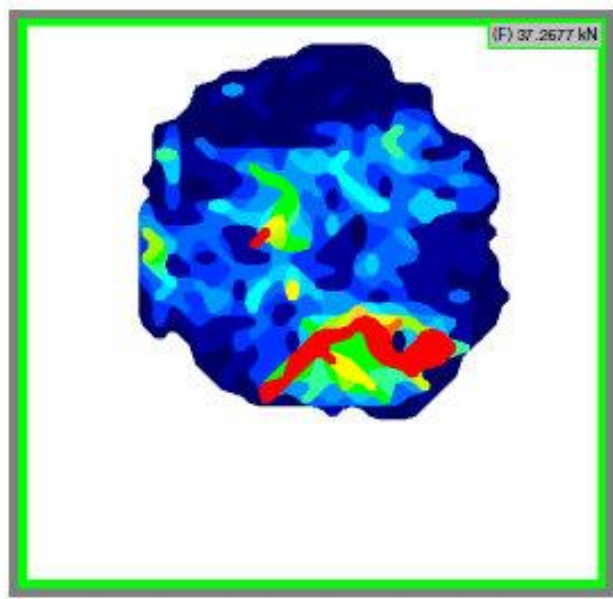

Force vs. Time

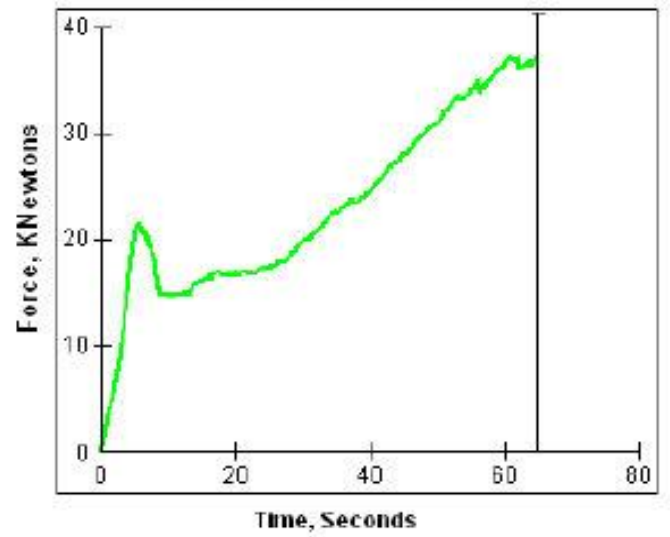

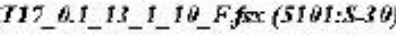

$11 / 28 / 201210: 47: 35.792 \mathrm{AM}$

$64.963 \mathrm{oso}$ (Tims)

$-\mathrm{F}=37.2677 \mathrm{kN}$

Calibration and sensor information for T17_0.1_13_1_10_F 
T19_1_13 1_10 F.fsx Frame 800 of 800 (11/28/2012 1:33-34.821PM)

Tekscan Pressure Measurement System 7.00-101

SENSOR TYPE 5101

ROWS 44

COLS 44

ROW SPACING 2.54 millimeters

COL SPACING 2.54 millimeters

SENSEL AREA $6.4516 \mathrm{~mm} 2$

NOISE THRESHOLD 3

SECONDS PER FRAME 0.01

MICRO SECOND D

UNITS $\mathrm{MPa}$

TIME 11/28/2012 1:33:26.834PM

SCALE FACTOR $0.834859 \mathrm{MPa} / \mathrm{raw}$

EXPONENT 0.597048

SATURATION PRESSURE $22.8262 \mathrm{MPa}$ (Exponential Extrapolation)

CALIBRATION_POINT_1 2.1153 (KNewtons) 1755 (Raw Sum) 52 (Number of Loaded Cells)

CALIBRATION MODE 1 Point

CALIBRATION POINT 239.3805 (KNewtons) 44902 (Raw Sum) 591 (Number of Loaded Cells)

CALIBRATION MODE 2 Point

CALIBRATION INFO C.IDocuments and Settings'kashfi'DesktopiLABITekscaniCalibrated files and moviesiNew FoldeniT19_1_13_1_10_F.fsx SENSITIVITY S-30
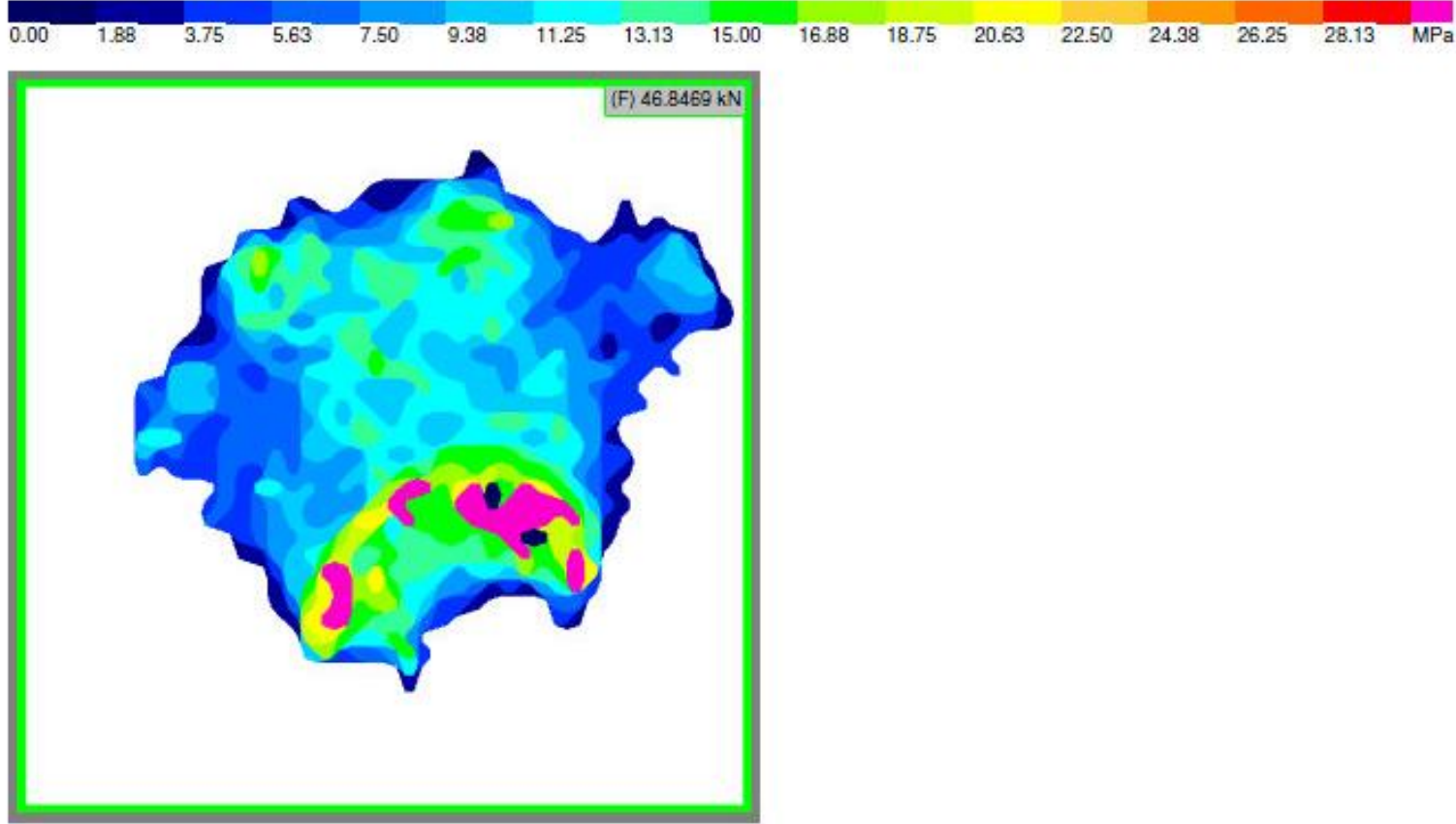

Force vs. Time

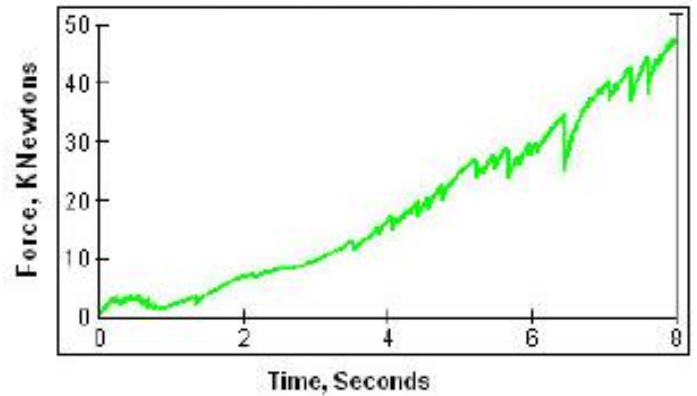

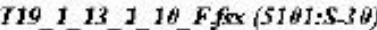

$11 / 28 / 201213934.821 \mathrm{FM}$

$7.987 \mathrm{se0}$ (Time)

$-F=46.8459 \mathrm{kN}$

Calibration and sensor information for T19_1_13_1_10_F 
T20_1_13_2_10_F.fsx Frame 1370 of 1370 (11/28/2012 1:51:39.001PM)

Tekscan Pressure Measurement System 7.00-101

SENSOR TYPE 5101

ROWS 44

COLS 44

ROW SPACING 2.54 millimeters

COL SPACING 2.54 millimeters

SENSEL AREA $6.4516 \mathrm{~mm} 2$

NOISE THRESHOLD 3

SECONDS PER FRAME 0.01

MICRO SECOND D

UNITS MPa

TIME 11/28/2012 1:51:25.316PM

SCALE FACTOR $0.200291 \mathrm{MPa} / \mathrm{raw}$

EXPONENT 0.933305

SATURATION PRESSURE $35.2938 \mathrm{MPa}$ (Exponential Extrapolation)

CALIBRATION POINT 10.9243 (KNewtons) 908 (Raw Sum) 35 (Number of Loaded Cells)

CALIBRATION MODE 1 Point

CALIBRATION_POINT2 44.6401 (KNewtons) 47439 (Raw Sum) 524 (Number of Loaded Cells)

CALIBRATION MODE 2 Point

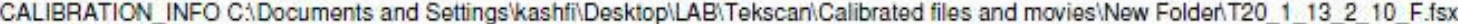
SENSITIVITY S-30

Analysis Type: Max Area Frame

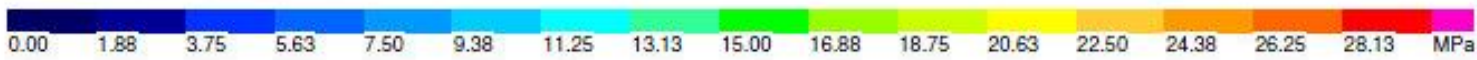

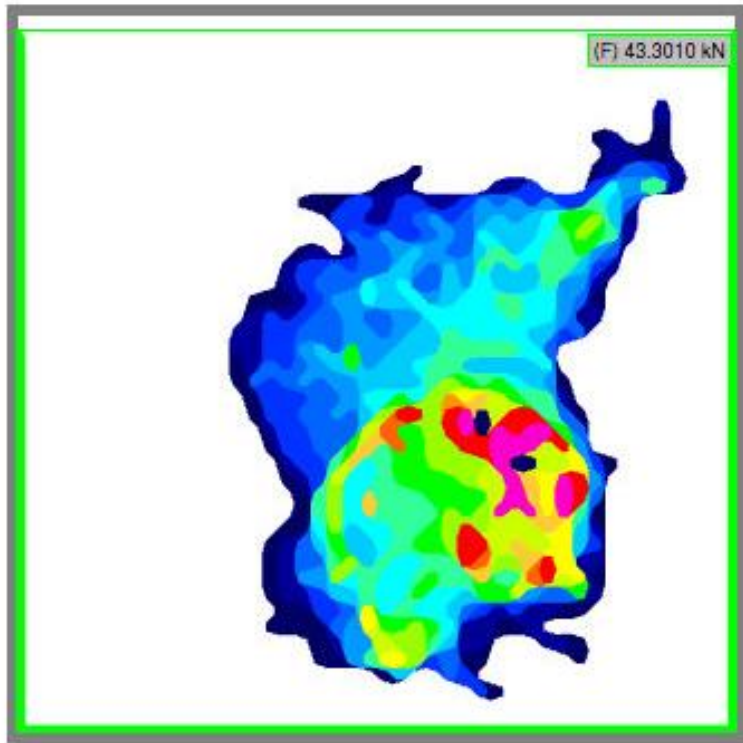

Force vs. Time

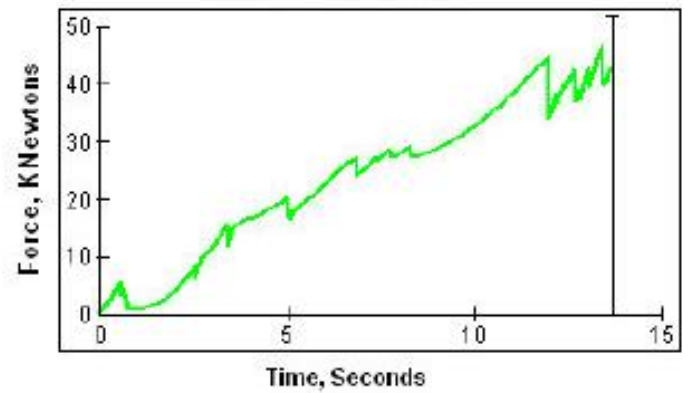

T26_13_2_1t_Ffor $\left(51 \theta 1: S_{-} 3 \theta\right)$

$11 / 28 / 2012151.39 .001 \mathrm{FM}$

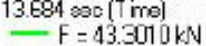

Calibration and sensor information for T20_1_13_2_10_F 
T21_10_13_1_10_F.fsx Frame 112 of 112 (11/28/2012 2:15:16.963PM)

Tekscan Pressure Measurement System 7.00-101

SENSOR TYPE 5101

ROWS 44

COLS 44

ROW SPACING 2.54 millimeters

COL SPACING 2.54 millimeters

SENSEL AREA $6.4516 \mathrm{~mm} 2$

NOISE THRESHOLD 3

SECONDS PER FRAME 0.01

MICRO SECOND 0

UNITS $\mathrm{MPa}$

TIME 11/28/2012 2:15:15.853PM

SCALE FACTOR $0.164738 \mathrm{MPa} / \mathrm{raw}$

EXPONENT 1.00458

SATURATION PRESSURE $43.0879 \mathrm{MPa}$ (Exponential Extrapolation)

CALIBRATION POINT 12.7388 (KNewtons) 2559 (Raw Sum) 54 (Number of Loaded Cells)

CALIBRATION_MODE_ 1 Point

CALIBRATION POINT2 35.0526 (KNewtons) 32333 (Raw Sum) 538 (Number of Loaded Cells)

CALIBRATION MODE 2 Point

CALIBRATION_INFO CIDocuments and Settingsikashfii.DesktopiLABITekscaniCalibrated files and moviesiNew FolderiT21_10_13_1_10_F.fs: SENSITIVITY S-30
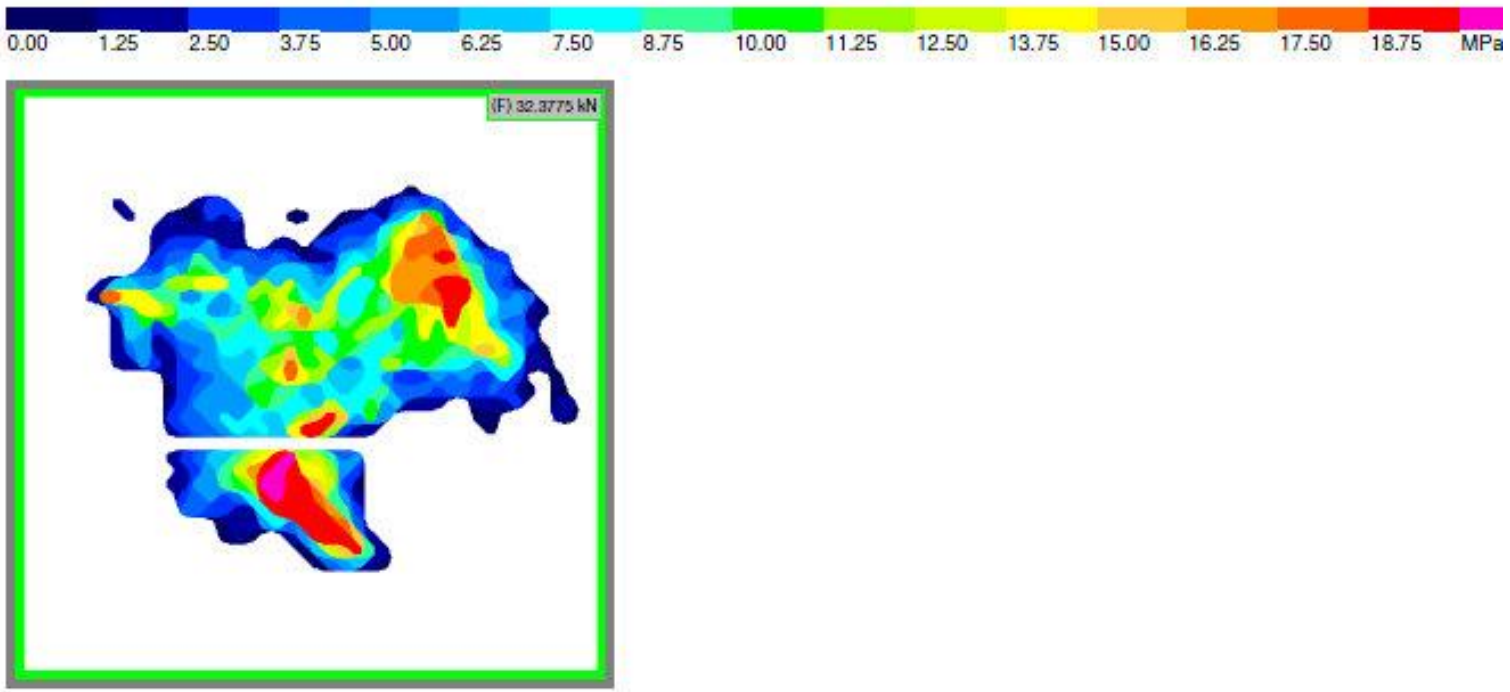

Force vs. Time

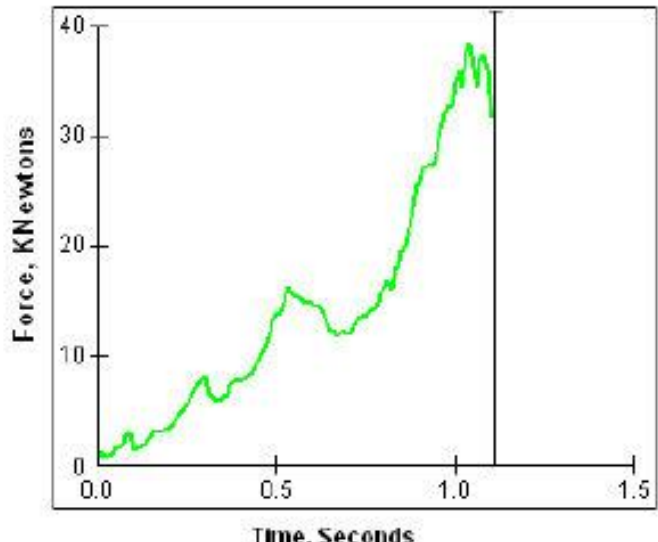

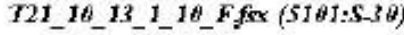

$11 / 28 / 201221516.963 \mathrm{FM}$

$1.11 \mathrm{sec}(\mathrm{T} \mathrm{me})$

$-\mathrm{F}=32.3775 \mathrm{kN}$

Calibration and sensor information for T21_10_13_1_10_F 
T22_10_13_2_10_F.fsx Frame 140 of 140 (1 1/28/2012 2:31:34.196PM)

Tekscan Pressure Measurement System 7.00-101

SENSOR TYPE 5101

ROWS 44

COLS 44

ROW SPACING 2.54 millimeters

COL SPACING 2.54 millimeters

SENSEL AREA $6.4516 \mathrm{~mm} 2$

NOISE THRESHOLD 3

SECONDS PER FRAME 0.01

MICRO SECOND O

UNITS $\mathrm{MPa}$

TIME 11/28/2012 2:31:32.807 PM

SCALE_FACTOR $0.0941507 \mathrm{MPa} /$ raw

EXPONENT 1.16019

SATURATION PRESSURE $58.3267 \mathrm{MPa}$ (Exponential Extrapolation)

CALIBRATION POINT 10.7197 (KNewtons) 697 (Raw Sum) 33 (Number of Loaded Cells)

CALIBRATION_MODE 1 Point

CALIBRATION_POINT2 19.9358 (KNewtons) 16031 (Raw Sum) 285 (Number of Loaded Cells)

CALIBRATION MODE 2 Point

CALIBRATION INFO CIDocuments and Settings'kashfilDesktop:LABITekscaniCalibrated files and moviesiNew Folden'T22_10_13_2_10_F.fs: SENSITIVITY S-30

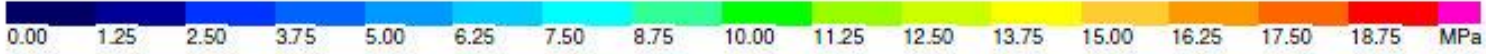

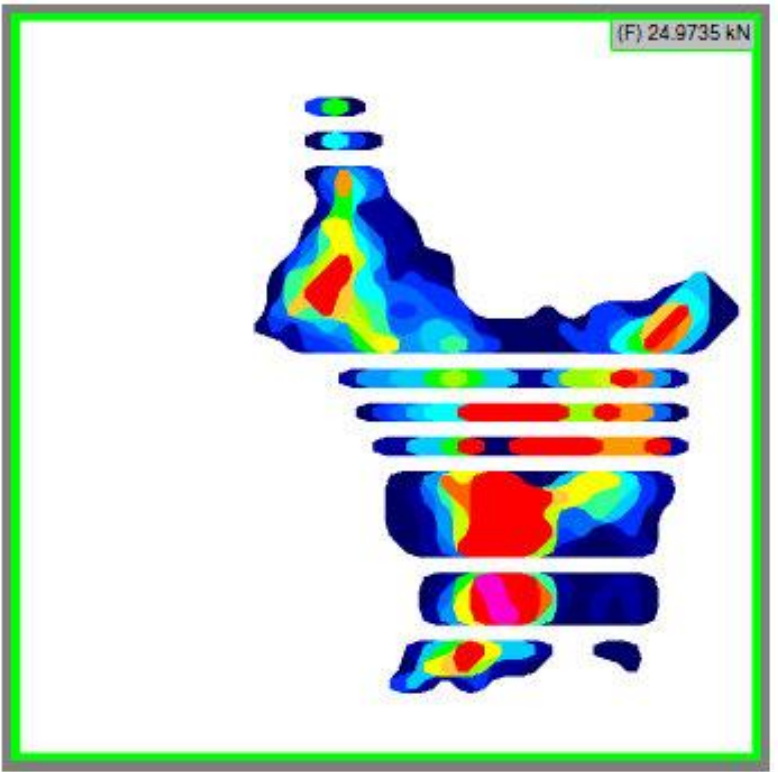

Force vs. Time

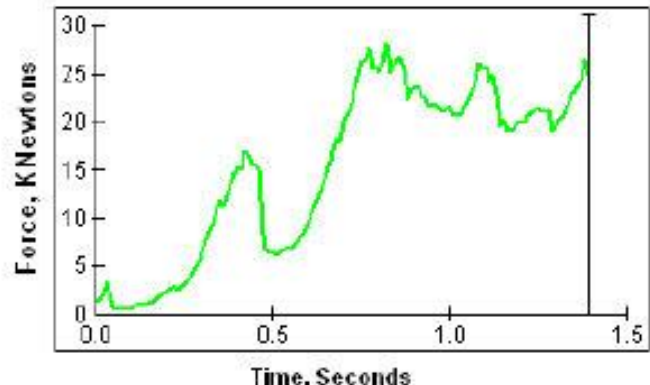

T22 10 13_2 10 Ffox (SIA1:S-3 $\theta)$

$11 / 28 / 2012231.34 .196 \mathrm{EM}$

$1.389 \mathrm{sec}$ (Time)

$-\mathrm{F}=24.9735 \mathrm{kN}$

Calibration and sensor information for T22_10_13_2_10_F 
T25 0.121 1 5 F.fsx Frame 9616 of 9616 (11/27/2012 2:31:34.869PM)

Tekścan Pressure Measurement System 7.00-101

SENSOR TYPE 5101

ROWS $44 j$

COLS 44

AOW SPACING 2.54 millimeters

COL SPACING 2.54 millimeters

SENSEL AREA $6.4516 \mathrm{~mm} 2$

NOISE THRESHOLD 3

SECONDS PER FRAME 0.01

MICRO SECOND 0

UNITS $\overline{M P a}$

TIME 11/27/2012 2-29:32.36PM

SATURATION PRESSURE $81.6868 \mathrm{MPa}$ ('Piece-wise' Linear Extrapolation)

CALIBRATION POINT 117.571 (KNewtons) 15158 (Raw Sum) 216 (Number of Loaded Cells)

CALIBRATION_MODE_ 1 Point

CALIBRATION_POINT_2 47.8206 (KNewtons) 76822 (Raw Sum) 1079 (Number of Loaded Cells)

CALIBRATION MODE 2 Point

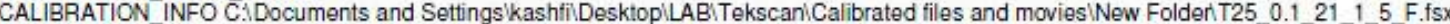
SENSITIVITY S-30

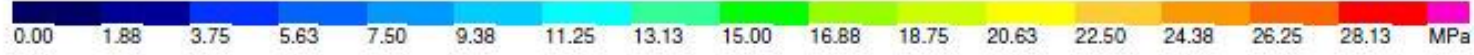

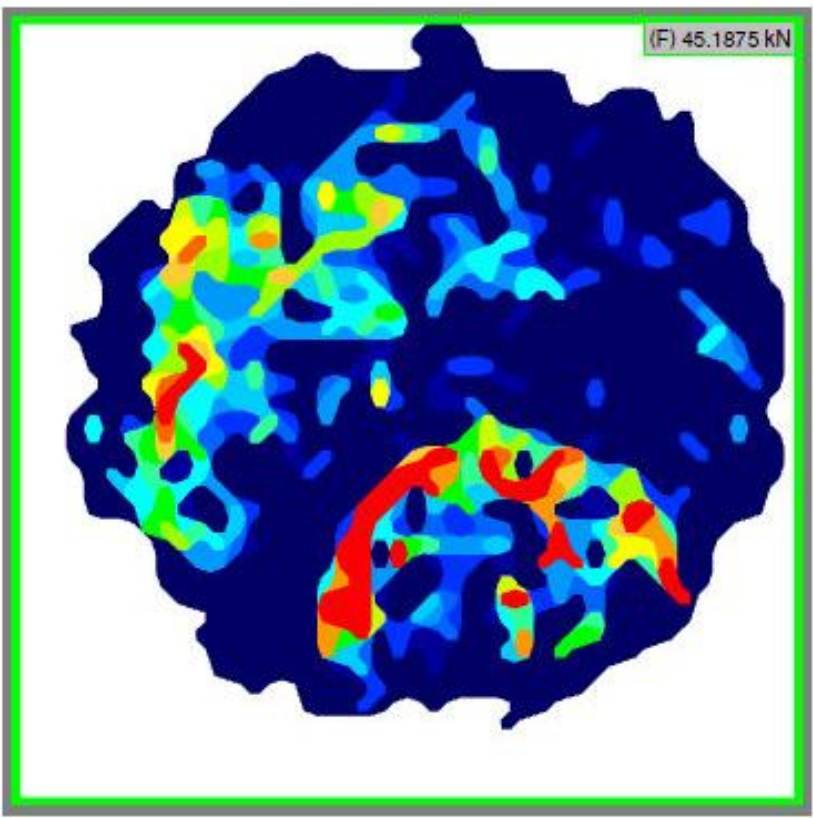

Force vs. Time

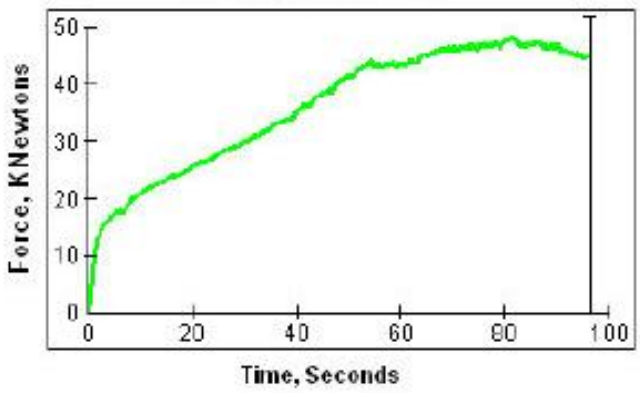

T25_0.I_2I_I_S_F.fs $(51 \theta 1: S-30)$

$11 / 27 / 2012233134.8699 \mathrm{M}$

$96.11 \mathrm{sec}[$ [Time]

$-\mathrm{F}=45.1875 \mathrm{kN}$

Calibration and sensor information for T25_0.1_21_1_5_F 
T26_1_21_1_5_F.fsx Frame 1420 of 1420 (11/27/2012 3:05-37.587PM)

Tekscan Pressure Measurement System 7.00-101

SENSOR TYPE 5101

ROWS 44

COLS 44

ROW SPACING 2.54 millimeters

COL SPACING 2.54 millimeters

SENSEL AREA $6.4516 \mathrm{~mm} 2$

NOISE THRESHOLD 3

SECONDS PER FRAME 0.01

MICRO SECOND 0

UNITS $\mathrm{MPa}$

TIME 11/27/2012 3:05:23.402PM

SCALE FACTOR $0.355835 \mathrm{MPa} / \mathrm{raw}$

EXPONENT 0.771662

SATURATION PRESSURE $25.6026 \mathrm{MPa}$ (Exponential Extrapolation)

CALIBRATION_POINT_1 23.3655 (KNewtons) 27537 (Raw Sum) 434 (Number of Loaded Cells)

CALIBRATION_MODE_ 1 Point

CALIBRATION_POINT_2 32.39 (KNewtons) 38760 (Raw Sum) 553 (Number of Loaded Cells)

CALIBRATION MODE 2 Point

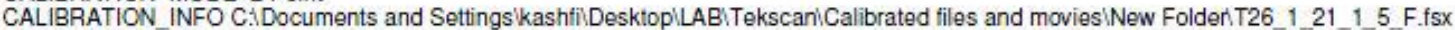
SENSITIVITY S-30
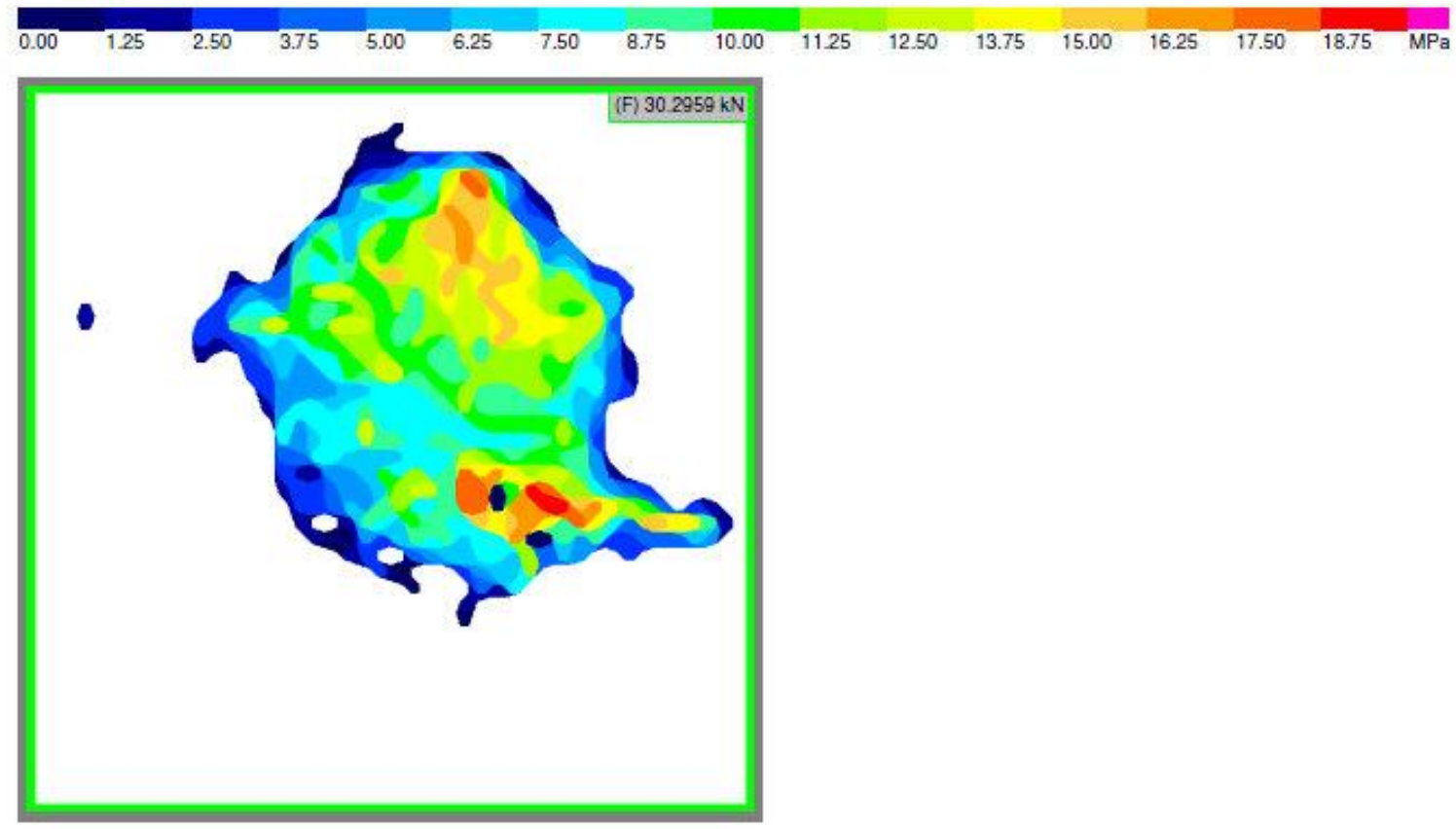

Force vs. Time

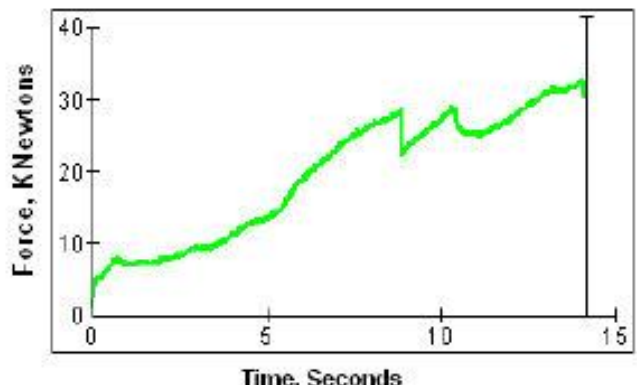

r26_1_21_1_S_Ffor $(S \operatorname{sit}: \mathrm{S}-3 \theta)$

$11 / 27 / 201230537.587 \mathrm{FN}$

$14.184 \sec ($ Timel

$-F=30.2959 \mathrm{kN}$

Calibration and sensor information for T26_1_21_1_5_F 
T27_10_21_1_5_F.fsx Frame 140 of 140 (11/27/2012 3:22:40.287PM)

Tekścan Pressure Measurement System 7.00-101

SENSOR TYPE 5101

ROWS 44

COLS 44

ROW SPACING 2.54 millimeters

COL SPACING 2.54 millimeters

SENSEL AREA $6.4516 \mathrm{~mm} 2$

NOISE THRESHOLD 3

SECONDS PER FRAME 0.01

MICRO SECOND O

UNITS MPa

TIME 11/27/2012 3-22:38.897PM

SCALE FACTOR $0.637187 \mathrm{MPa} / \mathrm{raw}$

EXPONENT 0.616891

SATURATION PRESSURE $19.4464 \mathrm{MPa}$ (Exponential Extrapolation)

CALIBRATION POINT 11.0385 (KNewtons) 944 (Raw Sum) 38 (Number of Loaded Cells)

CALIBRATION MODE 1 Point

CALIBRATION POINT 218.9793 (KNewtons) 23297 (Raw Sum) 435 (Number of Loaded Cells)

CALIBRATION MODE 2 Point

CALIBRATION INFO CIDocuments and Settings'kashfiiDesktopiLABITekscaniCalibrated files and moviesiNew FolderiT27_10_21_1_5_F.fsx SENSITIVITY S-30

Analysis Type: Max Area Frame

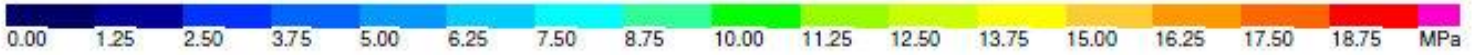

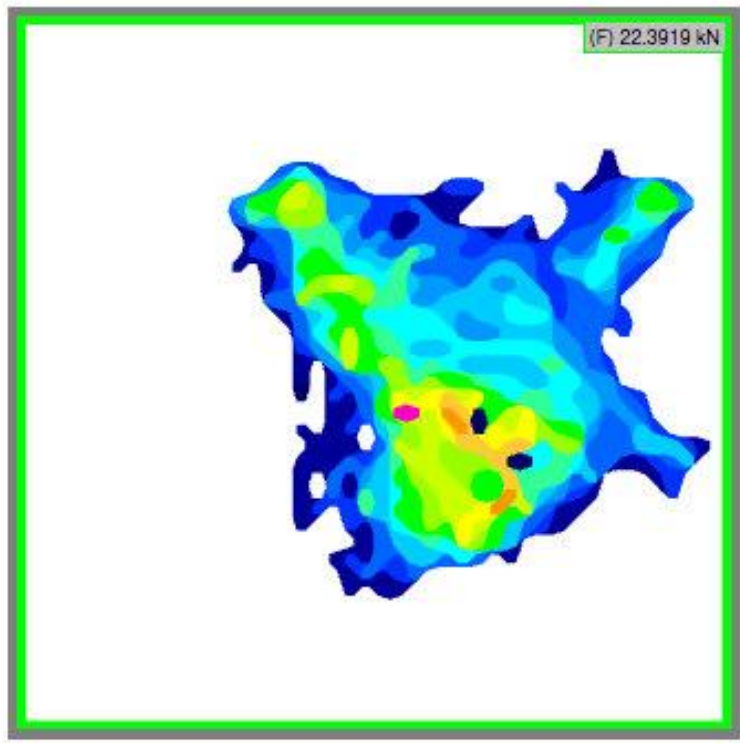

Force vs. Time

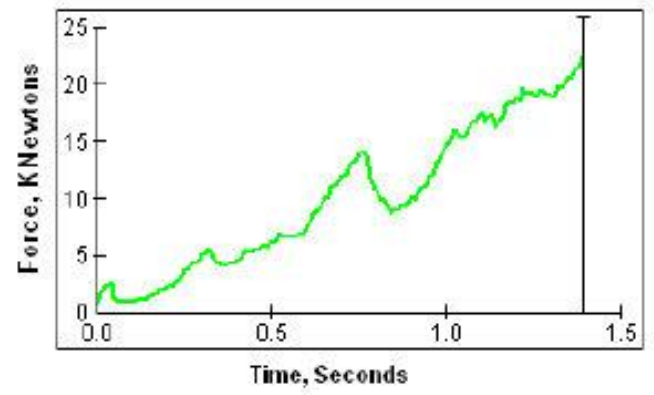

T25_10_21_1_S_Ffox $(51 \theta 1: \mathrm{S}-3 \theta)$

$11 / 27 / 201232240.287 \mathrm{FM}$

$1.389 \mathrm{sec}$ (Time)

$\mathrm{F}=22.3919 \mathrm{kN}$

Calibration and sensor information for T27_10_21_1_5_F 
T28 10212 5 F.fsx Frame 127 of 127 (11/27/2012 3:38:29.602PM)

Tekscan Pressure Measurement System 7.00-101

SENSOR TYPE 5101

ROWS 44

COLS 44

ROW_SPACING 2.54 millimeters

COL SPACING 2.54 millimeters

SENSEL AREA $6.4516 \mathrm{~mm} 2$

NOISE THRESHOLD 3

SECONDS PER FRAME 0.01

MICRO SECOND 0

UNITS $\mathrm{MPa}$

TIME 11/27/2012 3:38:28.342PM

SCALE FACTOR $0.522834 \mathrm{MPa} / \mathrm{raw}$

EXPONENT 0.738612

SATURATION PRESSURE $31.323 \mathrm{MPa}$ (Exponential Extrapolation)

CALIBRATION_POINT_1 0.809501 (KNewtons) 605 (Raw Sum) 26 (Number of Loaded Cells)

CALIBRATION MODE 1 Point

CALIBRATION_POINT2 21.19 (KNewtons) 18823 (Raw Sum) 362 (Number of Loaded Cells)

CALIBRATION MODE 2 Point

CALIBRATION INFO CIDocuments and Settings'kashti:DesktopiLAB TekscaniCalibrated files and moviesiNew Folden T28 102125 F fsX SENSITIVITY S-30

Analysis Type: Max Area Frame

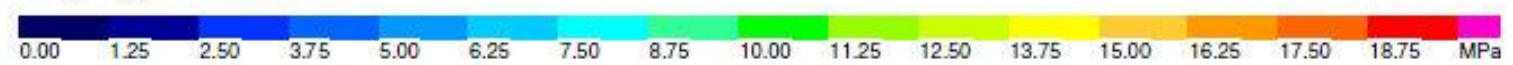

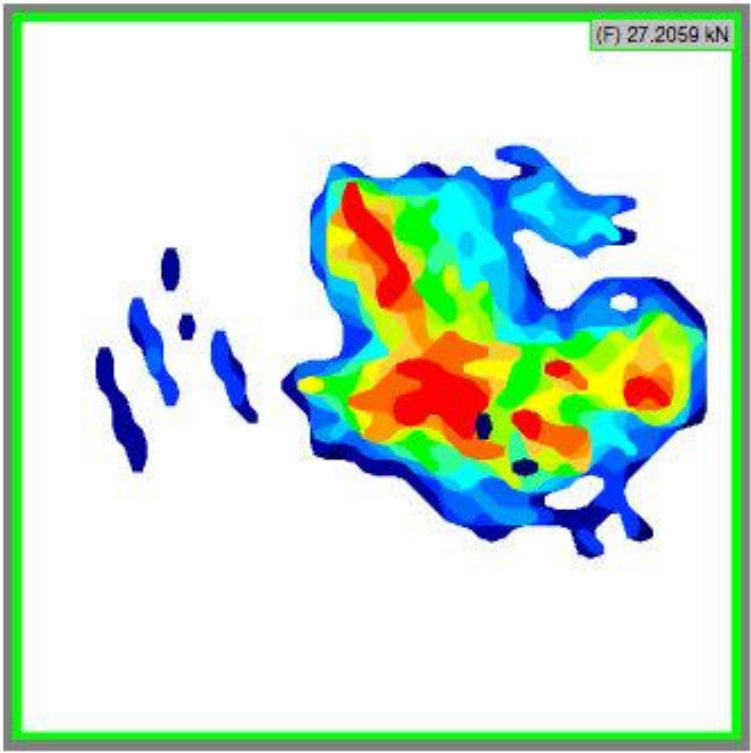

Force vs. Time

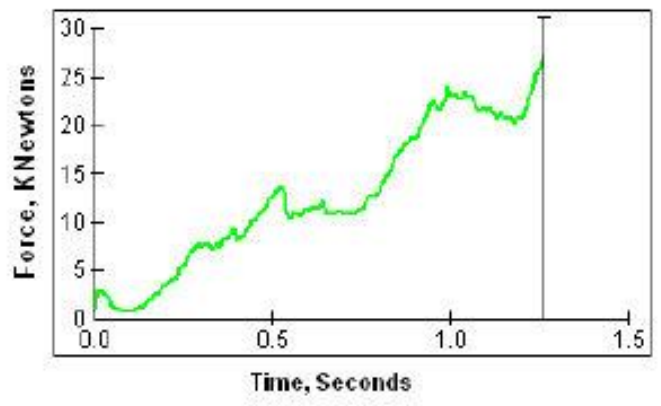

T28_10_21_2_S_Ffor $\left(51 \theta 1: S_{-} 3 \theta\right)$

$11 / 27 / 201233029.602 \mathrm{FM}$

$1.259 \mathrm{sec}$ (Time)

$-\mathrm{F}=27.2069 \mathrm{kN}$

Calibration and sensor information for T28_10_21_2_5_F 